Field Evaluation of Hopper Dredge Overflow for the Delaware River

Jerry L. Miller, Michael R. Palermo, and Thomas W. Groff
July 2002 
The contents of this report are not to be used for advertising, publication, or promotional purposes. Citation of trade names does not constitute an official endorsement or approval of the use of such commercial products.

The findings of this report are not to be construed as an official Department of the Army position, unless so designated by other authorized documents. 


\section{Field Evaluation of Hopper Dredge Overflow for the Delaware River}

by Jerry L. Miller, Michael R. Palermo

Environmental Laboratory

U.S. Army Engineer Research and Development Center

3909 Halls Ferry Road

Vicksburg, MS 39180-6199

Thomas W. Groff

Operations Division

U.S. Army Engineer District, Philadelphia

100 Penn Square East

Philadelphia, PA 19107-3390

Final report

Approved for public release; distribution is unlimited

Prepared for U.S. Army Engineer District, Philadelphia

Philadelphia, PA 19107-3390 


\section{Contents}

Preface ........................... vi

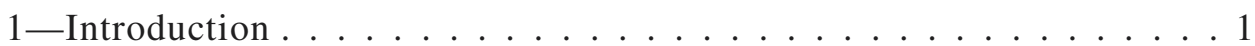

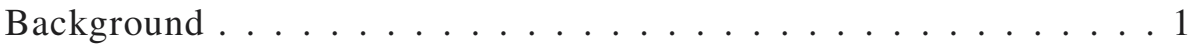

Study Location . . . . . . . . . . . . . . . . . 2

Purpose and Scope . . . . . . . . . . . . . . . . 4

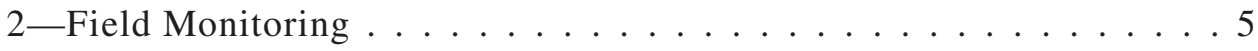

Dredging Equipment and Sampling Operations . . . . . . . . 5

Dredge Operation Variables . . . . . . . . . . . . . . 5

Collection of In Situ Sediment and Site Water . . . . . . . . . . 5

Hopper Inflow Monitoring . . . . . . . . . . . . . 6

Hopper Contents Monitoring . . . . . . . . . . . . . . 6

Hopper Overflow Monitoring . . . . . . . . . . . . 6

Plume Monitoring . . . . . . . . . . . . . . . . 7

Sedimentation Assessment . . . . . . . . . . . . 8

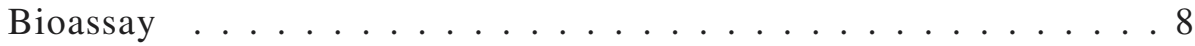

3-Data Analysis . . . . . . . . . . . . . . . . . 9

Hopper Loading Characteristics . . . . . . . . . . . . . . 9

Coarse-grained site . . . . . . . . . . . . 9

Fine-grained site . . . . . . . . . . . . 10

Economics ....................... 10

In Situ Sediment and Background Water Samples . . . . . . . . . 11

Coarse-grained site . . . . . . . . . . . . . 11

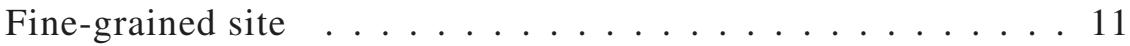

Hopper Inflow . . . . . . . . . . . . . . . . . . 17

Coarse-grained site . . . . . . . . . . . . . . . 17

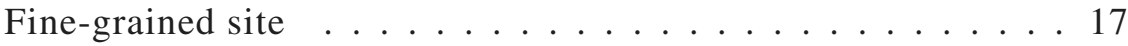

Hopper Contents . . . . . . . . . . . . . . . 17

Coarse-grained site . . . . . . . . . . . . . 17

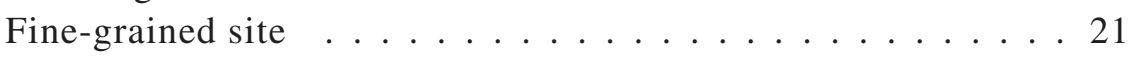

Hopper Overflow . . . . . . . . . . . . . . . 22

Coarse-grained site ................... 22 


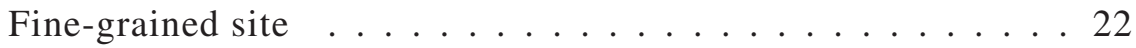

Plume Monitoring . . . . . . . . . . . . . . . . 27

Coarse-grained site . . . . . . . . . . . . 27

Fine-grained site . . . . . . . . . . . . 30

Sedimentation Results . . . . . . . . . . . . . . . 30

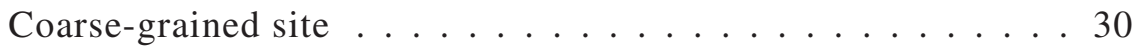

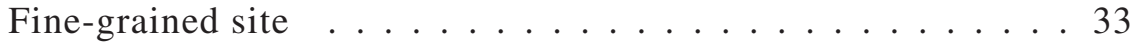

Standard Elutriate Tests . . . . . . . . . . . . . . 33

Coarse-grained site . . . . . . . . . . . . . 34

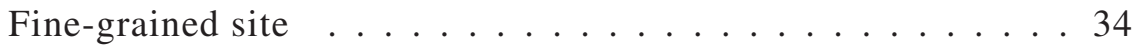

Technical Findings of a 96-hr Water Column Bioassay . . . . . 34

Coarse-grained site . . . . . . . . . . . . . 39

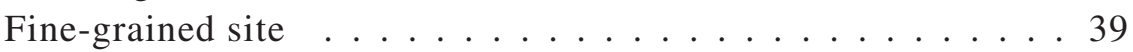

4 -Summary and Conclusions . . . . . . . . . . . . . . 40

Appendix A: Delaware River Sediment and Water Quality Analysis (Coarse- and Fine-Grained Sites) . . . . . . . A1

Appendix B: Plume Study Field Activities and Data Results . . . . . B1

Appendix C: Detection of Short-Term Sedimentation During Hopper Dredging Operations in Delaware Bay and the Delaware River . . . . . . . . . . . . C 1

Appendix D: Summary of Technical Findings: 96-hr Bioassay with Mysidopsis bahia and Menidia beryllina . . . . . . . D1

SF 298

\section{List of Figures}

Figure 1. Dredge McFarland ...............

Figure 2. Locations of the lower and upper hopper dredge overflow test study sites . . . . . . . . . . . . 3

Figure 3. Hopper loading at coarse-grained site . . . . . . . . . 9

Figure 4. Hopper loading at fine-grained site . . . . . . . . 11

Figure 5. Range of gradation curves from in situ sediment collected at the coarse-grained site . . . . . . . . 12

Figure 6. Range of gradation curves from in situ sediment collected at the fine-grained site . . . . . . . 18

Figure 7. Range of gradation curves from hopper inflow at the coarse-grained site . . . . . . . . . . . 19 
Figure 8. Range of gradation curves from hopper inflow at the fine-grained site . . . . . . . . . 20

Figure 9. Hopper contents-solids concentrations of coarse-grained material . . . . . . . . . . . 21

Figure 10. Hopper contents-solids concentrations of fine-grained material . . . . . . . . . . . . . 21

Figure 11. Range of gradation curves from hopper overflow at the coarse-grained site ............. 23

Figure 12. Range of gradation curves from hopper overflow composites at the coarse-grained site . . . . . . . 24

Figure 13. Range of gradation curves from hopper overflow at the fine-grained site . . . . . . . . . . 25

Figure 14. Range of gradation curves from hopper overflow composites at the fine-grained site . . . . . . . 26

Figure 15. Plume solids concentrations at surface . . . . . . . 28

Figure 16. Plume solids concentrations at middepth . . . . . . . 28

Figure 17. Plume solids concentrations at bottom . . . . . . . . . 29

Figure 18. Plume solids concentrations at coarse-grained site . . . . 29

Figure 19. Plume solids concentrations at surface . . . . . . . . 31

Figure 20. Plume solids concentrations at middepth . . . . . . . 31

Figure 21. Plume solids concentrations at bottom . . . . . . . . 32

Figure 22. Plume solids concentrations at fine-grained material . . . 32

\section{List of Tables}

Table 1 Delaware River Coarse- and Fine-Grained Loading Data . 10

Table 2 Delaware River Coarse-Grained Site, Summary of Sediment and Water Quality Data .......... 13

Table 3 Delaware River Fine-Grained Site, Summary of Sediment and Water Quality Data . . . . . . . . . 35 


\section{Preface}

This report describes the potential economic benefits and potential environmental effects from overflow dredging in the lower Delaware River. This work was conducted by the Environmental Laboratory (EL), U.S. Army Engineer Research and Development Center (ERDC), Vicksburg, MS. Funding for the study was provided by the U.S. Army Engineer District, Philadelphia.

This report was written by Mr. Jerry L. Miller, Ecological Resources Branch, Ecosystem Evaluation and Engineering Division (EEED), EL, Dr. Michael R. Palermo, Environmental Processes and Engineering Division (EPED), EL, and Mr. Thomas W. Groff, Operations Division, U.S. Army Engineer District, Philadelphia. Technical review of this report was provided by Messrs. Thomas R. Patin and Jerry J. Pasquale.

This study was conducted under the direct supervision of Dr. Michael F. Passmore, Chief, Ecological Resources Branch, Dr. Dave J. Tazik, Chief, EEED, and under the general supervision of Dr. Edwin A. Theriot, Director, EL.

At the time of publication of this report, Dr. James R. Houston was Director of ERDC, and COL John W. Morris III, EN, was Commander and Executive Director.

This report should be cited as follows:

Miller, J. L., Palermo, M. R., and Groff, T. W. (2002). "Field evaluation of hopper dredge overflow for the Delaware River," ERDC/EL TR-02-17, U.S. Army Engineer Research and Development Center, Vicksburg, MS.

The contents of this report are not to be used for advertising, publication, or promotional purposes. Citation of trade names does not constitute an official endorsement or approval of the use of such commercial products. 


\section{Introduction}

\section{Background}

The U.S. Army Engineer District (USAED), Philadelphia, has an extensive navigation responsibility throughout the Delaware River Basin. Maintenance dredging averages about $3,000,000 \mathrm{~m}^{3}\left(4,000,000 \mathrm{yd}^{3}\right)$ of material annually of which about $191,000 \mathrm{~m}^{3}\left(250,000 \mathrm{yd}^{3}\right)$ is removed by the Hopper Dredge McFarland (Figure 1). The dredging provides a safe navigation channel, which supports the shipping of nearly 136,000,000 metric tons $(150,000,000$ short tons) of cargo per year.

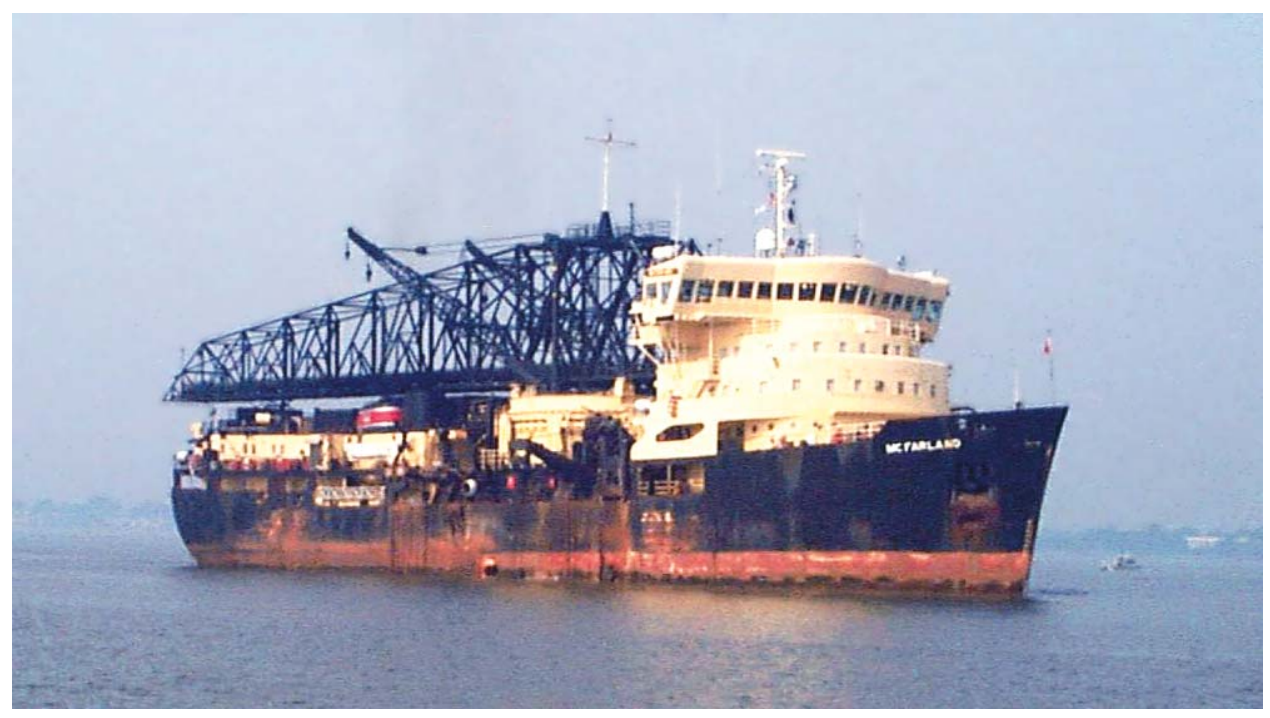

Figure 1. Dredge McFarland

Hopper dredges, like the McFarland, are self-propelled ships equipped with propulsion machinery, hoppers for dredged material storage, and dredge pumps. Dredged material is hydraulically raised through trailing dragarms in contact with the channel bottom and is discharged into the hoppers. The material is then held in the hoppers until placed at the disposal site. 
Hopper dredges are often loaded past the point of overflow for economic reasons. As the hopper is filled, dredged material is stored in the hopper bins until overflow begins. The density of the hopper contents is increased by allowing the low-density supernatant to overflow back into the waterway. As the low-density supernatant overflows, the average density of the hopper contents increase. Thus, more material can be transported per trip to the disposal site or facility. This practice of overflowing hoppers to achieve a high-density load is referred to as economic loading.

In considering overflow, there is normally a tradeoff between the potential economic benefits and potential environmental effects. Overflow results in increased water column turbidity, and supernatant solids may be redeposited near the dredge site. Also, if sediments are contaminated, the overflow may result in some release of contaminants to the water column. Therefore, the relationship between dredge production, density of the hopper load, and the rate of material overflow are important variables in maximizing the efficiency of the dredging operation while minimizing contaminant release.

State environmental resource agencies have expressed concerns regarding the turbidity, sedimentation of suspended solids, and potential contaminant release from overflow resulting from the presence of oyster seedbeds in some areas near the navigation channel. Currently, overflow is not permitted at any location within the Delaware River Basin.

There is a significant potential for economic benefits to overflow in certain reaches of the project if the impact resulting from overflow is environmentally acceptable. The USAED, Philadelphia, therefore, initiated an evaluation of the practice of overflow for select portions of the Delaware River and Delaware Bay to determine if overflow for those reaches can meet applicable water quality standards. The District requested assistance from the Environmental Laboratory (EL), U.S. Army Engineer Research and Development Center (ERDC), Vicksburg, MS, in conducting a study of overflow in the Delaware River/ Delaware Bay system.

This study helped to quantify the degree of turbidity, suspended solids, and contaminant release generated by overflow and the dispersion of the overflow plume in reaches near the oyster seedbeds. Reaches in the Delaware River Basin where overflow would be acceptable were determined.

\section{Study Location}

Two test areas were selected in the Delaware River in conjunction with recommendations from the New Jersey Department of Environmental Protection (NJDEP) and Delaware's Department of Natural Resources and Environmental Control (DNREC) (Figure 2). These areas were selected on the basis of historical knowledge of the Delaware Basin and known locations of material types (sand, silt, and clay) within the river. The first site 


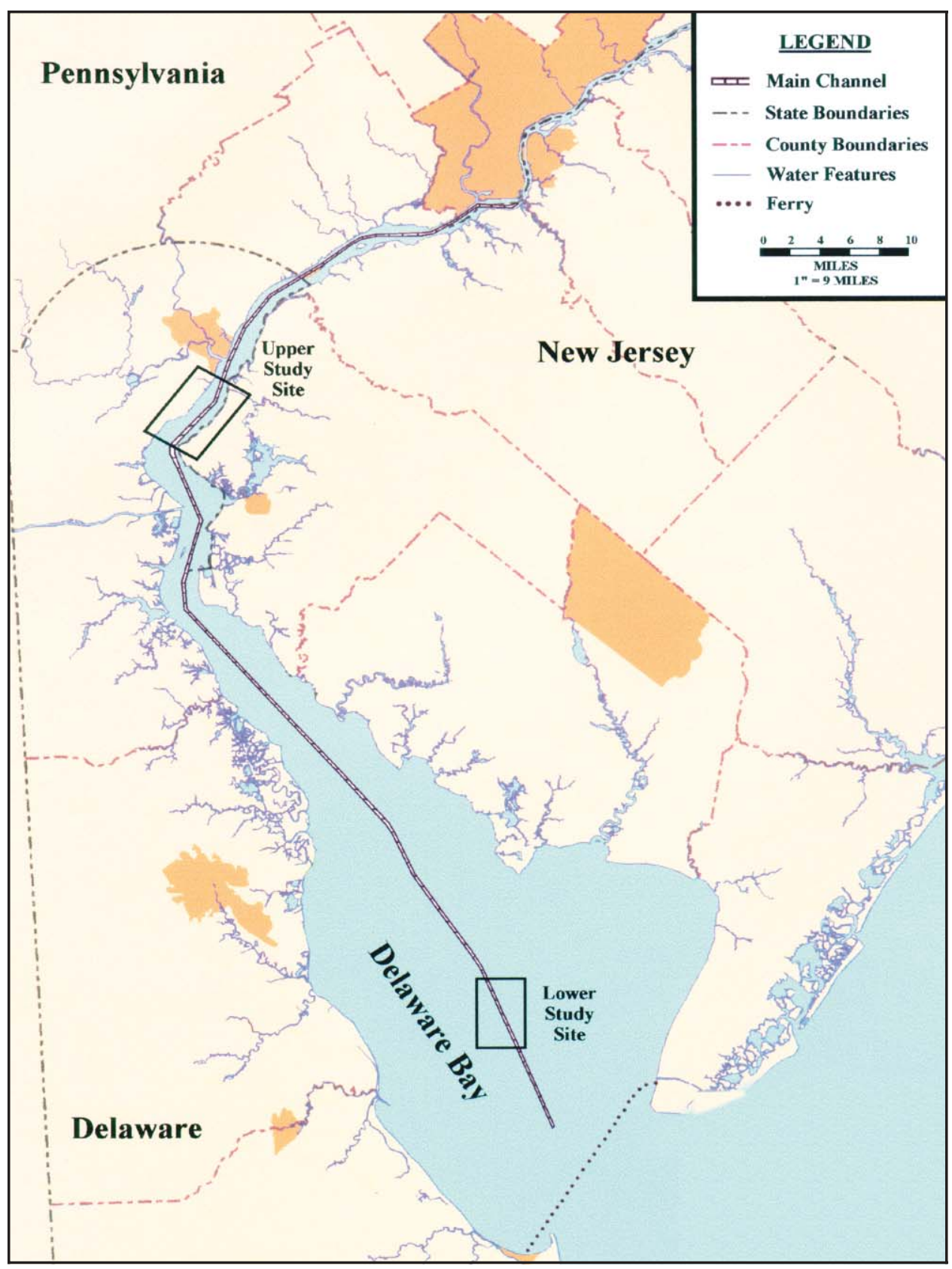

Figure 2. Locations of the lower and upper hopper dredge overflow test study sites

was located at the Brandywine range (Lower Study Site) in the lower Delaware Bay (mile marker 17.7) and was selected to represent a predominantly coarse-grained material. The second site was located at the Deepwater Point range (Upper Study Site) just below the Delaware Memorial Bridge (mile marker 67.9) and was selected to represent a typical fine-grained material. All the proposed activities for the study were reviewed with members of the Delaware River Fish Cooperative Technical Committee prior to submitting applications to the respective regulatory offices for Water Quality Certification (WQC) approvals. 


\section{Purpose and Scope}

The purpose of this study was to evaluate the efficiency of economic loading of a hopper dredge and the physical and chemical characteristics of hopper overflow for the Delaware River dredging project. The study was designed to evaluate the effectiveness of increasing the hopper load during overflow and to determine the physical and chemical characteristics of the overflow into the Delaware River.

The study involved the following activities:

a. Loading data collection - measurements of the load in the hopper at and following overflow.

$b$. Characterization of in situ sediment - physical and chemical analysis including elutriate testing.

c. Hopper inflow monitoring - physical and chemical analysis.

$d$. Hopper contents monitoring - physical and chemical analysis.

$e$. Hopper overflow monitoring - physical and chemical analysis.

f. Plume monitoring - physical and chemical analysis; and in situ turbidity measurements.

$g$. Sedimentation assessment - photo imagery of recent sediment deposits.

$h$. Elutriate and Bioassay Testing - elutriate tests and acute toxicity testing on a fish and a crustacean species were performed for purposes of prediction and potential effects of overflow for the entire project.

These activities provided information to characterize the in situ sediment, hopper inflow as pumped from the draghead, and hopper overflow. Measurement of the material density in the hopper, solids concentration, particle size, and rate of overflow provided information for the development of hopper filling relationships. Elutriate tests were performed to predict the contaminant release back into the water column. These test results were also compared with the data results of the hopper overflow for consistency in sample analysis. Samples taken from the water column defined the relative difference between sediment resuspended by the draghead and that caused by overflow. One overflow and one nonoverflow dredge pass or overflow event was monitored in each of the two reaches of the river. 


\section{Field Monitoring}

\section{Dredging Equipment and Sampling Operations}

The Dredge McFarland was used on September 15 and 16, 1998, to dredge in the two test reaches. The field sampling and monitoring was conducted during representative hopper operations with and without overflow in both reaches.

The tasks described in this technical report were the responsibility of the ERDC, Vicksburg, MS, with support provided by the USAED, Philadelphia. The USAED, Philadelphia, provided the necessary boats and personnel to assist the ERDC in all field monitoring, in situ data collection, and sample collection. ERDC staff members were present at the dredging site during the monitoring effort to direct the field efforts and assist in data and sample collection. ERDC performed all subsequent laboratory testing of samples, data analysis, and report preparation.

\section{Dredge Operation Variables}

At a minimum, it was necessary to have a complete record of the dredge operating variables during the monitoring and sampling periods. In addition to these standard dredge data, the time and duration of overflow during sampling events were recorded along with loading charts using the automated charts of the McFarland.

\section{Collection of In Situ Sediment and Site Water}

On September 14, in situ sediment and site water were collected at the two study sites prior to dredging to provide samples for sediment and water characterization and elutriate testing. Fifteen (15) sediment samples were taken at even intervals in a transect along which the dredge was expected to pass during overflow and nonoverflow conditions. Samples were collected with a grab-type apparatus. A $200-\mathrm{ml}$ portion of the sample was 
retained from each of the 15 samples for water content and density analysis (15 individual analyses). The remaining material of the 15 samples, were composited for sediment and water characterization and elutriate tests.

Composited samples were also obtained for elutriate testing from three sampling locations. Thus, five buckets and fifteen $250-\mathrm{ml}$ bottles of sediment were obtained and shipped to the ERDC to characterize the in situ sediment. The five buckets of sediment were further composited to produce a single uniform composite. From this composite, standard elutriate testing was performed using the site water to prepare the samples. Density (or water content) estimates were made on all 15 samples, and the other physical and chemical tests were performed on the composite sediment sample.

\section{Hopper Inflow Monitoring}

The sediment slurry that was picked up by the draghead and transported through the hydraulic suction line was sampled as it entered the hopper (in 3-min intervals during filling and overflow). Grab samples at the inflow port(s) were collected and analyzed for solids concentration and appropriately composited and analyzed for grain size distribution, particle size distribution of fines, and chemical concentrations. The composited samples represented sediment from five equal time intervals during hopper loading.

\section{Hopper Contents Monitoring}

As material is pumped into the hoppers, a layer of high-density settled material is formed in the lower portion of the hopper with a layer of water with suspended material in the upper portion of the hopper. The vertical distribution of suspended material density or concentration in the upper portion of the hopper was measured. These data, in conjunction with overflow concentration data, can be used to determine when an economic load is achieved and when material density in the hopper is at a maximum. A second use for hopper vertical density measurements is to examine the potential for equipment modification, such as introducing settling tubes to enhance settling rates of solids in hopper bins. Hopper sampling at three depths was taken at the beginning of overflow and at the end of overflow. Three locations in the hopper were sampled.

\section{Hopper Overflow Monitoring}

Because of the variability in solids concentration at the hopper overflow, 40 samples were taken to determine suspended solids for each overflow 
period. Samples were composited for chemical contaminant determination of chemical concentrations, grain size, particle size distribution of fines, and toxicity testing.

\section{Plume Monitoring}

Plume monitoring provided an evaluation of the amount of sediment in the water column resuspended by the operating draghead vs. the amount of sediment contributed by overflow. Data on plume concentrations as a function of distance and time provided information to determine an appropriate buffer distance from the oyster beds in which overflow should be restricted. Differentiation between the magnitude of sediment plumes caused by the draghead and plumes from overflow materials required monitoring both overflow and nonoverflow periods. Monitoring one dredge pass without overflow and one dredge pass with overflow was the minimal plume monitoring effort. To reduce the variability of results between tests, the dredge was required to be moving in the same direction relative to the current flow for every overflow and nonoverflow test monitored. Plume monitoring also provided information on contaminant dispersion in the water column.

Plume monitoring required two boats. One boat was positioned behind the hopper dredge in its path immediately after it passed and began sampling the water column to evaluate the rate of settling of the plume. The other boat towed a turbidimeter (in situ-type probe) across the plume to give information on lateral plume dispersion. Thus, the duration and geometry of the plume could be estimated. Both boats in the monitoring area carried out background sampling immediately before the dredging began.

Lateral plume dispersion measurements were made at middepth by locating the turbidimeter probe at the midpoint of the water column. Background turbidity was extensively measured. The boat towing the turbidimeter monitored distance from the dredge, using a range finder and hand bearing compass, and distance from the anchored sample boat. The whole plume was traversed, going outside of the plume at each extreme of the turbidity plume.

While the mobile boat was measuring lateral plume dispersion, the anchored boat measured decay of the plume as it settled through the water column. Water samples were taken at the surface (less than $1 \mathrm{~m}$ deep), middepth, and near bottom (within 1 to $2 \mathrm{~m}$ of the bottom). Fifteen samples at three depths for a 50-min period were taken to characterize background total suspended solids (TSS) conditions, and about 30 samples at three depths in a 30-min time frame were taken to characterize the overflow plume after the dredging pass. The latter sampling protocol was also used for the nonoverflow sediment plume measurements.

TSS was measured for all plume samples and a compositing scheme was used to reduce the number of samples for chemical analysis. Three compos- 
ite samples for the plume monitoring were obtained (one at each of the three depths) by mixing portions of the samples taken at all three depths over one-third of the plume monitoring effort. Chemical analysis included heavy metals, polychlorinated biphenyls (PCBs) and polycyclic aromatic hydrocarbons (PAHs) and provided data on potential contamination of the water column by the dredging operation.

\section{Sedimentation Assessment}

One difficulty in assessing potential impacts of sedimentation resulting from hopper overflow is detection of thin overburdens in habitats in the vicinity of the dredging operation. Although thin $(<5 \mathrm{~cm})$ overburdens could have detrimental impacts, for example on the settlement and attachment of oyster larvae, this exceeds the detection limits of most conventional techniques. One method effective in measuring sedimentation events of less than $1 \mathrm{~cm}$ is sediment-profiling imagery using a sediment profile camera. This technique involves insertion of a prism into the substrate through which images of the sediment-water interface are obtained. The images provide rapid, accurate measures of recent sedimentation, particularly if the overburden sediments are dissimilar from the ambient substrate. The images also provide indications of impacts to benthic communities (e.g., distribution and position of annelid worms and bivalve mollusks relative to the relict and overburden surface) and changes in physical/chemical conditions of the sediment (e.g., altered redox potential discontinuity, evidence of hypoxia). This camera system is unaffected by ambient turbidity. An attached plan-view underwater camera also provided photographs at the sediment profile stations.

The sediment profiling camera system was deployed at the Delaware River overflow operation site. Because the area is tidally influenced, stations were occupied both up and down current from the dredging project. Stations were allocated to gather information for transects across several cross sections of the river reach potentially influenced by overflow, including any charted oyster bars.

\section{Bioassay}

Samples were taken at the hopper overflow for use in a 96-hr water column bioassay. This portion of the study will help in determining the possible biological effects of water column exposure to Delaware River sediment. 


\section{Data Analysis}

\section{Hopper Loading Characteristics}

\section{Coarse-grained site}

The loading data provided by the USAED, Philadelphia, for the coarsegrained site are shown in Figure 3, and the summary data for the load increase can be found in Table 1. Loading volumes are based on calculations using historical density data in the area being dredged.

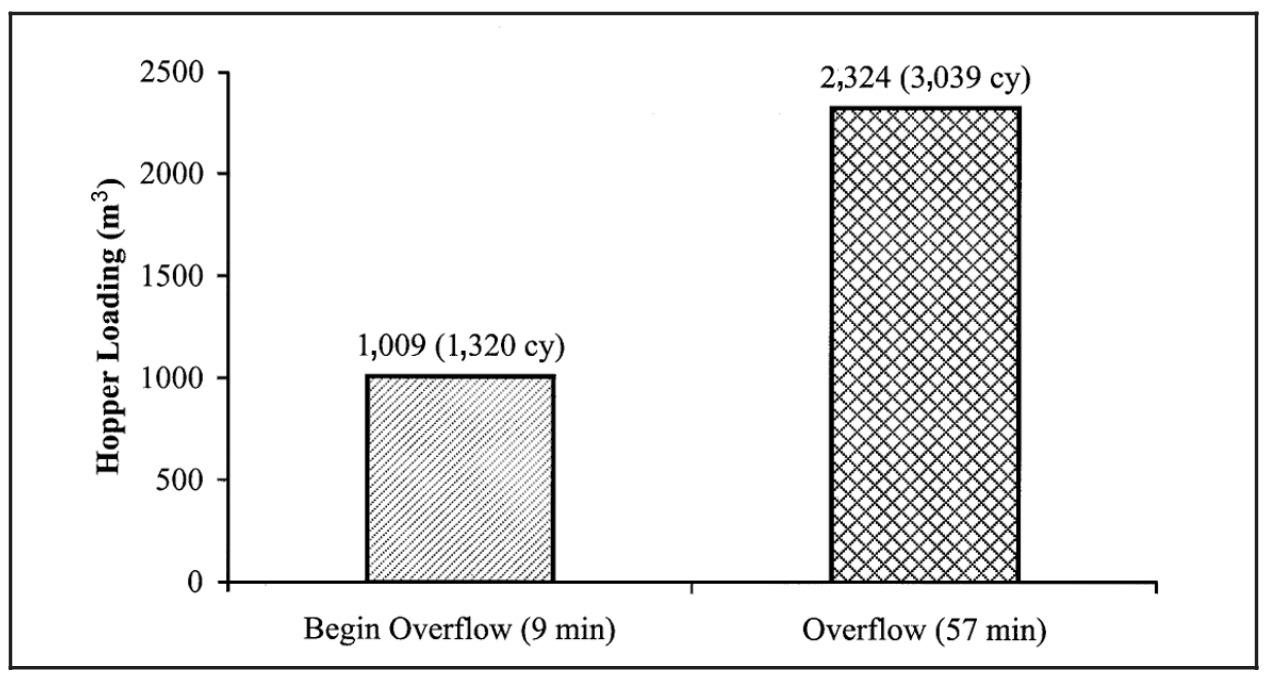

Figure 3. Hopper loading at coarse-grained site

It took 9 min of dredging to reach overflow status. During the first $9 \mathrm{~min}$, material increased at a rate of $112.4 \mathrm{~m}^{3} / \mathrm{min}\left(147 \mathrm{yd}^{3} / \mathrm{min}\right)$. Once overflow began, the increase in material loading was determined to be $22.9 \mathrm{~m}^{3} / \mathrm{min}\left(30 \mathrm{yd}^{3} / \mathrm{min}\right)$. Overflow continued for $57 \mathrm{~min}$ with a gain of 130 percent realized. At the end of the overflow period, the hopper was full of sediment. 


\section{Table 1}

Delaware River Coarse- and Fine-Grained Loading Data

\begin{tabular}{|c|c|c|c|c|c|c|c|}
\hline \multicolumn{4}{|c|}{ Coarse-Grained Material } & \multicolumn{4}{|c|}{ Fine-Grained Material } \\
\hline Time, $\min$ & \multicolumn{2}{|c|}{ Loading, $\mathrm{m}^{3}$} & Loading, $\mathrm{yd}^{3}$ & Time, $\min$ & \multicolumn{2}{|c|}{ Loading, $\mathrm{m}^{3}$} & Loading, $\mathrm{yd}^{3}$ \\
\hline $\begin{array}{r}0 \\
9 \\
66\end{array}$ & \multicolumn{2}{|c|}{$\begin{array}{l}0 \\
\text { 1,009 Begin overflow }(9 \mathrm{~min}) \\
2,324 \text { Overflow }(57 \mathrm{~min})\end{array}$} & $\begin{array}{l}1,320 \\
3,039\end{array}$ & $\begin{array}{r}0 \\
13 \\
34 \\
\\
0 \\
13 \\
18\end{array}$ & \multicolumn{2}{|c|}{$\begin{array}{l}0 \\
871 \text { Begin overflow }(13 \mathrm{~min}) \\
\text { 1,031 Overflow }(21 \mathrm{~min}) \\
0 \\
871 \text { Begin overflow }(13 \mathrm{~min}) \\
961 \text { Overflow }(5 \mathrm{~min})\end{array}$} & $\begin{array}{l}1,139 \\
1,348 \\
1,139 \\
1,257 \\
\end{array}$ \\
\hline Time, min & $\begin{array}{l}\text { Loading } \\
\mathrm{m}^{3} / \mathrm{min}\end{array}$ & $\begin{array}{l}\text { Losing to } \\
\text { Overflow } \\
\mathrm{m}^{3} / \mathrm{min}\end{array}$ & $\%$ Gain & Time, min & $\begin{array}{l}\text { Loading } \\
\mathrm{m}^{3} / \mathrm{min}\end{array}$ & $\begin{array}{l}\text { Losing to } \\
\text { Overflow } \\
\mathrm{m}^{3} / \mathrm{min}\end{array}$ & $\%$ Gain \\
\hline $\begin{array}{r}9 \\
57\end{array}$ & $\begin{array}{r}112.4 \\
22.9\end{array}$ & 89.5 & 130.3 & $\begin{array}{r}13 \\
21 \\
5\end{array}$ & $\begin{array}{r}67.0 \\
7.6 \\
18.0\end{array}$ & $\begin{array}{l}59.4 \\
49.0\end{array}$ & $\begin{array}{l}18.4 \\
10.3\end{array}$ \\
\hline
\end{tabular}

\section{Fine-grained site}

The loading diagram for the fine-grained site is shown in Figure 4 and the summary data for the load increase can be found in Table 1. For this site, the dredge operated 13 min before overflow began. During this first $13 \mathrm{~min}$ of dredging, material increased at a rate of $67.0 \mathrm{~m}^{3} / \mathrm{min}\left(87 \mathrm{yd}^{3} /\right.$ $\mathrm{min})$. Once overflow began, the increase in material loading was determined to be $7.6 \mathrm{~m}^{3} / \mathrm{min}\left(10 \mathrm{yd}^{3} / \mathrm{min}\right)$. Overflow continued for $21 \mathrm{~min}$ with a gain of 18 percent realized. The percent gain realized for the coarse reach was interpolated for $21 \mathrm{~min}$ and was 50 percent, so that a comparison could be made during the same time frame between the two sites.

\section{Economics}

These results are consistent with the material composition at the two sites. The coarse-grained site would be expected to settle at a more rapid rate, therefore, showing a significant gain in material. Whereas, the finegrained material would tend to stay in suspension, resulting in most of the sediment being discharged out the overflow. Because of the large amount of gain realized at the coarse-grained site, a rate of return of about 50 to 60 percent may be realized based on the amount of material retained in the hopper and the round-trip travel time required to the dump site. Basically, for every 3 days of nonoverflow dredging, approximately the same amount of material can be removed by allowing overflow dredging in a 2-day period. This percent return also assumes that the material being discharged in the overflow settles in the navigation channel and will require redredging the area. At the fine-grained site, the rate of return is negligible because of the small gain in load achieved. This is also based on round-trip travel time required to the pump-out site, material being discharged in the overflow settling in the navigation channel and requiring redredging of the area. If 


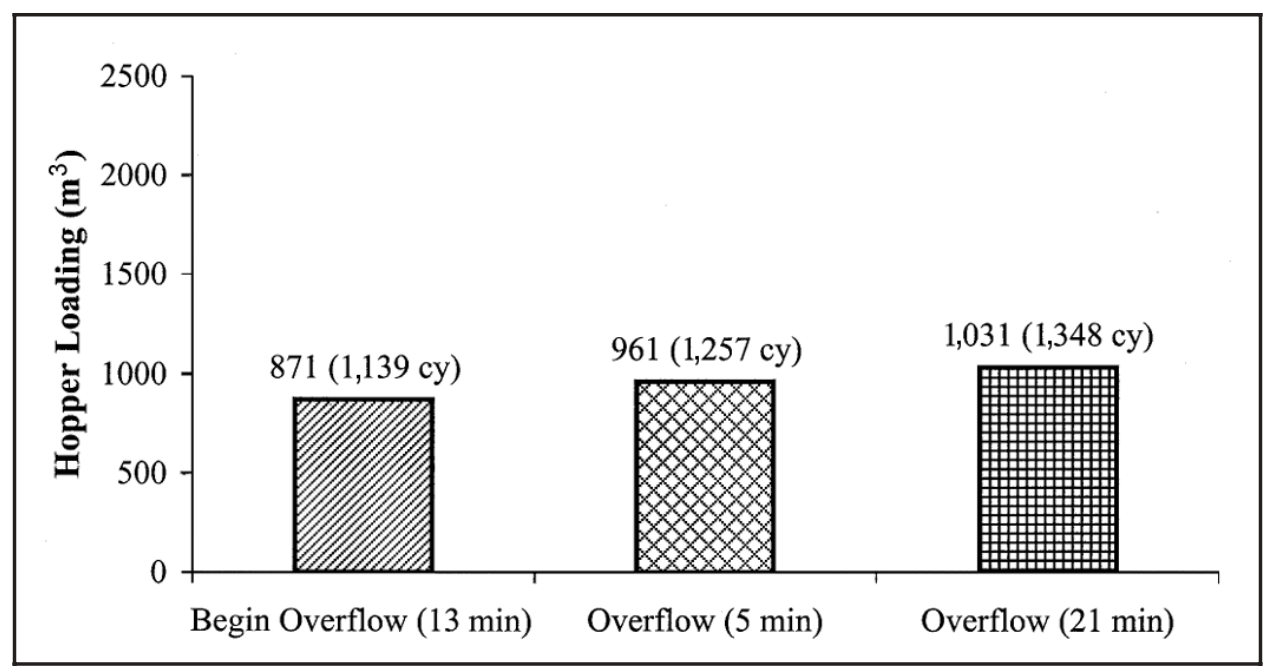

Figure 4. Hopper loading at fine-grained site

redredging the area at either site is not required, then the percent return estimated at those sites may increase.

\section{In Situ Sediment and Background Water Samples}

\section{Coarse-grained site}

The composited sediment samples at the coarse-grained site show the proposed dredged area to average 97 percent sand (Figure 5). The range was less than 1 percent \pm of the average value (96.5 to 97.7 percent). Background water chemical concentrations were compared with the contaminants of concern as listed in the acute marine objectives for toxic pollutants for the protection of aquatic life in the Delaware River estuary. This information can be found in the Delaware River Basin Commission West Trenton, New Jersey, Administrative Manual-Part III, Water Quality Regulations, October 23, 1996. The only parameter above the standard was background dissolved copper (Table 2). The standard for copper is $5.3 \mu \mathrm{g} / \mathrm{l}$, and the background value was $13 \mu \mathrm{g} / \mathrm{l}$. The water quality and sediment data for the coarse-grained site can be found in Appendix A.

\section{Fine-grained site}

The composited sediment samples at the fine-grained site show the proposed dredged area to average 33 percent sand (Figure 6). The range for sand was from 18 to 50 percent. Background water concentrations for the contaminants of concern were all below the more stringent of the freshwater or marine stream quality objectives for acute toxicity standards as 


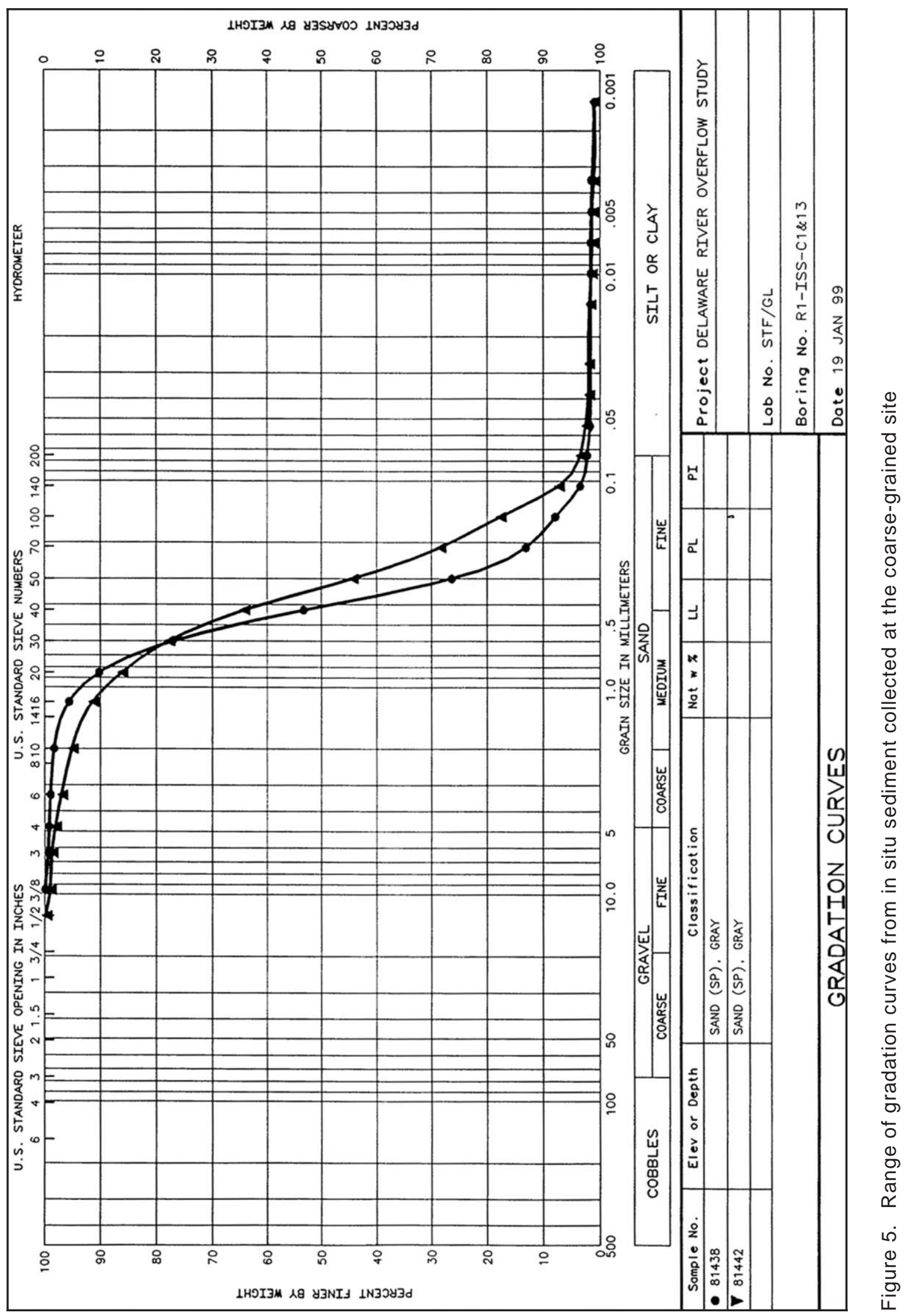




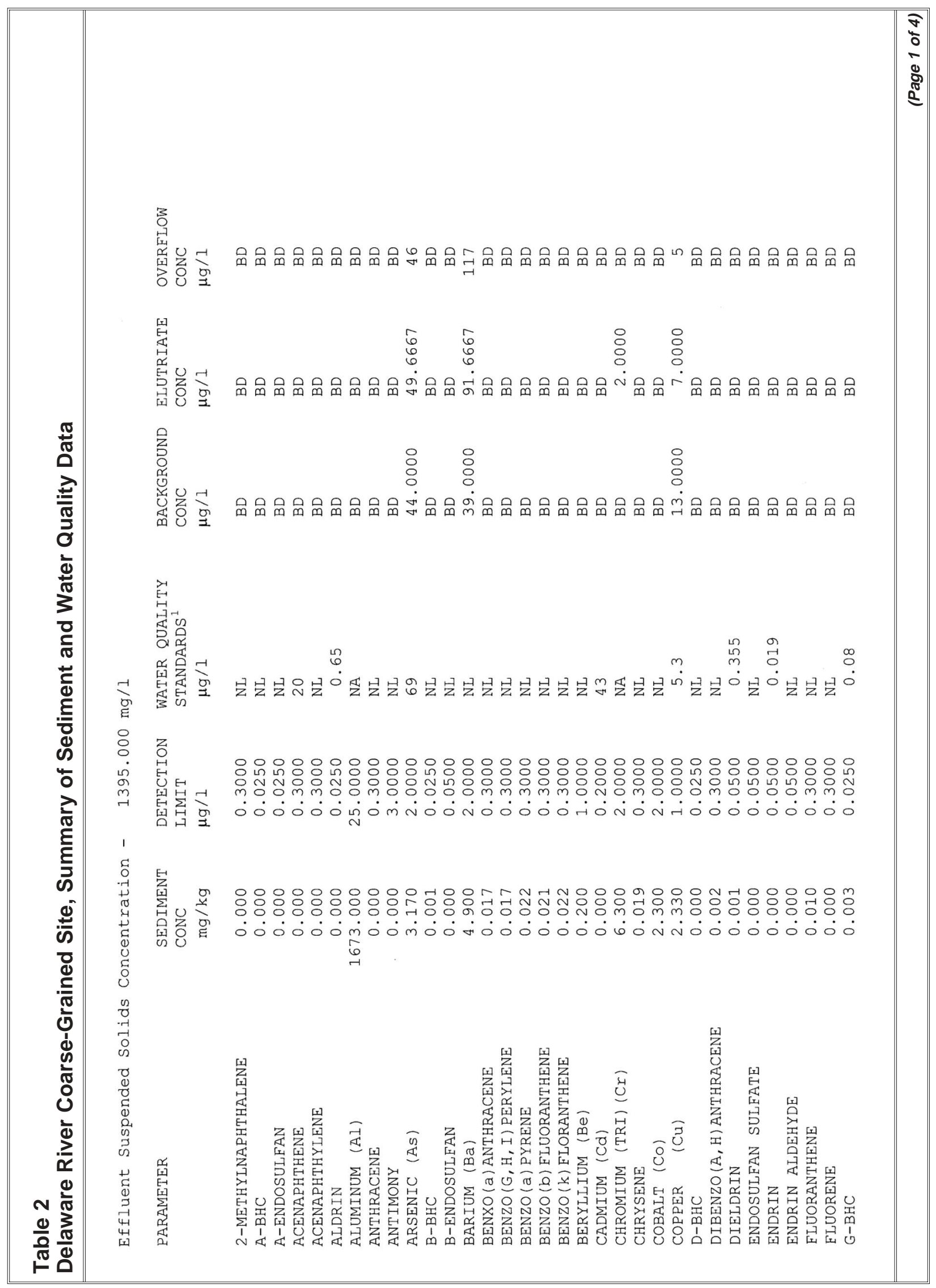




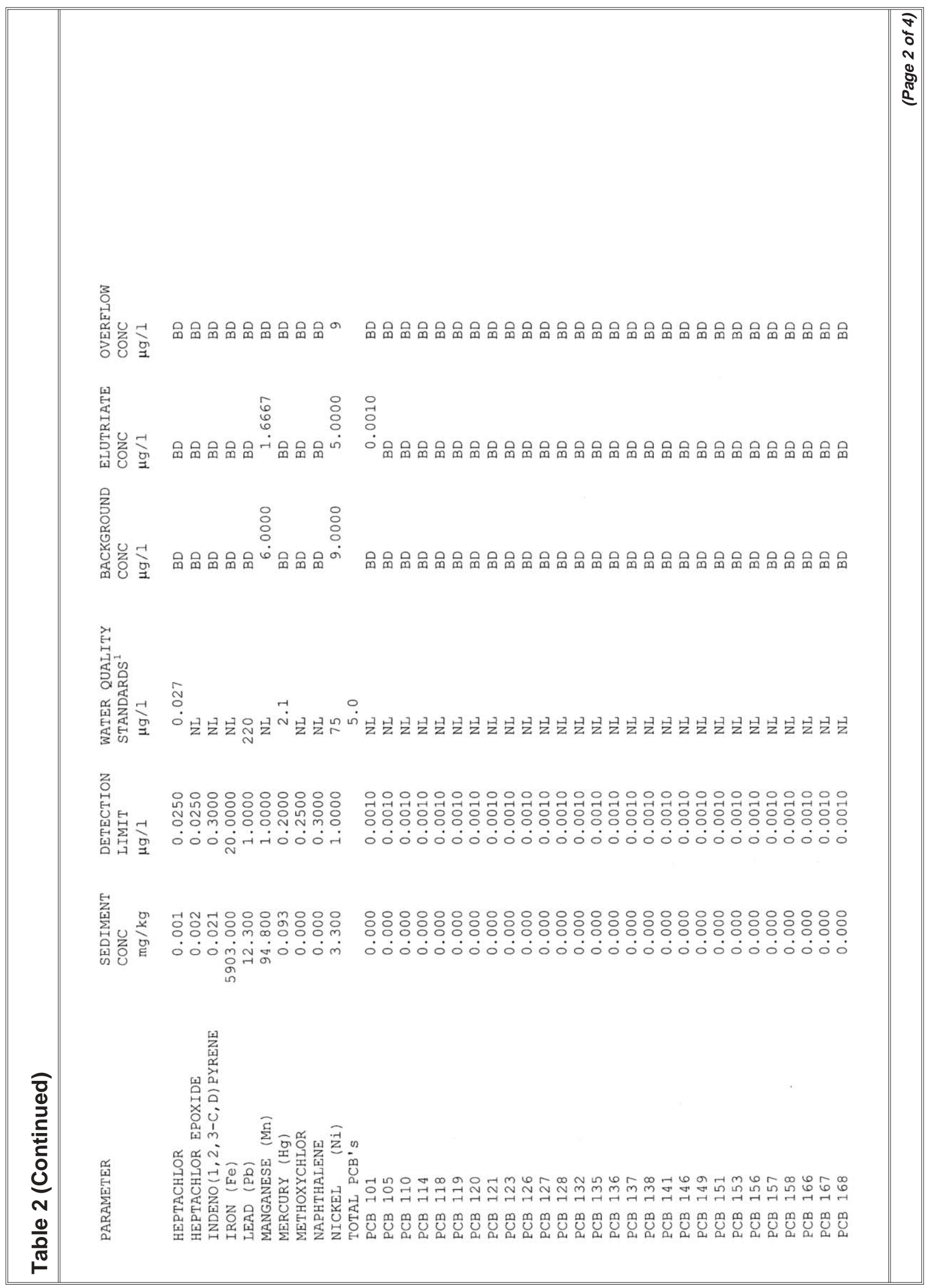




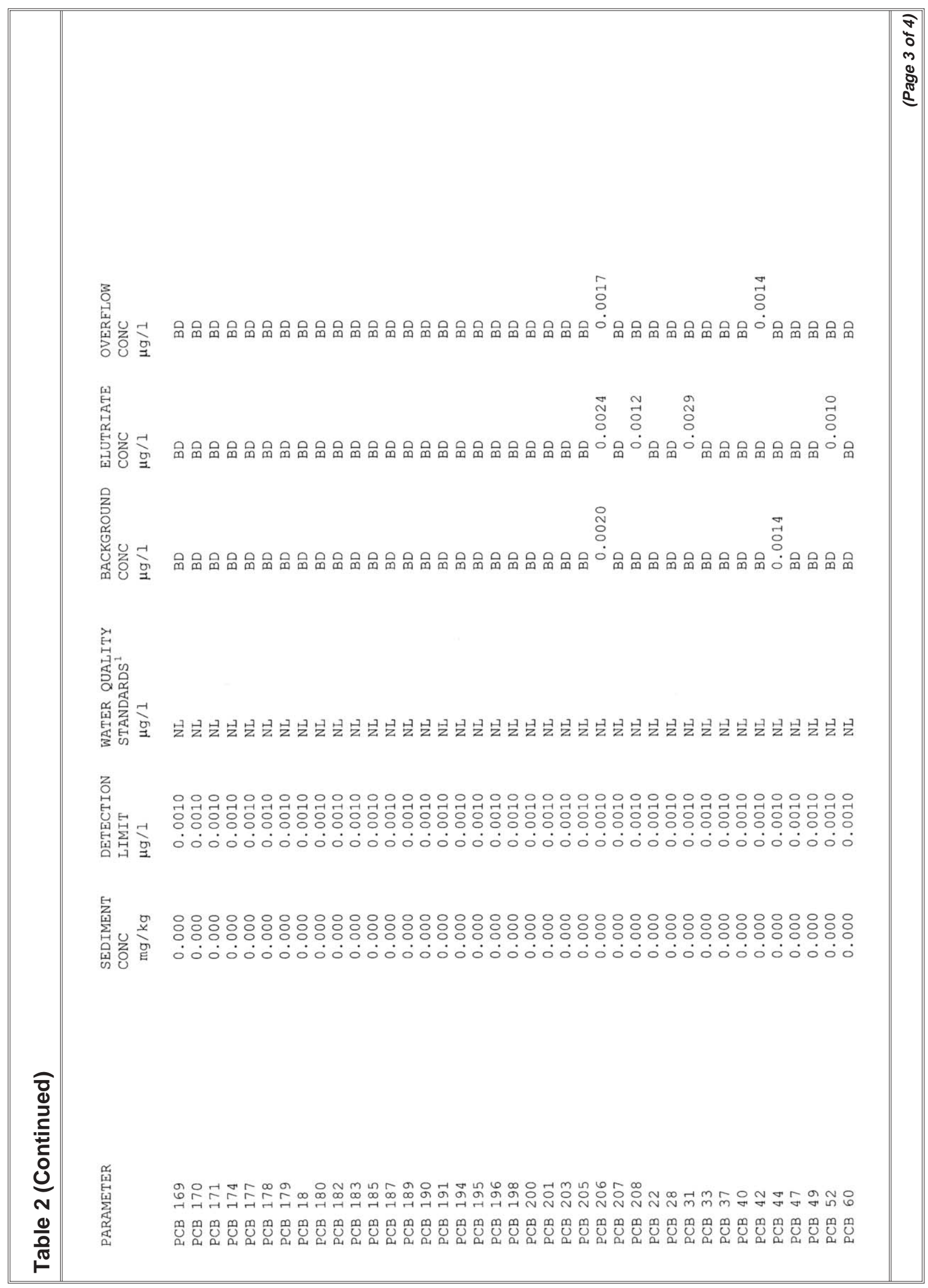




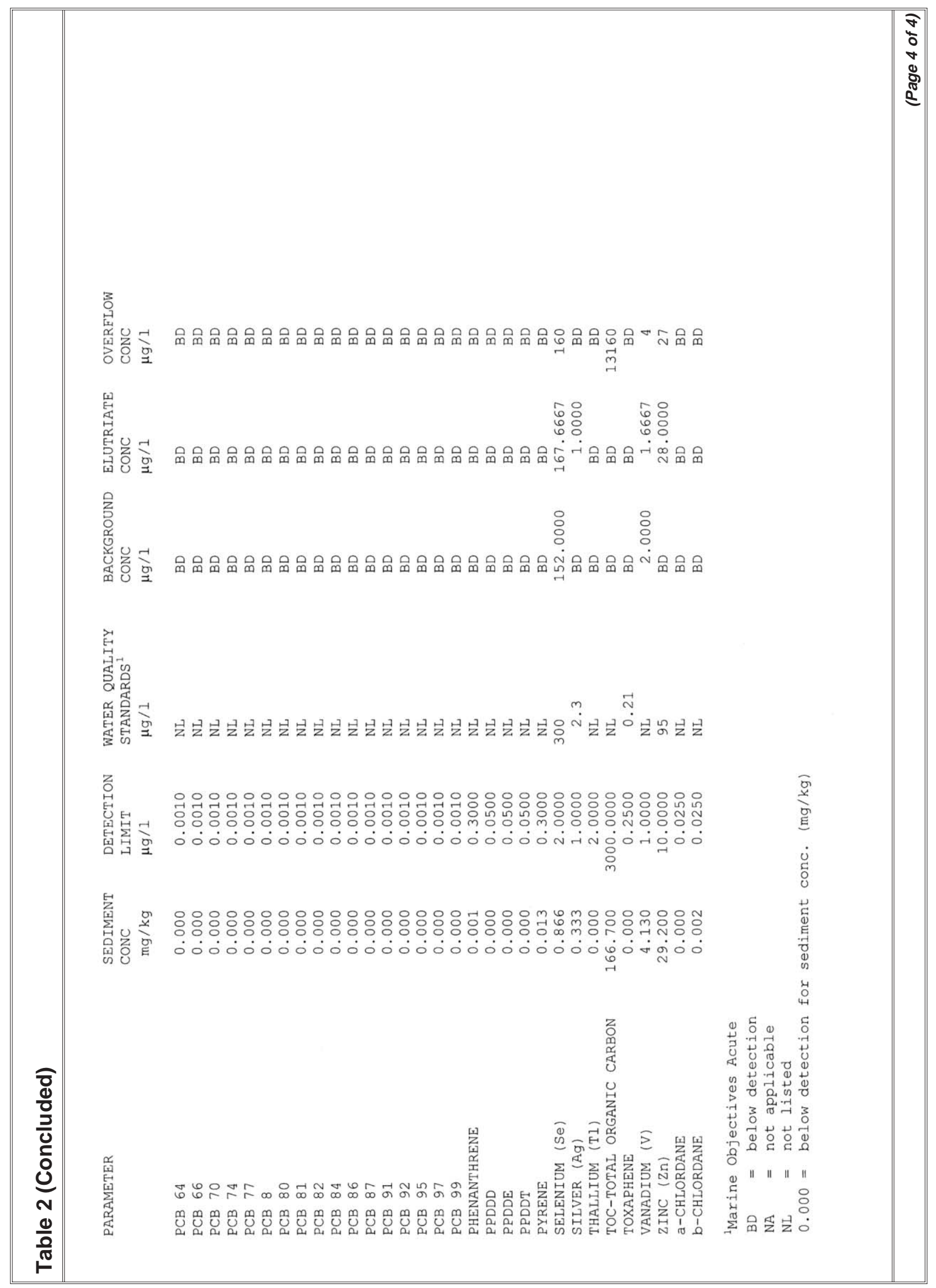


found in the Delaware River Basin Commission West Trenton, New Jersey, Administrative Manual-Part III, Water Quality Regulations, October 23, 1996. Only two exceedances were found in the dissolved overflow water (Table 3). Endrin was measured at a concentration of $0.0754 \mu \mathrm{g} / \mathrm{l}$ as compared to the standard of $0.019 \mu \mathrm{g} / \mathrm{l}$. Zinc was measured at a concentration of $131 \mu \mathrm{g} / \mathrm{l}$ as compared to the standard of $95 \mu \mathrm{g} / \mathrm{l}$. See Appendix A for the Delaware River water quality and sediment analysis for the fine-grained site.

\section{Hopper Inflow}

\section{Coarse-grained site}

Samples collected for grain-size distribution at the hopper inflow at the coarse-grained site averaged 84 percent sand (Figure 7). The range was from 52 to 98 percent. Eliminating the 52-percent sample resulted in a sandy composition of 92 percent with a range from 86 to 98 percent. This is more representative of that collected from the in situ sampling. Suspended solids concentrations in the hopper inflow could not be accurately determined because the coarse-grained material rapidly settled to the bottom of the sampling buckets, and the total bucket sample was not retained for analysis.

\section{Fine-grained site}

Samples collected for grain-size distribution at the hopper inflow at the fine-grained site averaged 12 percent sand (Figure 8). The range was from 9 to 15 percent. This is much less than the 33 percent represented by the in situ sampling.

\section{Hopper Contents}

\section{Coarse-grained site}

Suspended solids concentrations in the hopper at the coarse-grained site were $<15 \mathrm{~g} / \mathrm{l}$ (Figure 9). This indicates that settling was occurring very rapidly. Although the samples should be representative of the water column, it should be realized that the agitation occurring inside the hopper will keep the material in suspension for an extended period of time. Therefore, when the sample was collected, the material being agitated quickly settled and was not collected in the $250-\mathrm{ml}$ sample bottle. 


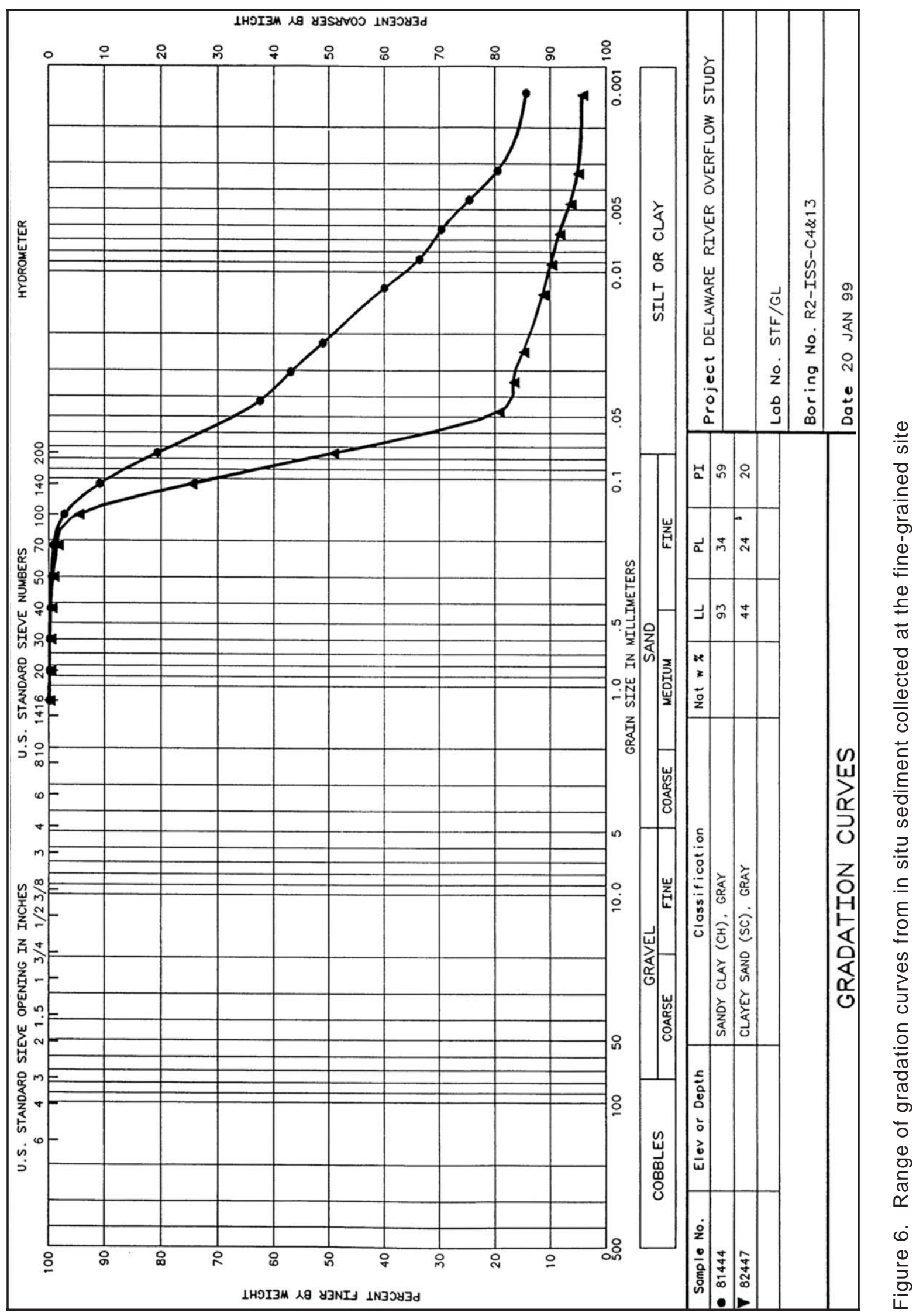




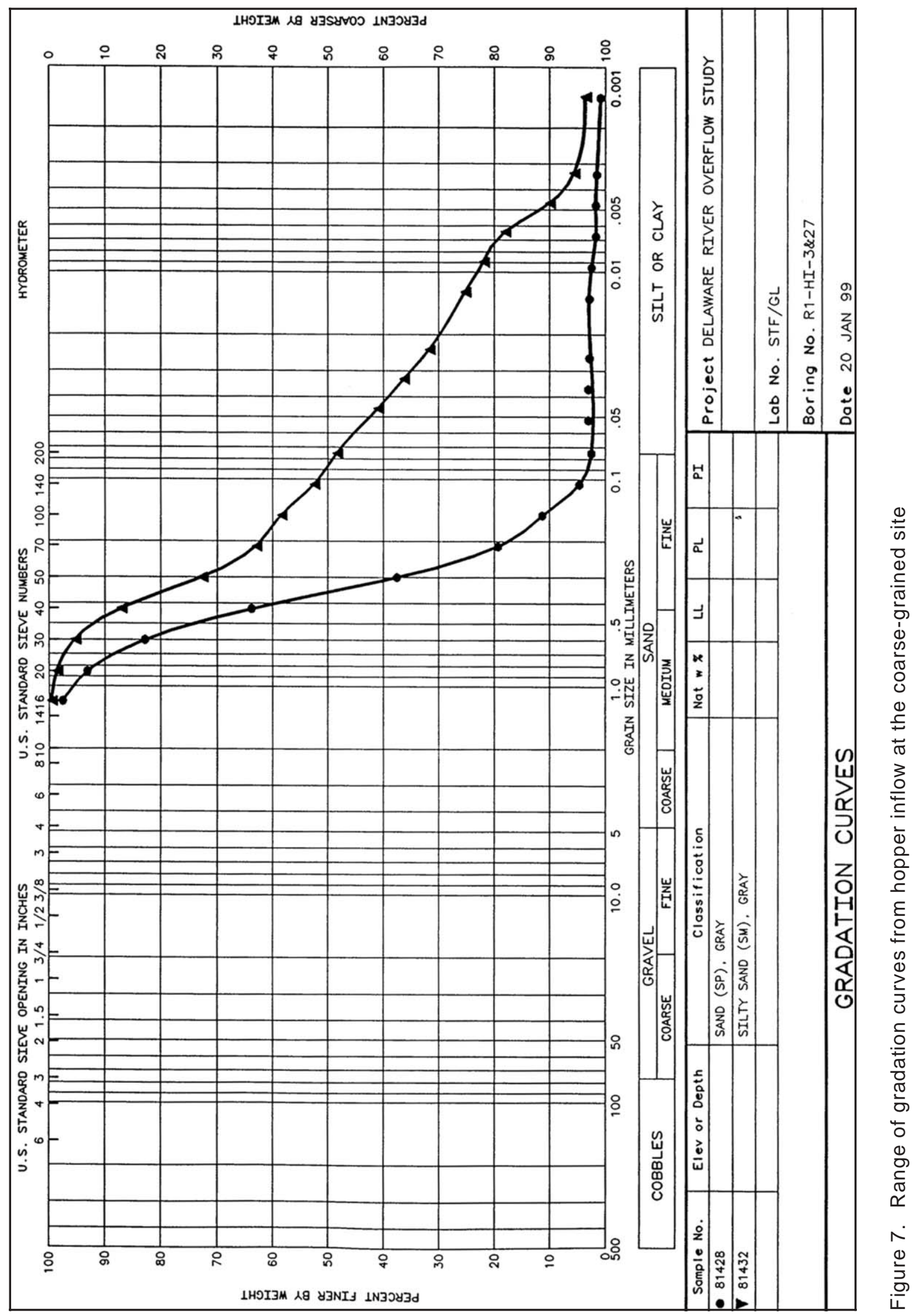




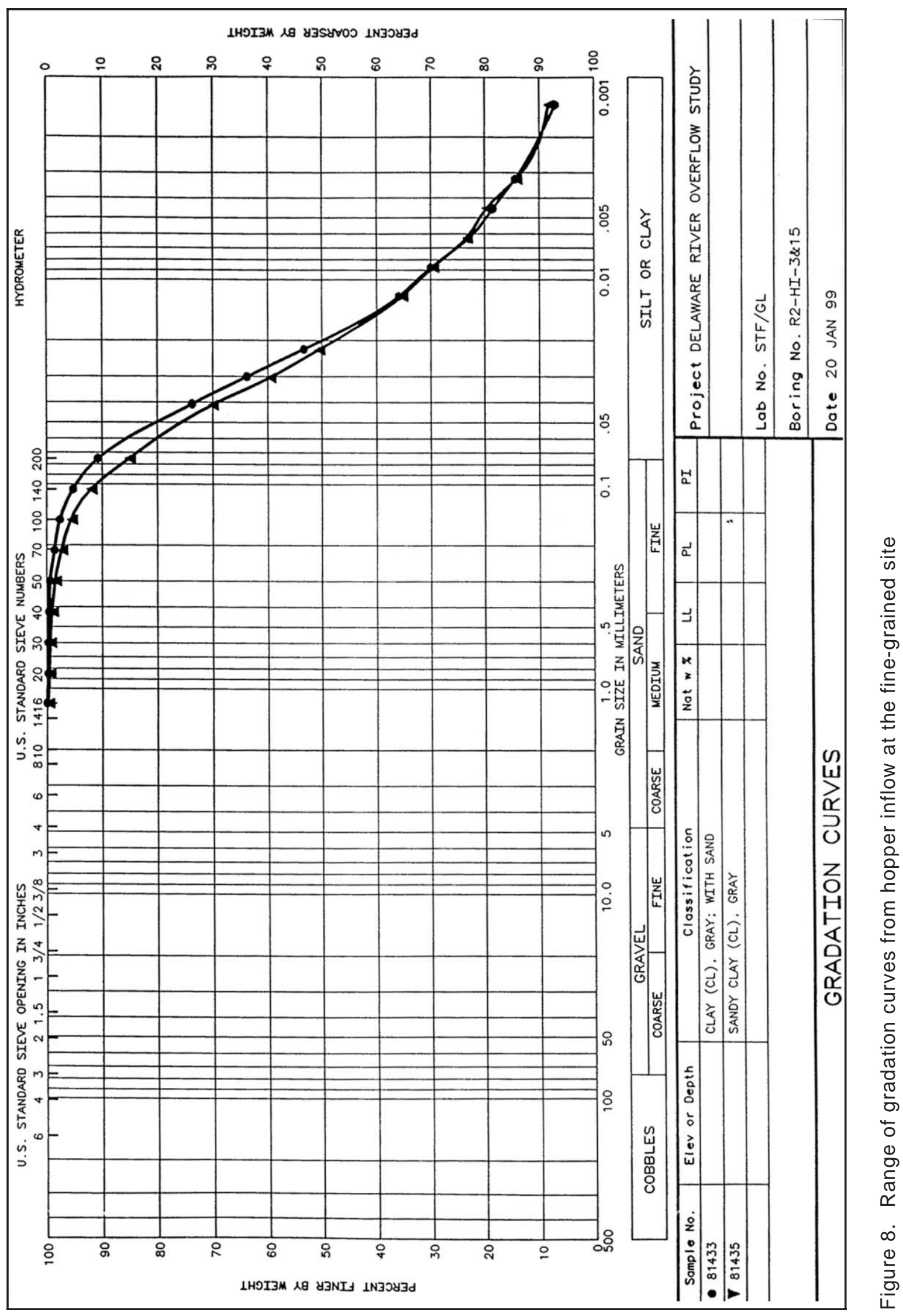




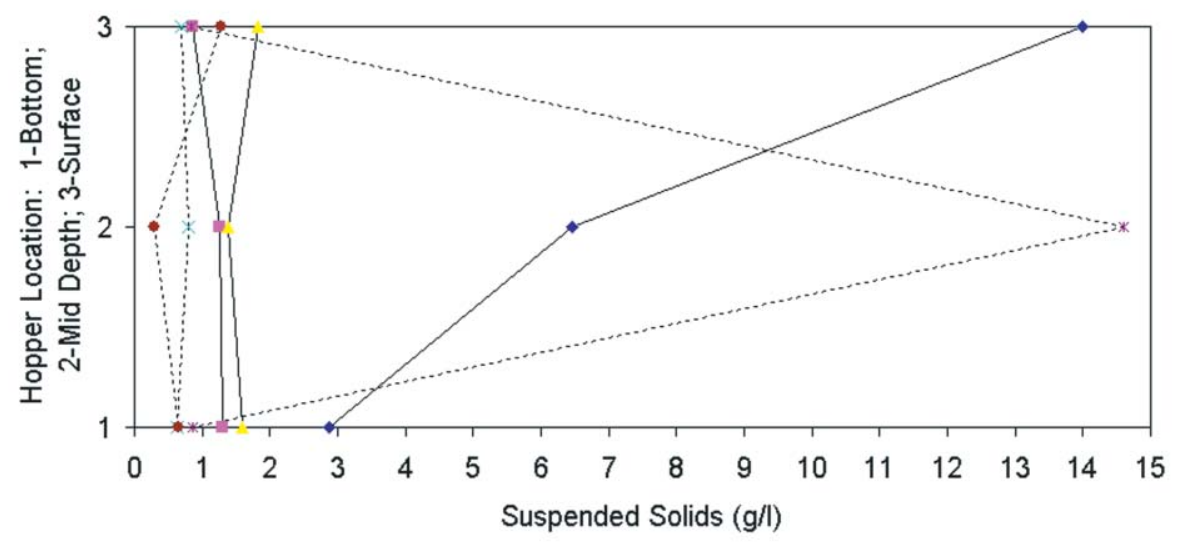

$\longrightarrow$ Begin Overflow Station $1-$ Begin Overflow Station $2-$ Begin Overflow Station 3 ......... End Overflow Station $1 \quad \ldots . \cdots * \cdots$ End Overflow Station $2 \quad \cdots . \cdots$.... End Overflow Station 3

Figure 9. Hopper contents—solids concentrations of coarse-grained material

\section{Fine-grained site}

Suspended solids concentrations in the hopper at the fine-grained site were upward of $150 \mathrm{~g} / \mathrm{l}$ at the bottom and approximately $80 \mathrm{~g} / \mathrm{l}$ at the surface (Figure 10). It is expected that high concentrations of suspended solids would be found in the water column as the hopper agitates the fine-grained material and keeps it in suspension. The high concentrations of suspended solids at the surface indicate that a large amount of the material was lost to overflow in the fine-grained site.

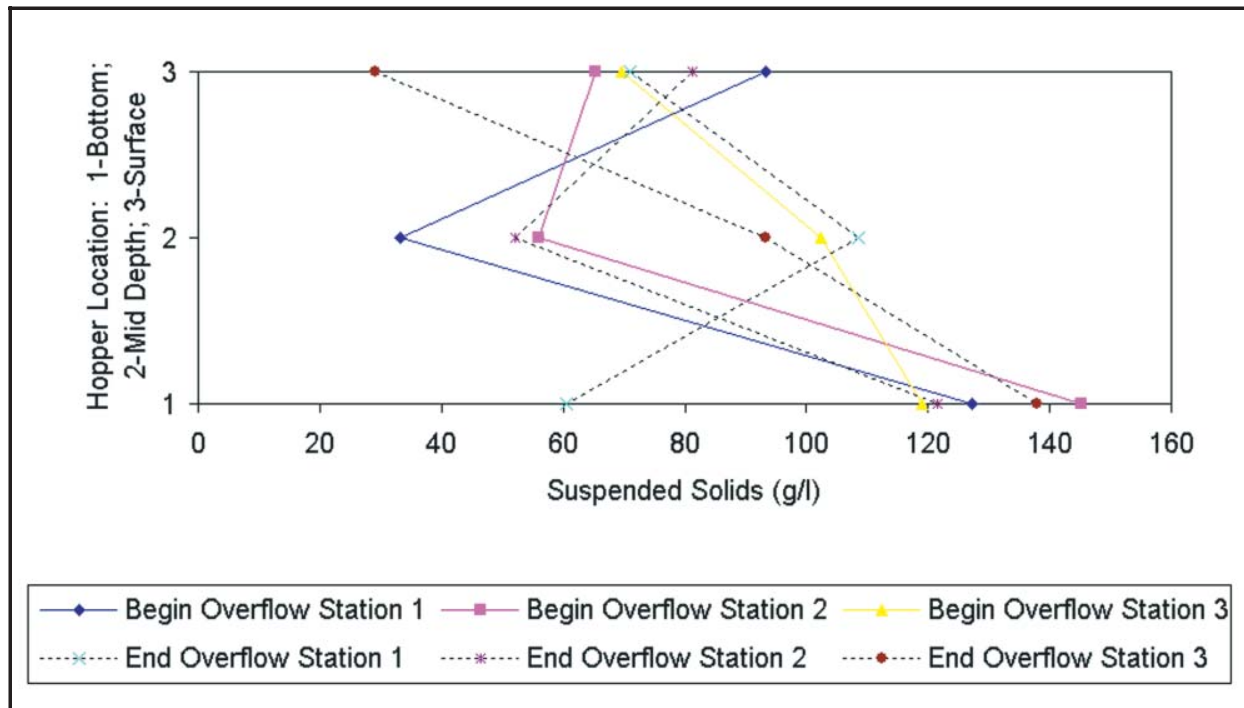

Figure 10. Hopper contents-solids concentrations of fine-grained material 


\section{Hopper Overflow}

\section{Coarse-grained site}

Samples collected for grain-size distribution at the hopper overflow at the coarse-grained site averaged 81.1 percent sand with a range from 24.4 to 96.1 percent (Figure 11). Composites of five samples were obtained and the average grain-size distribution was 78.1 percent with a range from 66.7 to 87.7 percent (Figure 12). This shows that a large amount of the sandy material was being agitated in the hopper and being washed out during overflow. This is consistent with the loading data that show a loading of about $112.4 \mathrm{~m}^{3} / \mathrm{min}\left(147 \mathrm{yd}^{3} / \mathrm{min}\right)$ before overflow and an average loading of about $22.9 \mathrm{~m}^{3} / \mathrm{min}\left(30 \mathrm{yd}^{3} / \mathrm{min}\right)$ over the 57 -min period during overflow. However, the rate of loading in the initial stages of overflow was likely much higher with the material in the overflow increasing as the hopper filled and retention time was decreased. None of the chemistry parameters analyzed in the overflow samples collected at the coarse-grained site exceeded marine acute objectives as listed in the Delaware River Basin Water Quality Regulations for dissolved criteria limits. Although the background value for copper $(13 \mu \mathrm{g} / \mathrm{l})$ exceeded the criteria $(5.3 \mu \mathrm{g} / \mathrm{l})$, the dissolved value for copper in the overflow was $5 \mu \mathrm{g} / 1$, indicating a scavenging of metals by the suspended material during the dredging and overflow process.

\section{Fine-grained site}

Samples collected for grain-size distribution at the hopper overflow at the fine-grained site averaged 12.2 percent sand with a range from 6.2 to 31.2 percent (Figure 13). Composites of five samples were obtained and the average grain-size distribution was 10.6 percent with a range from 9.3 to 11.6 percent (Figure 14). The suspended solids concentrations in the overflow averaged $110 \mathrm{~g} / \mathrm{l}$ over the total overflow period of $21 \mathrm{~min}$. The solids concentrations were essentially consistent throughout the overflow period, indicating little retention of the fine material in the hopper once overflow began. A large amount of material, about $59.4 \mathrm{~m}^{3} / \mathrm{min}\left(78 \mathrm{yd}^{3} / \mathrm{min}\right)$ or about 89 percent of the inflow is being lost to overflow. Zinc $(131 \mu \mathrm{g} / \mathrm{l})$ and endrin $(0.0754 \mu \mathrm{g} / \mathrm{l})$ were the only two chemical parameters measured in the overflow that exceeded the more stringent acute objectives of the freshwater and marine stream quality standards $(95 \mu \mathrm{g} / 1$ for zinc and $0.019 \mu \mathrm{g} / \mathrm{l}$ for endrin) as listed in the Delaware River Basin Water Quality Regulations for dissolved criteria limits. The value for endrin exceeded standards by a factor of 4 , indicating that both water quality objectives could be met a short distance from the point of overflow. None of the other chemistry parameters analyzed in the overflow samples collected at the fine-grained site exceeded the acute objectives. 


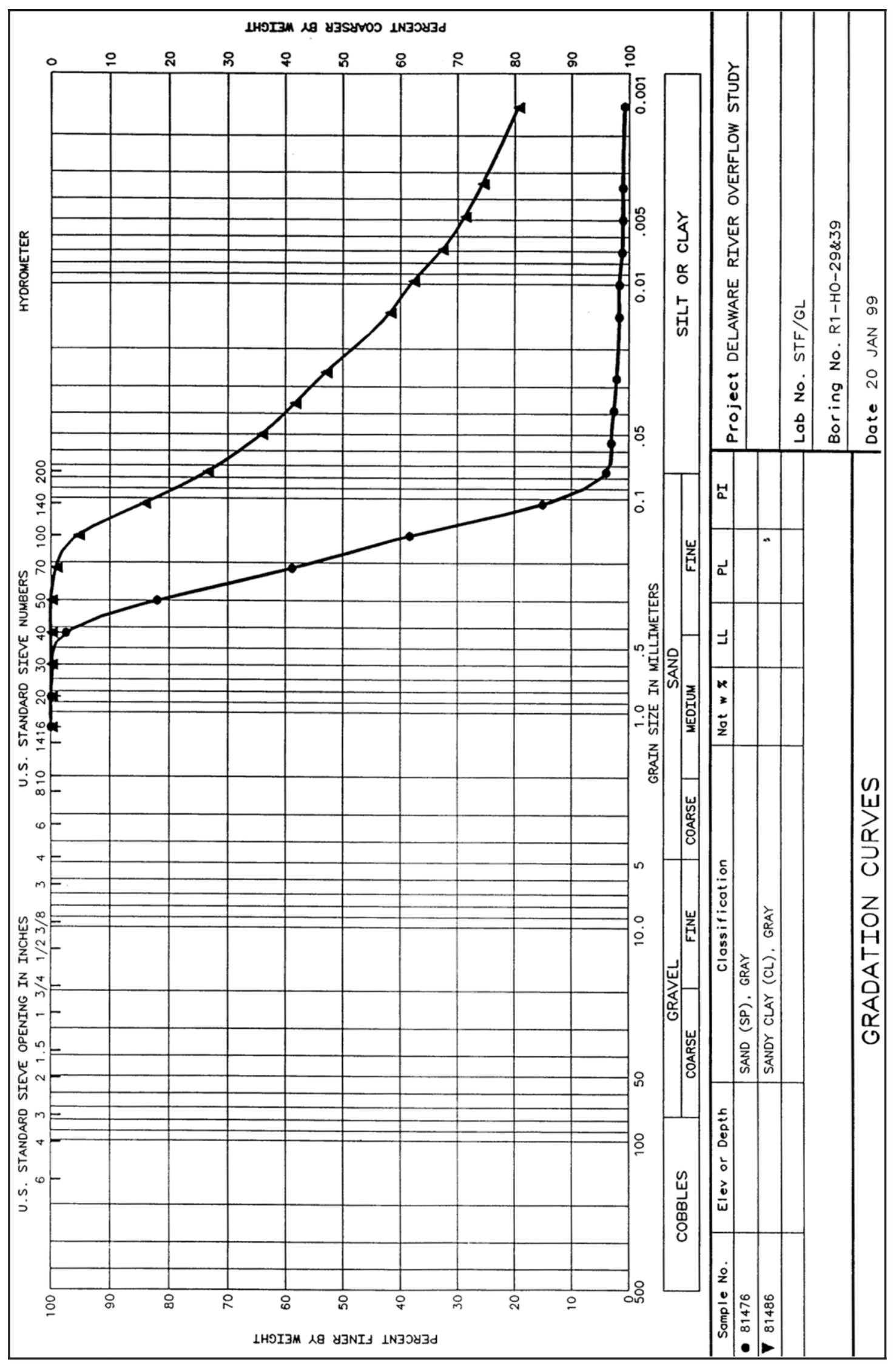

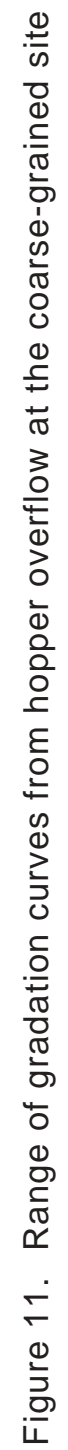




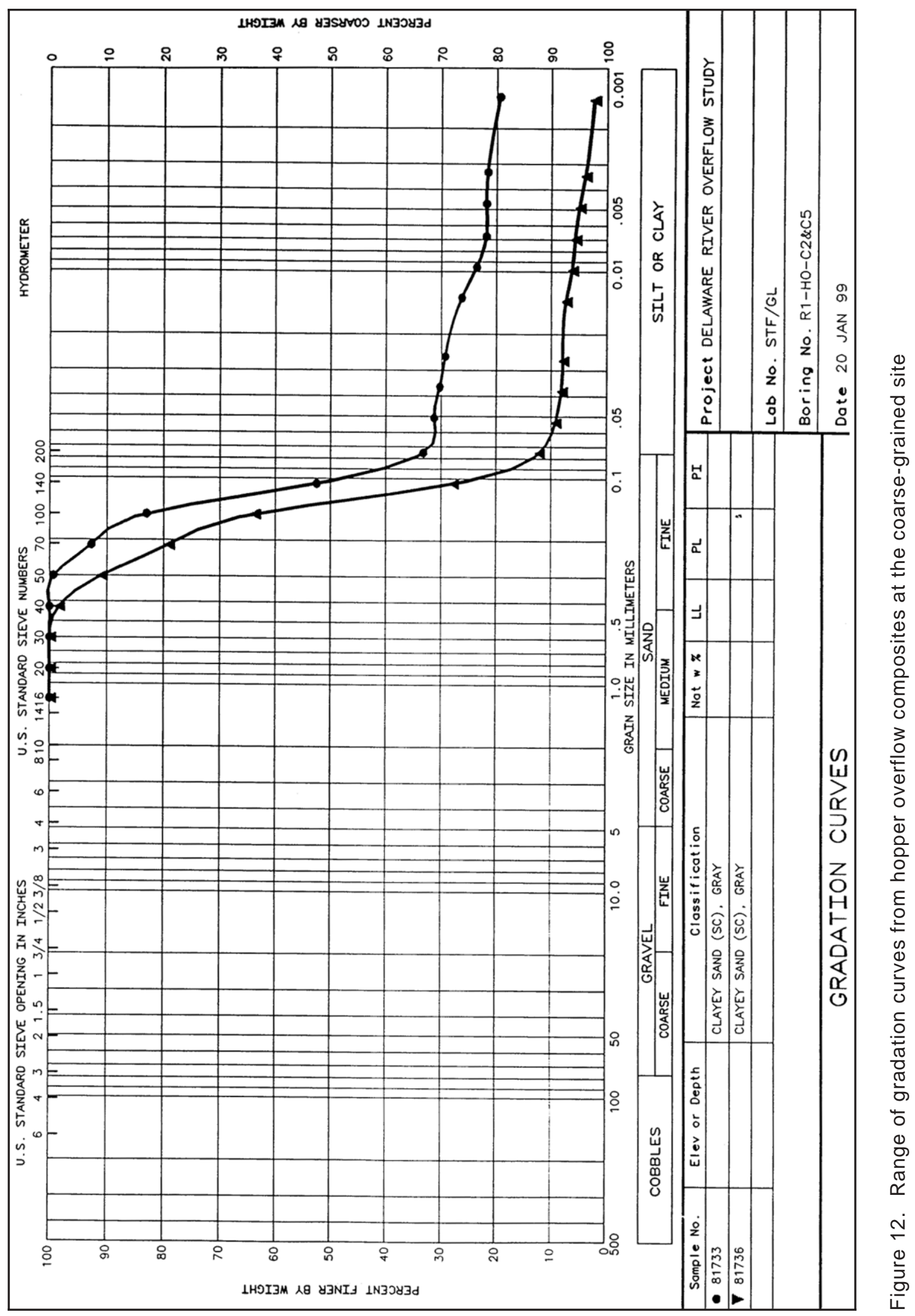




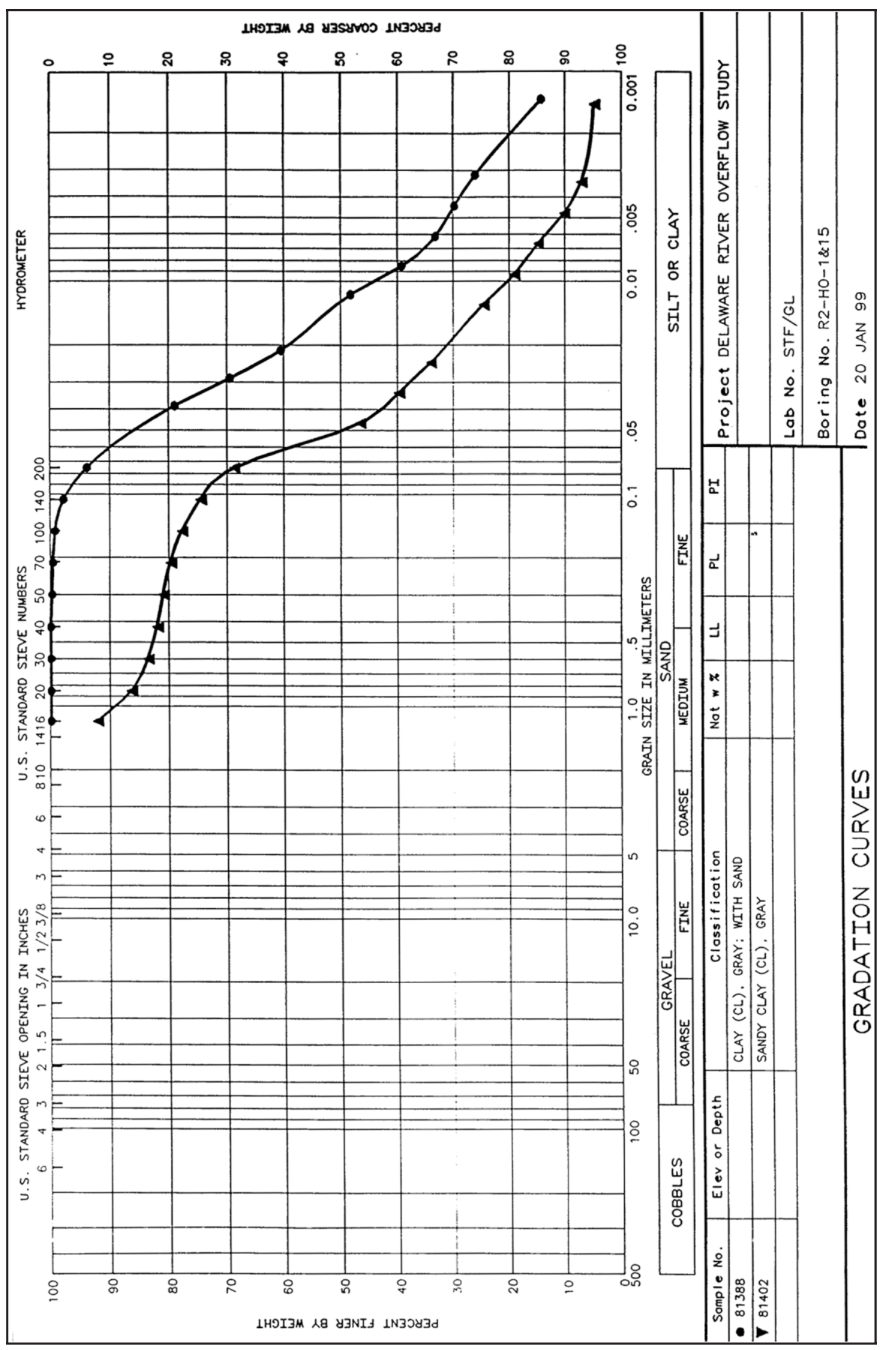

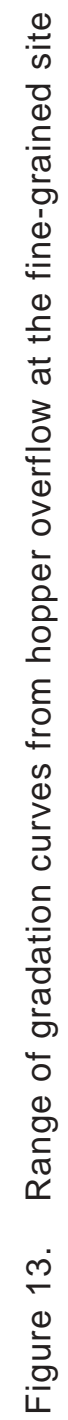




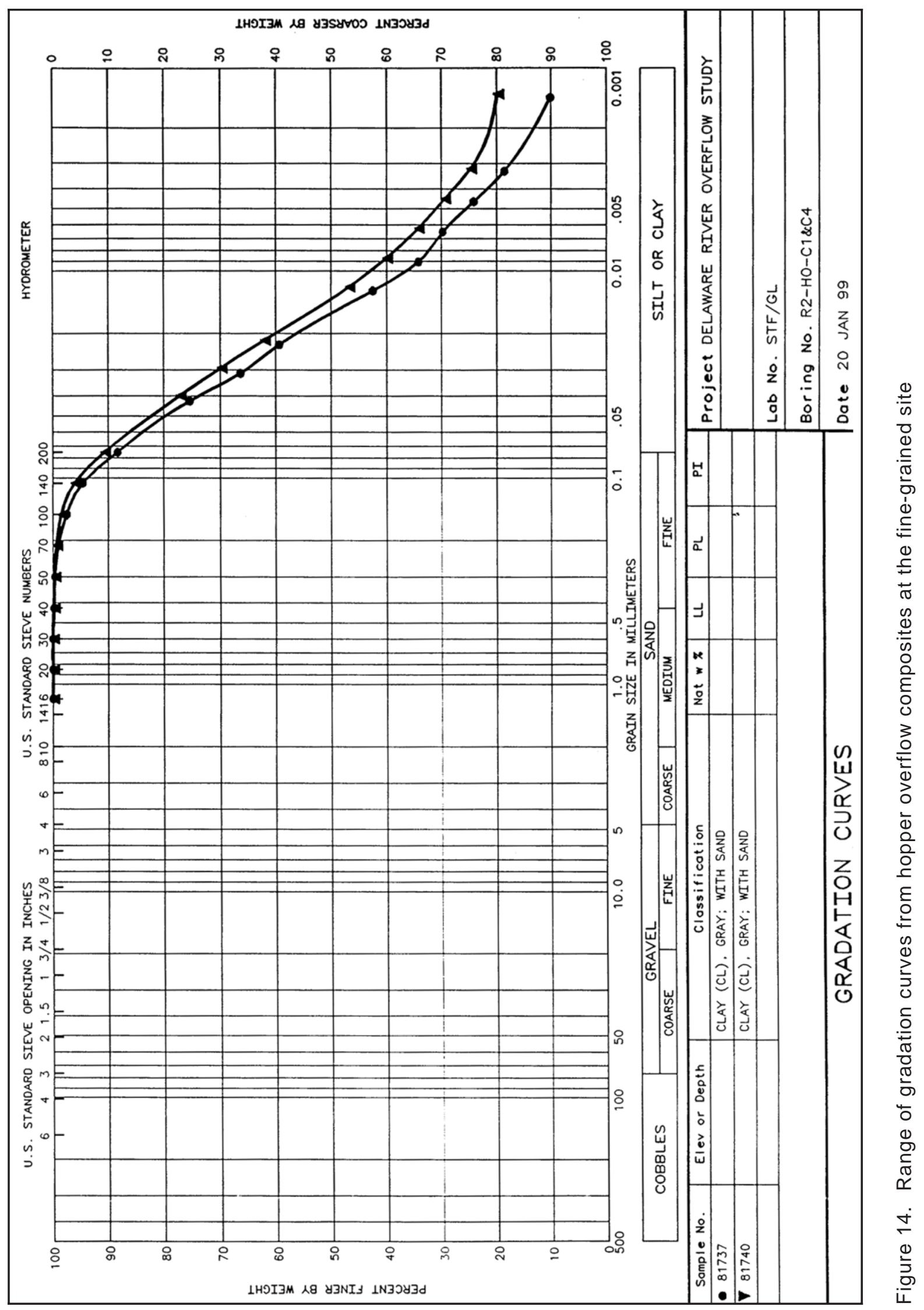




\section{Plume Monitoring}

Monitoring of the sediment plumes was accomplished using a boatmounted 1,200-kHz Broad-Band Acoustic Doppler Current Profiler (ADCP). The instrument collects velocity vectors in the water column together with backscatter levels to determine the position and relative intensity of the sediment plume. Along with the ADCP, a MicroLite recording instrument with an Optical Backscatterance (OBS) Sensor was towed by the vessel at a depth of $15 \mathrm{ft}$. The MicroLite recorded data at 0.5 -sec intervals. Navigation data for monitoring were obtained by a Starlink differential Global Positioning System (GPS). The GPS monitors the boat position from the starting and ending points along each transect.

\section{Coarse-grained site}

Transects were monitored in each test area to obtain the background levels of suspended materials prior to dredging activities. A period of 8 min following the dredge passing during nonoverflow dredging shows the level of suspended material to be returning to background levels. No lateral dispersion of the plume out of the channel was observed during the nonoverflow dredging operation.

During overflow dredging, a wider transect was performed to determine the lateral extent of the plume. No significant change above background levels could be detected. At 1-hr elapsed time following the end of the overflow dredging operation, the levels of suspended material returned to background conditions. Again, no lateral dispersion of the plume out of the channel area was observed. A complete analysis of the plume study can be found in Appendix B.

Figure 15 is a surface profile of the solids concentrations measured during nonoverflow and overflow conditions. Both sets of data fall within the minimum and maximum range of the background solids concentrations measured prior to dredging. Figure 16 is a middepth profile of the solids concentrations. Because of the narrow range between the measured values of the minimum and maximum range, both the nonoverflow and the overflow measured solids concentrations were above the maximum range. Figure 17 is a bottom profile of the solids concentrations and can be described much like that of the surface profile in that both sets of data fall within the minimum and maximum range of the background solids concentrations. In all three instances, there is not a significant difference in the solids concentrations measured during nonoverflow and the solids concentrations measured during overflow. Figure 18 shows that all solids concentrations measured during nonoverflow and overflow fell within the total minimum and maximum range measured in the background prior to dredging. 


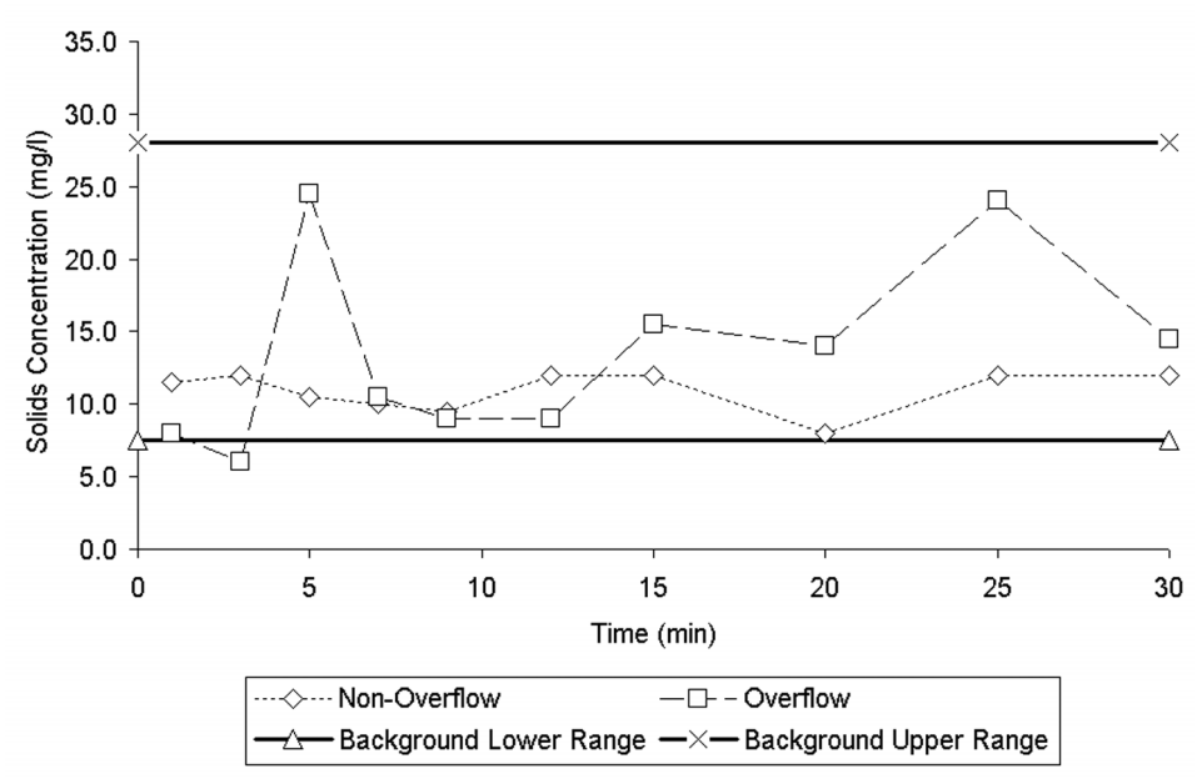

Figure 15. Plume solids concentrations at surface (coarse-grained material)

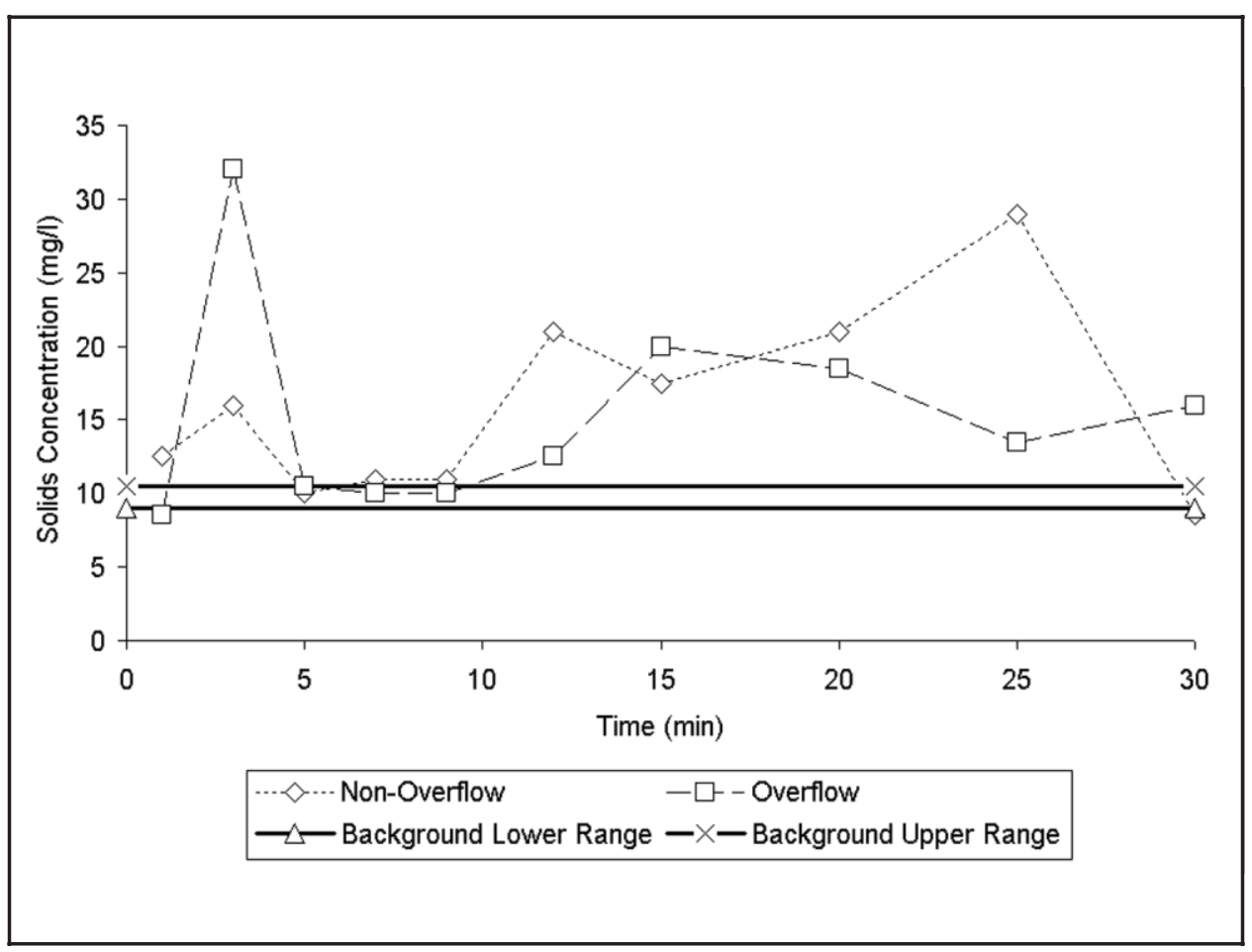

Figure 16. Plume solids concentrations at middepth (coarse-grained material) 


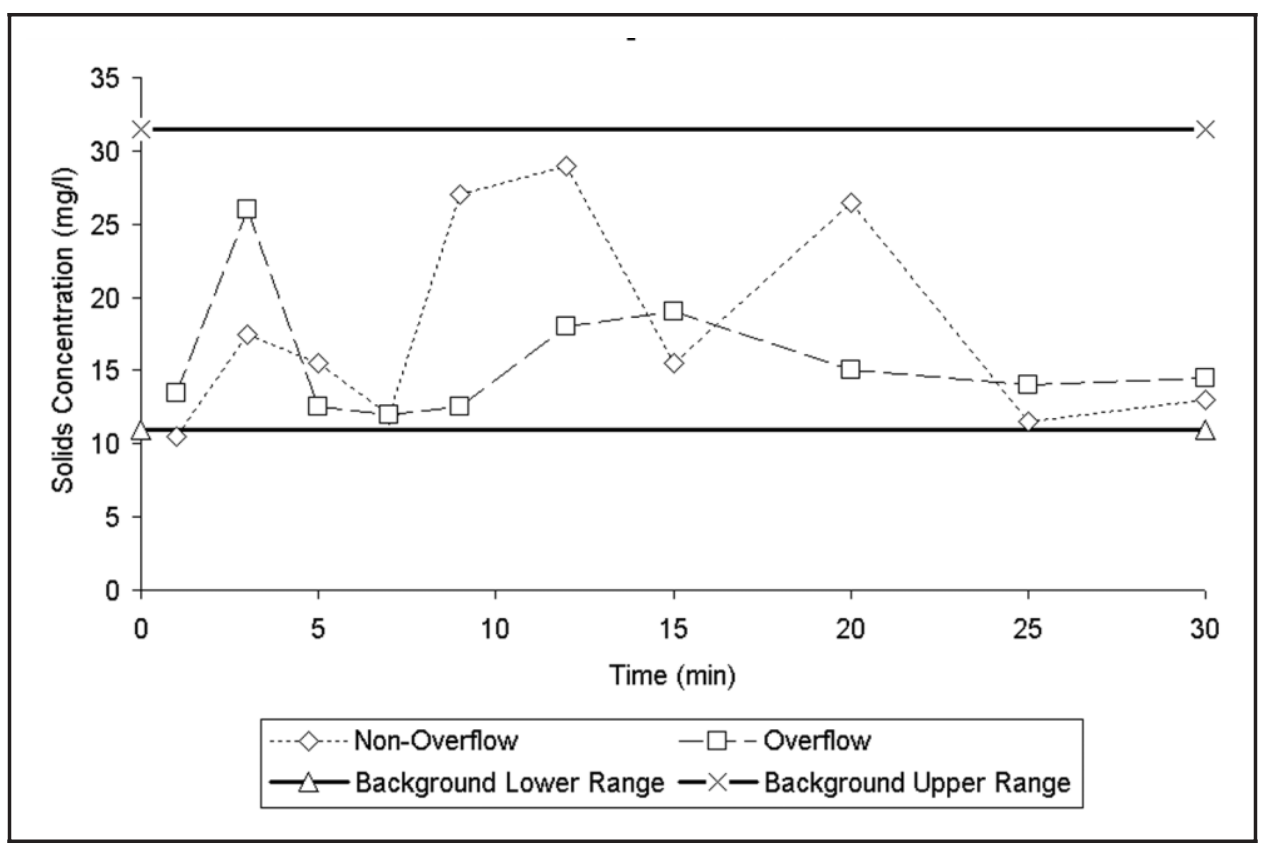

Figure 17. Plume solids concentrations at bottom (coarse-grained material)

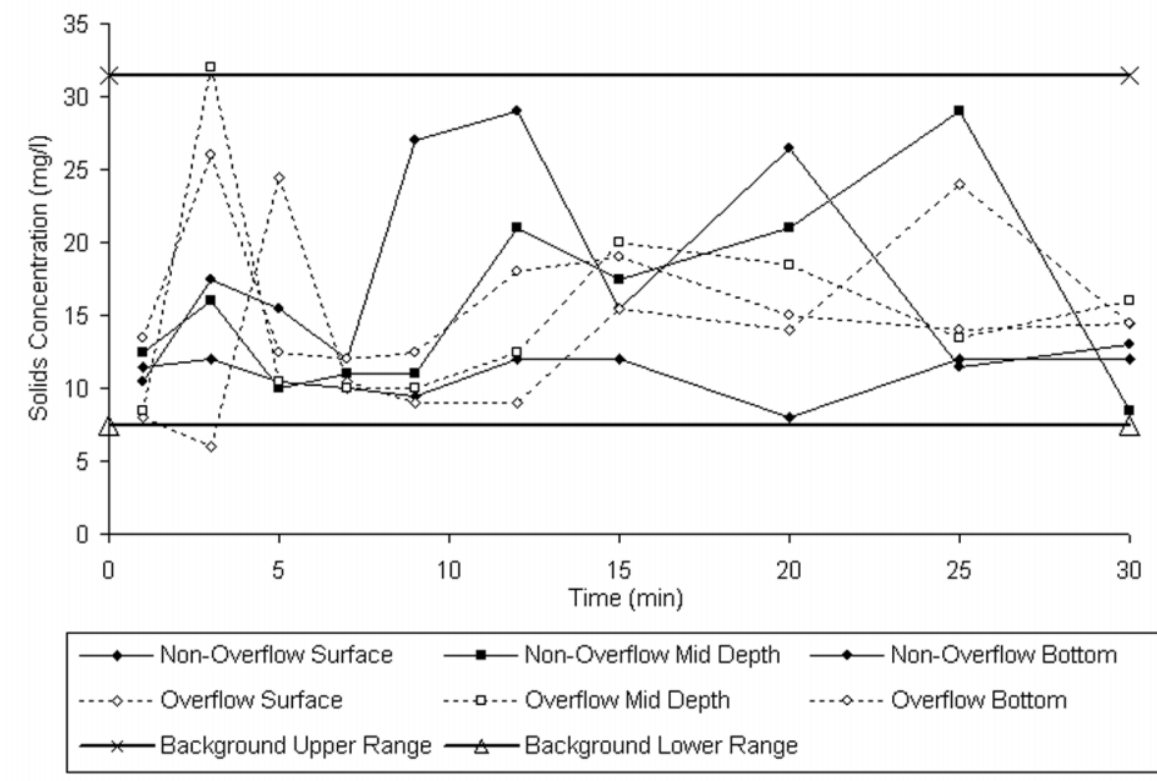

Figure 18. Plume solids concentrations at coarse-grained site 


\section{Fine-grained site}

During the nonoverflow dredging operation, the tidal flow in the dredging area reversed from flood flow to ebb flow conditions. This accounts for the relative change in observed background levels taken before the nonoverflow and overflow test dredging. At 19 min following the end of nonoverflow dredging, the levels of suspended material had returned to background conditions. Despite the change in direction of flow in the dredging area, no lateral movement of the plume beyond the channel limits was observed.

Immediately prior to overflow conditions, an increase in the background suspended material was observed. This increase is assumed to be the result of the increase in the ebb flow velocities and the resulting disturbance of bottom materials from near-bottom velocities and not dredge plume dispersion. When hopper overflow conditions began, the width of the transect was increased to observe the lateral extent of the dispersion of the dredge plume. After an elapsed time of $1 \mathrm{hr}$ following the completion of the overflow dredging operation, levels of suspended materials had returned to background conditions. As in the previous dredge operations, no lateral dispersion of the dredge plume beyond the channel limits was observed. A complete analysis of the plume study can be found in Appendix B.

Figure 19 shows the solids concentrations as measured at the surface during nonoverflow and overflow conditions. The overflow solids concentrations oscillate outside the maximum background solids concentration. Toward the end of overflow, the concentrations fall back within the background range. Figure 20 shows the solids concentration as measured at middepth. The same pattern as the surface profile is exhibited. Figure 21 shows the solids concentration as measured at the bottom. The nonoverflow solids concentrations remain within the measured range of the background; however, the overflow solids concentrations remain above the maximum background range throughout the duration of overflow. Figure 22 shows the maximum background range of solids concentration measured. The nonoverflow solids measured are well within the total range while the overflow solids concentrations oscillate outside the maximum range. This is consistent since 70 percent or more of the material is fine-grained and would settle slowly.

\section{Sedimentation Results}

\section{Coarse-grained site}

Sediment profile images from a total of 14 stations were analyzed from the coarse-grained site as shown in Figure 2 of Appendix C. There was evidence that recent sedimentation had occurred at several of the stations 


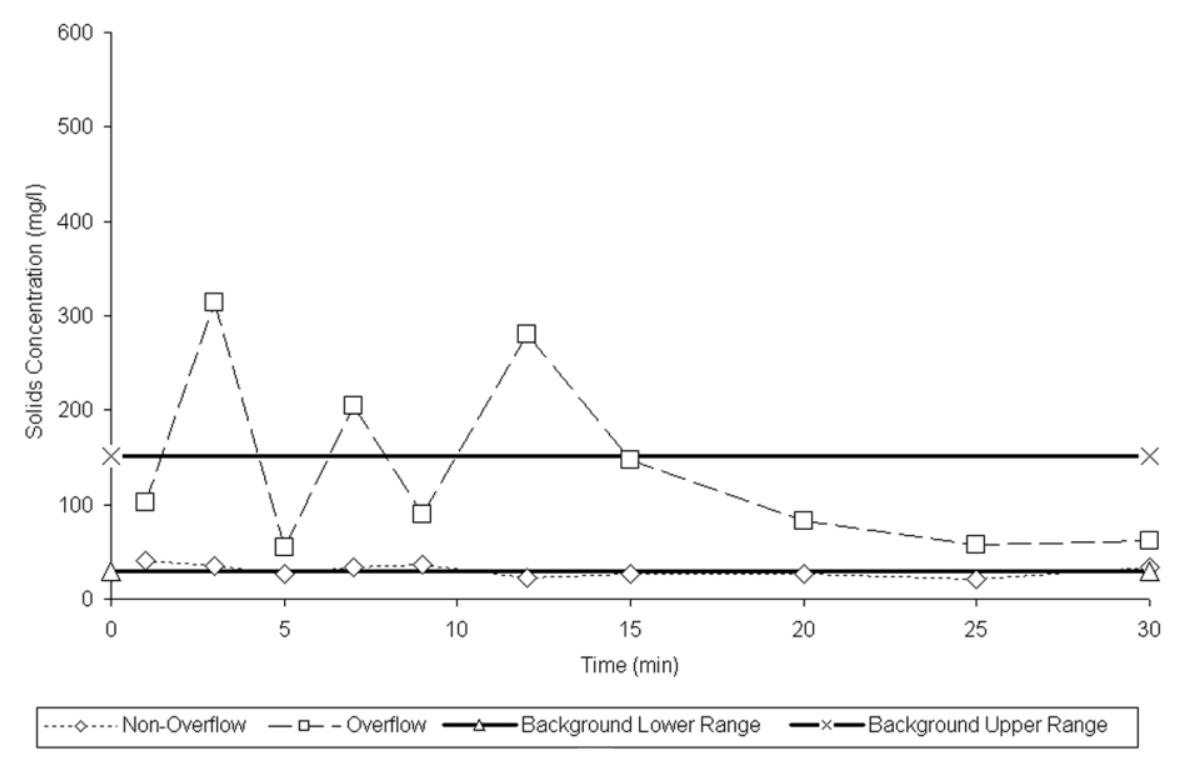

Figure 19. Plume solids concentrations at surface (fine-grained material)

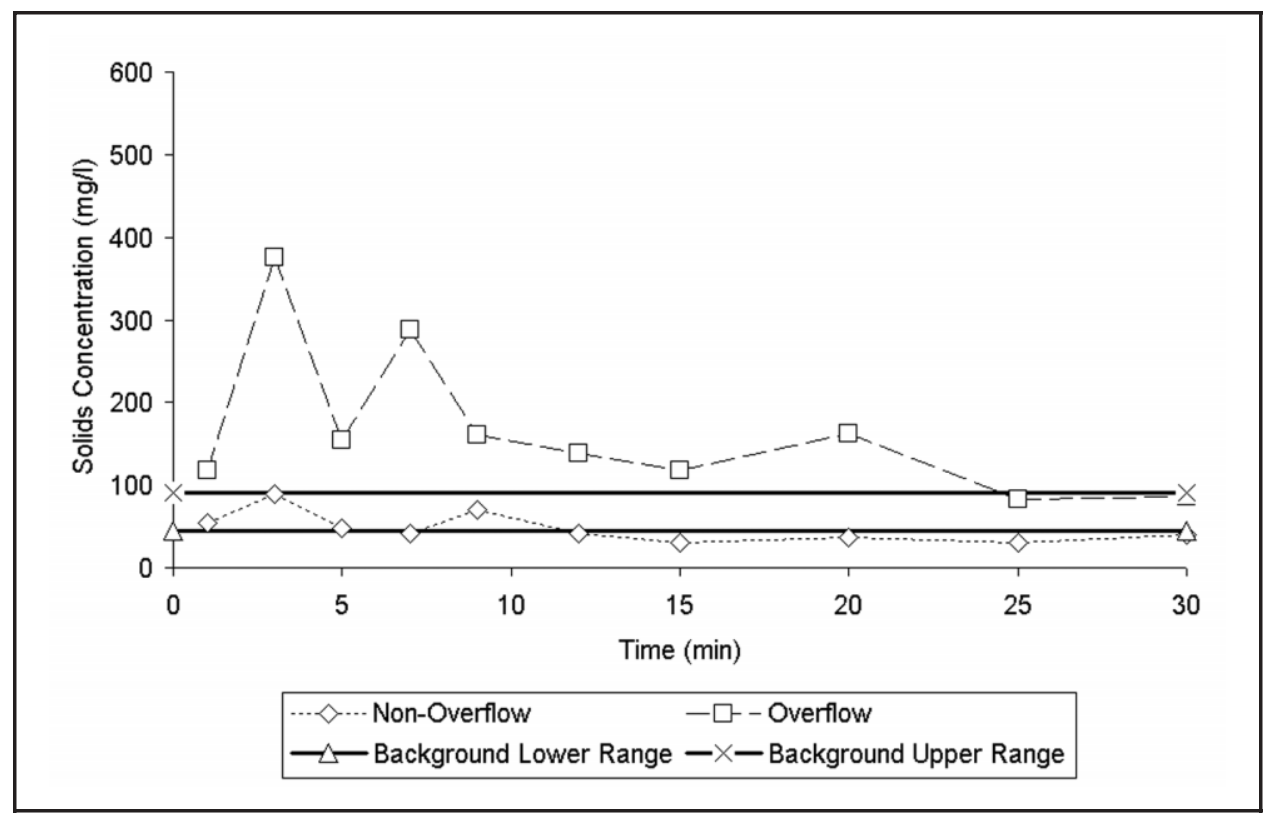

Figure 20. Plume solids concentrations at middepth (fine-grained material) 


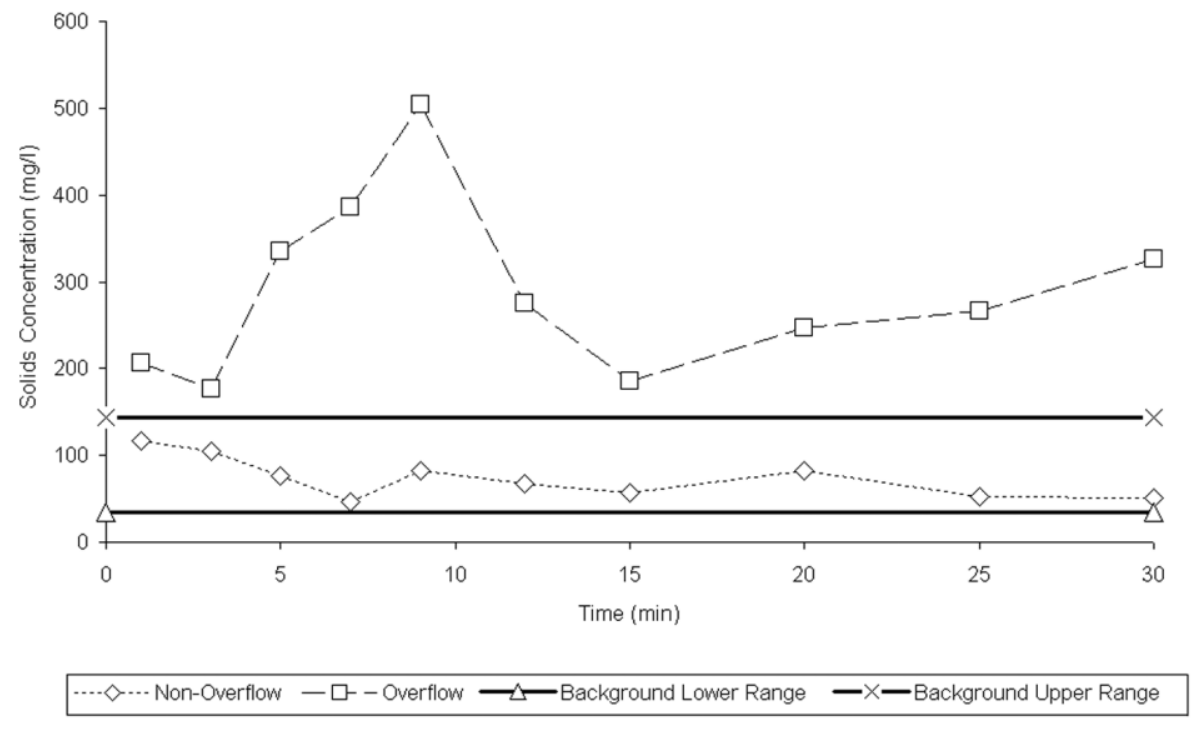

Figure 21. Plume solids concentrations at bottom (fine-grained material)
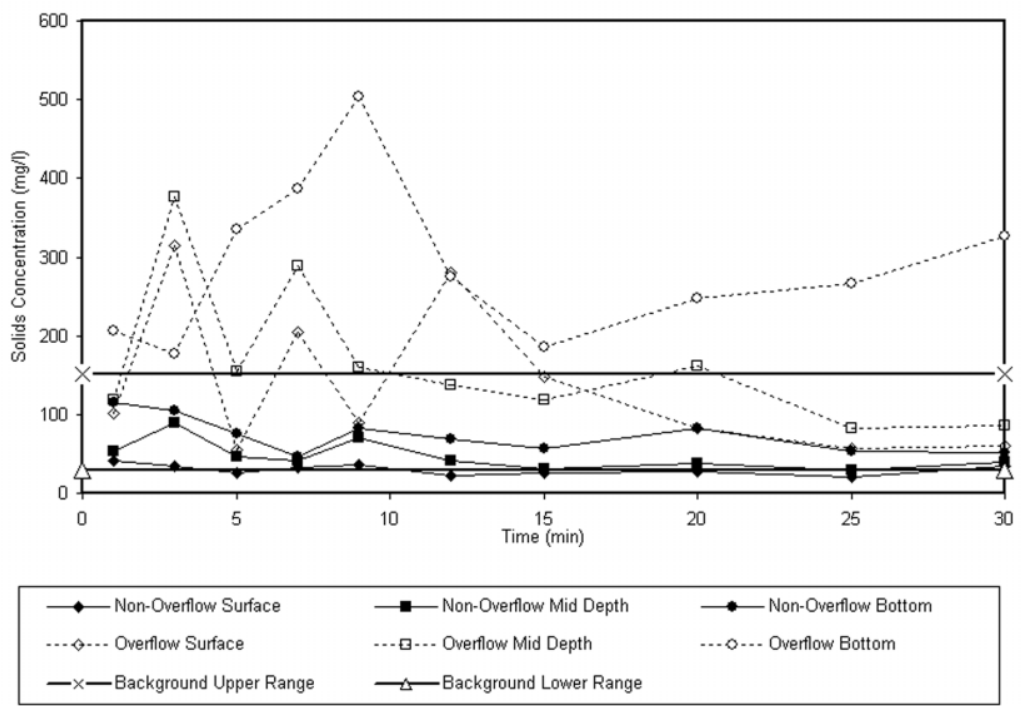

Figure 22. Plume solids concentrations of fine-grained material 
within the channel, possibly a result of the dredging operations. Gray colored suspended material, indicative of hopper overflow material, was observed at two of the stations. Four of the stations had layering from grain-size changes but are assumed to have occurred because of normal sediment transport processes rather than hopper overflow operations.

\section{Fine-grained site}

Sediment profile images from a total of 41 stations were analyzed from the fine-grained site as shown in Figure 3 of Appendix C. No evidence of recent physical disturbance was detected at any of the stations, but material that could have come from the hopper overflow was observed at one station. Five of the stations on the edge of the channel had grain-size layering with sands on the surface overlaying clayey sediments. Since the sediments in the channel were finer silts and clays, it was unlikely that the layers at the channel edge stations were the result of the dredging operations. Three of the stations on the edge of the channel had sediment layering with amphipod and worm tubes which could not have reestablished living position in the short interval between dredging and sampling. Flocculent sediment layers, thin layers of unconsolidated surface sediments, occurred at six shoal stations and one channel edge station. Based on their color tones, all flock layers appeared to be composed of background sediments and not hopper overflow or dredged material.

No indication of newly deposited dredged material was observed at stations outside the edge of the navigation channel at either study site. Although the sampling station coverage was not extensive, given the relatively short duration of the tests, the risk of significant sedimentation as a consequence of the hopper dredging operations appears largely restricted to the bottom and side slopes of the channel. The full report on the sedimentation analysis is attached as Appendix C.

\section{Standard Elutriate Tests}

The standard elutriate analysis was performed using the composited insitu sediment and site water. The purpose of the standard elutriate testing was to gain data on possible application of the test for prediction of overflow contaminant concentrations. The mean predicted dissolved values from the elutriates were calculated using the EFQUAL computer program, a module of the ADDAMS software package. ${ }^{1}$ The elutriate test was conducted using standard procedures. ${ }^{2}$

\footnotetext{
1 Palermo, M. R., and Schroder, P. R. (1991). "Documentation of the EFQUAL module for ADDAMS: Comparison of predicted effluent water quality with standards," Technical Note EEDP-06-13, U.S. Army Engineer Waterways Experiment Station, Vicksburg, MS

2 U.S. Environmental Protection Agency and U.S. Army Corps of Engineers. (1998).

"Evaluation of dredged material proposed for discharge in inland and near-coastal waters - Testing manual,” EPA-823-B-98-004, U.S. Environmental Protection Agency, Washington, DC.
} 


\section{Coarse-grained site}

At the coarse-grained site, background dissolved copper was the only contaminant of concern that was predicted to be above the standard (Table 2). The program predicted that copper would be discharged at $7 \mu \mathrm{g} / \mathrm{l}$ which is above the marine objective acute criteria but well below the background value of $13 \mu \mathrm{g} / \mathrm{l}$. Therefore, a dilution of the background with respect to copper would naturally occur as a result of the dredging operation, and a mixing zone would not be required. The actual value recorded at the hopper overflow (effluent) for copper was $5 \mu \mathrm{g} / \mathrm{l}$, which was below both the background and the standard of $5.3 \mu \mathrm{g} / \mathrm{l}$.

\section{Fine-grained site}

At the fine-grained site, the predicted dissolved value of selenium was $24.3 \mu \mathrm{g} / \mathrm{l}$ (Table 3). The more stringent acute value of the freshwater or marine stream quality standard for selenium is $20 \mu \mathrm{g} / \mathrm{l}$ and the background was $19 \mu \mathrm{g} / \mathrm{l}$. The actual value recorded at the hopper overflow for selenium was $14.2 \mu \mathrm{g} / \mathrm{l}$, which is below the criteria and the background value, which would indicate a natural dilution of the contaminant of concern during dredging operations. Again, because of this natural dilution, a mixing zone would not be required.

At both reaches, the predicted elutriate values appear somewhat conservative when compared with the overflow values. The close agreement of the elutriate values with the actual overflow values (Tables 2 and 3 ) indicate that the elutriate test can be used as a valid predictor of overflow quality for the Delaware River. Summaries of the standard elutriate and predicted effluent quality results for the two sites can be found in Tables 2 and 3. A complete listing of the water quality, sediment, and elutriate analysis for both sites can be found in Appendix A.

\section{Technical Findings of a 96-hr Water Column Bioassay}

This test was performed to determine the possible biological effects of water column exposure to Delaware River overflow. Two species were used in performing the bioassays, the mysid shrimp, a crustacean species, Mysidopsis bahia, and the inland silverside, a fish species, Menidia beryllina. These species were selected based on conversations with personnel from the Delaware Department of Natural Resources and Environmental Control. The filtered elutriate was diluted with standard laboratory control seawater (6-ppt salinity for the fine-grained site and 30-ppt salinity for the coarse-grained site) to yield the following concentrations: 0-, 6.25-, 12.5-, 25-, 50-, and 100-percent elutriate. Each treatment was replicated five 


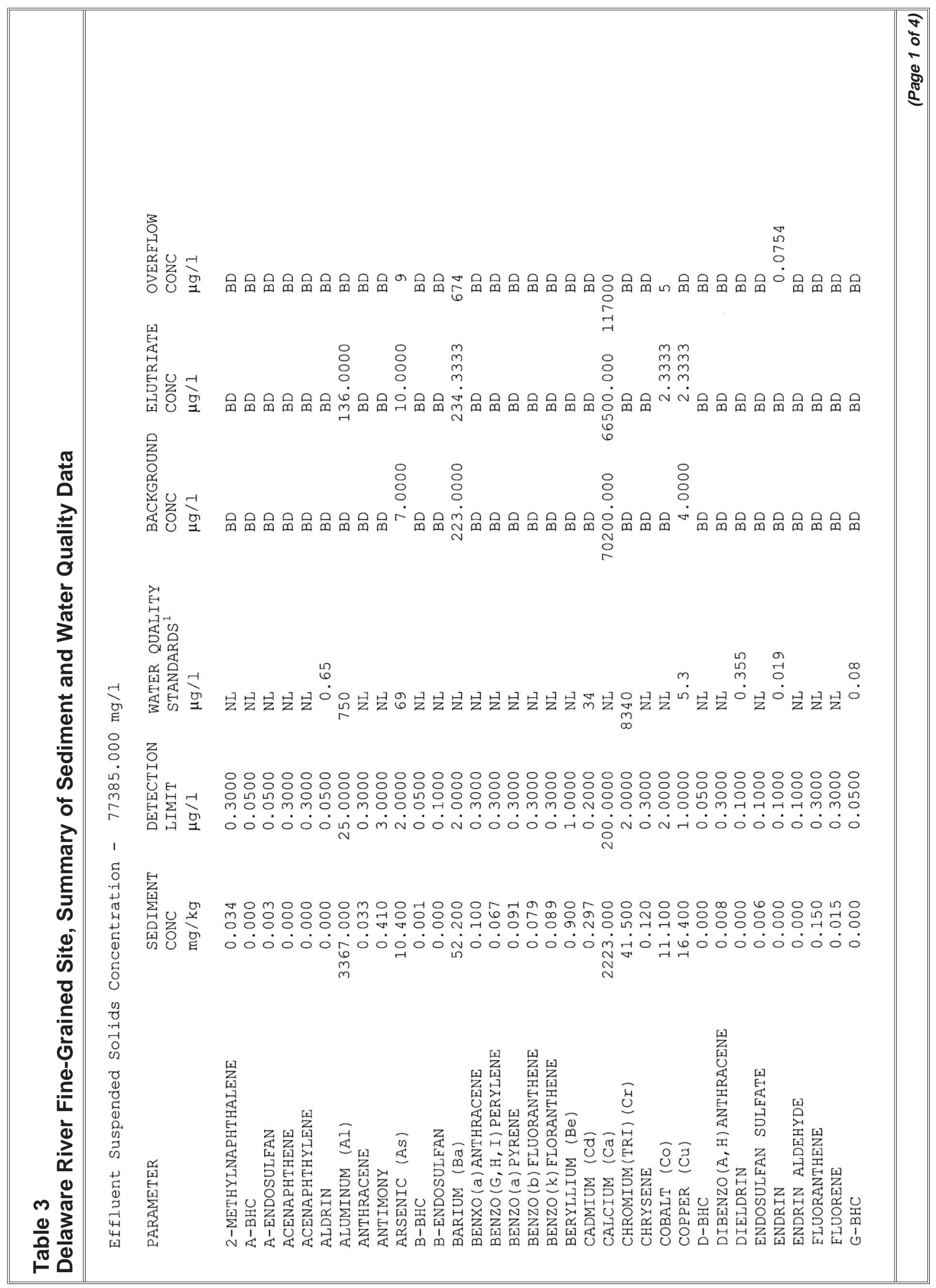




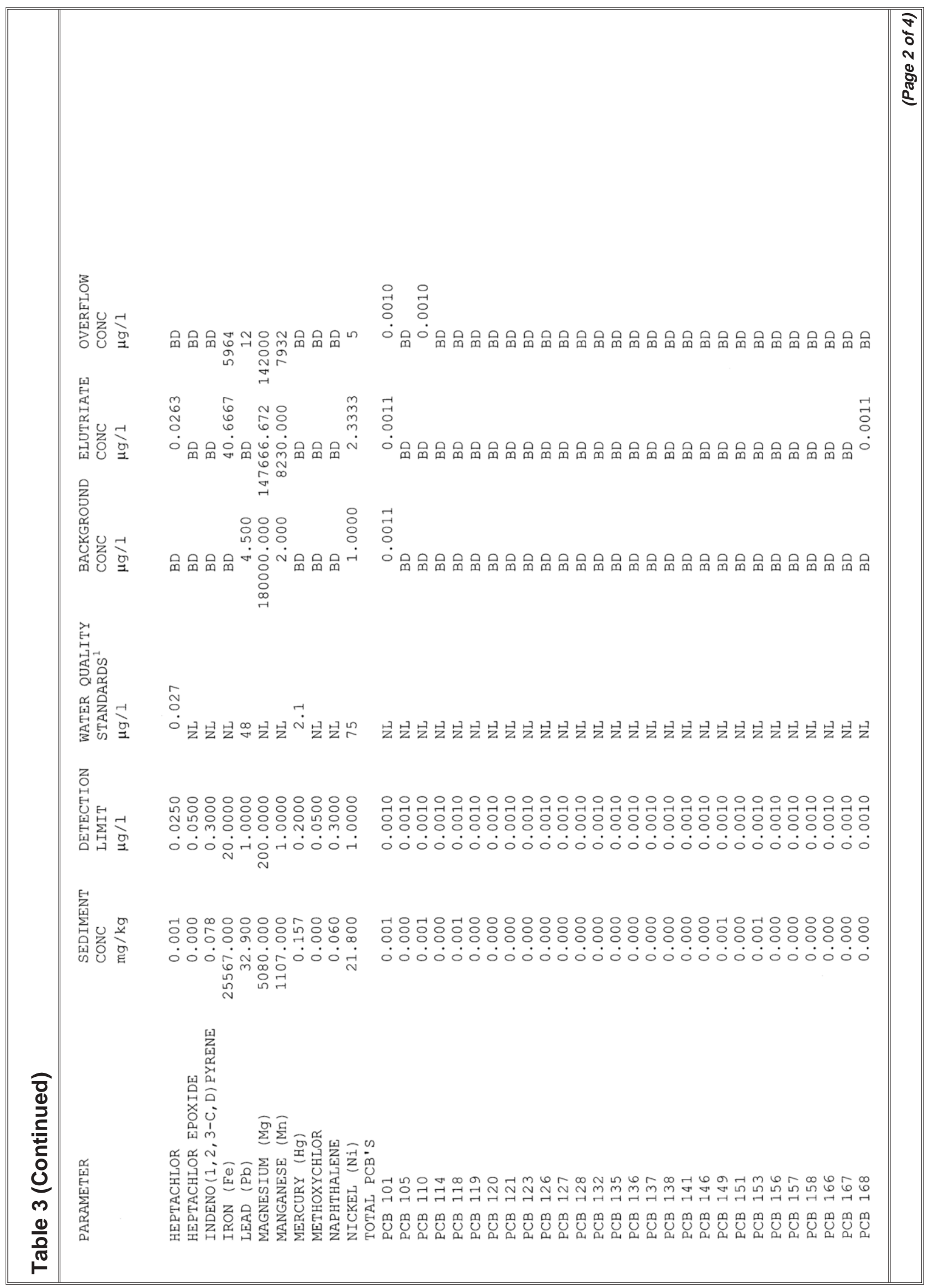




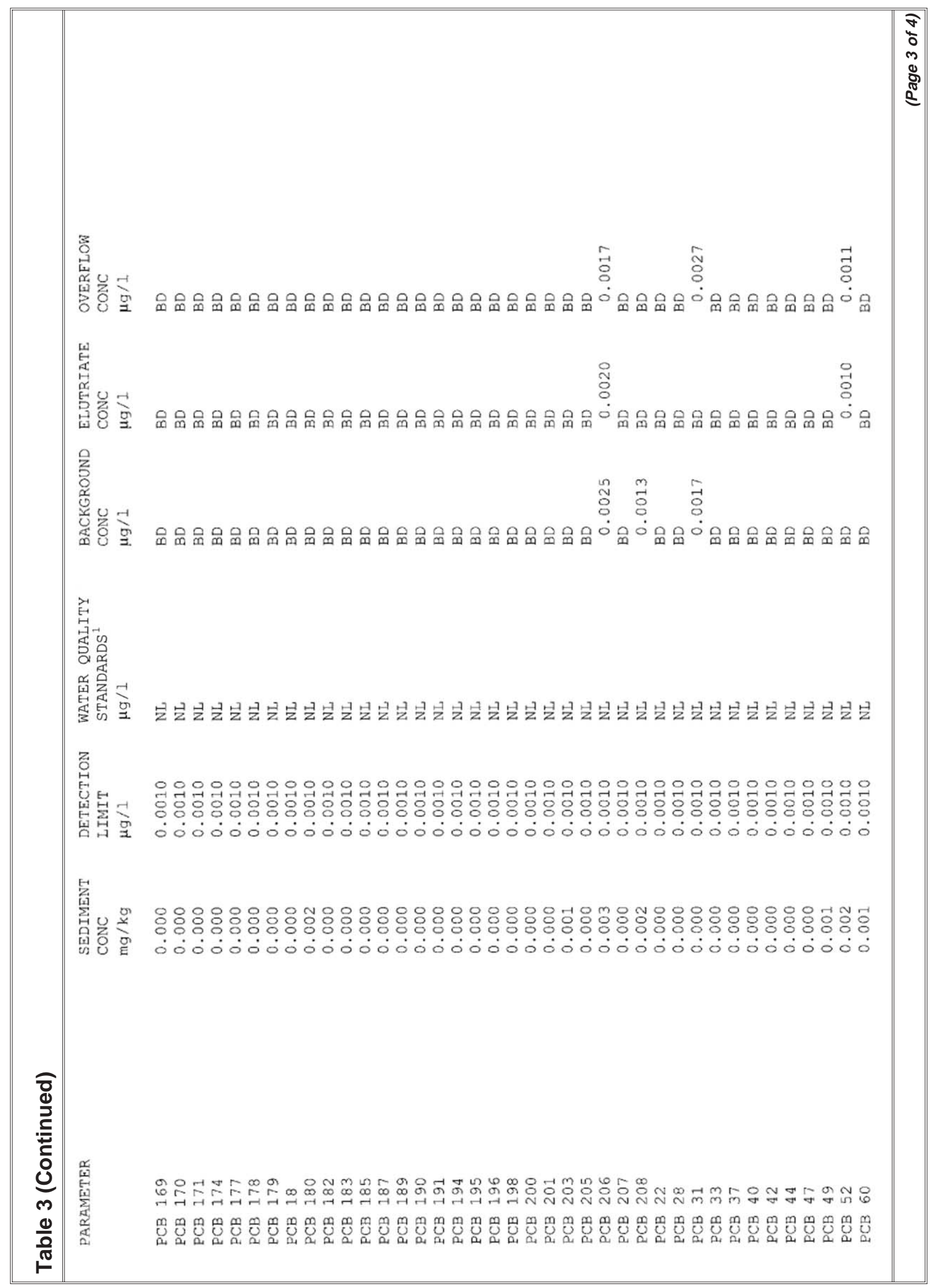




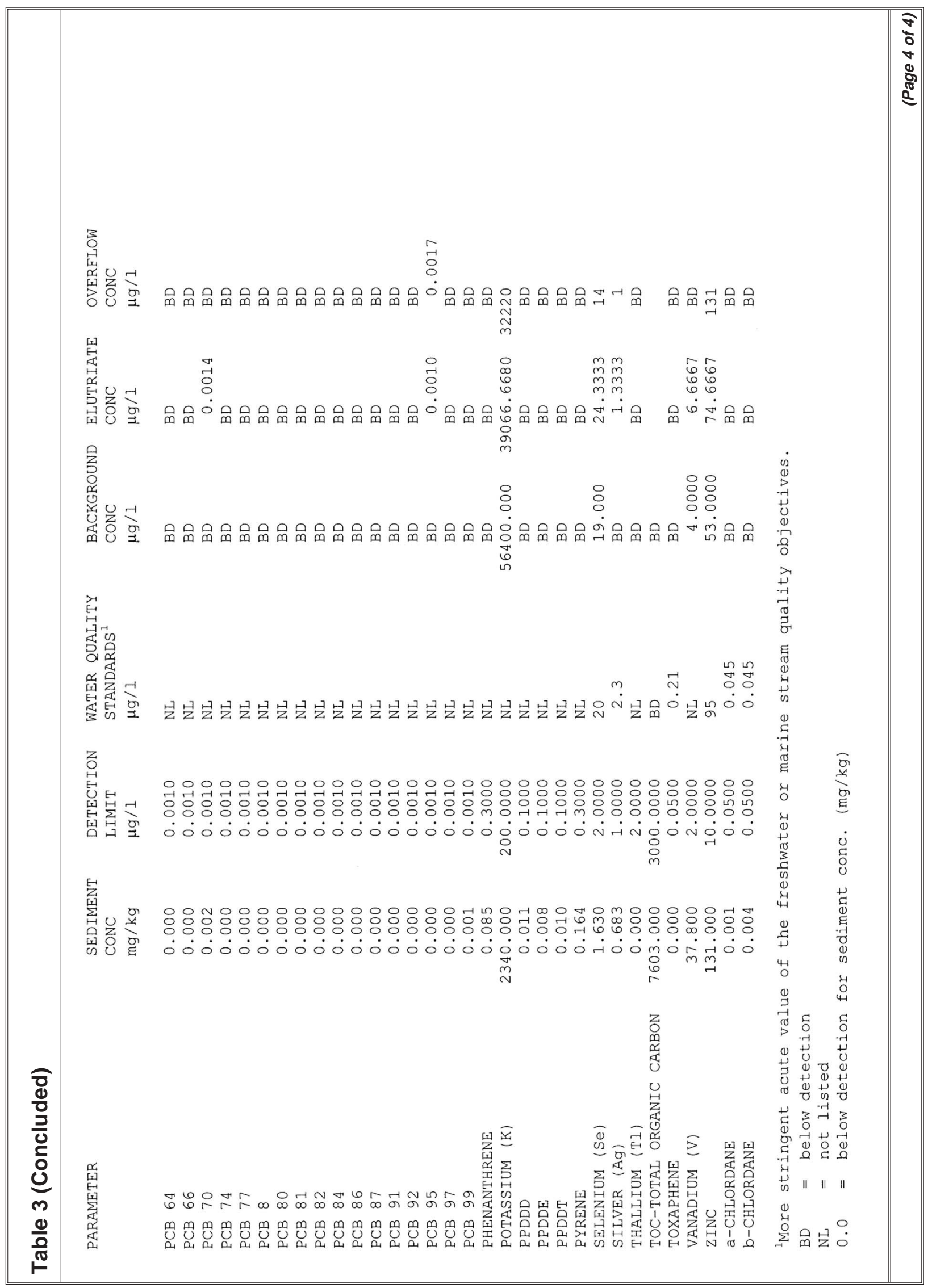


times. The trimmed Spearman-Karber method was used to calculate $\mathrm{LC}_{50}$ values. The bioassay report is attached as Appendix D.

\section{Coarse-grained site}

Survival in test concentrations from the coarse-grained site ranged from 100 to 88 percent for Mysidopsis bahia and from 88 to 68 percent for Menidia beryllina. Exposures in elutriate test concentrations from the coarsegrained site did not adversely affect survival of either test species. Since neither test species had mortality values greater than 50 percent, an $\mathrm{LC}_{50}$ value could not be calculated.

\section{Fine-grained site}

Survival in test concentrations from the fine-grained site ranged from 90 to 0 percent with 0 -percent survival in the 50- and 100-percent exposures for Mysidopsis bahia. Survival for Menidia beryllina ranged from 98 to 0 percent with 4 - to 0 -percent survival in the 50- and 100-percent elutriate treatments. An $\mathrm{LC}_{50}$ value of 30.04 percent was calculated for Mysidopsis bahia and an $\mathrm{LC}_{50}$ value of 31.66 percent was calculated for Menidia beryllina. Mortality observed from exposures in elutriate test concentrations was attributed to the high level of $\mathrm{NH}_{3}$. In the short term, high levels of $\mathrm{NH}_{3}$ are common in predominately fine-grained sites during dredging operations. 


\section{Summary and Conclusions}

Based on the results of the study, the following conclusions can be made:

a. Loading data at the coarse-grained site shows a gain of 130 percent over a period of $57 \mathrm{~min}$ after overflow began. Based on the roundtrip travel time required to the disposal site and the amount of material retained in the hopper, rates of return greater than 50 percent may be realized for the coarse-grained material. Loading data at the fine-grained site show a gain of 18 percent over a period of 21 min after overflow began. Based on the round-trip travel time required to the pump-out site and the amount of material retained in the hopper, there was no economic benefit to overflow for the fine-grained material. In both instances, rates of return are also based on the assumption that all material in the overflow will return to the channel and will require redredging.

$b$. Using the same economic assumptions as discussed above, about a 20-percent return may be realized from a material containing about 60 percent sand and about a 40 -percent return may be realized from a material containing about 80 percent sand.

$c$. Based on the water chemistry analysis at the two sites, no contaminants of concern caused a problem because of the dredging operation. None of the contaminants of concern exceeded water quality objectives in the overflow at the coarse-grained site. At the coarsegrained site, only dissolved copper was above the standard in the background. Samples taken for dissolved copper at the hopper overflow, however, were within standards. This indicates a scavenging of the metal by the suspended material occurred during the dredging and overflow process. At the fine-grained site, only zinc and endrin were measured at the overflow to be above the standard. However, the predicted elutriate for both zinc and endrin were measured at below detection levels.

$d$. The plume study results showed that the coarse-grained material settled quite rapidly and that no lateral dispersion of the plume out of the channel was observed. No significant change above background levels could be detected. At $1 \mathrm{hr}$ elapsed time following 
the end of the overflow dredging operation, the levels of suspended material had returned to background conditions. At the finegrained site, an increase in the suspended material was observed. However, after an elapsed time of $1 \mathrm{hr}$ following the completion of the overflow dredging operation, levels of suspended materials had returned to background conditions. Again, no lateral dispersion of the dredge plume beyond the channel limits was observed.

$e$. The sedimentation portion of the study confirmed what was observed during the plume study. At the coarse-grained site, there was evidence that recent sedimentation had occurred at several of the stations, possibly a result of dredging operations. But no indication of newly deposited dredged material was observed at stations outside the edge of the navigation channel. At the fine-grained site, some sediment layering was found even though no evidence of recent physical disturbance was detected at any of the stations. Again, no indication of newly deposited dredged material was observed at stations outside the edge of the navigation channel.

$f$. Although the sampling station coverage was not extensive, the risk of significant sedimentation as a consequence of the hopper dredging operations appears to be restricted to the bottom and side slopes of the channel.

g. The elutriate test results were consistent with and slightly conservative as compared to the overflow samples, indicating that the elutriate test is a valid prediction of overflow quality for the Delaware system.

$h$. The bioassay analysis showed no adverse effects to exposures of fish and crustaceans species being exposed to the elutriate samples from the coarse-grained site. Some species mortality were observed using elutriates from the fine-grained site, but was determined to be caused from high levels of $\mathrm{NH}_{3}$, which is a common short-term by-product of dredging in fine-grained material.

$i$. The overall results of the study indicate that overflow meets the applicable water quality objectives and has no measurable physical impact outside the navigation channels. The loading data indicate that overflow in coarse-grained reaches results in significant load gains, while load gains in fine-grained reaches are small. Based on these results, overflow in coarse-grained reaches should be considered for future operations. 


\section{Appendix A Delaware River Sediment and Water Quality Analysis}

Delaware River Sediment and Water Quality Analysis (Coarse and FineGrained Sites)

$\begin{array}{ll}\text { Metscoar } & \text { - Metals (Coarse-Grained Site) } \\ \text { PAHscoar } & \text { - PAH's (Coarse-Grained Site) } \\ \text { Pestcoar } & \text { - Pesticides (Coarse-Grained Site) } \\ \text { PCBscoar } & \text { - PCB's (Coarse-Grained Site) } \\ \text { Tsscoar } & \text { - Total Suspended Solids (Coarse-Grained Site) } \\ \text { Nutcoar } & \text { - Nutrients (Coarse-Grained Site) } \\ \text { Spgrcoar } & \text { - Specific Gravity and \%Moisture (Coarse-Grained Site) } \\ \text { Metsfine } & \text { - Metals (Fine-Grained Site) } \\ \text { PAHsfine } & \text { - PAH's (Fine-Grained Site) } \\ \text { Pestfine } & \text { - Pesticides (Fine-Grained Site) } \\ \text { PCBsfine } & \text { - PCB's (Fine-Grained Site) } \\ \text { Tssfine } & \text { - Total Suspended Solids (Fine-Grained Site) } \\ \text { Nutfine } & \text { - Nutrients (Fine-Grained Site) } \\ \text { Spgrfine } & \text { - Specific Gravity and \% Moisture (Fine-Grained Site) }\end{array}$


Metscoar

Delaware River Water Analysis (Coarse-Grained Site)

TYPE ID

Detection Limit (mg/l)

Plume Monitoring

$\begin{array}{lll}\text { Water } & 80827 & \text { Background, dissolved } \\ \text { Water } & 80735 & \text { Background, }\end{array}$

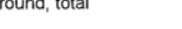

$\begin{array}{lll}\text { Water } & 80828 & 0-10 \mathrm{~min}, \text { overflow, dissolved } \\ \text { Water } & 80829 & 10-20 \mathrm{~min}, \text { overflow, dissolved }\end{array}$

$\begin{array}{lll}\text { Water } & 80829 & 10-20 \mathrm{~min}, \text { overflow, dissolved } \\ \text { Water } & 80830 & 20-30 \mathrm{~min}, \text { overflow, dissolved } \\ \text { Water } & 80736 & 0-10 \mathrm{~min} \text {, overflow, total }\end{array}$

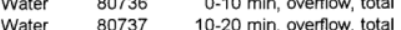

Water $\quad 80738 \quad 20-30 \mathrm{~min}$, overflow, total

$\begin{array}{llr}\text { Water } & 80831 & \text { 0-10 min, non-overflow, dissolved } \\ \text { Water } & 80832 & 10-20 \mathrm{~min}, \text { non-overflow, dissolved }\end{array}$

$\begin{array}{lll}\text { Water } & 80832 & 10-20 \mathrm{~min} \text {, non-overflow, dissolved } \\ \text { Water } & 80833 & 20-30 \mathrm{~min} \text {, non-overflow, dissolved }\end{array}$

$\begin{array}{llr}\text { Water } & 80833 & 20-30 \mathrm{~min}, \text { non-overflow, dissolv } \\ \text { Water } & 80739 & 0-10 \mathrm{~min} \text {, non-overflow, total }\end{array}$

$\begin{array}{llr}\text { Water } & 80739 & 0-10 \mathrm{~min} \text {, non-overflow, total } \\ \text { Water } & 80740 & 10-20 \mathrm{~min} \text {, non-overflow, total }\end{array}$

$\begin{array}{lll}\text { Water } & 80740 & 10-20 \mathrm{~min}, \text { non-overflow, total } \\ \text { Water } & 80741 & 20-30 \mathrm{~min} \text {, non-overflow, total }\end{array}$

$\begin{array}{cc} & \\ \text { Water } & 80780 \quad 3 \& 6 \text { Inflow Monitoring }\end{array}$

Water $3 \& 6 \mathrm{~min}$, dissolved

$\begin{array}{lll}\text { Water } & 80781 & 9 \& 12 \mathrm{~min} \text {, dissolved } \\ \text { Water } & 80782 & 15 \& 18 \mathrm{~min} \text {, dissolved }\end{array}$

Water $80783 \quad 21824 \mathrm{~min}$, dissolved

Water $\quad 80784 \quad 27830 \mathrm{~min}$, dissolve

Water 80668

Water $\quad 80669$

$\begin{array}{ll}\text { Water } \quad 80670 \\ \text { Water } & 80671\end{array}$

$\begin{array}{ll}\text { Water } & 80671 \\ & 80672\end{array}$

3\& $6 \mathrm{~min}$, total

$9 \& 12 \mathrm{~min}$, total

$15 \& 18 \mathrm{~min}$, total

$21 \& 24 \mathrm{~min}$, total
$27 \& 30 \mathrm{~min}$, total

$\begin{array}{lll} & \text { Hopper Overflow Monitoring } \\ \text { Water } & 80785 \quad 2 \& 4 \mathrm{~min} \text {, dissolved }\end{array}$

Water $80786 \quad 6 \& 8 \mathrm{~min}$, dissolved

Water $\quad 80787 \quad 10 \% 12 \mathrm{~min}$, dissolved

Water $\quad 80788 \quad 14816 \mathrm{~min}$, dissolved

$\begin{array}{lll}\text { Water } & 80789 & 18820 \mathrm{~min} \text {, dissolv } \\ \text { Water } & 80674 & 284 \mathrm{~min} \text {, total }\end{array}$

$\begin{array}{lll}\text { Water } & 80674 & 2 \& 4 \mathrm{~min} \text {, total } \\ \text { Water } & 80675 & 6 \& 8 \mathrm{~min} \text {, total }\end{array}$

Water $\quad 80676 \quad 10812 \mathrm{~min}$, total

Water $80677 \quad 14 \& 16 \mathrm{~min}$, total

Water $80678 \quad 18820 \mathrm{~min}$, total

Water $81648 \quad$ Site Water

Water $81648 \quad$ Sample 1 Total

Water 81649 Sample 2 Total

(2)

Water $81654 \quad$ Elutriate

Water 81655 Sample 2 Dissolved

Water $81656 \quad$ Sample 3 Dissolve

Water $81651 \quad$ Sample 1 Total

$\begin{array}{lll}\text { Water } & 81652 & \text { Sample 2 Total } \\ \text { Water } & 81653 & \text { Sample 3 Total }\end{array}$

SAMPLE SAMPLE DESCRIPTION

TYPE ID

$\begin{array}{lll} & & \begin{array}{l}\text { Detection Limit }(\mathrm{mg} / \mathrm{kg}) \\ \text { Insitu Sediment }\end{array} \\ \text { Sediment } & 81726 & \text { Sample \#1 } \\ \text { Sediment } & 81727 & \text { Sample \#2 } \\ \text { Sediment } 81728 & \text { Sample \#3 }\end{array}$

$\begin{array}{rr}\text { SB } & \text { AS } \\ & \\ 0.003 & 0.002 \\ & \\ & \\ \mathbf{0 . 0 0 3} & 0.044 \\ \mathbf{0 . 0 0 3} & 0.044 \\ & \\ \mathbf{0 . 0 0 3} & 0.044 \\ \mathbf{0 . 0 0 3} & 0.044 \\ \mathbf{0 . 0 0 3} & 0.046 \\ \mathbf{0 . 0 0 6} & 0.045 \\ \mathbf{0 . 0 0 3} & 0.046 \\ \mathbf{0 . 0 0 3} & 0.045 \\ \mathbf{0 . 0 0 3} & 0.047 \\ \mathbf{0 . 0 0 3} & 0.048 \\ \mathbf{0 . 0 0 3} & 0.047 \\ \mathbf{0 . 0 0 3} & 0.044 \\ \mathbf{0 . 0 0 3} & 0.048 \\ \mathbf{0 . 0 0 3} & 0.044\end{array}$

$\begin{array}{ll}0.002 & 0.0002 \\ & \\ 0.002 & 0.0002 \\ 0.001 & 0.0002 \\ & \\ 0.002 & 0.0002 \\ 0.002 & 0.0002 \\ 0.002 & 0.0002 \\ 0.001 & 0.0002 \\ 0.001 & 0.0002 \\ 0.001 & 0.0002 \\ & \\ 0.002 & 0.0002 \\ 0.002 & 0.0004 \\ 0.002 & 0.0002 \\ 0.001 & 0.0002 \\ 0.001 & 0.0002 \\ 0.001 & 0.0002\end{array}$

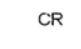

$\mathrm{cu}$

PB

HG

$0.003 \quad 0.044$

$0.003 \quad 0.051$

0.003

0.003

0.003
0.003

0.003
0.003

0.003
0.003

0.003
0.003
0.003

0.003
0.003

0.045

0.046

0.048
0.050

0.076

0.070
0.069

0.069
0.105

0.115

0.001

0.001

0.001
0.001

0.001
0.001
0.002

0.002
0.002

0.002
0.002

0.007
0.008

$\mathbf{0 . 0 0 0 2}$
$\mathbf{0 . 0 0 0 2}$
$\mathbf{0 . 0 0 0 2}$
$\mathbf{0 . 0 0 0 2}$
$\mathbf{0 . 0 0 0 2}$
0.0029
0.0010
0.0005
0.0008
0.0009

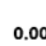

0.003

$0.045 \quad 0.001$

0.048
0.047
0.046

0.046
0.045

0.047

0.052

0.066

0.056
0.046

0.001
0.00
0.00
0.00
0.00
0.00
0.00
0.001
0.001

0.0002

\subsection{0002}

0.0002

0.0002

0.0027

0.0013

0.0034

$\mathbf{0 . 0 0 0 2}$

$\begin{array}{llll}0.002 & 0.001 & 0.001 & 0.00020\end{array}$

$\begin{array}{llll}\mathbf{0 . 0 0 2} & 0.013 & \mathbf{0 . 0 0 1} & \mathbf{0 . 0 0 0 2 0} \\ \mathbf{0 . 0 0 2} & 0.012 & 0.003 & \mathbf{0 . 0 0 0 2 0}\end{array}$

$\begin{array}{llll}0.002 & 0.011 & 0.001 & \mathbf{0 . 0 0 0 2 0}\end{array}$

$\begin{array}{llll}0.002 & 0.010 & 0.001 & \mathbf{0 . 0 0 0 2 0}\end{array}$

$\begin{array}{llll}0.002 & 0.011 & 0.001 & 0.00020 \\ 0.002 & 0.011 & 0.0020\end{array}$

$\begin{array}{llll}0.002 & 0.012 & 0.001 & \mathbf{0 . 0 0 0 2 0}\end{array}$

$\begin{array}{ll}0.001 & 0.00020\end{array}$

$\mathbf{0 . 0 0 2} \quad 0.012$

0.002

0.002

$\begin{array}{lll}0.012 & 0.001 & 0.00020\end{array}$

$0.011-0.002-0.00020$

$0.011-0.001-0.00020$

0.00020

$\begin{array}{llll}\mathbf{0 . 0 0 2} & 0.007 & \mathbf{0 . 0 0 1} & \mathbf{0 . 0 0 0 2 0} \\ \mathbf{0 . 0 0 2} & 0.005 & \mathbf{0 . 0 0 1} & \mathbf{0 . 0 0 0 2 0} \\ \mathbf{0 . 0 0 2} & 0.006 & \mathbf{0 . 0 0 1} & \mathbf{0 . 0 0 0 2 0} \\ \mathbf{0 . 0 0 2} & 0.007 & 0.001 & \mathbf{0 . 0 0 0 2 0} \\ \mathbf{0 . 0 0 2} & 0.006 & \mathbf{0 . 0 0 1} & \mathbf{0 . 0 0 0 2 0} \\ \mathbf{0 . 0 8 8} & 0.090 & 0.168 & 0.00083 \\ 0.088 & 0.062 & 0.140 & 0.00064 \\ 0.140 & 0.094 & 0.132 & 0.00129 \\ 0.332 & 0.127 & 0.292 & 0.00369 \\ 0.392 & 0.158 & \mathbf{0 . 2 0 8} & 0.00126\end{array}$

$\begin{array}{llll}\mathbf{0 . 0 0 2} & 0.005 & \mathbf{0 . 0 0 1} & \mathbf{0 . 0 0 0 2 0} \\ \mathbf{0 . 0 0 2} & 0.005 & \mathbf{0 . 0 0 1} & \mathbf{0 . 0 0 0 2 0} \\ \mathbf{0 . 0 0 2} & 0.005 & \mathbf{0 . 0 0 1} & \mathbf{0 . 0 0 0 2 0} \\ \mathbf{0 . 0 0 2} & 0.005 & \mathbf{0 . 0 0 1} & \mathbf{0 . 0 0 0 2 0} \\ \mathbf{0 . 0 0 2} & 0.005 & \mathbf{0 . 0 0 1} & \mathbf{0 . 0 0 0 2 0} \\ 0.059 & 0.031 & 0.080 & 0.00053 \\ 0.074 & 0.030 & 0.104 & 0.00113 \\ 0.080 & 0.035 & 0.114 & 0.00086 \\ 0.040 & 0.028 & 0.049 & 0.00056 \\ \mathbf{0 . 0 4 8} & 0.018 & 0.040 & 0.00048\end{array}$

$\begin{array}{lll}0.048-0.018 & 0.040-0.00056 \\ -0.00048\end{array}$

$\begin{array}{llllllll}0.003 & 0.034 & \mathbf{0 . 0 0 1} & \mathbf{0 . 0 0 0 2} & 0.005 & 0.027 & 0.003 & \mathbf{0 . 0 0 0 2 0} \\ 0.003 & 0.037 & 0.001 & \mathbf{0 . 0 0 0 2} & 0.002 & 0.021 & 0.002 & 0.00020\end{array}$

$\begin{array}{llllllll}\mathbf{0} .003 & 0.037 & \mathbf{0 . 0 0 1} & \mathbf{0 . 0 0 0 2} & \mathbf{0 . 0 0 2} & 0.021 & 0.002 & \mathbf{0 . 0 0 0 2 0} \\ \mathbf{0 . 0 0 3} & 0.037 & \mathbf{0 . 0 0 1} & \mathbf{0 . 0 0 0 2} & \mathbf{0 . 0 0 2} & 0.016 & \mathbf{0 . 0 0 1} & \mathbf{0 . 0 0 0 2 0}\end{array}$

$\begin{array}{llllllll}\mathbf{0 . 0 0 3} & 0.050 & \mathbf{0 . 0 0 1} & \mathbf{0 . 0 0 0 2} & \mathbf{0 . 0 0 2} & 0.005 & \mathbf{0 . 0 0 1} & \mathbf{0 . 0 0 0 2 0} \\ \mathbf{0 . 0 0 3} & 0.052 & \mathbf{0 . 0 0 1} & \mathbf{0 . 0 0 0 2} & 0.002 & 0.006 & \mathbf{0 . 0 0 1} & \mathbf{0 . 0 0 0 2 0} \\ \mathbf{0 . 0 0 3} & 0.047 & \mathbf{0 . 0 0 1} & \mathbf{0 . 0 0 0 2} & 0.002 & 0.010 & \mathbf{0 . 0 0 1} & \mathbf{0 . 0 0 0 2 0} \\ \mathbf{0 . 0 0 3} & 0.040 & \mathbf{0 . 0 0 1} & \mathbf{0 . 0 0 0 2} & 0.002 & 0.006 & 0.002 & \mathbf{0 . 0 0 0 2 0} \\ \mathbf{0 . 0 0 3} & 0.042 & \mathbf{0 . 0 0 1} & \mathbf{0 . 0 0 0 2} & \mathbf{0 . 0 0 2} & 0.005 & 0.001 & \mathbf{0 . 0 0 0 2 0} \\ \mathbf{0 . 0 0 3} & 0.043 & \mathbf{0 . 0 0 1} & \mathbf{0 . 0 0 0 2} & \mathbf{0 . 0 0 2} & 0.005 & 0.001 & \mathbf{0 . 0 0 0 2 0}\end{array}$

$\begin{array}{llllllllll}S B & A S & B E & C D & C R & C U & \text { PB } & \text { HG }\end{array}$

$\begin{array}{llllllll}0.30 & 0.20 & 0.1 & 0.020 & 0.2 & 0.10 & 1.0 & 0.020\end{array}$

$\begin{array}{llllllll}\mathbf{0 . 3 0} & 3.50 & 0.1 & \mathbf{0 . 0 2 0} & 5.6 & 3.00 & 12.9 & 0.084 \\ \mathbf{0 . 3 0} & 2.90 & 0.2 & \mathbf{0 . 0 2 0} & 6.3 & 1.30 & 12.1 & 0.110\end{array}$

$\begin{array}{llllllll}0.30 & 2.90 & 0.2 & 0.020 & 6.3 & 1.30 & 12.1 & 0.110 \\ \mathbf{0 . 3 0} & 3.10 & 0.2 & \mathbf{0 . 0 2 0} & 7.0 & 2.70 & 12.0 & 0.084\end{array}$

SB-Antimony AS-Arsenic BE-Beryllium CD-Cadmium CR-Chromium CU-Copper PB-Lead HG-Mercury BOLD - less than values

Values below less than values are estimated results. Results are less than the reporting limit. 
Metscoar

Delaware River Water Analysis (Coarse-Grained Site)

$\begin{array}{ll}\text { SAMPLE } & \text { SAMPL } \\ \text { TYPE } & \text { ID }\end{array}$

Detection Limit (mg/l)

Plume Monitoring

Water 80827 Background, dissolved

Water 80735 Background, total

Water $\quad 80828 \quad 0-10 \mathrm{~min}$, overflow, dissolved

Water $\quad 80829 \quad 10-20 \mathrm{~min}$, overflow, dissolved

Water $\quad 80830 \quad 20-30 \mathrm{~min}$, overflow, dissolved

Water $80736 \quad 0-10 \mathrm{~min}$, overflow, total

10-20 min, overflow, tota

Water $80831 \quad 0-10 \mathrm{~min}$, non-overflow, dissolved

$\begin{array}{lll}\text { Water } & 80832 & 10-20 \mathrm{~min}, \text { non-overflow, dissolved } \\ \text { Water } & 80833 & 20-30 \mathrm{~min} \text {, non-overflow, dissolved }\end{array}$

$\begin{array}{lll}\text { Water } & 80833 & 20-30 \mathrm{~min} \text {, non-overflow, dissolv } \\ \text { Water } & 80739 & 0-10 \mathrm{~min} \text {, non-overflow, total }\end{array}$

Water $\quad 80740 \quad 10-20 \mathrm{~min}$, non-overflow, total

Water $\quad 80741 \quad 20-30 \mathrm{~min}$, non-overflow, total

NI
0.001
0.009
0.009
0.008
0.008
0.012
0.010
0.010
0.008
0.009
0.009
0.008
0.007
0.009
0.008

\section{Hopper Inflow Monitoring}

3\& $6 \mathrm{~min}$, dissolved

$9812 \mathrm{~min}$, dissolve

$15 \& 18 \mathrm{~min}$, dissolved

$21 \& 24 \mathrm{~min}$, dissolved

$27830 \mathrm{~min}$, dissolved

$3 \& 6 \mathrm{~min}$, total

$9 \& 12 \mathrm{~min}$, total

$15 \& 18 \mathrm{~min}$, total

$21824 \mathrm{~min}$, total

$27 \& 30 \mathrm{~min}$, total

Hopper Overflow Monitoring

2\&. $4 \mathrm{~min}$, dissolved

6\& $8 \mathrm{~min}$, dissolved

$10 \& 12 \mathrm{~min}$, dissolved

$14 \& 16 \mathrm{~min}$, dissolved

$18 \& 20 \mathrm{~min}$, dissolved

2\& 4 min, total

$6 \& 8 \mathrm{~min}$, total
$10 \& 12 \mathrm{~min}$, total

$10 \& 12 \mathrm{~min}$, total

$18820 \mathrm{~min}$, total

\section{Site Water}

$\begin{array}{ccl}\begin{array}{c}\text { Water } \\ \text { Water } \\ \text { Water }\end{array} & 81648 & \begin{array}{l}\text { Site Water } \\ \text { Sample 1 Total } \\ \text { Sample 2 Total } \\ \text { Sample 3 Total }\end{array} \\ & 81650 & \\ & & \begin{array}{l}\text { Elutriate } \\ \text { Water }\end{array} \\ \text { Water } & 81654 & \text { Sample 1 Dissolved } \\ \text { Water } & 81655 & \text { Sample 2 Dissolved } \\ \text { Water } & 81651 & \text { Sample 3 Dissolved } \\ \text { Water } & 81652 & \text { Sample 1 Total } \\ \text { Water } & 81653 & \text { Sample 3 Total } \\ \end{array}$

SAMPLE SAMPLE DESCRIPTION

TYPE ID

Detection Limit $(\mathrm{mg} / \mathrm{kg})$

Insitu Sediment

Sediment 81726 Insitu Sediment

Sediment $81727 \quad$ Sample \#1

Sediment 81728 Sample \#3

$\mathrm{NI}$ - Nickel SE - Selen
BOLD - less than values

Values below less than values are estimated results. Results are less than the reporting limit.

Page 2

$0.800 \quad 0.100$
SE

0.002

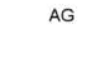

$0.152 \quad 0.001$

$0.138 \quad 0.003$

$0.150 \quad 0.001$

$\begin{array}{ll}0.1468 & 0.001\end{array}$

$0.157 \quad 0.004$

$0.153 \quad 0.004$

$\begin{array}{ll}0.157 & 0.003\end{array}$

$\begin{array}{ll}0.158 & 0.001 \\ 0.160 & 0.001\end{array}$

0.160

0.153

0.149

0.154

0.001

0.001

0.003

0.003

$\begin{array}{rrrrr}\text { TL } & \text { ZN } & \text { AL } & \text { BA } & \text { CA } \\ & & & & \\ 0.002 & 0.010 & 0.025 & 0.002 & 0.200 \\ & & & & \\ \mathbf{0 . 0 0 2} & \mathbf{0 . 0 1 0} & \mathbf{0 . 0 2 5} & 0.039 & 323 \\ \mathbf{0 . 0 0 2} & 0.017 & 0.644 & 0.016 & 254 \\ \mathbf{0 . 0 0 2} & \mathbf{0 . 0 1 0} & \mathbf{0 . 0 2 5} & 0.042 & 334 \\ \mathbf{0 . 0 0 2} & \mathbf{0 . 0 1 0} & \mathbf{0 . 0 2 5} & 0.061 & 369 \\ \mathbf{0 . 0 0 2} & 0.011 & \mathbf{0 . 0 2 5} & 0.077 & 331 \\ \mathbf{0 . 0 0 2} & 0.015 & 0.864 & 0.016 & 253 \\ \mathbf{0 . 0 0 2} & 0.017 & 0.984 & 0.016 & 261 \\ \mathbf{0 . 0 0 2} & 0.013 & 0.676 & 0.016 & 260 \\ \mathbf{0 . 0 0 2} & \mathbf{0 . 0 1 0} & \mathbf{0 . 0 2 5} & 0.038 & 319 \\ \mathbf{0 . 0 0 2} & \mathbf{0 . 0 1 0} & \mathbf{0 . 0 2 5} & 0.062 & 314 \\ \mathbf{0 . 0 0 2} & \mathbf{0 . 0 1 0} & \mathbf{0 . 0 2 5} & 0.043 & 319 \\ \mathbf{0 . 0 0 2} & \mathbf{0 . 0 1 0} & 0.716 & 0.016 & 243 \\ \mathbf{0 . 0 0 2} & 0.017 & 1.100 & 0.016 & 242 \\ \mathbf{0 . 0 0 2} & 0.012 & 0.664 & 0.016 & 247\end{array}$

$\begin{array}{lll}0.175 & 0.001 & 0.002\end{array}$

$0.149 \quad 0.001$

$\begin{array}{ll}0.161 & 0.001 \\ 0.163 & \mathbf{0 . 0 0 1}\end{array}$

$\begin{array}{ll}0.167 & \mathbf{0 . 0 0 1}\end{array}$

$\begin{array}{ll}0.069 & 0.006 \\ 0.062 & 0.012\end{array}$

$\begin{array}{ll}0.062 & 0.012 \\ 0.103 & 0.014\end{array}$

$\begin{array}{ll}0.103 & 0.014 \\ 0.113 & 0.015\end{array}$

$\begin{array}{ll}0.113 & 0.015 \\ 0.129 & 0.019\end{array}$

0.002

$0.002 \quad 0.034$

0.002
0.002

0.002

0.002

0.002

0.002

0.002

$\begin{array}{ll}0.155 & 0.001\end{array}$

$0.165 \quad 0.001$

0.166

0.162

0.153

$0.053 \quad 0.007$

$0.064 \quad 0.006$

$\begin{array}{ll}0.089 & 0.006 \\ 0.113 & 0.054\end{array}$

$\begin{array}{ll}0.118 & 0.011\end{array}$

0.002

0.002
0.002

0.002
0.002

0.002

0.002

0.002

0.002

0.002

$\begin{array}{ll}0.115 & 0.003\end{array}$

$\begin{array}{ll}0.124 & 0.005 \\ 0.134 & 0.004\end{array}$

0.002

0.002

$0.012-1.260$

0.010

0.056

0.012
0.012

305

$0.172 \quad 0.001$

$0.170-0.001$

0.161

0.135

0.140

0.001
0.001

0.004

0.003
0.003

0.002

0.002

0.002

0.002
0.002

0.002
0.002

0.031

0.031

0.010
0.010

0.010

$\begin{array}{ll}\mathbf{0 . 0 2 5} & 0.073 \\ 0.025 & 0.097\end{array}$

$\begin{array}{ll}0.025 & 0.105\end{array}$

$\begin{array}{ll}1.140 & 0.018\end{array}$

$\begin{array}{ll}1.140 & 0.018\end{array}$

SE $\quad A G$

TL

ZN

AL

$0.200 \quad 0.100$

0.200

1.0

1

BA

0.200

$29.9 \quad 1580$

$\begin{array}{rrrrrrr}0.900 & 0.400 & \mathbf{0 . 2 0 0} & 29.0 & 1720 & 4.8 & 11600 \\ 0.899 & 0.499 & \mathbf{0 . 2 0 0} & 28.7 & 1720 & 4.3 & 9820\end{array}$

0.1

20

$\begin{array}{rrr}5.7 & 19600 \\ 720 & 4.8 & 11600\end{array}$

AL-Aluminum BA-Barium CA-Calcium

(1)$$
\text { (1) }
$$ 
Metscoar

Delaware River Water Analysis (Coarse-Grained Site)

TYPE

Detection Limit (mg/l)

Plume Monitoring

$\begin{array}{lll}\text { Water } & 80827 & \text { Background, dissolved } \\ \text { Water } & 80735 & \end{array}$

Background, total

0-10 min, overflow, dissolved

10-20 min, overflow, cissolved

$0.10 \mathrm{~min}$, overflow, total

10-20 min, overflow, total

20-30 min, overflow, total

0-10 min, non-overflow, dissolved 10-20 min, non-overflow, dissolved 20-30 min, non-overflow, dissolved

$20-30 \mathrm{~min}$, non-overflow, dissol
$0.10 \mathrm{~min}$, non-overflow, total

0-10 min, non-overflow, total

20-30 min, non-overflow, total

Hopper Inflow Monitoring

3\& $6 \mathrm{~min}$, dissolved

$9 \& 12 \mathrm{~min}$, dissolved
$15 \& 18 \mathrm{~min}$, dissolved

$21824 \mathrm{~min}$, dissolved

$27 \& 30 \mathrm{~min}$, dissolved

3\& $6 \mathrm{~min}$, total

$9 \& 12 \mathrm{~min}$, total

$15 \& 18 \mathrm{~min}$, total

$21224 \mathrm{~min}$, total

$27 \& 30 \mathrm{~min}$, total

Hopper Overflow Monitoring

2\& 4 min, dissolved

6\& $8 \mathrm{~min}$, dissolved

$10812 \mathrm{~min}$, dissolved

$14 \& 16 \mathrm{~min}$, dissolved

$28,4 \mathrm{~min}$, total

$6 \& 8 \mathrm{~min}$, total

$10 \& 12 \mathrm{~min}$, total

$14 \& 16 \mathrm{~min}$, total

$18 \& 20 \mathrm{~min}$, total

Site Water
Sample 1 Total

Sample 2 Total

Sample 3 Total

Elutriate
Sample 1 Dissolved

Sample 1 Dissolved
Sample 2 Dissolved

Sample 2 Dissolved

Sample 1 Total

Sample 2 Total

$\begin{array}{lll}\text { Water } & 81652 & \text { Sample 2 Total } \\ \text { Water } & 81653 & \text { Sample 3 Total }\end{array}$

SAMPLE SAMPLE DESCRIPTION

TYPE ID

Detection Limit $(\mathrm{mg} / \mathrm{kg}$ )

Insitu Sediment

Sample \#1

Sample \#2

Sample \#3

$\begin{array}{rrrrrrr}\text { CO } & \text { FE } & \text { MG } & \text { MN } & K & \text { NA } & V \\ & & & & & & \\ 0.002 & 0.020 & 0.200 & 0.001 & 0.200 & 0.200 & 0.001 \\ & & & & & & \\ \mathbf{0 . 0 0 1} & \mathbf{0 . 0 2 0} & 961 & 0.006 & 284 & 9,540 & 0.002 \\ \mathbf{0 . 0 0 1} & \mathbf{0 . 2 0 4} & 968 & 0.012 & 291 & 7,970 & 0.004 \\ \mathbf{0 . 0 0 1} & \mathbf{0 . 0 2 0} & 994 & 0.002 & 294 & 8,280 & 0.002 \\ \mathbf{0 . 0 0 1} & \mathbf{0 . 0 2 0} & 985 & 0.002 & 290 & 9,230 & 0.002 \\ \mathbf{0 . 0 0 1} & \mathbf{0 . 0 2 0} & 1030 & 0.004 & 302 & 8,890 & 0.002 \\ \mathbf{0 . 0 0 1} & 0.312 & 940 & 0.012 & 300 & 9,560 & 0.004 \\ \mathbf{0 . 0 0 1} & 0.364 & 1030 & 0.015 & 368 & 8,580 & 0.004 \\ \mathbf{0 . 0 0 1} & 0.184 & 944 & 0.009 & 324 & 7,880 & 0.004 \\ & & & & & & \\ \mathbf{0 . 0 0 1} & \mathbf{0 . 0 2 0} & 992 & 0.005 & 292 & 8,930 & 0.002 \\ \mathbf{0 . 0 0 1} & \mathbf{0 . 0 2 0} & 986 & 0.006 & 288 & 8,510 & 0.002 \\ \mathbf{0 . 0 0 1} & \mathbf{0 . 0 2 0} & 957 & 0.005 & 282 & 9,040 & 0.002 \\ \mathbf{0 . 0 0 1} & 0.256 & 908 & 0.014 & 318 & 7,480 & 0.005 \\ \mathbf{0 . 0 0 1} & 0.572 & 912 & 0.015 & 272 & 8,480 & 0.004 \\ \mathbf{0 . 0 0 1} & 0.192 & 916 & 0.008 & 334 & 8,120 & 0.003\end{array}$

$\begin{array}{lll}\mathbf{0 . 0 2 0} & 1003 & 0.011\end{array}$

0.020

0.029
0.020

0.074

98.0
31.3

31.3
71.7

288.0

218.0

1007

$\begin{array}{ll}1023 & 0.002\end{array}$

$\begin{array}{ll}1035 & 0.002 \\ 1035 & 3.099\end{array}$

$1050 \quad 3.770$

$1040 \quad 2.170$

$1090-4.440$

4.440
1020 $\quad 3.200$

$8,120-0.003$

$\begin{array}{rrrrrr}\mathbf{0 . 0 2 0} & 993 & \mathbf{0 . 0 0 1} & 299 & 8,750 & 0.004 \\ \mathbf{0 . 0 2 0} & 962 & \mathbf{0 . 0 0 1} & 290 & 9,240 & 0.004 \\ \mathbf{0 . 0 2 0} & 999 & \mathbf{0 . 0 0 1} & 302 & 8,750 & 0.004 \\ \mathbf{0 . 0 2 0} & 1,000 & \mathbf{0 . 0 0 1} & 303 & 8,960 & 0.004 \\ \mathbf{0 . 0 2 0} & 990 & \mathbf{0 . 0 0 1} & 294 & 8,870 & 0.004 \\ 66.4 & 1,060 & 1.130 & 333 & 8,800 & 0.084 \\ 70.4 & 1,060 & 1.470 & 335 & 9,170 & 0.104 \\ 92.0 & 968 & 1.420 & 308 & 7,980 & 0.105 \\ 29.8 & 1,000 & 0.608 & 309 & 8,640 & 0.064 \\ 28.2 & 984 & 0.544 & 310 & 9,170 & 0.060\end{array}$

$\begin{array}{lllllll}0.002 & 0.837 & 998 & 0.028 & 306 & 8,370 & 0.003\end{array}$

$\begin{array}{lllllll}0.002 & 0.048 & 974 & 0.004 & 289 & 8,780 & 0.001\end{array}$

$\begin{array}{llllll}0.039 & 968 & 0.005 & 289 & 8,980 & 0.001\end{array}$

$\begin{array}{lrrrrrr}\mathbf{0 . 0 0 2} & \mathbf{0 . 0 2 0} & 1,020 & 0.002 & 303 & 8,820 & 0.002 \\ \mathbf{0 . 0 0 2} & \mathbf{0 . 0 2 0} & 1,030 & 0.001 & 304 & 9,250 & 0.001 \\ \mathbf{0 . 0 0 2} & \mathbf{0 . 0 2 0} & 1,030 & 0.002 & 304 & 9,040 & 0.002 \\ \mathbf{0 . 0 0 2} & 0.742 & 982 & 0.024 & 292 & 8,620 & 0.004 \\ \mathbf{0 . 0 0 2} & 0.632 & 991 & 0.019 & 294 & 8,760 & 0.003 \\ \mathbf{0 . 0 0 2} & 0.799 & 1,400 & 0.024 & 423 & 12,300 & 0.004\end{array}$

$\begin{array}{lllllllllll}C O & \mathrm{FE} & \mathrm{MG} & \mathrm{MN} & \mathrm{K} & \mathrm{NA} & \mathrm{V} & \% \text { Moisture }\end{array}$

$\begin{array}{lllllll}0.1 & 2 & 20 & 0.1 & 20 & 20 & 0.10\end{array}$

$\begin{array}{llllllll}2.2 & 5,810 & 1,260 & 91.7 & 443 & 2180 & 4.10 & 13.8 \\ 2.4 & 6,040 & 1,330 & 95.2 & 474 & 1920 & 4.30 & 13.8\end{array}$

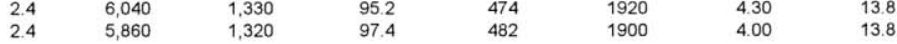

Co- Cobalt FE-Iron MG-Magnesium MN-Manganese $\quad K$-Potassium NA-Sodium $\quad V$-Vanadium BOLD - less than values

Values below less than values are estimated results. Results are less than the reporting limit. 
PAHscoar

Delaware River Water Analysis (Coarse-Grained Site)

\begin{tabular}{|c|c|c|c|c|c|c|c|c|c|}
\hline \multirow[t]{2}{*}{$\begin{array}{l}\text { SAMPLE } \\
\text { TYPE }\end{array}$} & $\begin{array}{l}\text { SAMPLE } \\
\text { ID }\end{array}$ & DESCRIPTION & NAPHTH & ACENAY & ACENAP & FLUORE & PHENAN & ANTRAC & FLANTHE \\
\hline & & Detection Limit (mg/) & 0.0003 & 0.0003 & 0.0003 & 0.0003 & 0.00030 & 0.00030 & 0.00030 \\
\hline & & Plume Monitoring & & & & & & & \\
\hline Water & 80848 & Background, dissolved & 0.0003 & 0.0003 & 0.0003 & 0.0003 & 0.00030 & 0.00030 & 0.00030 \\
\hline Water & 80763 & Background, total & 0.0003 & 0.0003 & 0.0003 & 0.0003 & 0.00030 & 0.00030 & 0.00030 \\
\hline Water & 80849 & 0-10 min, overflow, dissolved & 0.0003 & 0.0003 & 0.0003 & 0.0003 & 0.00030 & 0.00030 & 0.00030 \\
\hline Water & 80850 & 10-20 min, overflow, dissolved & 0.0003 & 0.0003 & 0.0003 & 0.0003 & 0.00030 & 0.00030 & 0.00030 \\
\hline Water & 80851 & 20-30 min, overflow, dissolved & 0.0003 & 0.0003 & 0.0003 & 0.0003 & 0.00030 & 0.00030 & 0.00030 \\
\hline Water & 80764 & $0-10 \mathrm{~min}$, overflow, total & 0.0003 & 0.0003 & 0.0003 & 0.0003 & 0.00030 & 0.00030 & 0.00030 \\
\hline Water & 80765 & $10-20 \mathrm{~min}$, overflow, total & 0.0003 & 0.0003 & 0.0003 & 0.0003 & 0.00030 & 0.00030 & 0.00030 \\
\hline Water & 80766 & 20-30 min, overflow, total & 0.0003 & 0.0003 & 0.0003 & 0.0003 & 0.00030 & 0.00030 & 0.00030 \\
\hline Water & 80852 & 0-10 min, non-overflow, dissolved & 0.0003 & 0.0003 & 0.0003 & 0.0003 & 0.00030 & 0.00030 & 0.00030 \\
\hline Water & 80853 & $10-20 \mathrm{~min}$, non-overflow, dissolved & 0.0003 & 0.0003 & 0.0003 & 0.0003 & 0.00030 & 0.00030 & 0.00030 \\
\hline Water & 80854 & 20-30 min, non-overflow, dissolved & 0.0003 & 0.0003 & 0.0003 & 0.0003 & 0.00030 & 0.00030 & 0.00030 \\
\hline Water & 80767 & 0-10 min, non-overflow, total & 0.0003 & 0.0003 & 0.0003 & 0.0003 & 0.00030 & 0.00030 & 0.00030 \\
\hline Water & 80768 & 10-20 min, non-overflow, total & 0.0003 & 0.0003 & 0.0003 & 0.0003 & 0.00030 & 0.00030 & 0.00030 \\
\hline \multirow[t]{2}{*}{ Water } & 80769 & 20-30 min, non-overflow, total & 0.0003 & 0.0003 & 0.0003 & 0.0003 & 0.00030 & 0.00030 & 0.00030 \\
\hline & & Hopper Inflow Monitoring & & & & & & & \\
\hline Water & 80810 & 3\& $6 \mathrm{~min}$, dissolved & 0.0003 & 0.0003 & 0.0003 & 0.0003 & 0.00030 & 0.00030 & 0.00030 \\
\hline Water & 80811 & $9 \& 12 \mathrm{~min}$, dissolved & 0.0003 & 0.0003 & 0.0003 & 0.0003 & 0.00030 & 0.00030 & 0.00030 \\
\hline Water & 80812 & $15 \& 18 \mathrm{~min}$, dissolved & 0.0003 & 0.0003 & 0.0003 & 0.0003 & 0.00030 & 0.00030 & 0.00030 \\
\hline Water & 80813 & $21824 \mathrm{~min}$, dissolved & 0.0003 & 0.0003 & 0.0003 & 0.0003 & 0.00030 & 0.00030 & 0.00030 \\
\hline Water & 80814 & $27 \& 30 \mathrm{~min}$, dissolved & 0.0003 & 0.0003 & 0.0003 & 0.0003 & 0.00030 & 0.00030 & 0.00030 \\
\hline Water & 80716 & $3 \& 6 \mathrm{~min}$, total & 0.0003 & 0.0003 & 0.0003 & 0.0003 & 0.00030 & 0.00030 & 0.00030 \\
\hline Water & 80717 & $9 \& 12 \mathrm{~min}$, total & 0.0003 & 0.0003 & 0.0003 & 0.0003 & 0.00030 & 0.00030 & 0.00030 \\
\hline Water & 80718 & $15 \& 18 \mathrm{~min}$, total & 0.0003 & 0.0003 & 0.0003 & 0.0003 & 0.00030 & 0.00030 & 0.00015 \\
\hline Water & 80719 & $21824 \mathrm{~min}$, total & 0.0003 & 0.0003 & 0.0003 & 0.0003 & 0.00016 & 0.00030 & 0.00024 \\
\hline \multirow[t]{2}{*}{ Water } & 80720 & $27 \& 30 \mathrm{~min}$, total & 0.0003 & 0.0003 & 0.0003 & 0.0003 & 0.00012 & 0.00030 & 0.00019 \\
\hline & & Hopper Overflow Monitoring & & & & & & & \\
\hline Water & 80815 & 2\& $4 \mathrm{~min}$, dissolved & 0.0003 & 0.0003 & 0.0003 & 0.0003 & 0.00030 & 0.00030 & 0.00030 \\
\hline Water & 80816 & $6 \& 8 \mathrm{~min}$, dissolved & 0.0003 & 0.0003 & 0.0003 & 0.0003 & 0.00030 & 0.00030 & 0.00030 \\
\hline Water & 80817 & $10 \& 12 \mathrm{~min}$, dissolved & 0.0003 & 0.0003 & 0.0003 & 0.0003 & 0.00030 & 0.00030 & 0.00030 \\
\hline Water & 80818 & $14 \& 16 \mathrm{~min}$, dissolved & 0.0003 & 0.0003 & 0.0003 & 0.0003 & 0.00030 & 0.00030 & 0.00030 \\
\hline Water & 80819 & $18 \& 20 \mathrm{~min}$, dissolved & 0.0003 & 0.0003 & 0.0003 & 0.0003 & 0.00030 & 0.00030 & 0.00030 \\
\hline Water & 80722 & 2\& $4 \mathrm{~min}$, total & 0.0006 & 0.0003 & 0.0003 & 0.0003 & 0.00030 & 0.00030 & 0.00014 \\
\hline Water & 80723 & 6\&. $8 \mathrm{~min}$, total & 0.0006 & 0.0003 & 0.0003 & 0.0003 & 0.00030 & 0.00030 & 0.00030 \\
\hline Water & 80724 & $10 \& 12 \mathrm{~min}$, total & 0.0006 & 0.0003 & 0.0003 & 0.0003 & 0.00073 & 0.00018 & 0.00075 \\
\hline Water & 80725 & $14 \& 16 \mathrm{~min}$, total & 0.0006 & 0.0003 & 0.0003 & 0.0003 & 0.00030 & 0.00030 & 0.00030 \\
\hline \multirow[t]{2}{*}{ Water } & 80726 & $18 \& 20 \mathrm{~min}$, total & 0.0006 & 0.0003 & 0.0003 & 0.0003 & 0.00030 & 0.00030 & 0.00030 \\
\hline & & Site Water & & & & & & & \\
\hline Water & 81630 & Sample 1 Total & 0.0003 & 0.0003 & 0.0003 & 0.0003 & 0.00030 & 0.00030 & 0.00030 \\
\hline Water & 81631 & Sample 2 Total & 0.0003 & 0.0003 & 0.0003 & 0.0003 & 0.00030 & 0.00030 & 0.00030 \\
\hline \multirow[t]{2}{*}{ Water } & 81632 & Sample 3 Total & 0.0003 & 0.0003 & 0.0003 & 0.0003 & 0.00030 & 0.00030 & 0.00030 \\
\hline & & Elutriate & & & & & & & \\
\hline Water & 81636 & Sample 1 Dissolved & 0.0003 & 0.0003 & 0.0003 & 0.0003 & 0.00030 & 0.00030 & 0.00030 \\
\hline Water & 81637 & Sample 2 Dissolved & 0.0003 & 0.0003 & 0.0003 & 0.0003 & 0.00030 & 0.00030 & 0.00030 \\
\hline Water & 81638 & Sample 3 Dissolved & 0.0003 & 0.0003 & 0.0003 & 0.0003 & 0.00030 & 0.00030 & 0.00030 \\
\hline Water & 81633 & Sample 1 Total & 0.0003 & 0.0003 & 0.0003 & 0.0003 & 0.00030 & 0.00030 & 0.00030 \\
\hline Water & 81634 & Sample 2 Total & 0.0003 & 0.0003 & 0.0003 & 0.0003 & 0.00030 & 0.00030 & 0.00030 \\
\hline Water & 81635 & Sample 3 Total & 0.0003 & 0.0003 & 0.0003 & 0.0003 & 0.00030 & 0.00030 & 0.00030 \\
\hline \multirow{3}{*}{$\begin{array}{l}\text { SAMPLE } \\
\text { TYPE }\end{array}$} & SAMPLE & DESCRIPTION & NAPHTH & ACENAY & ACENAP & FLUORE & PHENAN & ANTRAC & FLANTHE \\
\hline & & Detection Limit (mg/kg) & 0.011 & 0.011 & 0.011 & 0.011 & 0.0110 & 0.011 & 0.0110 \\
\hline & & Insitu Sediment & & & & & & & \\
\hline Sediment & 81702 & Sample\#1 & 0.011 & 0.011 & 0.011 & 0.011 & 0.0110 & 0.011 & 0.0110 \\
\hline Sediment & 81703 & Sample \#2 & 0.011 & 0.011 & 0.011 & 0.011 & 0.0038 & 0.011 & 0.0267 \\
\hline Sediment & 81704 & Sample \#3 & 0.011 & 0.011 & 0.011 & 0.011 & 0.0110 & 0.011 & 0.0042 \\
\hline
\end{tabular}

NAPHTH - Naphthalene ACENAY - Acenaphthylene ACENAP-Acenaphthene FLUORE - Fluorene PHENAN-Phenanthrene ANTRAC - Anthracene FLANTHE - Fluoranthene

$B O L D$ - less than values

Values below less than values are estimated results. Results are less than the reporting limit.

Page 1 
PAHscoar

Delaware River Water Analysis (Coarse-Grained Site)

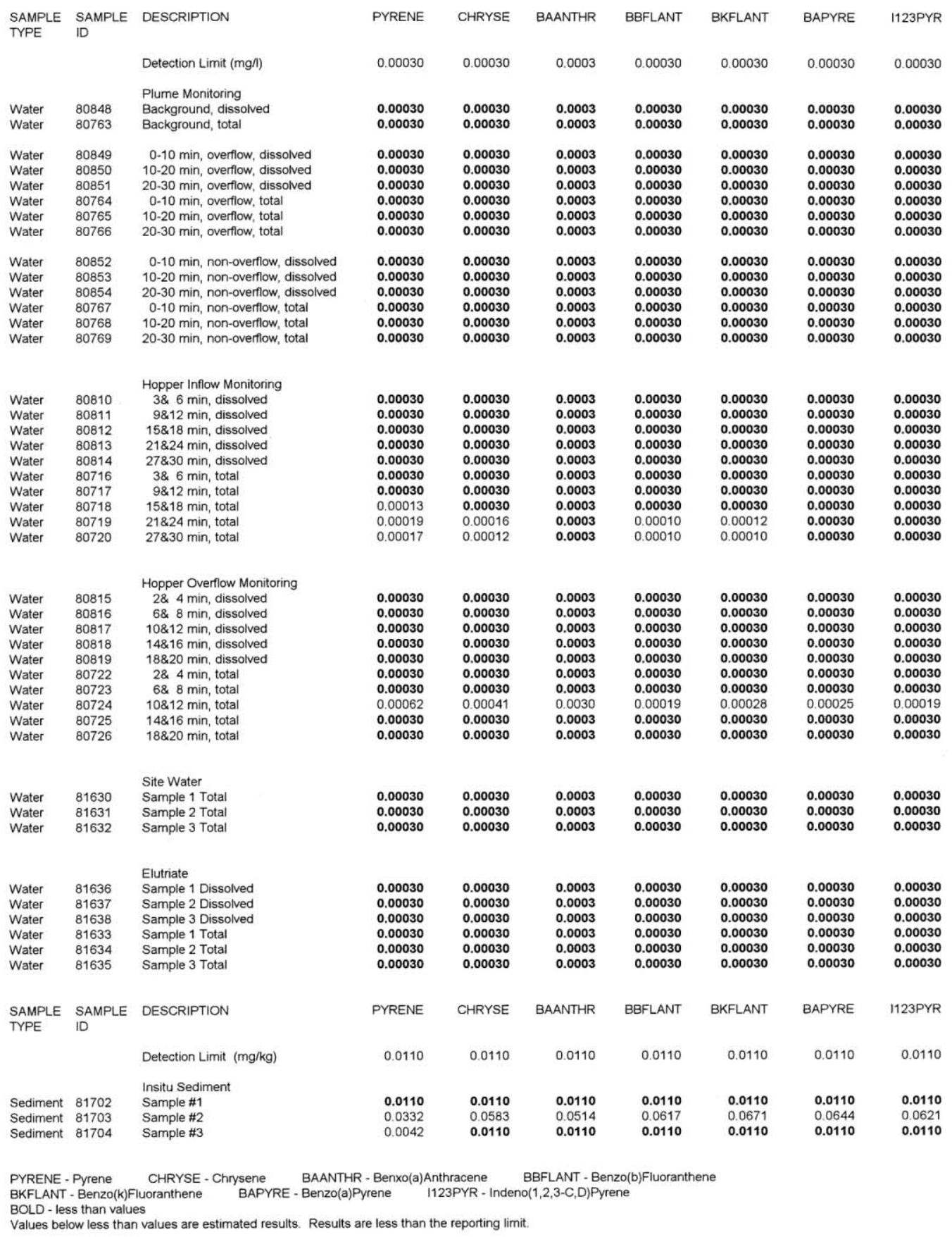

Page 2 
PAHscoar

Delaware River Water Analysis (Coarse-Grained Site)

\begin{tabular}{|c|c|c|c|c|c|c|c|}
\hline \multirow[t]{2}{*}{$\begin{array}{l}\text { SAMPLE } \\
\text { TYPE }\end{array}$} & $\begin{array}{l}\text { SAMPLE } \\
\text { ID }\end{array}$ & DESCRIPTION & DBAHANT & B-GHI-PY & 2MeNAPH & 2FIBP-S & PTERP-S \\
\hline & & Detection Limit (mg/l) & 0.0003 & 0.00030 & 0.0003 & & \\
\hline & & Plume Monitoring & & & & & \\
\hline Water & 80848 & Background, dissolved & 0.0003 & 0.00030 & 0.0003 & $89.8 \%$ & $71.9 \%$ \\
\hline Water & 80763 & Background, total & 0.0003 & 0.00030 & 0.0003 & $95.4 \%$ & $73.8 \%$ \\
\hline Water & 80849 & 0-10 min, overflow, dissolved & 0.0003 & 0.00030 & 0.0003 & $88.8 \%$ & $68.8 \%$ \\
\hline Water & 80850 & 10-20 min, overflow, dissolved & 0.0003 & 0.00030 & 0.0003 & $91.2 \%$ & $76.0 \%$ \\
\hline Water & 80851 & 20-30 min, overflow, dissolved & 0.0003 & 0.00030 & 0.0003 & $89.0 \%$ & $65.8 \%$ \\
\hline Water & 80764 & 0-10 min, overflow, total & 0.0003 & 0.00030 & 0.0003 & $59.1 \%$ & $76.3 \%$ \\
\hline Water & 80765 & $10-20 \mathrm{~min}$, overflow, total & 0.0003 & 0.00030 & 0.0003 & $36.6 \%$ & $31.1 \%$ \\
\hline Water & 80766 & 20-30 min, overflow, total & 0.0003 & 0.00030 & 0.0003 & $92.1 \%$ & $71.0 \%$ \\
\hline Water & 80852 & 0-10 min, non-overflow, dissolved & 0.0003 & 0.00030 & 0.0003 & $94.3 \%$ & $74.7 \%$ \\
\hline Water & 80853 & 10-20 min, non-overflow, dissolved & 0.0003 & 0.00030 & 0.0003 & $83.1 \%$ & $65.0 \%$ \\
\hline Water & 80854 & 20-30 min, non-overflow, dissolved & 0.0003 & 0.00030 & 0.0003 & $90.4 \%$ & $69.1 \%$ \\
\hline Water & 80767 & 0-10 min, non-overflow, total & 0.0003 & 0.00030 & 0.0003 & $36.5 \%$ & $27.1 \%$ \\
\hline Water & 80768 & 10-20 min, non-overflow, total & 0.0003 & 0.00030 & 0.0003 & $77.5 \%$ & $69.9 \%$ \\
\hline \multirow[t]{2}{*}{ Water } & 80769 & $20-30 \mathrm{~min}$, non-overflow, total & 0.0003 & 0.00030 & 0.0003 & $73.2 \%$ & $72.0 \%$ \\
\hline & & Hopper Inflow Monitoring & & & & & \\
\hline Water & 80810 & $3 \& 6 \mathrm{~min}$, dissolved & 0.0003 & 0.00030 & 0.0003 & $76.0 \%$ & $67.4 \%$ \\
\hline Water & 80811 & $9 \& 12 \mathrm{~min}$, dissolved & 0.0003 & 0.00030 & 0.0003 & $77.5 \%$ & $69.2 \%$ \\
\hline Water & 80812 & $15 \& 18 \mathrm{~min}$, dissolved & 0.0003 & 0.00030 & 0.0003 & $94.5 \%$ & $76.8 \%$ \\
\hline Water & 80813 & $21 \& 24 \mathrm{~min}$, dissolved & 0.0003 & 0.00030 & 0.0003 & $83.0 \%$ & $63.8 \%$ \\
\hline Water & 80814 & $27830 \mathrm{~min}$, dissolved & 0.0003 & 0.00030 & 0.0003 & $61.7 \%$ & $54.8 \%$ \\
\hline Water & 80716 & $3 \& 6 \mathrm{~min}$, total & 0.0003 & 0.00030 & 0.0003 & $48.0 \%$ & $60.3 \%$ \\
\hline Water & 80717 & $9 \& 12 \mathrm{~min}$, total & 0.0003 & 0.00030 & 0.0003 & $60.0 \%$ & $58.4 \%$ \\
\hline Water & 80718 & $15 \& 18 \mathrm{~min}$, total & 0.0003 & 0.00030 & 0.0003 & $72.2 \%$ & $66.1 \%$ \\
\hline Water & 80719 & $21 \& 24 \mathrm{~min}$, total & 0.0003 & 0.00030 & 0.0003 & $67.0 \%$ & $62.9 \%$ \\
\hline \multirow[t]{2}{*}{ Water } & 80720 & $27 \& 30 \mathrm{~min}$, total & 0.0003 & 0.00030 & 0.0003 & $58.0 \%$ & $66.6 \%$ \\
\hline & & Hopper Overflow Monitoring & & & & & \\
\hline Water & 80815 & 28 $4 \mathrm{~min}$, dissolved & 0.0003 & 0.00030 & 0.0003 & $63.0 \%$ & $60.7 \%$ \\
\hline Water & 80816 & $6 \& 8 \mathrm{~min}$, dissolved & 0.0003 & 0.00030 & 0.0003 & $63.8 \%$ & $84.8 \%$ \\
\hline Water & 80817 & $10 \& 12 \mathrm{~min}$, dissolved & 0.0003 & 0.00030 & 0.0003 & $64.4 \%$ & $67.7 \%$ \\
\hline Water & 80818 & $14816 \mathrm{~min}$, dissolved & 0.0003 & 0.00030 & 0.0003 & $75.4 \%$ & $81.3 \%$ \\
\hline Water & 80819 & $18 \& 20 \mathrm{~min}$, dissolved & 0.0003 & 0.00030 & 0.0003 & $48.3 \%$ & $65.8 \%$ \\
\hline Water & 80722 & $2 \& 4 \mathrm{~min}$, total & 0.0003 & 0.00030 & 0.0003 & $56.5 \%$ & $69.7 \%$ \\
\hline Water & 80723 & 6\& $8 \mathrm{~min}$, total & 0.0003 & 0.00030 & 0.0003 & $66.9 \%$ & $70.5 \%$ \\
\hline Water & 80724 & $10 \& 12 \mathrm{~min}$, total & 0.0003 & 0.00014 & 0.0003 & $60.8 \%$ & $67.6 \%$ \\
\hline Water & 80725 & $14 \& 16 \mathrm{~min}$, total & 0.0003 & 0.00030 & 0.0003 & $74.2 \%$ & $65.0 \%$ \\
\hline \multirow[t]{2}{*}{ Water } & 80726 & $18 \& 20 \mathrm{~min}$, total & 0.0003 & 0.00030 & 0.0003 & $57.7 \%$ & $68.1 \%$ \\
\hline & & Site Water & & & & & \\
\hline Water & 81630 & Sample 1 Total & 0.0003 & 0.00030 & 0.0003 & $39.7 \%$ & $61.9 \%$ \\
\hline Water & 81631 & Sample 2 Total & 0.0003 & 0.00030 & 0.0003 & $61.3 \%$ & $62.4 \%$ \\
\hline \multirow[t]{2}{*}{ Water } & 81632 & Sample 3 Total & 0.0003 & 0.00030 & 0.0003 & $68.0 \%$ & $66.8 \%$ \\
\hline & & Elutriate & & & & & \\
\hline Water & 81636 & Sample 1 Dissolved & 0.0003 & 0.00030 & 0.0003 & $55.8 \%$ & $678.0 \%$ \\
\hline Water & 81637 & Sample 2 Dissolved & 0.0003 & 0.00030 & 0.0003 & $79.2 \%$ & $56.1 \%$ \\
\hline Water & 81638 & Sample 3 Dissolved & 0.0003 & 0.00030 & 0.0003 & $63.3 \%$ & $58.3 \%$ \\
\hline Water & 81633 & Sample 1 Total & 0.0003 & 0.00030 & 0.0003 & $65.9 \%$ & $64.0 \%$ \\
\hline Water & 81634 & Sample 2 Total & 0.0003 & 0.00030 & 0.0003 & $37.7 \%$ & $71.0 \%$ \\
\hline Water & 81635 & Sample 3 Total & 0.0003 & 0.00030 & 0.0003 & $53.8 \%$ & $71.2 \%$ \\
\hline \multirow{3}{*}{$\begin{array}{l}\text { SAMPLE } \\
\text { TYPE }\end{array}$} & SAMPLE & DESCRIPTION & DBAHANT & B-GHI-PY & 2MeNAPH & 2FIBP-S & PTERP-S \\
\hline & & Detection Limit (mg/kg) & 0.0110 & 0.0110 & 0.011 & & \\
\hline & & Insitu Sediment & & & & & \\
\hline Sediment & 81702 & Sample \#1 & 0.0110 & 0.0110 & 0.011 & $62.7 \%$ & $46.1 \%$ \\
\hline Sediment & 81703 & Sample \#2 & 0.0046 & 0.0514 & 0.011 & $76.0 \%$ & $49.7 \%$ \\
\hline Sediment & 81704 & Sample \#3 & 0.0110 & 0.0110 & 0.011 & $68.8 \%$ & $52.0 \%$ \\
\hline
\end{tabular}

DBAHANT - Dibenzo(A,H)Anthracene B-GHI-PY - Benzo(G,H,I)Perylene 2MeNAPH - 2-Methylnaphthalene 2FIBP-S - 2-Fluorobiphenyl(Surrogate (43-116 W)) PTERP-S - p-Terphenyl-D14(Surrogate (33-141 W))

BOLD - less than values

Values below less than values are estimated results. Results are less than the reporting limit.

Page 3 
Delaware River Water Analysis (Coarse-Grained Site)

\begin{tabular}{|c|c|c|c|c|c|c|c|c|}
\hline \multirow[t]{2}{*}{$\begin{array}{l}\text { SAMPLE } \\
\text { TYPE }\end{array}$} & $\begin{array}{l}\text { SAMPLE } \\
\text { ID }\end{array}$ & DESCRIPTION & ALDRIN & A-BHC & B-BHC & G-BHC & D-BHC & PPDDD \\
\hline & & Detection Limit (mg/l) & 0.000035 & 0.000035 & 0.000035 & 0.000035 & 0.000035 & 0.000070 \\
\hline & & Plume Monitoring & & & & & & \\
\hline Water & 80841 & Background, dissolved & 0.000025 & 0.000025 & 0.000025 & 0.000025 & 0.000025 & 0.000050 \\
\hline Water & 80756 & Background, total & 0.000025 & 0.000025 & 0.000025 & 0.000025 & 0.000025 & 0.000050 \\
\hline Water & 80842 & 0-10 min, overflow, dissolved & 0.000025 & 0.000025 & 0.000025 & 0.000025 & 0.000025 & 0.000050 \\
\hline Water & 80843 & 10-20 min, overflow, dissolved & 0.000025 & 0.000025 & 0.000025 & 0.000025 & 0.000025 & 0.000050 \\
\hline Water & 80344 & 20-30 min, overflow, dissolved & 0.000024 & 0.000024 & 0.000024 & 0.000024 & 0.000024 & 0.000049 \\
\hline Water & 80757 & 0-10 min, overflow, total & 0.000025 & 0.000025 & 0.000025 & 0.000025 & 0.000025 & 0.000050 \\
\hline Water & 80758 & $10-20 \mathrm{~min}$, overflow, total & 0.000025 & 0.000025 & 0.000025 & 0.000025 & 0.000025 & 0.000050 \\
\hline Water & 80759 & 20-30 min, overflow, total & 0.000025 & 0.000025 & 0.000025 & 0.000025 & 0.000025 & 0.000050 \\
\hline Water & 80845 & 0-10 min, non-overflow, dissolved & 0.000025 & 0.000025 & 0.000025 & 0.000025 & 0.000025 & 0.000050 \\
\hline Water & 80846 & $10-20 \mathrm{~min}$, non-overflow, dissolved & 0.000025 & 0.000025 & 0.000025 & 0.000025 & 0.000025 & 0.000050 \\
\hline Water & 80847 & $20-30$ min, non-overflow, dissolved & 0.000035 & 0.000035 & 0.000035 & 0.000035 & 0.000035 & 0.000070 \\
\hline Water & 80760 & $0-10 \mathrm{~min}$, non-overflow, total & 0.000025 & 0.000025 & 0.000025 & 0.000025 & 0.000025 & 0.000050 \\
\hline Water & 80761 & $10-20 \mathrm{~min}$, non-overflow, total & 0.000025 & 0.000025 & 0.000025 & 0.000025 & 0.000025 & 0.000050 \\
\hline \multirow[t]{2}{*}{ Water } & 70762 & 20-30 min, non-overflow, total & 0.000028 & 0.000028 & 0.000028 & 0.000028 & 0.000028 & 0.000055 \\
\hline & & Hopper Inflow Monitoring & & & & & & \\
\hline Water & 80800 & $3 \& 6 \mathrm{~min}$, dissolved & 0.000025 & 0.000025 & 0.000025 & 0.000025 & 0.000025 & 0.000050 \\
\hline Water & 80801 & $9 \& 12 \mathrm{~min}$, dissolved & 0.000025 & 0.000020 & 0.000025 & 0.000025 & 0.000025 & 0.000050 \\
\hline Water & 80802 & $15 \& 18 \mathrm{~min}$, dissolved & 0.000025 & 0.000025 & 0.000046 & 0.000025 & 0.000025 & 0.000050 \\
\hline Water & 80803 & $21 \& 24 \mathrm{~min}$, dissolved & 0.000025 & 0.000011 & 0.000036 & 0.000025 & 0.000025 & 0.000050 \\
\hline Water & 80804 & $27830 \mathrm{~min}$, dissolved & 0.000025 & 0.000025 & 0.000043 & 0.000025 & 0.000025 & 0.000050 \\
\hline Water & 80704 & $3 \& 6 \mathrm{~min}$, total & 0.000025 & 0.000031 & 0.000075 & 0.000021 & 0.000039 & 0.000035 \\
\hline Water & 80705 & $9 \& 12 \mathrm{~min}$, total & 0.000025 & 0.000018 & 0.000025 & 0.000025 & 0.000022 & 0.000095 \\
\hline Water & 80706 & $15 \& 18 \mathrm{~min}$, total & 0.000027 & 0.000046 & 0.000027 & 0.000027 & 0.000023 & 0.000060 \\
\hline Water & 80707 & $21824 \mathrm{~min}$, total & 0.000027 & 0.000027 & 0.000027 & 0.000027 & 0.000027 & 0.000110 \\
\hline \multirow[t]{2}{*}{ Water } & 80708 & $27830 \mathrm{~min}$, total & 0.000025 & 0.000025 & 0.000025 & 0.000025 & 0.000025 & 0.000016 \\
\hline & & Hopper Overflow Monitoring & & & & & & \\
\hline Water & 80805 & 2\&. $4 \mathrm{~min}$, dissolved & 0.000025 & 0.000025 & 0.000025 & 0.000025 & 0.000025 & 0.000050 \\
\hline Water & 80806 & 6\& $8 \mathrm{~min}$, dissolved & 0.000025 & 0.000025 & 0.000025 & 0.000025 & 0.000025 & 0.000050 \\
\hline Water & 80807 & $10 \& 12 \mathrm{~min}$, dissolved & 0.000025 & 0.000013 & 0.000025 & 0.000025 & 0.000025 & 0.000050 \\
\hline Water & 80808 & $148.16 \mathrm{~min}$, dissolved & 0.000025 & 0.000025 & 0.000025 & 0.000025 & 0.000025 & 0.000050 \\
\hline Water & 80809 & $18 \& 20 \mathrm{~min}$, dissolved & 0.000025 & 0.000025 & 0.000025 & 0.000025 & 0.000025 & 0.000050 \\
\hline Water & 80710 & $2 \& 4 \mathrm{~min}$, total & 0.000025 & 0.000025 & 0.000025 & 0.000025 & 0.000025 & 0.000050 \\
\hline Water & 80711 & $6 \& 8 \mathrm{~min}$, total & 0.000025 & 0.000025 & 0.000025 & 0.000025 & 0.000025 & 0.000050 \\
\hline Water & 80712 & $10 \& 12 \mathrm{~min}$, total & 0.000027 & 0.000027 & 0.000027 & 0.000027 & 0.000027 & 0.000053 \\
\hline Water & 80713 & $14 \& 16 \mathrm{~min}$, total & 0.000027 & 0.000027 & 0.000027 & 0.000027 & 0.000027 & 0.000012 \\
\hline \multirow[t]{2}{*}{ Water } & 80714 & $18 \& 20 \mathrm{~min}$, total & 0.000025 & 0.000025 & 0.000025 & 0.000025 & 0.000025 & 0.000050 \\
\hline & & Site Water & & & & & & \\
\hline Water & 81612 & Sample 1 Total & 0.000025 & 0.000025 & 0.000025 & 0.000025 & 0.000025 & 0.000050 \\
\hline Water & 81613 & Sample 2 Total & 0.000025 & 0.000025 & 0.000025 & 0.000025 & 0.000025 & 0.000050 \\
\hline \multirow[t]{2}{*}{ Water } & 81614 & Sample 3 Total & 0.000025 & 0.000025 & 0.000025 & 0.000025 & 0.000025 & 0.000050 \\
\hline & & Elutriate & & & & & & \\
\hline Water & 81618 & Sample 1 Dissolved & 0.000025 & 0.000025 & 0.000025 & 0.000025 & 0.000025 & 0.000050 \\
\hline Water & 81619 & Sample 2 Dissolved & 0.000025 & 0.000025 & 0.000025 & 0.000011 & 0.000025 & 0.000050 \\
\hline Water & 81620 & Sample 3 Dissolved & 0.000025 & 0.000025 & 0.000025 & 0.000025 & 0.000025 & 0.000050 \\
\hline Water & 81615 & Sample 1 Total & 0.000025 & 0.000025 & 0.000025 & 0.000025 & 0.000025 & 0.000050 \\
\hline Water & 81616 & Sample 2 Total & 0.000025 & 0.000025 & 0.000025 & 0.000025 & 0.000025 & 0.000050 \\
\hline Water & 81617 & Sample 3 Total & 0.000025 & 0.000025 & 0.000025 & 0.000025 & 0.000025 & 0.000050 \\
\hline \multirow[t]{3}{*}{$\begin{array}{l}\text { SAMPLE } \\
\text { TYPE }\end{array}$} & $\begin{array}{l}\text { SAMPLE } \\
\text { ID }\end{array}$ & DESCRIPTION & ALDRIN & $\mathrm{A}-\mathrm{BHC}$ & B-BHC & G-BHC & $\mathrm{D}-\mathrm{BHC}$ & PPDDD \\
\hline & & Detection Limit ( $\mathrm{mg} / \mathrm{kg})$ & 0.00096 & 0.00096 & 0.00096 & 0.0018 & 0.00096 & 0.0019 \\
\hline & & Insitu Sediment & & & & & & \\
\hline Sediment & 81708 & Sample \#1 & 0.00096 & 0.00096 & 0.0012 & 0.0021 & 0.00096 & 0.0019 \\
\hline Sediment & 81709 & Sample \#2 & 0.00096 & 0.00096 & 0.0013 & 0.0034 & 0.00096 & 0.0019 \\
\hline Sediment & 81710 & Sample \#3 & 0.00096 & 0.00096 & 0.0012 & 0.0027 & 0.00096 & 0.0019 \\
\hline A & rin & $A-B H C \cdot A-B H C$ & G-BHC - G-BHC & D-BHC - D-BHC & PPD & PDDD & & \\
\hline
\end{tabular}

Page 1 
Delaware River Water Analysis (Coarse-Grained Site)

\begin{tabular}{|c|c|c|c|c|c|c|c|c|}
\hline \multirow{2}{*}{$\begin{array}{l}\text { SAMPLE } \\
\text { TYPE }\end{array}$} & & DESCRIPTION & PPDDE & PPDDT & HPTCL & DIELDRIN & ENDOI & ENDOII \\
\hline & & Detection Limit (mg/l) & 0.000070 & 0.000070 & 0.0000350 & 0.000070 & 0.000035 & 0.006070 \\
\hline & & Plume Monitoring & & & & & & \\
\hline Water & 80841 & Background, dissolved & 0.000050 & 0.000050 & 0.0000250 & 0.000050 & 0.000025 & 0.000050 \\
\hline Water & 80756 & Background, total & 0.000050 & 0.000050 & 0.0000250 & 0.000050 & 0.000025 & 0.000050 \\
\hline Water & 80842 & 0-10 min, overflow, dissolved & 0.000050 & 0.000050 & 0.0000250 & 0.000050 & 0.000025 & 0.000050 \\
\hline Water & 80843 & 10-20 min, overflow, dissolved & 0.000050 & 0.000050 & 0.0000250 & 0.000050 & 0.000025 & 0.000050 \\
\hline Water & 80844 & 20-30 min, overflow, dissolved & 0.000050 & 0.000049 & 0.0000240 & 0.000049 & 0.000024 & 0.000049 \\
\hline Water & 80757 & 0-10 min, overflow, total & 0.000050 & 0.000050 & 0.0000250 & 0.000050 & 0.000025 & 0.000050 \\
\hline Water & 80758 & 10-20 min, overflow, total & 0.000050 & 0.000050 & 0.0000250 & 0.000050 & 0.000025 & 0.000050 \\
\hline Water & 80759 & 20-30 min, overflow, total & 0.000050 & 0.000050 & 0.0000250 & 0.000050 & 0.000025 & 0.000050 \\
\hline Water & 80845 & 0-10 min, non-overflow, dissolved & 0.000050 & 0.000050 & 0.0000250 & 0.000050 & 0.000025 & 0.000050 \\
\hline Water & 80846 & 10-20 min, non-overflow, dissolved & 0.000050 & 0.000050 & 0.0000250 & 0.000050 & 0.000025 & 0.000050 \\
\hline Water & 80847 & 20-30 min, non-overflow, dissolved & 0.000070 & 0.000070 & 0.0000350 & 0.000070 & 0.000035 & 0.000070 \\
\hline Water & 80760 & 0-10 min, non-overflow, total & 0.000050 & 0.000050 & 0.0000250 & 0.000050 & 0.000025 & 0.000050 \\
\hline Water & 80761 & 10-20 min, non-overflow, total & 0.000050 & 0.000050 & 0.0000250 & 0.000050 & 0.000025 & 0.000050 \\
\hline \multirow[t]{2}{*}{ Water } & 70762 & 20-30 min, non-overflow, total & 0.000055 & 0.000055 & 0.0000280 & 0.000055 & 0.000028 & 0.000055 \\
\hline & & Hopper Inflow Monitoring & & & & & & \\
\hline Water & 80800 & $3 \& 6 \mathrm{~min}$, dissolved & 0.000050 & 0.000050 & 0.0000100 & 0.000050 & 0.000025 & 0.000050 \\
\hline Water & 80801 & $9 \& 12 \mathrm{~min}$, dissolved & 0.000050 & 0.000050 & 0.0000140 & 0.000050 & 0.000025 & 0.000050 \\
\hline Water & 80802 & $15 \& 18 \mathrm{~min}$, dissolved & 0.000050 & 0.000050 & 0.0000270 & 0.000050 & 0.000025 & 0.000050 \\
\hline Water & 80803 & 21824 min, dissolved & 0.000050 & 0.000050 & 0.0000100 & 0.000050 & 0.000025 & 0.000050 \\
\hline Water & 80804 & $27 \& 30 \mathrm{~min}$, dissolved & 0.000050 & 0.000050 & 0.0000130 & 0.000050 & 0.000010 & 0.000050 \\
\hline Water & 80704 & $3 \& 6 \mathrm{~min}$, total & 0.000050 & 0.000042 & 0.0000270 & 0.000050 & 0.000025 & 0.000050 \\
\hline Water & 80705 & $9 \& 12 \mathrm{~min}$, total & 0.000024 & 0.000660 & 0.0000160 & 0.000050 & 0.000025 & 0.000050 \\
\hline Water & 80706 & $15 \& 18 \mathrm{~min}$, total & 0.000023 & 0.000053 & 0.0000190 & 0.000053 & 0.000027 & 0.000053 \\
\hline Water & 80707 & $21224 \mathrm{~min}$, total & 0.000017 & 0.000053 & 0.0000070 & 0.000053 & 0.000027 & 0.000053 \\
\hline \multirow[t]{2}{*}{ Water } & 80708 & $27830 \mathrm{~min}$, total & 0.000029 & 0.000036 & 0.0000130 & 0.000027 & 0.000025 & 0.000050 \\
\hline & & Hopper Overflow Monitoring & & & & & & \\
\hline Water & 80805 & 2\& $4 \mathrm{~min}$, dissolved & 0.000050 & 0.000050 & 0.0000250 & 0.000050 & 0.000025 & 0.000050 \\
\hline Water & 80806 & $6 \& 8 \mathrm{~min}$, dissolved & 0.000050 & 0.000050 & 0.0000250 & 0.000050 & 0.000025 & 0.000050 \\
\hline Water & 80807 & $10 \& 12 \mathrm{~min}$, dissolved & 0.000050 & 0.000050 & 0.0000250 & 0.000050 & 0.000025 & 0.000050 \\
\hline Water & 80808 & $14 \& 16 \mathrm{~min}$, dissolved & 0.000050 & 0.000050 & 0.0000250 & 0.000050 & 0.000025 & 0.000050 \\
\hline Water & 80809 & $18820 \mathrm{~min}$, dissolved & 0.000050 & 0.000050 & 0.0000250 & 0.000050 & 0.000025 & 0.000050 \\
\hline Water & 80710 & 2\& $4 \mathrm{~min}$, total & 0.000010 & 0.000024 & 0.0000040 & 0.000050 & 0.000025 & 0.000050 \\
\hline Water & 80711 & 6\& $8 \mathrm{~min}$, total & 0.000005 & 0.000050 & 0.0000250 & 0.000050 & 0.000025 & 0.000050 \\
\hline Water & 80712 & $10 \& 12 \mathrm{~min}$, total & 0.000053 & 0.000053 & 0.0000270 & 0.000053 & 0.000027 & 0.000053 \\
\hline \multirow{3}{*}{ Water } & 80713 & $148.16 \mathrm{~min}$, total & 0.001100 & 0.000053 & 0.0000270 & 0.000053 & 0.000027 & 0.000053 \\
\hline & 80714 & $18820 \mathrm{~min}$, total & 0.000050 & 0.000050 & 0.0000250 & 0.000050 & 0.000025 & 0.000050 \\
\hline & & Site Water & & & & & & \\
\hline Water & 81612 & Sample 1 Total & 0.000050 & 0.000050 & 0.0000250 & 0.000050 & 0.000025 & 0.000050 \\
\hline Water & 81613 & Sample 2 Total & 0.000050 & 0.000050 & 0.0000250 & 0.000050 & 0.000025 & 0.000050 \\
\hline \multirow[t]{2}{*}{ Water } & 81614 & Sample 3 Total & 0.000050 & 0.000050 & 0.0000250 & 0.000050 & 0.000025 & 0.000050 \\
\hline & & Elutriate & & & & & & \\
\hline Water & 81618 & Sample 1 Dissolved & 0.000050 & 0.000050 & 0.0000250 & 0.000050 & 0.000025 & 0.000050 \\
\hline Water & 81619 & Sample 2 Dissolved & 0.000050 & 0.000050 & 0.0000039 & 0.000050 & 0.000025 & 0.000050 \\
\hline Water & 81620 & Sample 3 Dissolved & 0.000050 & 0.000050 & 0.0000050 & 0.000050 & 0.000025 & 0.000050 \\
\hline Water & 81615 & Sample 1 Total & 0.000050 & 0.000050 & 0.0000250 & 0.000050 & 0.000025 & 0.000050 \\
\hline Water & 81616 & Sample 2 Total & 0.000050 & 0.000050 & 0.0000250 & 0.000050 & 0.000025 & 0.000050 \\
\hline Water & 81617 & Sample 3 Total & 0.000050 & 0.000050 & 0.0000250 & 0.000050 & 0.000025 & 0.000050 \\
\hline \multirow{4}{*}{$\begin{array}{l}\text { SAMPLE } \\
\text { TYPE }\end{array}$} & SAMPLE & DESCRIPTION & PPDDE & PPDDT & HPTCL & DIELDRIN & ENDOI & ENDOII \\
\hline & ID & & & & & & & \\
\hline & & Detection Limit (mg/kg) & 0.0019 & 0.0019 & 0.00096 & 0.0036 & 0.00096 & 0.0019 \\
\hline & & Insitu Sediment & & & & & & \\
\hline Sediment & 81708 & Sample \#1 & 0.0019 & 0.0019 & 0.00059 & 0.00046 & 0.00096 & 0.0019 \\
\hline Sediment & 81709 & Sample \#2 & 0.0019 & 0.0019 & 0.00052 & 0.00067 & 0.00096 & 0.0019 \\
\hline Sediment & 81710 & Sample \#3 & 0.0019 & 0.0019 & 0.00049 & 0.00058 & 0.00096 & 0.0019 \\
\hline
\end{tabular}

PPDDE-PPDDE PPDDT-PPDDT HPTCL-Heptachlor DIELDRIN-Dieldrin ENDOI-A-Endosulfan ENDOII-B-Endosulfan BOL.D - less than values

Values below less than values are estimated results. Results are less than the reporting limit

Page 2 
Pestcoar

Delaware River Water Analysis (Coarse-Grained Site)

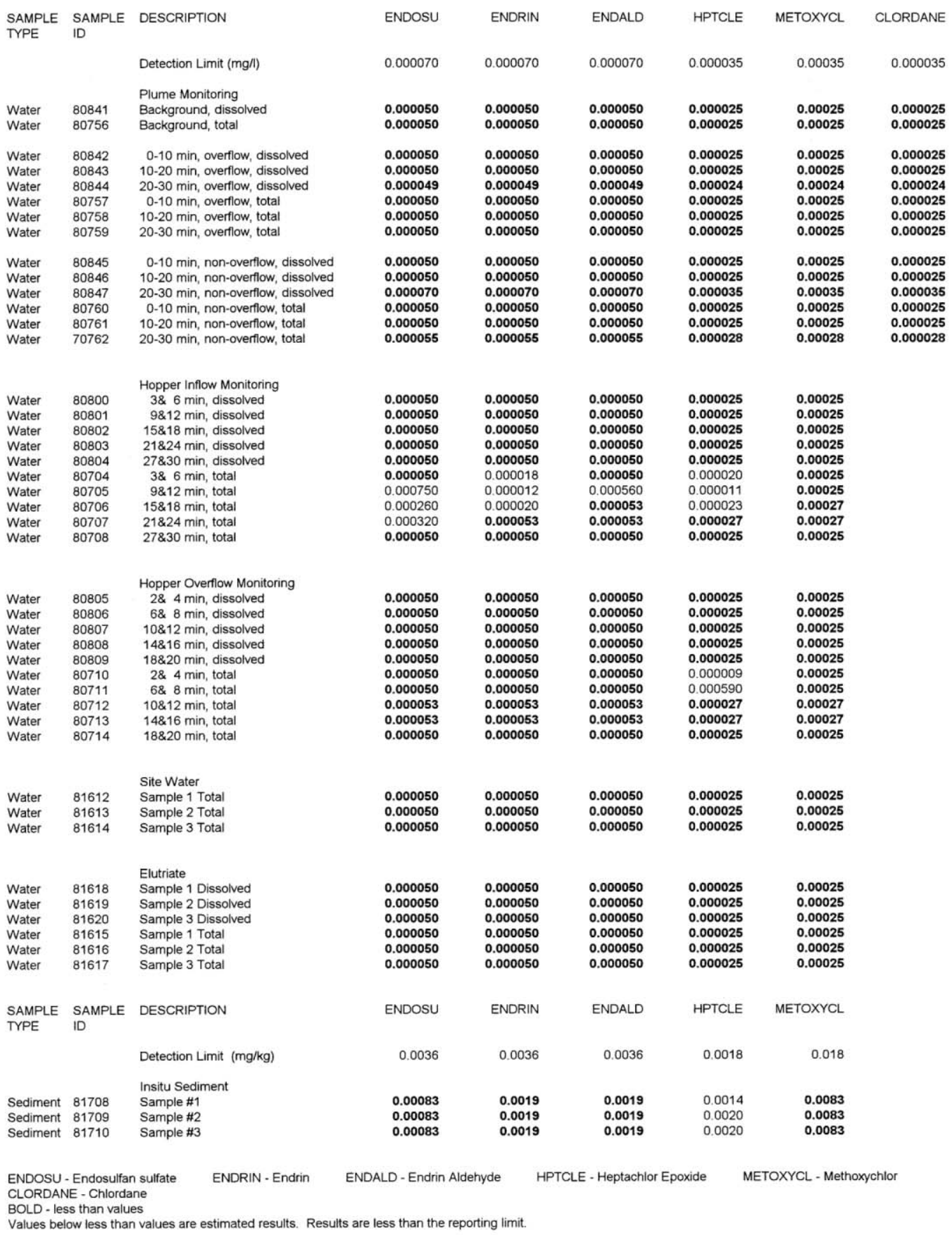

Page 3 
Pestcoar

Delaware River Water Analysis (Coarse-Grained Site)

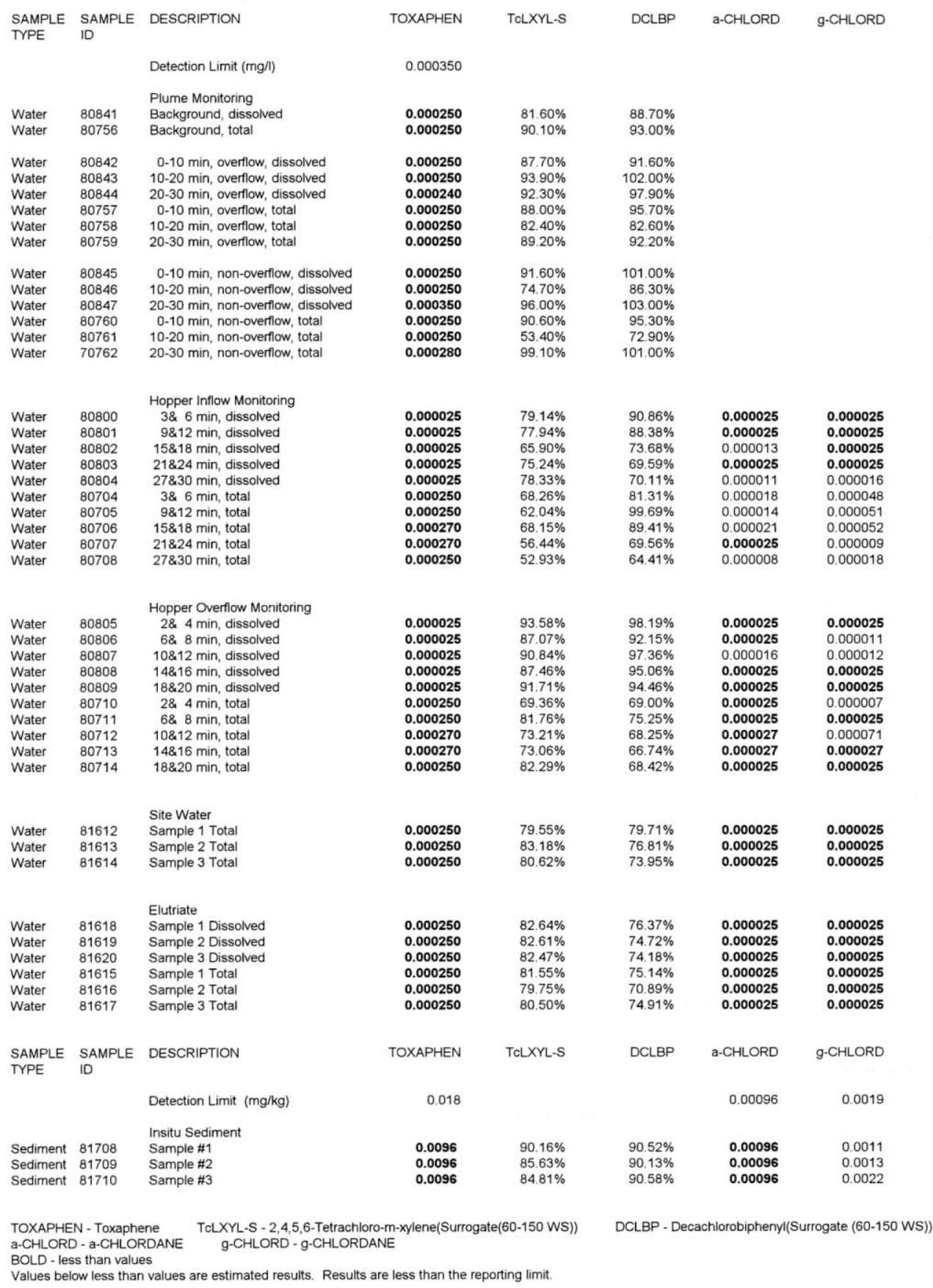

Page 4 
Delaware River Water Analysis (Coarse-Grained Site)

\begin{tabular}{|c|c|c|c|c|c|c|c|c|c|}
\hline \multirow[t]{2}{*}{$\begin{array}{l}\text { SAMPLE } \\
\text { TYPE }\end{array}$} & $\begin{array}{l}\text { SAMPLE } \\
\text { ID }\end{array}$ & DESCRIPTION & РCB 22 & РСВ 33 & РСB 37 & PCB 42 & PCB 47 & РCB 64 & PCB 74 \\
\hline & & Detection Limit (mg/l) & 0.0000011 & 0.0000011 & 0.0000011 & 0.0000011 & 0.0000011 & 0.0000011 & 0.0000011 \\
\hline & & Plume Monitoring & & & & & & & \\
\hline Water & 80834 & Background, dissolved & 0.0000011 & 0.0000011 & 0.0000011 & 0.0000011 & 0.0000011 & 0.0000011 & 0.0000011 \\
\hline Water & 80749 & Background, total & 0.0000010 & 0.0000010 & 0.0000010 & 0.0000010 & 0.0000010 & 0.0000010 & 0.0000010 \\
\hline Water & 80835 & 0-10 min, overflow, dissolved & 0.0000010 & 0.0000010 & 0.0000010 & 0.0000010 & 0.0000010 & 0.0000010 & 0.0000010 \\
\hline Water & 80836 & 10-20 min, overflow, dissolved & 0.0000010 & 0.0000010 & 0.0000010 & 0.0000010 & 0.0000010 & 0.0000010 & 0.0000010 \\
\hline Water & 80837 & 20-30 min, overflow, dissolved & 0.0000010 & 0.0000010 & 0.0000010 & 0.0000010 & 0.0000010 & 0.0000010 & 0.0000010 \\
\hline Water & 80750 & $0-10 \mathrm{~min}$, overflow, total & 0.0000011 & 0.0000011 & 0.0000011 & 0.0000011 & 0.0000011 & 0.0000011 & 0.0000011 \\
\hline Water & 80751 & 10-20 min, overflow, total & 0.0000004 & 0.0000010 & 0.0000010 & 0.0000010 & 0.0000010 & 0.0000010 & 0.0000010 \\
\hline Water & 80752 & $20-30$ min, overflow, total & 0.0000010 & 0.0000010 & 0.0000010 & 0.0000010 & 0.0000010 & 0.0000010 & 0.0000010 \\
\hline Water & 80838 & 0-10 min, non-overflow, dissolve & 0.0000010 & 0.0000010 & 0.0000010 & 0.0000010 & 0.0000010 & 0.0000010 & 0.0000010 \\
\hline Water & 80839 & 10-20 min, non-overflow, dissolve & 0.0000011 & 0.0000011 & 0.0000011 & 0.0000011 & 0.0000011 & 0.0000011 & 0.0000011 \\
\hline Water & 80840 & 20-30 min, non-overflow, dissolve & 0.0000011 & 0.0000011 & 0.0000011 & 0.0000011 & 0.0000011 & 0.0000011 & 0.0000011 \\
\hline Water & 80753 & $0-10 \mathrm{~min}$, non-overflow, total & 0.0000010 & 0.0000010 & 0.0000010 & 0.0000010 & 0.0000010 & 0.0000010 & 0.0000010 \\
\hline Water & 80754 & 10-20 min, non-overflow, total & 0.0000010 & 0.0000010 & 0.0000010 & 0.0000010 & 0.0000010 & 0.0000010 & 0.0000010 \\
\hline \multirow[t]{2}{*}{ Water } & 80755 & 20-30 min, non-overflow, total & 0.0000011 & 0.0000011 & 0.0000011 & 0.0000011 & 0.0000011 & 0.0000011 & 0.0000011 \\
\hline & & Hopper Inflow Monitoring & & & & & & & \\
\hline Water & 80790 & $3 \& 6 \mathrm{~min}$, dissolved & 0.0000010 & 0.0000010 & 0.0000010 & 0.0000022 & 0.0000010 & 0.0000010 & 0.0000010 \\
\hline Water & 80791 & $9 \& 12 \mathrm{~min}$, dissolved & 0.0000010 & 0.0000010 & 0.0000010 & 0.0000020 & 0.0000010 & 0.0000010 & 0.0000010 \\
\hline Water & 80792 & $15 \& 18 \mathrm{~min}$, dissolved & 0.0000010 & 0.0000010 & 0.0000010 & 0.0000014 & 0.0000005 & 0.0000010 & 0.0000010 \\
\hline Water & 80793 & $21 \& 24 \mathrm{~min}$, dissolved & 0.0000010 & 0.0000010 & 0.0000010 & 0.0000012 & 0.0000015 & 0.0000010 & 0.0000010 \\
\hline Water & 80794 & $27 \& 30 \mathrm{~min}$, dissolved & 0.0000010 & 0.0000010 & 0.0000010 & 0.0000009 & 0.0000008 & 0.0000010 & 0.0000010 \\
\hline Water & 80692 & $3 \& 6 \mathrm{~min}$, total & 0.0000010 & 0.0000010 & 0.0000010 & 0.0000010 & 0.0000010 & 0.0000010 & 0.0000010 \\
\hline Water & 80693 & $9 \& 12 \mathrm{~min}$, total & 0.0000010 & 0.0000010 & 0.0000010 & 0.0000010 & 0.0000010 & 0.0000010 & 0.0000010 \\
\hline Water & 80694 & $15 \& 18 \mathrm{~min}$, total & 0.0000010 & 0.0000010 & 0.0000010 & 0.0000010 & 0.0000010 & 0.0000010 & 0.0000010 \\
\hline Water & 80695 & $21 \& 24 \mathrm{~min}$, total & 0.0000011 & 0.0000011 & 0.0000011 & 0.0000011 & 0.0000011 & 0.0000027 & 0.0000011 \\
\hline \multirow[t]{2}{*}{ Water } & 80696 & $27 \& 30 \mathrm{~min}$, total & 0.0000010 & 0.0000010 & 0.0000010 & 0.0000010 & 0.0000010 & 0.0000020 & 0.0000010 \\
\hline & & Hopper Overflow Monitoring & & & & & & & \\
\hline Water & 80795 & 28. $4 \mathrm{~min}$, dissolved & 0.0000010 & 0.0000010 & 0.0000010 & 0.0000017 & 0.0000010 & 0.0000010 & 0.0000010 \\
\hline Water & 80796 & 6\& $8 \mathrm{~min}$, dissolved & 0.0000010 & 0.0000010 & 0.0000010 & 0.0000019 & 0.0000010 & 0.0000010 & 0.0000010 \\
\hline Water & 80797 & $10 \& 12 \mathrm{~min}$, dissolved & 0.0000010 & 0.0000010 & 0.0000010 & 0.0000011 & 0.0000010 & 0.0000010 & 0.0000010 \\
\hline Water & 80798 & $14 \& 16 \mathrm{~min}$, dissolved & 0.0000010 & 0.0000010 & 0.0000010 & 0.0000015 & 0.0000010 & 0.0000010 & 0.0000010 \\
\hline Water & 80799 & $18 \& 20 \mathrm{~min}$, dissolved & 0.0000010 & 0.0000010 & 0.0000010 & 0.0000010 & 0.0000010 & 0.0000010 & 0.0000010 \\
\hline Water & 80698 & 2\& $4 \mathrm{~min}$, total & 0.0000010 & 0.0000010 & 0.0000010 & 0.0000010 & 0.0000010 & 0.0000010 & 0.0000010 \\
\hline Water & 80699 & 6\& $8 \mathrm{~min}$, total & 0.0000010 & 0.0000010 & 0.0000010 & 0.0000010 & 0.0000010 & 0.0000010 & 0.0000010 \\
\hline Water & 80700 & $10 \& 12 \mathrm{~min}$, total & 0.0000010 & 0.0000010 & 0.0000010 & 0.0000010 & 0.0000010 & 0.0000010 & 0.0000010 \\
\hline Water & 80701 & $14 \& 16 \mathrm{~min}$, total & 0.0000011 & 0.0000011 & 0.0000011 & 0.0000011 & 0.0000011 & 0.0000011 & 0.0000011 \\
\hline \multirow[t]{2}{*}{ Water } & 80702 & $18 \& 20 \mathrm{~min}$, total & 0.0000010 & 0.0000010 & 0.0000010 & 0.0000010 & 0.0000010 & 0.0000010 & 0.0000010 \\
\hline & & Site Water & & & & & & & \\
\hline Water & 81594 & Sample 1 Total & 0.0000010 & 0.0000010 & 0.0000010 & 0.0000010 & 0.0000010 & 0.0000010 & 0.0000010 \\
\hline Water & 81595 & Sample 2 Total & 0.0000010 & 0.0000010 & 0.0000010 & 0.0000010 & 0.0000010 & 0.0000010 & 0.0000010 \\
\hline \multirow[t]{2}{*}{ Water } & 81596 & Sample 3 Total & 0.0000010 & 0.0000010 & 0.0000010 & 0.0000010 & 0.0000010 & 0.0000010 & 0.0000010 \\
\hline & & Elutriate & & & & & & & \\
\hline Water & 81600 & Sample 1 Dissolved & 0.0000010 & 0.0000010 & 0.0000010 & 0.0000010 & 0.0000010 & 0.0000010 & 0.0000010 \\
\hline Water & 81601 & Sample 2 Dissolved & 0.0000010 & 0.0000010 & 0.0000010 & 0.0000010 & 0.0000010 & 0.0000010 & 0.0000010 \\
\hline Water & 81602 & Sample 3 Dissolved & 0.0000010 & 0.0000010 & 0.0000010 & 0.0000010 & 0.0000010 & 0.0000010 & 0.0000010 \\
\hline Water & 81597 & Sample 1 Total & 0.0000010 & 0.0000010 & 0.0000010 & 0.0000010 & 0.0000010 & 0.0000010 & 0.0000010 \\
\hline Water & 81598 & Sample 2 Total & 0.0000010 & 0.0000010 & 0.0000010 & 0.0000010 & 0.0000010 & 0.0000010 & 0.0000010 \\
\hline Water & 81599 & Sample 3 Total & 0.0000010 & 0.0000010 & 0.0000010 & 0.0000010 & 0.0000010 & 0.0000010 & 0.0000010 \\
\hline \multirow[t]{3}{*}{ SAMPLE } & SAMPLE & DESCRIPTION & РСВ 22 & РСВ 33 & РСB 37 & РCB 42 & PCB 47 & PCB 64 & PCB 74 \\
\hline & & Detection Limit ( $\mathrm{mg} / \mathrm{kg}$ ) & 0.00033 & 0.00033 & 0.00033 & 0.00033 & 0.00033 & 0.00033 & 0.00033 \\
\hline & & Insitu Sediment & & & & & & & \\
\hline Sediment & 81714 & Sample \#1 & 0.00033 & 0.00033 & 0.00033 & 0.00033 & 0.00033 & 0.00033 & 0.00033 \\
\hline Sediment & 81715 & Sample \#2 & 0.00033 & 0.00033 & 0.00033 & 0.00033 & 0.00033 & 0.00033 & 0.00033 \\
\hline Sediment & 81716 & Sample \#3 & 0.00033 & 0.00033 & 0.00033 & 0.00033 & 0.00033 & 0.00033 & 0.00033 \\
\hline
\end{tabular}

BOLD - less than values

Values below less than values are estimated results. Results are less than the reporting limit. 
Delaware River Water Analysis (Coarse-Grained Site)

\begin{tabular}{|c|c|c|c|c|c|c|c|c|c|}
\hline \multirow[t]{2}{*}{$\begin{array}{l}\text { SAMPLE } \\
\text { TYPE }\end{array}$} & $\begin{array}{l}\text { SAMPLE } \\
\text { ID }\end{array}$ & DESCRIPTION & PCB 80 & PCB 81 & PCB 84 & PCB 91 & PCB 92 & PCB 95 & PCB 99 \\
\hline & & Detection Limit (mg/) & 0.0000011 & 0.0000011 & 0.00000110 & 0.0000011 & 0.0000011 & 0.00000110 & 0.00000110 \\
\hline & & Plume Monitoring & & & & & & & \\
\hline Water & 80834 & Background, dissolved & 0.0000011 & 0.0000011 & 0.00000110 & 0.0000011 & 0.0000011 & 0.00000080 & 0.00000110 \\
\hline Water & 80749 & Background, total & 0.0000010 & 0.0000010 & 0.00000100 & 0.0000010 & 0.0000010 & 0.00000060 & 0.00000100 \\
\hline Water & 80835 & 0-10 min, overflow, dissolved & 0.0000010 & 0.0000010 & 0.00000060 & 0.0000010 & 0.0000010 & 0.00000090 & 0.00000100 \\
\hline Water & 80836 & 10-20 min, overflow, dissolved & 0.0000010 & 0.0000010 & 0.00000060 & 0.0000010 & 0.0000010 & 0.00000080 & 0.00000040 \\
\hline Water & 80837 & 20-30 min, overflow, dissolved & 0.0000010 & 0.0000010 & 0.00000050 & 0.0000010 & 0.0000010 & 0.00000090 & 0.00000070 \\
\hline Water & 80750 & 0-10 $\mathrm{min}$, overflow, total & 0.0000011 & 0.0000011 & 0.00000110 & 0.0000011 & 0.0000011 & 0.00000110 & 0.00000110 \\
\hline Water & 80751 & 10-20 min, overflow, total & 0.0000010 & 0.0000010 & 0.00000040 & 0.0000010 & 0.0000010 & 0.00000090 & 0.00000100 \\
\hline Water & 80752 & 20-30 min, overflow, total & 0.0000010 & 0.0000010 & 0.00000100 & 0.0000010 & 0.0000010 & 0.00000050 & 0.00000100 \\
\hline Water & 80838 & 0-10 min, non-overflow, dissolve & 0.0000010 & 0.0000010 & 0.00000080 & 0.0000010 & 0.0000010 & 0.00000080 & 0.00000050 \\
\hline Water & 80839 & 10-20 $\mathrm{min}$, non-overflow, dissolve & 0.0000011 & 0.0000011 & 0.00000050 & 0.0000011 & 0.0000011 & 0.00000090 & 0.00000040 \\
\hline Water & 80840 & 20-30 min, non-overflow, dissolve & 0.0000011 & 0.0000011 & 0.00000050 & 0.0000011 & 0.0000011 & 0.00000110 & 0.00000040 \\
\hline Water & 80753 & 0-10 min, non-overflow, total & 0.0000010 & 0.0000010 & 0.00000100 & 0.0000010 & 0.0000010 & 0.00000050 & 0.00000100 \\
\hline Water & 80754 & 10-20 $\mathrm{min}$, non-overflow, total & 0.0000010 & 0.0000010 & 0.00000100 & 0.0000010 & 0.0000010 & 0.00000070 & 0.00000100 \\
\hline \multirow[t]{2}{*}{ Water } & 80755 & 20-30 min, non-overflow, total & 0.0000011 & 0.0000011 & 0.00000110 & 0.0000011 & 0.0000011 & 0.00000110 & 0.00000050 \\
\hline & & Hopper Inflow Monitoring & & & & & & & \\
\hline Water & 80790 & $3 \& 6 \mathrm{~min}$, dissolved & 0.0000010 & 0.0000010 & 0.00000140 & 0.0000010 & 0.0000010 & 0.00000120 & 0.00000050 \\
\hline Water & 80791 & $9 \& 12 \mathrm{~min}$, dissolved & 0.0000010 & 0.0000010 & 0.00000110 & 0.0000010 & 0.0000010 & 0.00000110 & 0.00000040 \\
\hline Water & 80792 & $15 \& 18 \mathrm{~min}$, dissolved & 0.0000010 & 0.0000010 & 0.00000100 & 0.0000010 & 0.0000010 & 0.00000100 & 0.00000100 \\
\hline Water & 80793 & $21 \& 24 \mathrm{~min}$, dissolved & 0.0000010 & 0.0000010 & 0.00000110 & 0.0000010 & 0.0000010 & 0.00000160 & 0.00000060 \\
\hline Water & 80794 & $27 \& 30 \mathrm{~min}$, dissolved & 0.0000010 & 0.0000010 & 0.00000170 & 0.0000010 & 0.0000010 & 0.00000160 & 0.00000100 \\
\hline Water & 80692 & 3\& $6 \mathrm{~min}$, total & 0.0000010 & 0.0000010 & 0.00000100 & 0.0000010 & 0.0000010 & 0.00000120 & 0.00000100 \\
\hline Water & 80693 & $9 \& 12 \mathrm{~min}$, total & 0.0000010 & 0.0000010 & 0.00000100 & 0.0000010 & 0.0000010 & 0.00000140 & 0.00000100 \\
\hline Water & 80694 & $15 \& 18 \mathrm{~min}$, total & 0.0000010 & 0.0000010 & 0.00000280 & 0.0000010 & 0.0000010 & 0.00000230 & 0.00000100 \\
\hline Water & 80695 & $21 \& 24 \mathrm{~min}$, total & 0.0000011 & 0.0000011 & 0.00000330 & 0.0000011 & 0.0000011 & 0.00000290 & 0.00000060 \\
\hline \multirow[t]{2}{*}{ Water } & 80696 & $27 \& 30 \mathrm{~min}$, total & 0.0000010 & 0.0000010 & 0.00000240 & 0.0000010 & 0.0000010 & 0.00000200 & 0.00000130 \\
\hline & & Hopper Overflow Monitoring & & & & & & & \\
\hline Water & 80796 & 2\& $4 \mathrm{~min}$, dissolved & 0.0000010 & 0.0000010 & 0.00000100 & 0.0000010 & 0.0000010 & 0.00000100 & 0.00000100 \\
\hline Water & 80797 & $\begin{array}{r}6 \& 8 \mathrm{~min} \text {, dissolved } \\
10 \& 12 \mathrm{~min} \text {, dissolved }\end{array}$ & 0.0000010 & 0.0000010 & 0.00000100 & 0.0000010 & $\begin{array}{l}0.0000010 \\
0.0000010\end{array}$ & $\begin{array}{l}0.00000050 \\
0.00000050\end{array}$ & $\begin{array}{l}0.00000100 \\
0.00000100\end{array}$ \\
\hline Water & 80798 & $14 \& 16 \mathrm{~min}$, dissolved & 0.0000010 & 0.0000010 & 0.00000100 & 0.0000010 & 0.0000010 & 0.00000060 & 0.00000100 \\
\hline Water & 80799 & $18 \& 20 \mathrm{~min}$, dissolved & 0.0000010 & 0.0000010 & 0.00000100 & 0.0000010 & 0.0000010 & 0.00000060 & 0.00000100 \\
\hline Water & 80698 & 2\& 4 min, total & 0.0000010 & 0.0000010 & 0.00000120 & 0.0000010 & 0.0000010 & 0.00000130 & 0.00000100 \\
\hline Water & 80699 & $6 \& 8 \mathrm{~min}$, total & 0.0000010 & 0.0000010 & 0.00000100 & 0.0000010 & 0.0000010 & 0.00000090 & 0.00000100 \\
\hline Water & 80700 & $10 \& 12 \mathrm{~min}$, total & 0.0000010 & 0.0000010 & 0.00000100 & 0.0000010 & 0.0000010 & 0.00000140 & 0.00000100 \\
\hline Water & 80701 & $14 \& 16 \mathrm{~min}$, total & 0.0000011 & 0.0000011 & 0.00000110 & 0.0000011 & 0.0000011 & 0.00000110 & 0.00000110 \\
\hline \multirow[t]{2}{*}{ Water } & 80702 & $18 \& 20 \mathrm{~min}$, total & 0.0000010 & 0.0000010 & 0.00000080 & 0.0000010 & 0.0000010 & 0.00000180 & 0.00000100 \\
\hline & & Site Water & & & & & & & \\
\hline Water & 81594 & Sample 1 Total & 0.0000010 & 0.0000010 & 0.00000100 & 0.0000010 & 0.0000010 & 0.00000100 & 0.00000100 \\
\hline Water & 81595 & Sample 2 Total & 0.0000010 & 0.0000010 & 0.00000100 & 0.0000010 & 0.0000010 & 0.00000090 & 0.00000047 \\
\hline \multirow[t]{2}{*}{ Water } & 81596 & Sample 3 Total & 0.0000010 & 0.0000010 & 0.00000100 & 0.0000010 & 0.0000010 & 0.00000100 & 0.00000100 \\
\hline & & Elutriate & & & & & & & \\
\hline Water & 81600 & Sample 1 Dissolved & 0.0000010 & 0.0000010 & 0.00000043 & 0.0000010 & 0.0000010 & 0.00000086 & 0.00000100 \\
\hline Water & 81601 & Sample 2 Dissolved & 0.0000010 & 0.0000010 & 0.00000034 & 0.0000010 & 0.0000010 & 0.00000065 & 0.00000100 \\
\hline Water & 81602 & Sample 3 Dissolved & 0.0000010 & 0.0000010 & 0.00000035 & 0.0000010 & 0.0000010 & 0.00000069 & 0.00000032 \\
\hline Water & 81597 & Sample 1 Total & 0.0000010 & 0.0000010 & 0.00000100 & 0.0000010 & 0.0000010 & 0.00000048 & 0.00000100 \\
\hline Water & 81598 & Sample 2 Total & 0.0000010 & 0.0000010 & 0.00000100 & 0.0000010 & 0.0000010 & 0.00000150 & 0.00000049 \\
\hline Water & 81599 & Sample 3 Total & 0.0000010 & 0.0000010 & 0.00000100 & 0.0000010 & 0.0000010 & 0.00000100 & 0.00000100 \\
\hline \multirow[t]{3}{*}{ SAMPLE } & $\begin{array}{l}\text { SAMPLE } \\
\text { ID }\end{array}$ & DESCRIPTION & PCB 80 & PCB 81 & PCB 84 & PCB 91 & РCB 92 & РСB 95 & РCB 99 \\
\hline & & Detection Limit (mg/kg) & 0.00033 & 0.00033 & 0.00033 & 0.00033 & 0.00033 & 0.00033 & 0.00033 \\
\hline & & Insitu Sediment & & & & & & & \\
\hline Sediment & 81714 & Sample \#1 & 0.00033 & 0.00033 & 0.00033 & 0.00033 & 0.00033 & 0.00033 & 0.00033 \\
\hline Sediment & 81715 & Sample \#2 & 0.00033 & 0.00033 & 0.00033 & 0.00033 & 0.00033 & 0.00033 & 0.00033 \\
\hline Sediment & 81716 & Sample \#3 & 0.00033 & 0.00033 & 0.00033 & 0.00033 & 0.00033 & 0.00033 & 0.00033 \\
\hline
\end{tabular}

BOLD - less than values

BOLD - less than values
Values below less than values are estimated results. Results are less than the reporting limit. 
Delaware River Water Analysis (Coarse-Grained Site)

\begin{tabular}{|c|c|c|c|c|c|c|c|c|c|}
\hline \multirow[t]{2}{*}{$\begin{array}{l}\text { SAMPLE } \\
\text { TYPE }\end{array}$} & $\begin{array}{l}\text { SAMPLE } \\
\text { ID }\end{array}$ & DESCRIPTION & PCB 110 & РСB 119 & РCB 120 & РCB 123 & РCB 126 & PCB 127 & PCB 132 \\
\hline & & Detection Limit (mg/l) & 0.00000110 & 0.0000011 & 0.0000011 & 0.0000011 & 0.0000011 & 0.0000011 & 0.0000011 \\
\hline & & Plume Monitoring & & & & & & & \\
\hline Water & 80834 & Background, dissolved & 0.00000100 & 0.0000011 & 0.0000011 & 0.0000011 & 0.0000011 & 0.0000011 & 0.0000011 \\
\hline Water & 80749 & Background, total & 0.00000050 & 0.0000010 & 0.0000010 & 0.0000010 & 0.0000010 & 0.0000010 & 0.0000010 \\
\hline Water & 80835 & 0-10 min, overflow, dissolved & 0.00000090 & 0.0000010 & 0.0000010 & 0.0000010 & 0.0000010 & 0.0000010 & 0.0000010 \\
\hline Water & 80836 & 10-20 min, overflow, dissolved & 0.00000110 & 0.0000010 & 0.0000010 & 0.0000010 & 0.0000010 & 0.0000010 & 0.0000010 \\
\hline Water & 80837 & 20-30 min, overflow, dissolved & 0.00000080 & 0.0000010 & 0.0000010 & 0.0000010 & 0.0000010 & 0.0000010 & 0.0000010 \\
\hline Water & 80750 & 0-10 min, overflow, total & 0.00000050 & 0.0000011 & 0.0000011 & 0.0000011 & 0.0000011 & 0.0000011 & 0.0000011 \\
\hline Water & 80751 & 10-20 min, overflow, total & 0.00000070 & 0.0000010 & 0.0000010 & 0.0000010 & 0.0000010 & 0.0000010 & 0.0000010 \\
\hline Water & 80752 & 20-30 min, overflow, total & 0.00000050 & 0.0000010 & 0.0000010 & 0.0000010 & 0.0000010 & 0.0000010 & 0.0000010 \\
\hline Water & 80838 & 0-10 min, non-overflow, dissolve & 0.00000110 & 0.0000010 & 0.0000010 & 0.0000010 & 0.0000010 & 0.0000010 & 0.0000010 \\
\hline Water & 80839 & 10-20 min, non-overflow, dissolve & 0.00000070 & 0.0000011 & 0.0000011 & 0.0000011 & 0.0000011 & 0.0000011 & 0.0000011 \\
\hline Water & 80840 & 20-30 min, non-overflow, dissolve & 0.00000110 & 0.0000011 & 0.0000011 & 0.0000011 & 0.0000011 & 0.0000011 & 0.0000011 \\
\hline Water & 80753 & 0-10 $\mathrm{min}$, non-overflow, total & 0.00000050 & 0.0000010 & 0.0000010 & 0.0000010 & 0.0000010 & 0.0000010 & 0.0000010 \\
\hline Water & 80754 & $10-20 \mathrm{~min}$, non-overflow, total & 0.00000060 & 0.0000010 & 0.0000010 & 0.0000010 & 0.0000010 & 0.0000010 & 0.0000010 \\
\hline \multirow[t]{2}{*}{ Water } & 80755 & $20-30 \mathrm{~min}$, non-overflow, total & 0.00000090 & 0.0000011 & 0.0000011 & 0.0000011 & 0.0000011 & 0.0000011 & 0.0000005 \\
\hline & & Hopper Inflow Monitoring & & & & & & & \\
\hline Water & 80790 & 3\& $6 \mathrm{~min}$, dissolved & 0.00000210 & 0.0000010 & 0.0000010 & 0.0000010 & 0.0000010 & 0.0000010 & 0.0000010 \\
\hline Water & 80791 & $9 \& 12 \mathrm{~min}$, dissolved & 0.00000160 & 0.0000010 & 0.0000010 & 0.0000010 & 0.0000010 & 0.0000010 & 0.0000010 \\
\hline Water & 80792 & $15 \& 18 \mathrm{~min}$, dissolved & 0.00000100 & 0.0000010 & 0.0000010 & 0.0000010 & 0.0000010 & 0.0000010 & 0.0000010 \\
\hline Water & 80793 & $21 \& 24 \mathrm{~min}$, dissolved & 0.00000160 & 0.0000010 & 0.0000010 & 0.0000010 & 0.0000010 & 0.0000010 & 0.0000010 \\
\hline Water & 80794 & $27 \& 30 \mathrm{~min}$, dissolved & 0.00000160 & 0.0000010 & 0.0000007 & 0.0000010 & 0.0000010 & 0.0000010 & 0.0000010 \\
\hline Water & 80692 & $3 \& 6 \mathrm{~min}$, total & 0.00000160 & 0.0000010 & 0.0000026 & 0.0000010 & 0.0000010 & 0.0000010 & 0.0000010 \\
\hline Water & 80693 & $9 \& 12 \mathrm{~min}$, total & 0.00000200 & 0.0000010 & 0.0000019 & 0.0000010 & 0.0000010 & 0.0000010 & 0.0000010 \\
\hline Water & 80694 & $15 \& 18 \mathrm{~min}$, total & 0.00000260 & 0.0000010 & 0.0000054 & 0.0000010 & 0.0000010 & 0.0000010 & 0.0000010 \\
\hline Water & 80695 & 21824 min, total & 0.00000260 & 0.0000011 & 0.0000061 & 0.0000011 & 0.0000011 & 0.0000011 & 0.0000011 \\
\hline \multirow[t]{2}{*}{ Water } & 80696 & $27 \& 30 \mathrm{~min}$, total & 0.00000230 & 0.0000010 & 0.0000071 & 0.0000010 & 0.0000010 & 0.0000010 & 0.0000010 \\
\hline & & Hopper Overflow Monitoring & & & & & & & \\
\hline Water & 80795 & 2\& $4 \mathrm{~min}$, dissolved & 0.00000070 & 0.0000010 & 0.0000010 & 0.0000010 & 0.0000010 & 0.0000010 & 0.0000010 \\
\hline Water & 80796 & 6\& $8 \mathrm{~min}$, dissolved & 0.00000100 & 0.0000010 & 0.0000010 & 0.0000010 & 0.0000010 & 0.0000010 & 0.0000010 \\
\hline Water & 80797 & $10 \& 12 \mathrm{~min}$, dissolved & 0.00000050 & 0.0000010 & 0.0000010 & 0.0000010 & 0.0000010 & 0.0000010 & 0.0000010 \\
\hline Water & 80798 & $14 \& 16 \mathrm{~min}$, dissolved & 0.00000060 & 0.0000010 & 0.0000010 & 0.0000010 & 0.0000010 & 0.0000010 & 0.0000010 \\
\hline Water & 80799 & $18 \& 20 \mathrm{~min}$, dissolved & 0.00000050 & 0.0000010 & 0.0000010 & 0.0000010 & 0.0000010 & 0.0000010 & 0.0000010 \\
\hline Water & 80698 & 2\& $4 \mathrm{~min}$, total & 0.00000150 & 0.0000010 & 0.0000008 & 0.0000010 & 0.0000010 & 0.0000010 & 0.0000010 \\
\hline Water & 80699 & $6 \& 8 \mathrm{~min}$, total & 0.00000120 & 0.0000010 & 0.0000005 & 0.0000010 & 0.0000010 & 0.0000010 & 0.0000010 \\
\hline Water & 80700 & $10 \& 12 \mathrm{~min}$, total & 0.00000150 & 0.0000010 & 0.0000010 & 0.0000010 & 0.0000010 & 0.0000010 & 0.0000010 \\
\hline Water & 80701 & $14 \& 16 \mathrm{~min}$, total & 0.00000110 & 0.0000011 & 0.0000006 & 0.0000011 & 0.0000011 & 0.0000011 & 0.0000011 \\
\hline \multirow[t]{2}{*}{ Water } & 80702 & $18 \& 20 \mathrm{~min}$, total & 0.00000160 & 0.0000010 & 0.0000005 & 0.0000010 & 0.0000010 & 0.0000010 & 0.0000010 \\
\hline & & Site Water & & & & & & & \\
\hline Water & 81594 & Sample 1 Total & 0.00000100 & 0.0000010 & 0.0000010 & 0.0000010 & 0.0000010 & 0.0000010 & 0.0000010 \\
\hline Water & 81595 & Sample 2 Total & 0.00000097 & 0.0000010 & 0.0000010 & 0.0000010 & 0.0000010 & 0.0000010 & 0.0000010 \\
\hline \multirow[t]{2}{*}{ Water } & 81596 & Sample 3 Total & 0.00000077 & 0.0000010 & 0.0000010 & 0.0000010 & 0.0000010 & 0.0000010 & 0.0000010 \\
\hline & & Elutriate & & & & & & & \\
\hline Water & 81600 & Sample 1 Dissolved & 0.00000091 & 0.0000010 & 0.0000010 & 0.0000010 & 0.0000010 & 0.0000010 & 0.0000010 \\
\hline Water & 81601 & Sample 2 Dissolved & 0.00000063 & 0.0000010 & 0.0000010 & 0.0000010 & 0.0000010 & 0.0000010 & 0.0000010 \\
\hline Water & 81602 & Sample 3 Dissolved & 0.00000064 & 0.0000010 & 0.0000010 & 0.0000010 & 0.0000010 & 0.0000010 & 0.0000010 \\
\hline Water & 81597 & Sample 1 Total & 0.00000061 & 0.0000010 & 0.0000010 & 0.0000010 & 0.0000010 & 0.0000010 & 0.0000010 \\
\hline Water & 81598 & Sample 2 Total & 0.00000110 & 0.0000010 & 0.0000010 & 0.0000010 & 0.0000010 & 0.0000010 & 0.0000010 \\
\hline Water & 81599 & Sample 3 Total & 0.00000059 & 0.0000010 & 0.0000010 & 0.0000010 & 0.0000010 & 0.0000010 & 0.0000010 \\
\hline \multirow{3}{*}{$\begin{array}{l}\text { SAMPLE } \\
\text { TYPE }\end{array}$} & SAMPLE & DESCRIPTION & PCB 110 & PCB 119 & PCB 120 & PCB 123 & PCB 126 & PCB 127 & РСB 132 \\
\hline & & Detection Limit (mg/kg) & 0.00033 & 0.00033 & 0.00033 & 0.00033 & 0.00033 & 0.00033 & 0.00033 \\
\hline & & Insitu Sediment & & & & & & & \\
\hline Sediment & 81714 & Sample \#1 & 0.00033 & 0.00033 & 0.00033 & 0.00033 & 0.00033 & 0.00033 & 0.00033 \\
\hline Sediment & 81715 & Sample \#2 & 0.00033 & 0.00033 & 0.00033 & 0.00033 & 0.00033 & 0.00033 & $\begin{array}{l}0.00033 \\
0.00033\end{array}$ \\
\hline Sediment & 81716 & Sample \#3 & & 0.00033 & 0.00033 & 0.00033 & 0.00033 & 0.00033 & 0.00033 \\
\hline
\end{tabular}

BOLD - less than values

Values below less than values are estimated results. Results are less than the reporting limit. 
Delaware River Water Analysis (Coarse-Grained Site)

\begin{tabular}{|c|c|c|c|c|c|c|c|c|c|}
\hline \multirow[t]{2}{*}{$\begin{array}{l}\text { SAMPLE } \\
\text { TYPE }\end{array}$} & $\begin{array}{l}\text { SAMPLE } \\
\text { ID }\end{array}$ & DESCRIPTION & PCB 135 & PCB 146 & РCB 149 & PCB 157 & PCB 158 & PCB 166 & PCB 168 \\
\hline & & Detection Limit (mg/l) & 0.0000011 & 0.00000110 & 0.00000110 & 0.0000011 & 0.0000011 & 0.0000011 & 0.00000110 \\
\hline & & Plume Monitoring & & & & & & & \\
\hline Water & 80834 & Background, dissolved & 0.0000011 & 0.00000110 & 0.00000110 & 0.0000011 & 0.0000011 & 0.0000011 & 0.00000110 \\
\hline Water & 80749 & Background, total & 0.0000010 & 0.00000100 & 0.00000100 & 0.0000010 & 0.0000010 & 0.0000010 & 0.00000100 \\
\hline Water & 80835 & 0-10 min, overflow, dissolved & 0.0000010 & 0.00000100 & 0.00000040 & 0.0000010 & 0.0000010 & 0.0000010 & 0.00000100 \\
\hline Water & 80836 & 10-20 min, overflow, dissolved & 0.0000010 & 0.00000050 & 0.00000040 & 0.0000010 & 0.0000010 & 0.0000010 & 0.00000100 \\
\hline Water & 80837 & 20-30 min, overflow, dissolved & 0.0000010 & 0.00000100 & 0.00000040 & 0.0000010 & 0.0000010 & 0.0000010 & 0.00000100 \\
\hline Water & 80750 & 0-10 min, overflow, total & 0.0000011 & 0.00000110 & 0.00000110 & 0.0000011 & 0.0000011 & 0.0000011 & 0.00000110 \\
\hline Water & 80751 & 10-20 min, overflow, total & 0.0000010 & 0.00000100 & 0.00000100 & 0.0000010 & 0.0000010 & 0.0000010 & 0.00000100 \\
\hline Water & 80752 & 20-30 min, overflow, total & 0.0000010 & 0.00000100 & 0.00000100 & 0.0000010 & 0.0000010 & 0.0000010 & 0.00000100 \\
\hline Water & 80838 & 0-10 min, non-overflow, dissolve & 0.0000010 & 0.00000100 & 0.00000100 & 0.0000010 & 0.0000010 & 0.0000010 & 0.00000100 \\
\hline Water & 80839 & & 0.0000011 & 0.00000110 & 0.00000110 & 0.0000011 & 0.0000011 & 0.0000011 & 0.00000110 \\
\hline Water & 80840 & 20-30 min, non-overflow, dissolve & 0.0000011 & 0.00000110 & 0.00000110 & 0.0000011 & 0.0000011 & 0.0000011 & 0.00000110 \\
\hline Water & 80753 & 0-10 min, non-overflow, total & 0.0000010 & 0.00000100 & 0.00000100 & 0.0000010 & 0.0000010 & 0.0000010 & 0.00000100 \\
\hline Water & 80754 & 10-20 min, non-overflow, total & 0.0000010 & 0.00000100 & 0.00000100 & 0.0000010 & 0.0000010 & 0.0000010 & 0.00000100 \\
\hline \multirow[t]{2}{*}{ Water } & 80755 & 20-30 $\mathrm{min}$, non-overflow, total & 0.0000011 & 0.00000110 & 0.00000110 & 0.0000011 & 0.0000011 & 0.0000011 & 0.00000110 \\
\hline & & Hopper Inflow Monitoring & & & & & & & \\
\hline Water & 80790 & $3 \& 6 \mathrm{~min}$, dissolved & 0.0000010 & 0.00000100 & 0.00000040 & 0.0000010 & 0.0000010 & 0.0000010 & 0.00000100 \\
\hline Water & 80791 & $9 \& 12 \mathrm{~min}$, dissolved & 0.0000010 & 0.00000100 & 0.00000040 & 0.0000010 & 0.0000010 & 0.0000010 & 0.00000100 \\
\hline Water & 80792 & $15 \& 18 \mathrm{~min}$, dissolved & 0.0000010 & 0.00000100 & 0.00000040 & 0.0000010 & 0.0000010 & 0.0000010 & 0.00000100 \\
\hline Water & 80793 & 21824 min, dissolved & 0.0000010 & 0.00000100 & 0.00000070 & 0.0000010 & 0.0000010 & 0.0000010 & 0.00000100 \\
\hline Water & 80794 & $27 \& 30 \mathrm{~min}$, dissolved & 0.0000010 & 0.00000100 & 0.00000130 & 0.0000010 & 0.0000010 & 0.0000010 & 0.00000100 \\
\hline Water & 80692 & $3 \& 6 \mathrm{~min}$, total & 0.0000010 & 0.00000100 & 0.00000100 & 0.0000010 & 0.0000010 & 0.0000010 & 0.00000075 \\
\hline Water & 80693 & $9 \& 12 \mathrm{~min}$, total & 0.0000010 & 0.00000100 & 0.00000130 & 0.0000010 & 0.0000010 & 0.0000010 & 0.00000075 \\
\hline Water & 80694 & $15 \& 18 \mathrm{~min}$, total & 0.0000010 & 0.00000100 & 0.00000290 & 0.0000010 & 0.0000010 & 0.0000010 & 0.00000160 \\
\hline Water & 80695 & $21 \& 24 \mathrm{~min}$, total & 0.0000011 & 0.00000100 & 0.00000300 & 0.0000011 & 0.0000011 & 0.0000011 & 0.00000140 \\
\hline \multirow[t]{2}{*}{ Water } & 80696 & $27 \& 30 \mathrm{~min}$, total & 0.0000010 & 0.00000097 & 0.00000300 & 0.0000010 & 0.0000010 & 0.0000010 & 0.00000100 \\
\hline & & Hopper Overflow Monitoring & & & & & & & \\
\hline Water & 80795 & 2\& $4 \mathrm{~min}$, dissolved & 0.0000010 & 0.00000100 & 0.00000100 & 0.0000010 & 0.0000010 & 0.0000010 & 0.00000100 \\
\hline Water & 80796 & 6\& $8 \mathrm{~min}$, dissolved & 0.0000010 & 0.00000100 & 0.00000100 & 0.0000010 & 0.0000010 & 0.0000010 & 0.00000100 \\
\hline Water & 80797 & $10 \& 12 \mathrm{~min}$, dissolved & 0.0000010 & 0.00000100 & 0.00000100 & 0.0000010 & 0.0000010 & 0.0000010 & 0.00000100 \\
\hline Water & 80798 & $14 \& 16 \mathrm{~min}$, dissolved & 0.0000010 & 0.00000100 & 0.00000100 & 0.0000010 & 0.0000010 & 0.0000010 & 0.00000100 \\
\hline Water & 80799 & $18 \& 20 \mathrm{~min}$, dissolved & 0.0000010 & 0.00000100 & 0.00000100 & 0.0000010 & 0.0000010 & 0.0000010 & 0.00000100 \\
\hline Water & 80698 & 2\& $4 \mathrm{~min}$, total & 0.0000010 & 0.00000070 & 0.00000160 & 0.0000010 & 0.0000010 & 0.0000010 & 0.00000061 \\
\hline Water & 80699 & $6 \& 8$ min, total & 0.0000010 & 0.00000100 & 0.00000088 & 0.0000010 & 0.0000010 & 0.0000010 & 0.00000100 \\
\hline Water & 80700 & $10 \& 12 \mathrm{~min}$, total & 0.0000010 & 0.00000100 & 0.00000120 & 0.0000010 & 0.0000010 & 0.0000010 & 0.00000100 \\
\hline Water & 80701 & $14 \& 16 \mathrm{~min}$, total & 0.0000011 & 0.00000067 & 0.00000240 & 0.0000011 & 0.0000011 & 0.0000011 & 0.00000110 \\
\hline \multirow[t]{2}{*}{ Water } & 80702 & $18 \& 20 \mathrm{~min}$, total & 0.0000010 & 0.00000084 & 0.00000110 & 0.0000010 & 0.0000010 & 0.0000010 & 0.00000100 \\
\hline & & Site Water & & & & & & & \\
\hline Water & 81594 & Sample 1 Total & 0.0000010 & 0.00000100 & 0.00000100 & 0.0000010 & 0.0000010 & 0.0000010 & 0.00000100 \\
\hline Water & 81595 & Sample 2 Total & 0.0000010 & 0.00000100 & 0.00000050 & 0.0000010 & 0.0000010 & 0.0000010 & 0.00000100 \\
\hline \multirow[t]{2}{*}{ Water } & 81596 & Sample 3 Total & 0.0000010 & 0.00000100 & 0.00000100 & 0.0000010 & 0.0000010 & 0.0000010 & 0.00000100 \\
\hline & & Elutriate & & & & & & & \\
\hline Water & 81600 & Sample 1 Dissolved & 0.0000010 & 0.00000100 & 0.00000038 & 0.0000010 & 0.0000010 & 0.0000010 & 0.00000100 \\
\hline Water & 81601 & Sample 2 Dissolved & 0.0000010 & 0.00000100 & 0.00000037 & 0.0000010 & 0.0000010 & 0.0000010 & 0.00000100 \\
\hline Water & 81602 & Sample 3 Dissolved & 0.0000010 & 0.00000100 & 0.00000100 & 0.0000010 & 0.0000010 & 0.0000010 & 0.00000100 \\
\hline Water & 81597 & Sample 1 Total & 0.0000010 & 0.00000100 & 0.00000100 & 0.0000010 & 0.0000010 & 0.0000010 & 0.00000100 \\
\hline Water & 81598 & Sample 2 Total & 0.0000010 & 0.00000100 & 0.00000066 & 0.0000010 & 0.0000010 & 0.0000010 & 0.00000100 \\
\hline Water & 81599 & Sample 3 Total & 0.0000010 & 0.00000100 & 0.00000100 & 0.0000010 & 0.0000010 & 0.0000010 & 0.00000100 \\
\hline \multirow{3}{*}{$\begin{array}{l}\text { SAMPLE } \\
\text { TYPE }\end{array}$} & SAMPLE & DESCRIPTION & PCB 135 & PCB 146 & РCB 149 & РСВ 157 & PCB 158 & РCB 166 & РСB 168 \\
\hline & & Detection Limit (mg/kg) & 0.00033 & 0.00033 & 0.00033 & 0.00033 & 0.00033 & 0.00033 & 0.00033 \\
\hline & & Insitu Sediment & & & & & & & \\
\hline Sediment & 81714 & Sample \#1 & 0.00033 & 0.00033 & 0.00033 & 0.00033 & 0.00033 & 0.00033 & 0.00033 \\
\hline Sediment & 81715 & Sample \#2 & 0.00033 & 0.00033 & 0.00033 & 0.00033 & 0.00033 & 0.00033 & 0.00033 \\
\hline Sediment & 81716 & Sample \#3 & 0.00033 & 0.00033 & 0.00033 & 0.00033 & 0.00033 & 0.00033 & 0.00033 \\
\hline
\end{tabular}

BOLD - less than values
Values below less than values are estimated results. Results are less than the reporting limit. 
Delaware River Water Analysis (Coarse-Grained Site)

\begin{tabular}{|c|c|c|c|c|c|c|c|c|c|}
\hline \multirow[t]{2}{*}{$\begin{array}{l}\text { SAMPLE } \\
\text { TYPE }\end{array}$} & $\begin{array}{l}\text { SAMPLE } \\
\text { ID }\end{array}$ & DESCRIPTION & PCB 169 & PCB 174 & PCB 177 & PCB 178 & PCB 179 & РСB 8 & PCB 18 \\
\hline & & Detection Limit (mg/) & 0.0000011 & 0.0000011 & 0.0000011 & 0.0000011 & 0.0000011 & 0.0000011 & 0.00000110 \\
\hline & & Plume Monitoring & & & & & & & \\
\hline Water & 80834 & Background, dissolved & 0.0000011 & 0.0000011 & 0.0000011 & 0.0000011 & 0.0000011 & 0.0000011 & 0.00000110 \\
\hline Water & 80749 & Background, total & 0.0000010 & 0.0000010 & 0.0000010 & 0.0000010 & 0.0000010 & 0.0000010 & 0.00000100 \\
\hline Water & 80835 & 0-10 min, overflow, dissolved & 0.0000010 & 0.0000010 & 0.0000010 & 0.0000010 & 0.0000010 & 0.0000010 & 0.00000100 \\
\hline Water & 80836 & $10-20 \mathrm{~min}$, overflow, dissolved & 0.0000010 & 0.0000010 & 0.0000010 & 0.0000010 & 0.0000010 & 0.0000010 & 0.00000100 \\
\hline Water & 80837 & $20-30 \mathrm{~min}$, overflow, dissolved & 0.0000010 & 0.0000010 & 0.0000010 & 0.0000010 & 0.0000010 & 0.0000010 & 0.00000100 \\
\hline Water & 80750 & 0-10 min, overflow, total & 0.0000011 & 0.0000011 & 0.0000011 & 0.0000011 & 0.0000011 & 0.0000011 & 0.00000110 \\
\hline Water & 80751 & 10-20 min, overflow, total & 0.0000010 & 0.0000010 & 0.0000010 & 0.0000010 & 0.0000010 & 0.0000010 & 0.00000100 \\
\hline Water & 80752 & $20-30 \mathrm{~min}$, overflow, total & 0.0000010 & 0.0000010 & 0.0000010 & 0.0000010 & 0.0000010 & 0.0000010 & 0.00000100 \\
\hline Water & 80838 & & 0.0000010 & 0.0000010 & 0.0000010 & 0.0000010 & 0.0000010 & 0.0000010 & 0.00000100 \\
\hline Water & 80839 & $10-20 \mathrm{~min}$, non-overflow, dissolve & 0.0000011 & 0.0000011 & 0.0000011 & 0.0000011 & 0.0000011 & 0.0000011 & 0.00000110 \\
\hline Water & 80840 & 20-30 min, non-overflow, dissolve & 0.0000011 & 0.0000011 & 0.0000011 & 0.0000011 & 0.0000011 & 0.0000011 & 0.00000110 \\
\hline Water & 80753 & 0-10 min, non-overflow, total & 0.0000010 & 0.0000010 & 0.0000010 & 0.0000010 & 0.0000010 & 0.0000010 & 0.00000100 \\
\hline Water & 80754 & $10-20 \mathrm{~min}$, non-overflow, total & 0.0000010 & 0.0000010 & 0.0000010 & 0.0000010 & 0.0000010 & 0.0000010 & 0.00000100 \\
\hline \multirow[t]{2}{*}{ Water } & 80755 & 20-30 min, non-overflow, total & 0.0000011 & 0.0000011 & 0.0000011 & 0.0000011 & 0.0000011 & 0.0000011 & 0.00000110 \\
\hline & & Hopper Inflow Monitoring & & & & & & & \\
\hline Water & 80790 & $3 \& 6 \mathrm{~min}$, dissolved & 0.0000010 & 0.0000010 & 0.0000010 & 0.0000010 & 0.0000010 & 0.0000010 & 0.00000460 \\
\hline Water & 80791 & $9 \& 12 \mathrm{~min}$, dissolved & 0.0000010 & 0.0000010 & 0.0000010 & 0.0000010 & 0.0000010 & 0.0000010 & 0.00000100 \\
\hline Water & 80792 & $15 \& 18 \mathrm{~min}$, dissolved & 0.0000010 & 0.0000010 & 0.0000010 & 0.0000010 & 0.0000010 & 0.0000010 & 0.00000100 \\
\hline Water & 80793 & $21 \& 24 \mathrm{~min}$, dissolved & 0.0000010 & 0.0000010 & 0.0000010 & 0.0000010 & 0.0000010 & 0.0000010 & 0.00000100 \\
\hline Water & 80794 & $27 \& 30 \mathrm{~min}$, dissolved & 0.0000010 & 0.0000010 & 0.0000010 & 0.0000010 & 0.0000010 & 0.0000010 & 0.00000100 \\
\hline Water & 80692 & $3 \& 6 \mathrm{~min}$, total & 0.0000010 & 0.0000010 & 0.0000010 & 0.0000010 & 0.0000010 & 0.0000010 & 0.00000100 \\
\hline Water & 80693 & $9 \& 12 \mathrm{~min}$, total & 0.0000010 & 0.0000010 & 0.0000010 & 0.0000010 & 0.0000010 & 0.0000010 & 0.00000100 \\
\hline Water & 80694 & $15 \& 18 \mathrm{~min}$, total & 0.0000010 & 0.0000084 & 0.0000010 & 0.0000010 & 0.0000010 & 0.0000010 & 0.00000100 \\
\hline Water & 80695 & $21 \& 24 \mathrm{~min}$, total & 0.0000011 & 0.0000058 & 0.0000011 & 0.0000011 & 0.0000011 & 0.0000011 & 0.00000110 \\
\hline \multirow[t]{2}{*}{ Water } & 80696 & $27 \& 30 \mathrm{~min}$, total & 0.0000010 & 0.0000042 & 0.0000010 & 0.0000010 & 0.0000010 & 0.0000010 & 0.00000100 \\
\hline & & Hopper Overflow Monitoring & & & & & & & \\
\hline Water & 80795 & $2 \& 4 \mathrm{~min}$, dissolved & 0.0000010 & 0.0000010 & 0.0000010 & 0.0000010 & 0.0000010 & 0.0000010 & 0.00000100 \\
\hline Water & 80796 & $6 \& 8 \mathrm{~min}$, dissolved & 0.0000010 & 0.0000010 & 0.0000010 & 0.0000010 & 0.0000010 & 0.0000010 & 0.00000100 \\
\hline Water & 80797 & $10 \& 12 \mathrm{~min}$, dissolved & 0.0000010 & 0.0000010 & 0.0000010 & 0.0000010 & 0.0000010 & 0.0000010 & 0.00000100 \\
\hline Water & 80798 & $14 \& 16 \mathrm{~min}$, dissolved & 0.0000010 & 0.0000010 & 0.0000010 & 0.0000010 & 0.0000010 & 0.0000010 & 0.00000100 \\
\hline Water & 80799 & $18 \& 20 \mathrm{~min}$, dissolved & 0.0000010 & 0.0000010 & 0.0000010 & 0.0000010 & 0.0000010 & 0.0000010 & 0.00000100 \\
\hline Water & 80698 & 2\& $4 \mathrm{~min}$, total & 0.0000010 & 0.0000010 & 0.0000010 & 0.0000010 & 0.0000010 & 0.0000010 & 0.00000100 \\
\hline Water & 80699 & 6\& $8 \mathrm{~min}$, total & 0.0000010 & 0.0000010 & 0.0000010 & 0.0000010 & 0.0000010 & 0.0000010 & 0.00000100 \\
\hline Water & 80700 & $10 \& 12 \mathrm{~min}$, total & 0.0000010 & 0.0000010 & 0.0000010 & 0.0000010 & 0.0000010 & 0.0000010 & 0.00000100 \\
\hline Water & 80701 & $14 \& 16 \mathrm{~min}$, total & 0.0000011 & 0.0000011 & 0.0000011 & 0.0000011 & 0.0000011 & 0.0000011 & 0.00000110 \\
\hline \multirow[t]{2}{*}{ Water } & 80702 & $18 \& 20 \mathrm{~min}$, total & 0.0000010 & 0.0000010 & 0.0000010 & 0.0000010 & 0.0000010 & 0.0000010 & 0.00000100 \\
\hline & & Site Water & & & & & & & \\
\hline Water & 81594 & Sample 1 Total & 0.0000010 & 0.0000010 & 0.0000010 & 0.0000010 & 0.0000010 & 0.0000010 & 0.00000070 \\
\hline Water & 81595 & Sample 2 Total & 0.0000010 & 0.0000010 & 0.0000010 & 0.0000010 & 0.0000010 & 0.0000010 & 0.00000063 \\
\hline \multirow[t]{2}{*}{ Water } & 81596 & Sample 3 Total & 0.0000010 & 0.0000010 & 0.0000010 & 0.0000010 & 0.0000010 & 0.0000010 & 0.00000082 \\
\hline & & Elutriate & & & & & & & \\
\hline Water & 81600 & Sample 1 Dissolved & 0.0000010 & 0.0000010 & 0.0000010 & 0.0000010 & 0.0000010 & 0.0000010 & 0.00000100 \\
\hline Water & 81601 & Sample 2 Dissolved & 0.0000010 & 0.0000010 & 0.0000010 & 0.0000010 & 0.0000010 & 0.0000010 & 0.00000100 \\
\hline Water & 81602 & Sample 3 Dissolved & 0.0000010 & 0.0000010 & 0.0000010 & 0.0000010 & 0.0000010 & 0.0000010 & 0.00000100 \\
\hline Water & 81597 & Sample 1 Total & 0.0000010 & 0.0000010 & 0.0000010 & 0.0000010 & 0.0000010 & 0.0000010 & 0.00000052 \\
\hline Water & 81598 & Sample 2 Total & 0.0000010 & 0.0000010 & 0.0000010 & 0.0000010 & 0.0000010 & 0.0000010 & 0.00000058 \\
\hline Water & 81599 & Sample 3 Total & 0.0000010 & 0.0000010 & 0.0000010 & 0.0000010 & 0.0000010 & 0.0000010 & 0.00000100 \\
\hline \multirow{3}{*}{$\begin{array}{l}\text { SAMPLE } \\
\text { TYPE }\end{array}$} & SAMPLE & DESCRIPTION & РCB 169 & РCB 174 & РCB 177 & РСВ 178 & РСB 179 & PCB 8 & PCB 18 \\
\hline & & Detection Limit $(\mathrm{mg} / \mathrm{kg})$ & 0.00033 & 0.00033 & 0.00033 & 0.00033 & 0.00033 & 0.00033 & 0.00033 \\
\hline & & Insitu Sediment & & & & & & & \\
\hline Sediment & 81714 & Sample \#1 & 0.00033 & 0.00033 & 0.00033 & 0.00033 & 0.00033 & 0.00033 & 0.00033 \\
\hline Sediment & 81715 & Sample \#2 & 0.00033 & 0.00033 & 0.00033 & 0.00033 & 0.00033 & 0.00033 & 0.00033 \\
\hline Sediment & 81716 & Sample \#3 & 0.00033 & 0.00033 & 0.00033 & 0.00033 & 0.00033 & 0.00033 & 0.00033 \\
\hline
\end{tabular}

BOLD - less than values

BOLD - less than values
Values below less than values are estimated results. Results are less than the reporting limit. 
Delaware River Water Analysis (Coarse-Grained Site)

\begin{tabular}{|c|c|c|c|c|c|c|c|c|c|}
\hline \multirow[t]{2}{*}{$\begin{array}{l}\text { SAMPLE } \\
\text { TYPE }\end{array}$} & $\begin{array}{l}\text { SAMPLE } \\
\text { ID }\end{array}$ & DESCRIPTION & РСВ 28 & РСВ 31 & РСB 40 & РСB 44 & РСB 49 & РСB 52 & PCB 60 \\
\hline & & Detection Limit (mg/) & 0.0000011 & 0.0000011 & 0.0000011 & 0.00000110 & 0.0000011 & 0.00000110 & 0.0000011 \\
\hline & & Plume Monitoring & & & & & & & \\
\hline Water & 80834 & Background, dissolved & 0.0000011 & 0.0000011 & 0.0000011 & 0.00000140 & 0.0000011 & 0.00000090 & 0.0000011 \\
\hline Water & 80749 & Background, total & 0.0000010 & 0.0000010 & 0.0000010 & 0.00000100 & 0.0000010 & 0.00000060 & 0.0000010 \\
\hline Water & 80835 & $0-10 \mathrm{~min}$, overflow, dissolved & 0.0000010 & 0.0000010 & 0.0000010 & 0.00000100 & 0.0000010 & 0.00000090 & 0.0000010 \\
\hline Water & 80836 & 10-20 min, overflow, dissolved & 0.0000010 & 0.0000010 & 0.0000010 & 0.00000100 & 0.0000010 & 0.00000110 & 0.0000010 \\
\hline Water & 80837 & 20-30 min, overflow, dissolved & 0.0000010 & 0.0000010 & 0.0000010 & 0.00000080 & 0.0000010 & 0.00000100 & 0.0000010 \\
\hline Water & 80750 & 0-10 min, overflow, total & 0.0000011 & 0.0000011 & 0.0000011 & 0.00000110 & 0.0000011 & 0.00000060 & 0.0000011 \\
\hline Water & 80751 & $10-20 \mathrm{~min}$, overflow, total & 0.0000010 & 0.0000051 & 0.0000010 & 0.00000100 & 0.0000010 & 0.00000090 & 0.0000010 \\
\hline Water & 80752 & 20-30 min, overflow, total & 0.0000010 & 0.0000010 & 0.0000010 & 0.00000100 & 0.0000010 & 0.00000050 & 0.0000010 \\
\hline Water & 80838 & 0-10 min, non-overflow, dissolve & 0.0000010 & 0.0000010 & 0.0000010 & 0.00000130 & 0.0000010 & 0.00000090 & 0.0000010 \\
\hline Water & 80839 & $10-20 \mathrm{~min}$, non-overflow, dissolve & 0.0000011 & 0.0000011 & 0.0000011 & 0.00000110 & 0.0000011 & 0.00000090 & 0.0000011 \\
\hline Water & 80840 & 20-30 min, non-overflow, dissolve & 0.0000011 & 0.0000011 & 0.0000011 & 0.00000070 & 0.0000011 & 0.00000100 & 0.0000011 \\
\hline Water & 80753 & 0-10 min, non-overflow, total & 0.0000010 & 0.0000010 & 0.0000010 & 0.00000100 & 0.0000010 & 0.00000060 & 0.0000010 \\
\hline Water & 80754 & 10-20 min, non-overflow, total & 0.0000010 & 0.0000010 & 0.0000010 & 0.00000100 & 0.0000010 & 0.00000080 & 0.0000010 \\
\hline \multirow[t]{2}{*}{ Water } & 80755 & 20-30 min, non-overflow, total & 0.0000011 & 0.0000011 & 0.0000010 & 0.00000110 & 0.0000011 & 0.00000110 & 0.0000011 \\
\hline & & Hopper Inflow Monitoring & & & & & & & \\
\hline Water & 80790 & 3\& $6 \mathrm{~min}$, dissolved & 0.0000010 & 0.0000010 & 0.0000010 & 0.00000100 & 0.0000010 & 0.00000050 & 0.0000010 \\
\hline Water & 80791 & $9 \& 12 \mathrm{~min}$, dissolved & 0.0000010 & 0.0000010 & 0.0000010 & 0.00000100 & 0.0000010 & 0.00000080 & 0.0000010 \\
\hline Water & 80792 & $15 \& 18 \mathrm{~min}$, dissolved & 0.0000010 & 0.0000010 & 0.0000010 & 0.00000100 & 0.0000010 & 0.00000440 & 0.0000010 \\
\hline Water & 80793 & $21 \& 24 \mathrm{~min}$, dissolved & 0.0000010 & 0.0000010 & 0.0000010 & 0.00000100 & 0.0000010 & 0.00000000 & 0.0000010 \\
\hline Water & 80794 & $27830 \mathrm{~min}$, dissolved & 0.0000010 & 0.0000010 & 0.0000010 & 0.00000100 & 0.0000010 & 0.00000100 & 0.0000010 \\
\hline Water & 80692 & $3 \& 6 \mathrm{~min}$, total & 0.0000010 & 0.0000010 & 0.0000010 & 0.00000100 & 0.0000010 & 0.00000100 & 0.0000010 \\
\hline Water & 80693 & $9 \& 12 \mathrm{~min}$, total & 0.0000010 & 0.0000010 & 0.0000010 & 0.00000110 & 0.0000041 & 0.00000100 & 0.0000010 \\
\hline Water & 80694 & $15 \& 18 \mathrm{~min}$, total & 0.0000010 & 0.0000010 & 0.0000010 & 0.00000170 & 0.0000010 & 0.00000200 & 0.0000010 \\
\hline Water & 80695 & $21 \& 24 \mathrm{~min}$, total & 0.0000011 & 0.0000011 & 0.0000011 & 0.00000210 & 0.0000011 & 0.00000280 & 0.0000011 \\
\hline \multirow[t]{2}{*}{ Water } & 80696 & $27 \& 30 \mathrm{~min}$, total & 0.0000010 & 0.0000010 & 0.0000010 & 0.00000470 & 0.0000010 & 0.00000390 & 0.0000010 \\
\hline & & Hopper Overflow Monitoring & & & & & & & \\
\hline Water & 80795 & 2\& $4 \mathrm{~min}$, dissolved & 0.0000010 & 0.0000010 & 0.0000010 & 0.00000100 & 0.0000010 & 0.00000100 & 0.0000010 \\
\hline Water & 80796 & $6 \& 8 \mathrm{~min}$, dissolved & 0.0000010 & 0.0000010 & 0.0000010 & 0.00000100 & 0.0000010 & 0.00000060 & 0.0000010 \\
\hline Water & 80797 & $10 \& 12 \mathrm{~min}$, dissolved & 0.0000010 & 0.0000010 & 0.0000010 & 0.00000100 & 0.0000010 & 0.00000050 & 0.0000010 \\
\hline Water & 80798 & $14816 \mathrm{~min}$, dissolved & 0.0000010 & 0.0000010 & 0.0000010 & 0.00000100 & 0.0000010 & 0.00000060 & 0.0000010 \\
\hline Water & 80799 & $18 \& 20 \mathrm{~min}$, dissolved & 0.0000010 & 0.0000010 & 0.0000010 & 0.00000100 & 0.0000010 & 0.00000060 & 0.0000010 \\
\hline Water & 80698 & 2\& $4 \mathrm{~min}$, total & 0.0000010 & 0.0000010 & 0.0000010 & 0.00000100 & 0.0000010 & 0.00000100 & 0.0000010 \\
\hline Water & 80699 & 6\& $8 \mathrm{~min}$, total & 0.0000010 & 0.0000010 & 0.0000010 & 0.00000100 & 0.0000010 & 0.00000094 & 0.0000010 \\
\hline Water & 80700 & $10 \& 12 \mathrm{~min}$, total & 0.0000010 & 0.0000010 & 0.0000010 & 0.00000063 & 0.0000010 & 0.00000100 & 0.0000010 \\
\hline Water & 80701 & $14 \& 16 \mathrm{~min}$, total & 0.0000011 & 0.0000011 & 0.0000011 & 0.00000110 & 0.0000011 & 0.00000089 & 0.0000011 \\
\hline \multirow[t]{2}{*}{ Water } & 80702 & $18 \& 20 \mathrm{~min}$, total & 0.0000010 & 0.0000010 & 0.0000010 & 0.00000060 & 0.0000010 & 0.00000140 & 0.0000010 \\
\hline & & Site Water & & & & & & & \\
\hline Water & 81594 & Sample 1 Total & 0.0000010 & 0.0000022 & 0.0000010 & 0.00000100 & 0.0000010 & 0.00000082 & 0.0000010 \\
\hline Water & 81595 & Sample 2 Total & 0.0000010 & 0.0000033 & 0.0000010 & 0.00000064 & 0.0000010 & 0.00000096 & 0.0000010 \\
\hline \multirow[t]{2}{*}{ Water } & 81596 & Sample 3 Total & 0.0000010 & 0.0000036 & 0.0000010 & 0.00000073 & 0.0000010 & 0.00000078 & 0.0000010 \\
\hline & & Elutriate & & & & & & & \\
\hline Water & 81600 & Sample 1 Dissolved & 0.0000010 & 0.0000032 & 0.0000010 & 0.00000100 & 0.0000010 & 0.00000100 & 0.0000010 \\
\hline Water & 81601 & Sample 2 Dissolved & 0.0000010 & 0.0000022 & 0.0000010 & 0.00000100 & 0.0000010 & 0.00000084 & 0.0000010 \\
\hline Water & 81602 & Sample 3 Dissolved & 0.0000010 & 0.0000032 & 0.0000010 & 0.00000100 & 0.0000010 & 0.00000095 & 0.0000010 \\
\hline Water & 81597 & Sample 1 Total & 0.0000010 & 0.0000021 & 0.0000010 & 0.00000100 & 0.0000010 & 0.00000054 & 0.0000010 \\
\hline Water & 81598 & Sample 2 Total & 0.0000010 & 0.0000040 & 0.0000010 & 0.00000130 & 0.0000010 & 0.00000170 & 0.0000010 \\
\hline Water & 81599 & Sample 3 Total & 0.0000010 & 0.0000025 & 0.0000010 & 0.00000100 & 0.0000010 & 0.00000058 & 0.0000010 \\
\hline \multirow{3}{*}{$\begin{array}{l}\text { SAMPLE } \\
\text { TYPE }\end{array}$} & SAMPLE & DESCRIPTION & РСВ 28 & PCB 31 & РСв 40 & РCB 44 & РСВ 49 & РСB 52 & PCB 60 \\
\hline & & Detection Limit ( $\mathrm{mg} / \mathrm{kg}$ ) & 0.00033 & 0.00033 & 0.00033 & 0.00033 & 0.00033 & 0.00033 & 0.00033 \\
\hline & & Insitu Sediment & & & & & & & \\
\hline Sediment & 81714 & Sample \#1 & 0.00033 & 0.00033 & 0.00033 & 0.00033 & 0.00033 & 0.00033 & 0.00033 \\
\hline Sediment & 81715 & Sample \#2 & 0.00033 & 0.00033 & 0.00033 & 0.00033 & 0.00033 & 0.00033 & 0.00033 \\
\hline Sediment & 81716 & Sample \#3 & 0.00033 & 0.00033 & 0.00033 & 0.00033 & 0.00033 & 0.00033 & 0.00033 \\
\hline
\end{tabular}

BOLD - less than values

Values below less than values are estimated results. Results are less than the reporting limit. 
Delaware River Water Analysis (Coarse-Grained Site)

\begin{tabular}{|c|c|c|c|c|c|c|c|c|c|}
\hline \multirow[t]{2}{*}{$\begin{array}{l}\text { SAMPLE } \\
\text { TYPE }\end{array}$} & $\begin{array}{l}\text { SAMPLE } \\
\text { ID }\end{array}$ & DESCRIPTION & РCB 70 & РCB 77 & РCB 82 & РСB 86 & РСB 87 & РСB 97 & PCB 101 \\
\hline & & Detection Limit (mg/l) & 0.00000110 & 0.0000011 & 0.0000011 & 0.00000110 & 0.00000110 & 0.00000110 & 0.00000110 \\
\hline & & Plume Monitoring & & & & & & & \\
\hline Water & 80834 & Background, dissolved & 0.00000110 & 0.0000011 & 0.0000011 & 0.00000090 & 0.00000080 & 0.00000090 & 0.00000090 \\
\hline Water & 80749 & Background, total & 0.00000100 & 0.0000010 & 0.0000010 & 0.00000080 & 0.00000050 & 0.00000080 & 0.00000050 \\
\hline Water & 80835 & 0-10 min, overflow, dissolved & 0.00000040 & 0.0000010 & 0.0000010 & 0.00000070 & 0.00000080 & 0.00000070 & 0.00000090 \\
\hline Water & 80836 & $10-20 \mathrm{~min}$, overflow, dissolved & 0.00000040 & 0.0000010 & 0.0000010 & 0.00000070 & 0.00000050 & 0.00000070 & 0.00000110 \\
\hline Water & 80837 & 20-30 min, overflow, dissolved & 0.00000040 & 0.0000010 & 0.0000010 & 0.00000060 & 0.00000040 & 0.00000060 & 0.00000090 \\
\hline Water & 80750 & 0-10 min, overflow, total & 0.00000050 & 0.0000011 & 0.0000011 & 0.00000110 & 0.00000040 & 0.00000110 & 0.00000060 \\
\hline Water & 80751 & $10-20 \mathrm{~min}$, overflow, total & 0.00000050 & 0.0000010 & 0.0000010 & 0.00000040 & 0.00000080 & 0.00000040 & 0.00000060 \\
\hline Water & 80752 & 20-30 min, overflow, total & 0.00000040 & 0.0000010 & 0.0000010 & 0.00000050 & 0.00000100 & 0.00000050 & 0.00000050 \\
\hline Water & 80838 & 0-10 min, non-overflow, dissolve & 0.00000050 & 0.0000010 & 0.0000010 & 0.00000080 & 0.00000070 & 0.00000080 & 0.00000090 \\
\hline Water & 80839 & 10-20 min, non-overflow, dissolve & 0.00000050 & 0.0000011 & 0.0000011 & 0.00000060 & 0.00000070 & 0.00000060 & 0.00000090 \\
\hline Water & 80840 & 20-30 min, non-overflow, dissolve & 0.00000060 & 0.0000011 & 0.0000011 & 0.00000100 & 0.00000080 & 0.00000100 & 0.00000090 \\
\hline Water & 80753 & 0-10 min, non-overflow, total & 0.00000100 & 0.0000010 & 0.0000010 & 0.00000040 & 0.00000050 & 0.00000040 & 0.00000050 \\
\hline Water & 80754 & 10-20 $\mathrm{min}$, non-overflow, total & 0.00000100 & 0.0000010 & 0.0000010 & 0.00000040 & 0.00000050 & 0.00000040 & 0.00000070 \\
\hline \multirow[t]{2}{*}{ Water } & 80755 & $20-30 \mathrm{~min}$, non-overflow, total & 0.00000050 & 0.0000010 & 0.0000011 & 0.00000100 & 0.00000070 & 0.00000110 & 0.00000110 \\
\hline & & Hopper Inflow Monitoring & & & & & & & \\
\hline Water & 80790 & $3 \& 6 \mathrm{~min}$, dissolved & 0.00000040 & 0.0000010 & 0.0000010 & 0.00000080 & 0.00000110 & 0.00000080 & 0.00000140 \\
\hline Water & 80791 & $9 \& 12 \mathrm{~min}$, dissolved & 0.00000100 & 0.0000010 & 0.0000010 & 0.00000060 & 0.00000090 & 0.00000060 & 0.00000120 \\
\hline Water & 80792 & $15 \& 18 \mathrm{~min}$, dissolved & 0.00000040 & 0.0000010 & 0.0000010 & 0.00000100 & 0.00000130 & 0.00000100 & 0.00000100 \\
\hline Water & 80793 & $21824 \mathrm{~min}$, dissolved & 0.00000060 & 0.0000010 & 0.0000010 & 0.00000130 & 0.00000150 & 0.00000130 & 0.00000030 \\
\hline Water & 80794 & $27 \& 30 \mathrm{~min}$, dissolved & 0.00000090 & 0.0000010 & 0.0000010 & 0.00000180 & 0.00000110 & 0.00000180 & 0.00000100 \\
\hline Water & 80692 & $3 \& 6 \mathrm{~min}$, total & 0.00000280 & 0.0000010 & 0.0000010 & 0.00000190 & 0.00000100 & 0.00000190 & 0.00000430 \\
\hline Water & 80693 & $9 \& 12 \mathrm{~min}$, total & 0.00000180 & 0.0000010 & 0.0000010 & 0.00000088 & 0.00000100 & 0.00000088 & 0.00000300 \\
\hline Water & 80694 & $15 \& 18 \mathrm{~min}$, total & 0.00000250 & 0.0000010 & 0.0000010 & 0.00000290 & 0.00000100 & 0.00000290 & 0.00000390 \\
\hline Water & 80695 & $21824 \mathrm{~min}$, total & 0.00000150 & 0.0000011 & 0.0000011 & 0.00000049 & 0.00000110 & 0.00000049 & 0.00000360 \\
\hline \multirow[t]{2}{*}{ Water } & 80696 & $27 \& 30 \mathrm{~min}$, total & 0.00000140 & 0.0000010 & 0.0000010 & 0.00000220 & 0.00000100 & 0.00000220 & 0.00000380 \\
\hline & & Hopper Overflow Monitoring & & & & & & & \\
\hline Water & 80795 & 22. $4 \mathrm{~min}$, dissolved & 0.00000100 & 0.0000010 & 0.0000010 & 0.00000100 & 0.00000100 & 0.00000100 & 0.00000080 \\
\hline Water & 80796 & 6\& $8 \mathrm{~min}$, dissolved & 0.00000100 & 0.0000010 & 0.0000010 & 0.00000100 & 0.00000100 & 0.00000100 & 0.00000100 \\
\hline Water & 80797 & $10 \& 12 \mathrm{~min}$, dissolved & 0.00000030 & 0.0000010 & 0.0000010 & 0.00000100 & 0.00000050 & 0.00000100 & 0.00000100 \\
\hline Water & 80798 & $14 \& 16 \mathrm{~min}$, dissolved & 0.00000050 & 0.0000010 & 0.0000010 & 0.00000040 & 0.00000100 & 0.00000040 & 0.00000100 \\
\hline Water & 80799 & $18 \& 20 \mathrm{~min}$, dissolved & 0.00000040 & 0.0000010 & 0.0000010 & 0.00000100 & 0.00000100 & 0.00000100 & 0.00000100 \\
\hline Water & 80698 & 2\& 4 min, total & 0.00000100 & 0.0000010 & 0.0000010 & 0.00000061 & 0.00000100 & 0.00000061 & 0.00000240 \\
\hline Water & 80699 & 6\& $8 \mathrm{~min}$, total & 0.00000073 & 0.0000010 & 0.0000010 & 0.00000044 & 0.00000100 & 0.00000044 & 0.00000140 \\
\hline Water & 80700 & $10 \& 12 \mathrm{~min}$, total & 0.00000100 & 0.0000010 & 0.0000010 & 0.00000051 & 0.00000063 & 0.00000051 & 0.00000200 \\
\hline Water & 80701 & $14 \& 16 \mathrm{~min}$, total & 0.00000110 & 0.0000011 & 0.0000011 & 0.00000052 & 0.00000110 & 0.00000052 & 0.00000210 \\
\hline \multirow[t]{2}{*}{ Water } & 80702 & $18 \& 20 \mathrm{~min}$, total & 0.00000100 & 0.0000010 & 0.0000010 & 0.00000063 & 0.00000100 & 0.00000063 & 0.00000240 \\
\hline & & Site Water & & & & & & & \\
\hline Water & 81594 & Sample 1 Total & 0.00000058 & 0.0000010 & 0.0000010 & 0.00000100 & 0.00000100 & 0.00000100 & 0.00000079 \\
\hline Water & 81595 & Sample 2 Total & 0.00000078 & 0.0000010 & 0.0000010 & 0.00000100 & 0.00000100 & 0.00000100 & 0.00000130 \\
\hline \multirow[t]{2}{*}{ Water } & 81596 & Sample 3 Total & 0.00000078 & 0.0000010 & 0.0000010 & 0.00000100 & 0.00000100 & 0.00000100 & 0.00000110 \\
\hline & & Elutriate & & & & & & & \\
\hline Water & 81600 & Sample 1 Dissolved & 0.00000100 & 0.0000010 & 0.0000010 & 0.00000100 & 0.00000038 & 0.00000100 & 0.00000100 \\
\hline Water & 81601 & Sample 2 Dissolved & 0.00000100 & 0.0000010 & 0.0000010 & 0.00000100 & 0.00000062 & 0.00000100 & 0.00000071 \\
\hline Water & 81602 & Sample 3 Dissolved & 0.00000049 & 0.0000010 & 0.0000010 & 0.00000100 & 0.00000100 & 0.00000100 & 0.00000077 \\
\hline Water & 81597 & Sample 1 Total & 0.00000053 & 0.0000010 & 0.0000010 & 0.00000100 & 0.00000100 & 0.00000100 & 0.00000054 \\
\hline Water & 81598 & Sample 2 Total & 0.00000120 & 0.0000010 & 0.0000010 & 0.00000057 & 0.00000068 & 0.00000057 & 0.00000160 \\
\hline Water & 81599 & Sample 3 Total & 0.00000052 & 0.0000010 & 0.0000010 & 0.00000100 & 0.00000100 & 0.00000100 & 0.00000078 \\
\hline \multirow{3}{*}{$\begin{array}{l}\text { SAMPLE } \\
\text { TYPE }\end{array}$} & SAMPLE & DESCRIPTION & PCB 70 & РCB 77 & РСB 82 & РCB 86 & РСB 87 & РCB 97 & РСB 101 \\
\hline & & Detection Limit (mg/kg) & 0.00033 & 0.00033 & 0.00033 & 0.00033 & 0.00033 & 0.00033 & 0.00033 \\
\hline & & Insitu Sediment & & & & & & & \\
\hline Sediment & 81714 & Sample \#1 & 0.00033 & 0.00033 & 0.00033 & 0.00033 & 0.00033 & 0.00033 & 0.00033 \\
\hline Sediment & 81715 & Sample \#2 & 0.00033 & 0.00033 & 0.00033 & 0.00033 & 0.00033 & 0.00033 & 0.00033 \\
\hline Sediment & 81716 & Sample \#3 & 0.00033 & 0.00033 & 0.00033 & 0.00033 & 0.00033 & 0.00033 & 0.00033 \\
\hline
\end{tabular}

BOLD - less than values

Values below less than values are estimated results. Results are less than the reporting limit. 
Delaware River Water Analysis (Coarse-Grained Site)

\begin{tabular}{|c|c|c|c|c|c|c|c|c|c|}
\hline \multirow{2}{*}{$\begin{array}{l}\text { SAMPLE } \\
\text { TYPE }\end{array}$} & $\begin{array}{l}\text { SAMPLE } \\
\text { ID }\end{array}$ & DESCRIPTION & PCB 105 & PCB 114 & PCB 118 & PCB 121 & PCB 128 & PCB 136 & PCB 137 \\
\hline & & Detection Limit (mg/l) & 0.00000110 & 0.0000011 & 0.00000110 & 0.0000011 & 0.0000011 & 0.0000011 & 0.0000011 \\
\hline & & Plume Monitoring & & & & & & & \\
\hline Water & 80834 & Background, dissolved & 0.00000110 & 0.0000011 & 0.00000110 & 0.0000011 & 0.0000011 & 0.0000011 & 0.0000011 \\
\hline Water & 80749 & Background, total & 0.00000100 & 0.0000010 & 0.00000100 & 0.0000010 & 0.0000010 & 0.0000010 & 0.0000010 \\
\hline Water & 80835 & 0-10 min, overflow, dissolved & 0.00000100 & 0.0000010 & 0.00000100 & 0.0000010 & 0.0000010 & 0.0000010 & 0.0000010 \\
\hline Water & 80836 & 10-20 min, overflow, dissolved & 0.00000100 & 0.0000010 & 0.00000100 & 0.0000010 & 0.0000010 & 0.0000010 & 0.0000010 \\
\hline Water & 80837 & 20-30 min, overflow, dissolved & 0.00000100 & 0.0000010 & 0.00000100 & 0.0000010 & 0.0000010 & 0.0000010 & 0.0000010 \\
\hline Water & 80750 & $0-10 \mathrm{~min}$, overflow, total & 0.00000110 & 0.0000011 & 0.00000110 & 0.0000011 & 0.0000011 & 0.0000011 & 0.0000011 \\
\hline Water & 80751 & 10-20 min, overflow, total & 0.00000100 & 0.0000010 & 0.00000100 & 0.0000010 & 0.0000010 & 0.0000010 & 0.0000010 \\
\hline Water & 80752 & 20-30 min, overflow, total & 0.00000100 & 0.0000010 & 0.00000100 & 0.0000010 & 0.0000010 & 0.0000010 & 0.0000010 \\
\hline Water & 80838 & 0-10 min, non-overflow, dissolve & 0.00000100 & 0.0000010 & 0.00000100 & 0.0000010 & 0.0000010 & 0.0000010 & 0.0000010 \\
\hline Water & 80839 & 10-20 min, non-overflow, dissolve & 0.00000110 & 0.0000011 & 0.00000110 & 0.0000011 & 0.0000011 & 0.0000011 & 0.0000011 \\
\hline Water & 80840 & 20-30 min, non-overflow, dissolve & 0.00000110 & 0.0000011 & 0.00000110 & 0.0000011 & 0.0000011 & 0.0000011 & 0.0000011 \\
\hline Water & 80753 & $0.10 \mathrm{~min}$, non-overflow, total & 0.00000100 & 0.0000010 & 0.00000100 & 0.0000010 & 0.0000010 & 0.0000010 & 0.0000010 \\
\hline Water & 80754 & 10-20 min, non-overflow, total & 0.00000100 & 0.0000010 & 0.00000100 & 0.0000010 & 0.0000010 & 0.0000010 & 0.0000010 \\
\hline \multirow[t]{2}{*}{ Water } & 80755 & 20-30 min, non-overflow, total & 0.00000110 & 0.0000011 & 0.00000110 & 0.0000011 & 0.0000011 & 0.0000011 & 0.0000011 \\
\hline & & Hopper Inflow Monitoring & & & & & & & \\
\hline Water & 80790 & 3\& $6 \mathrm{~min}$, dissolved & 0.00000100 & 0.0000010 & 0.00000100 & 0.0000010 & 0.0000010 & 0.0000010 & 0.0000010 \\
\hline Water & 80791 & $9 \& 12 \mathrm{~min}$, dissolved & 0.00000100 & 0.0000010 & 0.00000100 & 0.0000010 & 0.0000010 & 0.0000007 & 0.0000010 \\
\hline Water & 80792 & $15 \& 18 \mathrm{~min}$, dissolved & 0.00000100 & 0.0000010 & 0.00000100 & 0.0000010 & 0.0000010 & 0.0000010 & 0.0000010 \\
\hline Water & 80793 & 21824 min, dissolved & 0.00000100 & 0.0000010 & 0.00000100 & 0.0000010 & 0.0000010 & 0.0000010 & 0.0000010 \\
\hline Water & 80794 & $27830 \mathrm{~min}$, dissolved & 0.00000100 & 0.0000010 & 0.00000100 & 0.0000010 & 0.0000010 & 0.0000010 & 0.0000010 \\
\hline Water & 80692 & $3 \& 6 \mathrm{~min}$, total & 0.00000100 & 0.0000010 & 0.00000100 & 0.0000010 & 0.0000010 & 0.0000010 & 0.0000010 \\
\hline Water & 80693 & $9 \& 12 \mathrm{~min}$, total & 0.00000100 & 0.0000010 & 0.00000100 & 0.0000010 & 0.0000010 & 0.0000010 & 0.0000010 \\
\hline Water & 80694 & $15 \& 18 \mathrm{~min}$, total & 0.00000250 & 0.0000010 & 0.00000100 & 0.0000010 & 0.0000010 & 0.0000010 & 0.0000010 \\
\hline Water & 80695 & $21 \& 24 \mathrm{~min}$, total & 0.00000240 & 0.0000011 & 0.00000110 & 0.0000011 & 0.0000011 & 0.0000011 & 0.0000011 \\
\hline \multirow[t]{2}{*}{ Water } & 80696 & $27 \& 30 \mathrm{~min}$, total & 0.00000250 & 0.0000010 & 0.00000100 & 0.0000010 & 0.0000010 & 0.0000010 & 0.0000010 \\
\hline & & Hopper Overflow Monitoring & & & & & & & \\
\hline Water & 80795 & 2\& $4 \mathrm{~min}$, dissolved & 0.00000100 & 0.0000010 & 0.00000100 & 0.0000010 & 0.0000010 & 0.0000010 & 0.0000010 \\
\hline Water & 80796 & 6\& $8 \mathrm{~min}$, dissolved & 0.00000100 & 0.0000010 & 0.00000100 & 0.0000010 & 0.0000010 & 0.0000010 & 0.0000010 \\
\hline Water & 80797 & $10 \& 12 \mathrm{~min}$, dissolved & 0.00000100 & 0.0000010 & 0.00000100 & 0.0000010 & 0.0000010 & 0.0000010 & 0.0000010 \\
\hline Water & 80798 & $14 \& 16 \mathrm{~min}$, dissolved & 0.00000100 & 0.0000010 & 0.00000100 & 0.0000010 & 0.0000010 & 0.0000010 & 0.0000010 \\
\hline Water & 80799 & $18 \& 20 \mathrm{~min}$, dissolved & 0.00000100 & 0.0000010 & 0.00000100 & 0.0000010 & 0.0000010 & 0.0000010 & 0.0000010 \\
\hline Water & 80698 & 28 $4 \mathrm{~min}$, total & 0.00000100 & 0.0000010 & 0.00000100 & 0.0000010 & 0.0000010 & 0.0000010 & 0.0000010 \\
\hline Water & 80699 & $6 \& 8 \mathrm{~min}$, total & 0.00000100 & 0.0000010 & 0.00000100 & 0.0000010 & 0.0000010 & 0.0000010 & 0.0000010 \\
\hline Water & 80700 & $10 \& 12 \mathrm{~min}$, total & 0.00000100 & 0.0000010 & 0.00000100 & 0.0000010 & 0.0000010 & 0.0000010 & 0.0000010 \\
\hline Water & 80701 & $14 \& 16 \mathrm{~min}$, total & 0.00000110 & 0.0000011 & 0.00000110 & 0.0000011 & 0.0000011 & 0.0000011 & 0.0000011 \\
\hline \multirow[t]{2}{*}{ Water } & 80702 & $18 \& 20 \mathrm{~min}$, total & 0.00000100 & 0.0000010 & 0.00000100 & 0.0000010 & 0.0000010 & 0.0000010 & 0.0000010 \\
\hline & & Site Water & & & & & & & \\
\hline Water & 81594 & Sample 1 Total & 0.00000100 & 0.0000010 & 0.00000054 & 0.0000010 & 0.0000010 & 0.0000010 & 0.0000010 \\
\hline Water & 81595 & Sample 2 Total & 0.00000047 & 0.0000010 & 0.00000097 & 0.0000010 & 0.0000010 & 0.0000010 & 0.0000010 \\
\hline \multirow[t]{2}{*}{ Water } & 81596 & Sample 3 Total & 0.00000100 & 0.0000010 & 0.00000057 & 0.0000010 & 0.0000010 & 0.0000010 & 0.0000010 \\
\hline & & Elutriate & & & & & & & \\
\hline Water & 81600 & Sample 1 Dissolved & 0.00000036 & 0.0000010 & 0.00000062 & 0.0000010 & 0.0000010 & 0.0000010 & 0.0000010 \\
\hline Water & 81601 & Sample 2 Dissolved & 0.00000032 & 0.0000010 & 0.00000046 & 0.0000010 & 0.0000010 & 0.0000010 & 0.0000010 \\
\hline Water & 81602 & Sample 3 Dissolved & 0.00000042 & 0.0000010 & 0.00000065 & 0.0000010 & 0.0000010 & 0.0000010 & 0.0000010 \\
\hline Water & 81597 & Sample 1 Total & 0.00000100 & 0.0000010 & 0.00000049 & 0.0000010 & 0.0000010 & 0.0000010 & 0.0000010 \\
\hline Water & 81598 & Sample 2 Total & 0.00000100 & 0.0000010 & 0.00000086 & 0.0000010 & 0.0000010 & 0.0000010 & 0.0000010 \\
\hline Water & 81599 & Sample 3 Total & 0.00000100 & 0.0000010 & 0.00000049 & 0.0000010 & 0.0000010 & 0.0000010 & 0.0000010 \\
\hline \multirow{3}{*}{$\begin{array}{l}\text { SAMPLE } \\
\text { TYPE }\end{array}$} & SAMPLE & DESCRIPTION & PCB 105 & PCB 114 & PCB 118 & PCB 121 & PCB 128 & PCB 136 & PCB 137 \\
\hline & & Detection Limit $(\mathrm{mg} / \mathrm{kg})$ & 0.00033 & 0.00033 & 0.00033 & 0.00033 & 0.00033 & 0.00033 & 0.00033 \\
\hline & & Insitu Sediment & & & & & & & \\
\hline Sediment & 81714 & Sample \#1 & 0.00033 & 0.00033 & 0.00010 & 0.00033 & 0.00033 & 0.00033 & 0.00033 \\
\hline Sediment & 81715 & Sample \#2 & 0.00033 & 0.00033 & 0.00016 & 0.00033 & 0.00033 & 0.00033 & 0.00033 \\
\hline Sediment & 81716 & Sample \#3 & 0.00033 & 0.00033 & 0.00020 & 0.00033 & 0.00033 & 0.00033 & 0.00033 \\
\hline
\end{tabular}

BOLD - less than values

Values below less than values are estimated results. Results are less than the reporting limit. 
Delaware River Water Analysis (Coarse-Grained Site)

\begin{tabular}{|c|c|c|c|c|c|c|c|c|c|}
\hline \multirow[t]{3}{*}{$\begin{array}{l}\text { SAMPLE } \\
\text { TYPE }\end{array}$} & $\begin{array}{l}\text { SAMPLE } \\
\text { ID }\end{array}$ & DESCRIPTION & PCB 138 & РCB 141 & PCB 151 & РCB 153 & РCB 156 & PCB 167 & РСB 170 \\
\hline & & Detection Limit (mg/) & 0.0000011 & 0.0000011 & 0.0000011 & 0.0000011 & 0.0000011 & 0.0000011 & 0.00000110 \\
\hline & & Plume Monitoring & & & & & & & \\
\hline Water & 80834 & Background, dissolved & 0.0000011 & 0.0000011 & 0.0000011 & 0.0000007 & 0.0000011 & 0.0000011 & 0.00000110 \\
\hline Water & 80749 & Background, total & 0.0000010 & 0.0000010 & 0.0000010 & 0.0000010 & 0.0000010 & 0.0000010 & 0.00000100 \\
\hline Water & 80835 & 0-10 min, overflow, dissolved & 0.0000004 & 0.0000010 & 0.0000010 & 0.0000009 & 0.0000010 & 0.0000010 & 0.00000100 \\
\hline Water & 80836 & 10-20 min, overflow, dissolved & 0.0000010 & 0.0000010 & 0.0000010 & 0.0000010 & 0.0000010 & 0.0000010 & 0.00000100 \\
\hline Water & 80837 & 20-30 $\mathrm{min}$, overflow, dissolved & 0.0000010 & 0.0000010 & 0.0000010 & 0.0000005 & 0.0000010 & 0.0000010 & 0.00000100 \\
\hline Water & 80750 & 0-10 min, overflow, total & 0.0000011 & 0.0000011 & 0.0000011 & 0.0000011 & 0.0000011 & 0.0000011 & 0.00000110 \\
\hline Water & 80751 & 10-20 min, overflow, total & 0.0000010 & 0.0000010 & 0.0000010 & 0.0000010 & 0.0000010 & 0.0000010 & 0.00000100 \\
\hline Water & 80752 & 20-30 min, overflow, total & 0.0000010 & 0.0000010 & 0.0000010 & 0.0000010 & 0.0000010 & 0.0000010 & 0.00000100 \\
\hline Water & 80838 & 0-10 min, non-overflow, dissolve & 0.0000010 & 0.0000010 & 0.0000010 & 0.0000010 & 0.0000010 & 0.0000010 & 0.00000100 \\
\hline Water & 80839 & 10-20 min, non-overflow, dissolve & 0.0000011 & 0.0000011 & 0.0000011 & 0.0000011 & 0.0000011 & 0.0000011 & 0.00000110 \\
\hline Water & 80840 & 20-30 min, non-overflow, dissolve & 0.0000011 & 0.0000011 & 0.0000011 & 0.0000011 & 0.0000011 & 0.0000011 & 0.00000110 \\
\hline Water & 80753 & 0-10 min, non-overflow, total & 0.0000010 & 0.0000010 & 0.0000010 & 0.0000010 & 0.0000010 & 0.0000010 & 0.00000100 \\
\hline Water & 80754 & $10-20 \mathrm{~min}$, non-overflow, total & 0.0000010 & 0.0000010 & 0.0000010 & 0.0000010 & 0.0000010 & 0.0000010 & 0.00000100 \\
\hline \multirow[t]{2}{*}{ Water } & 80755 & 20-30 $\mathrm{min}$, non-overflow, total & 0.0000011 & 0.0000011 & 0.0000011 & 0.0000010 & 0.0000011 & 0.0000011 & 0.00000110 \\
\hline & & Hopper Inflow Monitoring & & & & & & & \\
\hline Water & 80790 & 3\& $6 \mathrm{~min}$, dissolved & 0.0000006 & 0.0000010 & 0.0000010 & 0.0000010 & 0.0000010 & 0.0000010 & 0.00000100 \\
\hline Water & 80791 & $9 \& 12 \mathrm{~min}$, dissolved & 0.0000005 & 0.0000010 & 0.0000010 & 0.0000010 & 0.0000010 & 0.0000010 & 0.00000100 \\
\hline Water & 80792 & $15 \& 18 \mathrm{~min}$, dissolved & 0.0000006 & 0.0000010 & 0.0000010 & 0.0000010 & 0.0000010 & 0.0000010 & 0.00000100 \\
\hline Water & 80793 & 21824 min, dissolved & 0.0000007 & 0.0000010 & 0.0000010 & 0.0000010 & 0.0000010 & 0.0000010 & 0.00000100 \\
\hline Water & 80794 & $27830 \mathrm{~min}$, dissolved & 0.0000014 & 0.0000010 & 0.0000010 & 0.0000010 & 0.0000010 & 0.0000010 & 0.00000100 \\
\hline Water & 80692 & 3\& $6 \mathrm{~min}$, total & 0.0000019 & 0.0000010 & 0.0000010 & 0.0000030 & 0.0000010 & 0.0000010 & 0.00000074 \\
\hline Water & 80693 & $9 \& 12 \mathrm{~min}$, total & 0.0000019 & 0.0000010 & 0.0000010 & 0.0000028 & 0.0000010 & 0.0000010 & 0.00000100 \\
\hline Water & 80694 & $15 \& 18 \mathrm{~min}$, total & 0.0000043 & 0.0000010 & 0.0000010 & 0.0000048 & 0.0000010 & 0.0000010 & 0.00000140 \\
\hline Water & 80695 & $21824 \mathrm{~min}$, total & 0.0000034 & 0.0000011 & 0.0000011 & 0.0000055 & 0.0000011 & 0.0000011 & 0.00000110 \\
\hline \multirow[t]{2}{*}{ Water } & 80696 & $27 \& 30 \mathrm{~min}$, total & 0.0000029 & 0.0000010 & 0.0000010 & 0.0000036 & 0.0000010 & 0.0000010 & 0.00000100 \\
\hline & & Hopper Overflow Monitoring & & & & & & & \\
\hline Water & 80795 & 2\& $4 \mathrm{~min}$, dissolved & 0.0000010 & 0.0000010 & 0.0000010 & 0.0000010 & 0.0000010 & 0.0000010 & 0.00000100 \\
\hline Water & 80796 & $6 \& 8 \mathrm{~min}$, dissolved & 0.0000010 & 0.0000010 & 0.0000010 & 0.0000010 & 0.0000010 & 0.0000010 & 0.00000100 \\
\hline Water & 80797 & $10 \& 12 \mathrm{~min}$, dissolved & 0.0000010 & 0.0000010 & 0.0000010 & 0.0000010 & 0.0000010 & 0.0000010 & 0.00000100 \\
\hline Water & 80798 & $14 \& 16 \mathrm{~min}$, dissolved & 0.0000010 & 0.0000010 & 0.0000010 & 0.0000010 & 0.0000010 & 0.0000010 & 0.00000100 \\
\hline Water & 80799 & $18 \& 20 \mathrm{~min}$, dissolved & 0.0000010 & 0.0000010 & 0.0000010 & 0.0000010 & 0.0000010 & 0.0000010 & 0.00000100 \\
\hline Water & 80698 & 2\& $4 \mathrm{~min}$, total & 0.0000018 & 0.0000010 & 0.0000010 & 0.0000026 & 0.0000010 & 0.0000010 & 0.00000100 \\
\hline Water & 80699 & 6\& $8 \mathrm{~min}$, total & 0.0000013 & 0.0000010 & 0.0000010 & 0.0000010 & 0.0000010 & 0.0000010 & 0.00000100 \\
\hline Water & 80700 & $10 \& 12 \mathrm{~min}$, total & 0.0000011 & 0.0000010 & 0.0000010 & 0.0000010 & 0.0000010 & 0.0000010 & 0.00000100 \\
\hline Water & 80701 & $14 \& 16 \mathrm{~min}$, total & 0.0000020 & 0.0000011 & 0.0000011 & 0.0000011 & 0.0000011 & 0.0000011 & 0.00000110 \\
\hline \multirow[t]{2}{*}{ Water } & 80702 & $18 \& 20 \mathrm{~min}$, total & 0.0000015 & 0.0000010 & 0.0000010 & 0.0000010 & 0.0000010 & 0.0000010 & 0.00000100 \\
\hline & & Site Water & & & & & & & \\
\hline Water & 81594 & Sample 1 Total & 0.0000010 & 0.0000010 & 0.0000010 & 0.0000010 & 0.0000010 & 0.0000010 & 0.00000100 \\
\hline Water & 81595 & Sample 2 Total & 0.0000010 & 0.0000010 & 0.0000010 & 0.0000010 & 0.0000010 & 0.0000010 & 0.00000100 \\
\hline \multirow[t]{2}{*}{ Water } & 81596 & Sample 3 Total & 0.0000010 & 0.0000010 & 0.0000010 & 0.0000010 & 0.0000010 & 0.0000010 & 0.00000100 \\
\hline & & Elutriate & & & & & & & \\
\hline Water & 81600 & Sample 1 Dissolved & 0.0000010 & 0.0000010 & 0.0000010 & 0.0000010 & 0.0000010 & 0.0000010 & 0.00000100 \\
\hline Water & 81601 & Sample 2 Dissolved & 0.0000010 & 0.0000010 & 0.0000010 & 0.0000010 & 0.0000010 & 0.0000010 & 0.00000100 \\
\hline Water & 81602 & Sample 3 Dissolved & 0.0000010 & 0.0000010 & 0.0000010 & 0.0000010 & 0.0000010 & 0.0000010 & 0.00000100 \\
\hline Water & 81597 & Sample 1 Total & 0.0000010 & 0.0000010 & 0.0000010 & 0.0000010 & 0.0000010 & 0.0000010 & 0.00000100 \\
\hline Water & 81598 & Sample 2 Total & 0.0000010 & 0.0000010 & 0.0000010 & 0.0000010 & 0.0000010 & 0.0000010 & 0.00000100 \\
\hline Water & 81599 & Sample 3 Total & 0.0000010 & 0.0000010 & 0.0000010 & 0.0000010 & 0.0000010 & 0.0000010 & 0.00000100 \\
\hline \multirow{3}{*}{$\begin{array}{l}\text { SAMPLE } \\
\text { TYPE }\end{array}$} & SAMPLE & DESCRIPTION & PCB 138 & PCB 141 & PCB 151 & PCB 153 & PCB 156 & PCB 167 & РСB 170 \\
\hline & & Detection Limit ( $\mathrm{mg} / \mathrm{kg})$ & 0.00033 & 0.00033 & 0.00033 & 0.00033 & 0.00033 & 0.00033 & 0.00033 \\
\hline & & Insitu Sediment & & & & & & & \\
\hline Sediment & 81714 & Sample \#1 & 0.00033 & 0.00033 & 0.00033 & 0.00033 & 0.00033 & 0.00033 & 0.00033 \\
\hline Sediment & $\begin{array}{l}81715 \\
81716\end{array}$ & Sample \#2 & 0.00033 & 0.00033 & 0.00033 & 0.00033 & 0.00033 & 0.00033 & 0.00033 \\
\hline Sediment & 81716 & Sample \#3 & 0.00033 & 0.00033 & 0.00033 & 0.00033 & 0.00033 & 0.00033 & 0.00033 \\
\hline
\end{tabular}

BOLD - less than values

Values below less than values are estimated results. Results are less than the reporting limit. 
Delaware River Water Analysis (Coarse-Grained Site)

SAMPLE SAMPLE DESCRIPTION

TYPE

Detection Limit (mg/)

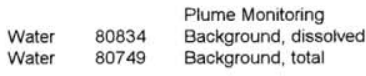

Water $80835 \quad 0-10 \mathrm{~min}$, overflow, dissolved

Water $\quad 80836$

Water 80837

Water 80750

$\begin{array}{ll}\text { Water } & 80751 \\ \text { Water } & 80752\end{array}$

Water 80838

Water 80839

Water 80840

Water 80753

$\begin{array}{ll}\text { Water } & 80754 \\ \text { Water } & 80755\end{array}$

$\begin{array}{ll}\text { Water } & 80790 \\ \text { Water } & 80791 \\ \text { Water } & 80792 \\ \text { Water } & 80793 \\ \text { Water } & 80794 \\ \text { Water } & 80692 \\ \text { Water } & 80693 \\ \text { Water } & 80694 \\ \text { Water } & 80695 \\ \text { Water } & 80696\end{array}$

Hopper Overflow Monitoring

Water $\quad 80795 \quad 2 \& 4 \mathrm{~min}$, dissolved

Water 80796

Water 80797

Water 80798

Water 80799

Water 80698

Water 80699

Water 80700

$\begin{array}{ll}\text { Water } & 80701 \\ \text { Water } & 80702\end{array}$

Water $81594 \quad$ Site Water

Water $81594 \quad$ Sample 1 Total

$\begin{array}{lll}\text { Water } & 81595 & \text { Sample 2 Total } \\ \text { Water } & 81596 & \text { Sample } 3 \text { Total }\end{array}$

Elutriate

Water 81600 Sample 1 Dissted

Water 81601 Sample 2 Dissolved

Water 81602 Sample 3 Dissolved

Water 81597 Sample 1 Total

$\begin{array}{lll}\text { Water } & 81598 & \text { Sample 2 Total } \\ \text { Water } & 81599 & \text { Sample 3 Total }\end{array}$

SAMPLE SAMPLE DESCRIPTION

TYPE ID

Detection Limit $(\mathrm{mg} / \mathrm{kg})$

Insitu Sediment

Sediment 81714 Sample\#1

$\begin{array}{ll}\text { Sediment } 81715 & \text { Sample \#2 } \\ \text { Sediment } 81716 & \text { Sample \#3 }\end{array}$
PCB 171
0.0000011

0.0000011
0.0000010
0.0000010
0.0000010
0.0000010
0.0000011
0.0000010
0.0000010
0.0000010
0.0000011
0.0000011
0.0000010
0.0000010
0.0000011

PCB 180

0.0000011

0.0000011

0.0000011

0.0000010

0.0000010

0.0000010

0.0000010
0.00000011

0.0000011
0.0000010

0.0000010

0.0000010

0.0000011

0.0000011

0.0000010

0.000001

0.000001

0.0000010

0.0000010

0.0000010

0.0000010
0.0000010

0.0000010
0.0000010

0.0000010
0.0000010

0.0000010

0.0000011

0.0000010

0.0000010

0.0000010

0.0000010

0.000001

0.0000010

0.0000010

0.0000010

0.000001

0.0000011

0.0000010

0.0000010

0.0000010

0.000001

0.0000010

0.0000010

0.0000010

0.0000010

0.0000010

0.0000011
0.0000010

0.0000010

0.000001

0.000001

0.0000010

0.0000010

0.0000015

0.000001

0.000001

0.000002

0.0000010

0.0000011
0.0000010

0.0000010

0.0000010

0.0000010
0.0000011

0.0000010

0.0000010

0.0000010

0.000001

0.000001

0.000001

0.000001

0.000001

0.0000010

0.0000010

0.000001

0.0000010

0.0000010

0.0000010

0.0000010

0.000001

0.000001

0.000001

0.0000010

0.0000010

0.0000010

0.0000010

0.0000010

0.0000010

0.0000010

0.000001

0.000001

0.0000010

0.0000010

0.0000010

0.0000010
0.0000010

0.0000010

0.0000010
0.0000010

0.000001

0.000001

0.0000010

0.0000010

0.000001

0.000001

0.0000010

0.000001

PCB 171

PCB 180

0.0000010

0.0000010

0.0000010

0.0000010

0.0000010

0.0000010

PCB 182

0.00033

0.00033

0.00033

0.00033

0.00033
0.00033

0.00033

0.00033

0.00033

0.00033

0.00033
0.00033

PCB 185

PCB 187

PCB 189

0.0000011

0.0000011

0.0000011

0.0000011

0.0000011

0.0000011

0.0000011

0.0000011

0.0000010

0.0000010

0.0000010

$\mathbf{0 . 0 0 0 0 0 1 0}$

0.0000011

0.0000011

0.0000010

0.000001

$0.0000010 \quad 0.0000010$

$0.0000010 \quad 0.0000010$

$\begin{array}{ll}0.0000010 & 0.0000010\end{array}$

$0.0000011 \quad 0.0000011$

$0.0000010 \quad 0.0000010$

0.0000010

0.0000011

0.0000011
0.0000010

0.0000010

0.0000010

0.000001

0.000001

0.0000011

0.000001

0.0000010

0.0000010

0.0000011

0.0000011

0.0000010

0.0000010
0.0000011

0.0000010

0.0000011

0.0000011

0.0000010

0.0000010

0.0000010

0.0000010

0.0000010

0.0000010

0.0000010

0.0000010

0.0000010

0.0000010

0.000001

0.000001

0.0000010

0.0000010

0.000001

0.0000010

0.0000010

0.0000010

0.0000010

0.000001

0.0000011

0.000001

0.0000010

0.0000010

0.0000010

0.0000010

0.0000010

0.0000021

0.0000017

0.0000033

0.0000011

0.000001

0.000002

0.0000010

0.0000010

0.0000010

0.0000010

0.0000010

0.0000010

0.0000010

0.000001

0.000001

0.0000010

0.000010

$\mathbf{0 . 0 0 0 0 0 1 0}$

0.0000010

0.0000010

0.0000010

0.0000010

0.0000010

0.000001

0.000001

0.0000010

0.0000010

0.0000010

0.0000010

0.0000010

0.0000010

0.0000010

$\mathbf{0 . 0 0 0 0 0 1 0}$

0.0000010

0.0000011

0.000001

0.0000010

0.0000010

0.0000010

0.0000010

0.0000010

0.0000010

0.0000010

0.0000010

0.0000010

0.0000010

0.0000010
0.0000010

0.0000010

0.0000010

0.0000010
0.0000010

0.0000010 
Delaware River Water Analysis (Coarse-Grained Site)

\begin{tabular}{|c|c|c|c|c|c|c|c|c|c|}
\hline \multirow[t]{2}{*}{$\begin{array}{l}\text { SAMPLE } \\
\text { TYPE }\end{array}$} & $\begin{array}{l}\text { SAMPLE } \\
\text { ID }\end{array}$ & DESCRIPTION & PCB 191 & PCB 194 & PCB 195 & PCB 196 & РCB 201 & РCB 203 & РCB 205 \\
\hline & & Detection Limit (mg/l) & 0.0000011 & 0.0000011 & 0.0000011 & 0.0000011 & 0.0000011 & 0.0000011 & 0.0000011 \\
\hline & & Plume Monitoring & & & & & & & \\
\hline Water & 80834 & Background, dissolved & 0.0000011 & 0.0000011 & 0.0000011 & 0.0000011 & 0.0000011 & 0.0000011 & 0.0000011 \\
\hline Water & 80749 & Background, total & 0.0000010 & 0.0000010 & 0.0000010 & 0.0000010 & 0.0000010 & 0.0000010 & 0.0000010 \\
\hline Water & 80835 & 0-10 min, overflow, dissolved & 0.0000010 & 0.0000010 & 0.0000010 & 0.0000010 & 0.0000010 & 0.0000010 & 0.0000010 \\
\hline Water & 80836 & 10-20 min, overflow, dissolved & 0.0000010 & 0.0000010 & 0.0000010 & 0.0000010 & 0.0000010 & 0.0000010 & 0.0000010 \\
\hline Water & 80837 & 20-30 min, overflow, dissolved & 0.0000010 & 0.0000010 & 0.0000010 & 0.0000010 & 0.0000010 & 0.0000010 & 0.0000010 \\
\hline Water & 80750 & $0-10 \mathrm{~min}$, overflow, total & 0.0000011 & 0.0000011 & 0.0000011 & 0.0000011 & 0.0000011 & 0.0000011 & 0.0000011 \\
\hline Water & 80751 & 10-20 min, overflow, total & 0.0000010 & 0.0000010 & 0.0000010 & 0.0000010 & 0.0000010 & 0.0000010 & 0.0000010 \\
\hline Water & 80752 & 20-30 min, overflow, total & 0.0000010 & 0.0000010 & 0.0000010 & 0.0000010 & 0.0000010 & 0.0000010 & 0.0000010 \\
\hline Water & 80838 & 0-10 min, non-overflow, dissolve & 0.0000010 & 0.0000010 & 0.0000010 & 0.0000010 & 0.0000010 & 0.0000010 & 0.0000010 \\
\hline Water & 80839 & 10-20 min, non-overflow, dissolve & 0.0000011 & 0.0000011 & 0.0000011 & 0.0000011 & 0.0000011 & 0.0000011 & 0.0000011 \\
\hline Water & 80840 & 20-30 min, non-overflow, dissolve & 0.0000011 & 0.0000011 & 0.0000011 & 0.0000011 & 0.0000011 & 0.0000011 & 0.0000011 \\
\hline Water & 80753 & 0-10 min, non-overflow, total & 0.0000010 & 0.0000010 & 0.0000010 & 0.0000010 & 0.0000010 & 0.0000010 & 0.0000010 \\
\hline Water & 80754 & 10-20 min, non-overflow, total & 0.0000010 & 0.0000010 & 0.0000010 & 0.0000010 & 0.0000010 & 0.0000010 & 0.0000010 \\
\hline \multirow[t]{2}{*}{ Water } & 80755 & $20-30 \mathrm{~min}$, non-overflow, total & 0.0000011 & 0.0000011 & 0.0000011 & 0.0000011 & 0.0000011 & 0.0000011 & 0.0000011 \\
\hline & & Hopper Inflow Monitoring & & & & & & & \\
\hline Water & 80790 & 3\& $6 \mathrm{~min}$, dissolved & 0.0000010 & 0.0000010 & 0.0000010 & 0.0000010 & 0.0000010 & 0.0000010 & 0.0000010 \\
\hline Water & 80791 & $9 \& 12 \mathrm{~min}$, dissolved & 0.0000010 & 0.0000010 & 0.0000010 & 0.0000010 & 0.0000010 & 0.0000010 & 0.0000010 \\
\hline Water & 80792 & $15 \& 18 \mathrm{~min}$, dissolved & 0.0000010 & 0.0000010 & 0.0000010 & 0.0000010 & 0.0000010 & 0.0000010 & 0.0000010 \\
\hline Water & 80793 & $21 \& 24 \mathrm{~min}$, dissolved & 0.0000010 & 0.0000010 & 0.0000010 & 0.0000010 & 0.0000010 & 0.0000010 & 0.0000010 \\
\hline Water & 80794 & $27 \& 30 \mathrm{~min}$, dissolved & 0.0000010 & 0.0000010 & 0.0000010 & 0.0000010 & 0.0000010 & 0.0000010 & 0.0000010 \\
\hline Water & 80692 & $3 \& 6 \mathrm{~min}$, total & 0.0000010 & 0.0000010 & 0.0000010 & 0.0000010 & 0.0000010 & 0.0000010 & 0.0000010 \\
\hline Water & 80693 & $9 \& 12 \mathrm{~min}$, total & 0.0000010 & 0.0000010 & 0.0000010 & 0.0000010 & 0.0000010 & 0.0000010 & 0.0000010 \\
\hline Water & 80694 & $15 \& 18 \mathrm{~min}$, total & 0.0000010 & 0.0000010 & 0.0000010 & 0.0000010 & 0.0000010 & 0.0000010 & 0.0000010 \\
\hline Water & 80695 & $21 \& 24 \mathrm{~min}$, total & 0.0000011 & 0.0000011 & 0.0000011 & 0.0000011 & 0.0000011 & 0.0000011 & 0.0000011 \\
\hline \multirow[t]{2}{*}{ Water } & 80696 & $27 \& 30 \mathrm{~min}$, total & 0.0000010 & 0.0000010 & 0.0000010 & 0.0000010 & 0.0000010 & 0.0000010 & 0.0000010 \\
\hline & & Hopper Overflow Monitoring & & & & & & & \\
\hline Water & 80795 & 2\& $4 \mathrm{~min}$, dissolved & 0.0000010 & 0.0000010 & 0.0000010 & 0.0000010 & 0.0000010 & 0.0000010 & 0.0000010 \\
\hline Water & 80796 & 6\& $8 \mathrm{~min}$, dissolved & 0.0000010 & 0.0000010 & 0.0000010 & 0.0000010 & 0.0000010 & 0.0000010 & 0.0000010 \\
\hline Water & 80797 & $10 \& 12 \mathrm{~min}$, dissolved & 0.0000010 & 0.0000010 & 0.0000010 & 0.0000010 & 0.0000010 & 0.0000010 & 0.0000010 \\
\hline Water & 80798 & $14 \& 16 \mathrm{~min}$, dissolved & 0.0000010 & 0.0000010 & 0.0000010 & 0.0000010 & 0.0000010 & 0.0000010 & 0.0000010 \\
\hline Water & 80799 & $18 \& 20 \mathrm{~min}$, dissolved & 0.0000010 & 0.0000010 & 0.0000010 & 0.0000010 & 0.0000010 & 0.0000010 & 0.0000010 \\
\hline Water & 80698 & 2\&. 4 min, total & 0.0000010 & 0.0000010 & 0.0000010 & 0.0000010 & 0.0000010 & 0.0000010 & 0.0000010 \\
\hline Water & 80699 & $6 \& 8 \mathrm{~min}$, total & 0.0000010 & 0.0000010 & 0.0000010 & 0.0000010 & 0.0000010 & 0.0000010 & 0.0000010 \\
\hline Water & 80700 & $10 \& 12 \mathrm{~min}$, total & 0.0000010 & 0.0000010 & 0.0000010 & 0.0000010 & 0.0000010 & 0.0000010 & 0.0000010 \\
\hline Water & 80701 & $14 \& 16 \mathrm{~min}$, total & 0.0000011 & 0.0000011 & 0.0000011 & 0.0000011 & 0.0000011 & 0.0000011 & 0.0000011 \\
\hline \multirow[t]{2}{*}{ Water } & 80702 & $18 \& 20 \mathrm{~min}$, total & 0.0000010 & 0.0000010 & 0.0000010 & 0.0000010 & 0.0000010 & 0.0000010 & 0.0000010 \\
\hline & & Site Water & & & & & & & \\
\hline Water & 81594 & Sample 1 Total & 0.0000010 & 0.0000010 & 0.0000010 & 0.0000010 & 0.0000010 & 0.0000010 & 0.0000010 \\
\hline Water & 81595 & Sample 2 Total & 0.0000010 & 0.0000010 & 0.0000010 & 0.0000010 & 0.0000010 & 0.0000010 & 0.0000010 \\
\hline \multirow[t]{2}{*}{ Water } & 81596 & Sample 3 Total & 0.0000010 & 0.0000010 & 0.0000010 & 0.0000010 & 0.0000010 & 0.0000010 & 0.0000010 \\
\hline & & Elutriate & & & & & & & \\
\hline Water & 81600 & Sample 1 Dissolved & 0.0000010 & 0.0000010 & 0.0000010 & 0.0000010 & 0.0000010 & 0.0000010 & 0.0000010 \\
\hline Water & 81601 & Sample 2 Dissolved & 0.0000010 & 0.0000010 & 0.0000010 & 0.0000010 & 0.0000010 & 0.0000010 & 0.0000010 \\
\hline Water & 81602 & Sample 3 Dissolved & 0.0000010 & 0.0000010 & 0.0000010 & 0.0000010 & 0.0000010 & 0.0000010 & 0.0000010 \\
\hline Water & 81597 & Sample 1 Total & 0.0000010 & 0.0000010 & 0.0000010 & 0.0000010 & 0.0000010 & 0.0000010 & 0.0000010 \\
\hline Water & 81598 & Sample 2 Total & 0.0000010 & 0.0000010 & 0.0000010 & 0.0000010 & 0.0000010 & 0.0000010 & 0.0000010 \\
\hline Water & 81599 & Sample 3 Total & 0.0000010 & 0.0000010 & 0.0000010 & 0.0000010 & 0.0000010 & 0.0000010 & 0.0000010 \\
\hline \multirow{3}{*}{$\begin{array}{l}\text { SAMPLE } \\
\text { TYPE }\end{array}$} & SAMPLE & DESCRIPTION & PCB 191 & РСB 194 & PCB 195 & РCB 196 & РCB 201 & РCB 203 & PCB 205 \\
\hline & & Detection Limit (mg/kg) & 0.00033 & 0.00033 & 0.00033 & 0.00033 & 0.00033 & 0.00033 & 0.00033 \\
\hline & & Insitu Sediment & & & & & & & \\
\hline Sediment & 81714 & Sample \#1 & 0.00033 & 0.00033 & 0.00033 & 0.00033 & 0.00033 & 0.00033 & 0.00033 \\
\hline Sediment & 81715 & Sample \#2 & 0.00033 & 0.00033 & 0.00033 & 0.00033 & 0.00033 & 0.00033 & 0.00033 \\
\hline Sediment & 81716 & Sample \#3 & 0.00033 & 0.00033 & 0.00033 & 0.00033 & 0.00033 & 0.00033 & 0.00033 \\
\hline
\end{tabular}

BOLD - less than values

Values below less than values are estimated results. Results are less than the reporting limit.

Page 11 
PCBscoar

Delaware River Water Analysis (Coarse-Grained Site)

\begin{tabular}{|c|c|c|c|c|c|c|c|c|c|}
\hline \multirow[t]{2}{*}{$\begin{array}{l}\text { SAMPLE } \\
\text { TYPE }\end{array}$} & $\begin{array}{l}\text { SAMPLE } \\
\text { ID }\end{array}$ & DESCRIPTION & PCB 206 & РСB 207 & PCB 208 & РCB 209 & PCB 66 & РСВ 190 & PCB 198 \\
\hline & & Detection Limit (mg/l) & 0.0000011 & 0.00000110 & 0.00000110 & & 0.0000011 & 0.0000011 & 0.0000011 \\
\hline & & Plume Monitoring & & & & & & & \\
\hline Water & 80834 & Background, dissolved & 0.0000020 & 0.00000040 & 0.00000080 & $110.02 \%$ & 0.0000011 & 0.0000011 & 0.0000011 \\
\hline Water & 80749 & Background, total & 0.0000015 & 0.00000100 & 0.00000060 & $95.61 \%$ & 0.0000010 & 0.0000010 & 0.0000010 \\
\hline Water & 80835 & 0-10 min, overflow, dissolved & 0.0000017 & 0.00000100 & 0.00000050 & $100.35 \%$ & 0.0000010 & 0.0000010 & 0.0000010 \\
\hline Water & 80836 & 10-20 min, overflow, dissolved & 0.0000018 & 0.00000100 & 0.00000080 & $110.93 \%$ & 0.0000010 & 0.0000010 & 0.0000010 \\
\hline Water & 80837 & 20-30 min, overflow, dissolved & 0.0000020 & 0.00000100 & 0.00000060 & $109.27 \%$ & 0.0000010 & 0.0000010 & 0.0000010 \\
\hline Water & 80750 & 0-10 min, overflow, total & 0.0000017 & 0.00000110 & 0.00000070 & $96.69 \%$ & 0.0000011 & 0.0000011 & 0.0000011 \\
\hline Water & 80751 & 10-20 min, overflow, total & 0.0000017 & 0.00000100 & 0.00000050 & $89.46 \%$ & 0.0000010 & 0.0000010 & 0.0000010 \\
\hline Water & 80752 & 20-30 min, overflow, total & 0.0000017 & 0.00000100 & 0.00000060 & $99.52 \%$ & 0.0000010 & 0.0000010 & 0.0000010 \\
\hline Water & 80838 & 0-10 min, non-overflow, dissolve & 0.0000020 & 0.00000100 & 0.00000070 & $111.00 \%$ & 0.0000010 & 0.0000010 & 0.0000010 \\
\hline Water & 80839 & 10-20 min, non-overflow, dissolve & 0.0000019 & 0.00000110 & 0.00000080 & $109.59 \%$ & 0.0000011 & 0.0000011 & 0.0000011 \\
\hline Water & 80840 & 20-30 min, non-overflow, dissolve & 0.0000022 & 0.00000110 & 0.00000070 & $105.27 \%$ & 0.0000011 & 0.0000011 & 0.0000011 \\
\hline Water & 80753 & 0-10 min, non-overflow, total & 0.0000017 & 0.00000100 & 0.00000060 & $94.00 \%$ & 0.0000010 & 0.0000010 & 0.0000010 \\
\hline Water & 80754 & 10-20 min, non-overflow, total & 0.0000016 & 0.00000100 & 0.00000050 & $92.43 \%$ & 0.0000010 & 0.0000010 & 0.0000010 \\
\hline \multirow[t]{2}{*}{ Water } & 80755 & 20-30 $\mathrm{min}$, non-overflow, total & 0.0000017 & 0.00000110 & 0.00000060 & $103.87 \%$ & 0.0000011 & 0.0000011 & 0.0000011 \\
\hline & & Hopper Inflow Monitoring & & & & & & & \\
\hline Water & 80790 & $3 \& 6 \mathrm{~min}$, dissolved & 0.0000016 & 0.00000100 & 0.00000060 & $102.69 \%$ & 0.0000010 & 0.0000010 & 0.0000010 \\
\hline Water & 80791 & $9 \& 12 \mathrm{~min}$, dissolved & 0.0000015 & 0.00000100 & 0.00000050 & $93.31 \%$ & 0.0000010 & 0.0000010 & 0.0000010 \\
\hline Water & 80792 & $15 \& 18 \mathrm{~min}$, dissolved & 0.0000014 & 0.00000100 & 0.00000050 & $83.49 \%$ & 0.0000010 & 0.0000010 & 0.0000010 \\
\hline Water & 80793 & $21824 \mathrm{~min}$, dissolved & 0.0000013 & 0.00000100 & 0.00000100 & $83.82 \%$ & 0.0000010 & 0.0000010 & 0.0000010 \\
\hline Water & 80794 & $27 \& 30 \mathrm{~min}$, dissolved & 0.0000013 & 0.00000100 & 0.00000040 & $74.65 \%$ & 0.0000011 & 0.0000010 & 0.0000010 \\
\hline Water & 80692 & $3 \& 6 \mathrm{~min}$, total & 0.0000013 & 0.00000100 & 0.00000100 & $75.42 \%$ & 0.0000010 & 0.0000010 & 0.0000010 \\
\hline Water & 80693 & $9 \& 12 \mathrm{~min}$, total & 0.0000015 & 0.00000100 & 0.00000040 & $83.26 \%$ & 0.0000010 & 0.0000010 & 0.0000010 \\
\hline Water & 80694 & $15 \& 18 \mathrm{~min}$, total & 0.0000019 & 0.00000100 & 0.00000100 & $74.76 \%$ & 0.0000010 & 0.0000010 & 0.0000010 \\
\hline Water & 80695 & $21 \& 24 \mathrm{~min}$, total & 0.0000022 & 0.00000130 & 0.00000048 & $77.49 \%$ & 0.0000011 & 0.0000011 & 0.0000011 \\
\hline \multirow[t]{2}{*}{ Water } & 80696 & $27 \& 30 \mathrm{~min}$, total & 0.0000018 & 0.00000150 & 0.00000064 & $61.74 \%$ & 0.0000010 & 0.0000010 & 0.0000010 \\
\hline & & $\begin{array}{l}\text { Hopper Overflow Monitoring } \\
28,4 \mathrm{~min} \text { dissolved }\end{array}$ & & & & & & & \\
\hline $\begin{array}{l}\text { Water } \\
\text { Water }\end{array}$ & 80795 & 2\& $4 \mathrm{~min}$, dissolved & 0.0000016 & $\begin{array}{l}0.00000100 \\
0.00000100\end{array}$ & 0.00000050 & $97.89 \%$ & 0.0000010 & 0.0000010 & 0.0000010 \\
\hline Water & 80797 & $\begin{array}{l}6 \& 8 \mathrm{~min} \text {, dissolved } \\
10 \& 12 \mathrm{~min} \text { dissolved }\end{array}$ & 0.0000017 & 0.00000100 & 0.00000050 & $99.86 \%$ & 0.0000010 & 0.0000010 & 0.0000010 \\
\hline Water & 80798 & $\begin{array}{l}10 \& 12 \mathrm{~min} \text {, dissolved } \\
14 \& 16 \mathrm{~min} \text {, dissolved }\end{array}$ & $\begin{array}{l}0.0000017 \\
0.0000017\end{array}$ & 0.00000100 & $\begin{array}{l}0.00000050 \\
0.00000050\end{array}$ & $100.24 \%$ & & 0.0000010 & 0.0000010 \\
\hline Water & 80799 & $\begin{array}{l}14 \& 16 \mathrm{~min}, \text { dissolved } \\
18 \& 20 \mathrm{~min} \text {, dissolved }\end{array}$ & $\begin{array}{l}0.0000017 \\
0.0000017\end{array}$ & 0.00000100 & 0.0000000050 & $\begin{array}{l}105.68 \% \\
105.68 \%\end{array}$ & $\begin{array}{l}0.0000010 \\
0.0000010\end{array}$ & $\begin{array}{l}0.0000010 \\
0.0000010\end{array}$ & $\begin{array}{l}0.0000010 \\
0.0000010\end{array}$ \\
\hline Water & 80698 & $2 \& 4 \mathrm{~min}$, total & 0.0000016 & 0.00000100 & 0.00000055 & $60.08 \%$ & 0.0000010 & 0.0000010 & 0.0000010 \\
\hline Water & 80699 & $68.8 \mathrm{~min}$, total & 0.0000015 & 0.00000100 & 0.00000100 & $51.08 \%$ & 0.0000010 & 0.0000010 & 0.0000010 \\
\hline Water & 80700 & $10 \& 12 \mathrm{~min}$, total & 0.0000031 & 0.00000100 & 0.00000100 & $145.37 \%$ & 0.0000010 & 0.0000010 & 0.0000010 \\
\hline Water & 80701 & $14 \& 16 \mathrm{~min}$, total & 0.0000020 & 0.00000110 & 0.00000110 & $98.00 \%$ & 0.0000011 & 0.0000011 & 0.0000011 \\
\hline \multirow[t]{2}{*}{ Water } & 80702 & $18 \& 20 \mathrm{~min}$, total & 0.0000016 & 0.00000100 & 0.00000100 & $88.00 \%$ & 0.0000010 & 0.0000010 & 0.0000010 \\
\hline & & Site Water & & & & & & & \\
\hline Water & 81594 & Sample 1 Total & 0.0000026 & 0.00000110 & 0.00000130 & $95.54 \%$ & 0.0000010 & 0.0000010 & 0.0000010 \\
\hline Water & 81595 & Sample 2 Total & 0.0000027 & 0.00000100 & 0.00000140 & $100.33 \%$ & 0.0000010 & 0.0000010 & 0.0000010 \\
\hline \multirow[t]{2}{*}{ Water } & 81596 & Sample 3 Total & 0.0000024 & 0.00000085 & 0.00000120 & $98.44 \%$ & 0.0000010 & 0.0000010 & 0.0000010 \\
\hline & & Elutriate & & & & & & & \\
\hline Water & 81600 & Sample 1 Dissolved & 0.0000025 & 0.00000088 & 0.00000140 & $110.94 \%$ & 0.0000010 & 0.0000010 & 0.0000010 \\
\hline Water & 81601 & Sample 2 Dissolved & 0.0000022 & 0.00000083 & 0.00000120 & $92.51 \%$ & 0.0000010 & 0.0000010 & 0.0000010 \\
\hline Water & 81602 & Sample 3 Dissolved & 0.0000024 & 0.00000066 & 0.00000110 & $98.83 \%$ & 0.0000010 & 0.0000010 & 0.0000010 \\
\hline Water & 81597 & Sample 1 Total & 0.0000022 & 0.00000064 & 0.00000110 & $97.19 \%$ & 0.0000010 & 0.0000010 & 0.0000010 \\
\hline Water & 81598 & Sample 2 Total & 0.0000021 & 0.00000051 & 0.00000097 & $100.67 \%$ & 0.0000010 & 0.0000010 & 0.0000010 \\
\hline Water & 81599 & Sample 3 Total & 0.0000022 & 0.00000100 & 0.00000110 & $103.83 \%$ & 0.0000010 & 0.0000010 & 0.0000010 \\
\hline \multirow{3}{*}{$\begin{array}{l}\text { SAMPLE } \\
\text { TYPE }\end{array}$} & SAMPLE & DESCRIPTION & РCB 206 & PCB 207 & PCB 208 & РСВ 209 & РCB 66 & РСВ 190 & PCB 198 \\
\hline & & Detection Limit (mg/kg) & 0.00033 & 0.00033 & 0.00033 & & 0.00033 & 0.00033 & 0.00033 \\
\hline & & Insitu Sediment & & & & & & & \\
\hline Sediment & 81714 & Sample \#1 & 0.00019 & 0.00033 & 0.00015 & $109.80 \%$ & 0.00033 & 0.00033 & 0.00033 \\
\hline Sediment & 81715 & Sampie \#2 & 0.00044 & 0.00033 & 0.00033 & $109.30 \%$ & 0.00033 & 0.00033 & 0.00033 \\
\hline Sediment & 81716 & Sample \#3 & 0.00020 & 0.00033 & 0.00015 & $106.21 \%$ & 0.00033 & 0.00033 & 0.00033 \\
\hline
\end{tabular}

BOLD - less than values

BOLD - less than values
Values below less than values are estimated results. Results are less than the reporting limit. 
Delaware River Water Analysis (Coarse-Grained Site)

\begin{tabular}{|c|c|c|c|}
\hline \multirow{2}{*}{$\begin{array}{l}\text { SAMPLE } \\
\text { TYPE }\end{array}$} & SAMPLE & DESCRIPTION & РCB 200 \\
\hline & & Detection Limit (mg/l) & 0.0000011 \\
\hline & & Plume Monitoring & \\
\hline Water & 80834 & Background, dissolved & 0.0000011 \\
\hline Water & 80749 & Background, total & 0.0000010 \\
\hline Water & 80835 & 0-10 min, overflow, dissolved & 0.0000010 \\
\hline Water & 80836 & 10-20 min, overflow, dissolved & 0.0000010 \\
\hline Water & 80837 & 20-30 min, overflow, dissolved & 0.0000010 \\
\hline Water & 80750 & 0-10 min, overflow, total & 0.0000011 \\
\hline Water & 80751 & $10-20 \mathrm{~min}$, overflow, total & 0.0000010 \\
\hline Water & 80752 & 20-30 min, overflow, total & 0.0000010 \\
\hline Water & 80838 & 0-10 min, non-overflow, dissolve & 0.0000010 \\
\hline Water & 80839 & $10-20 \mathrm{~min}$, non-overflow, dissolve & 0.0000011 \\
\hline Water & 80840 & $20-30 \mathrm{~min}$, non-overflow, dissolve & 0.0000011 \\
\hline Water & 80753 & 0-10 min, non-overflow, total & 0.0000010 \\
\hline Water & 80754 & $10-20 \mathrm{~min}$, non-overflow, total & 0.0000010 \\
\hline \multirow[t]{2}{*}{ Water } & 80755 & $20-30 \mathrm{~min}$, non-overflow, total & 0.0000011 \\
\hline & & Hopper Inflow Monitoring & \\
\hline Water & 80790 & $3 \& 6 \mathrm{~min}$, dissolved & 0.0000010 \\
\hline Water & 80791 & $9 \& 12 \mathrm{~min}$, dissolved & 0.0000010 \\
\hline Water & 80792 & $15 \& 18 \mathrm{~min}$, dissolved & 0.0000010 \\
\hline Water & 80793 & $21 \& 24$ min, dissolved & 0.0000010 \\
\hline Water & 80794 & $27 \& 30 \mathrm{~min}$, dissolved & 0.0000010 \\
\hline Water & 80692 & $3 \& 6 \mathrm{~min}$, total & 0.0000010 \\
\hline Water & 80693 & $9 \& 12 \mathrm{~min}$, total & 0.0000010 \\
\hline Water & 80694 & $15 \& 18 \mathrm{~min}$, total & 0.0000010 \\
\hline Water & 80695 & $21 \& 24 \mathrm{~min}$, total & 0.0000011 \\
\hline \multirow[t]{2}{*}{ Water } & 80696 & $27 \& 30 \mathrm{~min}$, total & 0.0000010 \\
\hline & & Hopper Overflow Monitoring & \\
\hline Water & 80795 & $2 \& 4 \mathrm{~min}$, dissolved & 0.0000010 \\
\hline Water & 80796 & 6\& $8 \mathrm{~min}$, dissolved & 0.0000010 \\
\hline Water & 80797 & $10 \& 12 \mathrm{~min}$, dissolved & 0.0000010 \\
\hline Water & 80798 & $14 \& 16 \mathrm{~min}$, dissolved & 0.0000010 \\
\hline Water & 80799 & $18 \& 20 \mathrm{~min}$, dissolved & 0.0000010 \\
\hline Water & 80698 & $2 \& 4 \mathrm{~min}$, total & 0.0000010 \\
\hline Water & 80699 & $6 \& 8 \mathrm{~min}$, total & 0.0000010 \\
\hline Water & 80700 & $10 \& 12 \mathrm{~min}$, total & 0.0000010 \\
\hline Water & 80701 & $14 \& 16 \mathrm{~min}$, total & 0.0000011 \\
\hline \multirow[t]{2}{*}{ Water } & 80702 & $18820 \mathrm{~min}$, total & 0.0000010 \\
\hline & & Site Water & \\
\hline Water & 81594 & Sample 1 Total & 0.0000010 \\
\hline Water & 81595 & Sample 2 Total & 0.0000010 \\
\hline \multirow[t]{2}{*}{ Water } & 81596 & Sample 3 Total & 0.0000010 \\
\hline & & Elutriate & \\
\hline Water & 81600 & Sample 1 Dissolved & 0.0000010 \\
\hline Water & 81601 & Sample 2 Dissolved & 0.0000010 \\
\hline Water & 81602 & Sample 3 Dissolved & 0.0000010 \\
\hline Water & 81597 & Sample 1 Total & 0.0000010 \\
\hline Water & 81598 & Sample 2 Total & 0.0000010 \\
\hline Water & 81599 & Sample 3 Total & 0.0000010 \\
\hline \multirow{3}{*}{$\begin{array}{l}\text { SAMPLE } \\
\text { TYPE }\end{array}$} & SAMPLE & DESCRIPTION & PCB 200 \\
\hline & & Detection Limit $(\mathrm{mg} / \mathrm{kg})$ & 0.00033 \\
\hline & & Insitu Sediment & \\
\hline Sediment & 81714 & Sample \#1 & 0.00033 \\
\hline Sediment & 81715 & Sample \#2 & 0.00033 \\
\hline Sediment & 81716 & Sample \#3 & 0.00033 \\
\hline
\end{tabular}

BOLD - less than values

Values below less than values are estimated results. Results are less than the reporting limit.

Page 13 


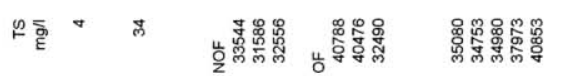

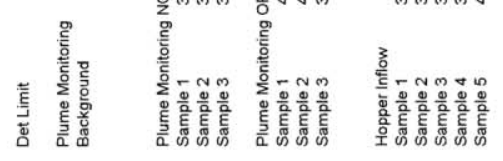

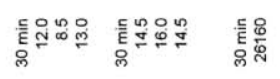

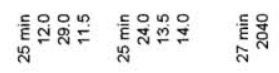

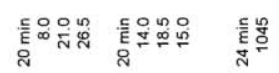

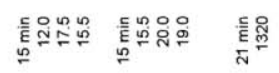

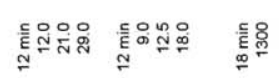

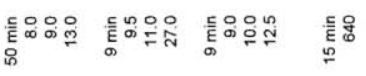

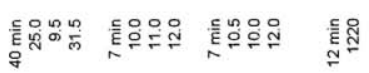

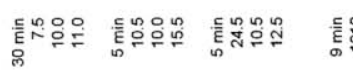

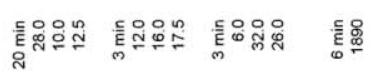

员 車

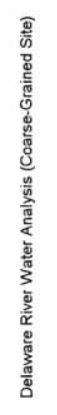

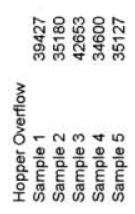

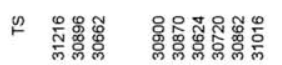

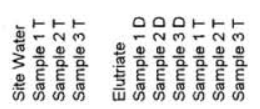

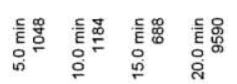

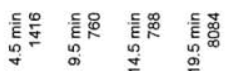

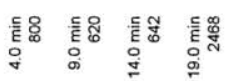

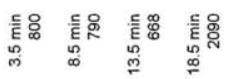

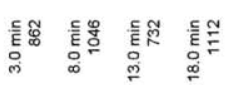

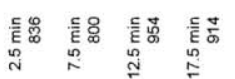

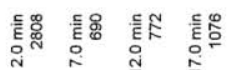

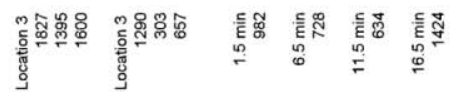

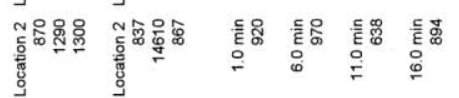

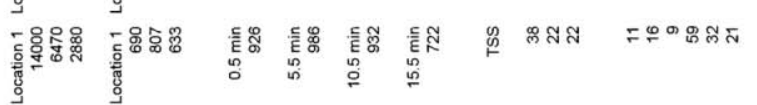

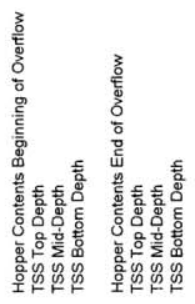

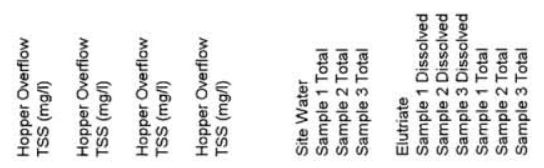

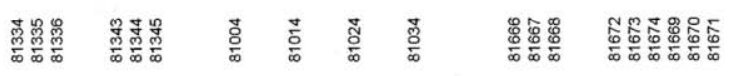

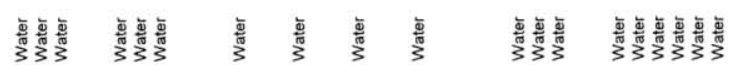


Delaware River Water Analysis (Coarse-Grained Site)

$\begin{array}{lll}\text { SAMPLE } & \text { SAMPLE } & \text { DESCRIPTION } \\ \text { TYPE } & \text { ID } & \text { TOC }\end{array}$

Detection Limit (mg/)

$\begin{array}{llll} & & \text { Plume Monitoring } \\ \text { Water } & 80820 & \text { Background, dissolved } & \\ \text { Water } & 80728 & \text { Background, total }\end{array}$

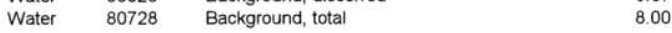

$\begin{array}{lrrr}\text { Water } & 80821 & 0-10 \mathrm{~min}, \text { overflow, dissolved } & 8.98 \\ \text { Water } & 80822 & 10-20 \mathrm{~min}, \text { overflow, dissolved } & 11.30\end{array}$

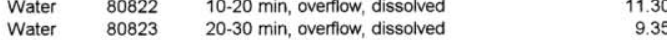

$\begin{array}{lll}\text { Water } & 80823 & 20-30 \mathrm{~min}, \text { overlow, dissolved } \\ \text { Water } & 80729 & 0-10 \mathrm{~min} \text {, overflow, total }\end{array}$

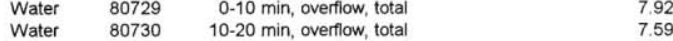

Water $80731 \quad 20-30 \mathrm{~min}$, overflow, total $\quad 8.86$

$\begin{array}{lll}\text { Water } & 80824 & 0-10 \mathrm{~min}, \text { non-overflow, dissolved }\end{array}$

$\begin{array}{lll}\text { Water } & 80825 & 10-20 \mathrm{~min}, \text { non-overflow, dissolved }\end{array}$

$\begin{array}{lll}\text { Water } & 80826 & 20-30 \mathrm{~min} \text {, non-overflow, dissolved }\end{array}$

$\begin{array}{lrrr}\text { Water } & 80732 & 0-10 \mathrm{~min} \text {, non-overflow, total } & 6.80 \\ \text { Water } & 80733 & 10-20 \mathrm{~min} \text {, non-overflow, total } & 10.30\end{array}$

Water $80734 \quad 20-30 \mathrm{~min}$, non-overflow, total

$\begin{array}{lll}\text { Water } & 80770 \quad \begin{array}{c}\text { Hopper Inflow Monitoring } \\ 3 \& 6 \mathrm{~min} \text {, dissolved }\end{array} & 14.80\end{array}$

Water $\quad 80771 \quad 9 \& 12 \mathrm{~min}$, dissolved $\quad 3.45$

Water $\quad 80772 \quad 15 \& 18 \mathrm{~min}$, dissolved $\quad 13.50$

$\begin{array}{llll}\text { Water } & 80773 & 21824 \mathrm{~min} \text {, dissolved } & 14.50 \\ \text { Water } & 80774 & 27830 \mathrm{~min} \text {, dissolved } & 16.20\end{array}$

Water $\quad 80656 \quad 3 \& 6 \mathrm{~min}$, total $\quad 216.00$

Water $\quad 80657 \quad 9 \& 12 \mathrm{~min}$, total $\quad 46.80$

Water $\quad 80658 \quad 15 \& 18 \mathrm{~min}$, total $\quad 16.50$

$\begin{array}{llll}\text { Water } & 80659 & 21 \& 24 \mathrm{~min} \text {, total } & 28.60 \\ \text { Water } & 80660 & 27830 \mathrm{~min} \text {, total } & 54.20\end{array}$

Water $\quad 80775 \quad$ Hopper Overflow Monitoring

$\begin{array}{llll}\text { Water } & 80775 & 2 \& 4 \mathrm{~min} \text {, dissolved } & 12.40 \\ \text { Water } & 80776 & 6 \& 8 \mathrm{~min} \text {, dissolved } & 11.20\end{array}$

Water $80777 \quad 10 \& 12 \mathrm{~min}$, dissolved $\quad 13.80$

$\begin{array}{lll}\text { Water } & 80778 & 14816 \mathrm{~min} \text {, dissolved } \\ \text { Water } & 80779 & 18820 \mathrm{~min} \text {, dissolved }\end{array}$

$\begin{array}{lll}\text { Water } & 80779 & 18 \& 20 \mathrm{~min} \text {, dissolved }\end{array}$

$\begin{array}{lll}\text { Water } & 80662 & 2 \& 4 \mathrm{~min} \text {, total }\end{array}$

$\begin{array}{lll}\text { Water } & 80664 & 10812 \mathrm{~min}, \text { total }\end{array}$

Water $80666 \quad 18820 \mathrm{~min}$, total

$\begin{array}{llll} & & \\ \text { Water } & 81684 & \text { Site Water } & \\ \text { Water } & 81685 & \text { Sample 1 Total } & 5.12 \\ \text { Water } & 81686 & \text { Sample 2 Total } & 1.21 \\ & & \end{array}$

$\begin{array}{ll} & \text { Elutriate } \\ \text { Water } & 81690 \text { Sample } 1 \text { Dissolved }\end{array}$

$\begin{array}{llll}\text { Water } & 81690 & \text { Sample 1 Dissolved } & 1.07 \\ \text { Water } & 81691 & \text { Sample 2 Dissolved } & 3.00\end{array}$

$\begin{array}{llll}\text { Water } & 81691 & \text { Sample 2 Dissolved } & \mathbf{3 . 0 0} \\ \text { Water } & 81692 & \text { Sample 3 Dissolved } & \mathbf{3 . 0 0}\end{array}$

$\begin{array}{lll}\text { Water } & 81687 & \text { Sample 1 Total }\end{array}$

$\begin{array}{llll}\text { Water } & 81688 & \text { Sample 2 Total } & \mathbf{3 . 0 0} \\ \text { Water } & 81689 & \text { Sample 3 Total } & \mathbf{3 . 0 0}\end{array}$

SAMPLE SAMPLE DESCRIPTION TOC

$\begin{array}{lll}\text { TYPE ID } & \\ & \text { Detection Limit }(\mathrm{mg} / \mathrm{kg})\end{array}$

Insitu Sediment

$\begin{array}{lll}\text { Sediment } 81720 & \begin{array}{l}\text { Insitu Sediment } \\ \text { Sample \#1 }\end{array} & 174.0\end{array}$

$\begin{array}{llll}\text { Sediment } & 81721 & \text { Sample \#2 } & 155.0 \\ \text { Sediment } & 81722 & \text { Sample \#3 } & 170.0\end{array}$

BOLD - less than values

Values below less than values are estimated results. Results are less than the reporting limit. 
spgrcoar

Delaware River Water Analysis (Coarse-Grained Site)

\begin{tabular}{|c|c|c|c|c|}
\hline $\begin{array}{l}\text { SAMPLE } \\
\text { TYPE }\end{array}$ & $\begin{array}{l}\text { SAMPLE } \\
\text { ID }\end{array}$ & DESCRIPTION & Sp. Gr. & \%Moisture \\
\hline & & Insitu Sediment & & \\
\hline Sediment & 81209 & Sample \#1 & 2.71 & $22.57 \%$ \\
\hline Sediment & 81210 & Sample \#2 & 2.70 & $25.39 \%$ \\
\hline Sediment & 81211 & Sample \#3 & 2.71 & $22.00 \%$ \\
\hline Sediment & 81212 & Sample \#4 & 2.71 & $23.83 \%$ \\
\hline Sediment & 81213 & Sample \#5 & 2.71 & $21.04 \%$ \\
\hline Sediment & 81214 & Sample \#6 & 2.72 & $20.33 \%$ \\
\hline Sediment & 81215 & Sample \#7 & 2.71 & $20.06 \%$ \\
\hline Sediment & 81216 & Sample \#8 & 2.72 & $21.82 \%$ \\
\hline Sediment & 81217 & Sample \#9 & 2.72 & $21.30 \%$ \\
\hline Sediment & 81218 & Sample \#10 & 2.72 & $19.87 \%$ \\
\hline Sediment & 81219 & Sample \#11 & 2.74 & $23.49 \%$ \\
\hline Sediment & 81220 & Sample \#12 & 2.74 & $20.47 \%$ \\
\hline Sediment & 81221 & Sample \#13 & 2.73 & $23.70 \%$ \\
\hline Sediment & 81222 & Sample \#14 & 2.74 & $20.90 \%$ \\
\hline Sediment & 81223 & Sample \#15 & 2.73 & $21.95 \%$ \\
\hline
\end{tabular}

Average $\quad 2.72 \quad 21.91 \%$

Page 1 
Metsfine

Delaware River Water Analysis (Fine-Grained Site)

SAMPLE SAMPLE DESCRIPTION

TYPE ID

$\begin{array}{ll}\text { Water } & 809 \\ \text { Water } & 80934 \\ & \\ \text { Water } & 809 \\ \text { Water } & 8097 \\ \text { Water } & 909 \\ \text { Water } & 809 \\ \text { Water } & 809 \\ \text { Water } & 809 \\ \text { Water } & 809 \\ \text { Water } & 809 \\ \text { Water } & 809 \\ \text { Water } & 8093 \\ \text { Water } & 90939 \\ \text { Water } & 809\end{array}$

Detection Limit (mg/)

Plume Monitoring

Background, dissolved

Background, total

0-10 min, overflow, dissolved

10-20 min, overflow, dissolve

20-30 min, overflow, dissolved

0-10 min, overflow, tota

$10-20$ min, overllow, tot

0-10 min, non-oventow, dissolved

10-20 min, non-overflow, dissolved

20-30 min, non-overflow, dissolved

$0.10 \mathrm{~min}$, non-overflow, total

10-20 min, non-everfow, total

20-30 min, non-overflow, total

Hopper Inflow Monitoring

3\& $6 \mathrm{~min}$, dissolved

$9 \& 12 \mathrm{~min}$, dissolve

$15 \& 18 \mathrm{~min}$, dissolve

$21824 \mathrm{~min}$, dissolved

$27 \& 30 \mathrm{~min}$, dissolve

$3 \& 6 \mathrm{~min}$, tota

$9 \& 12 \mathrm{~min}$, total

$15818 \mathrm{~min}$, tota

$21 \& 24 \mathrm{~min}$, total
$27830 \mathrm{~min}$, tota

Hopper Overflow Monitoring

2\& $4 \mathrm{~min}$, dissolved

$6 \& 8 \mathrm{~min}$, dissolved
$10 \& 12 \mathrm{~min}$, dissolve

$10 \& 12 \mathrm{~min}$, dissolved

$14816 \mathrm{~min}$, dissolved
$18820 \mathrm{~min}$, dissolved

$18 \& 20 \mathrm{~min}$, dissol
$2 \& 4 \mathrm{~min}$, total

2\& $4 \mathrm{~min}$, total
$6 \& 8 \mathrm{~min}$, total

$6 \& 8 \mathrm{~min}$, total
$10 \& 12 \mathrm{~min}$, total

$10 \& 12 \mathrm{~min}$, total

$18 \& 20 \mathrm{~min}$, total

Site Water
Sample 1 Tota

Sample 1 Total
Sample 2 Total

Sample 3 Total

Elutriate

Sample 1 Dissolved

Sample 2 Dissolved

Sample 1 Total

Sample 3 Total
SB

0.0030

0.0030

0.0030

0.003

0.0030

0.003

0.0030

0.0030

0.0030

0.0030

0.0030

.0030

0.0030

0.0030

0.0030
0.0030

0.0030
0.0030

0.0030
0.0030

0.0030

0.0156

050

0.0288

0.1090

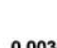

0.0030
0.0030

0.0030
0.0030

0.0030
0.0030

0.0030
0.0030

0.0030
0.0895

0.0950

.0840

0.0905
0.0815

0.0030

0.0030
0.0030
$\mathbf{0 . 0 0 3 0}$

0.010
0.009
$\mathbf{0 . 0 0 2}$

0.001
0.001
0.001

0.0030

0.0030
0.0030

0.003

0.0030
0.0030
0.0030

0.010
0.009
0.015
0.014
0.014

$\mathbf{0 . 0 0 1}$
$\mathbf{0 . 0 0 1}$
$\mathbf{0 . 0 0 1}$
$\mathbf{0 . 0 0 1}$
0.001
0.001

SB

AS

0.30

0.36

0.49
0.37

0.2

10.3
10.7
10.1

Insitu Sediment

Sediment 81729 Sample \#1

$\begin{array}{lll}\text { Sediment } & 81730 & \text { Sample \#2 } \\ \text { Sediment } & 81731 & \text { Sample \#3 }\end{array}$

SB - Antimony

BOLD - less than values

AS - Arsenic BE - Beryllium

CD - Cadmium

CR - Chromium

porting limit.

CU - Copper

B - Lead

Page 1 
Metsfine

Delaware River Water Analysis (Fine-Grained Site)

\begin{tabular}{|c|c|c|c|c|c|c|c|c|c|c|}
\hline \multirow[t]{2}{*}{$\begin{array}{l}\text { SAMPLE } \\
\text { TYPE }\end{array}$} & $\begin{array}{l}\text { SAMPLE } \\
\text { ID }\end{array}$ & DESCRIPTION & $\mathrm{NI}$ & SE & AG & TL & $\mathrm{ZN}$ & AL & $\mathrm{BA}$ & CA \\
\hline & & Detection Limit (mg/l) & 0.001 & 0.002 & 0.001 & 0.0020 & 0.010 & 0.025 & 0.002 & 0.200 \\
\hline & & Plume Monitoring & & & & & & & & \\
\hline Water & 80976 & Background, dissolved & 0.001 & 0.019 & 0.001 & 0.0020 & 0.053 & 0.025 & 0.223 & 70.2 \\
\hline Water & 80934 & Background, total & 0.004 & 0.025 & 0.001 & 0.0020 & 0.071 & 2.900 & 0.050 & 67.4 \\
\hline Water & 80977 & 0-10 min, overflow, dissolved & 0.001 & 0.013 & 0.001 & 0.0020 & 0.014 & 0.025 & 0.094 & 57.1 \\
\hline Water & 80978 & 10-20 min, overflow, dissolved & 0.001 & 0.013 & 0.001 & 0.0020 & 0.013 & 0.025 & 0.086 & 58.3 \\
\hline Water & 90979 & $20-30 \mathrm{~min}$, overflow, dissolved & 0.001 & 0.014 & 0.001 & 0.0020 & 0.013 & 0.025 & 0.089 & 53.1 \\
\hline Water & 80935 & 0-10 min, overflow, total & 0.007 & 0.019 & 0.001 & 0.0020 & 0.059 & 7.920 & 0.061 & 56.5 \\
\hline Water & 80936 & 10-20 min, overflow, total & 0.008 & 0.023 & 0.001 & 0.0020 & 0.060 & 7.640 & 0.065 & 57.7 \\
\hline Water & 80937 & 20-30 min, overflow, total & 0.004 & 0.021 & 0.001 & 0.0020 & 0.036 & 5.140 & 0.048 & 53.8 \\
\hline Water & 80980 & 0-10 min, non-overflow, dissolved & 0.001 & 0.021 & 0.001 & 0.0020 & 0.058 & 0.025 & 0.245 & 70.9 \\
\hline Water & 80981 & 10-20 min, non-overflow, dissolved & 0.001 & 0.023 & 0.001 & 0.0021 & 0.046 & 0.025 & 0.193 & 69.6 \\
\hline Water & 80982 & 20-30 min, non-overflow, dissolved & 0.001 & 0.021 & 0.001 & 0.0020 & 0.048 & 0.025 & 0.207 & 70.8 \\
\hline Water & 80938 & 0-10 min, non-overflow, total & 0.001 & 0.027 & 0.001 & 0.0020 & 0.013 & 1.800 & 0.170 & 70.6 \\
\hline Water & 90939 & 10-20 min, non-overflow, total & 0.003 & 0.027 & 0.001 & 0.0020 & 0.017 & 2.160 & 0.040 & 67.6 \\
\hline \multirow[t]{2}{*}{ Water } & 80940 & 20-30 min, non-overflow, total & 0.003 & 0.028 & 0.001 & 0.0020 & 0.010 & 1.790 & 0.038 & 66.1 \\
\hline & & Hopper Inflow Monitoring & & & & & & & & \\
\hline Water & 81094 & $3 \& 6 \mathrm{~min}$, dissolved & 0.003 & 0.015 & 0.001 & 0.0020 & 0.074 & 0.025 & 0.435 & 82.7 \\
\hline Water & 81095 & $9 \& 12 \mathrm{~min}$, dissolved & 0.004 & 0.015 & 0.002 & 0.0020 & 0.084 & 0.092 & 0.549 & 93.8 \\
\hline Water & 81096 & $15 \& 18 \mathrm{~min}$, dissolved & 0.005 & 0.014 & 0.001 & 0.0020 & 0.076 & 0.033 & 0.529 & 111.0 \\
\hline Water & 81097 & $21 \& 24 \mathrm{~min}$, dissolved & 0.003 & 0.014 & 0.001 & 0.0020 & 0.057 & 0.028 & 0.380 & 73.4 \\
\hline Water & 81098 & $27 \& 30 \mathrm{~min}$, dissolved & 0.005 & 0.015 & 0.001 & 0.0020 & 0.089 & 0.025 & 0.636 & 108.0 \\
\hline Water & 80867 & $3 \& 6 \mathrm{~min}$, total & 0.912 & 0.068 & 0.004 & 0.0110 & 5.880 & 744.0 & 3.000 & 178.0 \\
\hline Water & 80868 & $9 \& 12 \mathrm{~min}$, total & 1.950 & 0.116 & 0.076 & 0.0160 & 13.300 & 1856.0 & 6.440 & 392.0 \\
\hline Water & 80869 & $15 \& 18 \mathrm{~min}$, total & 3.240 & 0.180 & 0.098 & 0.0310 & 21.400 & 3320.0 & 10.200 & 640.0 \\
\hline Water & 80870 & $21 \& 24 \mathrm{~min}$, total & 1.270 & 0.084 & 0.044 & 0.0110 & 8.760 & 1110.0 & 4.200 & 241.0 \\
\hline \multirow[t]{2}{*}{ Water } & 80871 & $27 \& 30 \mathrm{~min}$, total & 4.750 & 0.255 & 0.150 & 0.0540 & 34.800 & 5440.0 & 16.800 & 1000.0 \\
\hline & & Hopper Overflow Monitoring & & & & & & & & \\
\hline Water & 81099 & 2\& $4 \mathrm{~min}$, dissolved & 0.005 & 0.015 & 0.001 & 0.0020 & 0.145 & 0.025 & 0.607 & 117.0 \\
\hline Water & 81100 & 6\& $8 \mathrm{~min}$, dissolved & 0.005 & 0.015 & 0.001 & 0.0020 & 0.157 & 0.025 & 0.713 & 121.0 \\
\hline Water & 81101 & $10 \& 12 \mathrm{~min}$, dissolved & 0.005 & 0.015 & 0.001 & 0.0020 & 0.130 & 0.025 & 0.748 & 112.0 \\
\hline Water & 81102 & $14 \& 16 \mathrm{~min}$, dissolved & 0.005 & 0.013 & 0.001 & 0.0020 & 0.138 & 0.025 & 0.749 & 119.0 \\
\hline Water & 81103 & $18 \& 20 \mathrm{~min}$, dissolved & 0.004 & 0.013 & 0.002 & 0.0020 & 0.085 & 0.025 & 0.551 & 116.0 \\
\hline Water & 80873 & 28 $4 \mathrm{~min}$, total & 3.350 & 0.010 & 0.007 & 0.0020 & 23.400 & 3450.0 & 11.000 & 590.0 \\
\hline Water & 80874 & $6 \& 8 \mathrm{~min}$, total & 3.310 & 0.010 & 0.005 & 0.0020 & 22.900 & 3080.0 & 10.700 & 615.0 \\
\hline Water & 80875 & $10 \& 12 \mathrm{~min}$, total & 3.030 & 0.141 & 0.053 & 0.0230 & 21.200 & 2860.0 & 10.000 & 565.0 \\
\hline Water & 80876 & $14 \& 16 \mathrm{~min}$, total & 3.820 & 0.195 & 0.114 & 0.0340 & 27.100 & 3930.0 & 12.800 & 745.0 \\
\hline \multirow[t]{2}{*}{ Water } & 80877 & $18 \& 20 \mathrm{~min}$, total & 3.760 & 0.191 & 0.078 & 0.0360 & 26.800 & 3740.0 & 12.500 & 760.0 \\
\hline & & Site Water & & & & & & & & \\
\hline Water & 81657 & Sample 1 Total & 0.002 & 0.026 & 0.003 & 0.0020 & 0.019 & 2.330 & 0.043 & 59.7 \\
\hline Water & 81658 & Sample 2 Total & 0.003 & 0.024 & 0.002 & 0.0020 & 0.019 & 2.060 & 0.042 & 60.7 \\
\hline \multirow[t]{2}{*}{ Water } & 81659 & Sample 3 Total & 0.001 & 0.002 & 0.002 & 0.0020 & 0.018 & 2.340 & 0.042 & 60.2 \\
\hline & & Elutriate & & & & & & & & \\
\hline Water & 81663 & Sample 1 Dissolved & 0.003 & 0.028 & 0.001 & 0.0020 & 0.073 & 0.185 & 0.280 & 65.8 \\
\hline Water & 81664 & Sample 2 Dissolved & 0.002 & 0.024 & 0.001 & 0.0020 & 0.075 & 0.118 & 0.209 & 67.6 \\
\hline Water & 81665 & Sample 3 Dissolved & 0.002 & 0.021 & 0.002 & 0.0020 & 0.076 & 0.105 & 0.214 & 66.1 \\
\hline Water & 81660 & Sample 1 Total & 0.011 & 0.030 & 0.002 & 0.0020 & 0.075 & 12.900 & 0.104 & 61.3 \\
\hline Water & 81661 & Sample 2 Total & 0.011 & 0.028 & 0.002 & 0.0020 & 0.074 & 13.200 & 0.107 & 62.8 \\
\hline Water & 81662 & Sample 3 Total & 0.012 & 0.030 & 0.002 & 0.0020 & 0.072 & 13.000 & 0.113 & 61.9 \\
\hline \multirow{3}{*}{$\begin{array}{l}\text { SAMPLE } \\
\text { TYPE }\end{array}$} & SAMPLE & DESCRIPTION & $\mathrm{NI}$ & SE & AG & $\mathrm{TL}$ & $\mathrm{ZN}$ & AL & BA & CA \\
\hline & & Detection Limit (mg/kg) & 0.5 & 0.20 & 0.100 & 0.200 & 1 & 1 & 0.1 & 20 \\
\hline & & Insitu Sediment & & & & & & & & \\
\hline Sediment & 81729 & Sample \#1 & 21.7 & 1.60 & 0.700 & 0.200 & 131 & 13300 & 51.4 & 2180 \\
\hline Sediment & 81730 & Sample \#2 & 22.2 & 1.60 & 0.700 & 0.200 & 133 & 13800 & 53.5 & 2260 \\
\hline Sediment & 81731 & Sample \#3 & 21.5 & 1.70 & 0.649 & 0.200 & 130 & 13000 & 51.7 & 2230 \\
\hline
\end{tabular}

NI-Nickel SE-Selenium AG-Silver TL-Thallium ZN-Zinc AL-Aluminum BA-Barium CA-Calcium

BOLD - less than values
Values below less than values are estimated results. Results are less than the reporting limit. 
Metsfine

Delaware River Water Analysis (Fine-Grained Site)

SAMPLE SAMPLE DESCRIPTION

TYPE ID

\begin{tabular}{|c|c|c|}
\hline & & Detection Limit (mg/l) \\
\hline & & Plume Monitoring \\
\hline Water & 80976 & Background, dissolved \\
\hline Water & 80934 & Background, total \\
\hline Water & 80977 & 0-10 min, overflow, dissolved \\
\hline Water & 80978 & 10-20 min, overflow, dissolved \\
\hline Water & 90979 & $20-30 \mathrm{~min}$, overflow, dissolved \\
\hline Water & 80935 & 0-10 min, overflow, total \\
\hline Water & 80936 & 10-20 min, overflow, total \\
\hline Water & 80937 & $20-30 \mathrm{~min}$, overflow, total \\
\hline Water & 80980 & 0-10 min, non-overflow, dissolved \\
\hline Water & 80981 & $10-20 \mathrm{~min}$, non-overflow, dissolved \\
\hline Water & 80982 & 20-30 min, non-overflow, dissolved \\
\hline Water & 80938 & $0-10 \mathrm{~min}$, non-overflow, total \\
\hline Water & 90939 & 10-20 min, non-overflow, total \\
\hline Water & 80940 & 20-30 min, non-overflow, total \\
\hline & & Hopper Inflow Monitoring \\
\hline Water & 81094 & $3 \& 6 \mathrm{~min}$, dissolved \\
\hline Water & 81095 & $9 \& 12 \mathrm{~min}$, dissolved \\
\hline Water & 81096 & $15 \& 18 \mathrm{~min}$, dissolved \\
\hline Water & 81097 & $21224 \mathrm{~min}$, dissolved \\
\hline Water & 81098 & $27 \& 30 \mathrm{~min}$, dissolved \\
\hline Water & 80867 & $3 \& 6 \mathrm{~min}$, total \\
\hline Water & 80868 & $9 \& 12 \mathrm{~min}$, total \\
\hline Water & 80869 & $15 \& 18 \mathrm{~min}$, total \\
\hline Water & 80870 & $21 \& 24 \mathrm{~min}$, total \\
\hline Water & 80871 & $27 \& 30$ min, total \\
\hline & & Hopper Overflow Monitoring \\
\hline Water & 81099 & 28 $4 \mathrm{~min}$, dissolved \\
\hline Water & 81100 & 6\& $8 \mathrm{~min}$, dissolved \\
\hline Water & 81101 & $10 \& 12 \mathrm{~min}$ dissolved \\
\hline Water & 81102 & $14 \& 16 \mathrm{~min}$, dissolved \\
\hline Water & 81103 & $18 \& 20 \mathrm{~min}$, dissolved \\
\hline Water & 80873 & 28 $4 \mathrm{~min}$, total \\
\hline Water & 80874 & $6 \& 8 \mathrm{~min}$, total \\
\hline Water & 80875 & $10 \& 12 \mathrm{~min}$, total \\
\hline Water & 80876 & $14 \& 16 \mathrm{~min}$, total \\
\hline Water & 80877 & $18820 \mathrm{~min}$, total \\
\hline & & Site Water \\
\hline Water & 81657 & Sample 1 Total \\
\hline Water & 81658 & Sample 2 Total \\
\hline Water & 81659 & Sample 3 Total \\
\hline & & Elutriate \\
\hline Water & 81663 & Sample 1 Dissolved \\
\hline Water & 81664 & Sample 2 Dissolved \\
\hline Water & 81665 & Sample 3 Dissolved \\
\hline Water & 81660 & Sample 1 Total \\
\hline Water & 81661 & Sample 2 Total \\
\hline Water & 81662 & Sample 3 Total \\
\hline
\end{tabular}

SAMPLE SAMPLE DESCRIPTION

TYPE ID

$\begin{array}{ll}C O & \\ & \\ 0.002 & 0.02 \\ & \\ \mathbf{0 . 0 0 1} & \mathbf{0 . 0 2 0} \\ \mathbf{0 . 0 0 1} & 2.4 \\ \mathbf{0 . 0 0 1} & \mathbf{0 . 0} \\ \mathbf{0 . 0 0 1} & \mathbf{0 . 0} \\ \mathbf{0 . 0 0 1} & \mathbf{0 . 0} \\ 0.002 & 9.7 \\ 0.002 & 9.2 \\ \mathbf{0 . 0 0 1} & 5.7 \\ \mathbf{0 . 0 0 1} & 0.027 \\ \mathbf{0 . 0 0 1} & \mathbf{0 . 0 2} \\ \mathbf{0 . 0 0 1} & \mathbf{0 . 0 2} \\ \mathbf{0 . 0 0 1} & 1.4 \\ \mathbf{0 . 0 0 1} & 1.8 \\ \mathbf{0 . 0 0 1} & 1.1 \\ \end{array}$

FE MG

$\begin{array}{llllll}0.020 & 0.200 & 0.001 & 0.20 & 0.20 & 0.002\end{array}$

$\begin{array}{llllll}\mathbf{0 . 0 2 0} & 180 & 0.002 & 56.4 & 1540 & 0.004\end{array}$

$\mathbf{0 . 0 2 0} \quad 121 \quad 0.062$

$\begin{array}{lll}0.020 & 116 & 0.027\end{array}$

$\begin{array}{lll}0.020 & 112 & 0.010\end{array}$

$\begin{array}{lll}9.260 & 121 & 0.465 \\ 5.730 & 129 & 0.450\end{array}$

0.450

0.278

0.011

0.011

0.005

0.073
0.098
0.061

$41.8 \quad 1030$

$\begin{array}{lll}41.1 & 973 & 0.003 \\ 37.4 & 9.003\end{array}$

$\begin{array}{lll}36.4 & 942 & 0.003\end{array}$

$916-020$
$920-0.020$

34.0

$57.8 \quad 1570$

$\begin{array}{lll}57.8 & 1570 & 0.003\end{array}$

$\begin{array}{lll}55.6 & 1520 & 0.003 \\ 55.7 & 1380 & 0.002\end{array}$

$\begin{array}{lll}54.9 & 1470 & 0.006\end{array}$

$\begin{array}{lll}53.8 & 1370 & 0.007\end{array}$

$1.140-175$

0.061

0.006

$\begin{array}{rr}0.002 & 0.928 \\ 0.004 & 6.850 \\ 0.006 & 10.200 \\ 0.004 & 0.467 \\ 0.006 & 11.600 \\ 0.480 & 1,180.0 \\ 1.030 & 2,860.0 \\ 1.700 & 5,130.0 \\ 0.684 & 1,630.0 \\ 2.510 & 9,200.0\end{array}$

\subsection{0}

9.200

11.200

3.840

6.920

58.0
132.0
244.0
74.8

244.0
74.8

412.0

$\begin{array}{rrr}45.1 & 1240 & \mathbf{0 . 0 0 1} \\ 38.9 & 1030 & 0.001 \\ 36.1 & 1030 & 0.001 \\ 40.0 & 1060 & \mathbf{0 . 0 0 1} \\ 33.6 & 940 & 0.002 \\ 160.0 & 1070 & 1.800 \\ 289.0 & 1104 & 3.950 \\ 451.0 & 1060 & 6.550 \\ 188.0 & 1020 & 3.130 \\ 700.0 & 970 & 9.650\end{array}$

0.004

0.004

0.004

0.005
0.006
0.006
1.760

$1.760 \quad 4,750.0$

$1.740 \quad 2,700$

$\begin{array}{ll}1.620 & 4,160.0 \\ 2.030 & 5,600.0\end{array}$

$\begin{array}{ll}2.030 & 5,600.0 \\ 1.980 & 6,150.0\end{array}$

$\begin{array}{lll}2.930 & 151 & 9.580\end{array}$

9.500
7.310

7.310
6.460

6.810

225.0

224.0

197.0
265.0

265.0
287.0

$\begin{array}{rrr}32.9 & 1000 & \mathbf{0 . 0 0 1} \\ 33.4 & 1030 & \mathbf{0 . 0 0 1} \\ 32.3 & 956 & \mathbf{0 . 0 0 1} \\ 30.0 & 932 & \mathbf{0 . 0 0 1} \\ 32.5 & 895 & \mathbf{0 . 0 0 1} \\ 474.0 & 885 & 6.650 \\ 389.0 & 970 & 6.600 \\ 415.0 & 960 & 5.900 \\ 520.0 & 930 & 7.400 \\ 510.0 & 960 & 7.350\end{array}$

2.420

$\begin{array}{ll}0.002 & 2.370 \\ 0.002 & 2.470\end{array}$

$135 \quad 0.120$

$\begin{array}{llll}0.120 & 39.80 & 1130 & 0.007 \\ 0.121 & 36.90 & 1140 & 0.007\end{array}$

$0.003-0.043-1400000$

$\begin{array}{lllllll}0.003 & 0.043 & 146 & 8.280 & 40.40 & 1140 & 0.006 \\ 0.002 & 0.042 & 153 & 8.100 & 40.40 & 1180 & 0.007\end{array}$

$\begin{array}{lrrrrrr}0.002 & 0.042 & 153 & 8.100 & 40.40 & 1180 & 0.007 \\ 0.002 & 0.037 & 144 & 8.310 & 36.40 & 1160 & 0.007\end{array}$

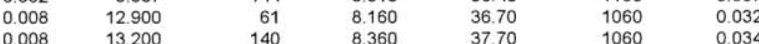

$\begin{array}{lllllll}0.008 & 13.200 & 140 & 8.360 & 37.70 & 1060 & 0.034 \\ 0.008 & 13.000 & 136 & 8.430 & 33.00 & 1020 & 0.034\end{array}$

$\begin{array}{rrrrrrrr}0.008 & 13.000 & 136 & 8.430 & 33.00 & 1020 & 0.034 & \\ \text { CO } & \text { FE } & \text { MG } & \text { MN } & \text { K } & \text { NA } & \text { V } & \% \text { Moisture }\end{array}$

$\begin{array}{lll} & & \begin{array}{l}\text { Detection Limit }(\mathrm{mg} / \mathrm{kg}) \\ \text { Insitu Sediment }\end{array} \\ \text { Sediment } & 81729 & \text { Sample \#1 } \\ \text { Sediment } & 81730 & \text { Sample \#2 } \\ \text { Sediment } 81731 & \text { Sample \#3 }\end{array}$

$\begin{array}{rrrrrrrr}0.1 & 2 & 20 & 0.1 & 20 & 20 & 0.1 & \\ & & & & & & & \\ 11.1 & 25,300 & 5,050 & 1,070.0 & 2,290 & 2110 & 33.8 & 65.6 \\ 11.2 & 26,200 & 5,120 & 1,130.0 & 2,380 & 2160 & 42.6 & 65.6 \\ 11.0 & 25,200 & 5,070 & 1,120.0 & 2,350 & 2140 & 37.1 & 65.6\end{array}$

CO- Cobalt FE-Iron MG-Magnesium

MN - Manganese

NA-Sodium $\quad V$-Vanadium

BOLD - less than values
Values below less than values are estimated results. Results are less than the reporting limit.

Page 3 
PAHsfine

Delaware River Water Analysis (Fine-Grained Site)

\begin{tabular}{|c|c|c|c|c|c|c|c|c|c|}
\hline \multirow{3}{*}{$\begin{array}{l}\text { SAMPLE } \\
\text { TYPE }\end{array}$} & $\begin{array}{l}\text { SAMPLE } \\
\text { ID }\end{array}$ & DESCRIPTION & NAPHTH & ACENAY & ACENAP & FLUORE & PHENAN & ANTRAC & FLANTHE \\
\hline & & Detection Limit (mg/l) & 0.00030 & 0.00030 & 0.00030 & 0.00030 & 0.00030 & 0.00030 & 0.00030 \\
\hline & & Plume Monitoring & & & & & & & \\
\hline Water & 80997 & Background, dissolved & 0.00030 & 0.00030 & 0.00030 & 0.00030 & 0.00030 & 0.00030 & 0.00030 \\
\hline Water & 80962 & Background, total & 0.00030 & 0.00030 & 0.00030 & 0.00030 & 0.00030 & 0.00030 & 0.00030 \\
\hline Water & 80998 & 0-10 min, overflow, dissolved & 0.00030 & 0.00030 & 0.00030 & 0.00030 & 0.00030 & 0.00030 & 0.00030 \\
\hline Water & 80999 & \multirow{2}{*}{$\begin{array}{l}\text { 10-20 min, overflow, dissolved } \\
20-30 \mathrm{~min} \text { overflow, dissolved }\end{array}$} & 0.00030 & 0.00030 & 0.00030 & 0.00030 & 0.00030 & 0.00030 & 0.00030 \\
\hline Water & 81000 & & 0.00030 & 0.00030 & 0.00030 & 0.00030 & 0.00030 & 0.00030 & 0.00030 \\
\hline Water & 80963 & & 0.00030 & 0.00030 & 0.00030 & 0.00030 & 0.00030 & 0.00030 & 0.00030 \\
\hline Water & 80964 & 10-20 min, overflow, total & 0.00030 & 0.00030 & 0.00030 & 0.00030 & 0.00030 & 0.00030 & 0.00030 \\
\hline Water & 80965 & 20-30 min, overflow, total & 0.00030 & 0.00030 & 0.00030 & 0.00030 & 0.00030 & 0.00030 & 0.00030 \\
\hline Water & 81001 & 0-10 min, non-overflow, dissolved & 0.00030 & 0.00030 & 0.00030 & 0.00030 & 0.00030 & 0.00030 & 0.00030 \\
\hline Water & 81002 & $10-20 \mathrm{~min}$, non-overflow, dissolved & 0.00030 & 0.00030 & 0.00030 & 0.00030 & 0.00030 & 0.00030 & 0.00030 \\
\hline Water & 81003 & 20-30 min, non-overflow, dissolved & 0.00030 & 0.00030 & 0.00030 & 0.00030 & 0.00030 & 0.00030 & 0.00030 \\
\hline Water & 80966 & \multirow{2}{*}{ 0-10 min, non-overflow, total } & 0.00030 & 0.00030 & 0.00030 & 0.00030 & 0.00030 & 0.00030 & 0.00030 \\
\hline Water & 80967 & & 0.00030 & 0.00030 & 0.00030 & 0.00030 & 0.00030 & 0.00030 & 0.00030 \\
\hline Water & 80968 & $\begin{array}{l}\text { 10-20 min, non-overflow, total } \\
20-30 \mathrm{~min} \text {, non-overflow, total }\end{array}$ & 0.00030 & 0.00030 & 0.00030 & 0.00030 & 0.00030 & 0.00030 & 0.00030 \\
\hline & & Hopper Inflow Monitoring & & & & & & & \\
\hline Water & 81124 & $3 \& 6 \mathrm{~min}$, dissolved & 0.00030 & 0.00030 & 0.00030 & 0.00030 & 0.00030 & 0.00030 & 0.00030 \\
\hline Water & 81125 & $9 \& 12 \mathrm{~min}$, dissolved & 0.00030 & 0.00030 & 0.00030 & 0.00030 & 0.00030 & 0.00030 & 0.00030 \\
\hline Water & 81126 & $15 \& 18 \mathrm{~min}$, dissolved & 0.00030 & 0.00030 & 0.00030 & 0.00030 & 0.00030 & 0.00030 & 0.00030 \\
\hline Water & 81127 & $21 \& 24 \mathrm{~min}$, dissolved & 0.00030 & 0.00030 & 0.00030 & 0.00030 & 0.00030 & 0.00030 & 0.00030 \\
\hline Water & 81128 & $27 \& 30 \mathrm{~min}$, dissolved & 0.00030 & 0.00030 & 0.00030 & 0.00030 & 0.00030 & 0.00030 & 0.00030 \\
\hline Water & 80915 & $3 \& 6 \mathrm{~min}$, total & 0.00043 & 0.00030 & 0.00030 & 0.00014 & 0.00054 & 0.00031 & 0.00221 \\
\hline Water & 80916 & $9 \& 12 \mathrm{~min}$, total & 0.00110 & 0.00030 & 0.00029 & 0.00057 & 0.00349 & 0.00103 & 0.00758 \\
\hline Water & 80917 & $15818 \mathrm{~min}$, total & 0.00057 & 0.00030 & 0.00017 & 0.00036 & 0.00217 & 0.00061 & 0.00484 \\
\hline Water & 80918 & $21 \& 24 \mathrm{~min}$, total & 0.00053 & 0.00030 & 0.00012 & 0.00023 & 0.00158 & 0.00048 & 0.00370 \\
\hline Water & 80919 & $27 \& 30 \mathrm{~min}$, total & 0.00183 & 0.00015 & 0.00047 & 0.00085 & 0.00582 & 0.00183 & 0.01320 \\
\hline & & Hopper Overflow Monitoring & & & & & & & \\
\hline Water & 81129 & 28 4 min, dissolved & 0.00030 & 0.00030 & 0.00030 & 0.00030 & 0.00030 & 0.00030 & 0.00030 \\
\hline Water & 81130 & 6\& $8 \mathrm{~min}$, dissolved & 0.00030 & 0.00030 & 0.00030 & 0.00030 & 0.00030 & 0.00030 & 0.00030 \\
\hline Water & 81131 & $10 \& 12 \mathrm{~min}$, dissolved & 0.00030 & 0.00030 & 0.00030 & 0.00030 & 0.00030 & 0.00030 & 0.00030 \\
\hline Water & 81132 & $14 \& 16 \mathrm{~min}$, dissolved & 0.00030 & 0.00030 & 0.00030 & 0.00030 & 0.00030 & 0.00030 & 0.00030 \\
\hline Water & 81133 & $18 \& 20 \mathrm{~min}$, dissolved & 0.00030 & 0.00030 & 0.00030 & 0.00030 & 0.00030 & 0.00030 & 0.00030 \\
\hline Water & 80921 & 2\& $4 \mathrm{~min}$, total & 0.00120 & 0.00011 & 0.00031 & 0.00051 & 0.00377 & 0.00104 & 0.00900 \\
\hline Water & 80922 & 6\& $8 \mathrm{~min}$, total & 0.00121 & 0.00012 & 0.00042 & 0.00068 & 0.00529 & 0.00311 & 0.01260 \\
\hline Water & 80923 & $10 \& 12 \mathrm{~min}$, total & 0.00062 & 0.00030 & 0.00020 & 0.00054 & 0.00325 & 0.00083 & 0.00838 \\
\hline Water & 80924 & $14 \& 16 \mathrm{~min}$, total & 0.00052 & 0.00030 & 0.00015 & 0.00047 & 0.00267 & 0.00074 & 0.00657 \\
\hline Water & 80925 & $18 \& 20 \mathrm{~min}$, total & 0.00257 & 0.00023 & 0.00073 & 0.00124 & 0.00923 & 0.00259 & 0.02030 \\
\hline & & Site Water & & & & & & & \\
\hline Water & 81639 & Sample 1 Total & 0.00030 & 0.00030 & 0.00030 & 0.00030 & 0.00030 & 0.00030 & 0.00030 \\
\hline Water & 81640 & Sample 2 Total & 0.00030 & 0.00030 & 0.00030 & 0.00030 & 0.00030 & 0.00030 & 0.00030 \\
\hline Water & 81641 & Sample 3 Total & 0.00030 & 0.00030 & 0.00030 & 0.00030 & 0.00030 & 0.00030 & 0.00030 \\
\hline & & Elutriate & & & & & & & \\
\hline Water & 81645 & Sample 1 Dissolved & 0.00030 & 0.00030 & 0.00030 & 0.00030 & 0.00030 & 0.00030 & 0.00030 \\
\hline Water & 81646 & Sample 2 Dissolved & 0.00030 & 0.00030 & 0.00030 & 0.00030 & 0.00030 & 0.00030 & 0.00030 \\
\hline Water & 81647 & Sample 3 Dissolved & 0.00030 & 0.00030 & 0.00030 & 0.00030 & 0.00030 & 0.00030 & 0.00030 \\
\hline Water & 81642 & Sample 1 Total & 0.00030 & 0.00030 & 0.00030 & 0.00030 & 0.00030 & 0.00030 & 0.00012 \\
\hline Water & 81643 & Sample 2 Total & 0.00030 & 0.00030 & 0.00030 & 0.00030 & 0.00030 & 0.00030 & 0.00011 \\
\hline Water & 81644 & Sample 3 Total & 0.00030 & 0.00030 & 0.00030 & 0.00030 & 0.00030 & 0.00030 & 0.00010 \\
\hline $\begin{array}{l}\text { SAMPLE } \\
\text { TYPE }\end{array}$ & SAMPLE & DESCRIPTION & NAPHTH & ACENAY & ACENAP & FLUORE & PHENAN & ANTRAC & FLANTHE \\
\hline & & Detection Limit (mg/kg) & 0.0220 & 0.022 & 0.022 & 0.022 & 0.0220 & 0.0220 & 0.022 \\
\hline & & Insitu Sediment & & & & & & & \\
\hline Sediment & 81705 & Sample \#1 & 0.0640 & 0.022 & 0.022 & 0.015 & 0.0921 & 0.0367 & 0.188 \\
\hline Sediment & 81706 & Sample \#2 & 0.0591 & 0.022 & 0.022 & 0.014 & 0.0800 & 0.0303 & 0.126 \\
\hline Sediment & 81707 & Sample \#3 & 0.0581 & 0.022 & 0.022 & 0.015 & 0.0828 & 0.0327 & 0.136 \\
\hline $\begin{array}{l}\text { NAPHTH - } \\
\text { ANTRAC- } \\
\text { BOLD - les }\end{array}$ & $\begin{array}{l}\text { Naphthalene } \\
\text { Anthracene } \\
\text { is than value }\end{array}$ & $\begin{array}{l}\text { ACENAY - Acenaphthylene } \\
\text { FLANTHE - Fluoranthene }\end{array}$ & ACENAP & enaphthene & FLUORE & luorene & PHENAN - Ph & anthrene & \\
\hline
\end{tabular}

Page 1 
PAHsfine

Delaware River Water Analysis (Fine-Grained Site)

\begin{tabular}{|c|c|c|c|c|c|c|c|c|c|}
\hline \multirow[t]{2}{*}{$\begin{array}{l}\text { SAMPLE } \\
\text { TYPE }\end{array}$} & $\begin{array}{l}\text { SAMPLE } \\
\text { ID }\end{array}$ & DESCRIPTION & PYRENE & CHRYSE & BAANTHR & BBFLANT & BKFLANT & BAPYRE & I123PYR \\
\hline & & Detection Limit (mg/l) & 0.00030 & 0.00030 & 0.00030 & 0.00030 & 0.00030 & 0.00030 & 0.00030 \\
\hline & & Plume Monitoring & & & & & & & \\
\hline Water & 80997 & Background, dissolved & 0.00030 & 0.00030 & 0.00030 & 0.00030 & 0.00030 & 0.00030 & 0.00030 \\
\hline Water & 80962 & Background, total & 0.00030 & 0.00030 & 0.00030 & 0.00030 & 0.00030 & 0.00030 & 0.00030 \\
\hline Water & 80998 & $0.10 \mathrm{~min}$, overflow, dissolved & 0.00030 & 0.00030 & 0.00030 & 0.00030 & 0.00030 & 0.00030 & 0.00030 \\
\hline Water & 80999 & 10-20 min, overflow, dissolved & 0.00030 & 0.00030 & 0.00030 & 0.00030 & 0.00030 & 0.00030 & 0.00030 \\
\hline Water & 81000 & 20-30 min, overflow, dissolved & 0.00030 & 0.00030 & 0.00030 & 0.00030 & 0.00030 & 0.00030 & 0.00030 \\
\hline Water & 80963 & 0-10 min, overflow, total & 0.00030 & 0.00030 & 0.00030 & 0.00030 & 0.00030 & 0.00030 & 0.00030 \\
\hline Water & 80964 & 10-20 min, overflow, total & 0.00030 & 0.00030 & 0.00030 & 0.00030 & 0.00030 & 0.00030 & 0.00030 \\
\hline Water & 80965 & 20-30 min, overflow, total & 0.00030 & 0.00030 & 0.00030 & 0.00030 & 0.00030 & 0.00030 & 0.00030 \\
\hline Water & 81001 & 0-10 min, non-overflow, dissolved & 0.00030 & 0.00030 & 0.00030 & 0.00030 & 0.00030 & 0.00030 & 0.00030 \\
\hline Water & 81002 & 10-20 min, non-overflow, dissolved & 0.00030 & 0.00030 & 0.00030 & 0.00030 & 0.00030 & 0.00030 & 0.00030 \\
\hline Water & 81003 & 20-30 min, non-overflow, dissolved & 0.00030 & 0.00030 & 0.00030 & 0.00030 & 0.00030 & 0.00030 & 0.00030 \\
\hline Water & 80966 & 0-10 min, non-overflow, total & 0.00030 & 0.00030 & 0.00030 & 0.00030 & 0.00030 & 0.00030 & 0.00030 \\
\hline Water & 80967 & 10-20 min, non-overflow, total & 0.00030 & 0.00030 & 0.00030 & 0.00030 & 0.00030 & 0.00030 & 0.00030 \\
\hline \multirow[t]{2}{*}{ Water } & 80968 & 20-30 min, non-overflow, total & 0.00030 & 0.00030 & 0.00030 & 0.00030 & 0.00030 & 0.00030 & 0.00030 \\
\hline & & Hopper Inflow Monitoring & & & & & & & \\
\hline Water & 81124 & $3 \& 6 \mathrm{~min}$, dissolved & 0.00030 & 0.00030 & 0.00030 & 0.00030 & 0.00030 & 0.00030 & 0.00030 \\
\hline Water & 81125 & $9 \& 12 \mathrm{~min}$, dissolved & 0.00030 & 0.00030 & 0.00030 & 0.00030 & 0.00030 & 0.00030 & 0.00030 \\
\hline Water & 81126 & $15 \& 18 \mathrm{~min}$, dissolved & 0.00030 & 0.00030 & 0.00030 & 0.00030 & 0.00030 & 0.00030 & 0.00030 \\
\hline Water & 81127 & $21824 \mathrm{~min}$, dissolved & 0.00030 & 0.00030 & 0.00030 & 0.00030 & 0.00030 & 0.00030 & 0.00030 \\
\hline Water & 81128 & $27 \& 30 \mathrm{~min}$, dissolved & 0.00030 & 0.00030 & 0.00030 & 0.00030 & 0.00030 & 0.00030 & 0.00030 \\
\hline Water & 80915 & $3 \& 6 \mathrm{~min}$, total & 0.00227 & 0.00159 & 0.00118 & 0.00177 & 0.00120 & 0.00088 & 0.00309 \\
\hline Water & 80916 & $9 \& 12 \mathrm{~min}$, total & 0.00782 & 0.00547 & 0.00451 & 0.00492 & 0.00385 & 0.00519 & 0.00549 \\
\hline Water & 80917 & $15 \& 18 \mathrm{~min}$, total & 0.00465 & 0.00341 & 0.00276 & 0.00371 & 0.00227 & 0.00360 & 0.00436 \\
\hline Water & 80918 & $21824 \mathrm{~min}$, total & 0.00364 & 0.00256 & 0.00204 & 0.00236 & 0.00183 & 0.00246 & 0.00255 \\
\hline \multirow[t]{2}{*}{ Water } & 80919 & $27 \& 30 \mathrm{~min}$, total & 0.01400 & 0.00948 & 0.00841 & 0.00785 & 0.00629 & 0.00838 & 0.00701 \\
\hline & & Hopper Overflow Monitoring & & & & & & & \\
\hline Water & 81129 & 2\& $4 \mathrm{~min}$, dissolved & 0.00030 & 0.00030 & 0.00030 & 0.00030 & 0.00030 & 0.00030 & 0.00030 \\
\hline Water & 81130 & 6\& $8 \mathrm{~min}$, dissolved & 0.00030 & 0.00030 & 0.00030 & 0.00030 & 0.00030 & 0.00030 & 0.00030 \\
\hline Water & 81131 & $10 \& 12 \mathrm{~min}$, dissolved & 0.00030 & 0.00030 & 0.00030 & 0.00030 & 0.00030 & 0.00030 & 0.00030 \\
\hline Water & 81132 & $14 \& 16 \mathrm{~min}$, dissolved & 0.00030 & 0.00030 & 0.00030 & 0.00030 & 0.00030 & 0.00030 & 0.00030 \\
\hline Water & 81133 & $18 \& 20 \mathrm{~min}$, dissolved & 0.00030 & 0.00030 & 0.00030 & 0.00030 & 0.00030 & 0.00030 & 0.00030 \\
\hline Water & 80921 & 2\& 4 min, total & 0.00903 & 0.00642 & 0.00576 & 0.00548 & 0.00433 & 0.00614 & 0.00542 \\
\hline Water & 80922 & $6 \& 8 \mathrm{~min}$, total & 0.01270 & 0.00907 & 0.00868 & 0.00823 & 0.00614 & 0.00838 & 0.00741 \\
\hline Water & 80923 & $10 \& 12 \mathrm{~min}$, total & 0.00711 & 0.00643 & 0.00543 & 0.00653 & 0.00438 & 0.00596 & 0.00579 \\
\hline Water & 80924 & $14 \& 16 \mathrm{~min}$, total & 0.00611 & 0.00566 & 0.00472 & 0.00555 & 0.00437 & 0.00019 & 0.00547 \\
\hline \multirow[t]{2}{*}{ Water } & 80925 & $18 \& 20 \mathrm{~min}$, total & 0.02000 & 0.01380 & 0.01290 & 0.01160 & 0.00953 & 0.01220 & 0.01050 \\
\hline & & Site Water & & & & & & & \\
\hline Water & 81639 & Sample 1 Total & 0.00030 & 0.00030 & 0.00030 & 0.00030 & 0.00030 & 0.00030 & 0.00030 \\
\hline Water & 81640 & Sample 2 Total & 0.00030 & 0.00030 & 0.00030 & 0.00030 & 0.00030 & 0.00030 & 0.00030 \\
\hline \multirow[t]{2}{*}{ Water } & 81641 & Sample 3 Total & 0.00030 & 0.00030 & 0.00030 & 0.00030 & 0.00030 & 0.00030 & 0.00030 \\
\hline & & Elutriate & & & & & & & \\
\hline Water & 81645 & Sample 1 Dissolved & 0.00030 & 0.00030 & 0.00030 & 0.00030 & 0.00030 & 0.00030 & 0.00030 \\
\hline Water & 81646 & Sample 2 Dissolved & 0.00030 & 0.00030 & 0.00030 & 0.00030 & 0.00030 & 0.00030 & 0.00030 \\
\hline Water & 81647 & Sample 3 Dissolved & 0.00030 & 0.00030 & 0.00030 & 0.00030 & 0.00030 & 0.00030 & 0.00030 \\
\hline Water & 81642 & Sample 1 Total & 0.00010 & 0.00030 & 0.00030 & 0.00030 & 0.00030 & 0.00030 & 0.00030 \\
\hline Water & 81643 & Sample 2 Total & 0.00010 & 0.00030 & 0.00030 & 0.00030 & 0.00030 & 0.00030 & 0.00030 \\
\hline Water & 81644 & Sample 3 Total & 0.00009 & 0.00030 & 0.00030 & 0.00030 & 0.00030 & 0.00030 & 0.00030 \\
\hline \multirow{3}{*}{$\begin{array}{l}\text { SAMPLE } \\
\text { TYPE }\end{array}$} & $\begin{array}{l}\text { SAMPLE } \\
\text { ID }\end{array}$ & DESCRIPTION & PYRENE & CHRYSE & BAANTHR & BBFLANT & BKFLANT & BAPYRE & I123PYR \\
\hline & & Detection Limit (mg/kg) & 0.022 & 0.022 & 0.0220 & 0.0220 & 0.0220 & 0.0220 & 0.0220 \\
\hline & & Insitu Sediment & & & & & & & \\
\hline Sediment & 81705 & Sample \#1 & 0.196 & 0.145 & 0.1290 & 0.0871 & 0.1090 & 0.1120 & 0.0899 \\
\hline Sediment & 81706 & Sample \#2 & 0.146 & 0.107 & 0.0865 & 0.0742 & 0.0850 & 0.0793 & 0.0699 \\
\hline Sediment & 81707 & Sample \#3 & 0.150 & 0.108 & 0.0858 & 0.0770 & 0.0727 & 0.0828 & 0.0749 \\
\hline PYRENE - & Pyrene & CHRYSE - Chrysene & Benxo(a)A & iracene & BBFLANT - E & b)Fluoran & & & \\
\hline $\begin{array}{l}\text { BKFLANT } \\
\text { BOLD - les }\end{array}$ & $\begin{array}{l}\text { Benzol } \\
\text { s than ve }\end{array}$ & $\begin{array}{l}\text { uoranthene } \\
\text { s }\end{array}$ & & & 11 200 & & & & \\
\hline
\end{tabular}

Page 2 
PAHsfine

Delaware River Water Analysis (Plume Monitoring)

\begin{tabular}{|c|c|c|c|c|c|c|c|}
\hline \multirow[t]{2}{*}{$\begin{array}{l}\text { SAMPLE } \\
\text { TYPE }\end{array}$} & $\begin{array}{l}\text { SAMPLE } \\
\text { ID }\end{array}$ & DESCRIPTION & DBAHANT & B-GHI-PY & 2MeNAPH & 2FIBP-S & PTERP-S \\
\hline & & Detection Limit (mg/) & 0.00030 & 0.00030 & 0.00030 & & \\
\hline & & Plume Monitoring & & & & & \\
\hline Water & 80997 & Background, dissolved & 0.00030 & 0.00030 & 0.00030 & $56.0 \%$ & $84.1 \%$ \\
\hline Water & 80962 & Background, total & 0.00030 & 0.00030 & 0.00030 & $73.2 \%$ & $85.1 \%$ \\
\hline Water & 80998 & 0-10 min, overflow, dissolved & 0.00030 & 0.00030 & 0.00030 & $64.7 \%$ & $87.3 \%$ \\
\hline Water & 80999 & 10-20 min, overflow, dissolved & 0.00030 & 0.00030 & 0.00030 & $83.0 \%$ & $87.8 \%$ \\
\hline Water & 81000 & 20-30 min, overflow, dissolved & 0.00030 & 0.00030 & 0.00030 & $65.1 \%$ & $81.5 \%$ \\
\hline Water & 80963 & $0-10 \mathrm{~min}$, overflow, total & 0.00030 & 0.00030 & 0.00030 & $63.2 \%$ & $79.3 \%$ \\
\hline Water & 80964 & 10-20 min, overflow, total & 0.00030 & 0.00030 & 0.00030 & $63.4 \%$ & $85.5 \%$ \\
\hline Water & 80965 & 20-30 min, overflow, total & 0.00030 & 0.00030 & 0.00030 & $58.7 \%$ & $88.4 \%$ \\
\hline Water & 81001 & 0-10 min, non-overflow, dissolved & 0.00030 & 0.00030 & 0.00030 & $69.4 \%$ & $84.7 \%$ \\
\hline Water & 81002 & 10-20 min, non-overflow, dissolved & 0.00030 & 0.00030 & 0.00030 & $63.4 \%$ & $87.5 \%$ \\
\hline Water & 81003 & 20-30 min, non-overflow, dissolved & 0.00030 & 0.00030 & 0.00030 & $61.6 \%$ & $86.0 \%$ \\
\hline Water & 80966 & 0-10 min, non-overflow, total & 0.00030 & 0.00030 & 0.00030 & $66.5 \%$ & $91.6 \%$ \\
\hline Water & 80967 & 10-20 min, non-overflow, total & 0.00030 & 0.00030 & 0.00030 & $68.1 \%$ & $88.6 \%$ \\
\hline \multirow[t]{2}{*}{ Water } & 80968 & 20-30 min, non-overflow, total & 0.00030 & 0.00030 & 0.00030 & $87.1 \%$ & $90.5 \%$ \\
\hline & & Hopper Inflow Monitoring & & & & & \\
\hline Water & 81124 & $3 \& 6 \mathrm{~min}$, dissolved & 0.00030 & 0.00030 & 0.00030 & $72.4 \%$ & $85.9 \%$ \\
\hline Water & 81125 & $9 \& 12 \mathrm{~min}$, dissolved & 0.00030 & 0.00030 & 0.00030 & $43.8 \%$ & $80.7 \%$ \\
\hline Water & 81126 & $15 \& 18 \mathrm{~min}$, dissolved & 0.00030 & 0.00030 & 0.00030 & $51.7 \%$ & $80.2 \%$ \\
\hline Water & 81127 & $21824 \mathrm{~min}$ dissolved & 0.00030 & 0.00030 & 0.00030 & $62.9 \%$ & $88.0 \%$ \\
\hline Water & 81128 & $27 \& 30 \mathrm{~min}$, dissolved & 0.00030 & 0.00030 & 0.00030 & $62.0 \%$ & $83.8 \%$ \\
\hline Water & 80915 & $3 \& 6 \mathrm{~min}$, total & 0.00019 & 0.00167 & 0.00029 & $58.2 \%$ & $48.6 \%$ \\
\hline Water & 80916 & $9 \& 12 \mathrm{~min}$, total & 0.00231 & 0.00424 & 0.00068 & $68.6 \%$ & $60.4 \%$ \\
\hline Water & 80917 & $15 \& 18 \mathrm{~min}$, total & 0.00217 & 0.00312 & 0.00038 & $64.5 \%$ & $60.7 \%$ \\
\hline Water & 80918 & $21 \& 24 \mathrm{~min}$, total & 0.00111 & 0.00197 & 0.00035 & $57.9 \%$ & $51.3 \%$ \\
\hline \multirow[t]{2}{*}{ Water } & 80919 & $27 \& 30 \mathrm{~min}$, total & 0.00169 & 0.00629 & 0.00116 & $67.5 \%$ & $53.6 \%$ \\
\hline & & Hopper Overflow Monitoring & & & & & \\
\hline Water & 81129 & $284 \mathrm{~min}$, dissolved & 0.00030 & 0.00030 & 0.00030 & $62.8 \%$ & $83.2 \%$ \\
\hline Water & 81130 & 6\& $8 \mathrm{~min}$ dissolved & 0.00030 & 0.00030 & 0.00030 & $76.4 \%$ & $82.0 \%$ \\
\hline Water & 81131 & $10 \& 12 \mathrm{~min}$, dissolved & 0.00030 & 0.00030 & 0.00030 & $47.2 \%$ & $65.7 \%$ \\
\hline Water & 81132 & $14 \& 16 \mathrm{~min}$, dissolved & 0.00030 & 0.00030 & 0.00030 & $70.7 \%$ & $71.0 \%$ \\
\hline Water & 81133 & $18820 \mathrm{~min}$, dissolved & 0.00030 & 0.00030 & 0.00030 & $46.2 \%$ & $66.7 \%$ \\
\hline Water & 80921 & 2\& 4 min, total & 0.00153 & 0.00460 & 0.00073 & $69.1 \%$ & $58.1 \%$ \\
\hline Water & 80922 & 6\& 8 min, total & 0.00204 & 0.00607 & 0.00076 & $67.1 \%$ & $62.3 \%$ \\
\hline Water & 80923 & $10 \& 12 \mathrm{~min}$, total & 0.00175 & 0.00473 & 0.00037 & $41.5 \%$ & $59.6 \%$ \\
\hline Water & 80924 & $14 \& 16 \mathrm{~min}$, total & 0.00165 & 0.00431 & 0.00030 & $36.4 \%$ & $61.5 \%$ \\
\hline \multirow[t]{2}{*}{ Water } & 80925 & $18 \& 20 \mathrm{~min}$, total & 0.00240 & 0.00883 & 0.00163 & $66.5 \%$ & $61.5 \%$ \\
\hline & & Site Water & & & & & \\
\hline Water & 81639 & Sample 1 Total & 0.00030 & 0.00030 & 0.00030 & $50.6 \%$ & $61.9 \%$ \\
\hline Water & 81640 & Sample 2 Total & 0.00030 & 0.00030 & 0.00030 & $60.2 \%$ & $62.4 \%$ \\
\hline \multirow[t]{2}{*}{ Water } & 81641 & Sample 3 Total & 0.00030 & 0.00030 & 0.00030 & $46.3 \%$ & $66.8 \%$ \\
\hline & & Elutriate & & & & & \\
\hline Water & 81645 & Sample 1 Dissolved & 0.00030 & 0.00030 & 0.00030 & $43.3 \%$ & $67.8 \%$ \\
\hline Water & 81646 & Sample 2 Dissolved & 0.00030 & 0.00030 & 0.00030 & $83.7 \%$ & $56.1 \%$ \\
\hline Water & 81647 & Sample 3 Dissolved & 0.00030 & 0.00030 & 0.00030 & $28.2 \%$ & $58.3 \%$ \\
\hline Water & 81642 & Sample 1 Total & 0.00030 & 0.00030 & 0.00030 & $62.7 \%$ & $64.0 \%$ \\
\hline Water & 81643 & Sample 2 Total & 0.00030 & 0.00030 & 0.00030 & $56.7 \%$ & $71.0 \%$ \\
\hline Water & 81644 & Sample 3 Total & 0.00030 & 0.00030 & 0.00030 & $65.9 \%$ & $71.2 \%$ \\
\hline \multirow{3}{*}{$\begin{array}{l}\text { SAMPLE } \\
\text { TYPE }\end{array}$} & SAMPLE & DESCRIPTION & DBAHANT & B-GHI-PY & 2MeNAPH & 2FIBP-S & PTERP-S \\
\hline & & Detection Limit $(\mathrm{mg} / \mathrm{kg})$ & 0.0220 & 0.0220 & 0.0220 & & \\
\hline & & Insitu Sediment & & & & & \\
\hline Sediment & 81705 & Sample \#1 & 0.0086 & 0.0748 & 0.0353 & $60.3 \%$ & $48.6 \%$ \\
\hline Sediment & 81706 & Sample \#2 & 0.0072 & 0.0605 & 0.0324 & $630 \%$ & $49.1 \%$ \\
\hline Sediment & 81707 & Sample \#3 & 0.0087 & 0.0647 & 0.0342 & $61.2 \%$ & $51.1 \%$ \\
\hline & & & & & $\mathrm{PH}$ - 2-Methyl & thalene & \\
\hline 2FIBP-S - & 2-Fluorobi & enyl(Surrogate $(43-116$ W)) & RP-S-p-Terph & yl-D14(Surr & ate $(33-141$ & & \\
\hline BOLD-les & & & & & & & \\
\hline
\end{tabular}

Page 3 
Pesttine

Delaware River Water Analysis (Fine-Grained Site)

\begin{tabular}{|c|c|c|c|c|c|c|c|c|}
\hline \multirow[t]{2}{*}{$\begin{array}{l}\text { SAMPLE } \\
\text { TYPE }\end{array}$} & $\begin{array}{l}\text { SAMPLE } \\
\text { ID }\end{array}$ & DESCRIPTION & ALDRIN & $\mathrm{A} \cdot \mathrm{BHC}$ & B-BHC & G-BHC & D-BHC & PPDDD \\
\hline & & Detection Limit (mg/l) & 0.000028 & 0.000028 & 0.000028 & 0.000028 & 0.000028 & 0.000055 \\
\hline & & Plume Monitoring & & & & & & \\
\hline Water & 80990 & Background, dissolved & 0.000025 & 0.000025 & 0.000025 & 0.000025 & 0.000025 & 0.000050 \\
\hline Water & 80955 & Background, total & 0.000028 & 0.000028 & 0.000028 & 0.000028 & 0.000028 & 0.000055 \\
\hline Water & 80991 & 0-10 min, overflow, dissolved & 0.000025 & 0.000025 & 0.000025 & 0.000025 & 0.000025 & 0.000050 \\
\hline Water & 80992 & 10-20 min, overflow, dissolved & 0.000025 & 0.000025 & 0.000025 & 0.000025 & 0.000025 & 0.000050 \\
\hline Water & 80993 & 20-30 min, overflow, dissolved & 0.000025 & 0.000025 & 0.000025 & 0.000025 & 0.000025 & 0.000050 \\
\hline Water & 80956 & 0-10 min, overflow, total & 0.000025 & 0.000025 & 0.000025 & 0.000025 & 0.000025 & 0.000050 \\
\hline Water & 80957 & 10-20 min, overflow, total & 0.000025 & 0.000025 & 0.000025 & 0.000025 & 0.000025 & 0.000050 \\
\hline Water & 80958 & 20-30 min, overflow, total & 0.000025 & 0.000025 & 0.000025 & 0.000025 & 0.000025 & 0.000050 \\
\hline Water & 80994 & 0-10 min, non-overflow, dissolved & 0.000025 & 0.000025 & 0.000025 & 0.000025 & 0.000025 & 0.000050 \\
\hline Water & 80995 & 10-20 min, non-overflow, dissolved & 0.000025 & 0.000025 & 0.000025 & 0.000025 & 0.000025 & 0.000050 \\
\hline Water & 80996 & 20-30 min, non-overflow, dissolved & 0.000025 & 0.000025 & 0.000025 & 0.000025 & 0.000025 & 0.000050 \\
\hline Water & 80959 & 0-10 min, non-overflow, total & 0.000025 & 0.000025 & 0.000025 & 0.000025 & 0.000025 & 0.000050 \\
\hline Water & 80960 & 10-20 min, non-overflow, total & 0.000025 & 0.000025 & 0.000025 & 0.000025 & 0.000025 & 0.000050 \\
\hline \multirow[t]{2}{*}{ Water } & 80961 & 20-30 $\mathrm{min}$, non-overflow, total & 0.000025 & 0.000025 & 0.000025 & 0.000025 & 0.000025 & 0.000050 \\
\hline & & Hopper Inflow Monitoring & & & & & & \\
\hline Water & 81114 & $3 \& 6 \mathrm{~min}$, dissolved & 0.000025 & 0.000025 & 0.000025 & 0.000025 & 0.000025 & 0.000050 \\
\hline Water & 81115 & $9 \& 12 \mathrm{~min}$, dissolved & 0.000026 & 0.000026 & 0.000026 & 0.000026 & 0.000026 & 0.000052 \\
\hline Water & 81116 & $15 \& 18 \mathrm{~min}$, dissolved & 0.000027 & 0.000027 & 0.000027 & 0.000027 & 0.000027 & 0.000054 \\
\hline Water & 81117 & $21 \& 24 \mathrm{~min}$, dissolved & 0.000025 & 0.000025 & 0.000025 & 0.000025 & 0.000025 & 0.000050 \\
\hline Water & 81118 & $27 \& 30 \mathrm{~min}$, dissolved & 0.000025 & 0.000025 & 0.000025 & 0.000025 & 0.000025 & 0.000050 \\
\hline Water & 80903 & $3 \& 6 \mathrm{~min}$, total & 0.000016 & 0.000025 & 0.000025 & 0.000025 & 0.000025 & 0.000120 \\
\hline Water & 80904 & $9 \& 12 \mathrm{~min}$, total & 0.000024 & 0.000025 & 0.000025 & 0.000009 & 0.000025 & 0.000160 \\
\hline Water & 80905 & $15 \& 18 \mathrm{~min}$, total & 0.000024 & 0.000027 & 0.000027 & 0.000027 & 0.000027 & 0.000200 \\
\hline Water & 80906 & $21824 \mathrm{~min}$, total & 0.000022 & 0.000027 & 0.000027 & 0.000014 & 0.000027 & 0.000130 \\
\hline \multirow[t]{2}{*}{ Water } & 80907 & $27 \& 30 \mathrm{~min}$, total & 0.000026 & 0.000025 & 0.000025 & 0.000025 & 0.000025 & 0.000130 \\
\hline & & Hopper Overflow Monitoring & & & & & & \\
\hline Water & 81119 & 2\& $4 \mathrm{~min}$, dissolved & 0.000026 & 0.000026 & 0.000026 & 0.000026 & 0.000026 & 0.000052 \\
\hline Water & 81120 & 6\& $8 \mathrm{~min}$, dissolved & 0.000025 & 0.000025 & 0.000025 & 0.000025 & 0.000025 & 0.000050 \\
\hline Water & 81121 & $10 \& 12 \mathrm{~min}$, dissolved & 0.000025 & 0.000025 & 0.000025 & 0.000025 & 0.000025 & 0.000050 \\
\hline Water & 81122 & $14 \& 16 \mathrm{~min}$, dissolved & 0.000025 & 0.000025 & 0.000025 & 0.000025 & 0.000025 & 0.000050 \\
\hline Water & 81123 & $18 \& 20 \mathrm{~min}$, dissolved & 0.000024 & 0.000024 & 0.000024 & 0.000024 & 0.000024 & 0.000049 \\
\hline Water & 80909 & 2\& $4 \mathrm{~min}$, total & 0.000030 & 0.000027 & 0.000027 & 0.000010 & 0.000027 & 0.000740 \\
\hline Water & 80910 & $6 \& 8 \mathrm{~min}$, total & 0.000045 & 0.000027 & 0.000027 & 0.000027 & 0.000027 & 0.000300 \\
\hline Water & 80911 & $10 \& 12 \mathrm{~min}$, total & Broken & Broken & Broken & Broken & Broken & Broken \\
\hline Water & 80912 & $14 \& 16 \mathrm{~min}$, total & 0.000062 & 0.000025 & 0.000025 & 0.000015 & 0.000025 & 0.000540 \\
\hline \multirow[t]{2}{*}{ Water } & 80913 & $18820 \mathrm{~min}$, total & 0.000043 & 0.000027 & 0.000027 & 0.000027 & 0.000027 & 0.000320 \\
\hline & & Site Water & & & & & & \\
\hline Water & 81621 & Sample 1 Total & 0.000025 & 0.000025 & 0.000025 & 0.000017 & 0.000025 & 0.000050 \\
\hline Water & 81622 & Sample 2 Total & 0.000025 & 0.000025 & 0.000025 & 0.000025 & 0.000025 & 0.000050 \\
\hline \multirow[t]{2}{*}{ Water } & 81623 & Sample 3 Total & 0.000025 & 0.000025 & 0.000025 & 0.000025 & 0.000025 & 0.000050 \\
\hline & & Elutriate & & & & & & \\
\hline Water & 81627 & Sample 1 Dissolved & 0.000050 & 0.000050 & 0.000050 & 0.000050 & 0.000050 & 0.000100 \\
\hline Water & 81628 & Sample 2 Dissolved & 0.000050 & 0.000050 & 0.000050 & 0.000050 & 0.000050 & 0.000100 \\
\hline Water & 81629 & Sample 3 Dissolved & 0.000050 & 0.000050 & 0.000050 & 0.000050 & 0.000050 & 0.000100 \\
\hline Water & 81624 & Sample 1 Total & 0.000025 & 0.000025 & 0.000025 & 0.000025 & 0.000025 & 0.000050 \\
\hline Water & 81625 & Sample 2 Total & 0.000025 & 0.000025 & 0.000025 & 0.000025 & 0.000025 & 0.000016 \\
\hline Water & 81626 & Sample 3 Total & 0.000025 & 0.000025 & 0.000025 & 0.000025 & 0.000025 & 0.000015 \\
\hline \multirow[t]{3}{*}{ SAMPLE } & SAMPLE & DESCRIPTION & ALDRIN & $\mathrm{A} \cdot \mathrm{BHC}$ & B-BHC & G-BHC & D-BHC & PPDDD \\
\hline & & Detection Limit (mg/kg) & 0.0018 & 0.0018 & 0.00096 & 0.0018 & 0.0018 & 0.0019 \\
\hline & & Insitu Sediment & & & & & & \\
\hline Sediment & 81711 & Sample \#1 & 0.0018 & 0.0018 & 0.00067 & 0.0018 & 0.0018 & 0.0063 \\
\hline Sediment & 81712 & Sample \#2 & 0.0018 & 0.0018 & 0.00062 & 0.0018 & 0.0018 & 0.0054 \\
\hline Sediment & 81713 & Sample \#3 & 0.0018 & 0.0018 & 0.00092 & 0.0018 & 0.0018 & 0.0210 \\
\hline
\end{tabular}

ALDRIN-Aldrin A-BHC-A-BHC B-BHC-B-BHC G-BHC-G-BHC D-BHC-D-BHC PPDDD-PPDDD $B O L D$ - less than values

Values below less than values are estimated results. Results are less than the reporting limit. 
Pestfine

Delaware River Water Analysis (Fine-Grained Site)

\begin{tabular}{|c|c|c|c|c|c|c|c|c|}
\hline \multirow{2}{*}{$\begin{array}{l}\text { SAMPLE } \\
\text { TYPE }\end{array}$} & & DESCRIPTION & PPDDE & PPDDT & HPTCL & DIELDRIN & ENDOI & ENDOII \\
\hline & & Detection Limit (mg/l) & 0.000055 & 0.000055 & 0.0000280 & 0.000055 & 0.000028 & 0.000055 \\
\hline & & Plume Monitoring & & & & & & \\
\hline Water & 80990 & Background, dissolved & 0.000050 & 0.000050 & 0.0000250 & 0.000050 & 0.000025 & 0.000050 \\
\hline Water & 80955 & Background, total & 0.000055 & 0.000055 & 0.0000280 & 0.000055 & 0.000028 & 0.000055 \\
\hline Water & 80991 & 0-10 min, overflow, dissolved & 0.000050 & 0.000050 & 0.0000250 & 0.000050 & 0.000025 & 0.000050 \\
\hline Water & 80992 & 10-20 min, overflow, dissolved & 0.000050 & 0.000050 & 0.0000250 & 0.000050 & 0.000025 & 0.000050 \\
\hline Water & 80993 & 20-30 min, overflow, dissolved & 0.000050 & 0.000050 & 0.0000250 & 0.000050 & 0.000025 & 0.000050 \\
\hline Water & 80956 & $0-10 \mathrm{~min}$, overflow, total & 0.000050 & 0.000050 & 0.0000250 & 0.000050 & 0.000025 & 0.000050 \\
\hline Water & 80957 & 10-20 min, overflow, total & 0.000050 & 0.000050 & 0.0000250 & 0.000050 & 0.000025 & 0.000050 \\
\hline Water & 80958 & $20-30 \mathrm{~min}$, overflow, total & 0.000050 & 0.000050 & 0.0000250 & 0.000050 & 0.000025 & 0.000050 \\
\hline Water & 80994 & 0-10 min, non-overflow, dissolved & 0.000050 & 0.000050 & 0.0000250 & 0.000050 & 0.000025 & 0.000050 \\
\hline Water & 80995 & $10-20 \mathrm{~min}$, non-overflow, dissolved & 0.000050 & 0.000050 & 0.0000250 & 0.000050 & 0.000025 & 0.000050 \\
\hline Water & 80996 & 20-30 min, non-overflow, dissolved & 0.000050 & 0.000050 & 0.0000250 & 0.000050 & 0.000025 & 0.000050 \\
\hline Water & 80959 & 0-10 min, non-overflow, total & 0.000050 & 0.000050 & 0.0000250 & 0.000050 & 0.000025 & 0.000050 \\
\hline Water & 80960 & 10-20 min, non-overflow, total & 0.000050 & 0.000050 & 0.0000250 & 0.000050 & 0.000025 & 0.000050 \\
\hline \multirow[t]{2}{*}{ Water } & 80961 & 20-30 min, non-overflow, total & 0.000050 & 0.000050 & 0.0000250 & 0.000050 & 0.000025 & 0.000050 \\
\hline & & Hopper Inflow Monitoring & & & & & & \\
\hline Water & 81114 & 3\& $6 \mathrm{~min}$, dissolved & 0.000050 & 0.000050 & 0.0000250 & 0.000050 & 0.000025 & 0.000050 \\
\hline Water & 81115 & $9 \& 12 \mathrm{~min}$, dissolved & 0.000052 & 0.000052 & 0.0000260 & 0.000052 & 0.000026 & 0.000052 \\
\hline Water & 81116 & $15 \& 18 \mathrm{~min}$, dissolved & 0.000054 & 0.000054 & 0.0000270 & 0.000054 & 0.000027 & 0.000054 \\
\hline Water & 81117 & $21 \& 24 \mathrm{~min}$, dissolved & 0.000050 & 0.000050 & 0.0000250 & 0.000050 & 0.000025 & 0.000050 \\
\hline Water & 81118 & $27830 \mathrm{~min}$, dissolved & 0.000050 & 0.000050 & 0.0000250 & 0.000050 & 0.000025 & 0.000050 \\
\hline Water & 80903 & $3 \& 6 \mathrm{~min}$, total & 0.000079 & 0.000050 & 0.0000250 & 0.000050 & 0.000025 & 0.000050 \\
\hline Water & 80904 & $9 \& 12 \mathrm{~min}$, total & 0.000110 & 0.000082 & 0.0000130 & 0.000050 & 0.000029 & 0.000050 \\
\hline Water & 80905 & $15 \& 18 \mathrm{~min}$, total & 0.000150 & 0.000050 & 0.0000130 & 0.000053 & 0.000033 & 0.000053 \\
\hline Water & 80906 & $21 \& 24 \mathrm{~min}$, total & 0.000120 & 0.000075 & 0.0000207 & 0.000053 & 0.000032 & 0.000053 \\
\hline \multirow[t]{2}{*}{ Water } & 80907 & $27830 \mathrm{~min}$, total & 0.000110 & 0.000068 & 0.0000290 & 0.000050 & 0.000032 & 0.000050 \\
\hline & & Hopper Overflow Monitoring & & & & & & \\
\hline Water & 81119 & $2 \& 4 \mathrm{~min}$, dissolved & 0.000052 & 0.000052 & 0.0000260 & 0.000052 & 0.000026 & 0.000052 \\
\hline Water & 81120 & 6\& $8 \mathrm{~min}$, dissolved & 0.000050 & 0.000066 & 0.0000250 & 0.000050 & 0.000025 & 0.000050 \\
\hline Water & 81121 & $10 \& 12 \mathrm{~min}$, dissolved & 0.000050 & 0.000069 & 0.0000250 & 0.000050 & 0.000025 & 0.000050 \\
\hline Water & 81122 & $14816 \mathrm{~min}$, dissolved & 0.000050 & 0.000065 & 0.0000250 & 0.000050 & 0.000025 & 0.000050 \\
\hline Water & 81123 & $18 \& 20 \mathrm{~min}$, dissolved & 0.000049 & 0.000049 & 0.0000240 & 0.000049 & 0.000024 & 0.000049 \\
\hline Water & 80909 & $2 \& 4 \mathrm{~min}$, total & 0.000190 & 0.000110 & 0.0000170 & 0.000053 & 0.000017 & 0.000053 \\
\hline Water & 80910 & 6\& $8 \mathrm{~min}$, total & 0.000180 & 0.000360 & 0.0000270 & 0.000053 & 0.000050 & 0.000053 \\
\hline Water & 80911 & $10 \& 12 \mathrm{~min}$, total & Broken & Broken & Broken & Broken & Broken & Broken \\
\hline Water & 80912 & $14 \& 16 \mathrm{~min}$, total & 0.000470 & 0.000340 & 0.0000280 & 0.000050 & 0.000033 & 0.000050 \\
\hline \multirow[t]{2}{*}{ Water } & 80913 & $18 \& 20 \mathrm{~min}$, total & 0.000300 & 0.000140 & 0.0000270 & 0.000053 & 0.000020 & 0.000053 \\
\hline & & Site Water & & & & & & \\
\hline Water & 81621 & Sample 1 Total & 0.000050 & 0.000050 & 0.0000037 & 0.000050 & 0.000025 & 0.000050 \\
\hline Water & 81622 & Sample 2 Total & 0.000050 & 0.000050 & 0.0000340 & 0.000050 & 0.000025 & 0.000050 \\
\hline \multirow[t]{2}{*}{ Water } & 81623 & Sample 3 Total & 0.000050 & 0.000050 & 0.0000370 & 0.000050 & 0.000025 & 0.000050 \\
\hline & & Elutriate & & & & & & \\
\hline Water & 81627 & Sample 1 Dissolved & 0.000100 & 0.000100 & 0.0000170 & 0.000100 & 0.000050 & 0.000100 \\
\hline Water & 81628 & Sample 2 Dissolved & 0.000100 & 0.000100 & 0.0000180 & 0.000100 & 0.000050 & 0.000100 \\
\hline Water & 81629 & Sample 3 Dissolved & 0.000100 & 0.000100 & 0.0000290 & 0.000100 & 0.000050 & 0.000100 \\
\hline Water & 81624 & Sample 1 Total & 0.000050 & 0.000050 & 0.0000130 & 0.000050 & 0.000025 & 0.000050 \\
\hline Water & 81625 & Sample 2 Total & 0.000050 & 0.000050 & 0.0000350 & 0.000050 & 0.000025 & 0.000050 \\
\hline Water & 81626 & Sample 3 Total & 0.000050 & 0.000050 & 0.0000330 & 0.000050 & 0.000025 & 0.000050 \\
\hline \multirow[t]{3}{*}{ SAMPLE } & SAMPLE & DESCRIPTION & PPDDE & PPDDT & HPTCL & DIELDRIN & ENDOI & ENDO॥ \\
\hline & & Detection Limit $(\mathrm{mg} / \mathrm{kg})$ & 0.0019 & 0.0019 & 0.00096 & 0.0036 & 0.00096 & 0.0036 \\
\hline & & Insitu Sediment & & & & & & \\
\hline Sediment & 81711 & Sample \#1 & 0.0061 & 0.0120 & 0.00058 & 0.0036 & 0.0030 & 0.0055 \\
\hline Sediment & 81712 & Sample \#2 & 0.0110 & 0.0059 & 0.00038 & 0.0036 & 0.0030 & 0.0036 \\
\hline Sediment & 81713 & Sample \#3 & 0.0075 & 0.0120 & 0.00051 & 0.0036 & 0.0030 & 0.0036 \\
\hline
\end{tabular}

PPDDE-PPDDE PPDDT-PPDDT HPTCL- Heptachlor DIELDRIN-Dieldrin ENDOI - A-Endosulfan ENDOII - B-Endosulfan $B O L D$ - less than values

Values below less than values are estimated results. Results are less than the reporting limit.

Page 2 
Pestfine

Delaware River Water Analysis (Fine-Grained Site)

\begin{tabular}{|c|c|c|c|c|c|c|c|c|}
\hline \multicolumn{2}{|c|}{$\begin{array}{l}\text { SAMPLE } \\
\text { TYPE }\end{array}$} & DESCRIPTION & ENDOSU & ENDRIN & ENDALD & HPTCLE & METOXYCL & CLORDANE \\
\hline & & Detection Limit (mg/l) & 0.000055 & 0.000050 & 0.000055 & 0.000028 & 0.00028 & 0.000028 \\
\hline & & Plume Monitoring & & & & & & \\
\hline Water & 80990 & Background, dissolved & 0.000050 & 0.000050 & 0.000050 & 0.000025 & 0.00025 & 0.000025 \\
\hline Water & 80955 & Background, total & 0.000055 & 0.000110 & 0.000055 & 0.000028 & 0.00028 & 0.000028 \\
\hline Water & 80991 & 0-10 min, overflow, dissolved & 0.000050 & 0.000050 & 0.000050 & 0.000025 & 0.00025 & 0.000025 \\
\hline Water & 80992 & 10-20 min, overflow, dissolved & 0.000050 & 0.000050 & 0.000050 & 0.000025 & 0.00025 & 0.000025 \\
\hline Water & 80993 & 20-30 min, overflow, dissolved & 0.000050 & 0.000050 & 0.000050 & 0.000025 & 0.00025 & 0.000025 \\
\hline Water & 80956 & $0.10 \mathrm{~min}$, overflow, total & 0.000050 & 0.000110 & 0.000050 & 0.000025 & 0.00025 & 0.000025 \\
\hline Water & 80957 & $10-20 \mathrm{~min}$, overflow, total & 0.000050 & 0.000120 & 0.000050 & 0.000025 & 0.00025 & 0.000025 \\
\hline Water & 80958 & 20-30 min, overflow, total & 0.000050 & 0.000100 & 0.000050 & 0.000025 & 0.00025 & 0.000025 \\
\hline Water & 80994 & 0-10 min, non-overflow, dissolved & 0.000050 & 0.000050 & 0.000050 & 0.000025 & 0.00025 & 0.000025 \\
\hline Water & 80995 & 10-20 min, non-overflow, dissolved & 0.000050 & 0.000110 & 0.000050 & 0.000025 & 0.00025 & 0.000025 \\
\hline Water & 80996 & 20-30 min, non-overflow, dissolved & 0.000050 & 0.000110 & 0.000050 & 0.000025 & 0.00025 & 0.000025 \\
\hline Water & 80959 & 0-10 min, non-overflow, total & 0.000050 & 0.000100 & 0.000050 & 0.000025 & 0.00025 & 0.000025 \\
\hline Water & 80960 & 10-20 min, non-overflow, total & 0.000050 & 0.000120 & 0.000050 & 0.000025 & 0.00025 & 0.000025 \\
\hline \multirow[t]{2}{*}{ Water } & 80961 & 20-30 min, non-overflow, total & 0.000050 & 0.000130 & 0.000050 & 0.000025 & 0.00025 & 0.000025 \\
\hline & & Hopper Inflow Monitoring & & & & & & \\
\hline Water & 81114 & 3\& $6 \mathrm{~min}$, dissolved & 0.000050 & 0.000081 & 0.000050 & 0.000025 & 0.00025 & 0.000025 \\
\hline Water & 81115 & $9 \& 12 \mathrm{~min}$, dissolved & 0.000052 & 0.000061 & 0.000052 & 0.000026 & 0.00026 & 0.000026 \\
\hline Water & 81116 & $15 \& 18 \mathrm{~min}$, dissolved & 0.000054 & 0.000060 & 0.000054 & 0.000027 & 0.00027 & 0.000027 \\
\hline Water & 81117 & $21824 \mathrm{~min}$, dissolved & 0.000050 & 0.000081 & 0.000050 & 0.000025 & 0.00025 & 0.000025 \\
\hline Water & 81118 & $27 \& 30 \mathrm{~min}$, dissolved & 0.000050 & 0.000069 & 0.000050 & 0.000025 & 0.00025 & 0.000025 \\
\hline Water & 80903 & $3 \& 6 \mathrm{~min}$, total & 0.000022 & 0.000050 & 0.000050 & 0.000013 & 0.00025 & \\
\hline Water & 80904 & $9 \& 12 \mathrm{~min}$, total & 0.000050 & 0.000050 & 0.000050 & 0.000025 & 0.00025 & \\
\hline Water & 80905 & $15 \& 18 \mathrm{~min}$, total & 0.000047 & 0.000053 & 0.000053 & 0.000027 & 0.00027 & \\
\hline Water & 80906 & $21824 \mathrm{~min}$, total & 0.000041 & 0.000053 & 0.000053 & 0.000027 & 0.00027 & \\
\hline \multirow[t]{2}{*}{ Water } & 80907 & $27 \& 30 \mathrm{~min}$, total & 0.000050 & 0.000050 & 0.000050 & 0.000025 & 0.00025 & \\
\hline & & Hopper Overflow Monitoring & & & & & & \\
\hline Water & 81119 & 2\& $4 \mathrm{~min}$, dissolved & 0.000052 & 0.000095 & 0.000052 & 0.000026 & 0.00026 & 0.000026 \\
\hline Water & 81120 & $6 \& 8 \mathrm{~min}$, dissolved & 0.000050 & 0.000079 & 0.000050 & 0.000025 & 0.00025 & 0.000025 \\
\hline Water & 81121 & $10 \& 12 \mathrm{~min}$, dissolved & 0.000050 & 0.000085 & 0.000050 & 0.000025 & 0.00025 & 0.000025 \\
\hline Water & 81122 & $14 \& 16 \mathrm{~min}$, dissolved & 0.000050 & 0.000063 & 0.000050 & 0.000025 & 0.00025 & 0.000025 \\
\hline Water & 81123 & $18 \& 20 \mathrm{~min}$, dissolved & 0.000049 & 0.000055 & 0.000049 & 0.000024 & 0.00024 & 0.000024 \\
\hline Water & 80909 & 2\& $4 \mathrm{~min}$, total & 0.000053 & 0.000053 & 0.000053 & 0.000014 & 0.00027 & \\
\hline Water & 80910 & 6\& $8 \mathrm{~min}$, total & 0.000053 & 0.000053 & 0.000053 & 0.000027 & 0.00027 & \\
\hline Water & 80911 & $10 \& 12 \mathrm{~min}$, total & Broken & Broken & Broken & Broken & Broken & \\
\hline Water & 80912 & $14 \& 16 \mathrm{~min}$, total & 0.000140 & 0.000050 & 0.000050 & 0.000020 & 0.00025 & \\
\hline \multirow[t]{2}{*}{ Water } & 80913 & $18 \& 20 \mathrm{~min}$, total & 0.000053 & 0.000053 & 0.000053 & 0.000027 & 0.00027 & \\
\hline & & Site Water & & & & & & \\
\hline Water & 81621 & Sample 1 Total & 0.000050 & 0.000050 & 0.000050 & 0.000025 & 0.00025 & \\
\hline Water & 81622 & Sample 2 Total & 0.000050 & 0.000050 & 0.000050 & 0.000025 & 0.00025 & \\
\hline Water & 81623 & Sample 3 Total & 0.000050 & 0.000050 & 0.000050 & 0.000025 & 0.00025 & \\
\hline
\end{tabular}

\begin{tabular}{|c|c|c|c|c|c|c|c|}
\hline & & Elutriate & & & & & \\
\hline Water & 81627 & Sample 1 Dissolved & 0.000100 & 0.000100 & 0.000100 & 0.000050 & 0.00050 \\
\hline Water & 81628 & Sample 2 Dissolved & 0.000100 & 0.000100 & 0.000100 & 0.000050 & 0.00050 \\
\hline Water & 81629 & Sample 3 Dissolved & 0.000100 & 0.000100 & 0.000100 & 0.000050 & 0.00050 \\
\hline Water & 81624 & Sample 1 Total & 0.000050 & 0.000050 & 0.000050 & 0.000025 & 0.00025 \\
\hline Water & 81625 & Sample 2 Total & 0.000050 & 0.000050 & 0.000050 & 0.000025 & 0.00025 \\
\hline Water & 81626 & Sample 3 Total & 0.000050 & 0.000050 & 0.000050 & 0.000025 & 0.00025 \\
\hline \multirow{3}{*}{$\begin{array}{l}\text { SAMPLE } \\
\text { TYPE }\end{array}$} & SAMPLE & DESCRIPTION & ENDOSU & ENDRIN & ENDALD & HPTCLE & METOXYCL \\
\hline & & Detection Limit $(\mathrm{mg} / \mathrm{kg}$ ) & 0.0036 & 0.0036 & 0.0036 & 0.0018 & 0.018 \\
\hline & & Insitu Sediment & & & & & \\
\hline Sediment & 81711 & Sample \#1 & 0.0036 & 0.0036 & 0.0036 & 0.0018 & 0.018 \\
\hline Sediment & 81712 & Sample \#2 & 0.0019 & 0.0036 & 0.0036 & 0.0018 & 0.018 \\
\hline Sediment & 81713 & Sample \#3 & 0.0036 & 0.0036 & 0.0036 & 0.0018 & 0.018 \\
\hline
\end{tabular}

ENDOSU - Endosulfan sulfate ENDRIN - Endrin ENDALD - Endrin Aldehyde HPTCLE - Heptachlor Epoxide METOXYCL - Methoxychlor CLORDANE - Chlordane

Values below less than values are estimated results. Results are less than the reporting limit 
Pestfine

Delaware River Water Analysis (Fine-Grained Site)

\begin{tabular}{|c|c|c|c|c|c|c|c|}
\hline \multirow[t]{2}{*}{$\begin{array}{l}\text { SAMPLE } \\
\text { TYPE }\end{array}$} & $\begin{array}{l}\text { SAMPLE } \\
\text { ID }\end{array}$ & DESCRIPTION & TOXAPHEN & TCLXYL-S & DCLBP & a-CHLORD & g-CHLORD \\
\hline & & Detection Limit (mg/l) & 0.00028 & & & 0.000025 & 0.000025 \\
\hline & & Plume Monitoring & & & & & \\
\hline Water & 80990 & Background, dissolved & 0.00025 & $83.00 \%$ & $92.60 \%$ & & \\
\hline Water & 80955 & Background, total & 0.00028 & $87.40 \%$ & $72.90 \%$ & & \\
\hline Water & 80991 & 0-10 min, overflow, dissolved & 0.00025 & $75.70 \%$ & $92.20 \%$ & & \\
\hline Water & 80992 & 10-20 min, overflow, dissolved & 0.00025 & $75.10 \%$ & $81.70 \%$ & & \\
\hline Water & 80993 & 20-30 min, overflow, dissolved & 0.00025 & $73.20 \%$ & $83.20 \%$ & & \\
\hline Water & 80956 & 0-10 min, overflow, total & 0.00025 & $83.50 \%$ & $70.90 \%$ & & \\
\hline Water & 80957 & $10-20 \mathrm{~min}$, overflow, total & 0.00025 & $92.40 \%$ & $72.10 \%$ & & \\
\hline Water & 80958 & $20-30 \mathrm{~min}$, overflow, total & 0.00025 & $74.40 \%$ & $63.90 \%$ & & \\
\hline Water & 80994 & 0-10 min, non-overflow, dissolved & 0.00025 & $73.30 \%$ & $84.10 \%$ & & \\
\hline Water & 80995 & 10-20 min, non-overflow, dissolved & 0.00025 & $79.50 \%$ & $87.40 \%$ & & \\
\hline Water & 80996 & 20-30 min, non-overflow, dissolved & 0.00025 & $73.40 \%$ & $84.10 \%$ & & \\
\hline Water & 80959 & 0-10 min, non-overflow, total & 0.00025 & $82.40 \%$ & $75.20 \%$ & & \\
\hline Water & 80960 & 10-20 min, non-overflow, total & 0.00025 & $86.80 \%$ & $79.50 \%$ & & \\
\hline \multirow[t]{2}{*}{ Water } & 80961 & 20-30 min, non-overflow, total & 0.00025 & $82.30 \%$ & $80.60 \%$ & & \\
\hline & & Hopper Inflow Monitoring & & & & & \\
\hline Water & 81114 & 3\& $6 \mathrm{~min}$, dissolved & 0.00025 & $80.80 \%$ & $78.00 \%$ & & \\
\hline Water & 81115 & $9 \& 12 \mathrm{~min}$, dissolved & 0.00026 & $82.20 \%$ & $75.20 \%$ & & \\
\hline Water & 81116 & $15 \& 18 \mathrm{~min}$, dissolved & 0.00027 & $82.10 \%$ & $73.50 \%$ & & \\
\hline Water & 81117 & $21 \& 24 \mathrm{~min}$, dissolved & 0.00025 & $95.30 \%$ & $85.80 \%$ & & \\
\hline Water & 81118 & $27 \& 30 \mathrm{~min}$, dissolved & 0.00025 & $91.60 \%$ & $80.70 \%$ & & \\
\hline Water & 80903 & $386 \mathrm{~min}$, total & 0.00025 & $35.50 \%$ & $45.20 \%$ & 0.000034 & 0.000026 \\
\hline Water & 80904 & $9 \& 12 \mathrm{~min}$, total & 0.00025 & $31.64 \%$ & $43.66 \%$ & 0.000043 & 0.000032 \\
\hline Water & 80905 & $15 \& 18 \mathrm{~min}$, total & 0.00027 & $39.39 \%$ & $59.50 \%$ & 0.000052 & 0.000032 \\
\hline Water & 80906 & $21 \& 24 \mathrm{~min}$, total & 0.00027 & $44.21 \%$ & $59.72 \%$ & 0.000046 & 0.000029 \\
\hline \multirow[t]{2}{*}{ Water } & 80907 & $27 \& 30 \mathrm{~min}$, total & 0.00025 & $44.04 \%$ & $41.07 \%$ & 0.000043 & 0.000025 \\
\hline & & Hopper Overflow Monitoring & & & & & \\
\hline Water & 81119 & $224 \mathrm{~min}$, dissolved & 0.00026 & $88.10 \%$ & $80.40 \%$ & & \\
\hline Water & 81120 & $6 \& 8 \mathrm{~min}$, dissolved & 0.00025 & $92.10 \%$ & $76.20 \%$ & & \\
\hline Water & 81121 & $10 \& 12 \mathrm{~min}$, dissolved & 0.00025 & $91.70 \%$ & $81.80 \%$ & & \\
\hline Water & 81122 & $14 \& 16 \mathrm{~min}$, dissolved & 0.00025 & $72.30 \%$ & $56.10 \%$ & & \\
\hline Water & 81123 & $18 \& 20 \mathrm{~min}$, dissolved & 0.00024 & $73.50 \%$ & $61.10 \%$ & & \\
\hline Water & 80909 & 28 $4 \mathrm{~min}$, total & 0.00027 & $40.11 \%$ & $58.27 \%$ & 0.000068 & 0.000050 \\
\hline Water & 80910 & $6 \& 8 \mathrm{~min}$, total & 0.00027 & $47.04 \%$ & $64.93 \%$ & 0.000069 & 0.000048 \\
\hline Water & 80911 & $10 \& 12 \mathrm{~min}$, total & Broken & Broken & Broken & Broken & Broken \\
\hline Water & 80912 & $14 \& 16 \mathrm{~min}$, total & 0.00025 & $48.41 \%$ & $78.19 \%$ & 0.000130 & 0.000093 \\
\hline \multirow[t]{2}{*}{ Water } & 80913 & $18 \& 20 \mathrm{~min}$, total & 0.00027 & $44.62 \%$ & $83.94 \%$ & 0.000088 & 0.000059 \\
\hline & & Site Water & & & & & \\
\hline Water & 81621 & Sample 1 Total & 0.00025 & $76.88 \%$ & $68.76 \%$ & 0.000025 & 0.000025 \\
\hline Water & 81622 & Sample 2 Total & 0.00025 & $60.17 \%$ & $69.83 \%$ & 0.000025 & 0.000025 \\
\hline \multirow[t]{2}{*}{ Water } & 81623 & Sample 3 Total & 0.00025 & $71.76 \%$ & $68.80 \%$ & 0.000025 & 0.000025 \\
\hline & & Elutriate & & & & & \\
\hline Water & 81627 & Sample 1 Dissolved & 0.00050 & $75.82 \%$ & $87.54 \%$ & 0.000050 & 0.000050 \\
\hline Water & 81628 & Sample 2 Dissolved & 0.00050 & $85.13 \%$ & $88.79 \%$ & 0.000050 & 0.000050 \\
\hline Water & 81629 & Sample 3 Dissolved & 0.00050 & $76.31 \%$ & $87.53 \%$ & 0.000050 & 0.000050 \\
\hline Water & 81624 & Sample 1 Total & 0.00025 & $81.58 \%$ & $64.33 \%$ & 0.000025 & 0.000025 \\
\hline Water & 81625 & Sample 2 Total & 0.00025 & $60.65 \%$ & $55.24 \%$ & 0.000025 & 0.000025 \\
\hline Water & 81626 & Sample 3 Total & 0.00025 & $67.60 \%$ & $61.90 \%$ & 0.000025 & 0.000025 \\
\hline \multirow[t]{3}{*}{ SAMPLE } & SAMPLE & DESCRIPTION & TOXAPHEN & TCLXYL-S & DCLBP & a-CHLORD & g-CHLORD \\
\hline & & Detection Limit (mg/kg) & 0.018 & & & 0.00096 & 0.0019 \\
\hline & & Insitu Sediment & & & & & \\
\hline Sediment & 81711 & Sample \#1 & 0.018 & $86.90 \%$ & $92.01 \%$ & 0.0011 & 0.0035 \\
\hline Sediment & 81712 & Sample \#2 & 0.018 & $91.01 \%$ & $92.77 \%$ & 0.0016 & 0.0035 \\
\hline Sediment & 81713 & Sample \#3 & 0.018 & $84.77 \%$ & $102.76 \%$ & 0.0011 & 0.0038 \\
\hline \multicolumn{5}{|c|}{$\begin{array}{l}\text { TOXAPHEN - Toxaphene } \\
\text { a-CHLORD - a-CHLORD } \\
\text { BOLD - less than values }\end{array}$} & \multicolumn{3}{|c|}{ DCLBP - Decachlorobiphenyl(Surrogate (60-150 WS)) } \\
\hline
\end{tabular}

Page 4 
PCBsfine

Delaware River Water Analysis (Fine-Grained Site)

\begin{tabular}{|c|c|c|c|c|c|c|c|c|c|}
\hline \multirow[t]{2}{*}{$\begin{array}{l}\text { SAMPLE } \\
\text { TYPE }\end{array}$} & $\begin{array}{l}\text { SAMPLE } \\
\text { ID }\end{array}$ & DESCRIPTION & РCB 22 & РСB 33 & РСВ 37 & РСB 42 & PCB 47 & РCB 64 & PCB 74 \\
\hline & & Detection Limit (mg/l) & 0.0000011 & 0.0000011 & 0.0000011 & 0.0000011 & 0.00000110 & 0.0000011 & 0.0000011 \\
\hline & & Plume Monitoring & & & & & & & \\
\hline Water & 80983 & Background, dissolved & 0.0000010 & 0.0000010 & 0.0000010 & 0.0000010 & 0.00000100 & 0.0000010 & 0.0000010 \\
\hline Water & 80948 & Background, total & 0.0000011 & 0.0000011 & 0.0000011 & 0.0000011 & 0.00000110 & 0.0000011 & 0.0000011 \\
\hline Water & 80984 & 0-10 min, overflow, dissolved & 0.0000010 & 0.0000010 & 0.0000010 & 0.0000010 & 0.00000100 & 0.0000010 & 0.0000010 \\
\hline Water & 80985 & 10-20 min, overflow, dissolved & 0.0000011 & 0.0000011 & 0.0000011 & 0.0000011 & 0.00000110 & 0.0000011 & 0.0000011 \\
\hline Water & 80986 & 20-30 min, overflow, dissolved & 0.0000010 & 0.0000010 & 0.0000010 & 0.0000010 & 0.00000100 & 0.0000010 & 0.0000010 \\
\hline Water & 80949 & 0-10 min, overflow, total & 0.0000010 & 0.0000010 & 0.0000010 & 0.0000010 & 0.00000100 & 0.0000010 & 0.0000010 \\
\hline Water & 80950 & 10-20 min, overflow, total & 0.0000010 & 0.0000010 & 0.0000010 & 0.0000010 & 0.00000100 & 0.0000010 & 0.0000010 \\
\hline Water & 80951 & 20-30 min, overflow, total & 0.0000010 & 0.0000010 & 0.0000010 & 0.0000010 & 0.00000100 & 0.0000010 & 0.0000010 \\
\hline Water & 80987 & 0-10 min, non-overflow, dissolve & 0.0000010 & 0.0000010 & 0.0000010 & 0.0000010 & 0.00000100 & 0.0000010 & 0.0000010 \\
\hline Water & 80988 & $10-20 \mathrm{~min}$, non-overflow, dissolve & 0.0000010 & 0.0000010 & 0.0000010 & 0.0000010 & 0.00000100 & 0.0000010 & 0.0000010 \\
\hline Water & 80989 & 20-30 min, non-overflow, dissolve & 0.0000010 & 0.0000010 & 0.0000010 & 0.0000010 & 0.00000100 & 0.0000010 & 0.0000010 \\
\hline Water & 80952 & 0-10 min, non-overflow, total & 0.0000010 & 0.0000010 & 0.0000010 & 0.0000010 & 0.00000100 & 0.0000010 & 0.0000010 \\
\hline Water & 80953 & 10-20 min, non-overflow, total & 0.0000010 & 0.0000010 & 0.0000010 & 0.0000010 & 0.00000100 & 0.0000010 & 0.0000010 \\
\hline \multirow[t]{2}{*}{ Water } & 80954 & 20-30 min, non-overflow, total & 0.0000010 & 0.0000010 & 0.0000010 & 0.0000010 & 0.00000100 & 0.0000010 & 0.0000010 \\
\hline & & Hopper Inflow Monitoring & & & & & & & \\
\hline Water & 81104 & $3 \& 6 \mathrm{~min}$, dissolved & 0.0000010 & 0.0000010 & 0.0000010 & 0.0000010 & 0.00000100 & 0.0000010 & 0.0000010 \\
\hline Water & 81105 & $9812 \mathrm{~min}$, dissolved & 0.0000010 & 0.0000010 & 0.0000010 & 0.0000010 & 0.00000100 & 0.0000010 & 0.0000010 \\
\hline Water & 81106 & $15 \& 18 \mathrm{~min}$, dissolved & 0.0000010 & 0.0000010 & 0.0000010 & 0.0000010 & 0.00000050 & 0.0000010 & 0.0000010 \\
\hline Water & 81107 & $21 \& 24 \mathrm{~min}$, dissolved & 0.0000010 & 0.0000010 & 0.0000010 & 0.0000010 & 0.00000100 & 0.0000010 & 0.0000010 \\
\hline Water & 81108 & $27 \& 30 \mathrm{~min}$, dissolved & 0.0000010 & 0.0000010 & 0.0000010 & 0.0000010 & 0.00000100 & 0.0000010 & 0.0000010 \\
\hline Water & 80891 & $3 \& 6 \mathrm{~min}$, total & 0.0000011 & 0.0000011 & 0.0000011 & 0.0000016 & 0.00000095 & 0.0000011 & 0.0000013 \\
\hline Water & 80892 & $9 \& 12 \mathrm{~min}$, total & 0.0000010 & 0.0000010 & 0.0000010 & 0.0000031 & 0.00000180 & 0.0000092 & 0.0000042 \\
\hline Water & 80893 & $15 \& 18 \mathrm{~min}$, total & 0.0000010 & 0.0000010 & 0.0000010 & 0.0000021 & 0.00000055 & 0.0000010 & 0.0000021 \\
\hline Water & 80894 & $21 \& 24 \mathrm{~min}$, total & 0.0000011 & 0.0000011 & 0.0000011 & 0.0000021 & 0.00000250 & 0.0000084 & 0.0000011 \\
\hline \multirow[t]{2}{*}{ Water } & 80895 & $27 \& 30 \mathrm{~min}$, total & 0.0000011 & 0.0000011 & 0.0000011 & 0.0000038 & 0.00000110 & 0.0000011 & 0.0000011 \\
\hline & & Hopper Overflow Monitoring & & & & & & & \\
\hline Water & 81109 & 2\& $4 \mathrm{~min}$, dissolved & 0.0000010 & 0.0000010 & 0.0000010 & 0.0000010 & 0.00000100 & 0.0000010 & 0.0000010 \\
\hline Water & 81110 & 6\& $8 \mathrm{~min}$, dissolved & 0.0000010 & 0.0000010 & 0.0000010 & 0.0000010 & 0.00000100 & 0.0000010 & 0.0000010 \\
\hline Water & 81111 & $10 \& 12 \mathrm{~min}$, dissolved & 0.0000010 & 0.0000010 & 0.0000010 & 0.0000010 & 0.00000100 & 0.0000010 & 0.0000010 \\
\hline Water & 81112 & $14 \& 16 \mathrm{~min}$, dissolved & 0.0000010 & 0.0000010 & 0.0000010 & 0.0000010 & 0.00000100 & 0.0000010 & 0.0000010 \\
\hline Water & 81113 & $18 \& 20 \mathrm{~min}$, dissolved & 0.0000010 & 0.0000010 & 0.0000010 & 0.0000010 & 0.00000100 & 0.0000010 & 0.0000010 \\
\hline Water & 80897 & 2\& 4 min, total & 0.0000011 & 0.0000011 & 0.0000011 & 0.0000046 & 0.00000830 & 0.0000011 & 0.0000011 \\
\hline Water & 80898 & $6 \& 8 \mathrm{~min}$, total & 0.0000011 & 0.0000011 & 0.0000011 & 0.0000011 & 0.00000110 & 0.0000150 & 0.0000061 \\
\hline Water & 80899 & $10 \& 12 \mathrm{~min}$, total & 0.0000011 & 0.0000011 & 0.0000011 & 0.0000011 & 0.00000110 & 0.0000160 & 0.0000064 \\
\hline Water & 80900 & $14 \& 16 \mathrm{~min}$, total & 0.0000011 & 0.0000011 & 0.0000011 & 0.0000069 & 0.00000110 & 0.0000200 & 0.0000080 \\
\hline \multirow[t]{2}{*}{ Water } & 80901 & $18 \& 20 \mathrm{~min}$, total & 0.0000010 & 0.0000010 & 0.0000010 & 0.0000058 & 0.00000100 & 0.0000190 & 0.0000074 \\
\hline & & Site Water & & & & & & & \\
\hline Water & 81603 & Sample 1 Total & 0.0000010 & 0.0000010 & 0.0000010 & 0.0000010 & 0.00000100 & 0.0000010 & 0.0000010 \\
\hline Water & 81604 & Sample 2 Total & 0.0000010 & 0.0000010 & 0.0000010 & 0.0000010 & 0.00000100 & 0.0000010 & 0.0000010 \\
\hline \multirow[t]{2}{*}{ Water } & 81605 & Sample 3 Total & 0.0000010 & 0.0000010 & 0.0000010 & 0.0000010 & 0.00000100 & 0.0000010 & 0.0000010 \\
\hline & & Elutriate & & & & & & & \\
\hline Water & 81609 & Sample 1 Dissolved & 0.0000010 & 0.0000010 & 0.0000010 & 0.0000010 & 0.00000100 & 0.0000010 & 0.0000010 \\
\hline Water & 81610 & Sample 2 Dissolved & 0.0000010 & 0.0000010 & 0.0000010 & 0.0000010 & 0.00000100 & 0.0000010 & 0.0000010 \\
\hline Water & 81611 & Sample 3 Dissolved & 0.0000010 & 0.0000010 & 0.0000010 & 0.0000010 & 0.00000100 & 0.0000010 & 0.0000010 \\
\hline Water & 81606 & Sample 1 Total & 0.0000010 & 0.0000010 & 0.0000010 & 0.0000010 & 0.00000100 & 0.0000010 & 0.0000010 \\
\hline Water & 81607 & Sample 2 Total & 0.0000010 & 0.0000010 & 0.0000010 & 0.0000010 & 0.00000057 & 0.0000010 & 0.0000010 \\
\hline Water & 81608 & Sample 3 Total & 0.0000010 & 0.0000010 & 0.0000010 & 0.0000010 & 0.00000051 & 0.0000010 & 0.0000010 \\
\hline \multirow[t]{3}{*}{ SAMPLE } & SAMPLE & DESCRIPTION & РCB 22 & РСв 33 & РCB 37 & РCB 42 & PCB 47 & РCB 64 & PCB 74 \\
\hline & & Detection Limit ( $\mathrm{mg} / \mathrm{kg}$ ) & 0.00077 & 0.00077 & 0.00077 & 0.00077 & 0.00077 & 0.00077 & 0.00077 \\
\hline & & Insitu Sediment & & & & & & & \\
\hline & 81717 & Sample \#1 & 0.00077 & 0.00077 & 0.00077 & 0.00077 & 0.00077 & 0.00077 & 0.00077 \\
\hline Sediment & 81718 & Sample \#2 & 0.00077 & 0.00077 & 0.00077 & 0.00077 & 0.00077 & 0.00077 & 0.00077 \\
\hline & & & 0.00077 & 0.00077 & 0.00077 & 0.00077 & 0.00077 & 0.00077 & 0.00077 \\
\hline
\end{tabular}

BOLD - less than values

Values below less than values are estimated results. Results are less than the reporting limit. 
Delaware River Water Analysis (Fine-Grained Site)

\begin{tabular}{|c|c|c|c|c|c|c|c|c|c|}
\hline \multirow{2}{*}{$\begin{array}{l}\text { SAMPLE } \\
\text { TYPE }\end{array}$} & $\begin{array}{l}\text { SAMPLE } \\
\text { ID }\end{array}$ & DESCRIPTION & PCB 80 & PCB 81 & PCB 84 & РСB 91 & РСB 92 & РСB 95 & PCB 99 \\
\hline & & Detection Limit (mg/l) & 0.0000011 & 0.0000011 & 0.00000110 & 0.00000110 & 0.0000011 & 0.00000110 & 0.00000110 \\
\hline & & Plume Monitoring & & & & & & & \\
\hline Water & 80983 & Background, dissolved & 0.0000010 & 0.0000010 & 0.00000100 & 0.00000100 & 0.0000010 & 0.00000058 & 0.00000100 \\
\hline Water & 80948 & Background, total & 0.0000011 & 0.0000011 & 0.00000110 & 0.00000110 & 0.0000011 & 0.00000110 & 0.00000110 \\
\hline Water & 80984 & 0-10 min, overflow, dissolved & 0.0000010 & 0.0000010 & 0.00000100 & 0.00000100 & 0.0000010 & 0.00000058 & 0.00000100 \\
\hline Water & 80985 & 10-20 min, overflow, dissolved & 0.0000011 & 0.0000011 & 0.00000110 & 0.00000110 & 0.0000011 & 0.00000085 & 0.00000110 \\
\hline Water & 80986 & 20-30 min, overflow, dissolved & 0.0000010 & 0.0000010 & 0.00000100 & 0.00000100 & 0.0000010 & 0.00000049 & 0.00000100 \\
\hline Water & 80949 & 0-10 min, overflow, total & 0.0000010 & 0.0000010 & 0.00000100 & 0.00000100 & 0.0000010 & 0.00000100 & 0.00000100 \\
\hline Water & 80950 & 10-20 min, overflow, total & 0.0000010 & 0.0000010 & 0.00000100 & 0.00000100 & 0.0000010 & 0.00000100 & 0.00000100 \\
\hline Water & 80951 & 20-30 min, overflow, total & 0.0000010 & 0.0000010 & 0.00000100 & 0.00000100 & 0.0000010 & 0.00000100 & 0.00000100 \\
\hline Water & 80987 & 0-10 min, non-overflow, dissolve & 0.0000010 & 0.0000010 & 0.00000100 & 0.00000100 & 0.0000010 & 0.00000049 & 0.00000100 \\
\hline Water & 80988 & 10-20 min, non-overflow, dissolve & 0.0000010 & 0.0000010 & 0.00000100 & 0.00000100 & 0.0000010 & 0.00000120 & 0.00000100 \\
\hline Water & 80989 & 20-30 min, non-overflow, dissolve & 0.0000010 & 0.0000010 & 0.00000100 & 0.00000100 & 0.0000010 & 0.00000056 & 0.00000100 \\
\hline Water & 80952 & 0-10 min, non-overflow, total & 0.0000010 & 0.0000010 & 0.00000100 & 0.00000100 & 0.0000010 & 0.00000100 & 0.00000100 \\
\hline Water & 80953 & 10-20 min, non-overflow, total & 0.0000010 & 0.0000010 & 0.00000100 & 0.00000100 & 0.0000010 & 0.00000100 & 0.00000100 \\
\hline \multirow[t]{2}{*}{ Water } & 80954 & 20-30 min, non-overflow, total & 0.0000010 & 0.0000010 & 0.00000100 & 0.00000100 & 0.0000010 & 0.00000100 & 0.00000100 \\
\hline & & Hopper Inflow Monitoring & & & & & & & \\
\hline Water & 81104 & $3 \& 6 \mathrm{~min}$, dissolved & 0.0000010 & 0.0000010 & 0.00000100 & 0.00000100 & 0.0000010 & 0.00000084 & 0.00000060 \\
\hline Water & 81105 & $9 \& 12 \mathrm{~min}$, dissolved & 0.0000010 & 0.0000010 & 0.00000100 & 0.00000100 & 0.0000010 & 0.00000110 & 0.00000100 \\
\hline Water & 81106 & $15 \& 18 \mathrm{~min}$, dissolved & 0.0000010 & 0.0000010 & 0.00000100 & 0.00000100 & 0.0000010 & 0.00000095 & 0.00000100 \\
\hline Water & 81107 & $21 \& 24 \mathrm{~min}$, dissolved & 0.0000010 & 0.0000010 & 0.00000100 & 0.00000100 & 0.0000010 & 0.00000084 & 0.00000045 \\
\hline Water & 81108 & $27830 \mathrm{~min}$, dissolved & 0.0000010 & 0.0000010 & 0.00000100 & 0.00000100 & 0.0000010 & 0.00000072 & 0.00000100 \\
\hline Water & 80891 & $3 \& 6 \mathrm{~min}$, total & 0.0000011 & 0.0000011 & 0.00000110 & 0.00000110 & 0.0000011 & 0.00000110 & 0.00000110 \\
\hline Water & 80892 & $9 \& 12 \mathrm{~min}$, total & 0.0000010 & 0.0000010 & 0.00000100 & 0.00000100 & 0.0000010 & 0.00000100 & 0.00000100 \\
\hline Water & 80893 & $15 \& 18 \mathrm{~min}$, total & 0.0000010 & 0.0000010 & 0.00000100 & 0.00000100 & 0.0000084 & 0.00000100 & 0.00000100 \\
\hline Water & 80894 & $21 \& 24 \mathrm{~min}$, total & 0.0000011 & 0.0000011 & 0.00000110 & 0.00000320 & 0.0000068 & 0.00001200 & 0.00000730 \\
\hline \multirow[t]{2}{*}{ Water } & 80895 & $27 \& 30 \mathrm{~min}$, total & 0.0000011 & 0.0000011 & 0.00000110 & 0,00000830 & 0.0000150 & 0.00002400 & 0.00001300 \\
\hline & 81109 & Hopper Overflow Monitoring & & & & & & & \\
\hline Water & 81109 & 2\& $4 \mathrm{~min}$, dissolved & 0.0000010 & 0.0000010 & 0.00000100 & 0.00000100 & 0.0000010 & 0.00000250 & 0.00000046 \\
\hline Water & $\begin{array}{l}81110 \\
81111\end{array}$ & $\begin{array}{l}6 \& 8 \mathrm{~min} \text {, dissolved } \\
10 \& 12 \mathrm{~min} \text { dissolved }\end{array}$ & 0.0000010 & $\begin{array}{l}0.0000010 \\
0.0000010\end{array}$ & $\begin{array}{l}0.00000100 \\
0.00000100\end{array}$ & $\begin{array}{l}0.00000100 \\
0.00000100\end{array}$ & 0.0000010 & 0.00000120 & 0.00000058 \\
\hline Water & 81112 & $\begin{array}{l}10 \& 12 \mathrm{~min} \text {, dissolved } \\
14 \& 16 \mathrm{~min} \text {, dissolved }\end{array}$ & $\begin{array}{l}0.0000010 \\
0.0000010\end{array}$ & 0.0000010 & $\begin{array}{l}0.00000100 \\
0.00000100\end{array}$ & 0.00000100 & $\begin{array}{l}0.0000010 \\
0.0000010\end{array}$ & 0.00000300 & 0.00000058 \\
\hline Water & 81113 & $18 \& 20 \mathrm{~min}$, dissolved & 0.0000010 & 0.0000010 & 0.00000100 & 0.00000100 & 0.0000010 & 0.00000170 & 0.00000063 \\
\hline Water & 80897 & 2\& $4 \mathrm{~min}$, total & 0.0000011 & 0.0000011 & 0.00000110 & 0.00001100 & 0.0000150 & 0.00002700 & 0.00001500 \\
\hline Water & 80898 & $6 \& 8 \mathrm{~min}$, total & 0.0000011 & 0.0000011 & 0.00000110 & 0.00000980 & 0.0000180 & 0.00002700 & 0.00001400 \\
\hline Water & 80899 & $10 \& 12 \mathrm{~min}$, total & 0.0000011 & 0.0000011 & 0.00000110 & 0.00001100 & 0.0000160 & 0.00003100 & 0.00001900 \\
\hline Water & 80900 & $14 \& 16 \mathrm{~min}$, total & 0.0000011 & 0.0000011 & 0.00000110 & 0.00001400 & 0.0000250 & 0.00003700 & 0.00002100 \\
\hline \multirow[t]{2}{*}{ Water } & 80901 & $18 \& 20 \mathrm{~min}$, total & 0.0000010 & 0.0000010 & 0.00000100 & 0.00001200 & 0.0000170 & 0.00003300 & 0.00001700 \\
\hline & & Site Water & & & & & & & \\
\hline Water & 81603 & Sample 1 Total & 0.0000010 & 0.0000010 & 0.00000100 & 0.00000046 & 0.0000010 & 0.00000093 & 0.00000100 \\
\hline Water & 81604 & Sample 2 Total & 0.0000010 & 0.0000010 & 0.00000100 & 0.00000100 & 0.0000010 & 0.00000100 & 0.00000100 \\
\hline \multirow[t]{2}{*}{ Water } & 81605 & Sample 3 Total & 0.0000010 & 0.0000010 & 0.00000100 & 0.00000046 & 0.0000010 & 0.00000095 & 0.00000100 \\
\hline & & Elutriate & & & & & & & \\
\hline Water & 81609 & Sample 1 Dissolved & 0.0000010 & 0.0000010 & 0.00000100 & 0.00000054 & 0.0000010 & 0.00000110 & 0.00000038 \\
\hline Water & 81610 & Sample 2 Dissolved & 0.0000010 & 0.0000010 & 0.00000100 & 0.00000068 & 0.0000010 & 0.00000094 & 0.00000047 \\
\hline Water & 81611 & Sample 3 Dissolved & 0.0000010 & 0.0000010 & 0.00000091 & 0.00000093 & 0.0000010 & 0.00000057 & 0.00000028 \\
\hline Water & 81606 & Sample 1 Total & 0.0000010 & 0.0000010 & 0.00000100 & 0.00000100 & 0.0000010 & 0.00000110 & 0.00000100 \\
\hline Water & 81607 & Sample 2 Total & 0.0000010 & 0.0000010 & 0.00000100 & 0.00000100 & 0.0000010 & 0.00000140 & 0.00000100 \\
\hline Water & 81608 & Sample 3 Total & 0.0000010 & 0.0000010 & 0.00000100 & 0.00000100 & 0.0000010 & 0.00000110 & 0.00000100 \\
\hline \multirow[t]{3}{*}{ SAMPLE } & SAMPLE & DESCRIPTION & PCB 80 & РCB 81 & РCB 84 & РСB 91 & РСВ 92 & РCB 95 & РСВ 99 \\
\hline & & Detection Limit (mg/kg) & 0.00077 & 0.00077 & 0.00077 & 0.00077 & 0.00077 & 0.00077 & 0.00077 \\
\hline & & Insitu Sediment & & & & & & & \\
\hline Sediment & 81717 & Sample \#1 & 0.00077 & 0.00077 & 0.00077 & 0.00077 & 0.00077 & 0.00077 & 0.00046 \\
\hline Sediment & 81718 & Sample \#2 & 0.00077 & 0.00077 & 0.00077 & 0.00040 & 0.00049 & 0.00077 & 0.00071 \\
\hline Sediment & 81719 & Sample \#3 & 0.00077 & 0.00077 & 0.00077 & 0.00052 & 0.00062 & 0.00077 & 0.00074 \\
\hline
\end{tabular}

BOLD - less than values
Values below less than values are estimated results. Results are less than the reporting limit. 
PCBsfine

Delaware River Water Analysis (Fine-Grained Site)

\begin{tabular}{|c|c|c|c|c|c|c|c|c|c|}
\hline \multirow[t]{2}{*}{$\begin{array}{l}\text { SAMPLE } \\
\text { TYPE }\end{array}$} & $\begin{array}{l}\text { SAMPLE } \\
\text { ID }\end{array}$ & DESCRIPTION & РСB 110 & РCB 119 & PCB 120 & PCB 123 & РCB 126 & РCB 127 & PCB 132 \\
\hline & & Detection Limit (mg/l) & 0.00000110 & 0.0000011 & 0.0000011 & 0.0000011 & 0.0000011 & 0.0000011 & 0.0000011 \\
\hline & & Plume Monitoring & & & & & & & \\
\hline Water & 80983 & Background, dissolved & 0.00000062 & 0.0000010 & 0.0000010 & 0.0000010 & 0.0000010 & 0.0000010 & 0.0000010 \\
\hline Water & 80948 & Background, total & 0.00000078 & 0.0000011 & 0.0000011 & 0.0000011 & 0.0000011 & 0.0000011 & 0.0000011 \\
\hline Water & 80984 & 0-10 min, overflow, dissolved & 0.00000060 & 0.0000010 & 0.0000010 & 0.0000010 & 0.0000010 & 0.0000010 & 0.0000010 \\
\hline Water & 80985 & $10-20 \mathrm{~min}$, overflow, dissolved & 0.00000087 & 0.0000011 & 0.0000011 & 0.0000011 & 0.0000011 & 0.0000011 & 0.0000011 \\
\hline Water & 80986 & 20-30 min, overflow, dissolved & 0.00000065 & 0.0000010 & 0.0000010 & 0.0000010 & 0.0000010 & 0.0000010 & 0.0000010 \\
\hline Water & 80949 & $0-10 \mathrm{~min}$, overflow, total & 0.00000100 & 0.0000010 & 0.0000010 & 0.0000010 & 0.0000010 & 0.0000010 & 0.0000010 \\
\hline Water & 80950 & 10-20 min, overflow, total & 0.00000110 & 0.0000010 & 0.0000010 & 0.0000010 & 0.0000010 & 0.0000010 & 0.0000010 \\
\hline Water & 80951 & 20-30 min, overflow, total & 0.00000110 & 0.0000010 & 0.0000010 & 0.0000010 & 0.0000010 & 0.0000010 & 0.0000010 \\
\hline Water & 80987 & 0-10 min, non-overflow, dissolve & 0.00000074 & 0.0000010 & 0.0000010 & 0.0000010 & 0.0000010 & 0.0000010 & 0.0000010 \\
\hline Water & 80988 & 10-20 min, non-overflow, dissolve & 0.00000068 & 0.0000010 & 0.0000010 & 0.0000010 & 0.0000010 & 0.0000010 & 0.0000010 \\
\hline Water & 80989 & 20-30 min, non-overflow, dissolve & 0.00000065 & 0.0000010 & 0.0000010 & 0.0000010 & 0.0000010 & 0.0000010 & 0.0000010 \\
\hline Water & 80952 & 0-10 min, non-overflow, total & 0.00000095 & 0.0000010 & 0.0000010 & 0.0000010 & 0.0000010 & 0.0000010 & 0.0000010 \\
\hline Water & 80953 & 10-20 min, non-overflow, total & 0.00000120 & 0.0000010 & 0.0000010 & 0.0000010 & 0.0000010 & 0.0000010 & 0.0000010 \\
\hline \multirow[t]{2}{*}{ Water } & 80954 & 20-30 min, non-overflow, total & 0.00000073 & 0.0000010 & 0.0000010 & 0.0000010 & 0.0000010 & 0.0000010 & 0.0000010 \\
\hline & & Hopper Inflow Monitoring & & & & & & & \\
\hline Water & 81104 & $3 \& 6 \mathrm{~min}$, dissolved & 0.00000095 & 0.0000010 & 0.0000010 & 0.0000010 & 0.0000010 & 0.0000010 & 0.0000010 \\
\hline Water & 81105 & $9 \& 12 \mathrm{~min}$, dissolved & 0.00000068 & 0.0000010 & 0.0000010 & 0.0000010 & 0.0000010 & 0.0000010 & 0.0000010 \\
\hline Water & 81106 & $15 \& 18 \mathrm{~min}$, dissolved & 0.00000076 & 0.0000010 & 0.0000010 & 0.0000010 & 0.0000010 & 0.0000010 & 0.0000010 \\
\hline Water & 81107 & $21 \& 24 \mathrm{~min}$, dissolved & 0.00000090 & 0.0000010 & 0.0000010 & 0.0000010 & 0.0000010 & 0.0000010 & 0.0000010 \\
\hline Water & 81108 & $27 \& 30 \mathrm{~min}$, dissolved & 0.00000076 & 0.0000010 & 0.0000010 & 0.0000010 & 0.0000010 & 0.0000010 & 0.0000010 \\
\hline Water & 80891 & $3 \& 6 \mathrm{~min}$, total & 0.00000990 & 0.0000011 & 0.0000011 & 0.0000011 & 0.0000011 & 0.0000011 & 0.0000011 \\
\hline Water & 80892 & $9 \& 12 \mathrm{~min}$, total & 0.00000250 & 0.0000010 & 0.0000010 & 0.0000010 & 0.0000010 & 0.0000010 & 0.0000088 \\
\hline Water & 80893 & $15 \& 18 \mathrm{~min}$, total & 0.00001200 & 0.0000010 & 0.0000010 & 0.0000010 & 0.0000010 & 0.0000010 & 0.0000010 \\
\hline Water & 80894 & $21 \& 24 \mathrm{~min}$, total & 0.00001400 & 0.0000011 & 0.0000011 & 0.0000011 & 0.0000011 & 0.0000011 & 0.0000066 \\
\hline \multirow[t]{2}{*}{ Water } & 80895 & $27 \& 30 \mathrm{~min}$, total & 0.00002900 & 0.0000011 & 0.0000011 & 0.0000011 & 0.0000011 & 0.0000011 & 0.0000110 \\
\hline & & Hopper Overflow Monitoring & & & & & & & \\
\hline Water & 81109 & 2\& $4 \mathrm{~min}$, dissolved & 0.00000095 & 0.0000010 & 0.0000010 & 0.0000010 & 0.0000010 & 0.0000010 & 0.0000010 \\
\hline Water & 81110 & 6\& $8 \mathrm{~min}$, dissolved & 0.00000100 & 0.0000010 & 0.0000010 & 0.0000010 & 0.0000010 & 0.0000010 & 0.0000010 \\
\hline Water & 81111 & $10 \& 12 \mathrm{~min}$, dissolved & 0.00000120 & 0.0000010 & 0.0000010 & 0.0000010 & 0.0000010 & 0.0000010 & 0.0000010 \\
\hline Water & 81112 & $14 \& 16 \mathrm{~min}$, dissolved & 0.00000095 & 0.0000010 & 0.0000010 & 0.0000010 & 0.0000010 & 0.0000010 & 0.0000010 \\
\hline Water & 81113 & $18 \& 20 \mathrm{~min}$, dissolved & 0.00000110 & 0.0000010 & 0.0000010 & 0.0000010 & 0.0000010 & 0.0000010 & 0.0000010 \\
\hline Water & 80897 & 2\& 4 min, total & 0.00003500 & 0.0000011 & 0.0000011 & 0.0000011 & 0.0000011 & 0.0000011 & 0.0000140 \\
\hline Water & 80898 & 6\& $8 \mathrm{~min}$, total & 0.00003500 & 0.0000011 & 0.0000011 & 0.0000011 & 0.0000011 & 0.0000011 & 0.0000230 \\
\hline Water & 80899 & $10 \& 12 \mathrm{~min}$, total & 0.00004100 & 0.0000011 & 0.0000011 & 0.0000011 & 0.0000011 & 0.0000011 & 0.0000150 \\
\hline Water & 80900 & $14 \& 16 \mathrm{~min}$, total & 0.00004900 & 0.0000011 & 0.0000011 & 0.0000011 & 0.0000011 & 0.0000011 & 0.0000300 \\
\hline \multirow[t]{2}{*}{ Water } & 80901 & $18 \& 20 \mathrm{~min}$, total & 0.00004100 & 0.0000010 & 0.0000010 & 0.0000010 & 0.0000010 & 0.0000010 & 0.0000170 \\
\hline & & Site Water & & & & & & & \\
\hline Water & 81603 & Sample 1 Total & 0.00000077 & 0.0000010 & 0.0000010 & 0.0000010 & 0.0000010 & 0.0000010 & 0.0000010 \\
\hline Water & 81604 & Sample 2 Total & 0.00000099 & 0.0000010 & 0.0000010 & 0.0000010 & 0.0000010 & 0.0000010 & 0.0000010 \\
\hline \multirow[t]{2}{*}{ Water } & 81605 & Sample 3 Total & 0.00000110 & 0.0000010 & 0.0000010 & 0.0000010 & 0.0000010 & 0.0000010 & 0.0000010 \\
\hline & & Elutriate & & & & & & & \\
\hline Water & 81609 & Sample 1 Dissolved & 0.00000091 & 0.0000010 & 0.0000010 & 0.0000010 & 0.0000010 & 0.0000010 & 0.0000010 \\
\hline Water & 81610 & Sample 2 Dissolved & 0.00000088 & 0.0000010 & 0.0000010 & 0.0000010 & 0.0000010 & 0.0000010 & 0.0000010 \\
\hline Water & 81611 & Sample 3 Dissolved & 0.00000057 & 0.0000010 & 0.0000010 & 0.0000010 & 0.0000010 & 0.0000010 & 0.0000010 \\
\hline Water & 81606 & Sample 1 Total & 0.00000120 & 0.0000010 & 0.0000010 & 0.0000010 & 0.0000010 & 0.0000010 & 0.0000010 \\
\hline Water & 81607 & Sample 2 Total & 0.00000130 & 0.0000010 & 0.0000010 & 0.0000010 & 0.0000010 & 0.0000010 & 0.0000010 \\
\hline Water & 81608 & Sample 3 Total & 0.00000110 & 0.0000010 & 0.0000010 & 0.0000010 & 0.0000010 & 0.0000010 & 0.0000010 \\
\hline \multirow{3}{*}{$\begin{array}{l}\text { SAMPLE } \\
\text { TYPE }\end{array}$} & SAMPLE & DESCRIPTION & PCB 110 & PCB 119 & PCB 120 & РCB 123 & РCB 126 & PCB 127 & РCB 132 \\
\hline & & Detection Limit (mg/kg) & 0.00077 & 0.00077 & 0.00077 & 0.00077 & 0.00077 & 0.00077 & 0.00077 \\
\hline & & Insitu Sediment & & & & & & & \\
\hline Sediment & 81717 & Sample \#1 & 0.00100 & 0.00077 & 0.00077 & 0.00077 & 0.00077 & 0.00077 & 0.00077 \\
\hline Sediment & 81718 & Sample \#2 & 0.00087 & 0.00077 & 0.00077 & 0.00077 & 0.00077 & 0.00077 & 0.00077 \\
\hline Sediment & 81719 & Sample \#3 & 0.00100 & 0.00077 & 0.00077 & 0.00077 & 0.00077 & 0.00077 & 0.00077 \\
\hline
\end{tabular}

BOLD - less than values

Values below less than values are estimated results. Results are less than the reporting limit 
PCBsfine

Delaware River Water Analysis (Fine-Grained Site)

\begin{tabular}{|c|c|c|c|c|c|c|c|c|c|}
\hline \multirow{2}{*}{$\begin{array}{l}\text { SAMPLE } \\
\text { TYPE }\end{array}$} & $\begin{array}{l}\text { SAMPLE } \\
\text { ID }\end{array}$ & DESCRIPTION & PCB 135 & PCB 146 & PCB 149 & PCB 157 & PCB 158 & PCB 166 & РСB 168 \\
\hline & & Detection Limit (mg/) & 0.0000011 & 0.00000110 & 0.00000110 & 0.0000011 & 0.0000011 & 0.0000011 & 0.00000110 \\
\hline & & Plume Monitoring & & & & & & & \\
\hline Water & 80983 & Background, dissolved & 0.0000010 & 0.00000100 & 0.00000100 & 0.0000010 & 0.0000010 & 0.0000010 & 0.00000100 \\
\hline Water & 80948 & Background, total & 0.0000011 & 0.00000110 & 0.00000110 & 0.0000011 & 0.0000011 & 0.0000011 & 0.00000110 \\
\hline Water & 80984 & 0-10 min, overflow, dissolved & 0.0000010 & 0.00000100 & 0.00000100 & 0.0000010 & 0.0000010 & 0.0000010 & 0.00000100 \\
\hline Water & 80985 & 10-20 min, overflow, dissolved & 0.0000011 & 0.00000110 & 0.00000110 & 0.0000011 & 0.0000011 & 0.0000011 & 0.00000110 \\
\hline Water & 80986 & 20-30 min, overflow, dissolved & 0.0000010 & 0.00000100 & 0.00000100 & 0.0000010 & 0.0000010 & 0.0000010 & 0.00000100 \\
\hline Water & 80949 & 0-10 min, overflow, total & 0.0000010 & 0.00000100 & 0.00000100 & 0.0000010 & 0.0000010 & 0.0000010 & 0.00000100 \\
\hline Water & 80950 & $10-20 \mathrm{~min}$, overflow, total & 0.0000010 & 0.00000064 & 0.00000100 & 0.0000010 & 0.0000010 & 0.0000010 & 0.00000100 \\
\hline Water & 80951 & 20-30 min, overflow, total & 0.0000010 & 0.00000057 & 0.00000100 & 0.0000010 & 0.0000010 & 0.0000010 & 0.00000100 \\
\hline Water & 80987 & 0-10 min, non-overflow, dissolve & 0.0000010 & 0.00000100 & 0.00000100 & 0.0000010 & 0.0000010 & 0.0000010 & 0.00000100 \\
\hline Water & 80988 & 10-20 min, non-overflow, dissolve & 0.0000010 & 0.00000100 & 0.00000100 & 0.0000010 & 0.0000010 & 0.0000010 & 0.00000100 \\
\hline Water & 80989 & $20-30 \mathrm{~min}$, non-overflow, dissolve & 0.0000010 & 0.00000100 & 0.00000100 & 0.0000010 & 0.0000010 & 0.0000010 & 0.00000100 \\
\hline Water & 80952 & 0-10 min, non-overflow, total & 0.0000010 & 0.00000140 & 0.00000100 & 0.0000010 & 0.0000010 & 0.0000010 & 0.00000100 \\
\hline Water & 80953 & 10-20 min, non-overflow, total & 0.0000010 & 0.00000100 & 0.00000100 & 0.0000010 & 0.0000010 & 0.0000010 & 0.00000100 \\
\hline \multirow[t]{2}{*}{ Water } & 80954 & 20-30 min, non-overflow, total & 0.0000010 & 0.00000100 & 0.00000100 & 0.0000010 & 0.0000010 & 0.0000010 & 0.00000100 \\
\hline & & Hopper Inflow Monitoring & & & & & & & \\
\hline Water & 81104 & 3\& $6 \mathrm{~min}$, dissolved & 0.0000010 & 0.00000100 & 0.00000100 & 0.0000010 & 0.0000010 & 0.0000010 & 0.00000100 \\
\hline Water & 81105 & $9 \& 12 \mathrm{~min}$, dissolved & 0.0000010 & 0.00000100 & 0.00000100 & 0.0000010 & 0.0000010 & 0.0000010 & 0.00000100 \\
\hline Water & 81106 & $15 \& 18 \mathrm{~min}$, dissolved & 0.0000010 & 0.00000100 & 0.00000100 & 0.0000010 & 0.0000010 & 0.0000010 & 0.00000100 \\
\hline Water & 81107 & $21 \& 24 \mathrm{~min}$, dissolved & 0.0000010 & 0.00000100 & 0.00000100 & 0.0000010 & 0.0000010 & 0.0000010 & 0.00000100 \\
\hline Water & 81108 & $27830 \mathrm{~min}$, dissolved & 0.0000010 & 0.00000100 & 0.00000100 & 0.0000010 & 0.0000010 & 0.0000010 & 0.00000100 \\
\hline Water & 80891 & $3 \& 6 \mathrm{~min}$, total & 0.0000011 & 0.00000260 & 0.00001100 & 0.0000011 & 0.0000011 & 0.0000011 & 0.00000110 \\
\hline Water & 80892 & $9 \& 12 \mathrm{~min}$, total & 0.0000010 & 0.00000660 & 0.00002500 & 0.0000010 & 0.0000010 & 0.0000010 & 0.00000100 \\
\hline Water & 80893 & $15 \& 18 \mathrm{~min}$, total & 0.0000010 & 0.00000460 & 0.00001800 & 0.0000010 & 0.0000010 & 0.0000010 & 0.00000100 \\
\hline Water & 80894 & $21 \& 24 \mathrm{~min}$, total & 0.0000011 & 0.00000110 & 0.00001800 & 0.0000011 & 0.0000011 & 0.0000011 & 0.00000110 \\
\hline \multirow[t]{2}{*}{ Water } & 80895 & $27 \& 30 \mathrm{~min}$, total & 0.0000076 & 0.00000110 & 0.00003200 & 0.0000011 & 0.0000011 & 0.0000011 & 0.00000110 \\
\hline & & Hopper Overflow Monitoring & & & & & & & \\
\hline Water & 81109 & 2\& $4 \mathrm{~min}$, dissolved & 0.0000010 & 0.00000100 & 0.00000100 & 0.0000010 & 0.0000010 & 0.0000010 & 0.00000100 \\
\hline Water & 81110 & 6\& $8 \mathrm{~min}$, dissolved & 0.0000010 & 0.00000100 & 0.00000043 & 0.0000010 & 0.0000010 & 0.0000010 & 0.00000100 \\
\hline Water & 81111 & $10 \& 12 \mathrm{~min}$, dissolved & 0.0000010 & 0.00000100 & 0.00000054 & 0.0000010 & 0.0000010 & 0.0000010 & 0.00000100 \\
\hline Water & 81112 & $14 \& 16 \mathrm{~min}$, dissolved & 0.0000010 & 0.00000100 & 0.00000056 & 0.0000010 & 0.0000010 & 0.0000010 & 0.00000100 \\
\hline Water & 81113 & $18 \& 20 \mathrm{~min}$, dissolved & 0.0000010 & 0.00000100 & 0.00000100 & 0.0000010 & 0.0000010 & 0.0000010 & 0.00000040 \\
\hline Water & 80897 & 2\& $4 \mathrm{~min}$, total & 0.0000088 & 0.00000110 & 0.00003800 & 0.0000011 & 0.0000011 & 0.0000011 & 0.00000110 \\
\hline Water & 80898 & $6 \& 8 \mathrm{~min}$, total & 0.0000083 & 0.00000110 & 0.00000110 & 0.0000011 & 0.0000011 & 0.0000011 & 0.00000110 \\
\hline Water & 80899 & $10 \& 12 \mathrm{~min}$, total & 0.0000100 & 0.00000110 & 0.00000110 & 0.0000011 & 0.0000011 & 0.0000011 & 0.00000110 \\
\hline Water & 80900 & $148.16 \mathrm{~min}$, total & 0.0000140 & 0.00000110 & 0.00000110 & 0.0000011 & 0.0000011 & 0.0000011 & 0.00000110 \\
\hline \multirow[t]{2}{*}{ Water } & 80901 & $18 \& 20 \mathrm{~min}$, total & 0.0000150 & 0.00000100 & 0.00000100 & 0.0000010 & 0.0000010 & 0.0000010 & 0.00000100 \\
\hline & & Site Water & & & & & & & \\
\hline Water & 81603 & Sample 1 Total & 0.0000010 & 0.00000100 & 0.00000051 & 0.0000010 & 0.0000010 & 0.0000010 & 0.00000100 \\
\hline Water & 81604 & Sample 2 Total & 0.0000010 & 0.00000100 & 0.00000053 & 0.0000010 & 0.0000010 & 0.0000010 & 0.00000056 \\
\hline \multirow[t]{2}{*}{ Water } & 81605 & Sample 3 Total & 0.0000010 & 0.00000100 & 0.00000055 & 0.0000010 & 0.0000010 & 0.0000010 & 0.00000043 \\
\hline & & Elutriate & & & & & & & \\
\hline Water & 81609 & Sample 1 Dissolved & 0.0000010 & 0.00000100 & 0.00000047 & 0.0000010 & 0.0000010 & 0.0000010 & 0.00000140 \\
\hline Water & 81610 & Sample 2 Dissolved & 0.0000010 & 0.00000100 & 0.00000046 & 0.0000010 & 0.0000010 & 0.0000010 & 0.00000100 \\
\hline Water & 81611 & Sample 3 Dissolved & 0.0000010 & 0.00000100 & 0.00000044 & 0.0000010 & 0.0000010 & 0.0000010 & 0.00000100 \\
\hline Water & 81606 & Sample 1 Total & 0.0000010 & 0.00000100 & 0.00000091 & 0.0000010 & 0.0000010 & 0.0000010 & 0.00000100 \\
\hline Water & 81607 & Sample 2 Total & 0.0000010 & 0.00000100 & 0.00000090 & 0.0000010 & 0.0000010 & 0.0000010 & 0.00000100 \\
\hline Water & 81608 & Sample 3 Total & 0.0000010 & 0.00000100 & 0.00000076 & 0.0000010 & 0.0000010 & 0.0000010 & 0.00000100 \\
\hline \multirow{3}{*}{$\begin{array}{l}\text { SAMPLE } \\
\text { TYPE }\end{array}$} & SAMPLE & DESCRIPTION & PCB 135 & PCB 146 & PCB 149 & PCB 157 & PCB 158 & РCB 166 & РСВ 168 \\
\hline & & Detection Limit (mg/kg) & 0.00077 & 0.00077 & 0.00077 & 0.00077 & 0.00077 & 0.00077 & 0.00077 \\
\hline & & Insitu Sediment & & & & & & & \\
\hline Sediment & 81717 & Sample \#1 & 0.00077 & 0.00077 & 0.00120 & 0.00077 & 0.00077 & 0.00077 & 0.00077 \\
\hline Sediment & 81718 & Sample \#2 & 0.00077 & 0.00077 & 0.00120 & 0.00077 & 0.00077 & 0.00077 & 0.00077 \\
\hline Sediment & 81719 & Sample \#3 & 0.00077 & 0.00077 & 0.00100 & 0.00077 & 0.00077 & 0.00077 & 0.00077 \\
\hline
\end{tabular}

BOLD - less than values

Values below less than values are estimated results. Results are less than the reporting limit. 
PCBsfine

Delaware River Water Analysis (Fine-Grained Site)

\begin{tabular}{|c|c|c|c|c|c|c|c|c|c|}
\hline \multirow{2}{*}{$\begin{array}{l}\text { SAMPLE } \\
\text { TYPE }\end{array}$} & $\begin{array}{l}\text { SAMPLE } \\
\text { ID }\end{array}$ & DESCRIPTION & PCB 169 & PCB 174 & PCB 177 & PCB 178 & РСВ 179 & РCB 8 & PCB 18 \\
\hline & & Detection Limit (mg/l) & 0.0000011 & 0,00000110 & 0.0000011 & 0.0000011 & 0.0000011 & 0.0000011 & 0.0000011 \\
\hline & & Plume Monitoring & & & & & & & \\
\hline Water & 80983 & Background, dissolved & 0.0000010 & 0.00000100 & 0.0000010 & 0.0000010 & 0.0000010 & 0.0000010 & 0.0000010 \\
\hline Water & 80948 & Background, total & 0.0000011 & 0.00000110 & 0.0000011 & 0.0000011 & 0.0000011 & 0.0000011 & 0.0000011 \\
\hline Water & 80984 & 0-10 min, overflow, dissolved & 0.0000010 & 0.00000100 & 0.0000010 & 0.0000010 & 0.0000010 & 0.0000010 & 0.0000010 \\
\hline Water & 80985 & 10-20 min, overflow, dissolved & 0.0000011 & 0.00000110 & 0.0000011 & 0.0000011 & 0.0000011 & 0.0000011 & 0.0000011 \\
\hline Water & 80986 & 20-30 min, overflow, dissolved & 0.0000010 & 0.00000100 & 0.0000010 & 0.0000010 & 0.0000010 & 0.0000010 & 0.0000010 \\
\hline Water & 80949 & 0-10 min, overflow, total & 0.0000010 & 0.00000100 & 0.0000010 & 0.0000010 & 0.0000010 & 0.0000010 & 0.0000010 \\
\hline Water & 80950 & 10-20 min, overflow, total & 0.0000010 & 0.00000100 & 0.0000010 & 0.0000010 & 0.0000010 & 0.0000010 & 0.0000010 \\
\hline Water & 80951 & 20-30 min, overflow, total & 0.0000010 & 0.00000100 & 0.0000010 & 0.0000010 & 0.0000010 & 0.0000010 & 0.0000010 \\
\hline Water & 80987 & 0-10 min, non-overflow, dissolve & 0.0000010 & 0.00000100 & 0.0000010 & 0.0000010 & 0.0000010 & 0.0000010 & 0.0000010 \\
\hline Water & 80988 & 10-20 min, non-overflow, dissolve & 0.0000010 & 0.00000100 & 0.0000010 & 0.0000010 & 0.0000010 & 0.0000010 & 0.0000010 \\
\hline Water & 80989 & 20-30 min, non-overflow, dissolve & 0.0000010 & 0.00000100 & 0.0000010 & 0.0000010 & 0.0000010 & 0.0000010 & 0.0000010 \\
\hline Water & 80952 & 0-10 min, non-overflow, total & 0.0000010 & 0.00000100 & 0.0000010 & 0.0000010 & 0.0000010 & 0.0000010 & 0.0000010 \\
\hline Water & 80953 & 10-20 min, non-overflow, total & 0.0000010 & 0.00000100 & 0.0000010 & 0.0000010 & 0.0000010 & 0.0000010 & 0.0000010 \\
\hline \multirow[t]{2}{*}{ Water } & 80954 & 20-30 min, non-overflow, total & 0.0000010 & 0.00000100 & 0.0000010 & 0.0000010 & 0.0000010 & 0.0000010 & 0.0000015 \\
\hline & & Hopper Inflow Monitoring & & & & & & & \\
\hline Water & 81104 & 3\& $6 \mathrm{~min}$, dissolved & 0.0000010 & 0.00000100 & 0.0000010 & 0.0000010 & 0.0000010 & 0.0000010 & 0.0000010 \\
\hline Water & 81105 & $9 \& 12 \mathrm{~min}$, dissolved & 0.0000010 & 0.00000100 & 0.0000010 & 0.0000010 & 0.0000010 & 0.0000010 & 0.0000010 \\
\hline Water & 81106 & $15 \& 18 \mathrm{~min}$, dissolved & 0.0000010 & 0.00000100 & 0.0000010 & 0.0000010 & 0.0000010 & 0.0000010 & 0.0000010 \\
\hline Water & 81107 & $21 \& 24 \mathrm{~min}$, dissolved & 0.0000010 & 0.00000100 & 0.0000010 & 0.0000010 & 0.0000010 & 0.0000010 & 0.0000010 \\
\hline Water & 81108 & $27830 \mathrm{~min}$, dissolved & 0.0000010 & 0.00000100 & 0.0000010 & 0.0000010 & 0.0000010 & 0.0000010 & 0.0000010 \\
\hline Water & 80891 & $3 \& 6 \mathrm{~min}$, total & 0.0000011 & 0.00000390 & 0.0000024 & 0.0000011 & 0.0000011 & 0.0000011 & 0.0000011 \\
\hline Water & 80892 & $9 \& 12 \mathrm{~min}$, total & 0.0000010 & 0.00000970 & 0.0000067 & 0.0000010 & 0.0000010 & 0.0000010 & 0.0000010 \\
\hline Water & 80893 & $15 \& 18 \mathrm{~min}$, total & 0.0000010 & 0.00000620 & 0.0000042 & 0.0000010 & 0.0000010 & 0.0000010 & 0.0000010 \\
\hline Water & 80894 & $21 \& 24 \mathrm{~min}$, total & 0.0000011 & 0.00000820 & 0.0000023 & 0.0000011 & 0.0000017 & 0.0000011 & 0.0000051 \\
\hline \multirow[t]{2}{*}{ Water } & 80895 & $27 \& 30 \mathrm{~min}$, total & 0.0000011 & 0.00000110 & 0.0000011 & 0.0000011 & 0.0000069 & 0.0000063 & 0.0000011 \\
\hline & & Hopper Overflow Monitoring & & & & & & & \\
\hline Water & 81109 & $2 \& 4 \mathrm{~min}$, dissolved & 0.0000010 & 0.00000049 & 0.0000010 & 0.0000010 & 0.0000010 & 0.0000010 & 0.0000010 \\
\hline Water & 81110 & 6\& $8 \mathrm{~min}$, dissolved & 0.0000010 & 0.00000100 & 0.0000010 & 0.0000010 & 0.0000010 & 0.0000010 & 0.0000010 \\
\hline Water & 81111 & $10 \& 12 \mathrm{~min}$, dissolved & 0.0000010 & 0.00000100 & 0.0000010 & 0.0000010 & 0.0000010 & 0.0000010 & 0.0000010 \\
\hline Water & 81112 & $14 \& 16 \mathrm{~min}$, dissolved & 0.0000010 & 0.00000100 & 0.0000010 & 0.0000010 & 0.0000010 & 0.0000010 & 0.0000010 \\
\hline Water & 81113 & $18 \& 20 \mathrm{~min}$, dissolved & 0.0000010 & 0.00000100 & 0.0000010 & 0.0000010 & 0.0000010 & 0.0000010 & 0.0000010 \\
\hline Water & 80897 & $2 \& 4 \mathrm{~min}$, total & 0.0000011 & 0.00000110 & 0.0000011 & 0.0000011 & 0.0000011 & 0.0000011 & 0.0000011 \\
\hline Water & 80898 & 6\& $8 \mathrm{~min}$, total & 0.0000011 & 0.00001700 & 0.0000011 & 0.0000011 & 0.0000011 & 0.0000011 & 0.0000011 \\
\hline Water & 80899 & $10 \& 12 \mathrm{~min}$, total & 0.0000011 & 0.00001700 & 0.0000011 & 0.0000011 & 0.0000011 & 0.0000140 & 0.0000011 \\
\hline Water & 80900 & $14 \& 16 \mathrm{~min}$, total & 0.0000011 & 0.00000110 & 0.0000011 & 0.0000011 & 0.0000011 & 0.0000011 & 0.0000011 \\
\hline \multirow[t]{2}{*}{ Water } & 80901 & $18 \& 20 \mathrm{~min}$, total & 0.0000010 & 0.00000100 & 0.0000010 & 0.0000010 & 0.0000010 & 0.0000010 & 0.0000010 \\
\hline & & Site Water & & & & & & & \\
\hline Water & 81603 & Sample 1 Total & 0.0000010 & 0.00000100 & 0.0000010 & 0.0000010 & 0.0000010 & 0.0000010 & 0.0000010 \\
\hline Water & 81604 & Sample 2 Total & 0.0000010 & 0.00000100 & 0.0000010 & 0.0000010 & 0.0000010 & 0.0000010 & 0.0000010 \\
\hline \multirow[t]{2}{*}{ Water } & 81605 & Sample 3 Total & 0.0000010 & 0.00000100 & 0.0000010 & 0.0000010 & 0.0000010 & 0.0000010 & 0.0000015 \\
\hline & & Elutriate & & & & & & & \\
\hline Water & 81609 & Sample 1 Dissolved & 0.0000010 & 0.00000100 & 0.0000010 & 0.0000010 & 0.0000010 & 0.0000010 & 0.0000010 \\
\hline Water & 81610 & Sample 2 Dissolved & 0.0000010 & 0.00000100 & 0.0000010 & 0.0000010 & 0.0000010 & 0.0000010 & 0.0000010 \\
\hline Water & 81611 & Sample 3 Dissolved & 0.0000010 & 0.00000100 & 0.0000010 & 0.0000010 & 0.0000010 & 0.0000010 & 0.0000010 \\
\hline Water & 81606 & Sample 1 Total & 0.0000010 & 0.00000100 & 0.0000010 & 0.0000010 & 0.0000010 & 0.0000010 & 0.0000010 \\
\hline Water & 81607 & Sample 2 Total & 0.0000010 & 0.00000100 & 0.0000010 & 0.0000010 & 0.0000010 & 0.0000010 & 0.0000010 \\
\hline Water & 81608 & Sample 3 Total & 0.0000010 & 0.00000100 & 0.0000010 & 0.0000010 & 0.0000010 & 0.0000010 & 0.0000010 \\
\hline \multirow{3}{*}{$\begin{array}{l}\text { SAMPLE } \\
\text { TYPE }\end{array}$} & $\begin{array}{l}\text { SAMPLE } \\
\text { ID }\end{array}$ & DESCRIPTION & PCB 169 & РСВ 174 & PCB 177 & РСВ 178 & РСВ 179 & РCB 8 & PCB 18 \\
\hline & & Detection Limit $(\mathrm{mg} / \mathrm{kg})$ & 0.00077 & 0.00077 & 0.00077 & 0.00077 & 0.00077 & 0.00077 & 0.00077 \\
\hline & & Insitu Sediment & & & & & & & \\
\hline Sediment & 81717 & Sample \#1 & 0.00077 & 0.00077 & 0.00077 & 0.00077 & 0.00077 & 0.00077 & 0.00077 \\
\hline Sediment & 81718 & Sample \#2 & 0.00077 & 0.00077 & 0.00077 & 0.00077 & 0.00077 & 0.00077 & 0.00077 \\
\hline Sedimen & 81719 & Sample \#3 & 0.00077 & 0.00077 & 0.00077 & 0.00077 & 0.00077 & 0.00077 & 0.00077 \\
\hline
\end{tabular}

BOLD - less than values

Values below less than values are estimated results. Results are less than the reporting limit

Page 5 
PCBsfine

Delaware River Water Analysis (Fine-Grained Site)

\begin{tabular}{|c|c|c|c|c|c|c|c|c|c|}
\hline \multirow{2}{*}{$\begin{array}{l}\text { SAMPLE } \\
\text { TYPE }\end{array}$} & $\begin{array}{l}\text { SAMPLE } \\
\text { ID }\end{array}$ & DESCRIPTION & РCB 28 & РCB 31 & PCB 40 & РCB 44 & РCB 49 & PCB 52 & PCB 60 \\
\hline & & Detection Limit (mg/l) & 0.0000011 & 0.0000011 & 0.0000011 & 0.0000011 & 0.00000110 & 0.00000110 & 0.00000110 \\
\hline & & Plume Monitoring & & & & & & & \\
\hline Water & 80983 & Background, dissolved & 0.0000010 & 0.0000017 & 0.0000010 & 0.0000010 & 0.00000100 & 0.00000046 & 0.00000100 \\
\hline Water & 80948 & Background, total & 0.0000011 & 0.0000011 & 0.0000011 & 0.0000011 & 0.00000110 & 0.00000110 & 0.00000110 \\
\hline Water & 80984 & 0-10 min, overflow, dissolved & 0.0000010 & 0.0000010 & 0.0000010 & 0.0000010 & 0.00000100 & 0.00000100 & 0.00000100 \\
\hline Water & 80985 & 10-20 min, overflow, dissolved & 0.0000011 & 0.0000011 & 0.0000011 & 0.0000011 & 0.00000110 & 0.00000110 & 0.00000110 \\
\hline Water & 80986 & 20-30 min, overflow, dissolved & 0.0000010 & 0.0000010 & 0.0000010 & 0.0000010 & 0.00000100 & 0.00000100 & 0.00000100 \\
\hline Water & 80949 & 0-10 min, overflow, total & 0.0000010 & 0.0000010 & 0.0000010 & 0.0000010 & 0.00000100 & 0.00000120 & 0.00000100 \\
\hline Water & 80950 & 10-20 min, overflow, total & 0.0000010 & 0.0000010 & 0.0000010 & 0.0000010 & 0.00000100 & 0.00000095 & 0.00000100 \\
\hline Water & 80951 & $20-30 \mathrm{~min}$, overflow, total & 0.0000010 & 0.0000010 & 0.0000010 & 0.0000010 & 0.00000100 & 0.00000077 & 0.00000100 \\
\hline Water & 80987 & 0-10 $\mathrm{min}$, non-overflow, dissolve & 0.0000010 & 0.0000010 & 0.0000010 & 0.0000010 & 0.00000085 & 0.00000100 & 0.00000100 \\
\hline Water & 80988 & 10-20 $\mathrm{min}$, non-overflow, dissolve & 0.0000010 & 0.0000010 & 0.0000010 & 0.0000010 & 0.00000044 & 0.00000100 & 0.00000100 \\
\hline Water & 80989 & 20-30 min, non-overflow, dissolve & 0.0000010 & 0.0000010 & 0.0000010 & 0.0000010 & 0.00000100 & 0.00000100 & 0.00000100 \\
\hline Water & 80952 & 0-10 min, non-overflow, total & 0.0000010 & 0.0000010 & 0.0000010 & 0.0000010 & 0.00000100 & 0.00000097 & 0.00000100 \\
\hline Water & 80953 & 10-20 min, non-overflow, total & 0.0000010 & 0.0000010 & 0.0000010 & 0.0000010 & 0.00000100 & 0.00000100 & 0.00000100 \\
\hline \multirow[t]{2}{*}{ Water } & 80954 & $20-30 \mathrm{~min}$, non-overflow, total & 0.0000010 & 0.0000010 & 0.0000010 & 0.0000010 & 0.00000100 & 0.00000074 & 0.00000100 \\
\hline & & Hopper Inflow Monitoring & & & & & & & \\
\hline Water & 81104 & $3 \& 6 \mathrm{~min}$, dissolved & 0.0000010 & 0.0000010 & 0.0000010 & 0.0000010 & 0.00000100 & 0.00000083 & 0.00000100 \\
\hline Water & 81105 & $9 \& 12 \mathrm{~min}$, dissolved & 0.0000010 & 0.0000010 & 0.0000010 & 0.0000010 & 0.00000100 & 0.00000093 & 0.00000100 \\
\hline Water & 81106 & $15 \& 18 \mathrm{~min}$, dissolved & 0.0000010 & 0.0000029 & 0.0000010 & 0.0000014 & 0.00000072 & 0.00000094 & 0.00000100 \\
\hline Water & 81107 & $21 \& 24 \mathrm{~min}$, dissolved & 0.0000010 & 0.0000010 & 0.0000010 & 0.0000010 & 0.00000180 & 0.00000080 & 0.00000100 \\
\hline Water & 81108 & $27 \& 30 \mathrm{~min}$, dissolved & 0.0000010 & 0.0000010 & 0.0000010 & 0.0000010 & 0.00000046 & 0.00000070 & 0.00000100 \\
\hline Water & 80891 & $3 \& 6 \mathrm{~min}$, total & 0.0000067 & 0.0000011 & 0.0000011 & 0.0000057 & 0.00000410 & 0.00001100 & 0.00000040 \\
\hline Water & 80892 & $9 \& 12 \mathrm{~min}$, total & 0.0000080 & 0.0000010 & 0.0000010 & 0.0000150 & 0.00000960 & 0.00002500 & 0.00000130 \\
\hline Water & 80893 & $15 \& 18 \mathrm{~min}$, total & 0.0000080 & 0.0000010 & 0.0000010 & 0.0000092 & 0.00000630 & 0.00001600 & 0.00000076 \\
\hline Water & 80894 & $21 \& 24$ min, total & 0.0000067 & 0.0000011 & 0.0000041 & 0.0000080 & 0.00000600 & 0.00001500 & 0.00000075 \\
\hline \multirow[t]{2}{*}{ Water } & 80895 & $27830 \mathrm{~min}$, total & 0.0000130 & 0.0000011 & 0.0000079 & 0.0000170 & 0.00001200 & 0.00002600 & 0.00000150 \\
\hline & & Hopper Overflow Monitoring & & & & & & & \\
\hline Water & 81109 & 2\& $4 \mathrm{~min}$, dissolved & 0.0000010 & 0.0000031 & 0.0000010 & 0.0000010 & 0.00000110 & 0.00000086 & 0.00000100 \\
\hline Water & 81110 & 6\& $8 \mathrm{~min}$, dissolved & 0.0000010 & 0.0000010 & 0.0000010 & 0.0000010 & 0.00000047 & 0.00000082 & 0.00000100 \\
\hline Water & 81111 & $10 \& 12 \mathrm{~min}$, dissolved & 0.0000010 & 0.0000038 & 0.0000010 & 0.0000010 & 0.00000077 & 0.00000120 & 0.00000100 \\
\hline Water & 81112 & $14 \& 16 \mathrm{~min}$, dissolved & 0.0000010 & 0.0000029 & 0.0000010 & 0.0000010 & 0.00000065 & 0.00000110 & 0.00000100 \\
\hline Water & 81113 & $18 \& 20 \mathrm{~min}$, dissolved & 0.0000010 & 0.0000037 & 0.0000010 & 0.0000010 & 0.00000060 & 0.00000140 & 0.00000100 \\
\hline Water & 80897 & 2\& $4 \mathrm{~min}$, total & 0.0001200 & 0.0000011 & 0.0000011 & 0.0000160 & 0.00001400 & 0.00003600 & 0.00002000 \\
\hline Water & 80898 & 6\& $8 \mathrm{~min}$, total & 0.0000011 & 0.0000011 & 0.0000088 & 0.0000200 & 0.00001400 & 0.00003400 & 0.00000190 \\
\hline Water & 80899 & $10 \& 12 \mathrm{~min}$, total & 0.0000130 & 0.0000011 & 0.0000120 & 0.0000260 & 0.00001600 & 0.00003700 & 0.00000220 \\
\hline Water & 80900 & $14 \& 16 \mathrm{~min}$, total & 0.0000180 & 0.0000011 & 0.0000140 & 0.0000300 & 0.00002000 & 0.00004300 & 0.00000270 \\
\hline \multirow[t]{2}{*}{ Water } & 80901 & $18 \& 20 \mathrm{~min}$, total & 0.0000180 & 0.0000010 & 0.0000130 & 0.0000270 & 0.00001800 & 0.00003900 & 0.00000270 \\
\hline & & Site Water & & & & & & & \\
\hline Water & 81603 & Sample 1 Total & 0.0000010 & 0.0000024 & 0.0000010 & 0.0000014 & 0.00000065 & 0.00000092 & 0.00000100 \\
\hline Water & 81604 & Sample 2 Total & 0.0000010 & 0.0000022 & 0.0000010 & 0.0000015 & 0.00000100 & 0.00000100 & 0.00000100 \\
\hline \multirow[t]{2}{*}{ Water } & 81605 & Sample 3 Total & 0.0000010 & 0.0000025 & 0.0000010 & 0.0000014 & 0.00000047 & 0.00000110 & 0.00000100 \\
\hline & & Elutriate & & & & & & & \\
\hline Water & 81609 & Sample 1 Dissolved & 0.0000010 & 0.0000010 & 0.0000010 & 0.0000010 & 0.00000100 & 0.00000100 & 0.00000100 \\
\hline Water & 81610 & Sample 2 Dissolved & 0.0000010 & 0.0000010 & 0.0000010 & 0.0000010 & 0.00000100 & 0.00000077 & 0.00000100 \\
\hline Water & 81611 & Sample 3 Dissolved & 0.0000010 & 0.0000010 & 0.0000010 & 0.0000010 & 0.00000100 & 0.00000054 & 0.00000100 \\
\hline Water & 81606 & Sample 1 Total & 0.0000010 & 0.0000039 & 0.0000010 & 0.0000010 & 0.00000300 & 0.00000150 & 0.00000100 \\
\hline Water & 81607 & Sample 2 Total & 0.0000010 & 0.0000010 & 0.0000010 & 0.0000010 & 0.00000330 & 0.00000180 & 0.00000100 \\
\hline Water & 81608 & Sample 3 Total & 0.0000010 & 0.0000010 & 0.0000010 & 0.0000010 & 0.00000290 & 0.00000160 & 0.00000100 \\
\hline \multirow{3}{*}{$\begin{array}{l}\text { SAMPLE } \\
\text { TYPE }\end{array}$} & SAMPLE & DESCRIPTION & РCB 28 & РСB 31 & РCB 40 & PCB 44 & РСВ 49 & PCB 52 & PCB 60 \\
\hline & & Detection Limit (mg/kg) & 0.00077 & 0.00077 & 0.00077 & 0.00077 & 0.00077 & 0.00077 & 0.00077 \\
\hline & & Insitu Sediment & & & & & & & \\
\hline Sediment & 81717 & Sample \#1 & 0.00077 & 0.00077 & 0.00077 & 0.00077 & 0.00061 & 0.00250 & 0.00057 \\
\hline Sediment & 81718 & Sample \#2 & 0.00077 & 0.00077 & 0.00077 & 0.00077 & 0.00081 & 0.00250 & 0.00047 \\
\hline Sediment & 81719 & Sample \#3 & 0.00077 & 0.00077 & 0.00077 & 0.00077 & 0.00069 & 0.00240 & 0.00039 \\
\hline
\end{tabular}

BOLD - less than values

Values below less than values are estimated results. Results are less than the reporting limit. 
PCBsfine

Delaware River Water Analysis (Fine-Grained Site)

\begin{tabular}{|c|c|c|c|c|c|c|c|c|c|}
\hline \multirow[t]{2}{*}{$\begin{array}{l}\text { SAMPLE } \\
\text { TYPE }\end{array}$} & $\begin{array}{l}\text { SAMPLE } \\
\text { ID }\end{array}$ & DESCRIPTION & PCB 70 & РCB 77 & PCB 82 & PCB 86 & PCB 87 & РCB 97 & РCB 101 \\
\hline & & Detection Limit (mg/l) & 0.00000110 & 0.0000011 & 0.0000011 & 0.00000110 & 0.00000110 & 0.00000110 & 0.00000110 \\
\hline & & Plume Monitoring & & & & & & & \\
\hline Water & 80983 & Background, dissolved & 0.00000040 & 0.0000010 & 0.0000010 & 0.00000100 & 0.00000100 & 0.00000100 & 0.00000110 \\
\hline Water & 80948 & Background, total & 0.00000110 & 0.0000011 & 0.0000011 & 0.00000057 & 0.00000110 & 0.00000057 & 0.00000160 \\
\hline Water & 80984 & 0-10 min, overflow, dissolved & 0.00000100 & 0.0000010 & 0.0000010 & 0.00000100 & 0.00000100 & 0.00000100 & 0.00000064 \\
\hline Water & 80985 & 10-20 min, overflow, dissolved & 0.00000051 & 0.0000011 & 0.0000011 & 0.00000110 & 0.00000110 & 0.00000110 & 0.00000093 \\
\hline Water & 80986 & 20-30 min, overflow, dissolved & 0.00000044 & 0.0000010 & 0.0000010 & 0.00000100 & 0.00000100 & 0.00000100 & 0.00000074 \\
\hline Water & 80949 & 0-10 min, overflow, total & 0.00000100 & 0.0000010 & 0.0000010 & 0.00000100 & 0.00000100 & 0.00000100 & 0.00000180 \\
\hline Water & 80950 & 10-20 min, overflow, total & 0.00000048 & 0.0000010 & 0.0000010 & 0.00000063 & 0.00000100 & 0.00000063 & 0.00000200 \\
\hline Water & 80951 & 20-30 min, overflow, total & 0.00000038 & 0.0000010 & 0.0000010 & 0.00000053 & 0.00000100 & 0.00000053 & 0.00000190 \\
\hline Water & 80987 & 0-10 min, non-overflow, dissolve & 0.00000037 & 0.0000010 & 0.0000010 & 0.00000100 & 0.00000100 & 0.00000100 & 0.00000093 \\
\hline Water & 80988 & 10-20 min, non-overflow, dissolve & 0.00000050 & 0.0000010 & 0.0000010 & 0.00000100 & 0.00000100 & 0.00000100 & 0.00000085 \\
\hline Water & 80989 & 20-30 min, non-overflow, dissolve & 0.00000056 & 0.0000010 & 0.0000010 & 0.00000100 & 0.00000100 & 0.00000100 & 0.00000091 \\
\hline Water & 80952 & 0-10 min, non-overflow, total & 0.00000041 & 0.0000010 & 0.0000010 & 0.00000041 & 0.00000100 & 0.00000041 & 0.00000150 \\
\hline Water & 80953 & 10-20 min, non-overflow, total & 0.00000038 & 0.0000010 & 0.0000010 & 0.00000047 & 0.00000100 & 0.00000047 & 0.00000230 \\
\hline \multirow[t]{2}{*}{ Water } & 80954 & 20-30 $\mathrm{min}$, non-overflow, total & 0.00000038 & 0.0000010 & 0.0000010 & 0.00000043 & 0.00000054 & 0.00000043 & 0.00000190 \\
\hline & & Hopper Inflow Monitoring & & & & & & & \\
\hline Water & 81104 & $3 \& 6 \mathrm{~min}$, dissolved & 0.00000062 & 0.0000010 & 0.0000010 & 0.00000100 & 0.00000038 & 0.00000100 & 0.00000120 \\
\hline Water & 81105 & $9 \& 12 \mathrm{~min}$, dissolved & 0.00000042 & 0.0000010 & 0.0000010 & 0.00000100 & 0.00000100 & 0.00000100 & 0.00000061 \\
\hline Water & 81106 & $15 \& 18 \mathrm{~min}$, dissolved & 0.00000053 & 0.0000010 & 0.0000010 & 0.00000100 & 0.00000100 & 0.00000100 & 0.00000085 \\
\hline Water & 81107 & $21824 \mathrm{~min}$, dissolved & 0.00000059 & 0.0000010 & 0.0000010 & 0.00000100 & 0.00000042 & 0.00000100 & 0.00000120 \\
\hline Water & 81108 & $27 \& 30 \mathrm{~min}$, dissolved & 0.00000041 & 0.0000010 & 0.0000010 & 0.00000100 & 0.00000100 & 0.00000100 & 0.00000074 \\
\hline Water & 80891 & 3\& $6 \mathrm{~min}$, total & 0.00000420 & 0.0000011 & 0.0000014 & 0.00000300 & 0.00000310 & 0.00000300 & 0.00000900 \\
\hline Water & 80892 & $9 \& 12 \mathrm{~min}$, total & 0.00000890 & 0.0000010 & 0.0000010 & 0.00000600 & 0.00000420 & 0.00000600 & 0.00001900 \\
\hline Water & 80893 & $15 \& 18 \mathrm{~min}$, total & 0.00000660 & 0.0000010 & 0.0000010 & 0.00000420 & 0.00000290 & 0.00000420 & 0.00001100 \\
\hline Water & 80894 & $21 \& 24 \mathrm{~min}$, total & 0.00000640 & 0.0000011 & 0.0000011 & 0.00000730 & 0.00000290 & 0.00000730 & 0.00001200 \\
\hline \multirow[t]{2}{*}{ Water } & 80895 & $27 \& 30 \mathrm{~min}$, total & 0.00001100 & 0.0000011 & 0.0000011 & 0.00000730 & 0.00000110 & 0.00000730 & 0.00002500 \\
\hline & & Hopper Overflow Monitoring & & & & & & & \\
\hline Water & 81109 & 2\& 4 min, dissolved & 0.00000086 & 0.0000010 & 0.0000010 & 0.00000100 & 0.00000064 & 0.00000100 & 0.00000090 \\
\hline Water & 81110 & 6\& $8 \mathrm{~min}$, dissolved & 0.00000120 & 0.0000010 & 0.0000010 & 0.00000100 & 0.00000058 & 0.00000100 & 0.00000100 \\
\hline Water & 81111 & $10 \& 12 \mathrm{~min}$, dissolved & 0.00000084 & 0.0000010 & 0.0000010 & 0.00000100 & 0.00000065 & 0.00000100 & 0.00000120 \\
\hline Water & 81112 & $14 \& 16 \mathrm{~min}$, dissolved & 0.00000059 & 0.0000010 & 0.0000010 & 0.00000100 & 0.00000036 & 0.00000100 & 0.00000097 \\
\hline Water & 81113 & $18 \& 20 \mathrm{~min}$, dissolved & 0.00000074 & 0.0000010 & 0.0000010 & 0.00000100 & 0.00000050 & 0.00000100 & 0.00000100 \\
\hline Water & 80897 & $224 \mathrm{~min}$, total & 0.00001200 & 0.0000011 & 0.0000011 & 0.00000870 & 0.00000110 & 0.00000870 & 0.00002900 \\
\hline Water & 80898 & $6 \& 8 \mathrm{~min}$, total & 0.00001300 & 0.0000011 & 0.0000011 & 0.00000950 & 0.00000110 & 0.00000950 & 0.00002900 \\
\hline Water & 80899 & $10 \& 12 \mathrm{~min}$, total & 0.00001500 & 0.0000011 & 0.0000011 & 0.00000960 & 0.00000110 & 0.00000960 & 0.00003400 \\
\hline Water & 80900 & $14 \& 16 \mathrm{~min}$, total & 0.00001600 & 0.0000011 & 0.0000011 & 0.00001100 & 0.00000980 & 0.00001100 & 0.00004000 \\
\hline \multirow[t]{2}{*}{ Water } & 80901 & $18 \& 20 \mathrm{~min}$, total & 0.00001600 & 0.0000010 & 0.0000010 & 0.00000980 & 0.00000100 & 0.00000980 & 0.00003400 \\
\hline & & Site Water & & & & & & & \\
\hline Water & 81603 & Sample 1 Total & 0.00000100 & 0.0000010 & 0.0000010 & 0.00000100 & 0.00000054 & 0.00000100 & 0.00000110 \\
\hline Water & 81604 & Sample 2 Total & 0.00000140 & 0.0000010 & 0.0000010 & 0.00000100 & 0.00000049 & 0.00000100 & 0.00000140 \\
\hline \multirow[t]{2}{*}{ Water } & 81605 & Sample 3 Total & 0.00000130 & 0.0000010 & 0.0000010 & 0.00000100 & 0.00000038 & 0.00000100 & 0.00000150 \\
\hline & & Elutriate & & & & & & & \\
\hline Water & 81609 & Sample 1 Dissolved & 0.00000110 & 0.0000010 & 0.0000010 & 0.00000100 & 0.00000052 & 0.00000100 & 0.00000120 \\
\hline Water & 81610 & Sample 2 Dissolved & 0.00000160 & 0.0000010 & 0.0000010 & 0.00000100 & 0.00000063 & 0.00000100 & 0.00000075 \\
\hline Water & 81611 & Sample 3 Dissolved & 0.00000150 & 0.0000010 & 0.0000010 & 0.00000100 & 0.00000100 & 0.00000100 & 0.00000060 \\
\hline Water & 81606 & Sample 1 Total & 0.00000100 & 0.0000010 & 0.0000010 & 0.00000100 & 0.00000100 & 0.00000100 & 0.00000075 \\
\hline Water & 81607 & Sample 2 Total & 0.00000100 & 0.0000010 & 0.0000010 & 0.00000100 & 0.00000100 & 0.00000100 & 0.00000083 \\
\hline Water & 81608 & Sample 3 Total & 0.00000100 & 0.0000010 & 0.0000010 & 0.00000100 & 0.00000100 & 0.00000100 & 0.00000079 \\
\hline \multirow{3}{*}{$\begin{array}{l}\text { SAMPLE } \\
\text { TYPE }\end{array}$} & SAMPLE & DESCRIPTION & РCB 70 & РСB 77 & РCB 82 & РCB 86 & РCB 87 & PCB 97 & РCB 101 \\
\hline & & Detection Limit (mg/kg) & 0.00077 & 0.00077 & 0.00077 & 0.00077 & 0.00077 & 0.00077 & 0.00077 \\
\hline & & Insitu Sediment & & & & & & & \\
\hline Sediment & 81717 & Sample \#1 & 0.00210 & 0.00077 & 0.00077 & 0.00077 & 0.00077 & 0.00077 & 0.00100 \\
\hline Sediment & 81718 & Sample $\# 2$ & 0.00200 & 0.00077 & 0.00077 & 0.00077 & 0.00077 & 0.00077 & 0.00120 \\
\hline Sediment & 81719 & Sample \#3 & 0.00180 & 0.00077 & 0.00077 & 0.00077 & 0.00077 & 0.00077 & 0.00100 \\
\hline
\end{tabular}

BOLD - less than values

Values below less than values are estimated results. Results are less than the reporting limit. 
PCBsfine

Delaware River Water Analysis (Fine-Grained Site)

\begin{tabular}{|c|c|c|c|c|c|c|c|c|c|}
\hline \multirow[t]{2}{*}{$\begin{array}{l}\text { SAMPLE } \\
\text { TYPE }\end{array}$} & $\begin{array}{l}\text { SAMPLE } \\
\text { ID }\end{array}$ & DESCRIPTION & PCB 105 & PCB 114 & PCB 118 & PCB 121 & PCB 128 & PCB 136 & PCB 137 \\
\hline & & Detection Limit (mg/) & 0.00000110 & 0.00000110 & 0.00000110 & 0.0000011 & 0.0000011 & 0.0000011 & 0.0000011 \\
\hline & & Plume Monitoring & & & & & & & \\
\hline Water & 80983 & Background, dissolved & 0.00000041 & 0.00000100 & 0.00000077 & 0.0000010 & 0.0000010 & 0.0000010 & 0.0000010 \\
\hline Water & 80948 & Background, total & 0.00000110 & 0.00000110 & 0.00000110 & 0.0000011 & 0.0000011 & 0.0000011 & 0.0000011 \\
\hline Water & 80984 & 0-10 min, overflow, dissolved & 0.00000037 & 0.00000066 & 0.00000051 & 0.0000010 & 0.0000010 & 0.0000010 & 0.0000010 \\
\hline Water & 80985 & 10-20 min, overflow, dissolved & 0.00000110 & 0.00000110 & 0.00000064 & 0.0000011 & 0.0000011 & 0.0000011 & 0.0000011 \\
\hline Water & 80986 & 20-30 min, overflow, dissolved & 0.00000041 & 0.00000060 & 0.00000045 & 0.0000010 & 0.0000010 & 0.0000010 & 0.0000010 \\
\hline Water & 80949 & $0-10 \mathrm{~min}$, overflow, total & 0.00000100 & 0.00000100 & 0.00000100 & 0.0000010 & 0.0000010 & 0.0000010 & 0.0000010 \\
\hline Water & 80950 & 10-20 min, overflow, total & 0.00000100 & 0.00000100 & 0.00000100 & 0.0000010 & 0.0000010 & 0.0000010 & 0.0000010 \\
\hline Water & 80951 & 20-30 min, overflow, total & 0.00000100 & 0.00000100 & 0.00000100 & 0.0000010 & 0.0000010 & 0.0000010 & 0.0000010 \\
\hline Water & 80987 & 0-10 min, non-overflow, dissolve & 0.00000100 & 0.00000074 & 0.00000050 & 0.0000010 & 0.0000010 & 0.0000010 & 0.0000010 \\
\hline Water & 80988 & 10-20 min, non-overflow, dissolve & 0.00000040 & 0.00000100 & 0.00000046 & 0.0000010 & 0.0000010 & 0.0000010 & 0.0000010 \\
\hline Water & 80989 & 20-30 min, non-overflow, dissolve & 0.00000039 & 0.00000100 & 0.00000045 & 0.0000010 & 0.0000010 & 0.0000010 & 0.0000010 \\
\hline Water & 80952 & 0-10 min, non-overflow, total & 0.00000100 & 0.00000100 & 0.00000100 & 0.0000010 & 0.0000010 & 0.0000010 & 0.0000010 \\
\hline Water & 80953 & 10-20 $\mathrm{min}$, non-overflow, total & 0.00000100 & 0.00000100 & 0.00000100 & 0.0000010 & 0.0000010 & 0.0000010 & 0.0000010 \\
\hline \multirow[t]{2}{*}{ Water } & 80954 & 20-30 min, non-overflow, total & 0.00000100 & 0.00000100 & 0.00000100 & 0.0000010 & 0.0000010 & 0.0000010 & 0.0000010 \\
\hline & & Hopper Inflow Monitoring & & & & & & & \\
\hline Water & 81104 & 3\& $6 \mathrm{~min}$, dissolved & 0.00000041 & 0.00000100 & 0.00000054 & 0.0000010 & 0.0000010 & 0.0000010 & 0.0000010 \\
\hline Water & 81105 & $9 \& 12 \mathrm{~min}$, dissolved & 0.00000056 & 0.00000100 & 0.00000045 & 0.0000010 & 0.0000010 & 0.0000010 & 0.0000010 \\
\hline Water & 81106 & $15 \& 18 \mathrm{~min}$, dissolved & 0.00000035 & 0.00000100 & 0.00000100 & 0.0000010 & 0.0000010 & 0.0000010 & 0.0000010 \\
\hline Water & 81107 & $21 \& 24 \mathrm{~min}$, dissolved & 0.00000043 & 0.00000100 & 0.00000060 & 0.0000010 & 0.0000010 & 0.0000010 & 0.0000010 \\
\hline Water & 81108 & $27830 \mathrm{~min}$, dissolved & 0.00000037 & 0.00000100 & 0.00000100 & 0.0000010 & 0.0000010 & 0.0000010 & 0.0000010 \\
\hline Water & 80891 & 3\& $6 \mathrm{~min}$, total & 0.00000110 & 0.00000110 & 0.00000750 & 0.0000011 & 0.0000011 & 0.0000011 & 0.0000011 \\
\hline Water & 80892 & $9 \& 12 \mathrm{~min}$, total & 0.00000100 & 0.00000100 & 0.00000100 & 0.0000010 & 0.0000010 & 0.0000010 & 0.0000010 \\
\hline Water & 80893 & $15 \& 18 \mathrm{~min}$, total & 0.00000100 & 0.00000100 & 0.00000100 & 0.0000010 & 0.0000010 & 0.0000010 & 0.0000010 \\
\hline Water & 80894 & $21 \& 24 \mathrm{~min}$, total & 0.00000110 & 0.00000110 & 0.00000110 & 0.0000011 & 0.0000011 & 0.0000011 & 0.0000011 \\
\hline \multirow[t]{2}{*}{ Water } & 80895 & $27 \& 30 \mathrm{~min}$, total & 0.00000110 & 0.00000110 & 0.00001900 & 0.0000011 & 0.0000011 & 0.0000011 & 0.0000011 \\
\hline & & Hopper Overflow Monitoring & & & & & & & \\
\hline Water & 81109 & 2\& $4 \mathrm{~min}$, dissolved & 0.00000047 & 0.00000100 & 0.00000065 & 0.0000010 & 0.0000010 & 0.0000010 & 0.0000010 \\
\hline Water & 81110 & 6\& $8 \mathrm{~min}$, dissolved & 0.00000090 & 0.00000100 & 0.00000065 & 0.0000010 & 0.0000010 & 0.0000010 & 0.0000010 \\
\hline Water & 81111 & $10 \& 12 \mathrm{~min}$, dissolved & 0.00000097 & 0.00000100 & 0.00000100 & 0.0000010 & 0.0000010 & 0.0000010 & 0.0000010 \\
\hline Water & 81112 & $14 \& 16 \mathrm{~min}$, dissolved & 0.00000036 & 0.00000100 & 0.00000068 & 0.0000010 & 0.0000010 & 0.0000010 & 0.0000010 \\
\hline Water & 81113 & $18 \& 20 \mathrm{~min}$, dissolved & 0.00000055 & 0.00000100 & 0.00000094 & 0.0000010 & 0.0000010 & 0.0000010 & 0.0000010 \\
\hline Water & 80897 & $2 \& 4$ min, total & 0.00000110 & 0.00000110 & 0.00002400 & 0.0000011 & 0.0000011 & 0.0000011 & 0.0000011 \\
\hline Water & 80898 & $6 \& 8 \mathrm{~min}$, total & 0.00000110 & 0.00000110 & 0.00002300 & 0.0000011 & 0.0000011 & 0.0000011 & 0.0000011 \\
\hline Water & 80899 & $10 \& 12 \mathrm{~min}$, total & 0.00000110 & 0.00000110 & 0.00003000 & 0.0000011 & 0.0000011 & 0.0000011 & 0.0000011 \\
\hline Water & 80900 & $14 \& 16 \mathrm{~min}$, total & 0.00000110 & 0.00000110 & 0.00003800 & 0.0000011 & 0.0000011 & 0.0000011 & 0.0000011 \\
\hline \multirow[t]{2}{*}{ Water } & 80901 & $18 \& 20 \mathrm{~min}$, total & 0.00000100 & 0.00000100 & 0.00003200 & 0.0000010 & 0.0000010 & 0.0000190 & 0.0000010 \\
\hline & & Site Water & & & & & & & \\
\hline Water & 81603 & Sample 1 Total & 0.00000043 & 0.00000140 & 0.00000096 & 0.0000010 & 0.0000010 & 0.0000010 & 0.0000010 \\
\hline Water & 81604 & Sample 2 Total & 0.00000054 & 0.00000140 & 0.00000093 & 0.0000010 & 0.0000010 & 0.0000010 & 0.0000010 \\
\hline \multirow[t]{2}{*}{ Water } & 81605 & Sample 3 Total & 0.00000050 & 0.00000120 & 0.00000100 & 0.0000010 & 0.0000010 & 0.0000010 & 0.0000010 \\
\hline & & Elutriate & & & & & & & \\
\hline Water & 81609 & Sample 1 Dissolved & 0.00000067 & 0.00000100 & 0.00000058 & 0.0000010 & 0.0000010 & 0.0000010 & 0.0000010 \\
\hline Water & 81610 & Sample 2 Dissolved & 0.00000054 & 0.00000100 & 0.00000048 & 0.0000010 & 0.0000010 & 0.0000010 & 0.0000010 \\
\hline Water & 81611 & Sample 3 Dissolved & 0.00000042 & 0.00000100 & 0.00000052 & 0.0000010 & 0.0000010 & 0.0000010 & 0.0000010 \\
\hline Water & 81606 & Sample 1 Total & 0.00000068 & 0.00000100 & 0.00000120 & 0.0000010 & 0.0000010 & 0.0000010 & 0.0000010 \\
\hline Water & 81607 & Sample 2 Total & 0.00000071 & 0.00000100 & 0.00000130 & 0.0000010 & 0.0000010 & 0.0000010 & 0.0000010 \\
\hline Water & 81608 & Sample 3 Total & 0.00000064 & 0.00000100 & 0.00000100 & 0.0000010 & 0.0000010 & 0.0000010 & 0.0000010 \\
\hline \multirow{3}{*}{$\begin{array}{l}\text { SAMPLE } \\
\text { TYPE }\end{array}$} & SAMPLE & DESCRIPTION & PCB 105 & РСB 114 & PCB 118 & PCB 121 & PCB 128 & PCB 136 & РСВ 137 \\
\hline & & Detection Limit (mg/kg) & 0.00077 & 0.00077 & 0.00077 & 0.00077 & 0.00077 & 0.00077 & 0.00077 \\
\hline & & Insitu Sediment & & & & & & & \\
\hline Sediment & 81717 & Sample \#1 & 0.00077 & 0.00077 & 0.00098 & 0.00077 & 0.00077 & 0.00077 & 0.00077 \\
\hline Sediment & 81718 & Sample \#2 & 0.00077 & 0.00077 & 0.00110 & 0.00077 & 0.00077 & 0.00077 & 0.00077 \\
\hline Sediment & 81719 & Sample \#3 & 0.00077 & 0.00077 & 0.00100 & 0.00077 & 0.00077 & 0.00077 & 0.00077 \\
\hline
\end{tabular}

BOLD - less than values

Values below less than values are estimated results. Results are less than the reporting limit. 
Delaware River Water Analysis (Fine-Grained Site)

\begin{tabular}{|c|c|c|c|c|c|c|c|c|c|}
\hline \multirow[t]{2}{*}{$\begin{array}{l}\text { SAMPLE } \\
\text { TYPE }\end{array}$} & $\begin{array}{l}\text { SAMPLE } \\
\text { ID }\end{array}$ & DESCRIPTION & РСB 138 & PCB 141 & РСB 151 & PCB 153 & PCB 156 & PCB 167 & РCB 170 \\
\hline & & Detection Limit (mg/l) & 0.00000110 & 0.0000011 & 0.0000011 & 0.0000011 & 0.00000110 & 0.0000011 & 0.0000011 \\
\hline & & Plume Monitoring & & & & & & & \\
\hline Water & 80983 & Background, dissolved & 0.00000100 & 0.0000010 & 0.0000010 & 0.0000010 & 0.00000100 & 0.0000010 & 0.0000010 \\
\hline Water & 80948 & Background, total & 0.00000045 & 0.0000011 & 0.0000011 & 0.0000011 & 0.00000110 & 0.0000011 & 0.0000011 \\
\hline Water & 80984 & 0-10 min, overflow, dissolved & 0.00000100 & 0.0000010 & 0.0000010 & 0.0000010 & 0.00000030 & 0.0000010 & 0.0000010 \\
\hline Water & 80985 & $10-20 \mathrm{~min}$, overflow, dissolved & 0.00000110 & 0.0000011 & 0.0000011 & 0.0000011 & 0.00000038 & 0.0000011 & 0.0000011 \\
\hline Water & 80986 & 20-30 min, overflow, dissolved & 0.00000100 & 0.0000010 & 0.0000010 & 0.0000010 & 0.00000100 & 0.0000010 & 0.0000010 \\
\hline Water & 80949 & 0-10 min, overflow, total & 0.00000077 & 0.0000010 & 0.0000010 & 0.0000010 & 0.00000100 & 0.0000010 & 0.0000010 \\
\hline Water & 80950 & 10-20 min, overflow, total & 0.00000066 & 0.0000010 & 0.0000010 & 0.0000010 & 0.00000100 & 0.0000010 & 0.0000010 \\
\hline Water & 80951 & 20-30 min, overflow, total & 0.00000066 & 0.0000010 & 0.0000010 & 0.0000010 & 0.00000100 & 0.0000010 & 0.0000010 \\
\hline Water & 80987 & 0-10 min, non-overflow, dissolve & 0.00000100 & 0.0000010 & 0.0000010 & 0.0000010 & 0.00000100 & 0.0000010 & 0.0000010 \\
\hline Water & 80988 & 10-20 min, non-overflow, dissolve & 0.00000100 & 0.0000010 & 0.0000010 & 0.0000010 & 0.00000100 & 0.0000010 & 0.0000010 \\
\hline Water & 80989 & 20-30 min, non-overflow, dissolve & 0.00000100 & 0.0000010 & 0.0000010 & 0.0000010 & 0.00000100 & 0.0000010 & 0.0000010 \\
\hline Water & 80952 & 0-10 min, non-overflow, total & 0.00000061 & 0.0000010 & 0.0000010 & 0.0000010 & 0.00000100 & 0.0000010 & 0.0000010 \\
\hline Water & 80953 & 10-20 min, non-overflow, total & 0.00000057 & 0.0000010 & 0.0000010 & 0.0000010 & 0.00000100 & 0.0000010 & 0.0000010 \\
\hline \multirow[t]{2}{*}{ Water } & 80954 & 20-30 min, non-overflow, total & 0.00000045 & 0.0000010 & 0.0000010 & 0.0000010 & 0.00000100 & 0.0000010 & 0.0000010 \\
\hline & & Hopper Inflow Monitoring & & & & & & & \\
\hline Water & 81104 & $3 \& 6 \mathrm{~min}$, dissolved & 0.00000100 & 0.0000010 & 0.0000010 & 0.0000010 & 0.00000100 & 0.0000010 & 0.0000010 \\
\hline Water & 81105 & $9 \& 12 \mathrm{~min}$, dissolved & 0.00000100 & 0.0000010 & 0.0000010 & 0.0000010 & 0.00000100 & 0.0000010 & 0.0000010 \\
\hline Water & 81106 & $15 \& 18 \mathrm{~min}$, dissolved & 0.00000100 & 0.0000010 & 0.0000010 & 0.0000010 & 0.00000100 & 0.0000010 & 0.0000010 \\
\hline Water & 81107 & $21 \& 24 \mathrm{~min}$, dissolved & 0.00000100 & 0.0000010 & 0.0000010 & 0.0000010 & 0.00000100 & 0.0000010 & 0.0000010 \\
\hline Water & 81108 & $27 \& 30 \mathrm{~min}$, dissolved & 0.00000100 & 0.0000010 & 0.0000010 & 0.0000010 & 0.00000100 & 0.0000010 & 0.0000010 \\
\hline Water & 80891 & $3 \& 6 \mathrm{~min}$, total & 0.00001400 & 0.0000011 & 0.0000027 & 0.0000011 & 0.00000110 & 0.0000011 & 0.0000011 \\
\hline Water & 80892 & $9 \& 12 \mathrm{~min}$, total & 0.00003400 & 0.0000010 & 0.0000058 & 0.0000010 & 0.00000100 & 0.0000010 & 0.0000010 \\
\hline Water & 80893 & $15 \& 18 \mathrm{~min}$, total & 0.00002300 & 0.0000010 & 0.0000062 & 0.0000010 & 0.00000100 & 0.0000010 & 0.0000010 \\
\hline Water & 80894 & $21824 \mathrm{~min}$, total & 0.00002400 & 0.0000011 & 0.0000045 & 0.0000011 & 0.00000350 & 0.0000021 & 0.0000086 \\
\hline \multirow[t]{2}{*}{ Water } & 80895 & $27830 \mathrm{~min}$, total & 0.00000110 & 0.0000011 & 0.0000089 & 0.0000011 & 0.00000110 & 0.0000011 & 0.0000011 \\
\hline & & Hopper Overflow Monitoring & & & & & & & \\
\hline Water & 81109 & 2\& $4 \mathrm{~min}$, dissolved & 0.00000100 & 0.0000010 & 0.0000010 & 0.0000010 & 0.00000100 & 0.0000010 & 0.0000010 \\
\hline Water & 81110 & $6 \& 8 \mathrm{~min}$, dissolved & 0.00000100 & 0.0000010 & 0.0000010 & 0.0000010 & 0.00000100 & 0.0000010 & 0.0000010 \\
\hline Water & 81111 & $10 \& 12 \mathrm{~min}$, dissolved & 0.00000100 & 0.0000010 & 0.0000010 & 0.0000010 & 0.00000100 & 0.0000010 & 0.0000010 \\
\hline Water & 81112 & $14 \& 16 \mathrm{~min}$, dissolved & 0.00000100 & 0.0000010 & 0.0000010 & 0.0000010 & 0.00000100 & 0.0000010 & 0.0000010 \\
\hline Water & 81113 & $18 \& 20 \mathrm{~min}$, dissolved & 0.00000100 & 0.0000010 & 0.0000010 & 0.0000010 & 0.00000100 & 0.0000010 & 0.0000010 \\
\hline Water & 80897 & 2\& $4 \mathrm{~min}$, total & 0.00000110 & 0.0000010 & 0.0000098 & 0.0000011 & 0.00000110 & 0.0000011 & 0.0000011 \\
\hline Water & 80898 & 6\& $8 \mathrm{~min}$, total & 0.00000110 & 0.0000010 & 0.0000094 & 0.0000011 & 0.00000760 & 0.0000011 & 0.0000011 \\
\hline Water & 80899 & $10 \& 12 \mathrm{~min}$, total & 0.00000110 & 0.0000010 & 0.0000120 & 0.0000011 & 0.00000790 & 0.0000011 & 0.0000011 \\
\hline Water & 80900 & $14 \& 16 \mathrm{~min}$, total & 0.00000110 & 0.0000010 & 0.0000190 & 0.0000011 & 0.00001200 & 0.0000011 & 0.0000011 \\
\hline \multirow[t]{2}{*}{ Water } & 80901 & $18 \& 20 \mathrm{~min}$, total & 0.00000100 & 0.0000010 & 0.0000110 & 0.0000010 & 0.00000790 & 0.0000010 & 0.0000010 \\
\hline & & Site Water & & & & & & & \\
\hline Water & 81603 & Sample 1 Total & 0.00000100 & 0.0000010 & 0.0000010 & 0.0000010 & 0.00000100 & 0.0000010 & 0.0000010 \\
\hline Water & 81604 & Sample 2 Total & 0.00000100 & 0.0000010 & 0.0000010 & 0.0000010 & 0.00000100 & 0.0000010 & 0.0000010 \\
\hline \multirow[t]{2}{*}{ Water } & 81605 & Sample 3 Total & 0.00000100 & 0.0000010 & 0.0000010 & 0.0000010 & 0.00000100 & 0.0000010 & 0.0000010 \\
\hline & & Elutriate & & & & & & & \\
\hline Water & 81609 & Sample 1 Dissolved & 0.00000100 & 0.0000010 & 0.0000010 & 0.0000010 & 0.00000100 & 0.0000010 & 0.0000010 \\
\hline Water & 81610 & Sample 2 Dissolved & 0.00000100 & 0.0000010 & 0.0000010 & 0.0000010 & 0.00000100 & 0.0000010 & 0.0000010 \\
\hline Water & 81611 & Sample 3 Dissolved & 0.00000100 & 0.0000010 & 0.0000010 & 0.0000010 & 0.00000100 & 0.0000010 & 0.0000010 \\
\hline Water & 81606 & Sample 1 Total & 0.00000100 & 0.0000010 & 0.0000010 & 0.0000012 & 0.00000100 & 0.0000010 & 0.0000010 \\
\hline Water & 81607 & Sample 2 Total & 0.00000100 & 0.0000010 & 0.0000010 & 0.0000012 & 0.00000100 & 0.0000010 & 0.0000010 \\
\hline Water & 81608 & Sample 3 Total & 0.00000100 & 0.0000010 & 0.0000010 & 0.0000013 & 0.00000100 & 0.0000010 & 0.0000010 \\
\hline \multirow{3}{*}{$\begin{array}{l}\text { SAMPLE } \\
\text { TYPE }\end{array}$} & SAMPLE & DESCRIPTION & PCB 138 & РСB 141 & РСВ 151 & РCB 153 & PCB 156 & PCB 167 & PCB 170 \\
\hline & & Detection Limit ( $\mathrm{mg} / \mathrm{kg})$ & 0.00077 & 0.00077 & 0.00077 & 0.00077 & 0.00077 & 0.00077 & 0.00077 \\
\hline & & Insitu Sediment & & & & & & & \\
\hline Sediment & 81717 & Sample \#1 & 0.00077 & 0.00077 & 0.00077 & 0.00150 & 0.00077 & 0.00077 & 0.00077 \\
\hline Sediment & 81718 & Sample \#2 & 0.00077 & 0.00077 & 0.00077 & 0.00077 & 0.00077 & 0.00077 & 0.00077 \\
\hline Sediment & 81719 & Sample \#3 & 0.00077 & 0.00077 & 0.00077 & 0.00077 & 0.00077 & 0.00077 & 0.00077 \\
\hline
\end{tabular}

BOLD - less than value

Values below less than values are estimated results. Results are less than the reporting limit. 
Delaware River Water Analysis (Fine-Grained Site)

\begin{tabular}{|c|c|c|c|c|c|c|c|c|c|}
\hline \multirow[t]{2}{*}{$\begin{array}{l}\text { SAMPLE } \\
\text { TYPE }\end{array}$} & $\begin{array}{l}\text { SAMPLE } \\
\text { ID }\end{array}$ & DESCRIPTION & PCB 171 & PCB 180 & PCB 182 & РCB 183 & РCB 185 & PCB 187 & PCB 189 \\
\hline & & Detection Limit (mg/l) & 0.0000011 & 0.0000011 & 0.0000011 & 0.0000011 & 0.0000011 & 0.0000011 & 0.0000011 \\
\hline & & Plume Monitoring & & & & & & & \\
\hline Water & 80983 & Background, dissolved & 0.0000010 & 0.0000010 & 0.0000010 & 0.0000010 & 0.0000010 & 0.0000010 & 0.0000010 \\
\hline Water & 80948 & Background, total & 0.0000011 & 0.0000011 & 0.0000011 & 0.0000011 & 0.0000011 & 0.0000011 & 0.0000011 \\
\hline Water & 80984 & 0-10 min, overflow, dissolved & 0.0000010 & 0.0000010 & 0.0000010 & 0.0000010 & 0.0000010 & 0.0000010 & 0.0000010 \\
\hline Water & 80985 & 10-20 min, overflow, dissolved & 0.0000011 & 0.0000011 & 0.0000011 & 0.0000011 & 0.0000011 & 0.0000011 & 0.0000011 \\
\hline Water & 80986 & 20-30 min, overflow, dissolved & 0.0000010 & 0.0000010 & 0.0000010 & 0.0000010 & 0.0000010 & 0.0000010 & 0.0000010 \\
\hline Water & 80949 & 0-10 min, overflow, total & 0.0000010 & 0.0000010 & 0.0000010 & 0.0000010 & 0.0000010 & 0.0000010 & 0.0000010 \\
\hline Water & 80950 & 10-20 min, overflow, total & 0.0000010 & 0.0000010 & 0.0000010 & 0.0000010 & 0.0000010 & 0.0000010 & 0.0000010 \\
\hline Water & 80951 & 20-30 min, overflow, total & 0.0000010 & 0.0000010 & 0.0000010 & 0.0000010 & 0.0000010 & 0.0000010 & 0.0000010 \\
\hline Water & 80987 & 0-10 min, non-overflow, dissolve & 0.0000010 & 0.0000010 & 0.0000010 & 0.0000010 & 0.0000010 & 0.0000010 & 0.0000010 \\
\hline Water & 80988 & 10-20 min, non-overflow, dissolve & 0.0000010 & 0.0000010 & 0.0000010 & 0.0000010 & 0.0000010 & 0.0000010 & 0.0000010 \\
\hline Water & 80989 & 20-30 min, non-overflow, dissolve & 0.0000010 & 0.0000010 & 0.0000010 & 0.0000010 & 0.0000010 & 0.0000010 & 0.0000010 \\
\hline Water & 80952 & 0-10 min, non-overflow, total & 0.0000010 & 0.0000010 & 0.0000010 & 0.0000010 & 0.0000010 & 0.0000010 & 0.0000010 \\
\hline Water & 80953 & 10-20 min, non-overflow, total & 0.0000010 & 0.0000010 & 0.0000010 & 0.0000010 & 0.0000010 & 0.0000010 & 0.0000010 \\
\hline \multirow[t]{2}{*}{ Water } & 80954 & 20-30 min, non-overflow, total & 0.0000010 & 0.0000010 & 0.0000010 & 0.0000010 & 0.0000010 & 0.0000010 & 0.0000010 \\
\hline & & Hopper Inflow Monitoring & & & & & & & \\
\hline Water & 81104 & $3 \& 6 \mathrm{~min}$, dissolved & 0.0000010 & 0.0000010 & 0.0000010 & 0.0000010 & 0.0000010 & 0.0000010 & 0.0000010 \\
\hline Water & 81105 & $9 \& 12 \mathrm{~min}$, dissolved & 0.0000010 & 0.0000010 & 0.0000010 & 0.0000010 & 0.0000010 & 0.0000010 & 0.0000010 \\
\hline Water & 81106 & $15 \& 18 \mathrm{~min}$, dissolved & 0.0000010 & 0.0000010 & 0.0000010 & 0.0000010 & 0.0000010 & 0.0000010 & 0.0000010 \\
\hline Water & 81107 & $21824 \mathrm{~min}$, dissolved & 0.0000010 & 0.0000010 & 0.0000010 & 0.0000010 & 0.0000010 & 0.0000010 & 0.0000010 \\
\hline Water & 81108 & $27 \& 30 \mathrm{~min}$, dissolved & 0.0000010 & 0.0000010 & 0.0000010 & 0.0000010 & 0.0000010 & 0.0000010 & 0.0000010 \\
\hline Water & 80891 & 3\& $6 \mathrm{~min}$, total & 0.0000012 & 0.0000011 & 0.0000011 & 0.0000029 & 0.0000046 & 0.0000092 & 0.0000011 \\
\hline Water & 80892 & $9 \& 12 \mathrm{~min}$, total & 0.0000034 & 0.0000010 & 0.0000010 & 0.0000068 & 0.0000010 & 0.0000210 & 0.0000010 \\
\hline Water & 80893 & $15 \& 18 \mathrm{~min}$, total & 0.0000022 & 0.0000010 & 0.0000010 & 0.0000038 & 0.0000010 & 0.0000140 & 0.0000010 \\
\hline Water & 80894 & $21224 \mathrm{~min}$, total & 0.0000011 & 0.0000280 & 0.0000011 & 0.0000011 & 0.0000011 & 0.0000011 & 0.0000011 \\
\hline \multirow[t]{2}{*}{ Water } & 80895 & $27830 \mathrm{~min}$, total & 0.0000011 & 0.0000490 & 0.0000011 & 0.0000022 & 0.0000011 & 0.0000011 & 0.0000011 \\
\hline & & Hopper Overflow Monitoring & & & & & & & \\
\hline Water & 81109 & 2\& $4 \mathrm{~min}$, dissolved & 0.0000010 & 0.0000010 & 0.0000010 & 0.0000010 & 0.0000010 & 0.0000010 & 0.0000010 \\
\hline Water & 81110 & 6\& $8 \mathrm{~min}$, dissolved & 0.0000010 & 0.0000010 & 0.0000010 & 0.0000010 & 0.0000010 & 0.0000010 & 0.0000010 \\
\hline Water & 81111 & $10 \& 12 \mathrm{~min}$, dissolved & 0.0000010 & 0.0000010 & 0.0000010 & 0.0000010 & 0.0000010 & 0.0000010 & 0.0000010 \\
\hline Water & 81112 & $14 \& 16 \mathrm{~min}$, dissolved & 0.0000010 & 0.0000010 & 0.0000010 & 0.0000010 & 0.0000010 & 0.0000010 & 0.0000010 \\
\hline Water & 81113 & $18 \& 20 \mathrm{~min}$, dissolved & 0.0000010 & 0.0000010 & 0.0000010 & 0.0000010 & 0.0000010 & 0.0000010 & 0.0000010 \\
\hline Water & 80897 & $284 \mathrm{~min}$, total & 0.0000011 & 0.0000590 & 0.0000011 & 0.0000011 & 0.0000011 & 0.0000011 & 0.0000011 \\
\hline Water & 80898 & $6 \& 8 \mathrm{~min}$, total & 0.0000011 & 0.0000011 & 0.0000011 & 0.0000011 & 0.0000011 & 0.0000011 & 0.0000011 \\
\hline Water & 80899 & $10 \& 12 \mathrm{~min}$, total & 0.0000069 & 0.0000011 & 0.0000011 & 0.0000011 & 0.0000011 & 0.0000011 & 0.0000011 \\
\hline Water & 80900 & $14816 \mathrm{~min}$, total & 0.0000011 & 0.0000011 & 0.0000011 & 0.0000011 & 0.0000011 & 0.0000011 & 0.0000011 \\
\hline \multirow[t]{2}{*}{ Water } & 80901 & $18 \& 20 \mathrm{~min}$, total & 0.0000010 & 0.0000010 & 0.0000010 & 0.0000010 & 0.0000010 & 0.0000010 & 0.0000010 \\
\hline & & Site Water & & & & & & & \\
\hline Water & 81603 & Sample 1 Total & 0.0000010 & 0.0000010 & 0.0000010 & 0.0000010 & 0.0000010 & 0.0000010 & 0.0000010 \\
\hline Water & 81604 & Sample 2 Total & 0.0000010 & 0.0000010 & 0.0000010 & 0.0000010 & 0.0000010 & 0.0000010 & 0.0000010 \\
\hline \multirow[t]{2}{*}{ Water } & 81605 & Sample 3 Total & 0.0000010 & 0.0000010 & 0.0000010 & 0.0000010 & 0.0000010 & 0.0000010 & 0.0000010 \\
\hline & & Elutriate & & & & & & & \\
\hline Water & 81609 & Sample 1 Dissolved & 0.0000010 & 0.0000010 & 0.0000010 & 0.0000010 & 0.0000010 & 0.0000010 & 0.0000010 \\
\hline Water & 81610 & Sample 2 Dissolved & 0.0000010 & 0.0000010 & 0.0000010 & 0.0000010 & 0.0000010 & 0.0000010 & 0.0000010 \\
\hline Water & 81611 & Sample 3 Dissolved & 0.0000010 & 0.0000010 & 0.0000010 & 0.0000010 & 0.0000010 & 0.0000010 & 0.0000010 \\
\hline Water & 81606 & Sample 1 Total & 0.0000010 & 0.0000016 & 0.0000010 & 0.0000010 & 0.0000010 & 0.0000010 & 0.0000010 \\
\hline Water & 81607 & Sample 2 Total & 0.0000010 & 0.0000018 & 0.0000010 & 0.0000010 & 0.0000010 & 0.0000010 & 0.0000010 \\
\hline Water & 81608 & Sample 3 Total & 0.0000010 & 0.0000016 & 0.0000010 & 0.0000010 & 0.0000010 & 0.0000010 & 0.0000010 \\
\hline \multirow{3}{*}{$\begin{array}{l}\text { SAMPLE } \\
\text { TYPE }\end{array}$} & SAMPLE & DESCRIPTION & РCB 171 & PCB 180 & PCB 182 & PCB 183 & PCB 185 & PCB 187 & РСB 189 \\
\hline & & Detection Limit (mg/kg) & 0.00077 & 0.00077 & 0.00077 & 0.00077 & 0.00077 & 0.00077 & 0.00077 \\
\hline & & Insitu Sediment & & & & & & & \\
\hline Sediment & 81717 & Sample \#1 & 0.00077 & 0.00150 & 0.00077 & 0.00077 & 0.00077 & 0.00077 & 0.00077 \\
\hline Sediment & 81718 & Sample \#2 & 0.00077 & 0.00140 & 0.00077 & 0.00077 & 0.00077 & 0.00077 & 0.00077 \\
\hline Sediment & 81719 & Sample \#3 & 0.00077 & 0.00160 & 0.00077 & 0.00077 & 0.00077 & 0.00100 & 0.00077 \\
\hline
\end{tabular}

BOLD - less than values

Values below less than values are estimated results. Results are less than the reporting limit. 
PCBsfine

Delaware River Water Analysis (Fine-Grained Site)

\begin{tabular}{|c|c|c|c|c|c|c|c|c|c|}
\hline \multirow[t]{2}{*}{$\begin{array}{l}\text { SAMPLE } \\
\text { TYPE }\end{array}$} & $\begin{array}{l}\text { SAMPLE } \\
\text { ID }\end{array}$ & DESCRIPTION & РСB 191 & PCB 194 & PCB 195 & РСВ 196 & PCB 201 & РСВ 203 & РСB 205 \\
\hline & & Detection Limit (mg/l) & 0.0000011 & 0.0000011 & 0.0000011 & 0.0000011 & 0.0000011 & 0.0000011 & 0.0000011 \\
\hline & & Plume Monitoring & & & & & & & \\
\hline Water & 80983 & Background, dissolved & 0.0000010 & 0.0000010 & 0.0000010 & 0.0000010 & 0.0000010 & 0.0000010 & 0.0000010 \\
\hline Water & 80948 & Background, total & 0.0000011 & 0.0000011 & 0.0000011 & 0.0000011 & 0.0000011 & 0.0000011 & 0.0000011 \\
\hline Water & 80984 & 0-10 min, overflow, dissolved & 0.0000010 & 0.0000010 & 0.0000010 & 0.0000010 & 0.0000010 & 0.0000010 & 0.0000010 \\
\hline Water & 80985 & 10-20 min, overflow, dissolved & 0.0000011 & 0.0000011 & 0.0000011 & 0.0000011 & 0.0000011 & 0.0000011 & 0.0000011 \\
\hline Water & 80986 & $20-30 \mathrm{~min}$, overflow, dissolved & 0.0000010 & 0.0000010 & 0.0000010 & 0.0000010 & 0.0000010 & 0.0000010 & 0.0000010 \\
\hline Water & 80949 & 0-10 min, overflow, total & 0.0000010 & 0.0000010 & 0.0000010 & 0.0000010 & 0.0000010 & 0.0000010 & 0.0000010 \\
\hline Water & 80950 & 10-20 min, overflow, total & 0.0000010 & 0.0000010 & 0.0000010 & 0.0000010 & 0.0000010 & 0.0000010 & 0.0000010 \\
\hline Water & 80951 & 20-30 min, overflow, total & 0.0000010 & 0.0000010 & 0.0000010 & 0.0000010 & 0.0000010 & 0.0000010 & 0.0000010 \\
\hline Water & 80987 & 0-10 min, non-overflow, dissolve & 0.0000010 & 0.0000010 & 0.0000010 & 0.0000010 & 0.0000010 & 0.0000010 & 0.0000010 \\
\hline Water & 80988 & 10-20 min, non-overflow, dissolve & 0.0000010 & 0.0000010 & 0.0000010 & 0.0000010 & 0.0000010 & 0.0000010 & 0.0000010 \\
\hline Water & 80989 & 20-30 min, non-overflow, dissolve & 0.0000010 & 0.0000010 & 0.0000010 & 0.0000010 & 0.0000010 & 0.0000010 & 0.0000010 \\
\hline Water & 80952 & 0-10 min, non-overflow, total & 0.0000010 & 0.0000010 & 0.0000010 & 0.0000010 & 0.0000010 & 0.0000010 & 0.0000010 \\
\hline Water & 80953 & 10-20 $\mathrm{min}$, non-overflow, total & 0.0000010 & 0.0000010 & 0.0000010 & 0.0000010 & 0.0000010 & 0.0000010 & 0.0000010 \\
\hline \multirow[t]{2}{*}{ Water } & 80954 & 20-30 min, non-overflow, total & 0.0000010 & 0.0000010 & 0.0000010 & 0.0000010 & 0.0000010 & 0.0000010 & 0.0000010 \\
\hline & & Hopper Inflow Monitoring & & & & & & & \\
\hline Water & 81104 & $3 \& 6 \mathrm{~min}$, dissolved & 0.0000010 & 0.0000010 & 0.0000010 & 0.0000010 & 0.0000010 & 0.0000010 & 0.0000010 \\
\hline Water & 81105 & $9 \& 12 \mathrm{~min}$, dissolved & 0.0000010 & 0.0000010 & 0.0000010 & 0.0000010 & 0.0000010 & 0.0000010 & 0.0000010 \\
\hline Water & 81106 & $15 \& 18 \mathrm{~min}$, dissolved & 0.0000010 & 0.0000010 & 0.0000010 & 0.0000010 & 0.0000010 & 0.0000010 & 0.0000010 \\
\hline Water & 81107 & $21 \& 24 \mathrm{~min}$, dissolved & 0.0000010 & 0.0000010 & 0.0000010 & 0.0000010 & 0.0000010 & 0.0000010 & 0.0000010 \\
\hline Water & 81108 & $27 \& 30 \mathrm{~min}$, dissolved & 0.0000010 & 0.0000010 & 0.0000010 & 0.0000010 & 0.0000010 & 0.0000010 & 0.0000010 \\
\hline Water & 80891 & 3\& $6 \mathrm{~min}$, total & 0.0000011 & 0.0000011 & 0.0000090 & 0.0000026 & 0.0000011 & 0.0000011 & 0.0000011 \\
\hline Water & 80892 & $9 \& 12 \mathrm{~min}$, total & 0.0000010 & 0.0000010 & 0.0000026 & 0.0000065 & 0.0000010 & 0.0000010 & 0.0000010 \\
\hline Water & 80893 & $15 \& 18 \mathrm{~min}$, total & 0.0000010 & 0.0000010 & 0.0000016 & 0.0000039 & 0.0000010 & 0.0000010 & 0.0000010 \\
\hline Water & 80894 & $21 \& 24 \mathrm{~min}$, total & 0.0000011 & 0.0000011 & 0.0000011 & 0.0000036 & 0.0000011 & 0.0000011 & 0.0000011 \\
\hline \multirow[t]{2}{*}{ Water } & 80895 & $27 \& 30 \mathrm{~min}$, total & 0.0000011 & 0.0000011 & 0.0000011 & 0.0000080 & 0.0000011 & 0.0000011 & 0.0000011 \\
\hline & & Hopper Overflow Monitoring & & & & & & & \\
\hline Water & 81109 & 2\& $4 \mathrm{~min}$, dissolved & 0.0000010 & 0.0000010 & 0.0000010 & 0.0000010 & 0.0000010 & 0.0000010 & 0.0000010 \\
\hline Water & 81110 & 6\& $8 \mathrm{~min}$, dissolved & 0.0000010 & 0.0000010 & 0.0000010 & 0.0000010 & 0.0000010 & 0.0000010 & 0.0000010 \\
\hline Water & 81111 & $10 \& 12 \mathrm{~min}$, dissolved & 0.0000010 & 0.0000010 & 0.0000010 & 0.0000010 & 0.0000010 & 0.0000010 & 0.0000010 \\
\hline Water & 81112 & $14 \& 16 \mathrm{~min}$, dissolved & 0.0000010 & 0.0000010 & 0.0000010 & 0.0000010 & 0.0000010 & 0.0000010 & 0.0000010 \\
\hline Water & 81113 & $18 \& 20 \mathrm{~min}$, dissolved & 0.0000010 & 0.0000010 & 0.0000010 & 0.0000010 & 0.0000010 & 0.0000010 & 0.0000010 \\
\hline Water & 80897 & 2\& $4 \mathrm{~min}$, total & 0.0000011 & 0.0000011 & 0.0000041 & 0.0000110 & 0.0000011 & 0.0000011 & 0.0000011 \\
\hline Water & 80898 & 6\& $8 \mathrm{~min}$, total & 0.0000011 & 0.0000011 & 0.0000081 & 0.0000067 & 0.0000011 & 0.0000011 & 0.0000011 \\
\hline Water & 80899 & $10 \& 12 \mathrm{~min}$, total & 0.0000011 & 0.0000011 & 0.0000044 & 0.0000100 & 0.0000011 & 0.0000011 & 0.0000011 \\
\hline Water & 80900 & $14 \& 16 \mathrm{~min}$, total & 0.0000011 & 0.0000011 & 0.0000110 & 0.0000190 & 0.0000011 & 0.0000011 & 0.0000011 \\
\hline \multirow[t]{2}{*}{ Water } & 80901 & $18 \& 20 \mathrm{~min}$, total & 0.0000010 & 0.0000010 & 0.0000150 & 0.0000097 & 0.0000010 & 0.0000010 & 0.0000010 \\
\hline & & Site Water & & & & & & & \\
\hline Water & 81603 & Sample 1 Total & 0.0000010 & 0.0000010 & 0.0000010 & 0.0000010 & 0.0000010 & 0.0000010 & 0.0000010 \\
\hline Water & 81604 & Sample 2 Total & 0.0000010 & 0.0000010 & 0.0000010 & 0.0000010 & 0.0000010 & 0.0000010 & 0.0000010 \\
\hline \multirow[t]{2}{*}{ Water } & 81605 & Sample 3 Total & 0.0000010 & 0.0000010 & 0.0000010 & 0.0000010 & 0.0000010 & 0.0000010 & 0.0000010 \\
\hline & & Elutriate & & & & & & & \\
\hline Water & 81609 & Sample 1 Dissolved & 0.0000010 & 0.0000010 & 0.0000010 & 0.0000010 & 0.0000010 & 0.0000010 & 0.0000010 \\
\hline Water & 81610 & Sample 2 Dissolved & 0.0000010 & 0.0000010 & 0.0000010 & 0.0000010 & 0.0000010 & 0.0000010 & 0.0000010 \\
\hline Water & 81611 & Sample 3 Dissolved & 0.0000010 & 0.0000010 & 0.0000010 & 0.0000010 & 0.0000010 & 0.0000010 & 0.0000010 \\
\hline Water & 81606 & Sample 1 Total & 0.0000010 & 0.0000010 & 0.0000010 & 0.0000010 & 0.0000010 & 0.0000010 & 0.0000010 \\
\hline Water & 81607 & Sample 2 Total & 0.0000010 & 0.0000010 & 0.0000010 & 0.0000010 & 0.0000010 & 0.0000010 & 0.0000010 \\
\hline Water & 81608 & Sample 3 Total & 0.0000010 & 0.0000010 & 0.0000010 & 0.0000010 & 0.0000010 & 0.0000010 & 0.0000010 \\
\hline \multirow{3}{*}{$\begin{array}{l}\text { SAMPLE } \\
\text { TYPE }\end{array}$} & SAMPLE & DESCRIPTION & РCB 191 & РСB 194 & PCB 195 & PCB 196 & PCB 201 & PCB 203 & PCB 205 \\
\hline & & Detection Limit (mg/kg) & 0.00077 & 0.00077 & 0.00077 & 0.00077 & 0.00077 & 0.00077 & 0.00077 \\
\hline & & Insitu Sediment & & & & & & & \\
\hline Sediment & 81717 & Sample \#1 & 0.00077 & 0.00077 & 0.00077 & 0.00077 & 0.00077 & 0.00077 & 0.00077 \\
\hline Sediment & 81718 & Sample \#2 & 0.00077 & 0.00077 & 0.00077 & 0.00033 & 0.00077 & 0.00082 & 0.00077 \\
\hline Sediment & 81719 & Sample \#3 & 0.00077 & 0.00077 & 0.00077 & 0.00025 & 0.00077 & 0.00084 & 0.00077 \\
\hline
\end{tabular}

BOLD - less than values

Values below less than values are estimated results. Results are less than the reporting limit

Page 11 
PCBsfine

Delaware River Water Analysis (Fine-Grained Site)

\begin{tabular}{|c|c|c|c|c|c|c|c|c|c|}
\hline \multirow[t]{2}{*}{$\begin{array}{l}\text { SAMPLE } \\
\text { TYPE }\end{array}$} & $\begin{array}{l}\text { SAMPLE } \\
\text { ID }\end{array}$ & DESCRIPTION & РСВ 206 & РCB 207 & РСB 208 & РСB 209 & РCB 66 & РCB 190 & РCB 198 \\
\hline & & Detection Limit (mg/l) & 0.0000011 & 0.00000110 & 0.00000110 & & 0.0000011 & 0.0000011 & 0.0000011 \\
\hline & & Plume Monitoring & & & & & & & \\
\hline Water & 80983 & Background, dissolved & 0.0000025 & 0.00000095 & 0.00000130 & $106.45 \%$ & 0.0000010 & 0.0000010 & 0.0000010 \\
\hline Water & 80948 & Background, total & 0.0000017 & 0.00000110 & 0.00000081 & $74.06 \%$ & 0.0000011 & 0.0000011 & 0.0000011 \\
\hline Water & 80984 & 0-10 min, overflow, dissolved & 0.0000024 & 0.00000090 & 0.00000150 & $92.43 \%$ & 0.0000010 & 0.0000010 & 0.0000010 \\
\hline Water & 80985 & 10-20 min, overflow, dissolved & 0.0000027 & 0.00000110 & 0.00000170 & $100.50 \%$ & 0.0000011 & 0.0000011 & 0.0000011 \\
\hline Water & 80986 & 20-30 min, overflow, dissolved & 0.0000026 & 0.00000110 & 0.00000140 & $99.77 \%$ & 0.0000010 & 0.0000010 & 0.0000010 \\
\hline Water & 80949 & 0-10 min, overflow, total & 0.0000020 & 0.00000100 & 0.00000092 & $64.53 \%$ & 0.0000010 & 0.0000010 & 0.0000010 \\
\hline Water & 80950 & 10-20 min, overflow, total & 0.0000020 & 0.00000100 & 0.00000085 & $75.26 \%$ & 0.0000010 & 0.0000010 & 0.0000010 \\
\hline Water & 80951 & 20-30 min, overflow, total & 0.0000020 & 0.00000100 & 0.00000089 & $81.36 \%$ & 0.0000010 & 0.0000010 & 0.0000010 \\
\hline Water & 80987 & 0-10 min, non-overflow, dissolve & 0.0000026 & 0.00000095 & 0.00000140 & $101.88 \%$ & 0.0000010 & 0.0000010 & 0.0000010 \\
\hline Water & 80988 & 10-20 min, non-overflow, dissolve & 0.0000025 & 0.00000074 & 0.00000130 & $104.87 \%$ & 0.0000010 & 0.0000010 & 0.0000010 \\
\hline Water & 80989 & 20-30 min, non-overflow, dissolve & 0.0000024 & 0.00000071 & 0.00000120 & $112.74 \%$ & 0.0000010 & 0.0000010 & 0.0000010 \\
\hline Water & 80952 & 0-10 min, non-overflow, total & 0.0000016 & 0.00000100 & 0.00000074 & $80.06 \%$ & 0.0000010 & 0.0000010 & 0.0000010 \\
\hline Water & 80953 & 10-20 min, non-overflow, total & 0.0000017 & 0.00000100 & 0.00000075 & $88.73 \%$ & 0.0000010 & 0.0000010 & 0.0000010 \\
\hline \multirow[t]{2}{*}{ Water } & 80954 & 20-30 $\mathrm{min}$, non-overflow, total & 0.0000017 & 0.00000100 & 0.00000083 & $87.12 \%$ & 0.0000010 & 0.0000010 & 0.0000010 \\
\hline & & Hopper Inflow Monitoring & & & & & & & \\
\hline Water & 81104 & $3 \& 6 \mathrm{~min}$, dissolved & 0.0000016 & 0.00000049 & 0.00000077 & $90.09 \%$ & 0.0000010 & 0.0000010 & 0.0000010 \\
\hline Water & 81105 & $9 \& 12 \mathrm{~min}$, dissolved & 0.0000019 & 0.00000056 & 0.00000087 & $86.49 \%$ & 0.0000010 & 0.0000010 & 0.0000010 \\
\hline Water & 81106 & $15 \& 18 \mathrm{~min}$, dissolved & 0.0000019 & 0.00000040 & 0.00000150 & $87.34 \%$ & 0.0000010 & 0.0000010 & 0.0000010 \\
\hline Water & 81107 & $218.24 \mathrm{~min}$, dissolved & 0.0000021 & 0.00000065 & 0.00000100 & $94.48 \%$ & 0.0000010 & 0.0000010 & 0.0000010 \\
\hline Water & 81108 & $27 \& 30 \mathrm{~min}$, dissolved & 0.0000017 & 0.00000054 & 0.00000094 & $72.89 \%$ & 0.0000010 & 0.0000010 & 0.0000010 \\
\hline Water & 80891 & $3 \& 6 \mathrm{~min}$, total & 0.0000410 & 0.00000280 & 0.00001700 & $97.66 \%$ & 0.0000011 & 0.0000011 & 0.0000011 \\
\hline Water & 80892 & $9 \& 12 \mathrm{~min}$, total & 0.0000900 & 0.00000530 & 0.00004100 & $58.62 \%$ & 0.0000010 & 0.0000010 & 0.0000010 \\
\hline Water & 80893 & $15 \& 18 \mathrm{~min}$, total & 0.0000580 & 0.00000650 & 0.00002500 & $108.63 \%$ & 0.0000010 & 0.0000010 & 0.0000010 \\
\hline Water & 80894 & $21 \& 24 \mathrm{~min}$, total & 0.0000460 & 0.00000190 & 0.00001900 & $108.06 \%$ & 0.0000011 & 0.0000011 & 0.0000011 \\
\hline \multirow[t]{2}{*}{ Water } & 80895 & $27 \& 30 \mathrm{~min}$, total & 0.0000920 & 0.00000620 & 0.00004000 & $124.79 \%$ & 0.0000011 & 0.0000011 & 0.0000011 \\
\hline & & Hopper Overflow Monitoring & & & & & & & \\
\hline Water & 81109 & $224 \mathrm{~min}$, dissolved & 0.0000019 & 0.00000037 & 0.00000061 & $81.94 \%$ & 0.0000010 & 0.0000010 & 0.0000010 \\
\hline Water & 81110 & 6\& $8 \mathrm{~min}$, dissolved & 0.0000016 & 0.00000100 & 0.00000061 & $78.71 \%$ & 0.0000010 & 0.0000010 & 0.0000010 \\
\hline Water & 81111 & $10 \& 12 \mathrm{~min}$, dissolved & 0.0000017 & 0.00000100 & 0.00000084 & $88.72 \%$ & 0.0000010 & 0.0000010 & 0.0000010 \\
\hline Water & 81112 & $14 \& 16 \mathrm{~min}$, dissolved & 0.0000018 & 0.00000043 & 0.00000086 & $83.24 \%$ & 0.0000010 & 0.0000010 & 0.0000010 \\
\hline Water & 81113 & $18 \& 20 \mathrm{~min}$, dissolved & 0.0000016 & 0.00000043 & 0.00000077 & $80.18 \%$ & 0.0000010 & 0.0000010 & 0.0000010 \\
\hline Water & 80897 & 28 4 min, total & 0.0001700 & 0.00000960 & 0.00007400 & $220.34 \%$ & 0.0000011 & 0.0000011 & 0.0000011 \\
\hline Water & 80898 & $6 \& 8 \mathrm{~min}$, total & 0.0001200 & 0.00000720 & 0.00005100 & $213.26 \%$ & 0.0000011 & 0.0000011 & 0.0000011 \\
\hline Water & 80899 & $10 \& 12 \mathrm{~min}$, total & 0.0001200 & 0.00000860 & 0.00005200 & $175.12 \%$ & 0.0000011 & 0.0000011 & 0.0000011 \\
\hline Water & 80900 & $14 \& 16 \mathrm{~min}$, total & 0.0001300 & 0.00001000 & 0.00005800 & $216.74 \%$ & 0.0000011 & 0.0000011 & 0.0000011 \\
\hline \multirow[t]{2}{*}{ Water } & 80901 & $18 \& 20 \mathrm{~min}$, total & 0.0001200 & 0.00000840 & 0.00005900 & $186.63 \%$ & 0.0000010 & 0.0000010 & 0.0000010 \\
\hline & & Site Water & & & & & & & \\
\hline Water & 81603 & Sample 1 Total & 0.0000028 & 0.00000062 & 0.00000110 & $101.92 \%$ & 0.0000010 & 0.0000010 & 0.0000010 \\
\hline Water & 81604 & Sample 2 Total & 0.0000024 & 0.00000100 & 0.00000100 & $90.51 \%$ & 0.0000010 & 0.0000010 & 0.0000010 \\
\hline \multirow[t]{2}{*}{ Water } & 81605 & Sample 3 Total & 0.0000022 & 0.00000100 & 0.00000092 & $90.22 \%$ & 0.0000010 & 0.0000010 & 0.0000010 \\
\hline & & Elutriate & & & & & & & \\
\hline Water & 81609 & Sample 1 Dissolved & 0.0000018 & 0.00000027 & 0.00000058 & $86.71 \%$ & 0.0000010 & 0.0000010 & 0.0000010 \\
\hline Water & 81610 & Sample 2 Dissolved & 0.0000021 & 0.00000029 & 0.00000085 & $96.44 \%$ & 0.0000010 & 0.0000010 & 0.0000010 \\
\hline Water & 81611 & Sample 3 Dissolved & 0.0000021 & 0.00000054 & 0.00000078 & $101.29 \%$ & 0.0000010 & 0.0000010 & 0.0000010 \\
\hline Water & 81606 & Sample 1 Total & 0.0000052 & 0.00000059 & 0.00000270 & $91.62 \%$ & 0.0000010 & 0.0000010 & 0.0000010 \\
\hline Water & 81607 & Sample 2 Total & 0.0000051 & 0.00000100 & 0.00000270 & $90.36 \%$ & 0.0000010 & 0.0000010 & 0.0000010 \\
\hline Water & 81608 & Sample 3 Total & 0.0000052 & 0.00000068 & 0.00000280 & $88.68 \%$ & 0.0000010 & 0.0000010 & 0.0000010 \\
\hline \multirow{3}{*}{$\begin{array}{l}\text { SAMPLE } \\
\text { TYPE }\end{array}$} & SAMPLE & DESCRIPTION & PCB 206 & PCB 207 & PCB 208 & РСB 209 & РСB 66 & PCB 190 & PCB 198 \\
\hline & & Detection Limit (mg/kg) & 0.00077 & 0.00077 & 0.00077 & & 0.00077 & 0.00077 & 0.00077 \\
\hline & & Insitu Sediment & & & & & & & \\
\hline Sediment & 81717 & Sample \#1 & 0.00390 & 0.00048 & 0.00220 & $106.21 \%$ & 0.00077 & 0.00077 & 0.00077 \\
\hline Sediment & 81718 & Sample \#2 & 0.00120 & 0.00077 & 0.00210 & $105.19 \%$ & 0.00077 & 0.00077 & 0.00077 \\
\hline Sediment & 81719 & Sample \#3 & 0.00370 & 0.00077 & 0.00180 & $98.01 \%$ & 0.00077 & 0.00077 & 0.00077 \\
\hline
\end{tabular}

BOLD - less than values
Values below less than values are estimated results. Results are less than the reporting limit.

Page 12 
Delaware River Water Analysis (Fine-Grained Site)

\begin{tabular}{|c|c|c|c|}
\hline \multirow{2}{*}{$\begin{array}{l}\text { SAMPLE } \\
\text { TYPE }\end{array}$} & SAMPLE & DESCRIPTION & РСВ 200 \\
\hline & & Detection Limit (mg/) & 0.0000011 \\
\hline & & Plume Monitoring & \\
\hline Water & 80983 & Background, dissolved & 0.0000010 \\
\hline Water & 80948 & Background, total & 0.0000011 \\
\hline Water & 80984 & 0-10 min, overflow, dissolved & 0.0000010 \\
\hline Water & 80985 & 10-20 min, overflow, dissolved & 0.0000011 \\
\hline Water & 80986 & $20-30 \mathrm{~min}$, overflow, dissolved & 0.0000010 \\
\hline Water & 80949 & $0-10 \mathrm{~min}$, overflow, total & 0.0000010 \\
\hline Water & 80950 & $10-20 \mathrm{~min}$, overflow, total & 0.0000010 \\
\hline Water & 80951 & $20-30$ min, overflow, total & 0.0000010 \\
\hline Water & 80987 & 0-10 min, non-overflow, dissolve & 0.0000010 \\
\hline Water & 80988 & $10-20 \mathrm{~min}$, non-overflow, dissolve & 0.0000010 \\
\hline Water & 80989 & 20-30 min, non-overflow, dissolve & 0.0000010 \\
\hline Water & 80952 & 0-10 min, non-overflow, total & 0.0000010 \\
\hline Water & 80953 & $10-20 \mathrm{~min}$, non-overflow, total & 0.0000010 \\
\hline \multirow[t]{2}{*}{ Water } & 80954 & $20-30 \mathrm{~min}$, non-overflow, total & 0.0000010 \\
\hline & & Hopper Inflow Monitoring & \\
\hline Water & 81104 & $3 \& 6 \mathrm{~min}$, dissolved & 0.0000010 \\
\hline Water & 81105 & $98.12 \mathrm{~min}$, dissolved & 0.0000010 \\
\hline Water & 81106 & $15 \& 18 \mathrm{~min}$, dissolved & 0.0000010 \\
\hline Water & 81107 & $21 \& 24 \mathrm{~min}$, dissolved & 0.0000010 \\
\hline Water & 81108 & $27830 \mathrm{~min}$, dissolved & 0.0000010 \\
\hline Water & 80891 & $3 \& 6 \mathrm{~min}$, total & 0.0000015 \\
\hline Water & 80892 & $9 \& 12 \mathrm{~min}$, total & 0.0000034 \\
\hline Water & 80893 & $15 \& 18 \mathrm{~min}$, total & 0.0000020 \\
\hline Water & 80894 & $21 \& 24 \mathrm{~min}$, total & 0.0000019 \\
\hline \multirow[t]{2}{*}{ Water } & 80895 & $27 \& 30 \mathrm{~min}$, total & 0.0000011 \\
\hline & & Hopper Overflow Monitoring & \\
\hline Water & 81109 & $2 \& 4 \mathrm{~min}$, dissolved & 0.0000010 \\
\hline Water & 81110 & $6 \& 8 \mathrm{~min}$, dissolved & 0.0000010 \\
\hline Water & 81111 & $10 \& 12 \mathrm{~min}$, dissolved & 0.0000010 \\
\hline Water & 81112 & $14816 \mathrm{~min}$, dissolved & 0.0000010 \\
\hline Water & 81113 & $18 \& 20 \mathrm{~min}$, dissolved & 0.0000010 \\
\hline Water & 80897 & 2\& $4 \mathrm{~min}$, total & 0.0000011 \\
\hline Water & 80898 & $6 \& 8 \mathrm{~min}$, total & 0.0000033 \\
\hline Water & 80899 & $10812 \mathrm{~min}$, total & 0.0000064 \\
\hline Water & 80900 & $14 \& 16 \mathrm{~min}$, total & 0.0000011 \\
\hline \multirow[t]{2}{*}{ Water } & 80901 & $18820 \mathrm{~min}$, total & 0.0000010 \\
\hline & & Site Water & \\
\hline Water & 81603 & Sample 1 Total & 0.0000010 \\
\hline Water & 81604 & Sample 2 Total & 0.0000010 \\
\hline \multirow[t]{2}{*}{ Water } & 81605 & Sample 3 Total & 0.0000010 \\
\hline & & Elutriate & \\
\hline Water & 81609 & Sample 1 Dissolved & 0.0000010 \\
\hline Water & 81610 & Sample 2 Dissolved & 0.0000010 \\
\hline Water & 81611 & Sample 3 Dissolved & 0.0000010 \\
\hline Water & 81606 & Sample 1 Total & 0.0000010 \\
\hline Water & 81607 & Sample 2 Total & 0.0000010 \\
\hline Water & 81608 & Sample 3 Total & 0.0000010 \\
\hline \multirow{4}{*}{$\begin{array}{l}\text { SAMPLE } \\
\text { TYPE }\end{array}$} & SAMPLE & DESCRIPTION & PCB 200 \\
\hline & & & \\
\hline & & Detection Limit (mg/kg) & 0.00077 \\
\hline & & Insitu Sediment & \\
\hline Sediment & 81717 & Sample \#1 & 0.00077 \\
\hline Sediment & 81718 & Sample \#2 & 0.00077 \\
\hline Sediment & 81719 & Sample \#3 & 0.00077 \\
\hline
\end{tabular}

BOLD - less than values

Values below less than values are estimated results. Results are less than the reporting limit

Page 13 


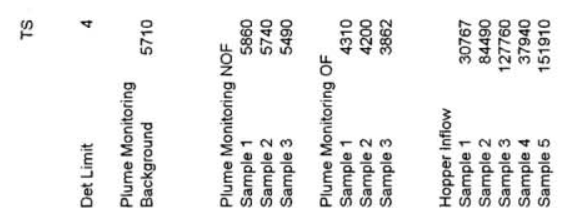

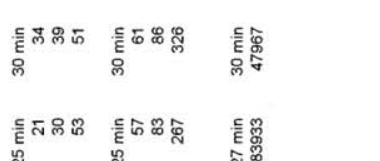

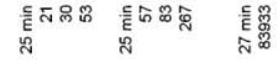

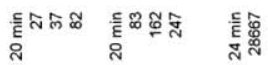

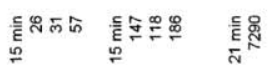

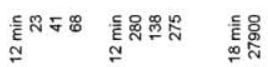

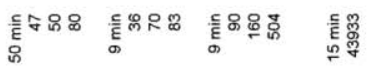

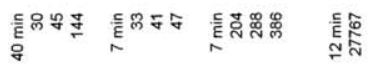

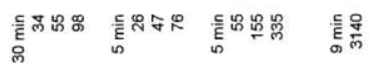

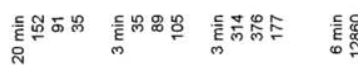

农

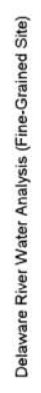

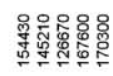

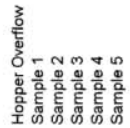

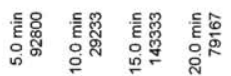

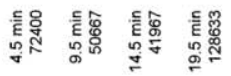

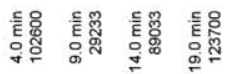

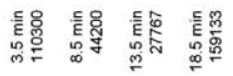

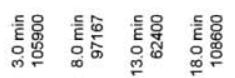

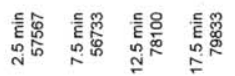

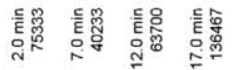

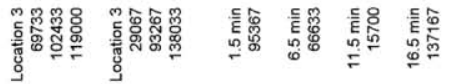

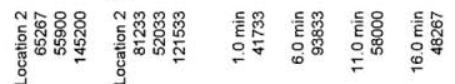

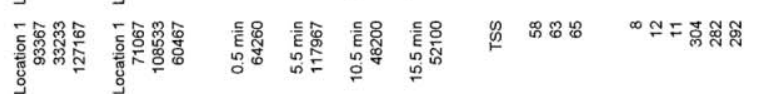

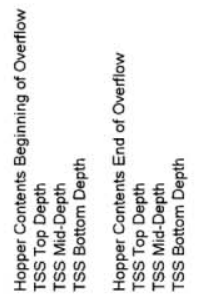

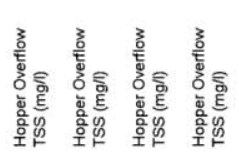

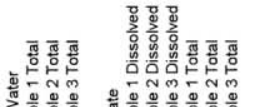

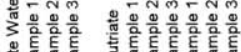

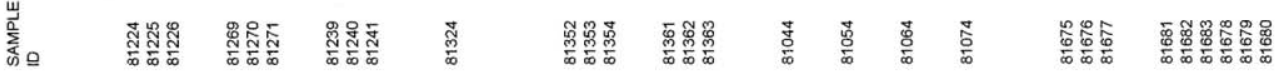

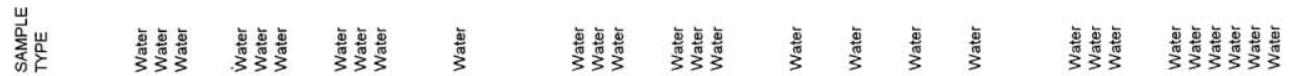


Delaware River Water Analysis (Fine-Grained Site)

\begin{tabular}{|c|c|c|c|}
\hline \multirow[t]{3}{*}{$\begin{array}{l}\text { SAMPLE } \\
\text { TYPE }\end{array}$} & $\begin{array}{l}\text { SAMPLE } \\
\text { ID }\end{array}$ & DESCRIPTION & TOC \\
\hline & & Detection Limit (mg/) & 3.00 \\
\hline & & Plume Monitoring & \\
\hline Water & 80969 & Background, dissolved & 6.12 \\
\hline Water & 80927 & Background, total & 7.30 \\
\hline Water & 80970 & 0-10 min, overflow, dissolved & 3.07 \\
\hline Water & 80971 & 10-20 min, overflow, dissolved & 2.91 \\
\hline Water & 80972 & 20-30 min, overflow, dissolved & 2.74 \\
\hline Water & 80928 & 0-10 min, overflow, total & 10.70 \\
\hline Water & 80923 & $10-20 \mathrm{~min}$, overflow, total & 11.30 \\
\hline Water & 80930 & $20-30 \mathrm{~min}$, overflow, total & 8.09 \\
\hline Water & 80973 & 0-10 $\mathrm{min}$, non-overflow, dissolved & 4.68 \\
\hline Water & 80974 & 10-20 min, non-overflow, dissolved & 6.35 \\
\hline Water & 80975 & 20-30 min, non-overflow, dissolved & 5.19 \\
\hline Water & 80931 & 0-10 min, non-overflow, total & 6.92 \\
\hline Water & 80932 & 10-20 min, non-overflow, total & 7.44 \\
\hline \multirow[t]{2}{*}{ Water } & 80933 & 20-30 min, non-overflow, total & 8.69 \\
\hline & & Hopper Inflow Monitoring & \\
\hline Water & 81084 & 3\& $6 \mathrm{~min}$, dissolved & 24.80 \\
\hline Water & 81085 & $9 \& 12 \mathrm{~min}$, dissolved & 47.30 \\
\hline Water & 81086 & $15 \& 18 \mathrm{~min}$, dissolved & 64.60 \\
\hline Water & 81087 & $21 \& 24 \mathrm{~min}$, dissolved & 19.00 \\
\hline Water & 81088 & $27 \& 30 \mathrm{~min}$, dissolved & 63.00 \\
\hline Water & 80855 & $3 \& 6 \mathrm{~min}$, total & 1010.00 \\
\hline Water & 80856 & $9 \& 12 \mathrm{~min}$, total & 3300.00 \\
\hline Water & 80857 & $15 \& 18 \mathrm{~min}$, total & 6030.00 \\
\hline Water & 80858 & $21 \& 24 \mathrm{~min}$, total & 1170.00 \\
\hline \multirow[t]{2}{*}{ Water } & 80859 & $27 \& 30 \mathrm{~min}$, total & 6460.00 \\
\hline & & Hopper Overflow Monitoring & \\
\hline Water & 81089 & 2\& $4 \mathrm{~min}$, dissolved & 14.10 \\
\hline Water & 81090 & $688 \mathrm{~min}$, dissolved & 11.90 \\
\hline Water & 81091 & $10 \& 12 \mathrm{~min}$, dissolved & 72.30 \\
\hline Water & 81092 & $14 \& 16 \mathrm{~min}$, dissolved & 79.20 \\
\hline Water & 81093 & $18 \& 20 \mathrm{~min}$, dissolved & 21.40 \\
\hline Water & 80861 & 2\& 4 min, total & 6660.00 \\
\hline Water & 80862 & $6 \& 8 \mathrm{~min}$, total & 5930.00 \\
\hline Water & 80863 & $10 \& 12 \mathrm{~min}$, total & 5280.00 \\
\hline Water & 80864 & $14 \& 16 \mathrm{~min}$, total & 6800.00 \\
\hline \multirow[t]{2}{*}{ Water } & 80865 & $18 \& 20 \mathrm{~min}$, total & 7150.00 \\
\hline & & Site Water & \\
\hline Water & 81693 & Sample 1 Total & 3.00 \\
\hline Water & 81694 & Sample 2 Total & 3.00 \\
\hline \multirow[t]{2}{*}{ Water } & 81695 & Sample 3 Total & 3.00 \\
\hline & & Elutriate & \\
\hline Water & 81699 & Sample 1 Dissolved & 3.00 \\
\hline Water & 81700 & Sample 2 Dissolved & 3.00 \\
\hline Water & 81701 & Sample 3 Dissolved & 3.00 \\
\hline Water & 81696 & Sample 1 Total & 1.48 \\
\hline Water & 81697 & Sample 2 Total & 1.43 \\
\hline Water & 81698 & Sample 3 Total & 1.92 \\
\hline \multirow{4}{*}{$\begin{array}{l}\text { SAMPLE } \\
\text { TYPE }\end{array}$} & SAMPLE & DESCRIPTION & TOC \\
\hline & & & \\
\hline & & Detection Limit (mg/kg) & 3.0 \\
\hline & & Insitu Sediment & \\
\hline Sediment & 81723 & Sample \#1 & 8090.0 \\
\hline Sediment & 81724 & Sample \#2 & 7200.0 \\
\hline Sediment & 81725 & Sample $\# 3$ & 7520.0 \\
\hline
\end{tabular}

BOLD - less than values

Values below less than values are estimated results. Results are less than the reporting limit.

Page 1 
spgrfine

Delaware River Water Analysis (Fine-Grained Site)

\begin{tabular}{|c|c|c|c|c|}
\hline $\begin{array}{l}\text { SAMPLE } \\
\text { TYPE }\end{array}$ & $\begin{array}{l}\text { SAMPLE } \\
\text { ID }\end{array}$ & DESCRIPTION & Sp. Gr. & \%Moisture \\
\hline & & Insitu Sediment & & \\
\hline Sediment & 81299 & Sample \#1 & 2.73 & $191.58 \%$ \\
\hline Sediment & 81300 & Sample \#2 & 2.75 & $254.93 \%$ \\
\hline Sediment & 81301 & Sample \#3 & 2.76 & $203.04 \%$ \\
\hline Sediment & 81302 & Sample \#4 & 2.74 & $181.93 \%$ \\
\hline Sediment & 81303 & Sample \#5 & 2.75 & $166.58 \%$ \\
\hline Sediment & 81304 & Sample \#6 & 2.72 & $117.93 \%$ \\
\hline Sediment & 81305 & Sample \#7 & 2.71 & $164.93 \%$ \\
\hline Sediment & 81306 & Sample \#8 & 2.72 & $108.57 \%$ \\
\hline Sediment & 81307 & Sample \#9 & 2.71 & $94.57 \%$ \\
\hline Sediment & 81308 & Sample \#10 & 2.73 & $103.87 \%$ \\
\hline Sediment & 81309 & Sample \#11 & 2.73 & $102.13 \%$ \\
\hline Sediment & 81310 & Sample \#12 & 2.73 & $130.69 \%$ \\
\hline Sediment & 81311 & Sample \#13 & 2.71 & $172.19 \%$ \\
\hline Sediment & 81312 & Sample \#14 & 2.72 & $156.47 \%$ \\
\hline Sediment & 81313 & Sample \#15 & 2.73 & $79.16 \%$ \\
\hline & & & 2.73 & $148.57 \%$ \\
\hline
\end{tabular}

Page 1 


\section{Appendix B Plume Study Field Activities and Data Results}

\section{Preface}

This section of the report describes field activities and data results from the relative acoustic backscatter channel cross sections with the OBS overlay. The investigators who participated in this part of the project were Messrs. Timothy L. Fagerburg, Howard A. Benson, and Terry N. Waller, U.S. Army Engineer Research and Development Center (ERDC), Coastal and Hydraulics Laboratory (CHL), Vicksburg, MS, and William H.

Dulaney, ERDC, Geotechnical and Structures Laboratory (GSL).

This section of the report was written by Messrs. Benson and Fagerburg, with assistance in data processing from Messrs. Waller, Martin T. Hebler, Mses. Clara J. Coleman and Jane M. Vaughan, CHL, and Mr. Daryl P. Cook, DIMCO, Inc., Vicksburg, MS.

\section{Field Procedure}

Two test areas were selected for monitoring. Reach 1, was a coarsegrained material site located near the Brandywine Range, in lower Delaware Bay. Reach 2, was a fine-grained material site located at the Deepwater Point Range near New Castle, DE (Figure B1). Channel cross-sectional transects were conducted with the 1,200-kHz Broad-Band Acoustic Doppler Current Profiler (ADCP) and Optical Backscatterance (OBS) sensor at several predetermined transect lines in the test areas for nonoverflow and overflow dredge operations. Several transects were monitored prior to the dredge passing to establish background conditions. The dredge would then begin dredging operations and the transect boat would run continuous transects behind it to determine the extent and dispersion of the plume. The first set of transects at each test area was made during the hopper dredge's being filled with no overflow. The dredge would then proceed to the dumping area, empty the load, and return to the site for the second test. 


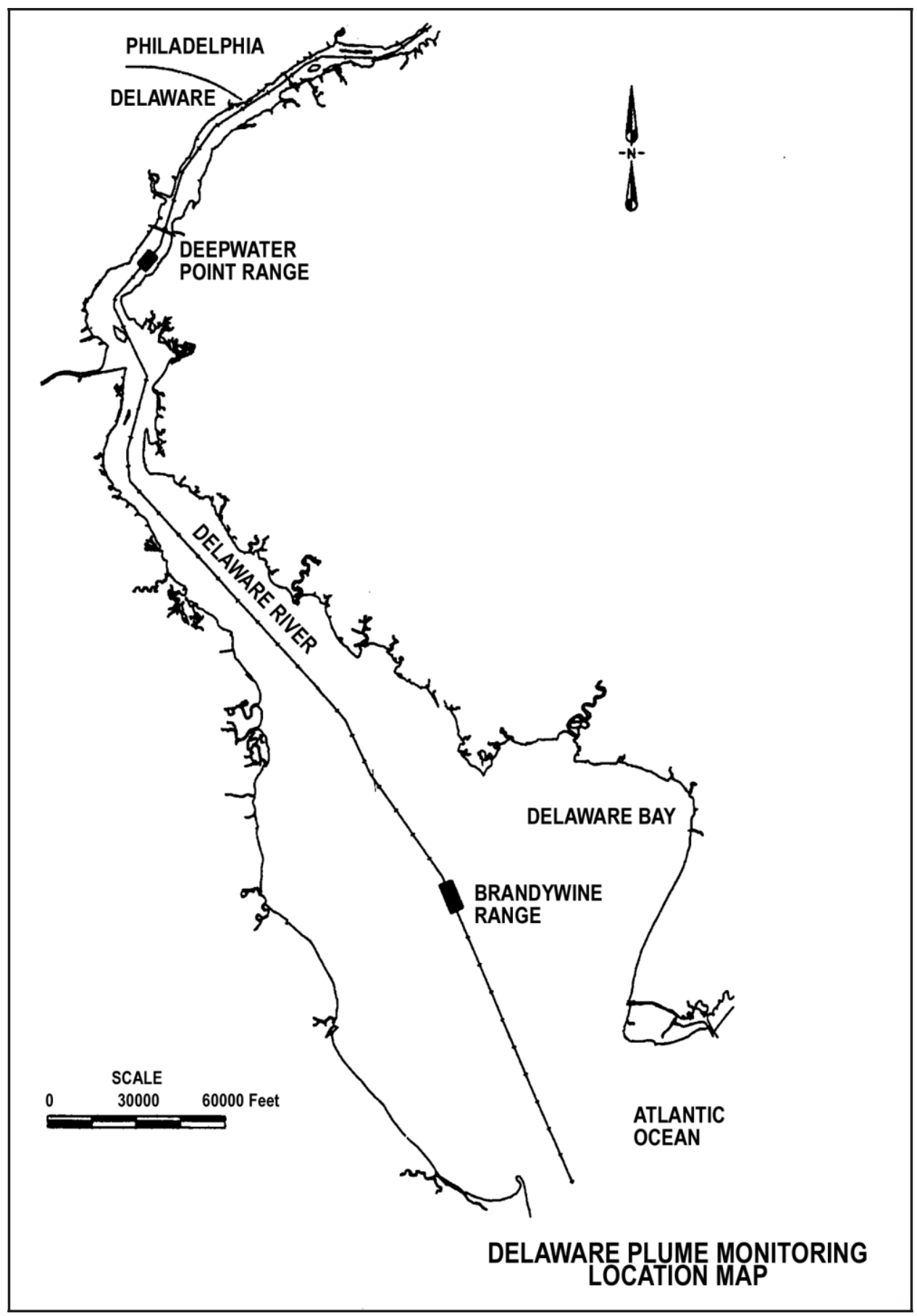

Figure B1. Delaware plume monitoring location map

Prior to the second test, data would be collected at several transect lines again to reestablish background conditions. The second dredging operation would include several minutes of hopper overflow while dredging. Again, the transect boat would run continuous transects behind it to determine the extent and dispersion of the plume. 
Monitoring the sediment plume was accomplished using a boat-mounted RD Instruments 1200-kHz Broad-Band ADCP. The instrument collects velocity vectors in the water column together with backscatter levels to determine the position and relative intensity of the sediment plume. A detailed description of the ADCP is presented in the Equipment Description section.

Along with the ADCP, a MicroLite recording instrument with an OBS Sensor was towed by the vessel at a depth of $4.6 \mathrm{~m}(15 \mathrm{ft})$. The MicroLite recorded data at 0.5 -sec intervals. A detailed description of the MicroLite is also presented in the Equipment Description section.

Navigation data for monitoring was obtained by a Starlink differential Global Positioning System (GPS). The GPS monitors the boat position from the starting and ending points along each transect. The manufacturer stated accuracy of the navigation system is $\pm 1 \mathrm{~m}$. The navigation data were recorded at $1-\mathrm{sec}$ intervals for merging with the ADCP and OBS data.

In situ sediment samples were collected prior to the dredging tests at both sites. Bottom samples were collected using a grab-type sampling bucket detailed in the Equipment Description section. Water samples for pore-water and toxicity tests were obtained using a portable pump sampler also described in the Equipment Description section. Types of samples, and the tests and analyses of the samples, are reported elsewhere in the report.

\section{Dredge Plume Monitoring}

The data presented in Figures B2 through B15 represent a time-history of the changes in suspended material levels in the water column resulting from dredge operations within each test area. The relative backscatter intensity of the ADCP acoustic signal is described as the strength of the return acoustic signal as it is affected by material suspended in the water column. Changes in levels of suspended material affect the acoustic reflectivity properties of the water column and, in turn, have an effect on the strength of the return signal intensity (decibels). High levels of suspended material in the water column result in high levels of acoustic intensity. The ADCP acoustic intensity data were utilized to identify levels of suspended material in the water column before, during, and following dredging operations.

As stated previously, transects were monitored in each test area to obtain the background levels of suspended materials prior to any dredging activities. The background levels shown in Figures B2 and B5 and in B9 and B12 are for the two test areas, Brandywine Range (Reach 1) and Deepwater Point Range (Reach 2), respectively.

Figures B2 through B4 illustrate the residence time of the sediment plume resulting from nonoverflow dredging operation in the Reach 1 . The background levels are shown in Figure B2. Figure B3 shows the vertical 


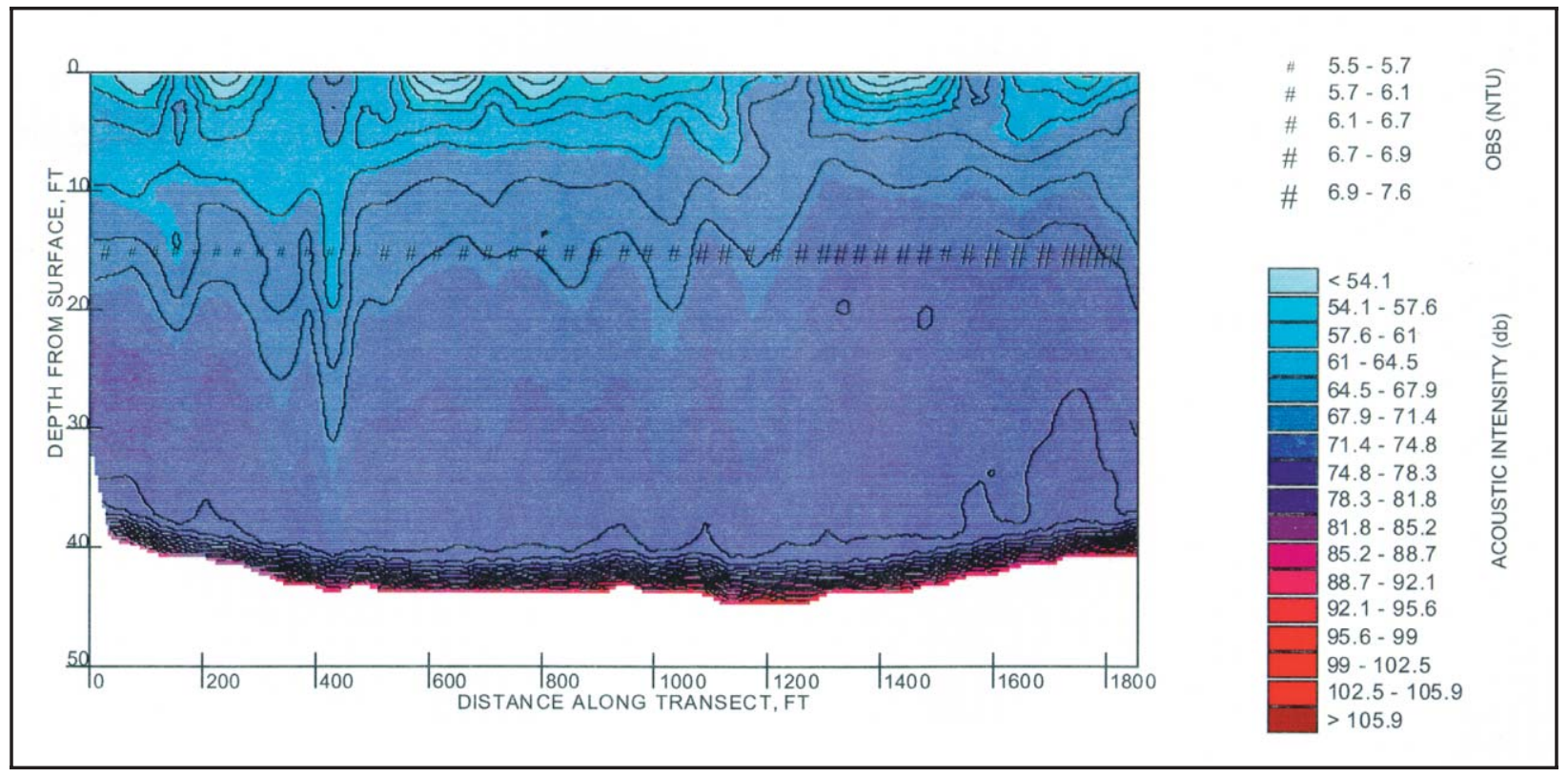

Figure B2. Relative acoustic intensity and OBS readings, Line 3, 1509 EST, Brandywine Range - Reach 1, $09 / 15 / 98$

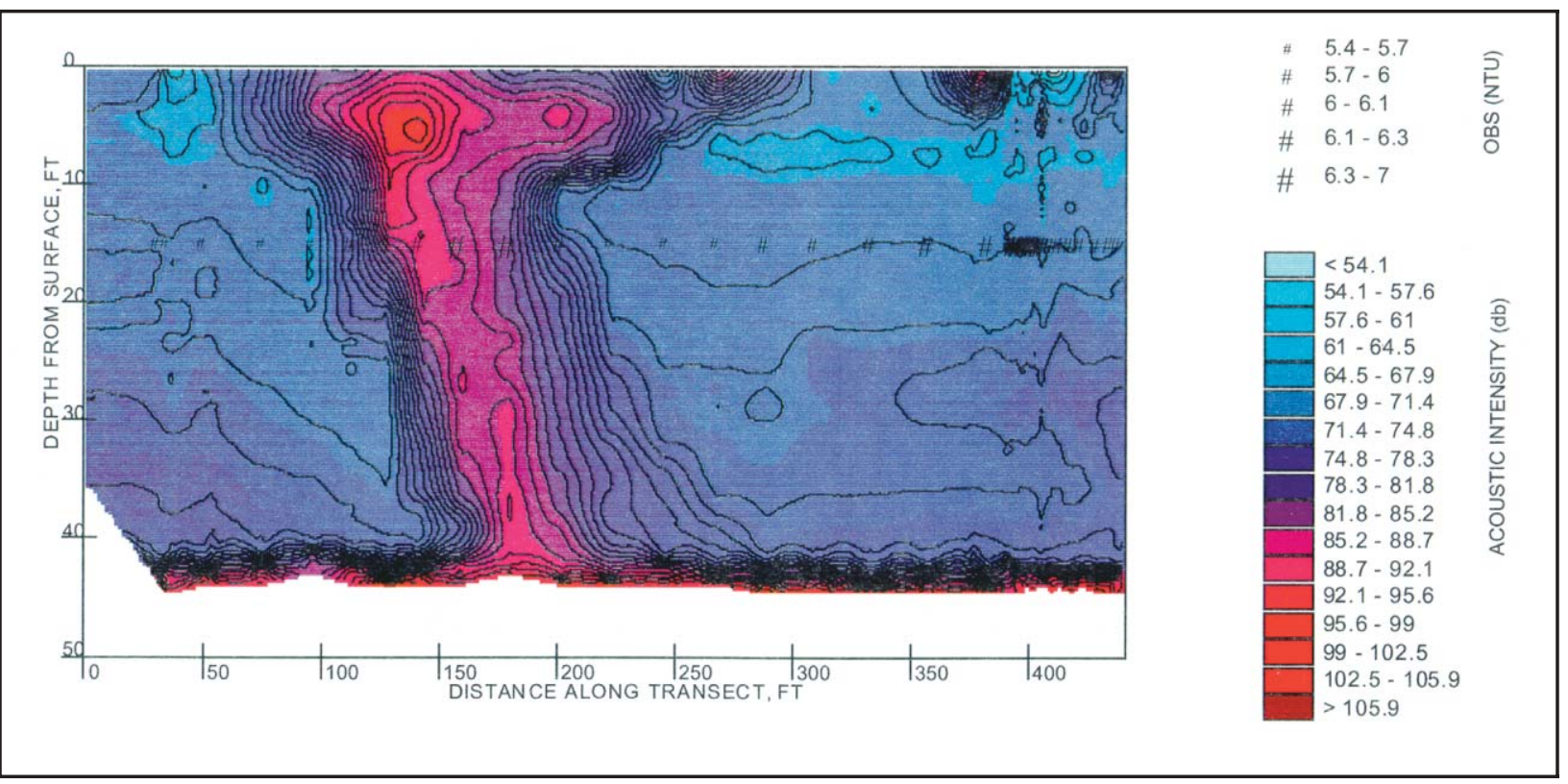

Figure B3. Relative acoustic intensity and OBS readings, Line 305, 1633 EST, Brandywine Range Reach 1, 09/15/98 


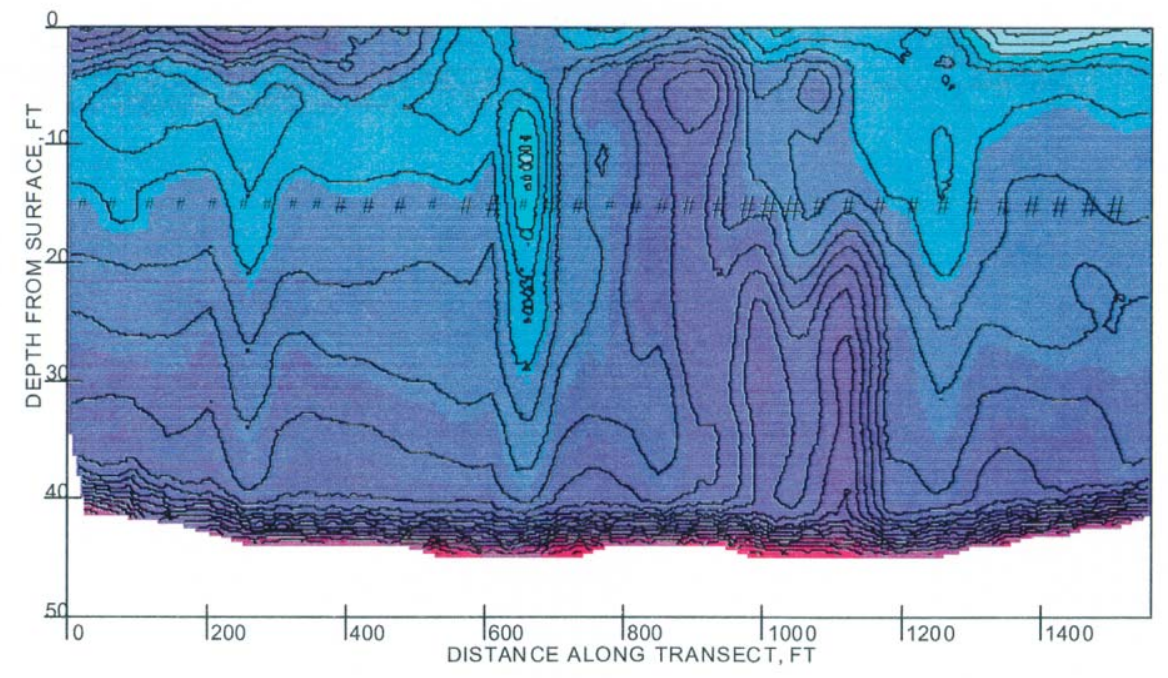

54.1 $54.1-57.6$ $57.6-61$

$61-64.5$

$64.5-67.9$ $67.9-71.4$

$71.4-74.8$

$74.8-78.3$

$78.3-81.8$

$81.8-85.2$

$85.2-88.7$

$88.7-92.1$

$92.1-95.6$

$95.6-99$

$99-102.5$

Figure B4. Relative acoustic intensity and OBS readings, Line 303, 1641 EST, Brandywine Range Reach 1, 09/15/98

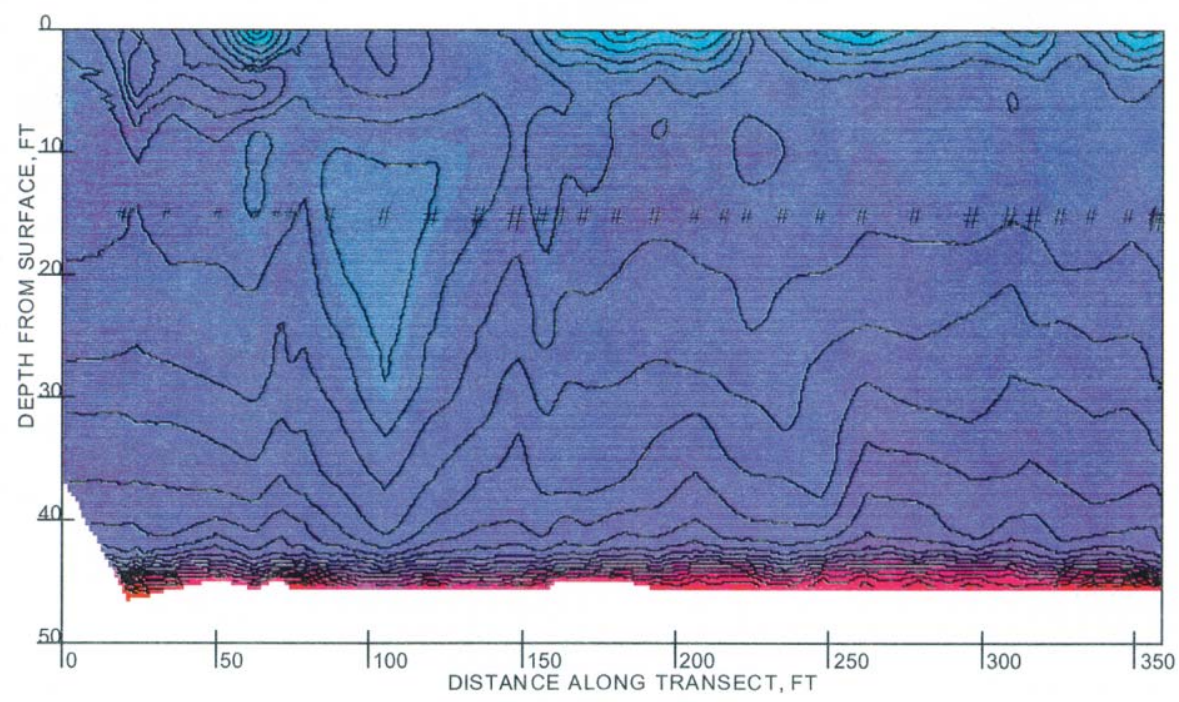

Figure B5. Relative acoustic intensity and OBS readings, Line 113, 1938 EST, Brandywine Range Reach 1, 09/15/98 


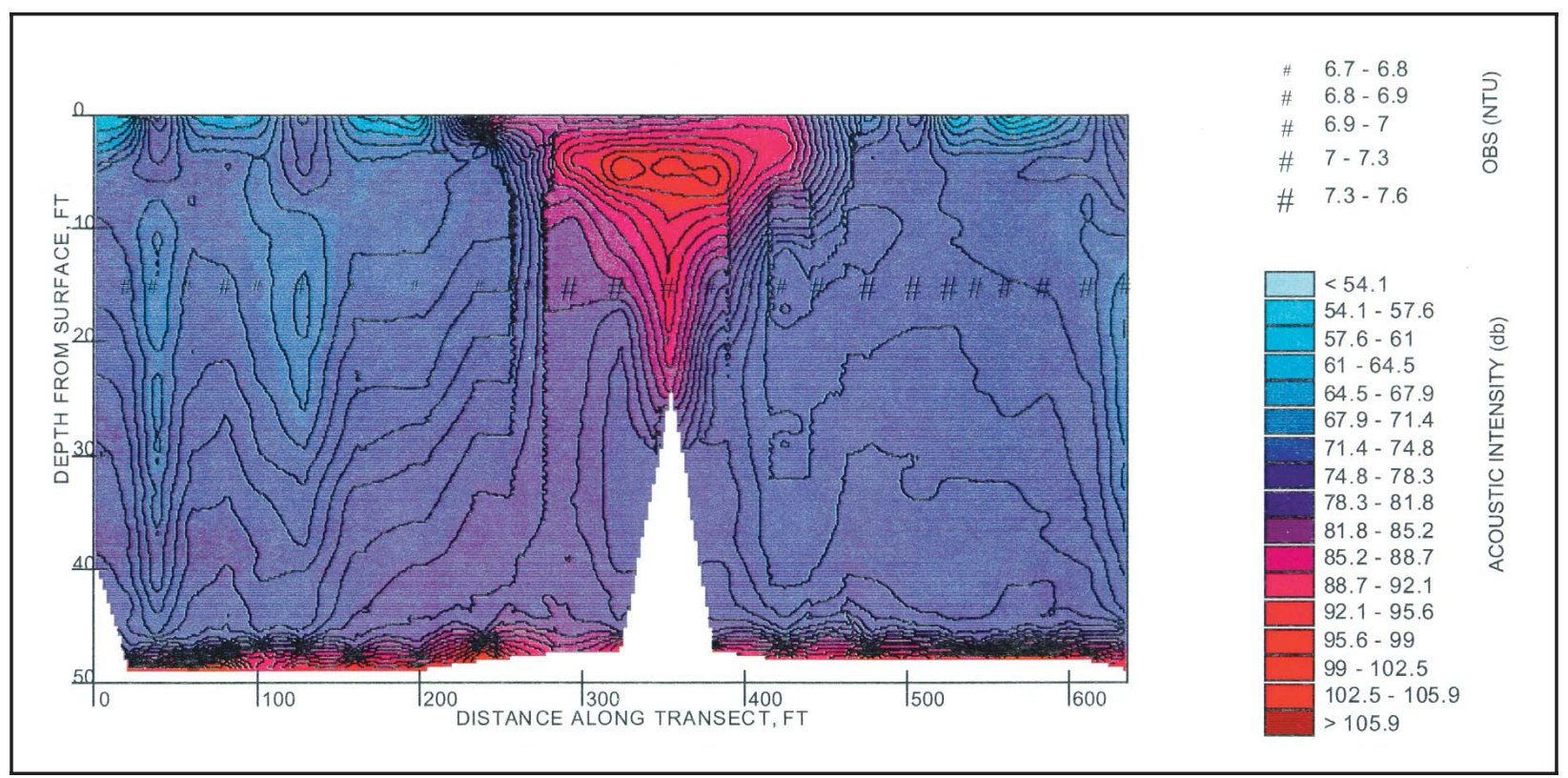

Figure B6. Relative acoustic intensity and OBS readings, Line 217, 1953 EST, Brandywine Range Reach 1, 09/15/98

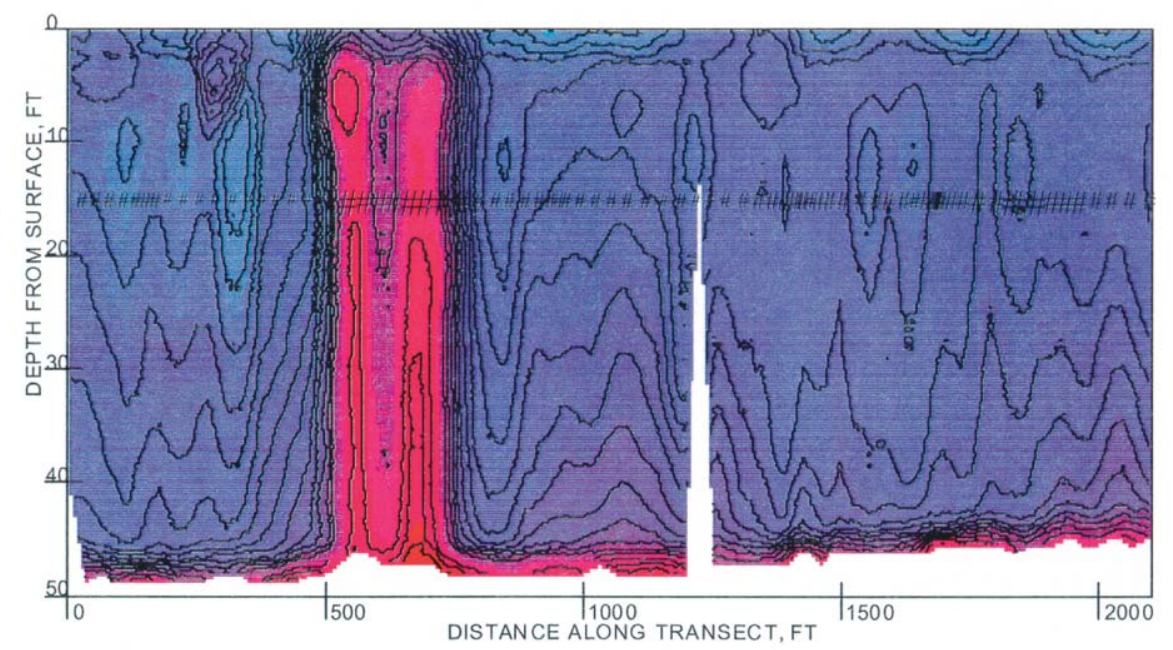

Figure B7. Relative acoustic intensity and OBS readings, Line 119, 1957 EST, Brandywine Range Reach 1, 09/15/98 


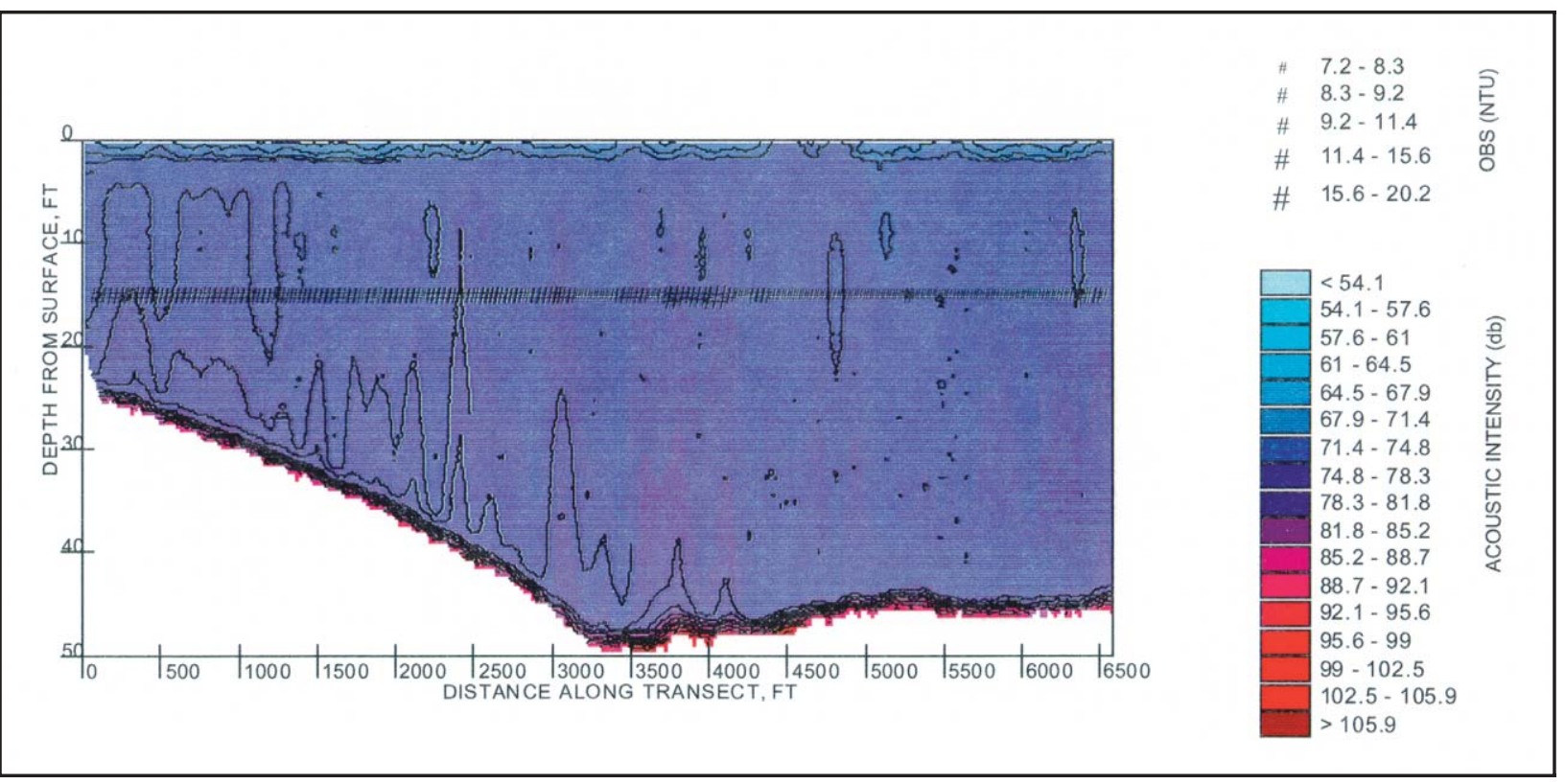

Figure B8. Relative acoustic intensity and OBS readings, Line 115, 2050 EST, Brandywine Range Reach 1, 09/15/98

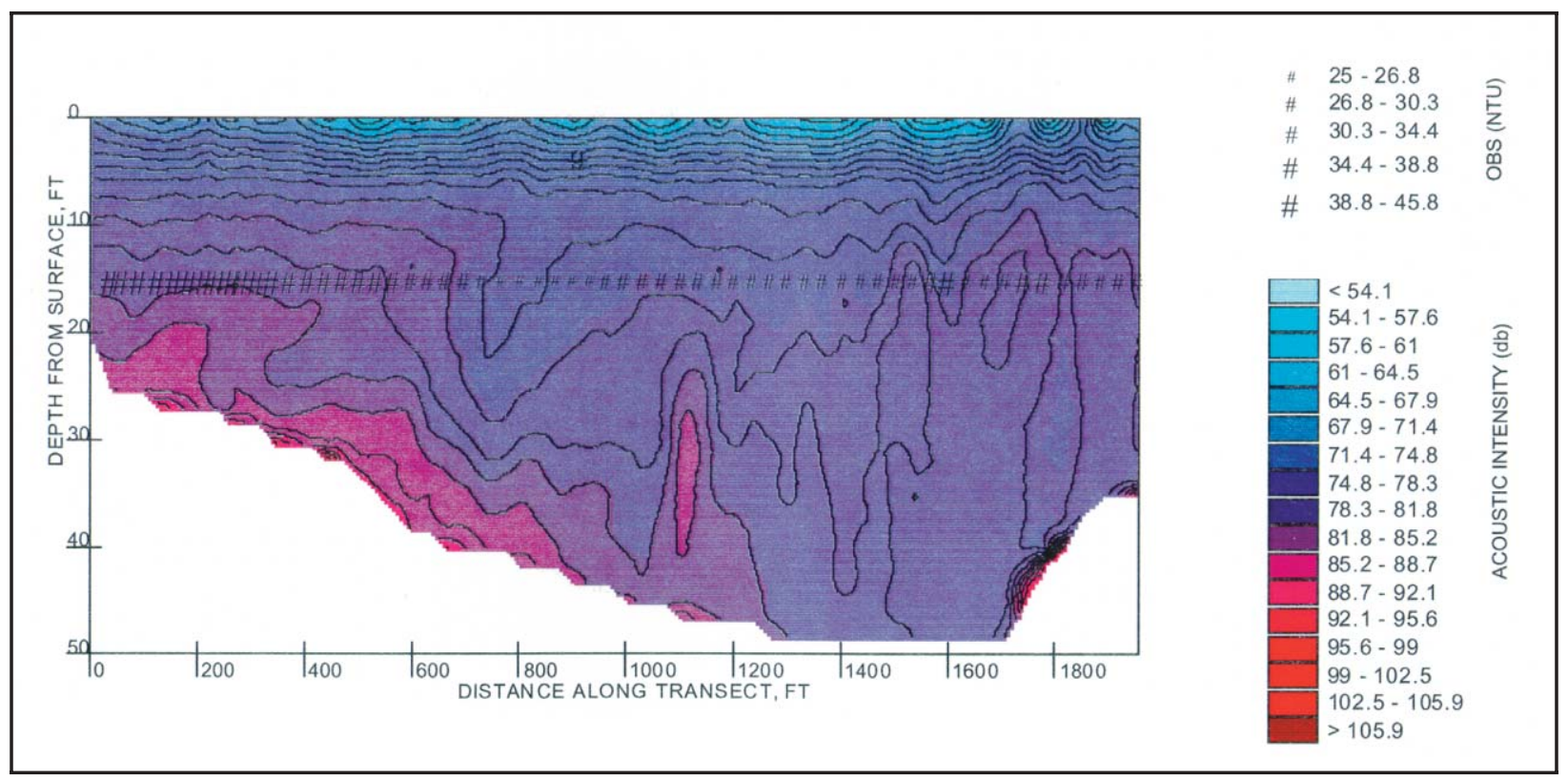

Figure B9. Relative acoustic intensity and OBS readings, Line 18, 1404 EST, Deepwater Point Range Reach 2, 09/16/98 


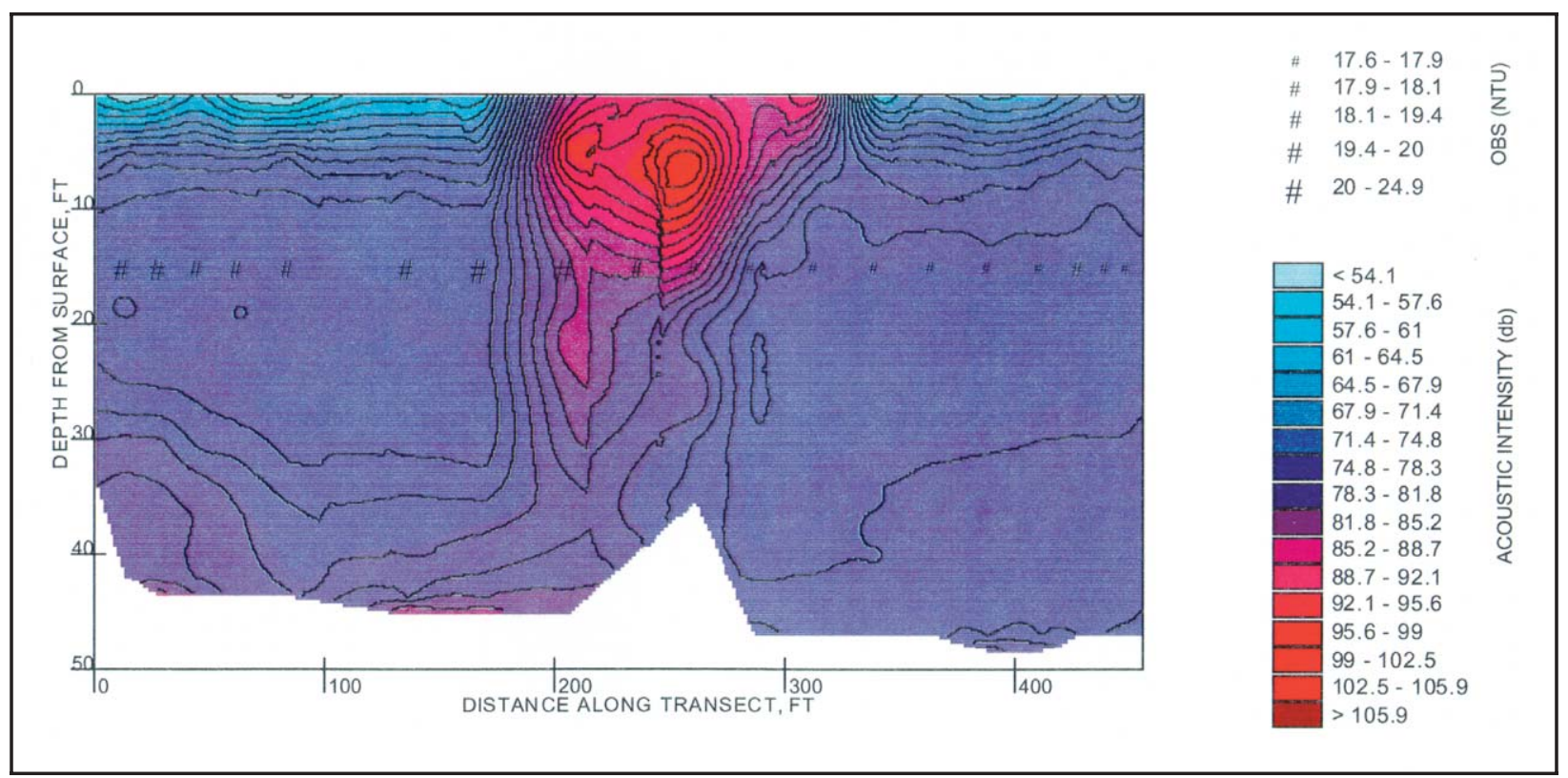

Figure B10. Relative acoustic intensity and OBS readings, Line 118, 1459 EST, Deepwater Point Range Reach 2, 09/16/98

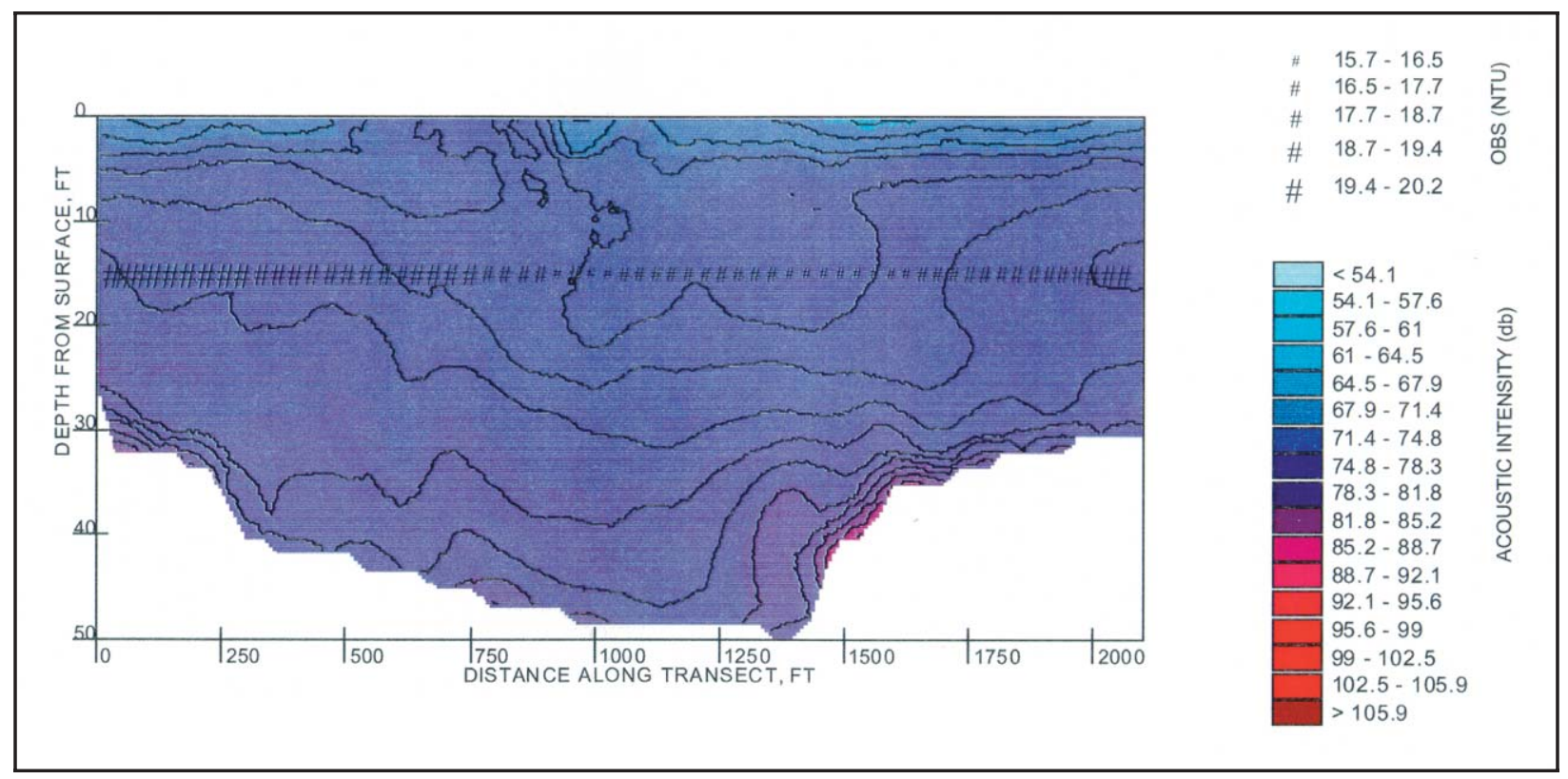

Figure B11. Relative acoustic intensity and OBS readings, Line 224, 1518 EST, Deepwater Point Range Reach 2, 09/16/98 


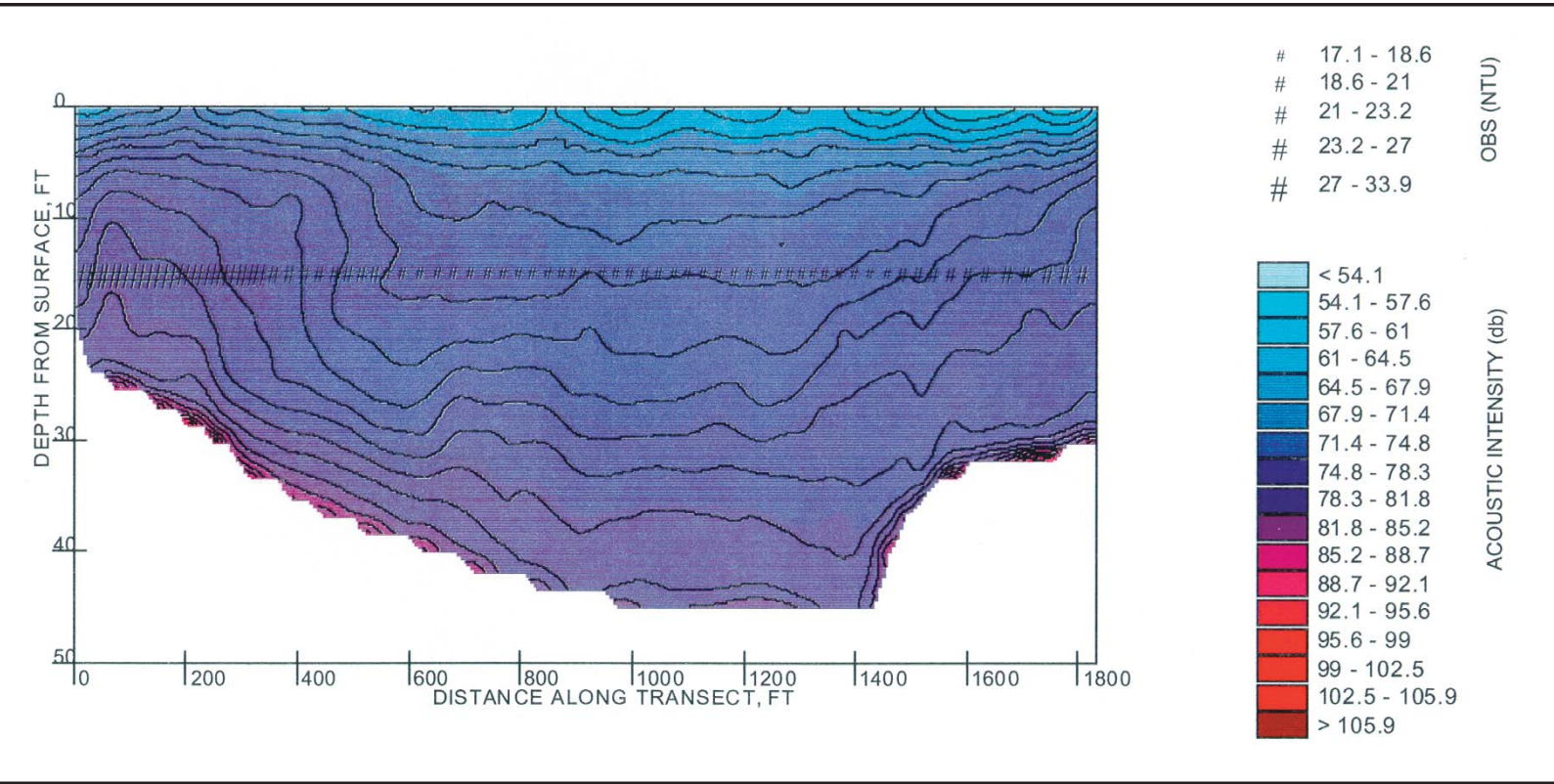

Figure B12. Relative acoustic intensity and OBS readings, Line 14, 1730 EST, Deepwater Point Range Reach 2, 09/16/98

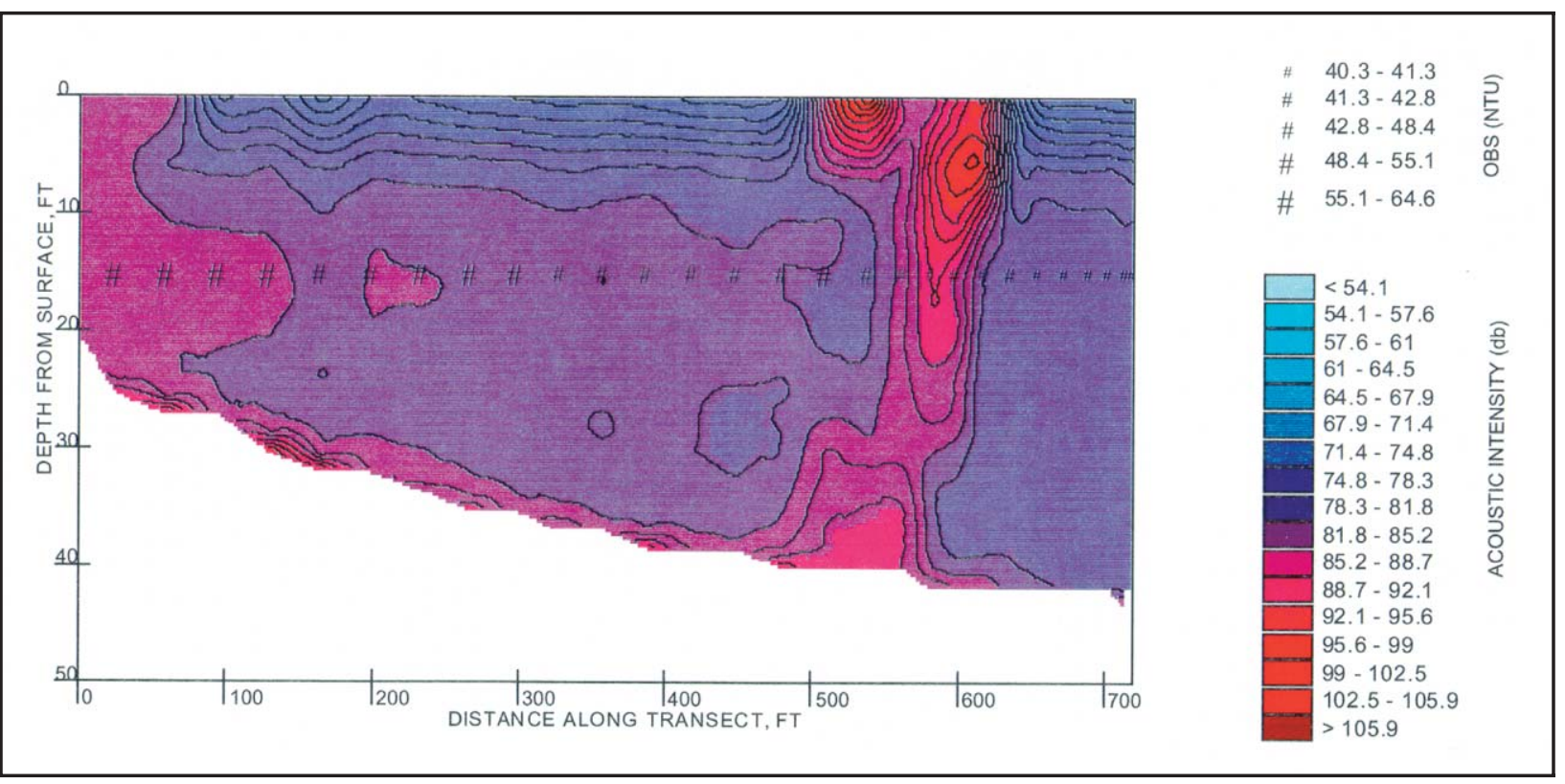

Figure B13. Relative acoustic intensity and OBS readings, Line 9, 1818 EST, Deepwater Point Range Reach 2, 09/16/98 


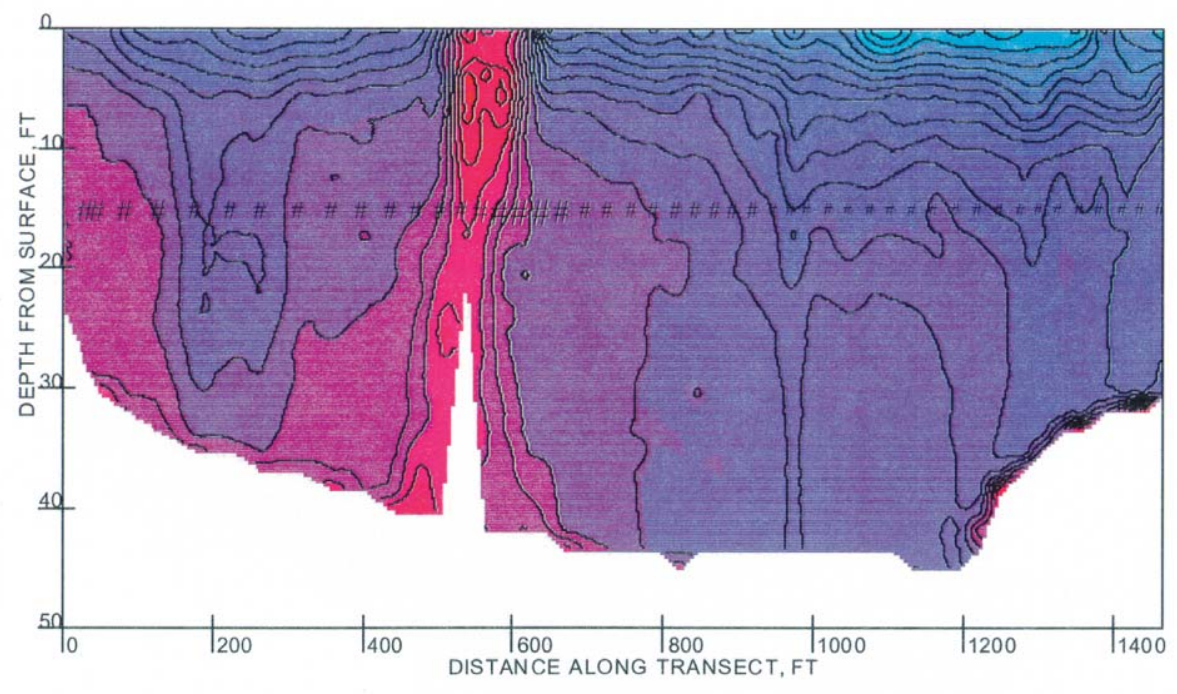

Figure B14. Relative acoustic intensity and OBS readings, Line 15, 1832 EST, Deepwater Point Range Reach 2, 09/16/98

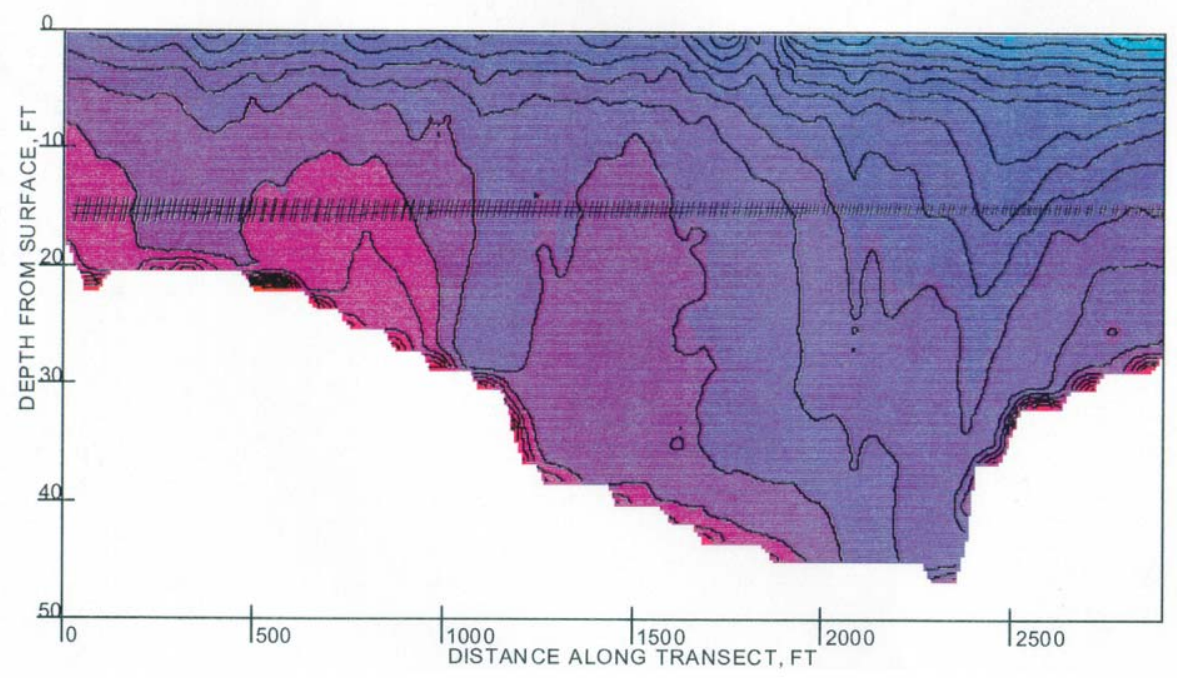

Figure B15. Relative acoustic intensity and OBS readings, Line 324, 2020 EST, Deepwater Point Range Reach 2, 09/16/98 
and horizontal dimensions of the sediment plume immediately behind the dredge. Figure B4 shows the level of suspended material in the water column 8 min following the dredge's passing, indicating that background levels of suspended material are returning to the site. No lateral dispersion of the plume out of channel was observed during the nonoverflow dredging operation.

Figures B5 through B8 illustrate the residence time of the sediment plume created with hopper-overflow conditions during dredging operations in Reach 1. Background levels of suspended materials prior to the dredging operations are shown in Figure B5. The vertical and horizontal dimensions of the sediment plume immediately behind the dredge while hopper over-flow conditions are occurring are shown in Figure B6. Plume dimensions 4 min after the dredge passed are shown in Figure B7. A wider transect was performed, as seen in the horizontal distance scale, to determine the lateral extent of the plume. No significant change above background levels could be detected. At $1 \mathrm{hr}$ elapsed time following the end of the overflow dredging operation, the levels of suspended material had returned to background conditions as shown in Figure B8. Again, no lateral dispersion of the plume out of the channel area was observed.

Figures B9 through B11 illustrate the residence time of the sediment plume created from nonoverflow conditions during dredging operations in the Reach 2 area. At the beginning of the dredging operations, background suspended material levels are shown in Figure B9. The plume dimensions in the lateral and vertical directions immediately behind the dredge at the start of dredging operations are shown in Figure B10. After an elapsed time of 19 min (Figure B11), following the end of dredging operations, the levels of suspended material had returned to background conditions. During this dredging operation, the tidal flow in the dredging area had reversed from flood flow to ebb flow conditions. This accounts for the relative change in background levels seen between Figure B9 and Figure B11. Despite the changes in background levels resulting from the change in direction of flow in the dredging area, no lateral movement of the plume beyond the channel limits was observed.

Figures B12 through B15 illustrate the residence time of the dredge plume resulting from hopper overflow dredging conditions in the Reach 2 area. Background levels prior to dredging operations are shown in Figure B12. The sediment plume dimensions immediately behind the dredge prior to overflow conditions can be seen in Figure B13. Note the increase in the suspended material levels within the first $400 \mathrm{ft}$ of the transect. The increase in these levels can be attributed to the increase in the ebb flow velocities and the resulting disturbance of bottom materials from near bottom velocities and not dredge plume dispersion. When hopper-overflow conditions began, another transect was performed located immediately behind the dredge as shown in Figure B14. The width of the transect was also increased, as indicated in the length of the horizontal distance scale, to observe the lateral extent of the dispersion of the dredge plume. After an elapsed time of $1 \mathrm{hr}$ following the completion of the overflow dredging 
operation, Figure B15 indicates that the levels of suspended materials had returned to background conditions. Note the increase in sediment disturbance near the bottom in the shallow portions of the transect which are due to the increase in the velocities during the ebb cycle of the tide. As in the previous dredge operations, no lateral dispersion of the dredge plume beyond the channel limits was observed.

The OBS data shown in Figures B2 through B15 were used to see if there is a correlation between the relative acoustic backscatter from the ADCP with different levels of turbidity for the OBS sensor. The figures indicate a fairly good correlation as increases in the ADCP relative acoustic intensities correspond to similar increases in the turbidity levels from the OBS sensor. Since the OBS sensor was deployed at a fixed depth, relative changes in turbidity throughout the water column were not measured.

\section{Equipment Description}

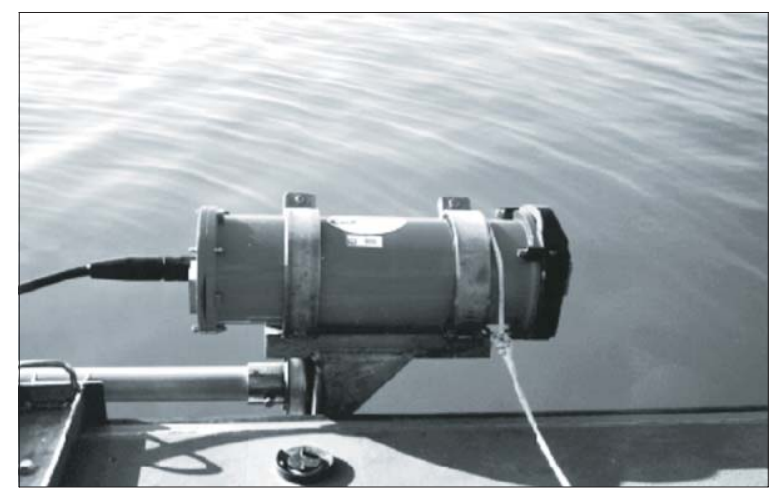

Figure B16. Acoustic Doppler Current Profiler

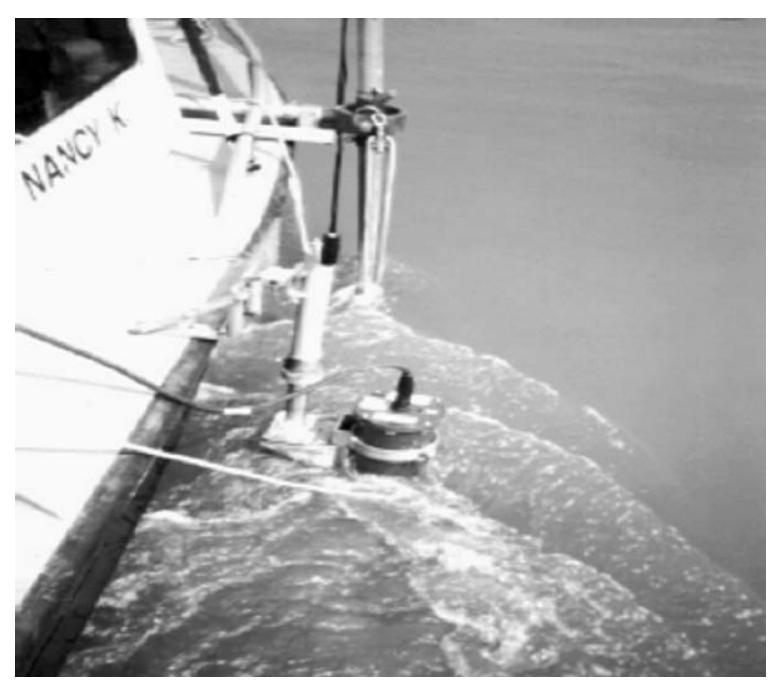

Figure B17. Vessel-mounted ADCP

\section{Acoustic Doppler Current Profiler (ADCP)}

Acoustic techniques are used to obtain current velocity and direction measurements for fast and accurate profiling in the field. The equipment used was a boat-mounted RD Instruments BroadBand Acoustic Doppler Current Profiler (ADCP) as shown in Figure B16. The RD instruments operating frequency was $1,200 \mathrm{kHz}$. The equipment can be mounted over the side of boat with the acoustic transducers submerged and data is collected while the vessel is underarey as shown in Figure B17.

The ADCP transmits sound bursts into the water column which are scattered back to the instrument by particulate matter suspended in the flowing water. The ADCP sensors listen for the returning signal and assigns depths and velocity to the received signal based on the change in the frequency caused by the moving particles. This change in frequency is referred to as a Doppler shift. 
The ADCP is also capable of measuring vessel direction, current direction, water temperature, and bottom depth. Communication with the instrument for setup and data recording are performed with a portable computer using manufacturer supplied software, hardware, and communication cables. The manufacturer stated accuracies for current speed measurement $\pm 0.2 \mathrm{~cm} / \mathrm{sec}$; for vessel direction, $\pm 2 \mathrm{deg}$; and for temperature, $\pm 5^{\circ} \mathrm{F}$.

\section{OBS Sensors}

The OBS sensor, a product of D\&A Instruments and Engineering, is a type of nephelometer for measuring turbidity and solids concentrations by detecting scattered infrared light from suspended matter. It consists of a high-intensity infrared emitting diode (IRED), a series of silicon photodiodes as detector and linear solid state temperature transducer. The IRED emits a beam at angles $50 \mathrm{deg}$ in the axial plane and $30 \mathrm{deg}$ in the radial plane to detect suspended particles by sensing the radiation they scatter, as shown in Figure B18. Scattering by particles is a strong function of the angle between the path of radiation from the sensor through the water and the signal return to the detector. OBS sensors detect only radiation scattered at angles greater than $140 \mathrm{deg}$. As with other optical turbidity sensors, the response of the OBS sensor depends on the size distribution, composition, and shape of particles suspended in the medium being monitored. For this reason, sensors must be calibrated with suspended solids from the waters being monitored. The OBS sensor is interfaced with Coastal Leasing, Inc., MicroLite solid-state microprocessor that controls samples, averaging, and data storage. The MicroLite uses Wizard portable PC software to provide user-friendly control of the instrument.

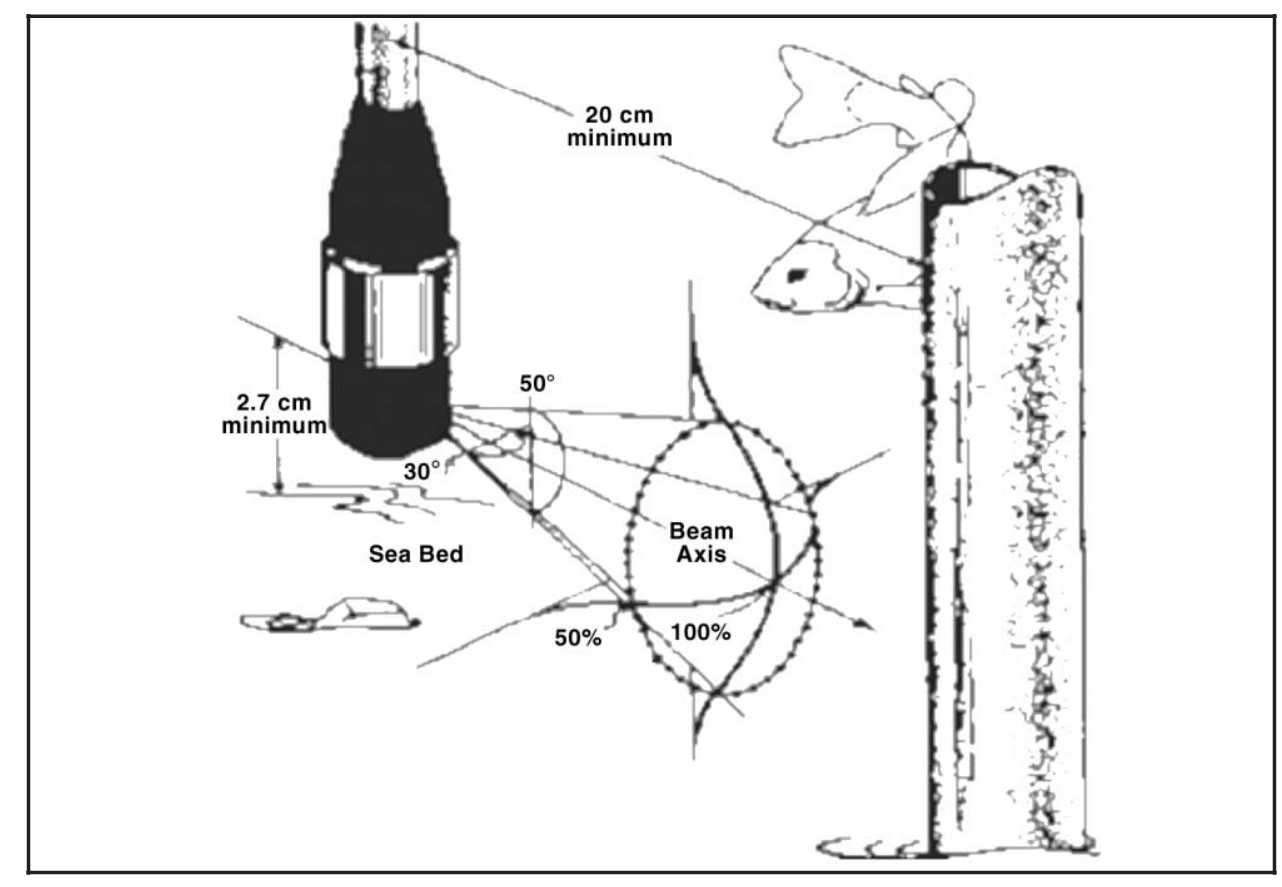

Figure B18. OBS sensor beam pattern 


\section{Tethered-drag sampler}

The Tethered-drag sampler is basically a 76-mm- (3-in.-) diam pipe cut on a 45-deg angle with a shackle mounted on one side. The sampler is

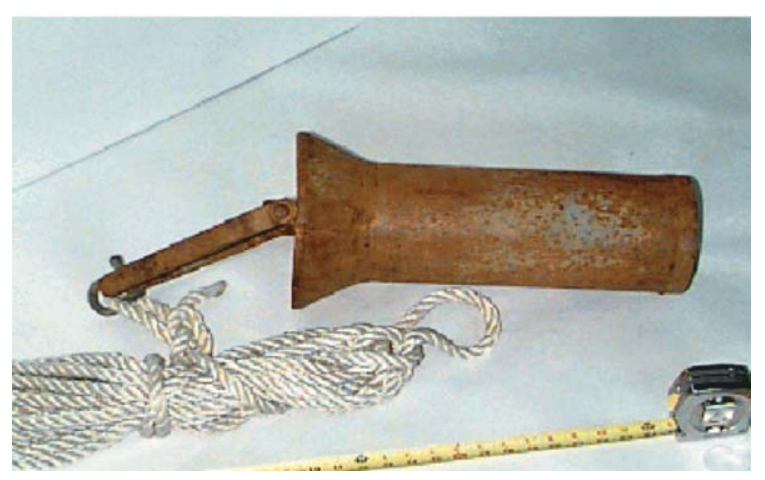

thrown over the side and dragged along the bottom. The sample accumulates inside the pipe. Samples are removed, inspected, and packaged in plastic bags or jars for further analysis once returned to ERDC. The Tethered-drag sampler is displayed in Figure B19.

Figure B19. Tethered-drag sampler

\section{Pumped water samples}

Water samples are obtained by pumping the sample from the desired depth to the surface collection point via a portable sampling pump. The pumping system consists of a 6-mm- (1/4-in.-) ID plastic tubing attached to a weighted "fish" for support. The weight is lowered by cable from a winch with a depth indicator. The opening of the sampling tubing is attached to a solid suspension bar above the weight and is pointed into the flow. A $12-\mathrm{V}$ DC pump is used to move the water through the tubing to the deck of the boat where each sample is then collected in appropriate glass or plastic containers. The pump and tubing are flushed for approximately $1 \mathrm{~min}$ at each depth before collecting the sample. 


\section{Appendix C \\ Detection of Short-Term Sedimentation During Hopper Dredging Operations in Delaware Bay and the Delaware River'}

\section{irury}

in:

US Army Corps of Engineers

Waterways Experiment Station

Vicksburg. MS 39180

February 1999

Detection of Short-Term Sedimentation During Hopper Dredging Operations in Delaware Bay and the Delaware River

by Robert J. Diaz

R. J. Diaz and Daughters

P. O. Box 114

Ware Neck, Virginia 23178

and

Douglas G. Clarke

Coastal Ecology Branch

U.S. Army Engineer Waterways Experiment Station

Adapted from unpublished draft report, Robert J. Diaz and Douglas G. Clarke, February 1999, R. J. Diaz and Daughters, Ware Neck, VA, and Coastal Ecology Branch, U.S. Army Engineer Waterways Experiment Station, Vicksburg, MS 


\section{Introduction}

Navigation channel maintenance dredging projects employing hopper dredges can produce substantial water-column turbidity when in situ sediments contain a high proportion of fines and overflow practices are used. Most of the sediment resuspended during overflow operations has been shown to settle within several hundred meters of the channel (Nichols, Diaz, and Schaffner 1990; Clarke et al. 1990). It has been hypothesized that even short-term pulses in sedimentation rates induced by overflow operations could negatively impact sensitive living resources, such as oyster beds, in the vicinity of dredged channels.

Detection and measurement of recently deposited thin layers of dredged material can be a severe technical challenge. Sediment overburdens with thicknesses on the scale of several millimeters can potentially raise concern for biological impacts. Precision bathymetry methods using acoustic technologies lack the sensitivity to detect low-density thin overburdens less than 5 to $10 \mathrm{~cm}$ thick, at best. However, direct in situ observations of very thin layers have effectively been done with sediment profile imaging (SPI). Nichols, Diaz, and Schaffner (1990) demonstrated that SPI techniques could detect thin layers of sediment deposited from hopper dredge overflow operations in the Chesapeake Bay. SPI has also proven to be very effective in mapping the distribution of thin layers resulting from open-water dredged material disposal operations in Mobile Bay (Diaz, Schafffner, and Kiley 1987a; Diaz and Schaffner 1988; Clarke and MillerWay 1992), Mississippi Sound (Diaz, Schafffner, and Kiley 1987b), and Long Island Sound (Morton, Parker, and Richmond 1985).

The primary focus of this study was to determine if short-term sedimentation of dredged material occurred as a consequence of resuspension by the dragheads or during overflow from the hopper dredge. A secondary objective, should sedimentation be detected in sediment profile images, was to determine the distances from the navigation channel at which measurable sedimentation occurred. Two areas were selected by the U.S. Army Engineer District, Philadelphia (CENAP), for conduct of the hopper dredging operations tests (Figure $\mathrm{C} 1$ ). These locations represent a relatively open-water site in the Delaware bay, designated the Lower Study site (LS), and a more riverine site in the Delaware River, designated the Upper Study site (US).

\section{Materials and Methods}

\section{Field methods}

On 15 and 16 September 1998, sediment profile images were collected at a series of stations at the two predetermined locations in Delaware Bay 
and the Delaware River (LS and US, respectively). SPI data were successfully collected at stations in the LS site (Figure C2) and stations in the US site (Figure C3). At each station a Hulcher Model Wrenn sediment profile camera was deployed. During each deployment the profile camera obtained two images (Fujichrome 100P 35-mm slides) at 5 and $15 \mathrm{sec}$ after

bottom contact. The two-image sequence helps to ensure that when deployment occurs in soft, unconsolidated sediments the sediment-water interface is captured in the image before the camera prism optical window descends too deeply into the substrate.

Stations were located based on considerations of prevailing wind, river discharge, and/or tidal flow conditions at the time of each hopper dredge test. At both study locations data were collected first while the dredge was operating without overflow, followed by a second test with overflow. Sampling proceeded for up to $2 \mathrm{hr}$ after dredging ceased.

\section{Image analysis}

The sediment profile images were first analyzed visually by projecting the images and recording all features seen into a preformatted, standardized spread sheet file. The images were then digitized using a Polaroid Sprint Scan 35 Plus scanner and analyzed using Adobe Photoshop and NTIS Image programs. Steps in the computer analysis of each image were standardized consistent with procedures described in Viles and Diaz (1991). Data from each image were sequentially saved to a spread sheet file for later analysis. Details of how these data were obtained can be found in Diaz and Schaffner (1988) and Rhoads and Germano (1986), and in the standardized image analysis procedures of Viles and Diaz (1991).

\section{Results and DIscussion}

SPI images from a total of 14 stations were analyzed from the LS site (Figure C2) and 41 stations from the US site (Figure C3). The approximate location of the hopper dredge in proximity to the sampling stations is shown in Figures C2 and C3. The LS site was sampled on 15 September from 1958 to $2208 \mathrm{hr}$. Tidal flows were flooding during the sampling period and winds were approximately 24 to $32 \mathrm{kph}$ ( 15 to $20 \mathrm{mph}$ ) out of the east. Sea conditions were marginal for successful deployment of the camera system, with approximate wave heights of 0.6 to $0.9 \mathrm{~m}$ ( 2 to $3 \mathrm{ft})$. The US site was sampled on 16 September 1998 from 1507 to $2102 \mathrm{hr}$. This reach of the Delaware River is influenced by tidal currents, which were ebbing during the sampling period. Sea conditions were mild with wave

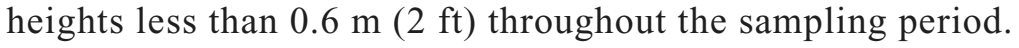

Presented below are explanations of each of the parameters produced from analysis of SPI images and an overview of observations of physical and biological features at the two study sites. Complete listings of visual 
and computer analysis data for each study site are given in Tables C1 and $\mathrm{C} 2$.

\section{Prism penetration}

This parameter provided a geotechnical estimate of sediment compaction, with the profile camera prism acting as a dead weight penetrometer. The depth of prism penetration is therefore related to the "softness" or degree of sediment compaction or water content. Penetration was simply measured as the distance the sediment interface moved up the $23-\mathrm{cm}$ length of the prism optical window as captured by the $15-\mathrm{sec}$ image. The weight of the camera frame was kept constant at $43 \mathrm{~kg}(95 \mathrm{lb})$ in order to allow comparisons of relative sediment compaction between stations.

Sand bottoms typical of the LS site had comparatively shallow penetration depths, ranging from 0.0 to $10.9 \mathrm{~cm}$ (Table $\mathrm{C} 1$ ). When sandy sediments are poorly sorted, as was the case at channel station LS-09 (Figure C4), prism penetration was deeper. Silty-clay sediments prominent at the US site had comparatively deep penetration (loosely compacted) values, ranging from 9.8 to $25.0 \mathrm{~cm}$ (Table $\mathrm{C} 2$ ). Compacted clay sediments, as indicated by very shallow penetration, can be seen in the image from station US-14 (Figure C5).

\section{Surface relief}

Surface relief or boundary roughness was measured as the difference between the maximum and minimum distance (relative to the sediment-water interface) the prism penetrated and provided qualitative and quantitative data on habitat characteristics useful for evaluating existing conditions. Small-scale bed roughness on the order of the width $(15 \mathrm{~cm})$ of the prism optical window can be estimated from the images. Factors contributing to observed roughness can often be inferred from visual analysis of the images.

In the open-water setting of the sandy LS site, physical factors (e.g., water current and wave generated turbulence) obviously dominated local sediment processes. Surface relief was typically present as small bed forms (e.g., LS-13, Figure C6) that ranged from 0.6 to $2.0 \mathrm{~cm}$ (Table C1). In contrast, the muddy habitats of the US site were primarily influenced by biological features, including mounds, pits, and tubes formed from the biogenic activity of benthic organisms (e.g., US-35, Figure C7). Here surface relief values ranged from 0.4 to $3.5 \mathrm{~cm}$ (Table $\mathrm{C} 2$ ).

\section{Apparent color redox potential discontinuity layer}

This parameter has been determined to be an important estimator of benthic habitat quality (Rhoads and Germano 1986, Diaz and Schaffner 1988), providing an estimate of the depth to which sediments are oxidized. The 
term "apparent" is used in describing this parameter because no direct chemical measurement is made of the redox potential. Rather an assumption is made that, given the complexities of iron and sulfate reduction-oxidation chemistry, reddish/greenish-brown sediment color tones (Diaz and Schaffner 1988) are indicative of oxic sediments, whereas reduced sediments have gray to black color tones. This is in accordance with the classical concept of redox potential discontinuity (RPD) depth, which associates RPD with sediment color (Fenchel 1969, Vismann 1991).

The depth of the apparent color RPD was defined as the area of all the pixels in the image discerned as being oxidized divided by the width of the digitized image. The area of the image with oxic sediment was obtained by digitally manipulating the image to enhance characteristics associated with oxic sediment (reddish/greenish-brown color tones). The enhanced area was then measured from a density slice of the image.

The apparent color RPD has been a very useful parameter in assessing the quality of a benthic habitat for infauna and epifauna from both physical and biological perspectives. Rhoads and Germano (1986); Revelas, Rhoads, and Germano (1987); Day, Schaffner, and Diaz (1988); Diaz and Schaffner (1988); Valente et al. (1992); and Bonsdorff et al. (1996) all found the depth of the RPD from profile images to be directly correlated to the quality of the benthic habitat in polyhaline and mesohaline estuarine zones. Controlling for differences in sediment type, habitats with relatively thin $(<5 \mathrm{~mm})$ RPD layers tend to be associated with some type of environmental stress. In contrast, habitats with relatively deep RPD values $(>2 \mathrm{~cm})$ usually have flourishing infaunal and epifaunal communities.

Porous sandy sediments (e.g., LS-09, Figure C4) and silty-clay sediments with evidence of high levels of biological activity (e.g., US-11, Figure C8) had the deepest RPD measurements in this study. Shallowest RPD measurements were associated with images that had signs of physical disturbance, possibly dredging related (e.g., LS-06, Figure C9), or were compact clays (e.g., US-33, Figure C10). In the LS site, average RPD depth ranged from 0.7 to $5.3 \mathrm{~cm}$, and from 0.1 to $6.6 \mathrm{~cm}$ in the US site (Tables C1 and $\mathrm{C} 2$ ).

\section{Sediment grain size}

Grain size is an important parameter for determining the nature of the physical forces acting on a sedimentary habitat. Grain size is also a major factor in determining benthic community structure (Rhoads 1974). The sediment type descriptors used for image analysis follow the Wentworth classification as described in Folk (1974) and represent the major modal class for each image. Grain size was determined by comparison of collected images with a set of standard images for which mean grain size had been determined in the laboratory. 
Grain size ranged from medium-sand gravel (e.g., US-21, Figure C11) to clay (e.g., US-35, Figure C7). Traces of sand were also seen at a few fine-grained stations (e.g., US-29, Figure C12) and traces of fines at coarsegrained stations (e.g., US-32, Figure C13). Within study site variation in sediment type for the LS site was low, with the modal grain size being finemedium-sand (e.g., LS-03, Figure C14). Shell hash was a major component of sediments in the LS site, particularly in the navigation channel (e.g., LS-06, Figure C9) (Table C1). In the US site sediments were more variable with the modal grain size being clay (e.g., US-09, Figure C4), which was closely followed by silty-clay (e.g., US-09, Figure C15). In addition to having finer sediments than the LS site, there was little evidence of shell hash in US site sediments (Table C2).

\section{Near-bottom turbidity}

The sediment profiling camera is also able to image water column turbidity immediately above the sediment-water interface. Light from the camera prism's internal strobe illuminates suspended sediment particles and allows qualitative estimation of turbidity. Turbidity was categorized as low (if the water column was clear with little or no suspended sediment, e.g., LS-02, Figure C16), moderate (e.g., US-09, Figure C15), and high (e.g., US-14, Figure C5). If plumes of resuspended sediment derived from either of the dragheads of overflow were present at the sampling station, the camera would capture the near-bottom turbidity. Such turbidity can be distinguished from other sources, such as that frequently caused by camera frame contact with the substrate, by color tones. Dredge-induced turbidity has a gray color because the bulk of the sediments dredged are from the anoxic zone and in a reduced redox chemical state. Reduced iron and manganese sulfide compounds are dark gray to black in color which contrasts well with the reddish to brown color tones of their oxidized compounds. Background turbidity or that caused by the camera frame landing on the bottom would be brown in color because the suspended sediments were disturbed from the uppermost few millimeters of surficial sediments, which are typically in an oxic redox state.

Two stations in the LS site (LS-07 and LS-12, Figures C17 and C18) had grayish colored suspended material. Station LS-07 was located on the edge of the navigation channel and could have been affected by passage of the dragheads. This station was occupied prior to initiation of overflow. LS-12 was located in the channel and appeared to have been recently disturbed. All other LS images had brownish suspended materials (Table C2).

The relative amount of suspended material showed no pattern relative to the dredging operation at either LS or US site. In the US site, high levels of turbidity seemed associated with shoal areas $(<5.5 \mathrm{~m}(<18 \mathrm{ft})$ deep) to the northwest of the channel (Figure C3). The four channel stations in the US site had low turbidity levels (Table C2). Only one of the four channel stations in the LS site had moderate turbidity, while the remaining three had low turbidities (Table C1). 


\section{Current scour}

While sitting on the bottom, the prism and camera housing assembly present an obstruction to bottom currents. Deflection of currents can erode the sediment-water interface at the edges of the prism. This erosion can be seen in SPI images as small dips in the sediment-water interface at the edges of the image. When these dips occur, it is reasonable to assume that bottom currents at the time the image was taken were $>10 \mathrm{~cm} / \mathrm{sec}$.

Evidence of scour was seen at three of the four channel stations in the LS site (e.g., LS-12, Figure C18) and one shoal station (LS-03, Figure C14) (Table C1). In the US site only one of the 41 stations (US-31, on the channel edge, Figure C19) showed evidence of scour (Table C2). Scour patterns indicated that bottom currents are likely stronger in the LS site relative to the US site.

\section{Dredged material}

When recently deposited, dredged sediments from hopper overflow or open-water disposal are distinct in color from background sediments (Diaz and Schaffner 1988; Nichols, Diaz, and Schaffner 1990), being grayer than background sediments. This is the result of in general, the more advanced diagenic state of deep sediments being dredged (Rhoads, SAIC, personal communication, as discussed in section on Near-Bottom Turbidity).

SPI images from three of the four channel stations in the LS site appear to be recently disturbed and likely dredged material (e.g., LS-06, Figure C9) (Table C1). The channel sediments are sands with shell hash that contain little fine sediment. It is not likely that the surface sediments are from hopper overflow, but more likely associated with disturbance from the dragheads. In addition, the test dredging and overflow were not of sufficient quantity or duration to produce extensive layering from sands.

No station from the US site appeared to have recently deposited dredged material. Sediments at all US site stations appeared to be undisturbed and representative of background conditions.

\section{Sediment layering}

Sediment layering as indicated by color or grain-size changes are readily seen in SPI images. The presence of layers is indicative of physical disturbances or episodic events. Sediment layering is characteristic of hopper overflow and open-water disposal operations and can be readily seen in SPI images (Diaz and Schaffner 1988; Nichols, Diaz, and Schaffner 1990).

In the LS site four stations had evidence of layering from grain-size changes (Table C1). Station LS-06 (Figure C9), in the channel, had a shell hash layer at $1.6 \mathrm{~cm}$ from the sediment surface. The other three stations, 
LS-11 (Figure C20) and LS-13 (Figure C6) on the edge of the channel and LS-10 (Figure C21) on the shoal near the channel, all had thin layers of sandy sediments overlaying silty sediments. Each case seemed indicative of recently deposited sediments, possibly from the dragheads or current induced transport of surface sands. The sediments were not likely from hopper overflow operations since little sand-size sediment would have been discharged from the hopper during a single loading process.

In the US site about half of the stations had sediment layers (Table C2). However, none of the four stations in the channel had sediment layering. About half of the stations ( 8 of 17) on the edge of the channel had layers, three with color layering and five with grain-size layering. All five of the grain-size layered channel edge stations had sands on the surface overlying clayey sediments. Since the sediments in the channel were fine silts and clays, it is unlikely that layers observed in these images were attributable to the dredging operations or overflow, which contained little or no sand. In addition, grain-size layered channel edge stations US-22 (Figure C22), US-23 (Figure C23), and US-33 (Figure C10) had amphipod and/or worm tubes which could not have reestablished living positions in the approximately 1-hr interval between dredging operations and sampling. Color layering was represented by varying hues of grays and was found deeper in the sediments, ranging from 2.5 to $9.0 \mathrm{~cm}$ from the surface (Table C2). These deeper color layers are not likely a result of recent dredging operations and may represent episodic events such as seasonal high river discharges or storm deposits. Detritus appeared to be mixed into the uppermost sediment layer at shoal stations US-09 (Figure C15), US-10 (Figure C24), and US-11 (Figure C8).

\section{Surface features}

Surface features include a variety of physical and biological parameters, each providing different information on the type of habitat and its quality for supporting benthic species. The presence of certain features is indicative of the overall nature of a habitat. For example, bed forms are always associated with physically dominated habitats, whereas the presence of worm tubes or feeding pits would be indicative of a more biologically accommodated habitat (Rhoads and Germano 1986, Diaz and Schaffner 1988). Surface features were visually evaluated from each image and compiled by type and frequency of occurrence.

The sediment surface at stations in the LS site was dominated by bed forms and shell hash (Table C1). In the US site, biogenic pits and mounds were the dominant surface features (Table C2). No epifauna were seen in either area. Flock layers, thin layers of unconsolidated sediments, occurred at six shoal stations (e.g., US-09, Figure C15) and one channel edge station (US-34, Figure C25) in the US site (Table C2). All flock layers appeared to be composed of background sediments and not dredged material, as evidenced by their respective color tones. 
Tubes were seen at only one of the LS site stations (LS-11, Figure C20). At the US site stations, worm or amphipod tubes occurred at 12 of 41 stations (Table C2). Amphipod tube mats occurred at US-05 (Figure C26), US-06 (Figure C27), and US-22 (Figure C22), which were channel and channel edge stations.

\section{Subsurface features}

These parameters include a wide variety of features and provide insights into physical and biological processes influencing the bottom. For example, the presence of methane gas voids has been an indication of anaerobic metabolism (Rhoads and Germano 1986) and associated with high rates of bacterial activity. Muddy habitats with large amounts of methane gas are generally associated with areas of oxygen stress or high organic loading. On the other hand, habitats with burrows, infaunal feeding voids, and/or actual infauna visible in SPI images are generally more biologically accommodated and considered "healthy" (Rhoads and Germano 1986, Diaz and Schaffner 1988, Valente et al. 1992). Subsurface features were visually evaluated from each image and compiled by type and frequency of occurrence.

No infauna, burrows, or voids were seen at the LS site stations (Table C1). This was the result, in part, of the prevalence of coarse sediments, which are not generally associated with fauna that form burrows or voids, and by shallow camera prism penetration.

In the US site, 4 stations had infaunal organisms, 12 had active burrows, 2 had active feeding voids, and 3 had anaerobic voids (Table C2). Gas filled voids occurred at nine stations and were abundant at most of these stations (e.g., US-26, Figure C28), indicating relatively high concentrations of organic matter in the sediments. Evidence of hydrocarbon contamination was seen at station US-03 (Figure C29) in the form of "oil spots." Diaz et al. (1993) found that sediments containing high concentrations of hydrocarbons had a unique signature in the SPI images and that this signature was significantly related to the occurrence of hydrocarbons.

\section{Summary and Conclusions}

The LS site was more physically accommodated than the US site which was more biologically accommodated (Table C3). Sediments in the LS site were coarser and had more shell hash than the US site which was characterized by finer sediments and more biologically reworked.

There was evidence that recent physical disturbance had occurred at several of the LS stations (LS-06, LS-09, LS-12), possibly a result of the dredging operations. Gray colored suspended material, indicative of hopper overflow material, was also observed at two stations (LS-07, LS-12). 
However, since this gray suspended material was also associated with recently disturbed sediments at LS-12, it could also have resulted from draghead activity. This leaves LS-07 as the station with the clearest signature of hopper overflow, but this was in the form of turbidity and not accumulation of overflow material on the sediment surface. Four LS stations had layering from grain-size changes. Station LS-06, in the channel, had a shell hash layer at $1.6 \mathrm{~cm}$ from the sediment surface. The other stations, LS-11 and LS-13 on the edge of the channel and LS-10 on the shoal near the channel, all had thin layers of sandy sediments overlying silty sediments. Although such layers are indicative of recently deposited sediments, those seen in the SPI images are likely the result of normal sediment transport processes rather than hopper overflow operations. Little sand would be discharged from overflow in a single pass of the hopper dredge.

In the US site, no evidence of recent physical disturbance was detected at any of the stations, but material that could have come from the hopper overflow was observed at one station (US-33). About half of the US stations had sediment layers, but none of the stations in the channel had sediment layers. About half of the stations on the edge of the channel had layers, three with color layering and five with grain-size layering. All five of the sediment layered channel edge stations had sands on the surface overlaying clayey sediments. Since the sediments in the channel were finer silts and clays, it was unlikely that the layers at the channel edge stations were the result of the dredging operations. In addition, sediment layered channel edge stations US-22, US-23, and US-33 had amphipod and/or worm tubes which could not have reestablished living position in the short interval between dredging and sampling. Flocculent sediment layers, thin layers of unconsolidated surface sediments, occurred at six shoal stations and one channel edge station in the US site. Based on their color tones, all flock layers appeared to be composed of background sediments and not hopper overflow or dredged material. Evidence of hydrocarbon contamination was seen at station US-03 in the form of "oil spots."

No indication of newly deposited dredged material was observed at stations outside the edge of the navigation channel at either study site. Although the sampling station coverage was not extensive, given the relatively short duration of the tests, the risk of significant sedimentation as a consequence of the hopper dredging operations appears largely restricted to the bottom and slide slopes of the channel.

\section{Literature Cited}

Bonsdorff, E., Diaz, R. J., Rosenberg, R. Norkko, A., and Cutter, G. R. (1996). "Characterization of soft-bottom benthic habitats of the Aland Islands, northern Baltic Sea," Marine Ecology Progress Series 142, 235-245. 
Clarke, D. G., Homziak, J., Lazor, R. L., Palermo, M. R., Banks, G. E., Benson, H. A., Johnson, B. H, Smith-Dozier, T., Revelas, G., and Dardeau, M. R. (1990). "Engineering design and environmental assessment of dredged material from hydraulically filled hopper barges in Mobile Bay, Alabama," Dredging Operations Technical Support Program Miscellaneous Paper D-90-4, U.S. Army Engineer Waterways Experiment Station, Vicksburg, MS.

Clarke, D. G., and Miller-Way, T. (1992). "An environmental assessment of the effects of open-water disposal of maintenance dredged material on benthic resources in Mobile Bay, Alabama," Dredging Operations Technical Support Program Miscellaneous Paper D-92-1, U.S. Army Engineer Waterways Experiment Station, Vicksburg, MS.

Day, M. E., Schaffner, L. C., and Diaz, R. J. (1988). "Long Island Sound sediment quality survey and analyses," Tetra Tec, Report to NOAA, NOS, OMA, Rockville, MD.

Diaz, R. J., Schaffner, L. C., and Kiley, K. (1987a). "Sediment profile camera survey of the Fowl River open-water dredged material disposal area, Mobile Bay, Alabama," Virginia Institute of Marine Science Contract Report to Taxonomic Associates, Mobile, AL.

. (1987b). "Sediment profile camera survey of the Gulfport, Mississippi, open-water dredged material disposal site," Science Applications International Inc. Report No. SAIC-88/5703-176 to Taxonomic Associates, Mobile, AL.

Diaz, R. J., and Schaffner, L. C. (1988). "Comparison of sediment landscapes in the Chesapeake Bay as seen by surface and profile imaging." Understanding the estuary; Advances in Chesapeake Bay research. M. P. Lynch and E. C. Krome, ed., Chesapeake Research Consortium Publication 129, CBP/TRS 24/88, 222-240.

Diaz, R. J., Hannsson, L. J., Rosenberg, R., Gapcynski, P., and Unger, M. (1993). "Rapid assessment of sedimentological and biological characteristics of a hydrocarbon pollution gradient," Water, Air and Soil Pollution 66, 251-266.

Fenchel, T. (1969). "The ecology of marine microbenthos. IV. Structure and function of the benthic ecosystem, its chemical and physical factors and microfauna communities with special reference to the ciliated Protozoa," Ophelia 6, 1-182.

Folk, R. L. (1974). Petrology of Sedimentary Rocks. Hemphill's, Austin, TX. 
Morton, R. W., Parker, J. H., and Richmond, W. H. (1985). "DAMOS. Summary of program results, 1981-1984," Science Applications International Inc. Report No. SAIC-84/7521-C46 to the U.S. Army Engineer Division, New England, Waltham, MA.

Nichols, M. M., Diaz, R. J., and Schaffner, L. C. (1990). "Effects of hopper dredging and sediment dispersion, Chesapeake Bay," Environmental Geology and Water Science 15,31-43.

Revelas, E. C., Rhoads, D. C., and Germano, J. D. (1987). "San Francisco Bay sediment quality survey and analysis," NOAA Technical Memorandum NOS OMA 35, Rockville, MD.

Rhoads, D. C. (1974). "Organism B sediment relations on the muddy sea floor," Oceanography and Marine Biology Annual Review 12, 263-300.

Rhoads, D. C., and Germano, J. D. (1982). "Characterization of organismsediment relations using sediment profile imaging: An efficient method of remote ecological monitoring of the sea floor (REMOTS system)," Marine Ecology Progress Series 8, 115-128.

. (1986). "Interpreting long-term changes in benthic community structure: A new protocol," Hydrobiologia 142, 291-308.

Valente, R. M.., Rhoads, D. C., Germano, J. D., and Cabelli, V. J. (1992). "Mapping of benthic enrichment patterns in Narragansett Bay, Rhode Island," Estuaries 15, 1-17.

Viles, C., and Diaz, R. J. (1991). "Bencore, an image analysis system for measuring sediment profile camera slides," School of Marine Science, Virginia Institute of Marine Science, College of William and Mary, Gloucester Point, VA.

Vismann, B. (1991). "Sulfide tolerance: Physiological mechanisms and ecological implications," Ophelia 34, 1-27. 


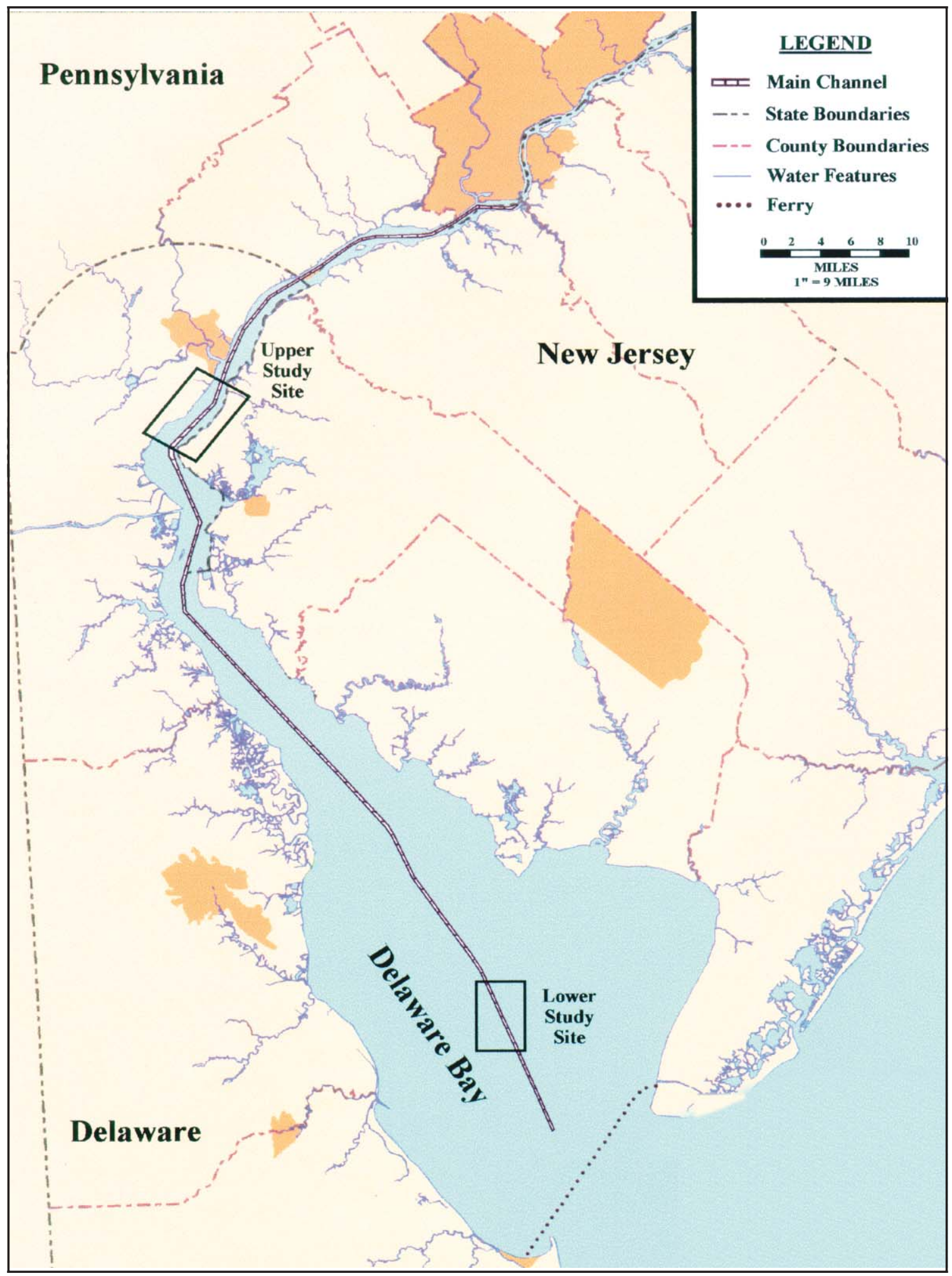

Figure C1. Locations of the lower and upper hopper dredge overflow test study sties 


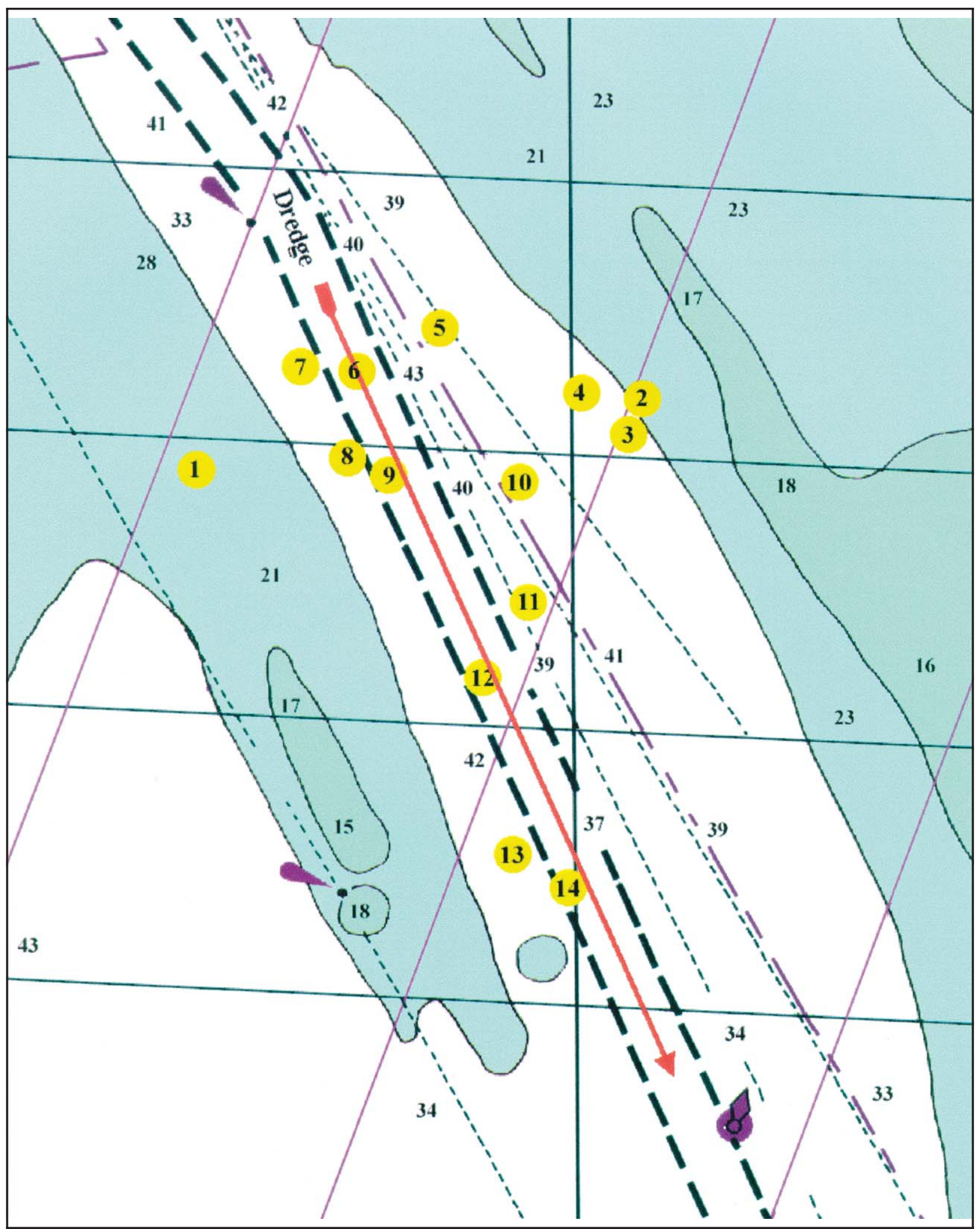

Figure C2. Sediment profiling imagery stations occupied during hopper dredge loading and overflow tests at the LS site. The figure has been modified from a NOAA navigaition chart with depths indicated in feet. Approximate start and end of test locations of the dredge are indicated by the red arrow 


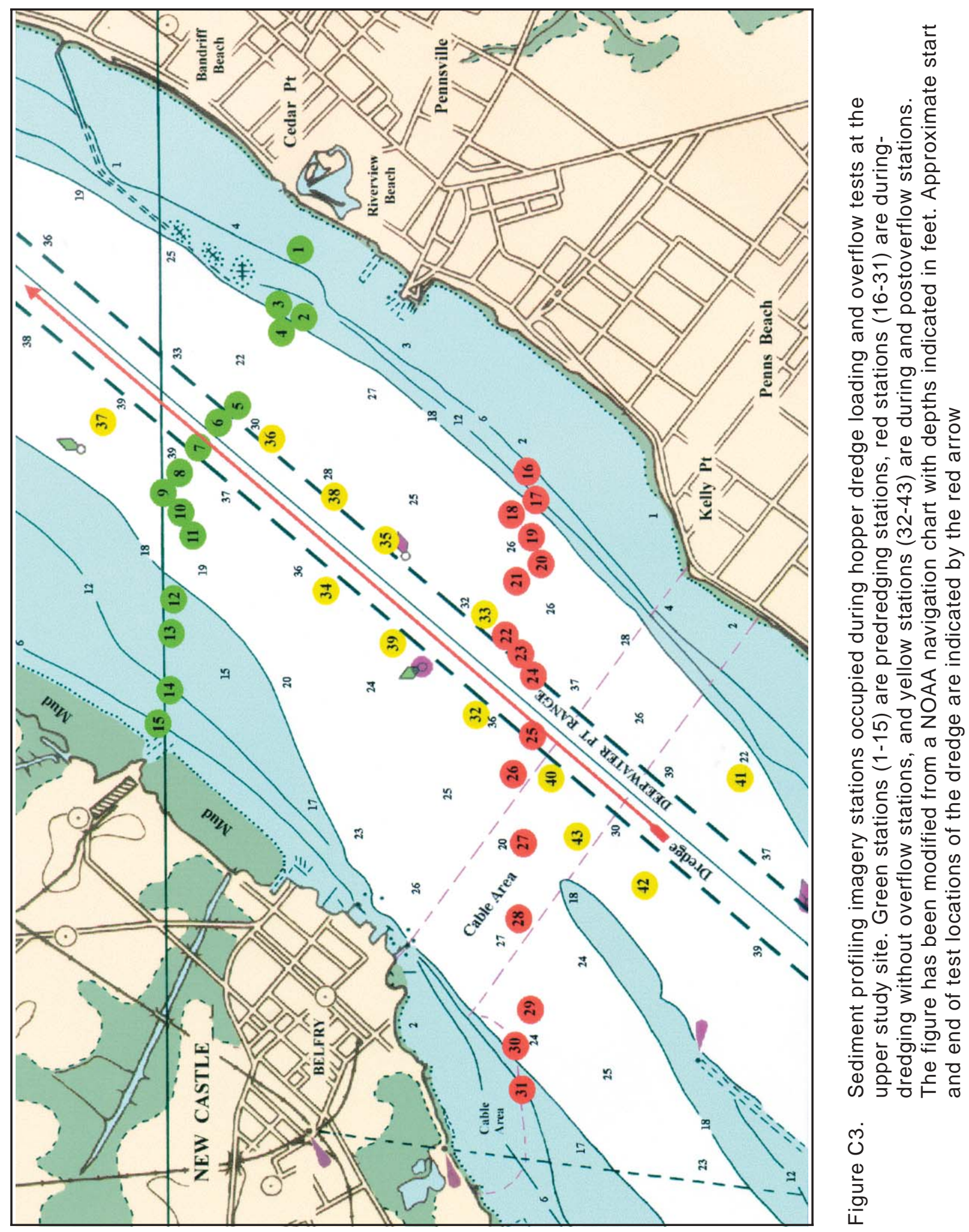




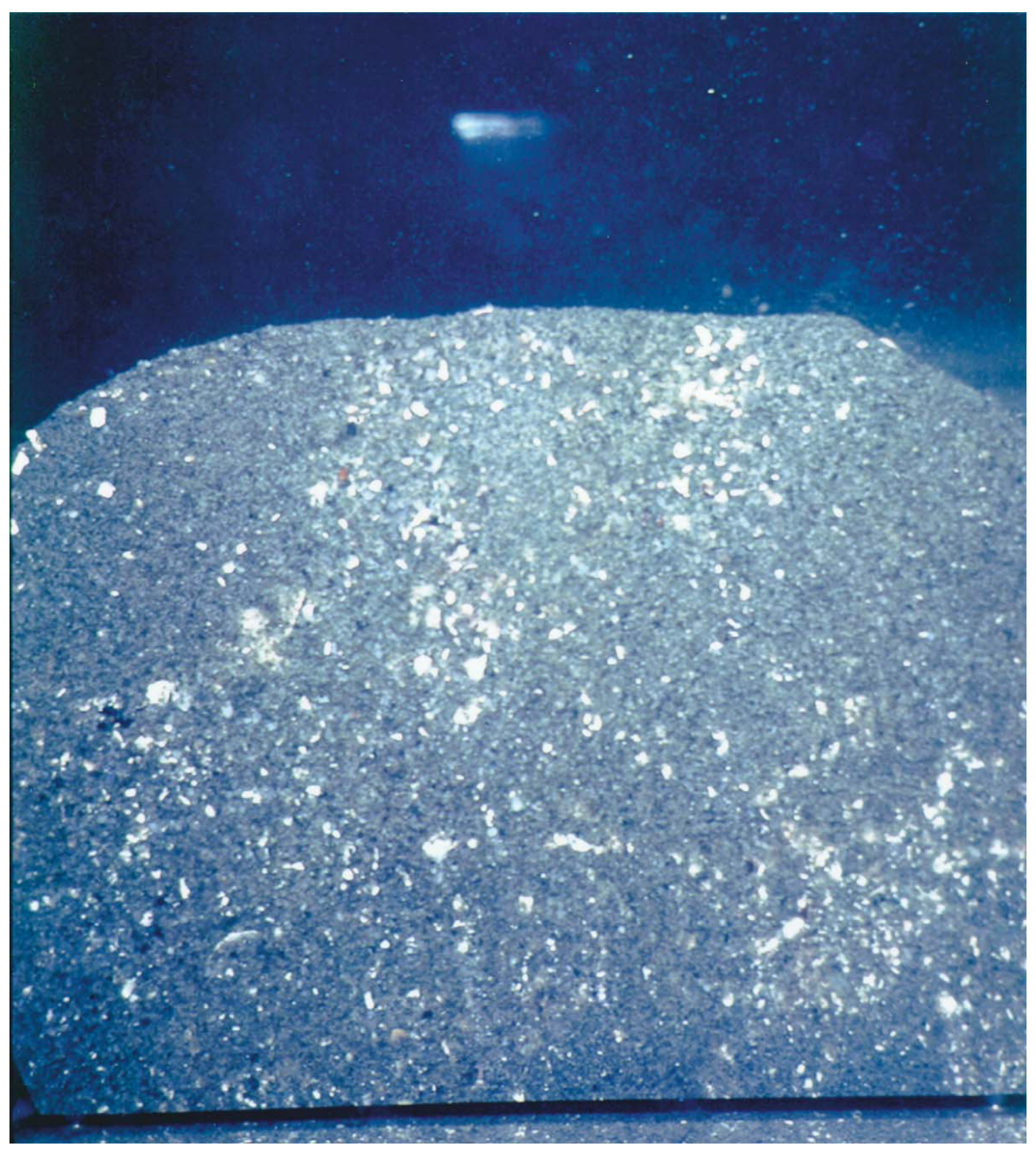

Figure C4. SPI image of Lower Study Site Station LS-09 


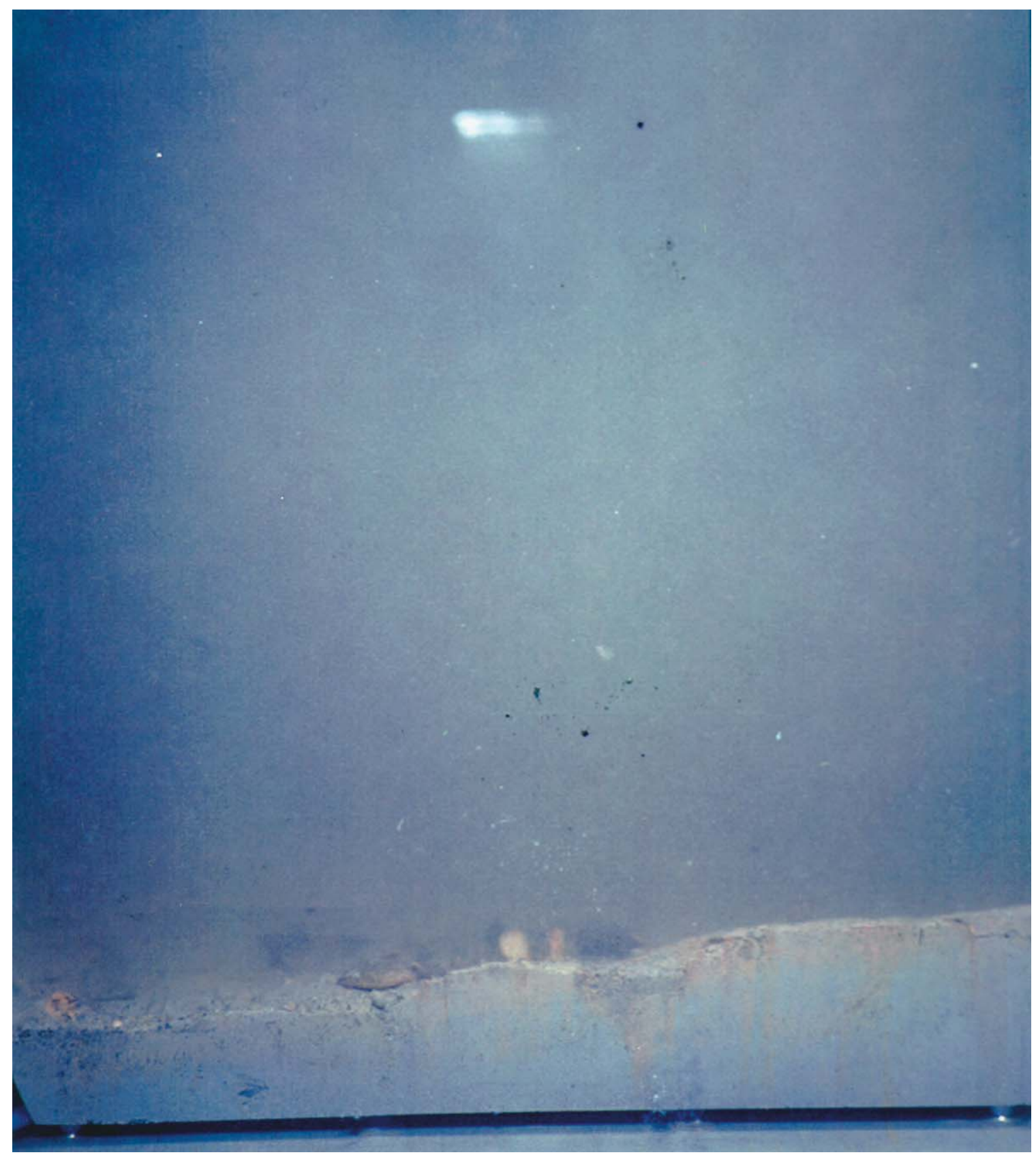

Figure C5. SPI image of Upper Study Site Station US-14 


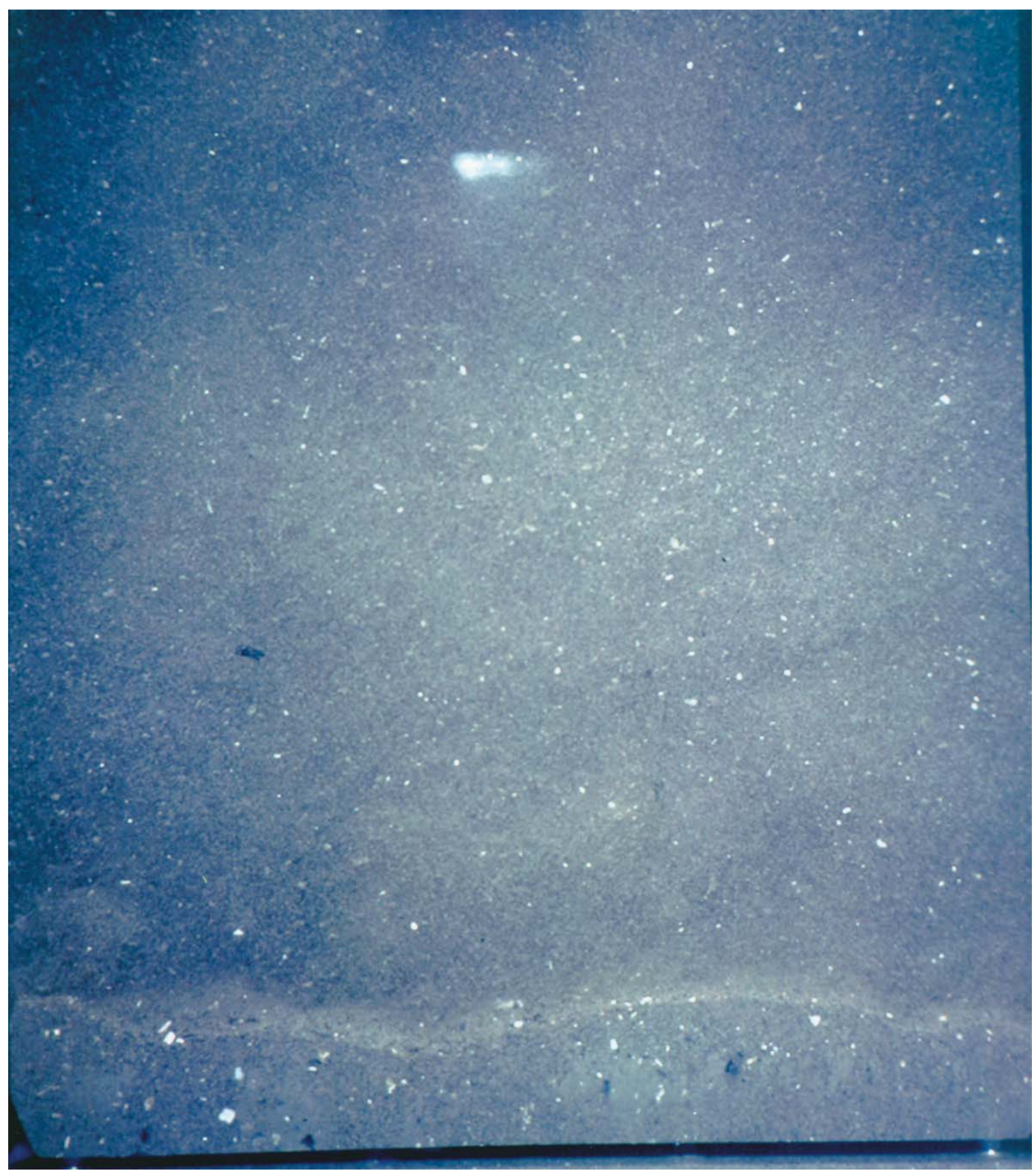

Figure C6. SPI image of Lower Study Site Station LS-13 


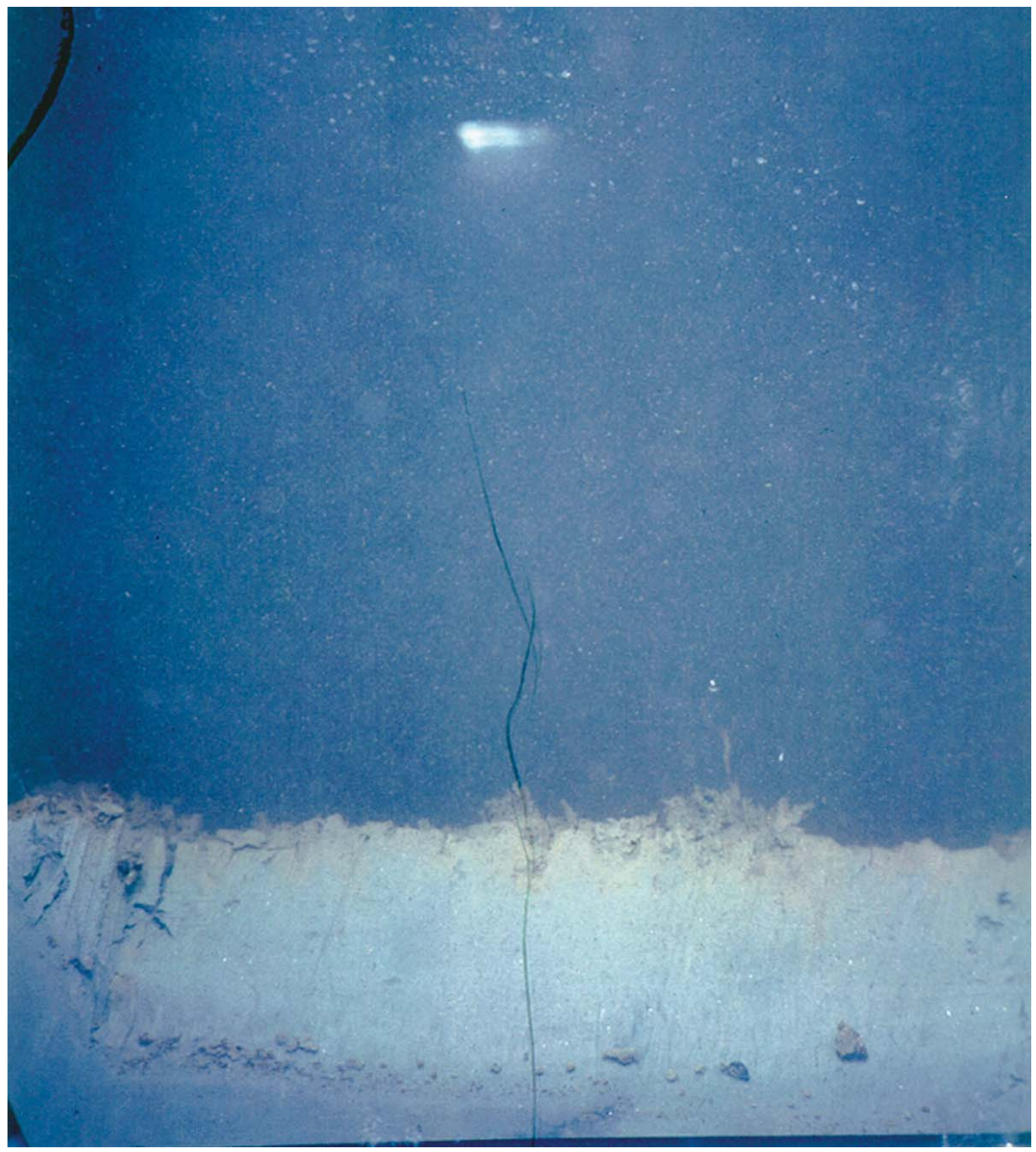

Figure C7. SPI image of Upper Study Site Station US-35 


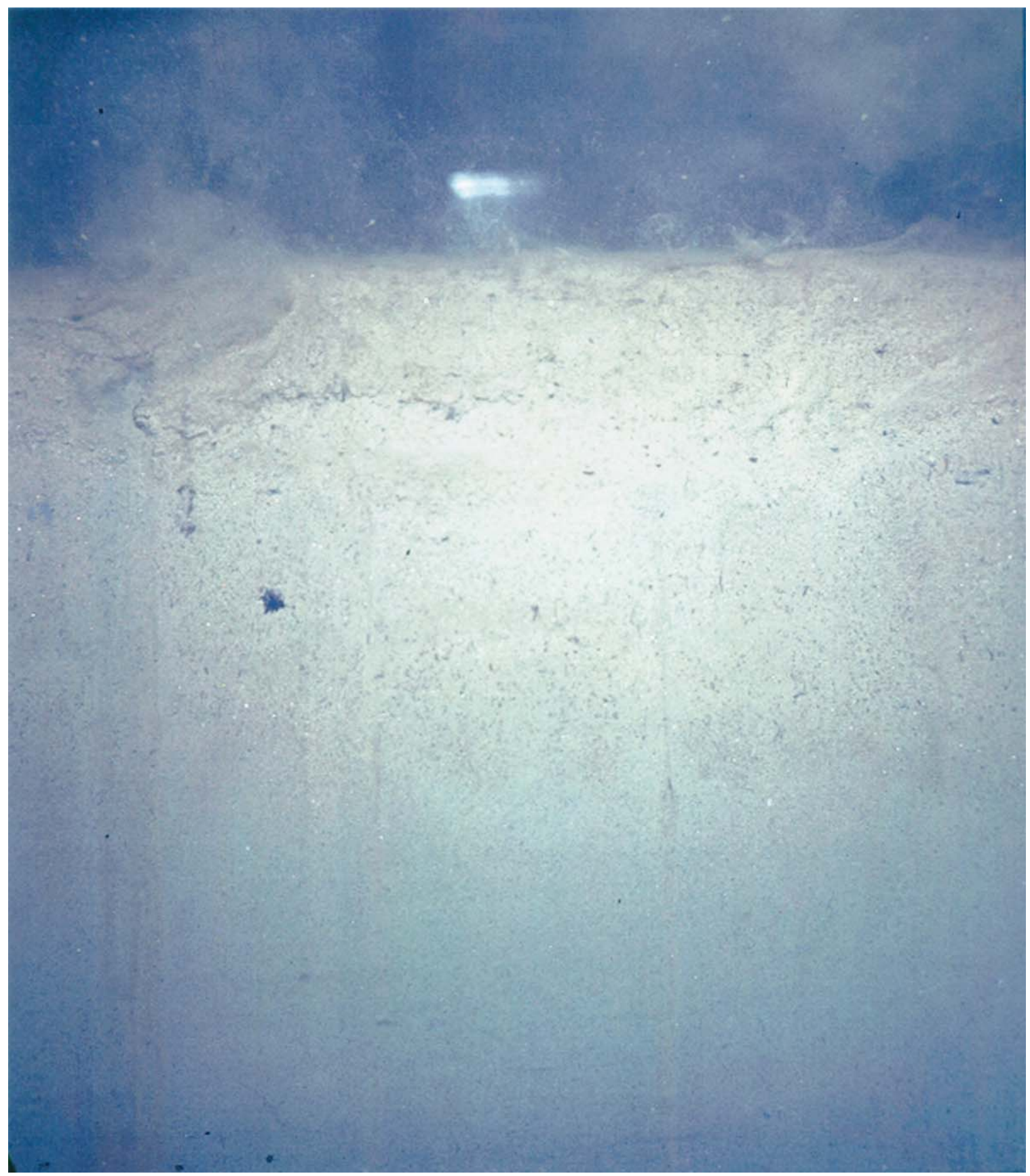

Figure C8. SPI image of Upper Study Site Station US-11 


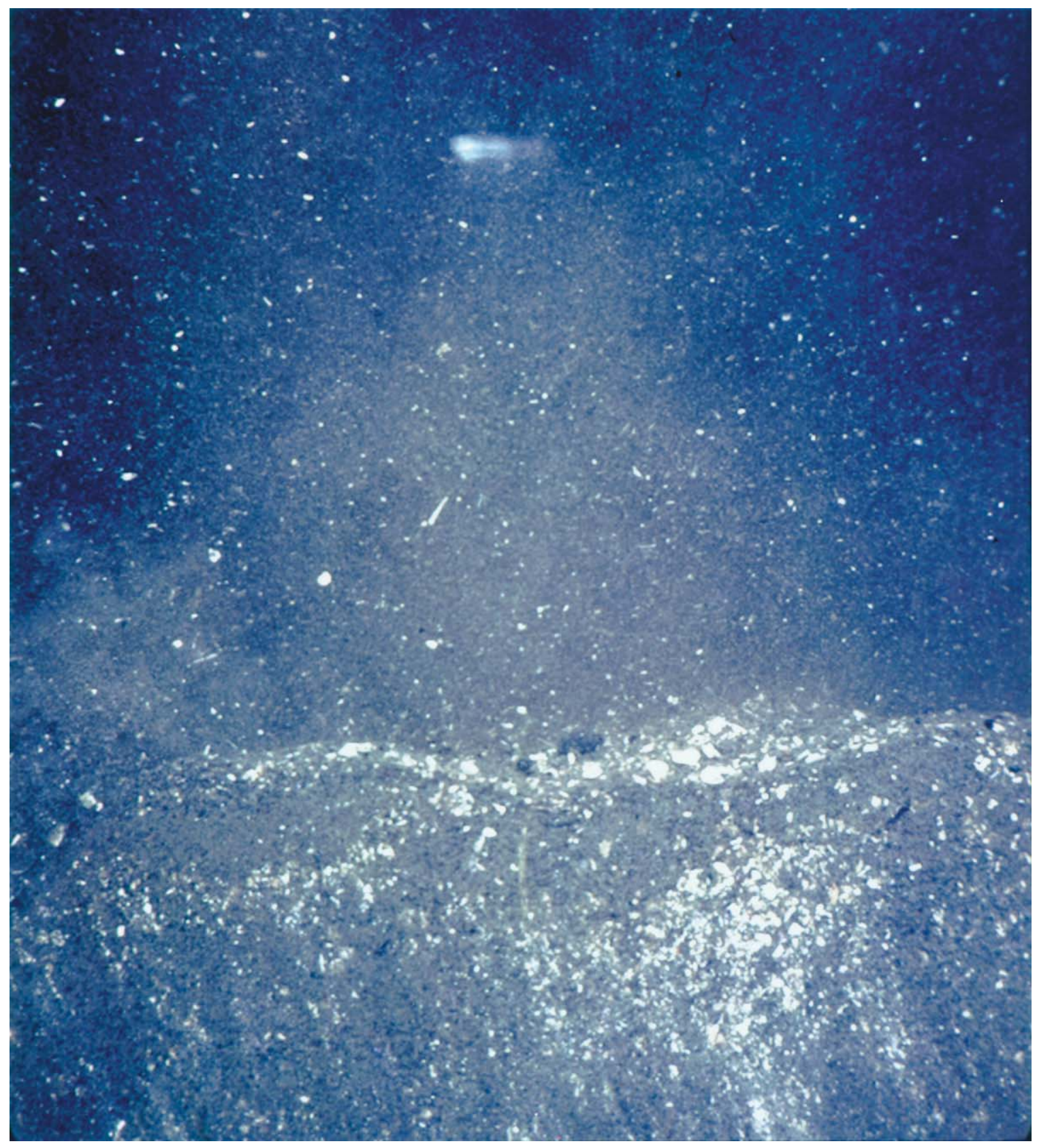

Figure C9. SPI image of Lower Study Site Station LS-06 


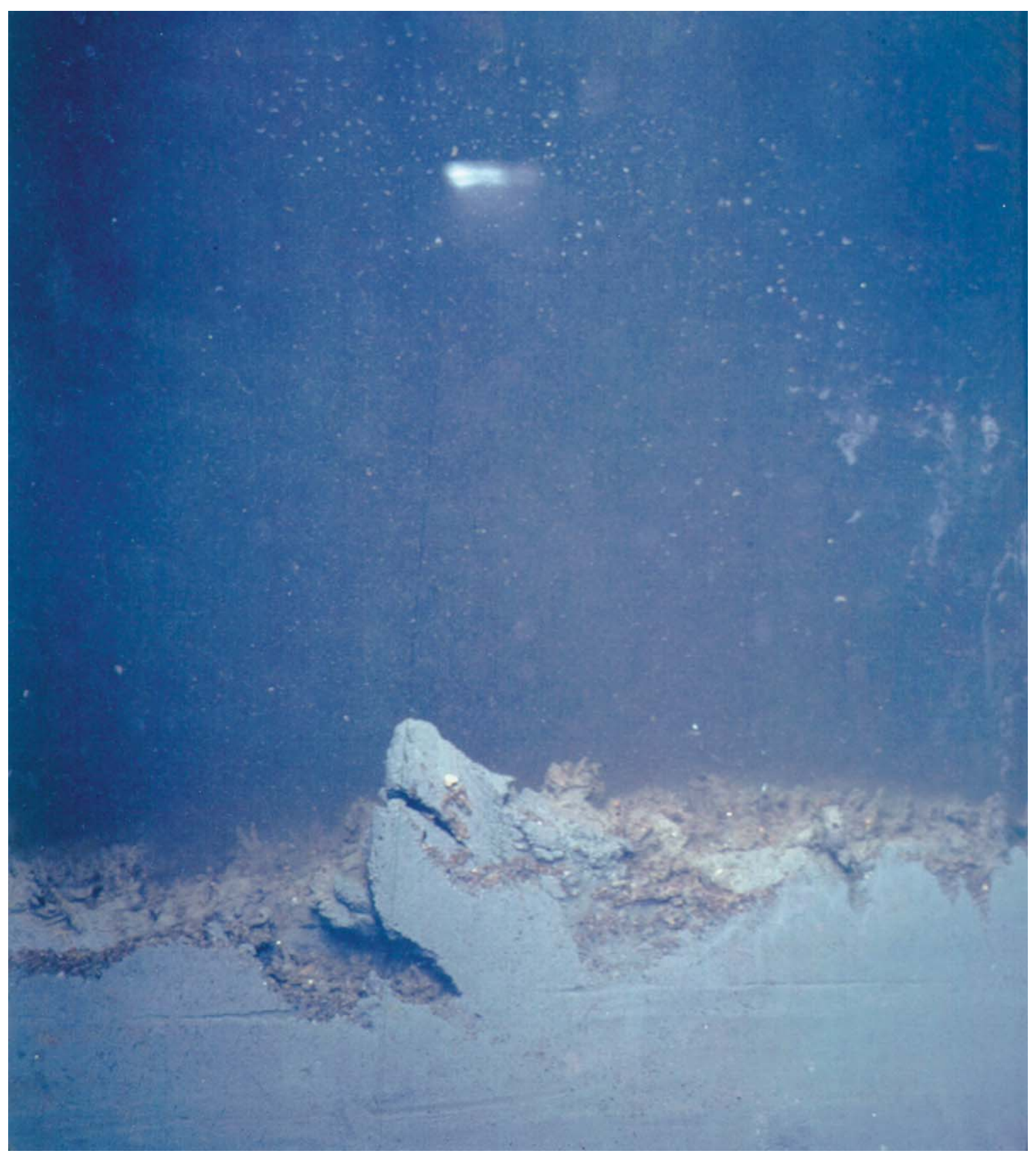

Figure C10. SPI image of Upper Study Site Station US-33 


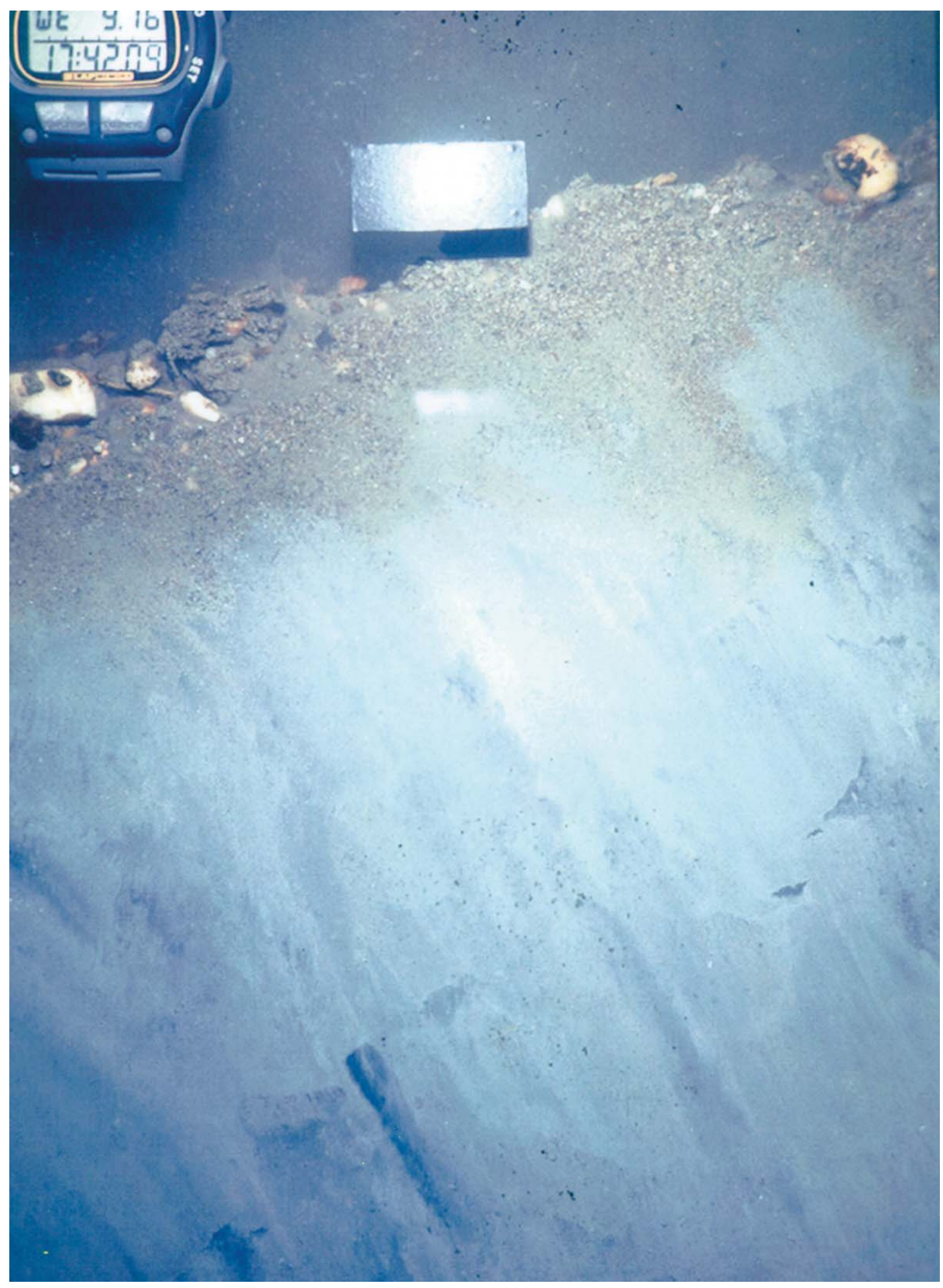

Figure C11. SPI image of Upper Study Site Station US-21 


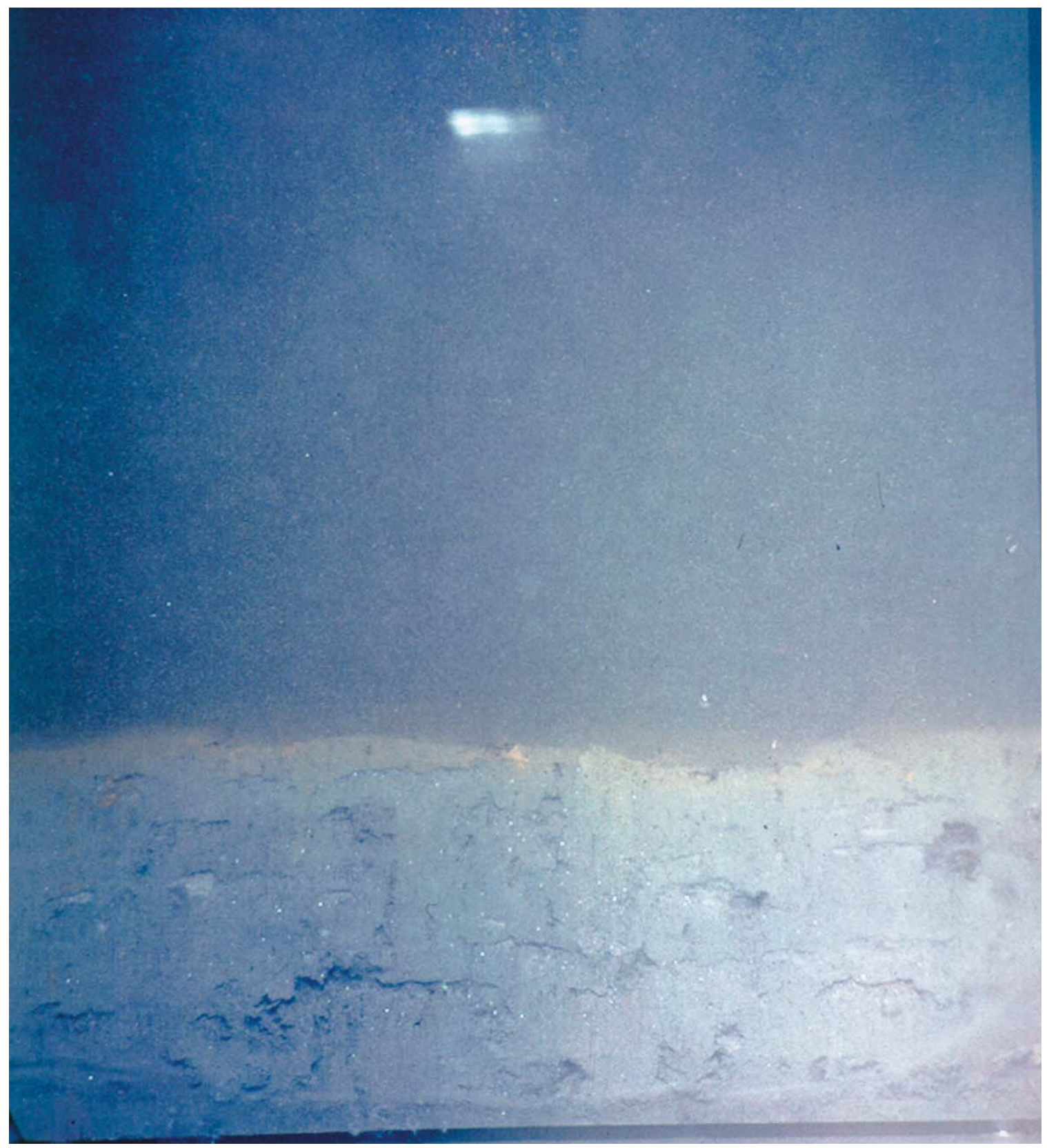

Figure C12. SPI image of Upper Study Site Station US-29 


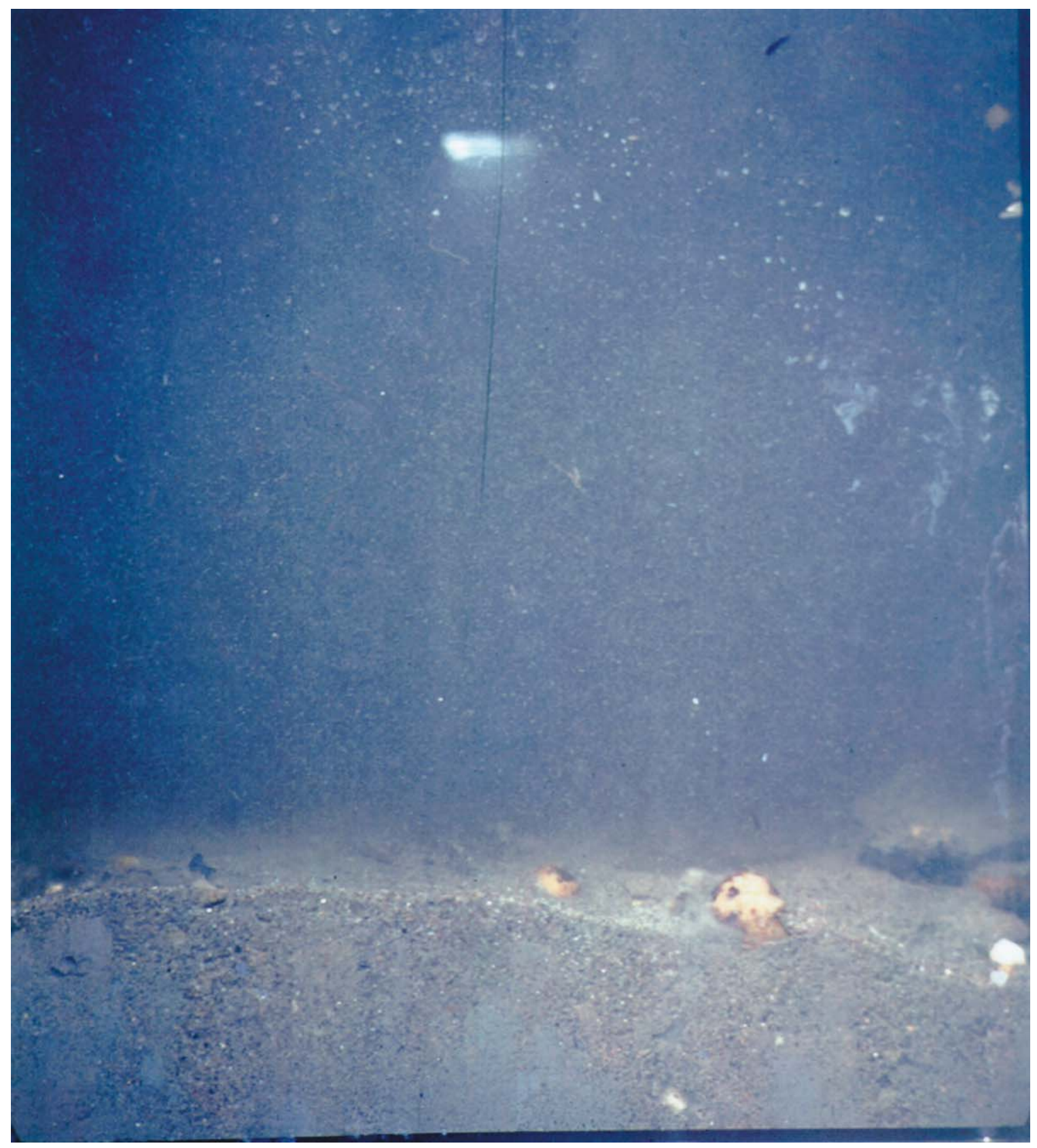

Figure C13. SPI image of Upper Study Site Station US-32 


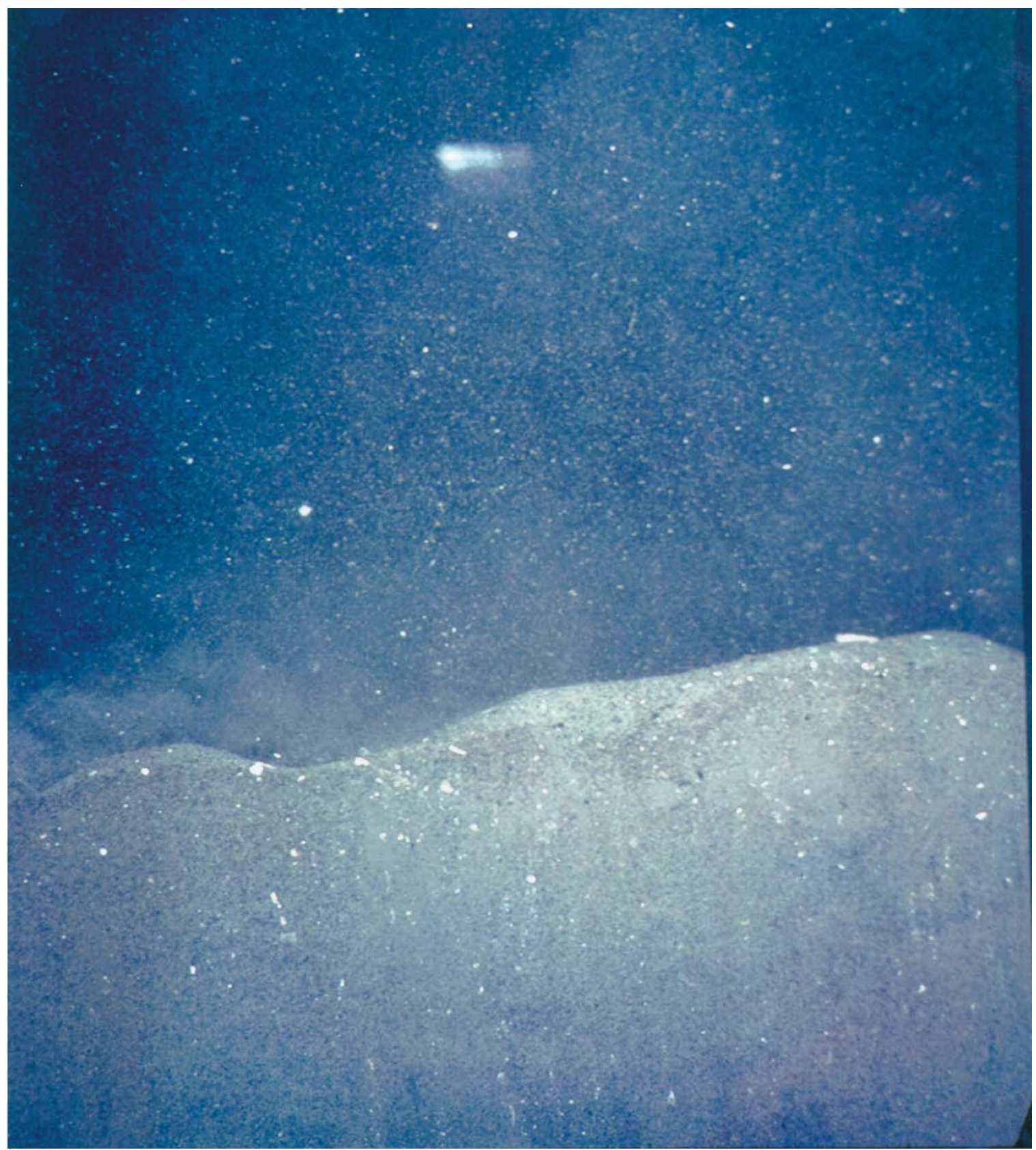

Figure C14. SPI image of Lower Study Site Station LS-03 


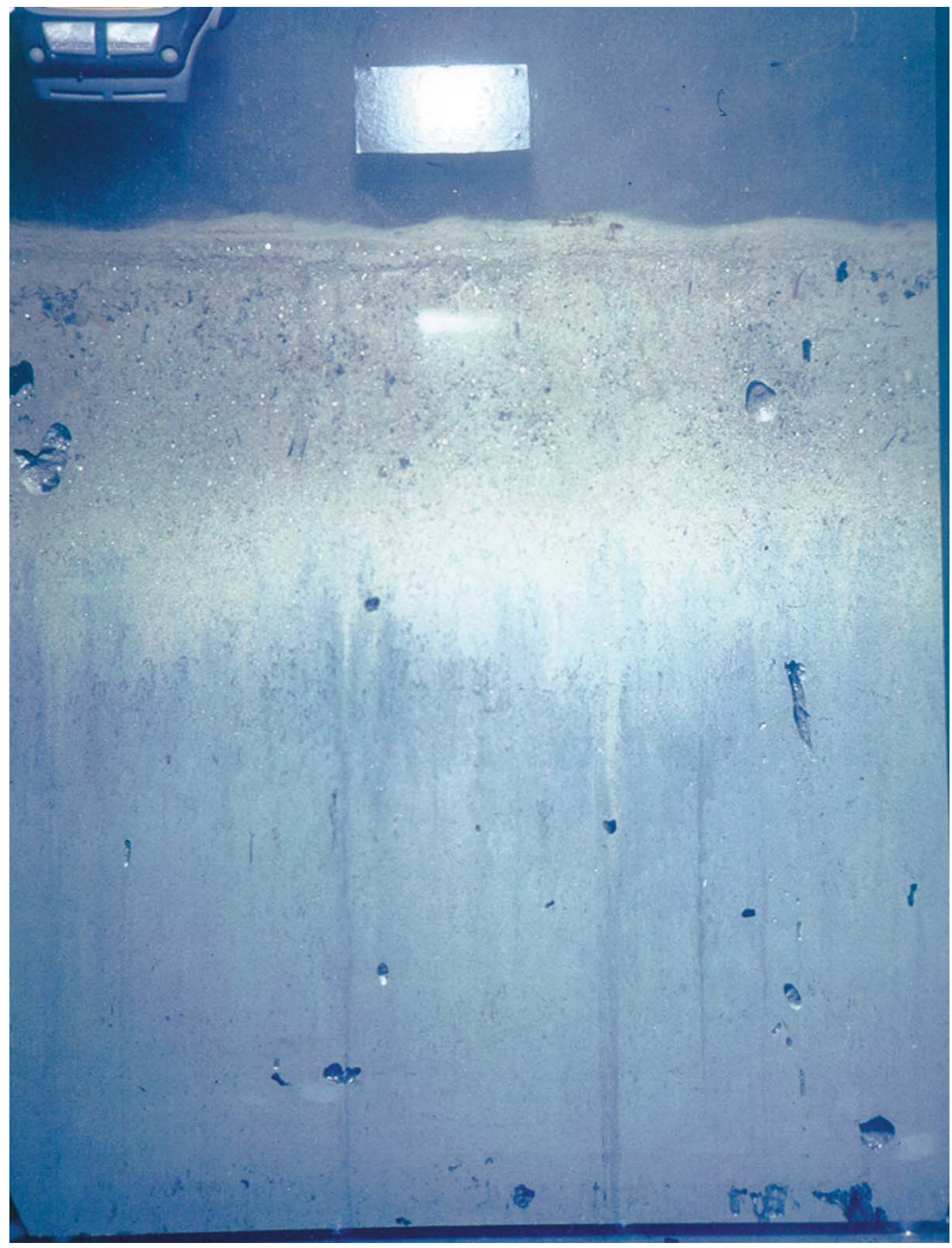

Figure C15. SPI image of Upper Study Site Station US-09 


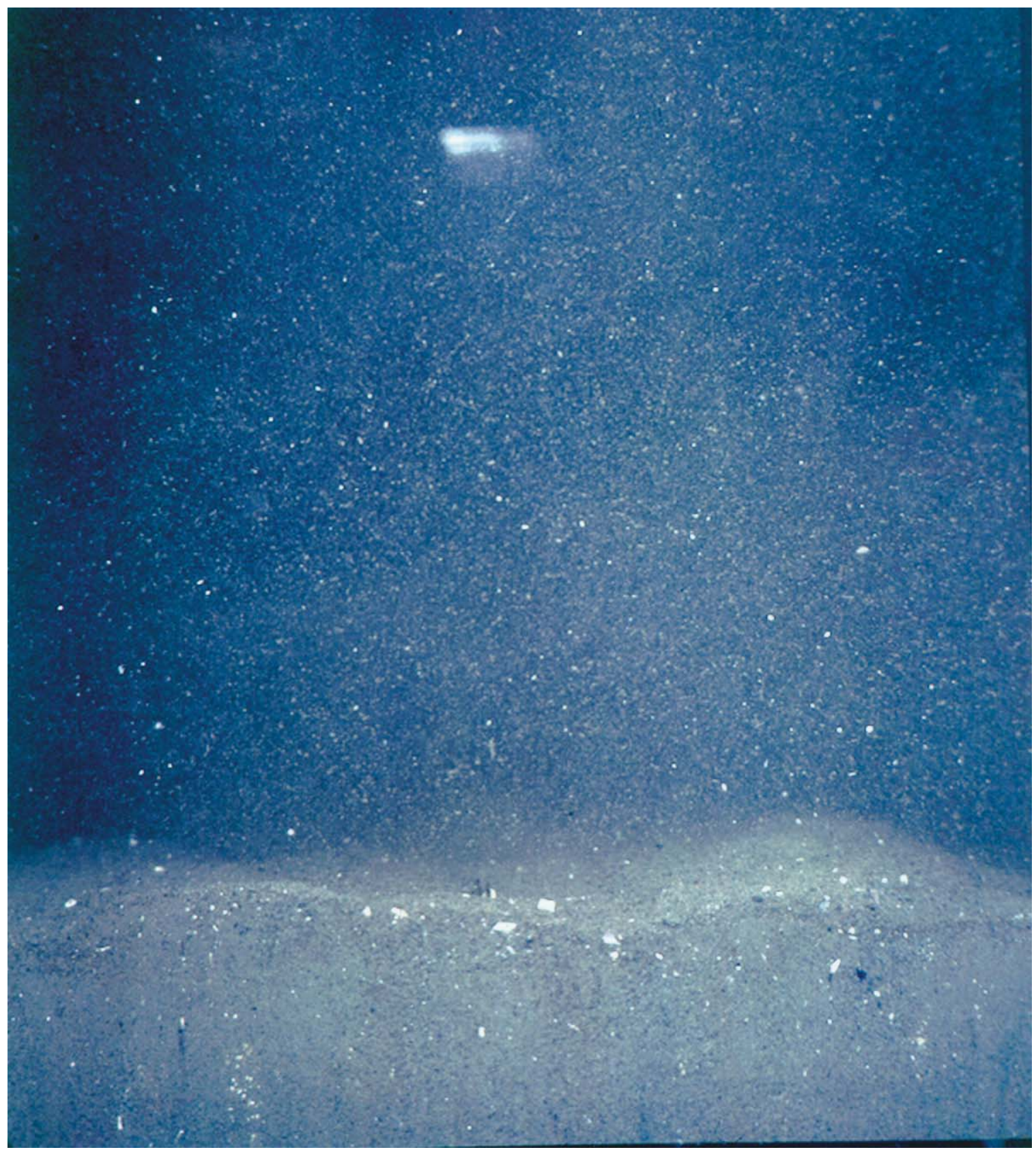

Figure C16. SPI image of Lower Study Site Station LS-02 


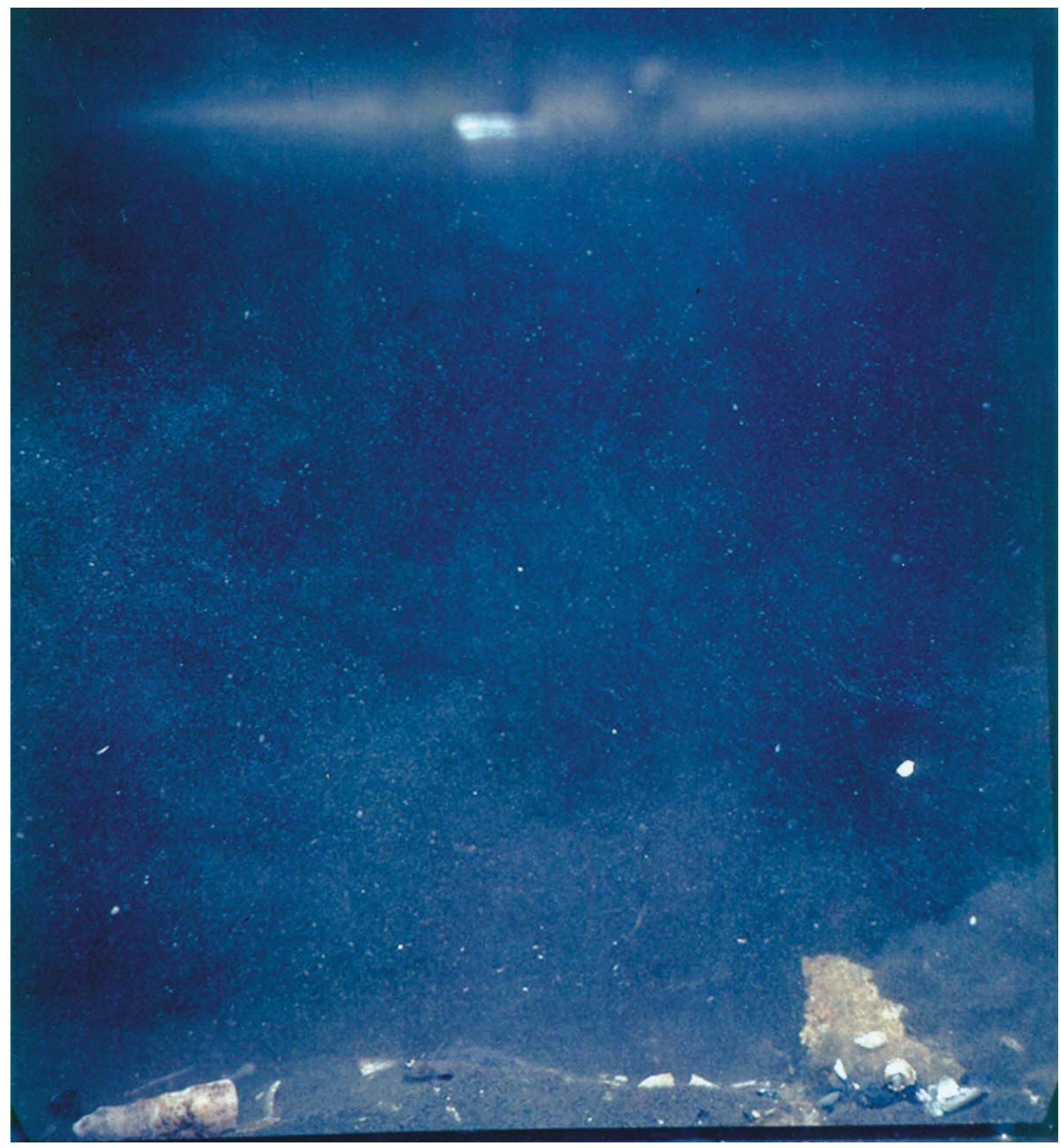

Figure C17. SPI image of Lower Study Site Station LS-07 


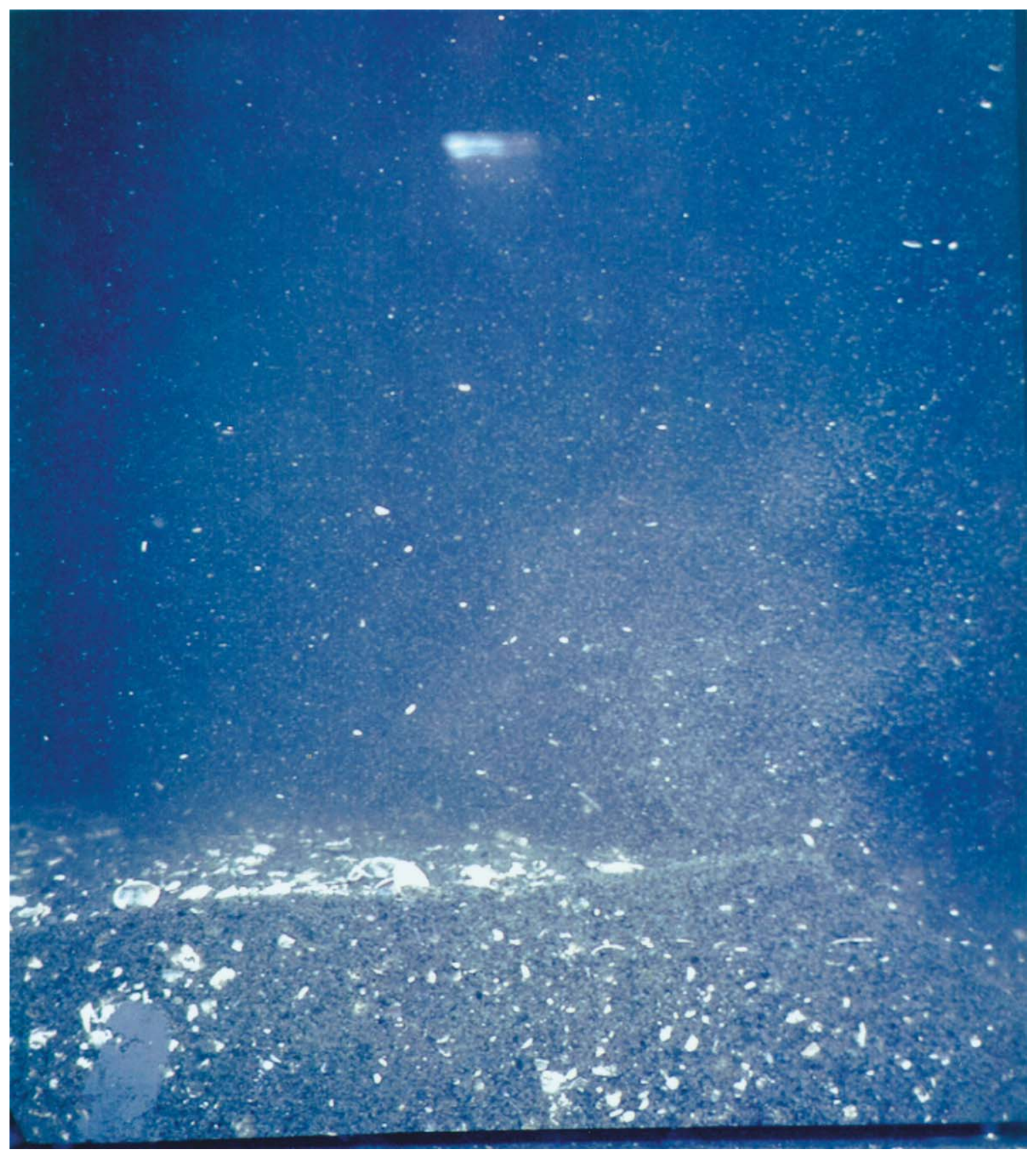

Figure C18. SPI image of Lower Study Site Station LS-12 


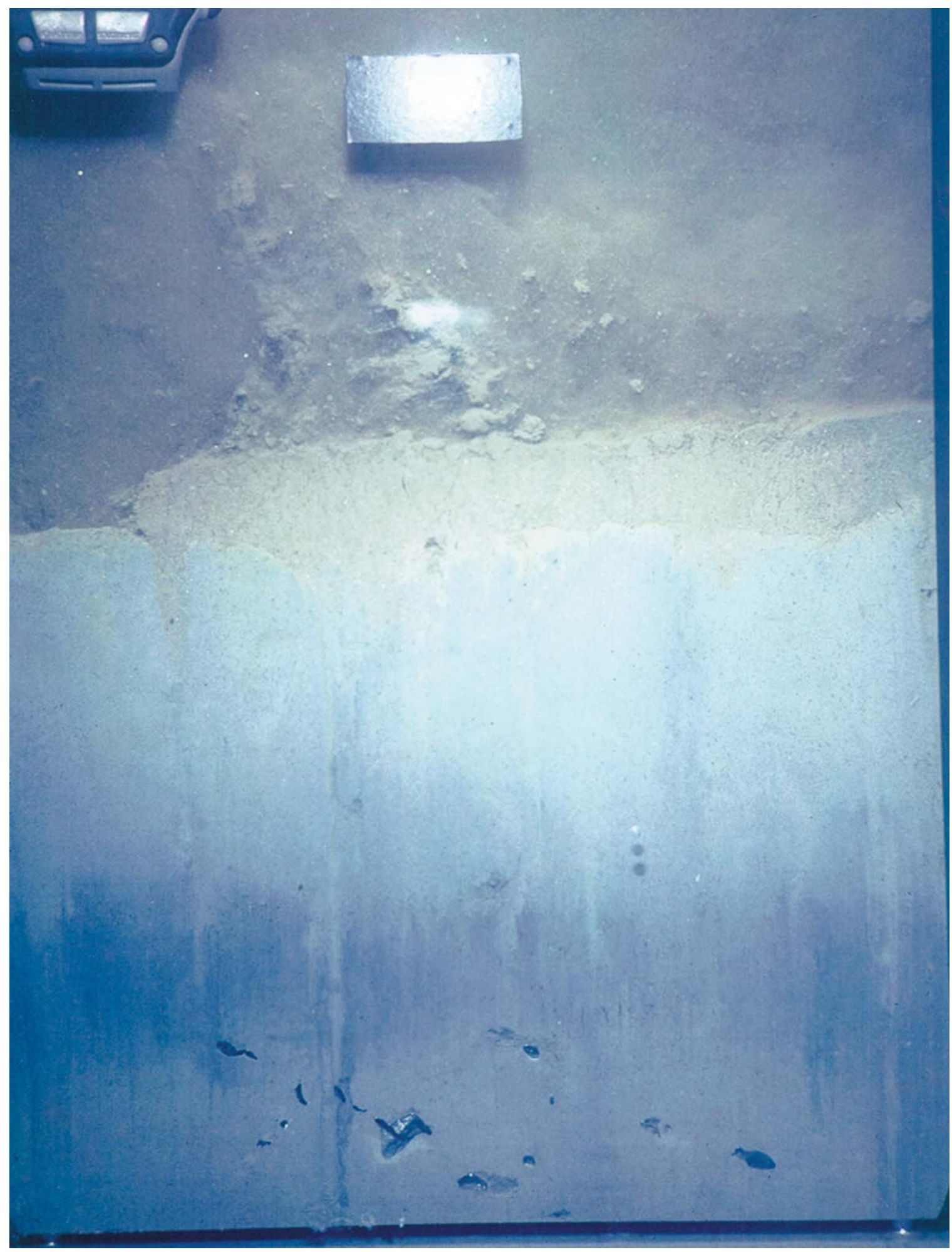

Figure C19. SPI image of Upper Study Site Station US-31 


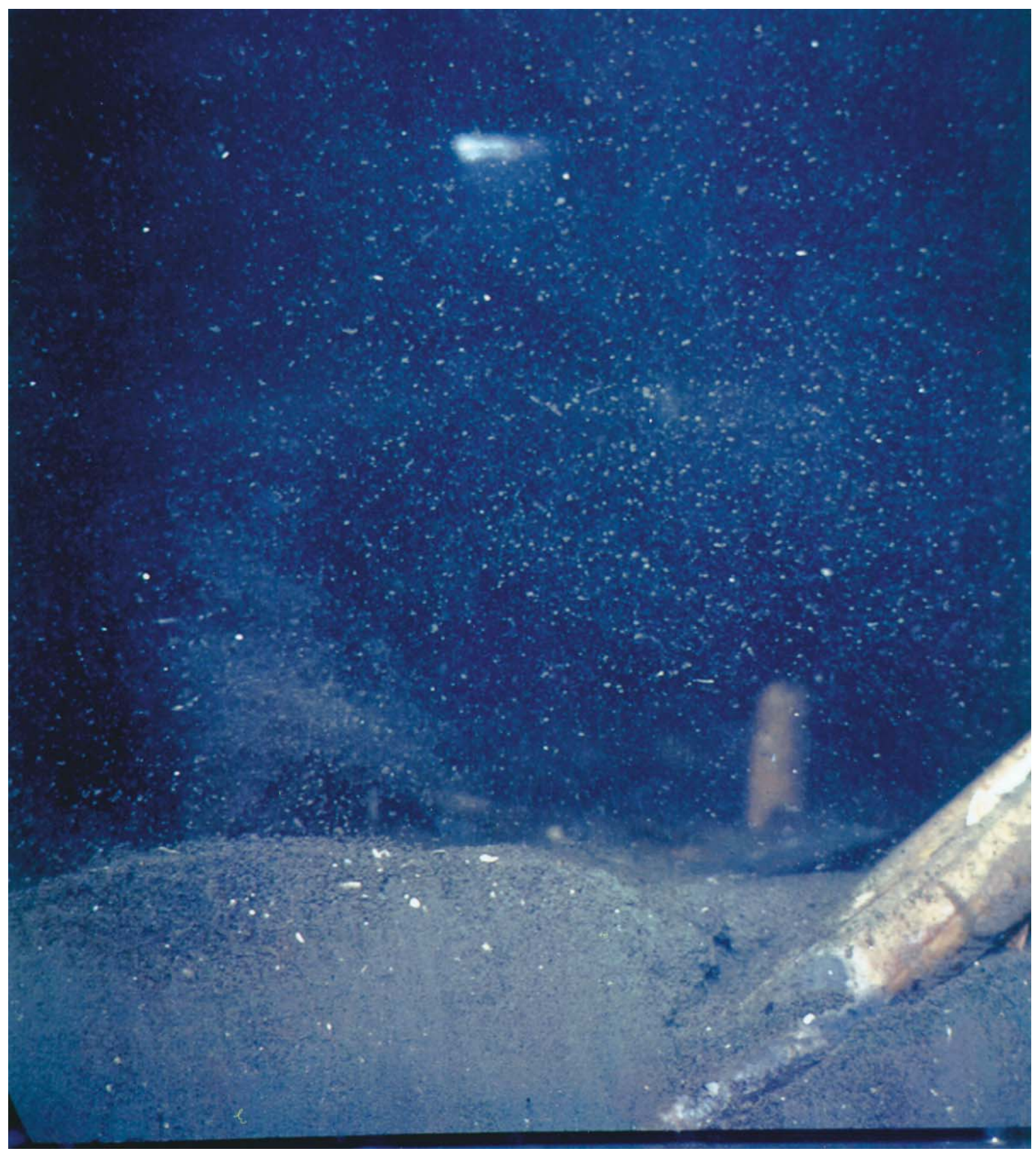

Figure C20. SPI image of Lower Study Site Station LS-11 


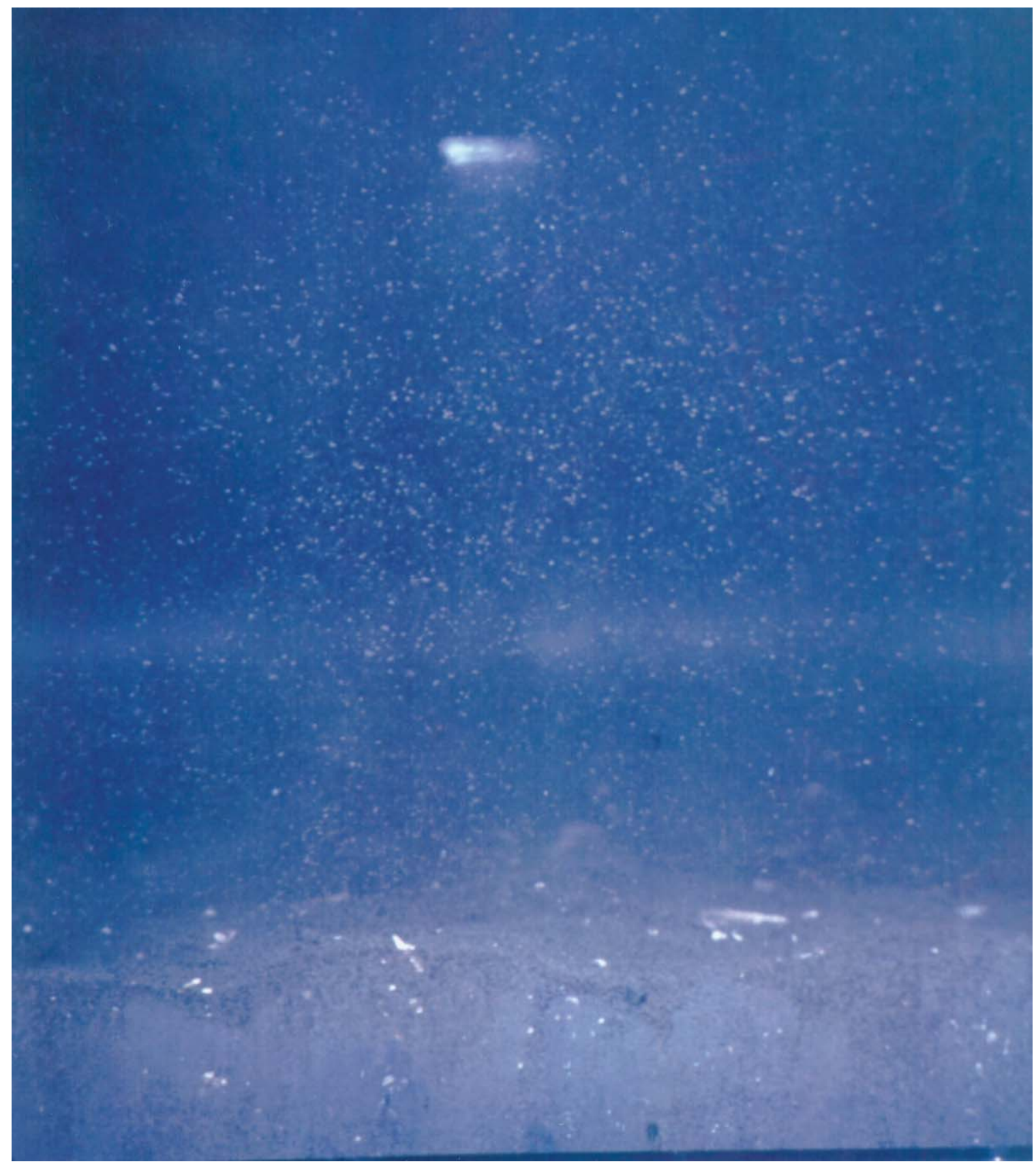

Figure C21. SPI image of Lower Study Site Station LS-10 


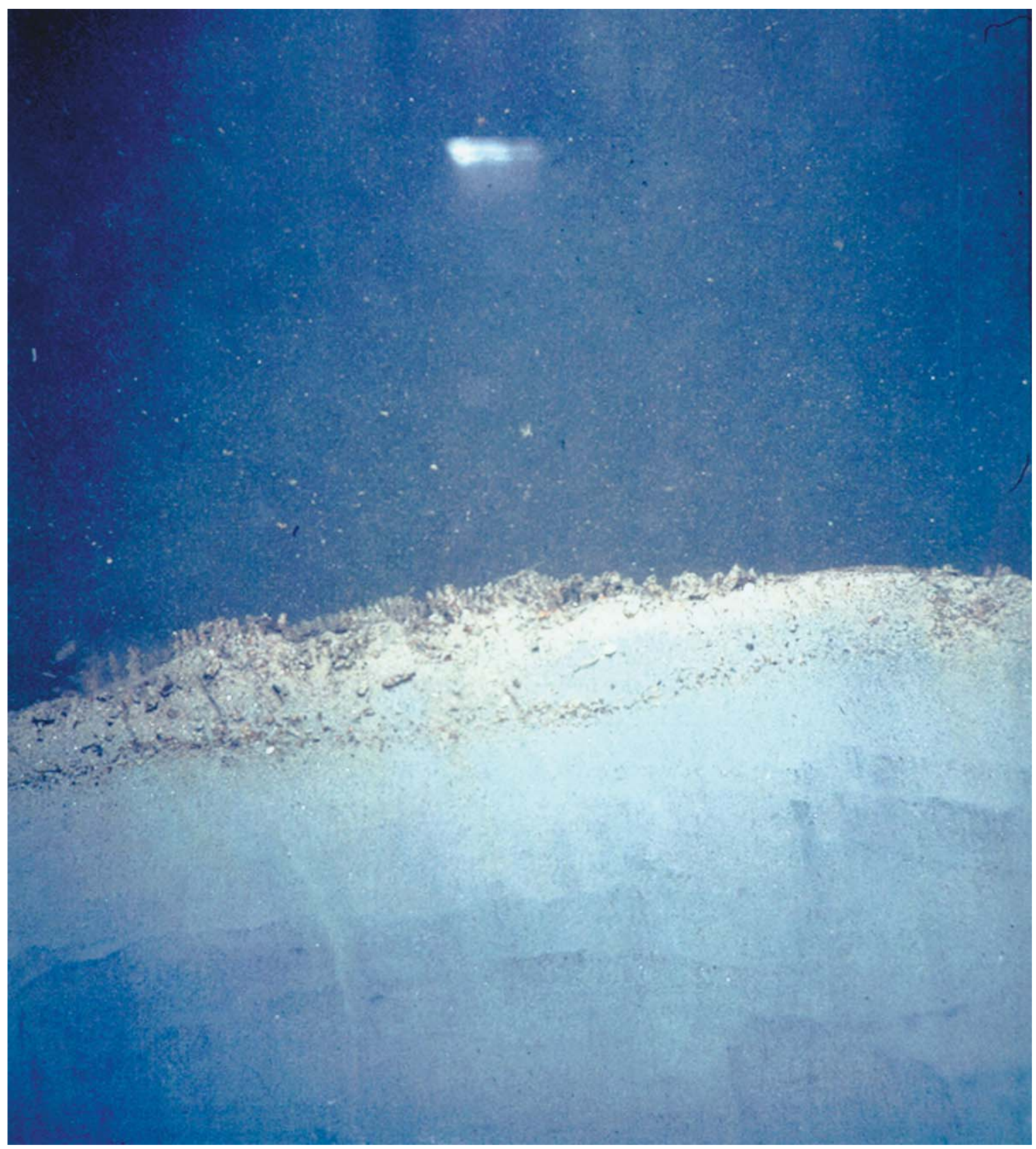

Figure C22. SPI image of Upper Study Site Station US-22 


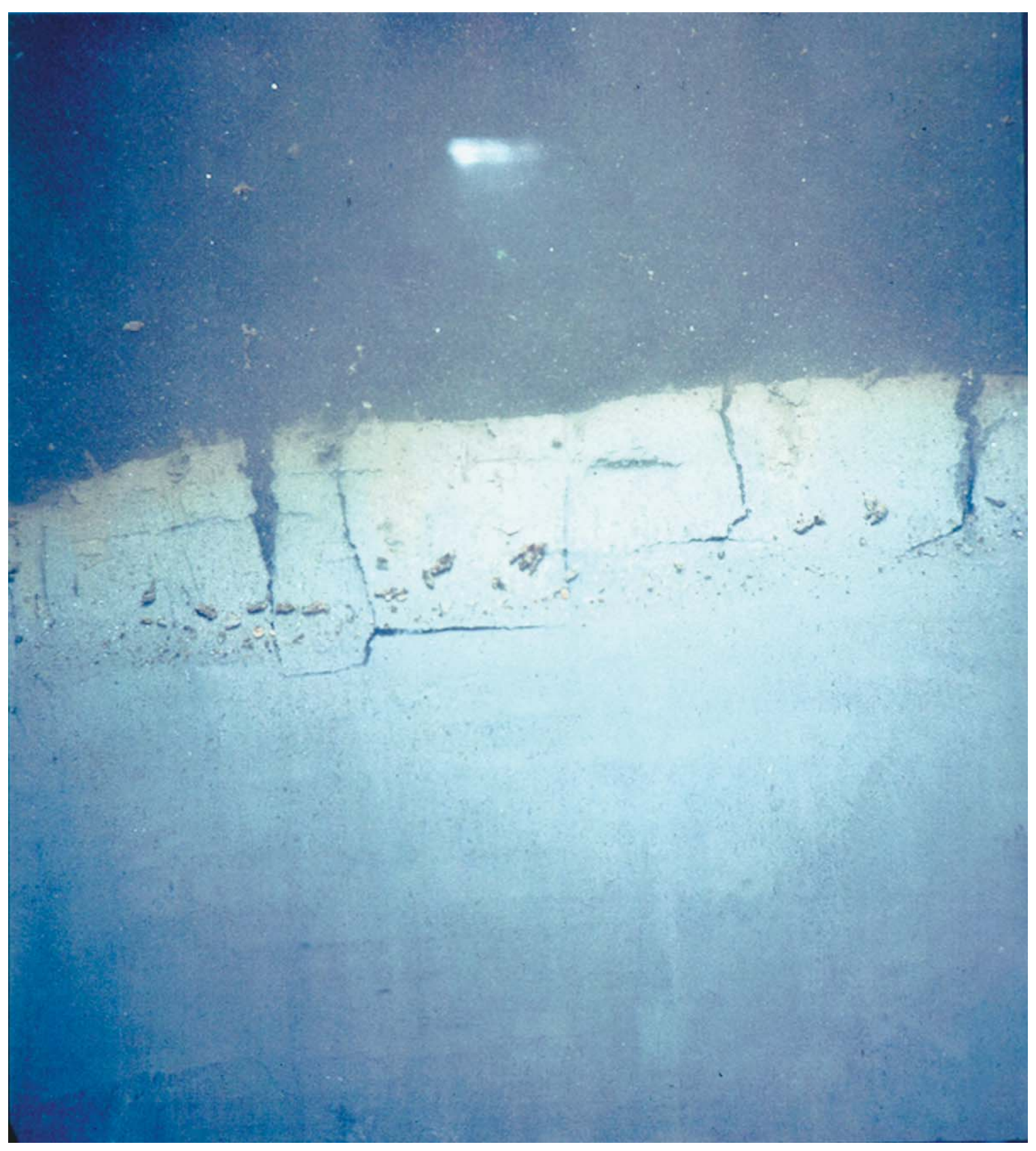

Figure C23. SPI image of Upper Study Site Station US-23 


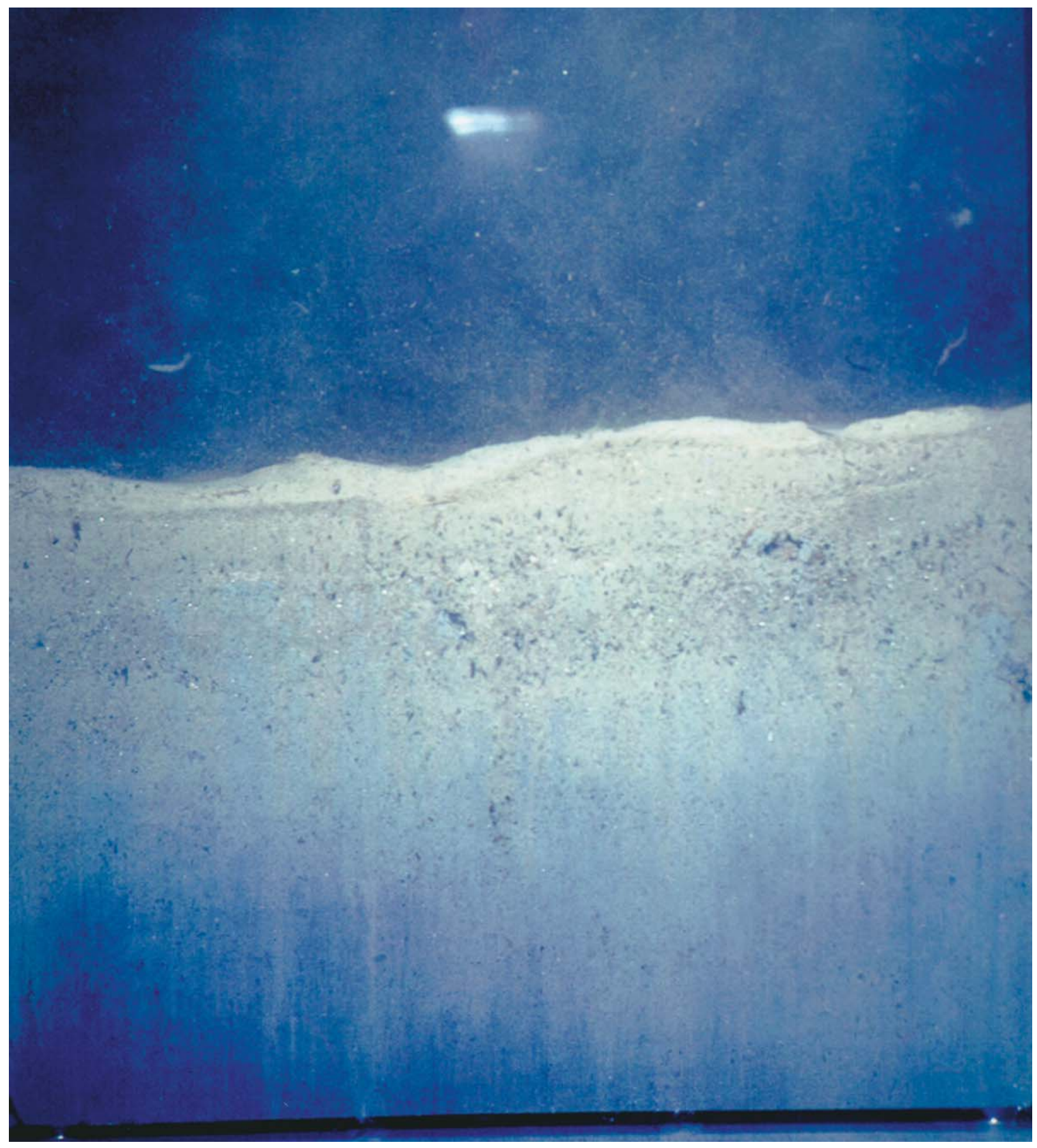

Figure C24. SPI image of Upper Study Site Station US-10 


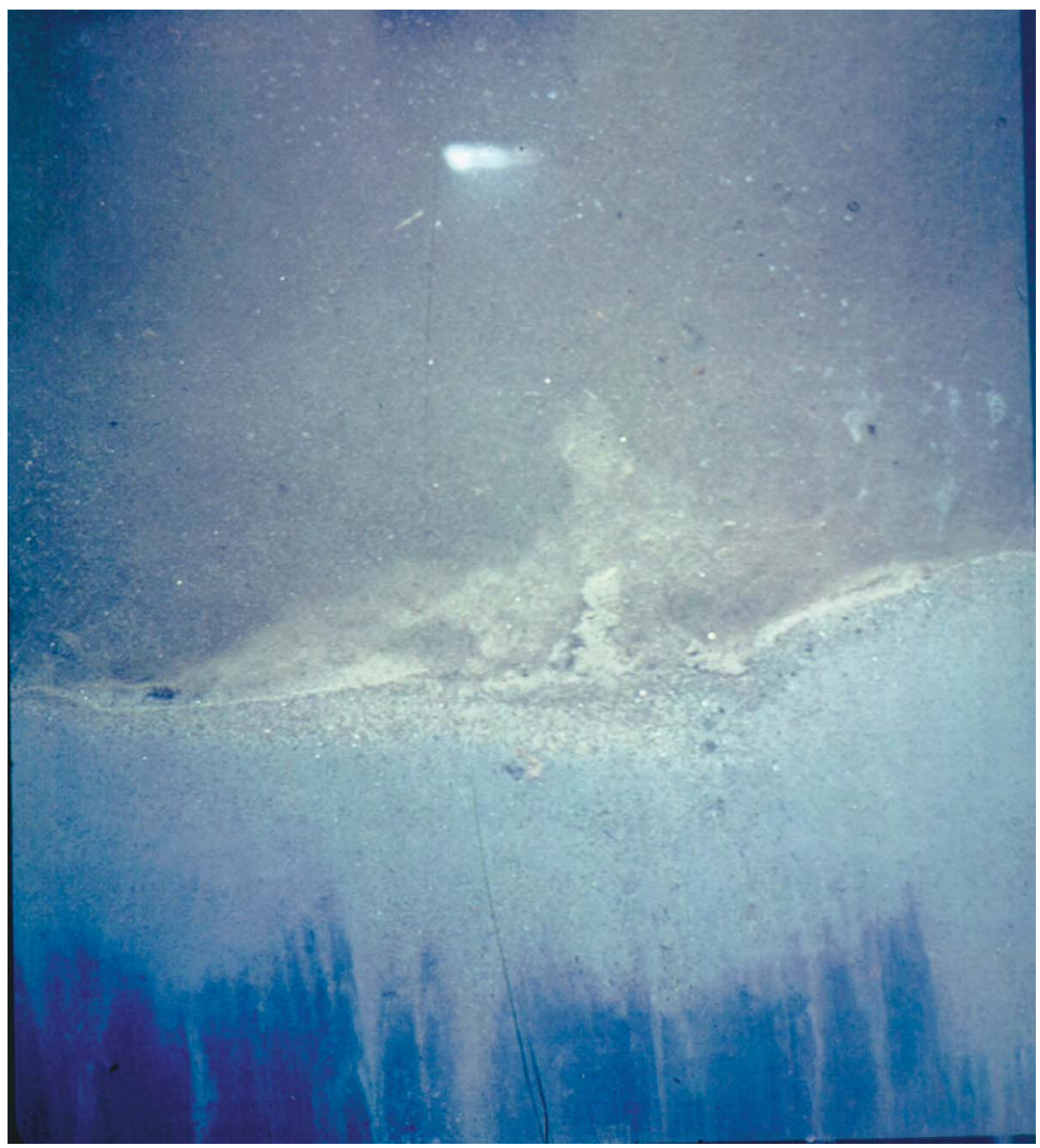

Figure C25. SPI image of Upper Study Site Station US-34 


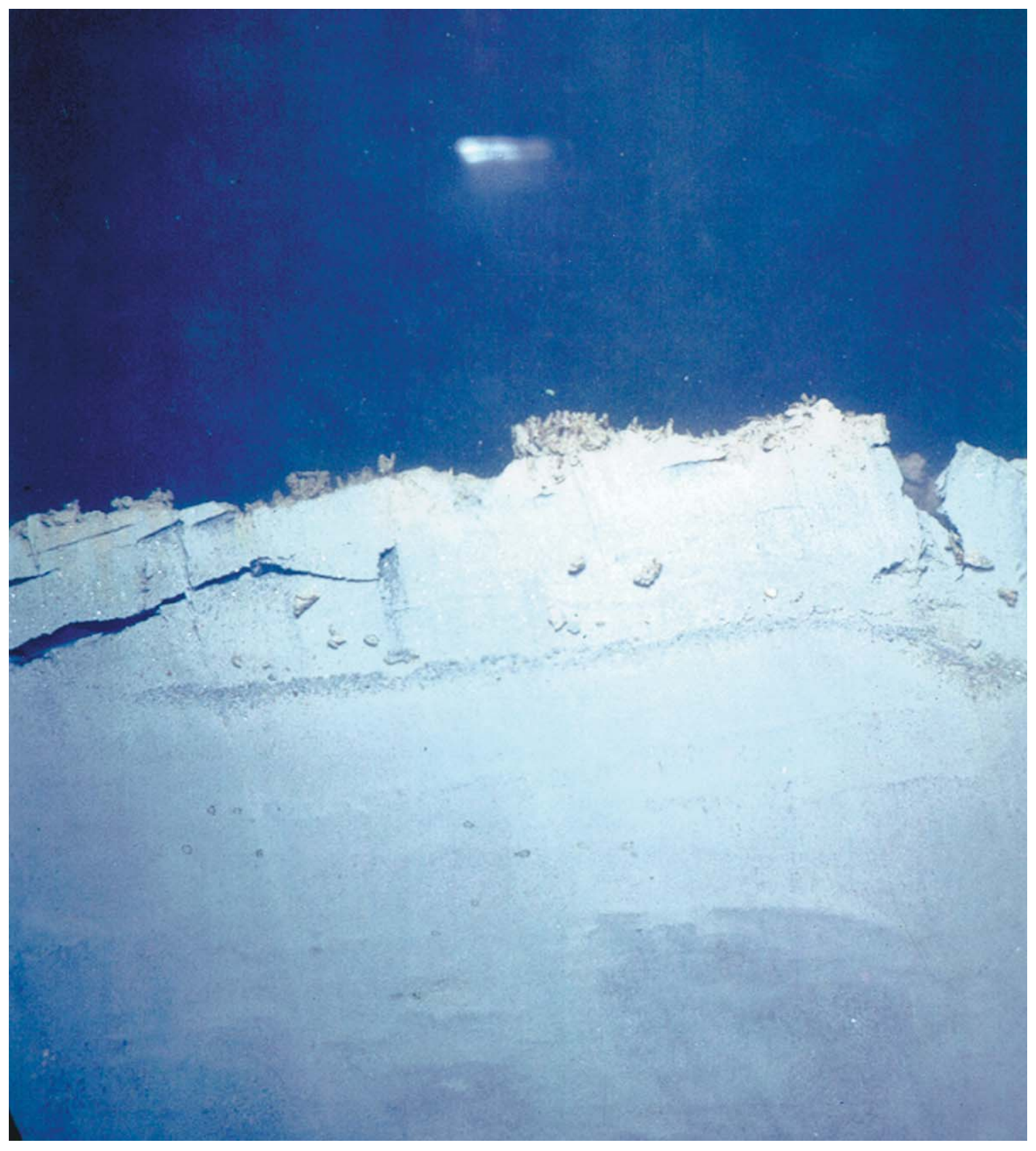

Figure C26. SPI image of Upper Study Site Station US-05 


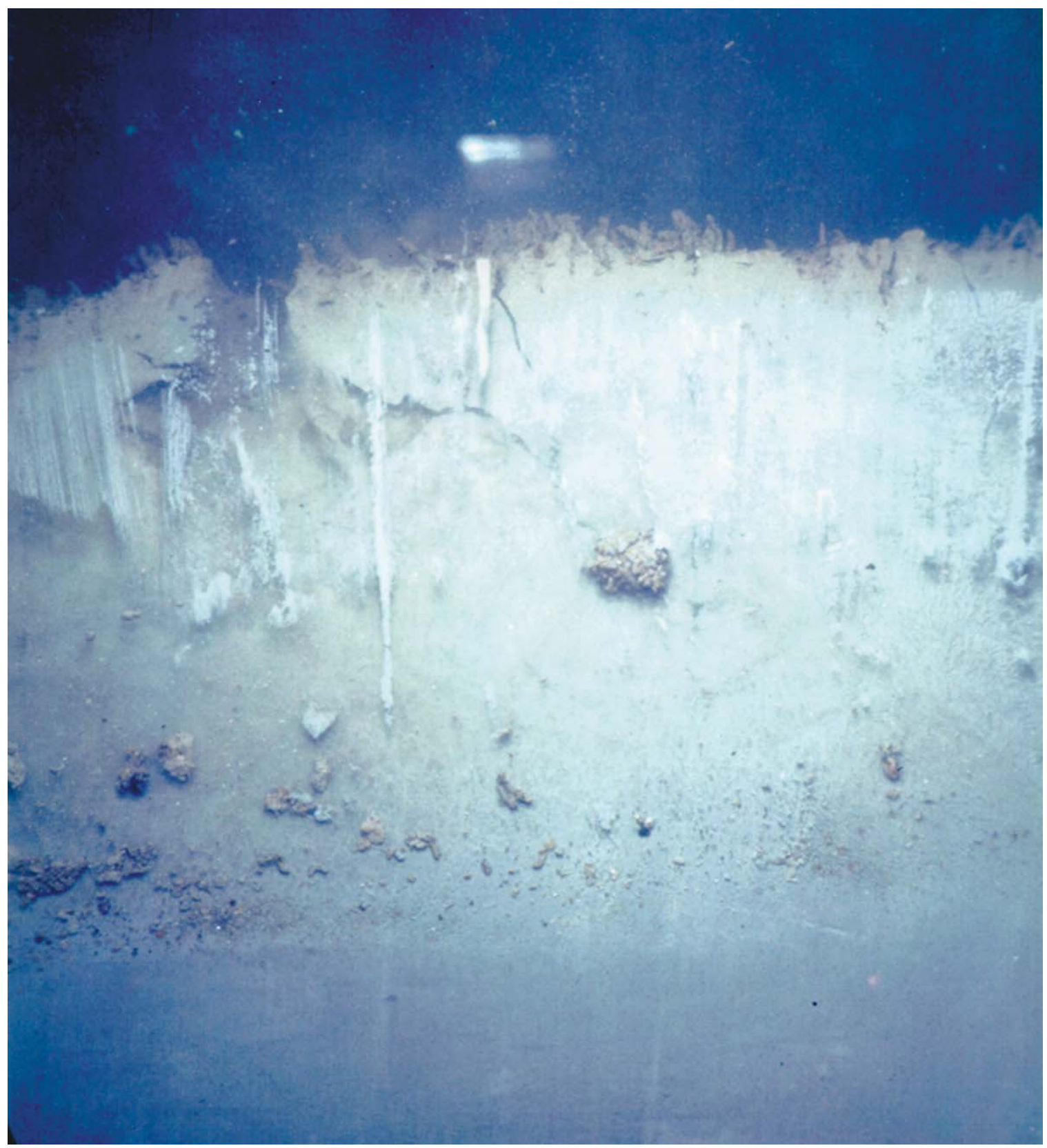

Figure C27. SPI image of Upper Study Site Station US-06 


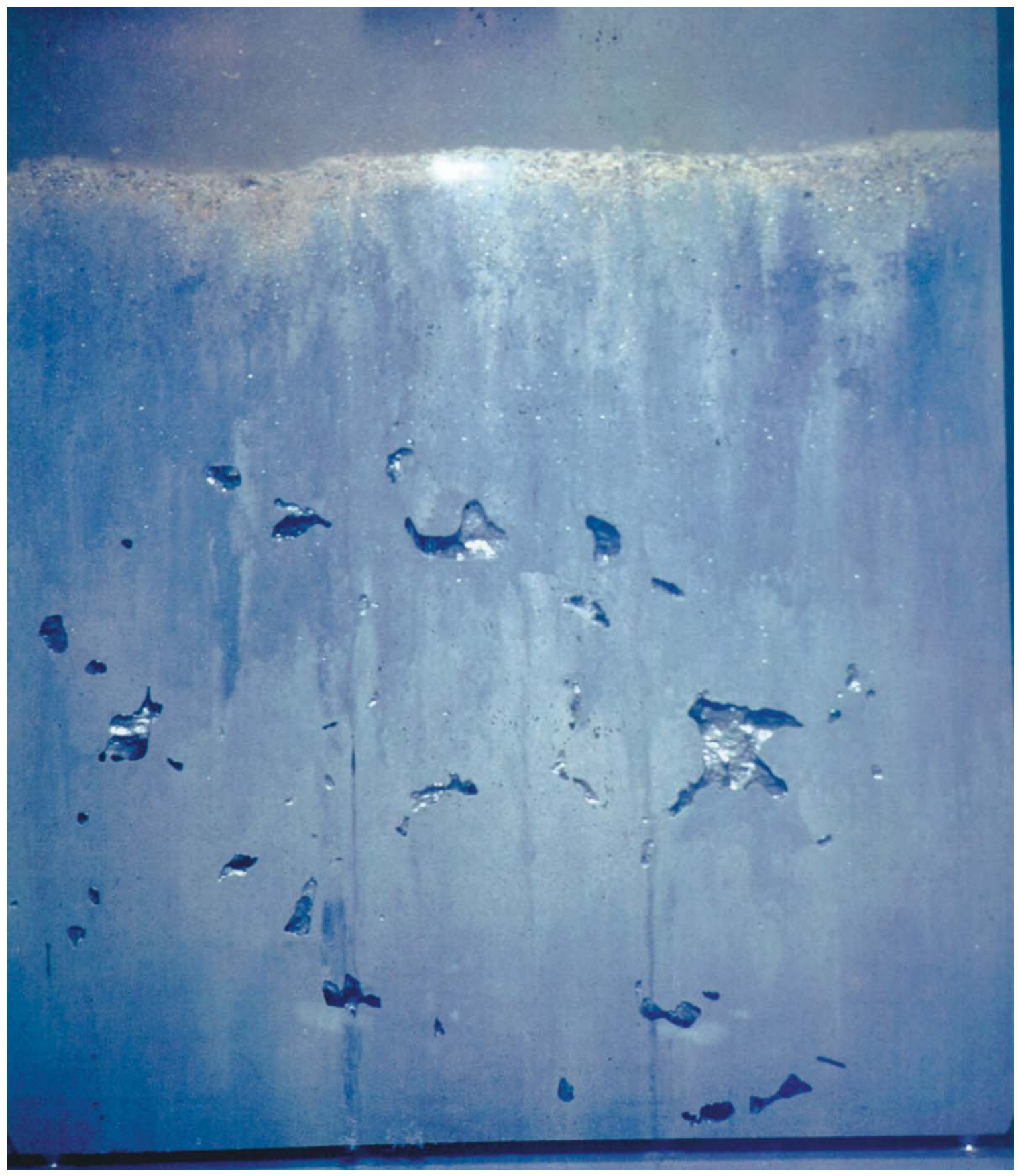

Figure C28. SPI image of Upper Study Site Station US-26 


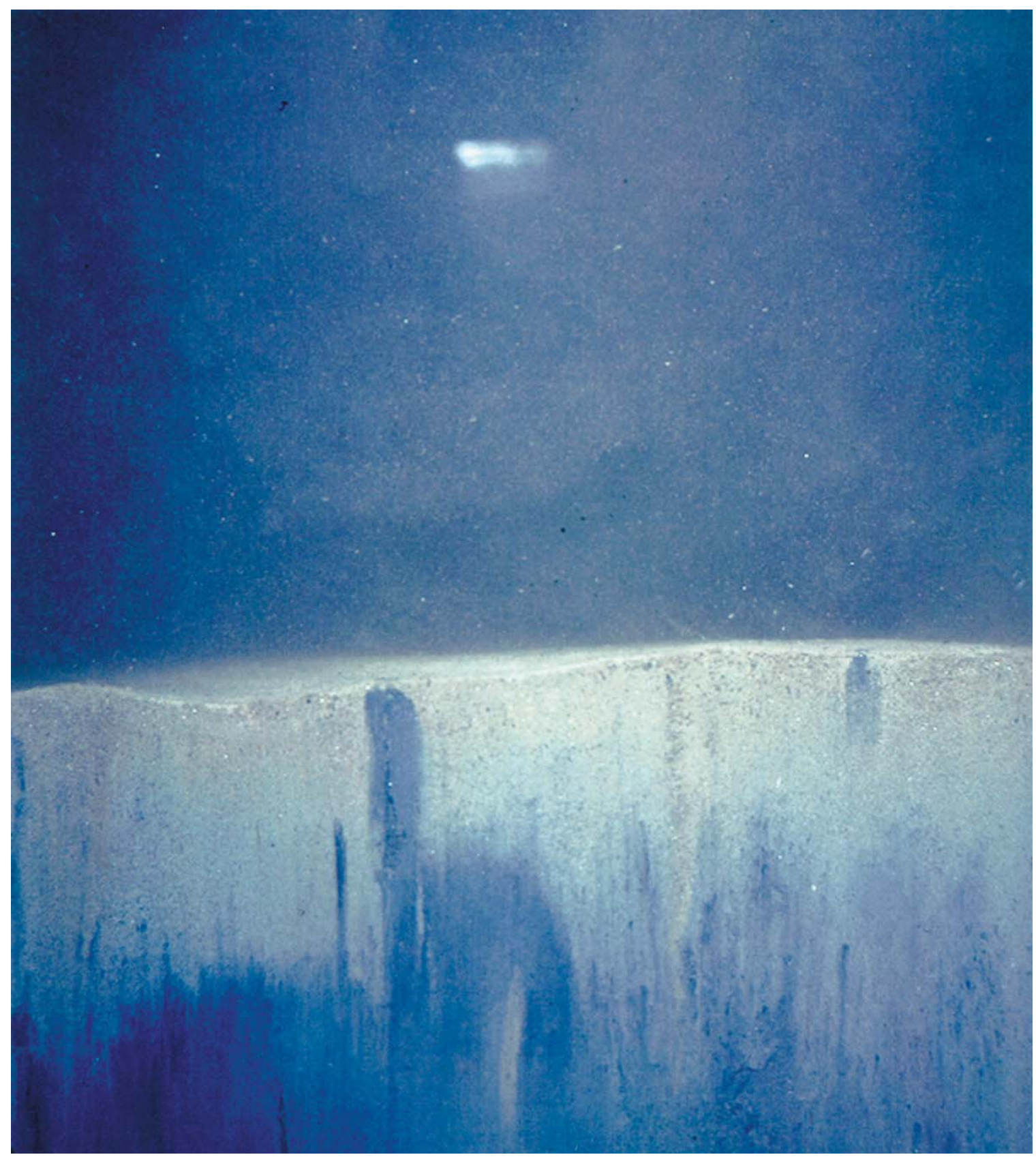

Figure C29. SPI image of Upper Study Site Station US-03 


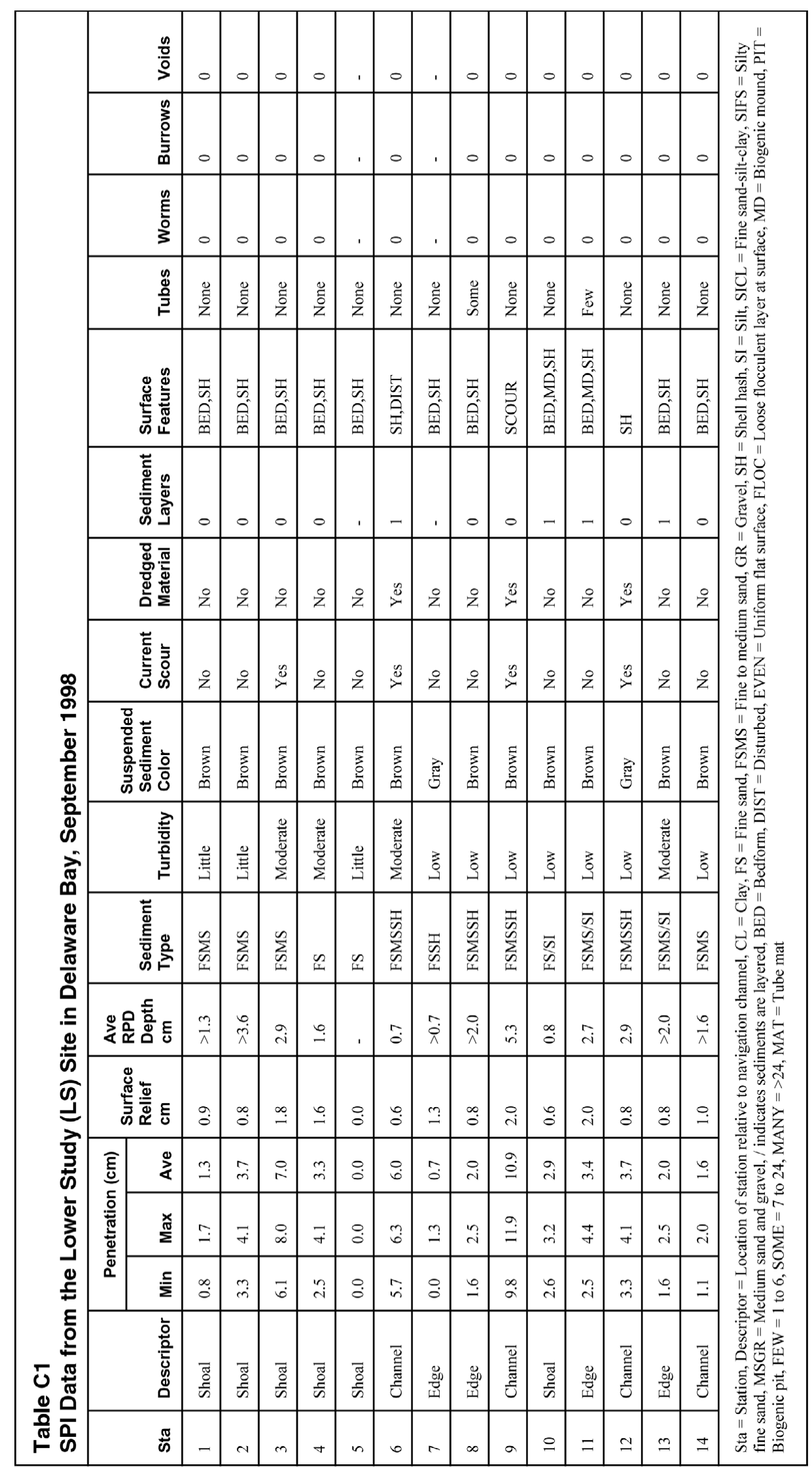




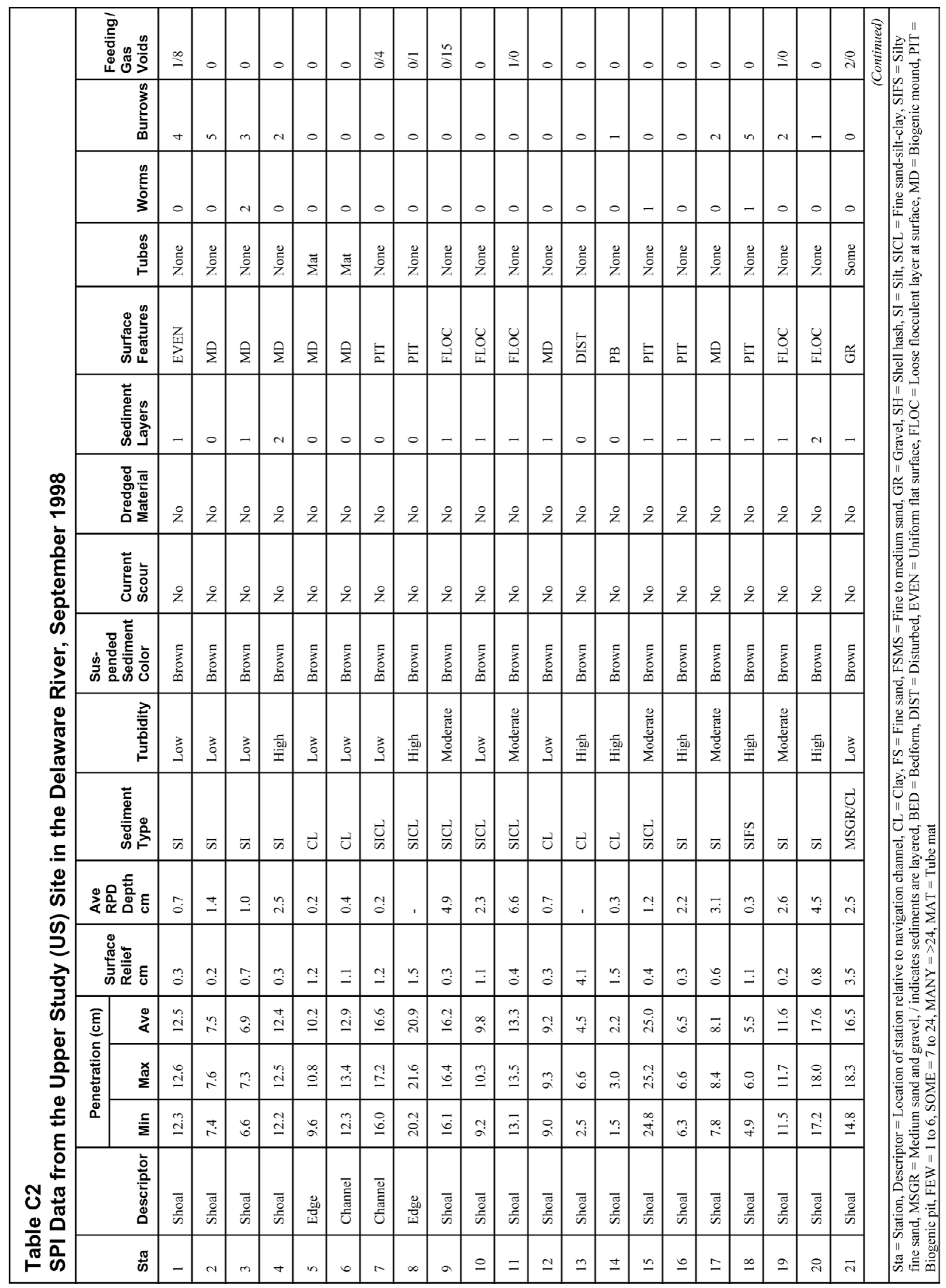




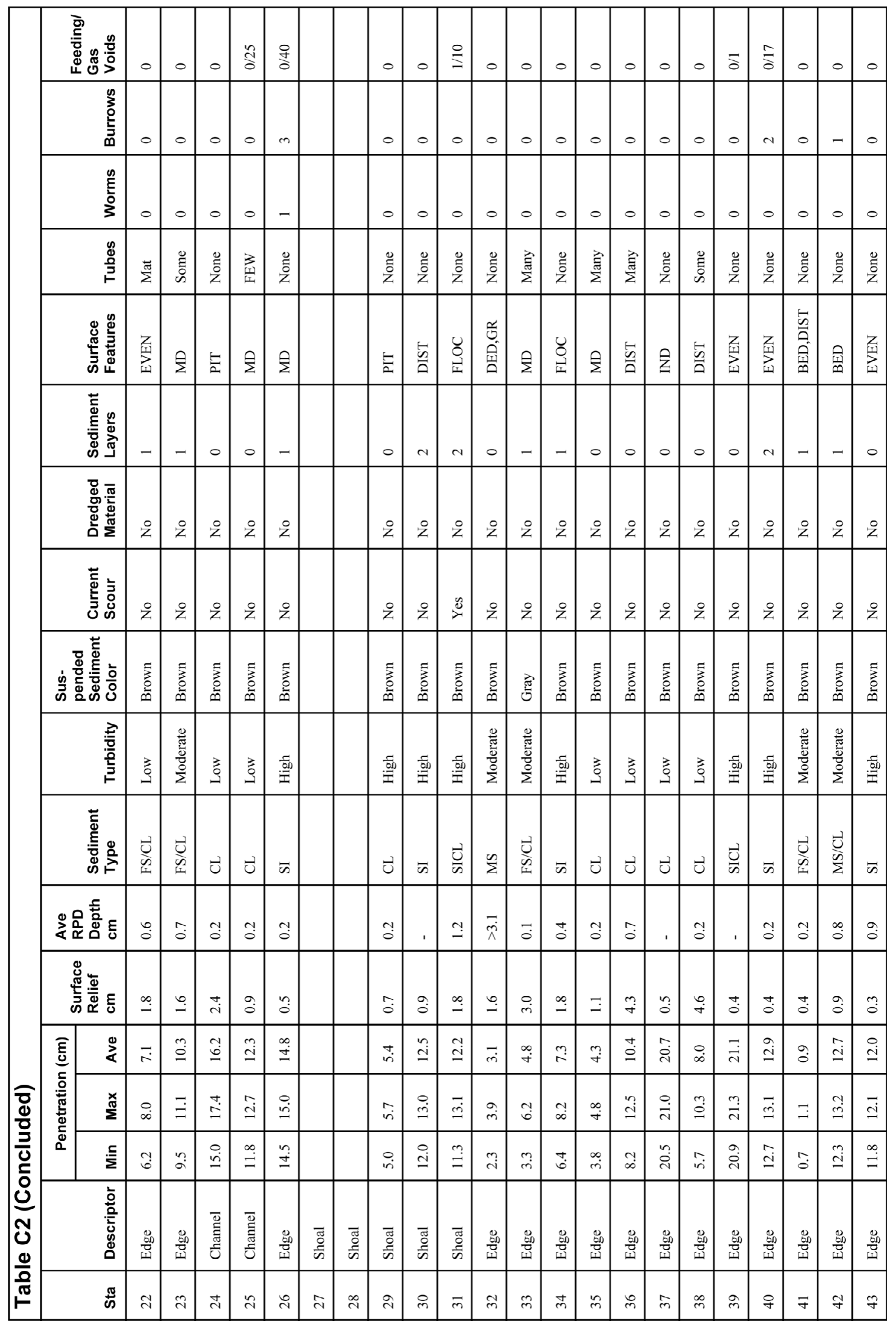




\begin{tabular}{|c|c|c|}
\hline \multicolumn{3}{|c|}{$\begin{array}{l}\text { Table C3 } \\
\text { General Comparison of Sediment Profile Image Data from the Lower Study Site (LS, } \\
\text { Delaware Bay) and Upper Study Site (US, Delaware River) Sampled During Hopper } \\
\text { Dredge Loading and Overflow Tests }\end{array}$} \\
\hline \multirow[b]{2}{*}{ Feature } & \multicolumn{2}{|r|}{ Location } \\
\hline & Lower Study Site & Upper Study Site \\
\hline Sediments & Homogeneous, Sands & Heterogeneous, Mainly Clays and Silt-Clays \\
\hline Sediment Layering & Sediment Grain Size Changes & Color and Sediment Grain Size Changes \\
\hline Prism Penetration & Shallow & Deep \\
\hline Surface Relief & Physical Bed Forms & Biogenic Pits and Mounds \\
\hline Suspended Material & Mostly Background Sediments & Mostly Background Sediments \\
\hline Dredged Material & Detected at 3 Stations & Not Detected \\
\hline Hopper Overflow & Detected at 1 Station & Detected at 1 Station \\
\hline Hydrocarbon Contamination & Not Detected & Detected at 1 Station \\
\hline Epifauna & Not Detected & Not Detected \\
\hline Amphipod or Worm Tubes & Scarce & Common \\
\hline Infauna & Not Detected & Common \\
\hline
\end{tabular}




\section{Appendix D Summary of Technical Findings: 96-hr Bioassay with Mysidopsis bahia and Menidia beryllina}


MEMORANDUM FOR: Mr. Jerry Miller, (CEWES-EE-A)

Thru:

Dr. Todd Bridges, (CEWES-ES-F)

SUBJECT: Narrative Summary of Technical Findings of a 96-hr Bioassay with Delaware River Sediment and Water.

1. Please find enclosed a letter report summarizing the results of bioassays conducted with Mysidopsis bahia and Menidia beryllina exposed to concentrations of filtered elutriate.

2. If you have any questions please call me at (601) 634-4027 or Dr. Todd Bridges at (601) 6343626.

\section{ALFREDA GIBSON \\ Research Biologist \\ CEWES-ES-F}




\section{Summary of Technical Findings: 96-hr bioassay with Mysidopsis bahia and Menidia beryllina}

1. Background: As part of an effort to determine the possible biological effects of water column exposure to Delaware River sediment, Mr. Jerry Miller (EED) requested that the Aquatic Biological Effects Team (ABET) conduct acute 96-hr elutriate bioassays on the material with survival being the observed endpoint. The two species used were Mysidopsis bahia and Menidia beryllina. This report summarizes the results of that study.

2. Technical Approach: 96-hr elutriate bioassays using the mysid shrimp Mysidopsis bahia and the inland silverside Menidia beryllina were conducted according to methods described in the CE/EPA Inland Testing Manual (1998) (Tables D1 and D2). Four treatments were evaluated: 1) Mysidopsis bahia exposed in R1-HO-TOX (coarse-grained material at 30 o/oo) (Table D3); Mysidopsis bahia exposed to R2-HO-TOX (finegrained material at $6 \mathrm{o} / \mathrm{oo}$ ) (Table D4); Menidia beryllina exposed to R2-HO-TOX (fine-grained material at 6 o/oo) (Table D5); and Menidia beryllina exposed to R1-HO-TOX (coarse-grained material at $30 \mathrm{o} / \mathrm{oo}$ ) (Table D6). The filtered elutriate was diluted with our standard laboratory control water 40 fathoms $(6 \mathrm{o} / \mathrm{oo}$ and $30 \mathrm{o} / \mathrm{oo})$ to yield the following concentrations: $0 ; 6.25 ; 12.5 ; 25 ; 50$; and $100 \%$ elutriate. Each treatment was replicated five times. The test was conducted using Mysidopsis bahia that were 5 days old and Menidia beryllina that were 9 days old. Mysidopsis bahia were fed newly hatched brine shrimp daily $(0.2 \mathrm{mg})$ and Menidia beryllina were fed newly hatched brine shrimp on day 2 of the test $(0.2 \mathrm{mg})$. Each beaker was provided trickle-flow aeration and covered with a watch glass to minimize evaporation.

3. Results: 96-hr survival of Mysidopsis bahia in the R1-HO-TOX (30 o/oo, coarse-grained material) exposures survival ranged from 100 to $88 \%$ (Table D1). Survival in R2-HO-TOX (6 o/oo fine-grained material) ranged from 90 to $0 \%$ with $0 \%$ survival in the 50 and $100 \%$ elutriate treatments (Table D1). 96-hr survival of Menidia beryllina in R1-HO-TOX (30 o/oo coarse-grained material) survival ranged from $88 \%-68 \%$. Survival in R2-HO-TOX (6 o/oo fine-grained material) with ranged from 98 to $0 \%$ with $4 \%-0 \%$ survival in the 50 and $100 \%$ exposures (Table D2). The trimmed spearman-karber method was used to calculate $\mathrm{LC}_{50}$ values (Hamilton et al. 1978). Mysidopsis bahia in R2-HO-TOX (6 o/oo) had an $\mathrm{LC}_{50}$ value of $30.04 \%$ (23.44 - 38.50 lower - upper confidence limit). Menidia beryllina in $\mathrm{R} 2-\mathrm{HO}-\mathrm{TOX}(6 \mathrm{o} / \mathrm{oo})$ had an $\mathrm{LC}_{50}$ value of $31.66 \%$ (27.54 - 36.40 lower -upper confidence limits). An $\mathrm{LC}_{50}$ value could not be calculated for Mysidopsis bahia or Menidia beryllina in R1-HO-TOX treatments because neither had mortality values greater than $50 \%$.

Survival met or exceeded the test acceptability criterion of $90 \%$ in the $6 \mathrm{o} / \mathrm{oo}$ and $30 \mathrm{o} / \mathrm{oo}$ Mysidopsis bahia controls, and also in the 6 o/oo Menidia beryllina control. Survival in the $30 \mathrm{o} / \mathrm{oo}$ Menidia beryllina control 
was slightly below the criterion at $88 \%$ but is not considered to render the test invalid.

Water quality data are presented in Tables D7 through D10. The $\mathrm{pH}$, dissolved oxygen, and temperature levels were within an acceptable range for conducting toxicity studies with the two test species. Ammonia levels (NH3) were exceedingly higher than the $\mathrm{LC}_{50}$ of $1.00 \mathrm{mg} / \mathrm{L}$ for 5 -day old Mysidopsis bahia or the $\mathrm{LC}_{50}$ of $1.24 \mathrm{mg} / \mathrm{L}$ for 9-days old Menidia beryllina (USEPA 1989).

In conclusion, R1-HO-TOX exposures did not adversely affect survival of either test species, whereas the mortality observed in R2-HO-TOX at 6 o/oo with both species can be attributed to the high level of $\mathrm{NH}_{3}$.

\section{References:}

Hamilton, M.A., Russo, R.C., and Thurston, R.V. (1978). "Trimmed Spearman-Karber Method for estimating median lethal concentration in toxicity bioassays," Environ. Sci. Tech. 12(4): 417.

USEPA. (1989). "Ambient water quality criteria for ammonia (Saltwater)-1989," Office of Water Regulations and Standards, Criteria and Standards Division, EPA 440/5-88-004, Washington, DC.

USEPA and USACE. (1998). "Evaluation of dredged material proposed for discharge in waters of the U.S. -Testing manual," EPA-823-B-98-004, Washington, DC. 


\section{Table D1}

Summary Survival Data for Mysidopsis bahia Exposed to Delaware River Elutriates

\begin{tabular}{|l|l|l|}
\hline & Elutriate Concentration & $\begin{array}{l}\text { Mean Percent Survival, } \\
\text { standard deviation }\end{array}$ \\
\hline R1-HO-TOX (30 o/oo) & 0 & $100(0.00)$ \\
\hline & 6 & $88(0.84)$ \\
\hline & 12 & $96(0.55)$ \\
\hline & 25 & $92(0.84)$ \\
\hline & 50 & $90(1.22)$ \\
\hline R2-HO-TOX (6 o/Oo) & 100 & $92(0.45)$ \\
\hline & 0 & $90(0.00)$ \\
\hline & 6 & $76(1.67)$ \\
\hline & 12 & $66(2.30)$ \\
\hline & 25 & $82(1.30)$ \\
\hline & 50 & $0(0.00)$ \\
\hline & 100 & $0(0.00)$ \\
\hline
\end{tabular}

\section{Table D2}

Summary Survival Data for Menidia beryllina Exposed to Delaware River Elutriates

\begin{tabular}{|c|c|c|}
\hline Treatment & Elutriate Concentration & $\begin{array}{l}\text { Mean Percent Survival, } \\
\text { standard deviation }\end{array}$ \\
\hline \multirow[t]{6}{*}{ R1-HO-TOX (30 o/oo) } & 0 & $88(0.84)$ \\
\hline & 6 & $70(1.58)$ \\
\hline & 12 & $68(1.64)$ \\
\hline & 25 & $78(1.79)$ \\
\hline & 50 & $80(1.22)$ \\
\hline & 100 & $74(1.82)$ \\
\hline \multirow[t]{6}{*}{ R2-HO-TOX (6 o/oo) } & 0 & $90(0.71)$ \\
\hline & 6 & $68(0.45)$ \\
\hline & 12 & $98(0.45)$ \\
\hline & 25 & $78(1.64)$ \\
\hline & 50 & $4(0.89)$ \\
\hline & 100 & $0(0.00)$ \\
\hline
\end{tabular}


Table D3

96-hr Survival Data for Mysidopsis bahia Exposed to R1-HO-TOX (coarsegrained material) Elutriates at $\mathbf{3 0}$ o/oo

\begin{tabular}{|c|c|c|}
\hline Treatment, \% & Replicate & Total Number Alive \\
\hline Control & 1 & 10 \\
\hline Control & 2 & 10 \\
\hline Control & 3 & 10 \\
\hline Control & 4 & 10 \\
\hline Control & 5 & 10 \\
\hline 6 & 1 & 8 \\
\hline 6 & 2 & 10 \\
\hline 6 & 3 & 9 \\
\hline 6 & 4 & 9 \\
\hline 6 & 5 & 8 \\
\hline 12 & 1 & 10 \\
\hline 12 & 2 & 9 \\
\hline 12 & 3 & 9 \\
\hline 12 & 4 & 10 \\
\hline 12 & 5 & 10 \\
\hline 25 & 1 & 9 \\
\hline 25 & 2 & 9 \\
\hline 25 & 3 & 8 \\
\hline 25 & 4 & 10 \\
\hline 25 & 5 & 10 \\
\hline 50 & 1 & 9 \\
\hline 50 & 2 & 9 \\
\hline 50 & 3 & 10 \\
\hline 50 & 4 & 7 \\
\hline 50 & 5 & 10 \\
\hline 100 & 1 & 9 \\
\hline 100 & 2 & 10 \\
\hline 100 & 3 & 9 \\
\hline 100 & 4 & 9 \\
\hline 100 & 5 & 9 \\
\hline
\end{tabular}


Table D4

96-hr Survival Data for Mysidopsis bahia Exposed to R2-HO-TOX (fine-grained material) Elutriates at 6 o/oo

\begin{tabular}{|c|c|c|}
\hline Treatment, \% & Replicate & Total Number Alive \\
\hline Control & 1 & 9 \\
\hline Control & 2 & 9 \\
\hline Control & 3 & 9 \\
\hline Control & 4 & 9 \\
\hline Control & 5 & 9 \\
\hline 6 & 1 & 8 \\
\hline 6 & 2 & 9 \\
\hline 6 & 3 & 7 \\
\hline 6 & 4 & 9 \\
\hline 6 & 5 & 5 \\
\hline 12 & 1 & 8 \\
\hline 12 & 2 & 6 \\
\hline 12 & 3 & 9 \\
\hline 12 & 4 & 7 \\
\hline 12 & 5 & 3 \\
\hline 25 & 1 & 7 \\
\hline 25 & 2 & 7 \\
\hline 25 & 3 & 9 \\
\hline 25 & 4 & 8 \\
\hline 25 & 5 & 10 \\
\hline 50 & 1 & 0 \\
\hline 50 & 2 & 0 \\
\hline 50 & 3 & 0 \\
\hline 50 & 4 & 0 \\
\hline 50 & 5 & 0 \\
\hline 100 & 1 & 0 \\
\hline 100 & 2 & 0 \\
\hline 100 & 3 & 0 \\
\hline 100 & 4 & 0 \\
\hline 100 & 5 & 0 \\
\hline
\end{tabular}




\section{Table D5}

96-hr Survival Data for Menidia beryllina Exposed to R2-HO-TOX

(fine-grained material) Elutriates at 6 o/oo

\begin{tabular}{|c|c|c|}
\hline Treatment, \% & Replicate & Total Number Alive \\
\hline Control & 1 & 9 \\
\hline Control & 2 & 9 \\
\hline Control & 3 & 9 \\
\hline Control & 4 & 8 \\
\hline Control & 5 & 10 \\
\hline 6 & 1 & 7 \\
\hline 6 & 2 & 7 \\
\hline 6 & 3 & 7 \\
\hline 6 & 4 & 6 \\
\hline 6 & 5 & 7 \\
\hline 12 & 1 & 10 \\
\hline 12 & 2 & 10 \\
\hline 12 & 3 & 9 \\
\hline 12 & 4 & 10 \\
\hline 12 & 5 & 10 \\
\hline 25 & 1 & 10 \\
\hline 25 & 2 & 7 \\
\hline 25 & 3 & 9 \\
\hline 25 & 4 & 7 \\
\hline 25 & 5 & 6 \\
\hline 50 & 1 & 0 \\
\hline 50 & 2 & 0 \\
\hline 50 & 3 & 0 \\
\hline 50 & 4 & 0 \\
\hline 50 & 5 & 2 \\
\hline 100 & 1 & 0 \\
\hline 100 & 2 & 0 \\
\hline 100 & 3 & 0 \\
\hline 100 & 4 & 0 \\
\hline 100 & 5 & 0 \\
\hline
\end{tabular}




\begin{tabular}{|c|c|c|}
\hline \multicolumn{3}{|c|}{$\begin{array}{l}\text { Table D6 } \\
\text { 96-hr Survival Data for Menidia beryllina Exposed to Elutriates } \\
\text { Made from R1-HO-TOX (coarse-grained material) at } 30 \text { o/oo }\end{array}$} \\
\hline Treatment, \% & Replicate & Total Number Alive \\
\hline Control & 1 & 9 \\
\hline Control & 2 & 8 \\
\hline Control & 3 & 10 \\
\hline Control & 4 & 8 \\
\hline Control & 5 & 9 \\
\hline 6 & 1 & 6 \\
\hline 6 & 2 & 9 \\
\hline 6 & 3 & 8 \\
\hline 6 & 4 & 5 \\
\hline 6 & 5 & 7 \\
\hline 12 & 1 & 8 \\
\hline 12 & 2 & 7 \\
\hline 12 & 3 & 8 \\
\hline 12 & 4 & 4 \\
\hline 12 & 5 & 7 \\
\hline 25 & 1 & 9 \\
\hline 25 & 2 & 10 \\
\hline 25 & 3 & 8 \\
\hline 25 & 4 & 6 \\
\hline 25 & 5 & 6 \\
\hline 50 & 1 & 8 \\
\hline 50 & 2 & 10 \\
\hline 50 & 3 & 7 \\
\hline 50 & 4 & 7 \\
\hline 50 & 5 & 8 \\
\hline 100 & 1 & 7 \\
\hline 100 & 2 & 7 \\
\hline 100 & 3 & 10 \\
\hline 100 & 4 & 8 \\
\hline 100 & 5 & 5 \\
\hline
\end{tabular}




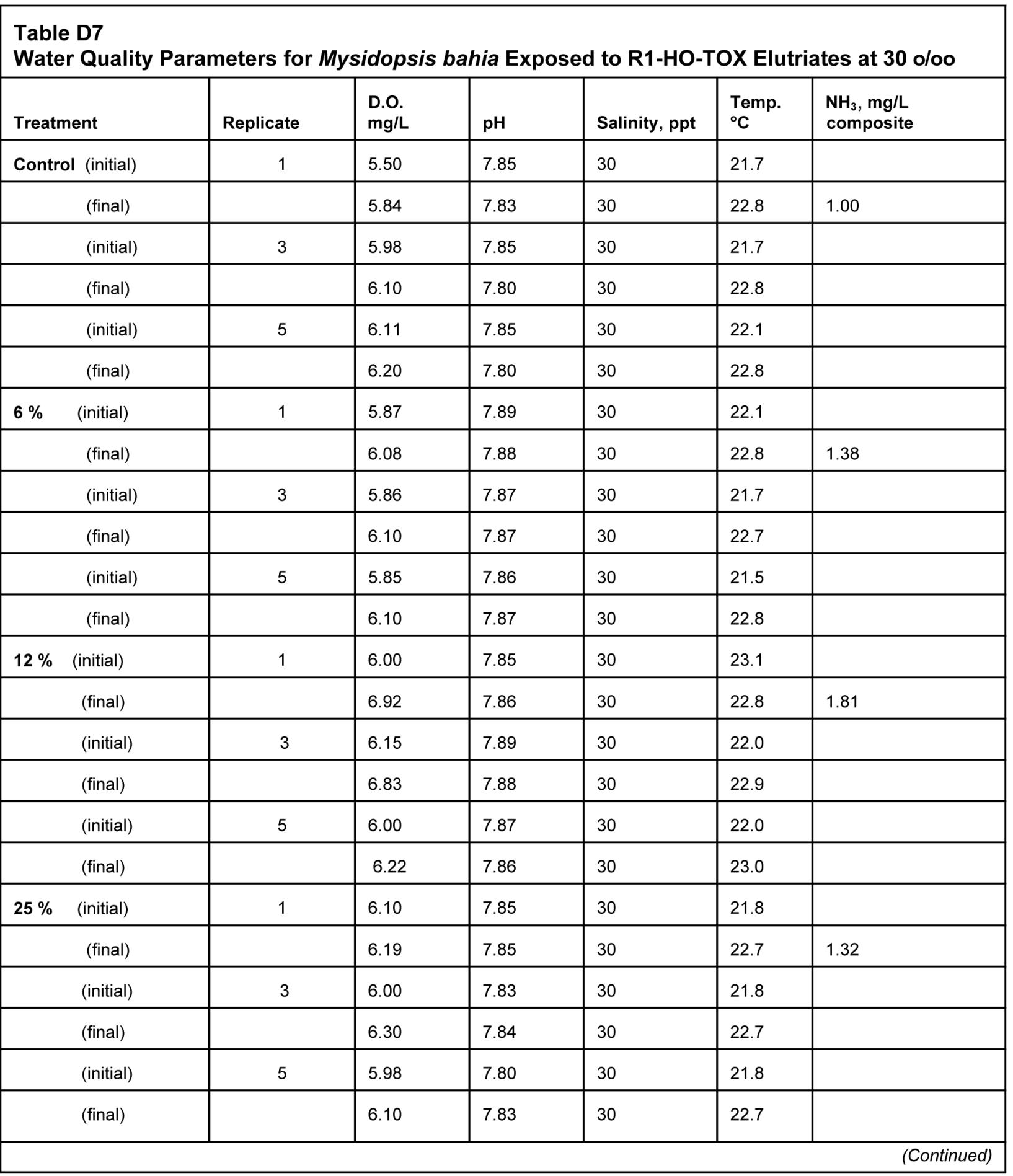




\begin{tabular}{|c|c|c|c|c|c|c|}
\hline Treatment & Replicate & $\begin{array}{l}\text { D.O. } \\
\mathrm{mg} / \mathrm{L}\end{array}$ & $\mathrm{pH}$ & Salinity, ppt & $\begin{array}{l}\text { Temp. } \\
{ }^{\circ} \mathrm{C}\end{array}$ & $\begin{array}{l}\mathrm{NH}_{3}, \mathrm{mg} / \mathrm{L} \\
\text { composite }\end{array}$ \\
\hline $\mathbf{5 0} \% \quad$ (initial) & 1 & 6.10 & 7.80 & 30 & 21.7 & \\
\hline (final) & & 6.20 & 7.80 & 30 & 22.7 & 0.67 \\
\hline (initial) & 3 & 5.95 & 7.82 & 30 & 21.7 & \\
\hline (final) & & 5.99 & 7.81 & 30 & 22.0 & \\
\hline (initial) & 5 & 5.97 & 7.70 & 30 & 21.7 & \\
\hline (final) & & 6.10 & 7.79 & 30 & 22.0 & \\
\hline $100 \% \quad$ (initial) & 1 & 5.97 & 7.65 & 28 & 21.7 & \\
\hline (final) & & 6.10 & 7.70 & 28 & 22.0 & 1.53 \\
\hline (initial) & 3 & 5.96 & 7.69 & 28 & 22.7 & \\
\hline (final) & & 6.10 & 7.70 & 28 & 22.8 & \\
\hline (initial) & 5 & 5.94 & 7.64 & 28 & 22.7 & \\
\hline (final) & & 6.05 & 7.69 & 28 & 22.8 & \\
\hline
\end{tabular}




\begin{tabular}{|c|c|c|c|c|c|c|}
\hline Treatment & Replicate & $\begin{array}{l}\text { D.O. } \\
\mathrm{mg} / \mathrm{L}\end{array}$ & $\mathrm{pH}$ & Salinity, ppt & $\begin{array}{l}\text { Temp. } \\
{ }^{\circ} \mathrm{C}\end{array}$ & $\begin{array}{l}\mathrm{NH}_{3}, \mathrm{mg} / \mathrm{L} \\
\text { composite }\end{array}$ \\
\hline Control (initial) & 1 & 6.10 & 7.34 & 6 & 22.0 & \\
\hline (final) & & 5.98 & 7.80 & 6 & 23.0 & 1.20 \\
\hline (initial) & 3 & 6.08 & 7.29 & 6 & 22.0 & \\
\hline (final) & & 6.00 & 7.70 & 6 & 23.1 & \\
\hline (initial) & 5 & 6.06 & 7.30 & 6 & 22.0 & \\
\hline (final) & & 6.00 & 7.77 & 6 & 23.0 & \\
\hline $6 \% \quad$ (initial) & 1 & 6.13 & 7.50 & 6 & 21.7 & \\
\hline (final) & & 5.35 & 7.83 & 6 & 23.0 & 3.63 \\
\hline (initial) & 3 & 6.13 & 7.55 & 6 & 21.7 & \\
\hline (final) & & 5.29 & 7.84 & 6 & 23.0 & \\
\hline (initial) & 5 & 6.19 & 7.55 & 6 & 21.7 & \\
\hline (final) & & 5.30 & 7.82 & 6 & 23.0 & \\
\hline $12 \% \quad$ (initial) & 1 & 6.21 & 7.53 & 6 & 21.6 & \\
\hline (final) & & 5.20 & 7.96 & 6 & 23.1 & 5.04 \\
\hline (initial) & 3 & 6.20 & 7.55 & 6 & 21.6 & \\
\hline (final) & & 5.30 & 7.97 & 6 & 23.0 & \\
\hline (initial) & 5 & 6.21 & 7.57 & 6 & 21.6 & \\
\hline (final) & & 5.75 & 7.97 & 6 & 23.0 & \\
\hline $25 \% \quad$ (initial) & 1 & 6.11 & 7.62 & 6 & 21.6 & \\
\hline (final) & & 5.30 & 8.10 & 6 & 23.0 & 7.33 \\
\hline (initial) & 3 & 6.10 & 7.60 & 6 & 21.6 & \\
\hline (final) & & 5.29 & 8.09 & 6 & 23.0 & \\
\hline (initial) & 5 & 6.10 & 7.64 & 6 & 21.6 & \\
\hline (final) & & 5.30 & 8.13 & 6 & 23.1 & \\
\hline
\end{tabular}




\begin{tabular}{|c|c|l|l|l|l|l|}
\hline \multicolumn{2}{|l|}{ Table D8 (Concluded) } \\
\hline Treatment & Replicate & $\begin{array}{l}\text { D.o. } \\
\mathbf{m g} / \mathbf{L}\end{array}$ & $\mathbf{p H}$ & $\begin{array}{l}\text { Salinity } \\
\text { ppt }\end{array}$ & $\begin{array}{l}\text { Temp. } \\
{ }^{\circ} \mathbf{C}\end{array}$ & $\begin{array}{l}\mathbf{N H}_{3}, \mathbf{m g} / \mathbf{L} \\
\text { composite }\end{array}$ \\
\hline $\mathbf{5 0} \% \quad$ (initial) & 1 & 6.00 & 7.65 & 5 & 21.7 & \\
\hline (final) & & 5.20 & 8.13 & 5 & 23.1 & 12.4 \\
\hline (initial) & 3 & 6.05 & 7.66 & 5 & 21.7 & \\
\hline (final) & & 5.40 & 8.15 & 5 & 23.0 & \\
\hline (initial) & 5 & 6.00 & 7.60 & 5 & 21.7 & \\
\hline (final) & & 5.30 & 8.16 & 5 & 23.0 & \\
\hline $\mathbf{1 0 0 \%}$ (initial) & 1 & 5.35 & 7.60 & 6 & 22.0 & \\
\hline (final) & & 5.50 & 8.20 & 6 & 23.0 & 21.2 \\
\hline (initial) & 3 & 5.45 & 7.67 & 6 & 22.0 & \\
\hline (final) & & 5.39 & 8.17 & 6 & 23.0 & \\
\hline (initial) & 5 & 5.39 & 7.67 & 6 & 21.9 & \\
\hline (final) & & 5.40 & 8.17 & 6 & 23.1 & \\
\hline
\end{tabular}




\begin{tabular}{|c|c|c|c|c|c|c|}
\hline \multicolumn{7}{|c|}{$\begin{array}{l}\text { Table D9 } \\
\text { Water Quality Parameters for Menidia beryllina Exposed to R1-HO-TOX Elutriates } \\
\text { at } 30 \text { o/00 }\end{array}$} \\
\hline Treatment & Replicate & $\begin{array}{l}\text { D.O. } \\
\mathrm{mg} / \mathrm{L}\end{array}$ & $\mathrm{pH}$ & $\begin{array}{l}\text { Salinity } \\
\text { ppt }\end{array}$ & $\begin{array}{l}\text { Temp. } \\
{ }^{\circ} \mathrm{C}\end{array}$ & $\begin{array}{l}\mathrm{NH}_{3}, \mathrm{mg} / \mathrm{L} \\
\text { composite }\end{array}$ \\
\hline Control (initial) & 1 & 5.45 & 7.83 & 30 & 21.7 & \\
\hline (final) & & 7.10 & 7.73 & 30 & 23.1 & 1.05 \\
\hline (initial) & 3 & 5.98 & 7.84 & 30 & 21.7 & \\
\hline (final) & & 7.06 & 7.67 & 30 & 23.1 & \\
\hline (initial) & 5 & 6.17 & 7.85 & 30 & 21.7 & \\
\hline (final) & & 7.08 & 7.74 & 30 & 23.0 & \\
\hline $6 \% \quad$ (initial) & 1 & 5.85 & 7.85 & 30 & 21.7 & 1.22 \\
\hline (final) & & 7.23 & 7.86 & 30 & 23.1 & \\
\hline (initial) & 3 & 5.88 & 7.85 & 30 & 21.5 & \\
\hline (final) & & 7.20 & 7.84 & 30 & 23.1 & \\
\hline (initial) & 5 & 5.93 & 7.86 & 30 & 21.6 & \\
\hline (final) & & 7.23 & 7.86 & 30 & 23.1 & \\
\hline $12 \% \quad$ (initial) & 1 & 5.98 & 7.85 & 30 & 22.0 & \\
\hline (final) & & 7.32 & 7.87 & 30 & 23.1 & 1.36 \\
\hline (initial) & 3 & 6.13 & 7.85 & 30 & 21.9 & \\
\hline (final) & & 6.95 & 7.88 & 30 & 23.1 & \\
\hline (initial) & 5 & 5.89 & 7.85 & 30 & 21.9 & \\
\hline (final) & & 6.65 & 7.87 & 30 & 23.1 & \\
\hline $25 \% \quad$ (initial) & 1 & 6.03 & 7.85 & 30 & 21.8 & \\
\hline (final) & & 6.07 & 7.91 & 30 & 23.1 & 1.27 \\
\hline (initial) & 3 & 5.95 & 7.83 & 30 & 21.8 & \\
\hline (final) & & 6.25 & 7.91 & 30 & 23.0 & \\
\hline (initial) & 5 & 6.02 & 7.80 & 30 & 21.8 & \\
\hline (final) & & 6.03 & 7.88 & 30 & 23.1 & \\
\hline
\end{tabular}




\begin{tabular}{|c|c|l|l|l|l|l|}
\hline \multicolumn{2}{|l|}{ Table D9 (Concluded) } \\
\hline Treatment & Replicate & $\begin{array}{l}\text { D.O. } \\
\mathbf{m g} / \mathbf{L}\end{array}$ & $\mathbf{p H}$ & $\begin{array}{l}\text { Salinity } \\
\mathbf{p p t}\end{array}$ & $\begin{array}{l}\text { Temp. } \\
{ }^{\circ} \mathbf{C}\end{array}$ & $\begin{array}{l}\mathbf{N H}_{3}, \mathbf{m g} / \mathbf{L} \\
\text { composite }\end{array}$ \\
\hline $\mathbf{5 0} \% \quad$ (initial) & 1 & 5.59 & 7.70 & 30 & 21.8 & \\
\hline (final) & & 5.88 & 7.93 & 30 & 23.1 & 1.22 \\
\hline (initial) & 3 & 5.95 & 7.80 & 30 & 21.8 & \\
\hline (final) & & 6.11 & 7.92 & 30 & 23.1 & \\
\hline (initial) & 5 & 5.85 & 7.80 & 30 & 21.7 & \\
\hline (final) & & 5.64 & 7.97 & 30 & 23.1 & \\
\hline $\mathbf{1 0 0 \%}$ (initial) & 1 & 5.95 & 7.64 & 30 & 21.7 & \\
\hline (final) & & 5.58 & 7.97 & 30 & 23.1 & 1.45 \\
\hline (initial) & 3 & 5.96 & 7.64 & 30 & 21.7 & \\
\hline (final) & & 5.54 & 7.96 & 30 & 23.1 & \\
\hline (initial) & 5 & 5.93 & 7.63 & 30 & 21.7 & \\
\hline (final) & & 5.69 & 7.95 & 30 & 23.1 & \\
\hline
\end{tabular}




\begin{tabular}{|c|c|c|c|c|c|c|}
\hline \multicolumn{7}{|c|}{$\begin{array}{l}\text { Table D10 } \\
\text { Water Quality Parameters for Menidia beryllina Exposed to R2-HO-TOX Elutriates at } 6 \text { o/o }\end{array}$} \\
\hline Treatment & Replicate & $\begin{array}{l}\text { D.O. } \\
\text { mg/L }\end{array}$ & $\mathrm{pH}$ & $\begin{array}{l}\text { Salinity } \\
\text { ppt }\end{array}$ & $\begin{array}{l}\text { Temp. } \\
{ }^{\circ} \mathrm{C}\end{array}$ & $\begin{array}{l}\mathrm{NH}_{3}, \mathrm{mg} / \mathrm{L} \\
\text { composite }\end{array}$ \\
\hline Control (initial) & 1 & 6.10 & 7.24 & 6 & 22.2 & \\
\hline (final) & & 5.08 & 7.65 & 6 & 23.1 & 1.81 \\
\hline (initial) & 3 & 6.10 & 7.26 & 6 & 22.2 & \\
\hline (final) & & 5.38 & 7.57 & 6 & 23.1 & \\
\hline (initial) & 5 & 6.06 & 7.30 & 6 & 22.2 & \\
\hline (final) & & 5.33 & 7.66 & 6 & 23.1 & \\
\hline $6 \% \quad$ (initial) & 1 & 6.13 & 7.47 & 6 & 21.7 & \\
\hline (final) & & 5.21 & 7.86 & 6 & 23.0 & 4.62 \\
\hline (initial) & 3 & 6.14 & 7.54 & 6 & 21.7 & \\
\hline (final) & & 5.30 & 7.84 & 6 & 23.1 & \\
\hline (initial) & 5 & 6.21 & 7.55 & 6 & 21.7 & \\
\hline (final) & & 5.25 & 7.86 & 6 & 23.1 & \\
\hline $12 \% \quad$ (initial) & 1 & 6.20 & 7.50 & 6 & 21.4 & \\
\hline (final) & & 5.00 & 7.97 & 6 & 23.0 & 6.20 \\
\hline (initial) & 3 & 6.25 & 7.55 & 6 & 21.4 & \\
\hline (final) & & 5.00 & 8.00 & 6 & 23.0 & \\
\hline (initial) & 5 & 6.20 & 7.55 & 6 & 21.4 & \\
\hline (final) & & 5.25 & 7.95 & 6 & 23.0 & \\
\hline $25 \% \quad$ (initial) & 1 & 6.10 & 7.63 & 6 & 21.7 & \\
\hline (final) & & 5.23 & 8.21 & 6 & 23.0 & 5.95 \\
\hline (initial) & 3 & 6.00 & 7.63 & 6 & 21.7 & \\
\hline (final) & & 5.00 & 8.20 & 6 & 23.1 & \\
\hline (initial) & 5 & 6.10 & 7.64 & 6 & 21.7 & \\
\hline (final) & & 5.25 & 8.16 & 6 & 23.1 & \\
\hline
\end{tabular}




\begin{tabular}{|c|c|l|l|l|l|l|}
\hline \multicolumn{2}{|l|}{ Table D10 (Concluded) } \\
\hline Treatment & Replicate & $\begin{array}{l}\text { D.O. } \\
\mathbf{m g} / \mathbf{L}\end{array}$ & $\mathbf{p H}$ & $\begin{array}{l}\text { Salinity } \\
\mathbf{p p t}\end{array}$ & $\begin{array}{l}\text { Temp. } \\
{ }^{\circ} \mathbf{C}\end{array}$ & $\begin{array}{l}\mathbf{N H}_{3}, \mathbf{m g} / \mathbf{L} \\
\text { composite }\end{array}$ \\
\hline $\mathbf{5 0} \% \quad$ (initial) & 1 & 6.00 & 7.65 & 6 & 21.4 & \\
\hline (final) & & 5.10 & 8.43 & 6 & 23.0 & 12.4 \\
\hline (initial) & 3 & 6.00 & 7.66 & 6 & 21.4 & \\
\hline (final) & & 5.01 & 8.44 & 6 & 23.0 & \\
\hline (initial) & 5 & 6.00 & 7.66 & 6 & 21.5 & \\
\hline (final) & & 5.00 & 8.46 & 6 & 23.0 & \\
\hline $\mathbf{1 0 0 \%}$ (initial) & 1 & 5.30 & 7.66 & 6 & 21.5 & \\
\hline (final) & & 5.00 & 8.75 & 6 & 23.1 & 22.3 \\
\hline (initial) & 3 & 5.31 & 7.67 & 6 & 21.5 & \\
\hline (final) & & 5.08 & 8.75 & 6 & 23.0 & \\
\hline (initial) & 5 & 5.23 & 7.67 & 6 & 21.7 & \\
\hline (final) & & 5.01 & 8.71 & 6 & 23.0 & \\
\hline
\end{tabular}




\begin{tabular}{|c|c|c|}
\hline \multicolumn{2}{|c|}{ REPORT DOCUMENTATION PAGE } & $\begin{array}{l}\text { Form Approved } \\
\text { OMB No. 0704-0188 }\end{array}$ \\
\hline \multicolumn{3}{|c|}{ 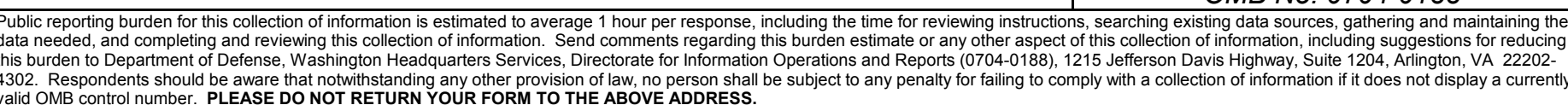 } \\
\hline $\begin{array}{l}\text { 1. REPORT } \\
\text { July } 2002\end{array}$ & $\begin{array}{l}\text { 2. REPORT TYPE } \\
\text { Final report }\end{array}$ & 3. DATES COVERED (From - To) \\
\hline \multirow{3}{*}{\multicolumn{2}{|c|}{$\begin{array}{l}\text { 4. TITLE AND SUBTITLE } \\
\text { Field Evaluation of Hopper Dredge Overflow for the Delaware River }\end{array}$}} & 5a. CONTRACT NUMBER \\
\hline & & 5b. GRANT NUMBER \\
\hline & & 5c. PROGRAM ELEMENT NUMBER \\
\hline \multirow{3}{*}{\multicolumn{2}{|c|}{$\begin{array}{l}\text { 6. AUTHOR(S) } \\
\text { Jerry L. Miller, Michael R. Palermo, Thomas W. Groff }\end{array}$}} & 5d. PROJECT NUMBER \\
\hline & & 5e. TASK NUMBER \\
\hline & & 5f. WORK UNIT NUMBER \\
\hline \multicolumn{2}{|c|}{$\begin{array}{l}\text { 7. PERFORMING ORGANIZATION NAME(S) AND ADDRESS(ES) } \\
\text { U.S. Army Engineer Research and Development Center } \\
\text { Environmental Laboratory } \\
3909 \text { Halls Ferry Road, Vicksburg, MS 39180-6199; } \\
\text { U.S. Army Engineer District, Philadelphia } \\
\text { Operations Division } \\
\text { Wanamaker Building } \\
100 \text { Penn Square East, Philadelphia, PA 19107-3390 }\end{array}$} & $\begin{array}{l}\text { 8. PERFORMING ORGANIZATION REPORT } \\
\text { NUMBER } \\
\text { ERDC/EL TR-02-17 }\end{array}$ \\
\hline \multirow{2}{*}{\multicolumn{2}{|c|}{$\begin{array}{l}\text { 9. SPONSORING / MONITORING AGENCY NAME(S) AND ADDRESS(ES) } \\
\text { U.S. Army Engineer District, Philadelphia } \\
\text { Philadelphia, PA } 19107-3390\end{array}$}} & 10. SPONSOR/MONITOR'S ACRONYM(S) \\
\hline & & $\begin{array}{l}\text { 11. SPONSOR/MONITOR'S REPORT } \\
\text { NUMBER(S) }\end{array}$ \\
\hline \multicolumn{3}{|c|}{$\begin{array}{l}\text { 12. DISTRIBUTION / AVAILABILITY STATEMENT } \\
\text { Approved for public release; distribution is unlimited. }\end{array}$} \\
\hline \multicolumn{3}{|c|}{ 13. SUPPLEMENTARY NOTES } \\
\hline
\end{tabular}

Hopper dredges are often loaded past the point of overflow for economic reasons. As the hopper is filled, dredged material is stored in the hopper until overflow begins. The density of the hopper contents is increased by allowing the low-density supernatant to overflow back into the waterway. As the low-density supernatant overflows, the average density of the hopper contents increases. Thus, more material can be transported per trip to the disposal site or facility resulting in an economical loading.

There is normally a tradeoff between the potential economic benefits and potential environmental effects. Overflow results in increased water column turbidity, and supernatant solids may be redeposited near the dredge site. Also, if sediments are contaminated, the overflow may result in some release of contaminants to the water column. Therefore, the relationship between dredge production, density of the hopper load, and the rate of material overflow are important variables in maximizing the efficiency of the dredging operation while minimizing harmful contaminant release.

(Continued)

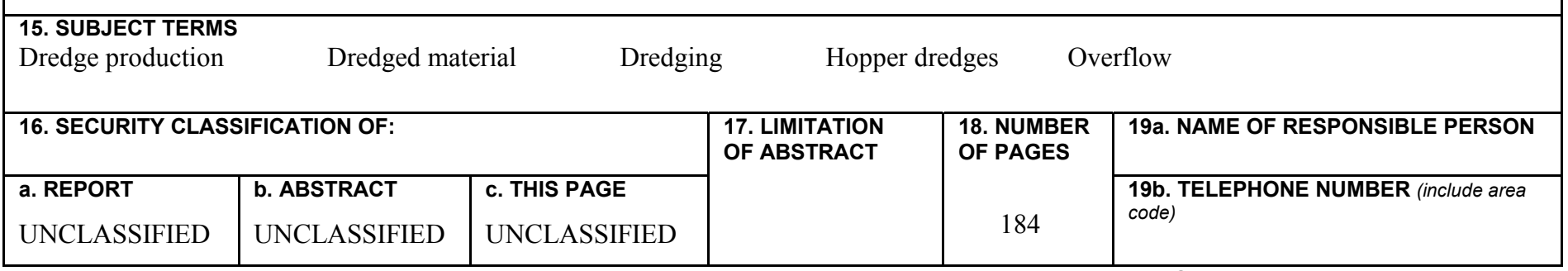




\section{ABSTRACT (Concluded).}

A field study was conducted during hopper dredging operations in the Delaware River and Delaware Bay area to quantify the potential load gains realized by overflow, the degree of suspended solids and contaminant release generated by overflow, and the dispersion of the overflow plume. Monitoring was conducted at two sites, one of predominately fine-grained material in the Delaware River, and the other of predominately coarse-grained material in Delaware Bay. This report summarizes the results of the study and describes the potential economic and environmental considerations for overflow at these sites. 